Alexandra N. Lenz und Albrecht Plewnia (Hrsg.)

Variation - Normen - Identitäten 


\section{Germanistische Sprachwissenschaft um 2020}

Herausgegeben von

Albrecht Plewnia und Andreas Witt

\section{Band 4}




\section{Variation - \\ Normen \\ Identitäten}

Herausgegeben von

Alexandra N. Lenz und Albrecht Plewnia 
Die Open-Access-Publikation dieses Bandes wurde gefördert vom Institut für Deutsche Sprache, Mannheim.

\section{(cc) BY}

Dieses Werk ist lizenziert unter der Creative Commons Attribution 4.0 Lizenz. Weitere Informationen finden Sie unter http://creativecommons.org/licenses/by/4.0/.

\section{Bibliografische Information der Deutschen Nationalbibliothek}

Die Deutsche Nationalbibliothek verzeichnet diese Publikation in der Deutschen Nationalbibliografie; detaillierte bibliografische Daten sind im Internet über http://dnb.dnb.de abrufbar.

(C) 2018 Alexandra N. Lenz und Albrecht Plewnia, publiziert von Walter de Gruyter GmbH, Berlin/Boston

Foto Einbandabbildung: (๑) Oliver Schonefeld, Institut für Deutsche Sprache, Mannheim Portrait Ludwig M. Eichinger, Seite V: ( David Ausserhofer, Leibniz-Gemeinschaft Satz: Meta Systems Publishing \& Printservices GmbH, Wustermark Druck und Bindung: CPI books GmbH, Leck

www.degruyter.com 


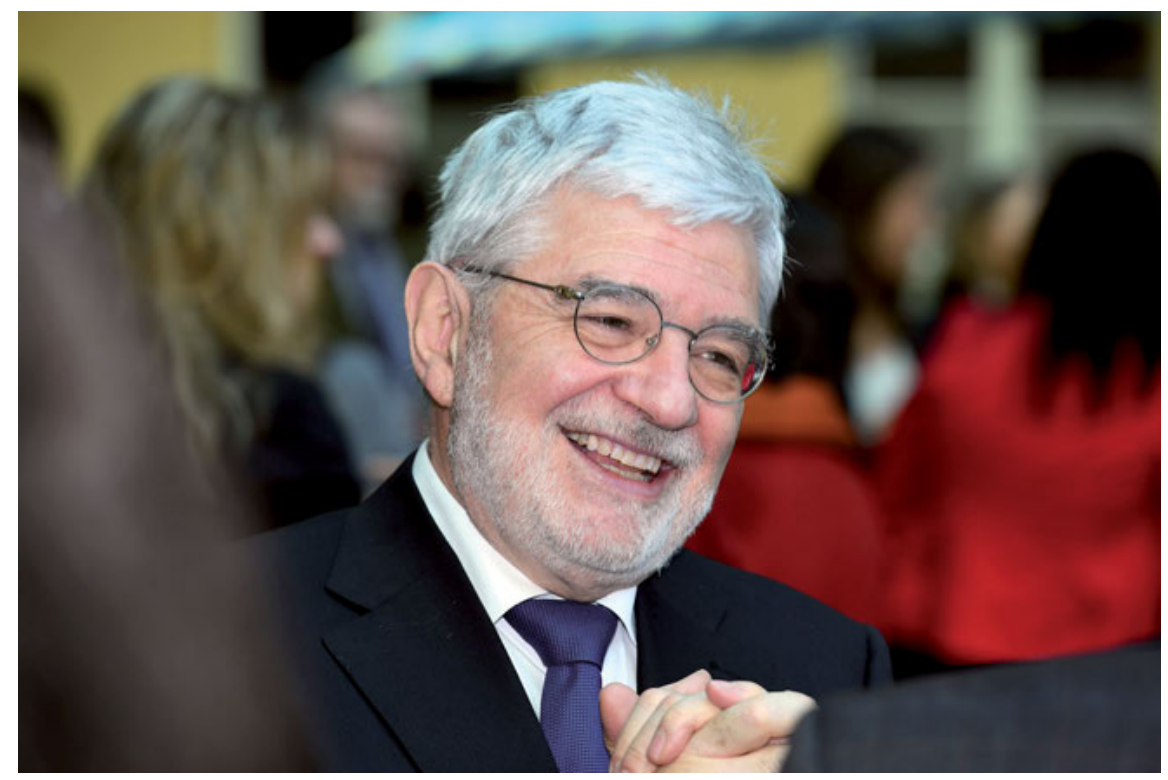

Ludwig M. Eichinger gewidmet 



\section{Vorwort}

Wo steht die germanistische Sprachwissenschaft aktuell? Der vorliegende Band mit dem Titel „Variation - Normen - Identitäten“ ist der vierte Teil einer auf sechs Bände angelegten Reihe, die eine zwar nicht exhaustive, aber doch umfassende Bestandsaufnahme derjenigen Themenfelder innerhalb der germanistischen Linguistik bieten will, die im Kontext der Arbeiten des Instituts für Deutsche Sprache in den letzten Jahren für das Fach von Bedeutung waren und in den kommenden Jahren von Bedeutung sein werden (und von denen nicht wenige auch vom Institut für Deutsche Sprache bedient wurden und werden). Jeder einzelne Band behandelt ein abgeschlossenes Themengebiet und steht insofern für sich; in der Zusammenschau aller Bände ergibt sich ein Panorama der „Germanistischen Sprachwissenschaft um 2020“.

Anlass des Erscheinens dieser Bände ist der Eintritt des langjährigen Direktors des Instituts für Deutsche Sprache, Ludwig M. Eichinger, in den Ruhestand. Ludwig M. Eichinger leitete das Institut von 2002 bis 2018. Seine akademische Laufbahn begann er als Wissenschaftlicher Assistent an der Universität Bayreuth; anschließend war er Heisenberg-Stipendiat an der LudwigMaximilians-Universität München. Ab 1990 hatte er eine Fiebiger-Professur für Deutsche Sprachwissenschaft an der Universität Passau inne, 1997 wurde er auf den Lehrstuhl für Deutsche Philologie an der Christian-Albrechts-Universität zu Kiel berufen. Mit seiner Ernennung zum Direktor des Instituts für Deutsche Sprache im Jahr 2002 wurde er auch Ordinarius für Germanistische Linguistik an der Universität Mannheim. Ludwig M. Eichinger ist Ehrendoktor der Pannonischen Universität Veszprém und der Universität Bukarest. Er ist Mitglied der Akademie der Wissenschaften und der Literatur zu Mainz und der Österreichischen Akademie der Wissenschaften; außerdem ist er Ständiger Gastprofessor an der Beijing Foreign Studies University.

Ludwig M. Eichinger hat das Institut in den Jahren seines Wirkens entscheidend geprägt; in Anerkennung und Dankbarkeit seien ihm diese Bände gewidmet.

Albrecht Plewnia und Andreas Witt

- Reihenherausgeber - 



\section{Inhalt}

Vorwort - VII

Alexandra N. Lenz und Albrecht Plewnia

Einleitung - 1

Peter Auer

1 Das Beste zweier Welten: Das Bild elsässischer Dialektsprecher von den Deutschen, den Franzosen und sich selbst -5

Ingrid Schröder und Lara Neumann

2 „Denn hebbt wi ok mal Platt schnackt.“ Codeswitching

in sprachbiographischen Interviews - 41

Astrid Adler und Albrecht Plewnia

3 Möglichkeiten und Grenzen der quantitativen

Spracheinstellungsforschung - 63

Markus Hundt

4 Wahrnehmungsdialektologie - quo vadis? - 99

Evelyn Ziegler

5 Visuelle Mehrsprachigkeit in Migrationsgesellschaften:

monolinguale Norm vs. plurilinguale Norm -127

Stefan Kleiner und Ralf Knöbl

6 Zur Aussprache nicht haupttoniger Vorsilben mit $\langle\mathrm{e}\rangle$ in Lehnwörtern im deutschen Gebrauchsstandard — 157

Winifred V. Davies

7 Sprachnormen in der Schule aus der Perspektive der „Critical Language Awareness" - 177

Elisabeth Knipf-Komlósi

8 Das Dilemma zwischen Norm und Variation im Deutschunterricht in Ungarn - und wie Lehrende darüber denken - 197 
$\mathbf{X}$ Inhalt

Rüdiger Harnisch

9 Reanalyse durch Varietätenkontakt - Morphogenese durch Hyperkorrektion -219

Claudia Maria Riehl

10 Simplifizierungsprozesse revisited -241

Peter Rosenberg

11 Überflutete Sprachinseln: Sprachvariation, Sprachwechsel und Sprachwandel in deutschen Sprachinseln in Russland und Brasilien — 263

Hans C. Boas und Katrin Fuchs

12 Zum Einfluss des Standarddeutschen auf das Texasdeutsche im 19. und 20. Jahrhundert: Empirische und methodologische Probleme - 283

Péter Maitz und Siegwalt Lindenfelser

13 Gesprochenes Alltagsdeutsch im Bismarck-Archipel um $1900-305$

Register -339

Autorinnen und Autoren - 341 


\section{Alexandra N. Lenz und Albrecht Plewnia Einleitung}

Wer spricht, wählt aus; das Ergebnis ist Variation. Sie ist die sichtbare Folge der Existenz von regulären Optionen im sprachlichen Möglichkeitsraum eines Sprechers. Einer jeden sprachlichen Handlung gehen zahlreiche Auswahlentscheidungen, bewusste und unbewusste (und halbbewusste), voran. Das betrifft die verschiedensten Ebenen, von der Phonologie bis zur Systemgrammatik; auf diesen verschiedenen Ebenen finden dann Auswahlentscheidungen statt, die u. a. auch der sozialen Positionierung der Sprecher dienen. Dabei existiert Variation in verschiedenen Maßstäben: einerseits gibt es überindividuelle Variationsräume, d. h. Unterschiede in den sprachlichen DefaultEntscheidungen zwischen verschiedenen Gruppen, die ihrerseits intern relativ homogen sind; am sichtbarsten wird dies wohl im Bereich der arealen Variation, die für die meisten Sprecher des Deutschen eine sehr unmittelbare Form der alltagssprachlichen Differenzerfahrung darstellt. Andererseits gibt es sie immer auch auf der individuellen Sprecherebene, jeder Sprecher hat die Wahl, und jeder Sprecher kann und muss wählen.

Dieser Band geht der Frage nach, welche linguistischen und soziolinguistischen Steuerungsfaktoren den Auswahlentscheidungen, die zu Variation führen, zugrunde liegen. Zwei Hauptlinien, die zugleich eng miteinander verschränkt sind, werden dabei verfolgt: Zum einen orientieren sich Sprecher bei ihren Entscheidungen immer an Normvorstellungen. Da sprachliches Handeln immer soziales Handeln ist und soziales Handeln sozialen Normen unterliegt, treffen Sprecher ihre Auswahlen entlang bestimmter (statuierter oder subsistenter) Normen. In diesem Sinne ist sprachliche Variation ein Ergebnis von Normenpluralität. Da die (Sprach-)Welt komplex ist, kommt es regelmäßig vor, dass konkurrierende Normsysteme konfligieren; gerade in solchen Fällen muss ein Sprecher sich entscheiden.

Der zweite thematische Faden geht aus von der Feststellung, dass die jeweiligen sprachlichen Auswahlentscheidungen nicht zuletzt zur Konstruktion von Identitäten eingesetzt werden. Sprache ist zentraler Identitätsanker; Identität ermöglicht es, Gruppenzugehörigkeiten wie auch Gruppengrenzen nach außen zu markieren, und dient der sozialen Selbstvergewisserung. Die Optionen, die der sprachliche Handlungsraum eröffnet, bieten hier Spielraum für Selbstpositionierungen und Fremdzuschreibungen, der systematisch genutzt wird.

Diesen beiden Hauptlinien - Normen und Identitäten - folgen die Beiträge dieses Bandes mit unterschiedlichen Schwerpunkten und je eigenen Akzentuierungen. In einem ersten thematischen Block werden Fragestellungen

○ Open Access. () 2018 Alexandra N. Lenz und Albrecht Plewnia, publiziert von De Gruyter. (๔)BY Dieses Werk ist lizenziert unter der Creative Commons Attribution 4.0 Lizenz.

https://doi.org/10.1515/9783110538625-001 
fokussiert, die sich mit Einstellungen von Sprechern zu verschiedenen Varietäten, Sprachformen/-mustern und den mit ihnen assoziierten Sprechergruppen befassen. Peter Auer zeigt in seinem Beitrag „Das Beste zweier Welten: Das Bild elsässischer Dialektsprecher von den Deutschen, den Franzosen und sich selbst“, wie (vermeintliche) Wissensausschnitte um regionalsprachliche Differenzen genutzt werden, um (durchaus entlang nationaler Stereotype) eine spezifische Regionalidentität zu konstruieren, wonach es den Elsässern gelingt, die jeweiligen Vorzüge der deutschen und der französischen Welt miteinander zu vereinen. - Von narrativen Identitätskonstruktionen berichten auch Ingrid Schröder und Lara Neumann in ihrem Beitrag „Denn hebbt wi ok mal Platt schnackt. Codeswitching in sprachbiographischen Interviews“. Sie weisen an Daten aus dem Projekt „Einstellungen gegenüber regionalen Sprachformen in der Großstadt: Niederdeutsch in Hamburg (NiH)“ nach, dass Codeswitching zwischen Hochdeutsch und Niederdeutsch neben der Herstellung von Nähe zu vielerlei weiteren kommunikativen Zwecken genutzt wird. - Eine methodische Perspektive nehmen Astrid Adler und Albrecht Plewnia in ihrem Beitrag „Möglichkeiten und Grenzen der quantitativen Spracheinstellungsforschung“ ein; sie berichten über die verschiedenen Spracheinstellungserhebungen, die in jüngerer Zeit am IDS durchgeführt wurden, und exemplifizieren an ausgewählten Daten verschiedene Auswertungszugänge. - Für linguistische Laien gehört areale Variation zu den prominentesten Varianzphänomenen. Markus Hundt liefert in seinem Beitrag „Wahrnehmungsdialektologie - quo vadis?“ eine Bestandsaufnahme der auf den deutschsprachigen Raum bezogenen Wahrnehmungsdialektologie, diskutiert ihre Methoden und benennt ihre Desiderate. - Eine andere Form sprachlichen Heterogenitätserlebens ermöglicht und erzwingt migrationsinduzierte Mehrsprachigkeit. In ihrem Beitrag „Visuelle Mehrsprachigkeit in Migrationsgesellschaften: monolinguale Norm vs. plurilinguale Norm“ macht Evelyn Ziegler anhand von Daten aus dem Projekt „Metropolenzeichen: Visuelle Mehrsprachigkeit in der Metropole Ruhr“ die verschiedenen Formen der Normorientierung bei der sprachlichen Gestaltung des öffentlichen Raums nachvollziehbar. Ihr Beitrag positioniert sich damit am Übergang der beiden thematischen Hauptstränge des Bandes.

Mit dem Spannungsfeld von regionalen Oralisierungsnormen und der Annahme einer hypostasierten, stark schriftorientierten Standardnorm befasst sich die zweite Gruppe von Beiträgen. Stefan Kleiner und Ralf Knöbl, die ein am Sprachgebrauch orientiertes Standardkonzept verfolgen, zeigen in ihrem Beitrag „Zur Aussprache nicht haupttoniger Vorsilben mit <e > in Lehnwörtern im deutschen Gebrauchsstandard“, dass die Sprachwirklichkeit um einiges komplexer ist, als die traditionellen Aussprachekodizes sie abbilden; neben vor allem phonologisch erklärbarer Variation gibt es hier auch areale Variation, die 
bis in den Gebrauchsstandard hineinreicht. - Ein Feld, in dem die Standardsprache eine hohe soziale Relevanz erfährt, ist die Schule. Winifred V. Davies stellt in ihrem Beitrag „Sprachnormen in der Schule aus der Perspektive der Critical Language Awareness“ die in Schulkontexten übliche starke Orientierung auf einen überregionalen Standard, mit der eine Abwertung arealsprachlicher Varietäten einhergeht, in Frage und plädiert für einen liberaleren und reflektierteren Umgang mit Nichtstandardvarietäten. - Ebenfalls von der Schule, allerdings aus der Perspektive des Unterrichts von Deutsch als Fremdsprache, handelt der Beitrag von Elisabeth Knipf-Komlósi mit dem Titel „Das Dilemma zwischen Norm und Variation im Deutschunterricht in Ungarn und wie Lehrende darüber denken“. Ausgehend von einer empirischen Erhebung unter Deutschlehrkräften diskutiert sie die Schwierigkeiten des Umgangs mit konkurrierenden (einerseits stärker an standardschriftsprachlichen Normen bzw. andererseits mehr an der gesprochenen „Alltagssprache“ ausgerichteten) Zielnormen. - Um die spannungsreichen Beziehungen zwischen Dialekt und Standard geht es auch im Beitrag „Reanalyse durch Varietätenkontakt Morphogenese durch Hyperkorrektion“ von Rüdiger Harnisch, allerdings stehen hier nicht die soziolinguistischen, sondern die systemlinguistischen Aspekte im Vordergrund. Er zeigt, wie die Koexistenz von Dialekt und Standard zu hyperkorrekten, typischerweise durch Analogie gebildeten Formen führt, die von den Sprechern einer rückwirkenden morphologischen Analyse unterzogen werden, wodurch neue Muster und Reihen entstehen können.

Ein auf andere Weise komplexes Verhältnis von Norm und Variation begegnet uns, wenn wir uns Aspekten des Sprachkontakts außerhalb des deutschsprachigen Raums zuwenden. Einerseits sind Sprachkontaktkonstellationen besonders geeignet, die Frage der sprachlichen Identität virulent werden zu lassen. Andererseits sind auch die durch Sprachkontakt bedingte Variation und die aus ihr resultierenden Sprachwandelprozesse von großem Interesse. Die dritte Gruppe von Beiträgen behandelt daher eine Reihe außereuropäischer Sprachkontaktsituationen des Deutschen. Einen grammatischen Fokus wählt Claudia Maria Riehl in ihrem Beitrag „Simplifizierungsprozesse revisited“. Sie untersucht das Phänomen des Dativabbaus an Daten aus dem Barossadeutschen (in Australien) und dem Russlanddeutschen; während das Russlanddeutsche noch über ein einigermaßen intaktes Pronominalsystem verfügt, hat sich im Barossadeutschen ein Zwei-Kasus-System etabliert. - Auch in den deutschen Sprachinseln in Brasilien zeigt sich der Kasusabbau. Peter Rosenberg zieht in seinem Beitrag „Überflutete Sprachinseln: Sprachvariation, Sprachwechsel und Sprachwandel in deutschen Sprachinseln in Russland und Brasilien“ Daten aus dem Süden Brasiliens und aus Sibirien heran; er findet ebenfalls ein differenziertes Bedingungsgefüge für die verschiedenen Umbau- 
und Abbauprozesse im Bereich der nominalen und pronominalen Flexionsmorphologie. - Das Dialekt-Standard-Problem gibt bzw. gab es auch in Sprachinseln. Wie das Verhältnis zwischen Dialekt und Standard im Texasdeutschen ausgesehen haben dürfte, ist das Thema des Beitrags von Hans C. Boas und Katrin Fuchs, „Zum Einfluss des Standarddeutschen auf das Texasdeutsche im 19. und 20. Jahrhundert: Empirische und methodologische Probleme“. Sie diskutieren die Schwierigkeiten der Datengewinnung und machen plausibel, dass die Standardkompetenz der meisten Sprecher des Texasdeutschen nicht überschätzt werden sollte. - Den Abschluss dieses Bandes bildet der Beitrag „Gesprochenes Alltagsdeutsch im Bismarck-Archipel um 1900“ von Péter Maitz und Siegwalt Lindenfelser. Auch hier geht es um die schwierige Rekonstruktion eines historischen Zustands; die Autoren weisen anhand von zeitgenössischen Quellen nach, dass (anders als bisher angenommen) als Superstrat von Unserdeutsch eine nordwestdeutsch-westfälisch geprägte, vergleichsweise standardnahe Varietät anzusetzen ist. 


\title{
1 Das Beste zweier Welten: Das Bild elsässischer Dialektsprecher von den Deutschen, den Franzosen und sich selbst
}

\begin{abstract}
Vor dem Hintergrund der zunehmenden sprachlichen Divergenz des Alemannischen im Elsass und im badischen Oberrheingebiet untersucht dieser Beitrag die Vorstellungen elsässischer Dialektsprecher zur Sprache und zu den sprachlichen Verständigungsmöglichkeiten mit den Deutschen auf der anderen Rheinseite („driwwe“) sowie ihre Einstellungen zu den Deutschen. Es wird gezeigt, dass die sprachliche Verständigung trotz wahrgenommener Differenzen nach wie vor nicht als Problem gesehen wird, jedoch keinesfalls zur Konstruktion einer gemeinsamen, grenzüberschreitenden Identität beiträgt. Der Beitrag diskutiert verschiedene ideologische Konstruktionen der Beziehung zwischen Franzosen, Elsässern und Deutschen (Badenern). Zu den elsässischen représentations von sich selbst gehört auch die Idee, „das Beste“ aus der französischen und der deutschen Welt zu vereinen.
\end{abstract}

Keywords: Alemannisch, Einstellungen, Elsass, Oberrheingebiet, Rheingrenze, Staatsgrenzen als Sprachgrenzen, Stereotypen

\section{Einleitung}

Jüngste Erhebungen zur Dialektphonologie auf beiden Seiten der deutschfranzösischen Grenze im Rahmen eines deutsch-französischen Kooperationsprojekts (vgl. Auer, Breuninger \& Pfeiffer 2017) haben gezeigt, dass die Staatsgrenze immer mehr zu einer Sprachgrenze zwischen dem Elsass und den badischen Gebieten im Oberrheingebiet wird. Diese Divergenz ist in erster Linie ein Epiphänomen der unterschiedlichen makrosoziolinguistischen Entwicklungen, also der jeweiligen Repertoirestruktur. Auf der deutschen Seite führt der allgemein im Oberdeutschen $\mathrm{zu}$ beobachtende Übergang von traditionellen $\mathrm{zu}$

Peter Auer, Albert-Ludwigs-Universität Freiburg, Abtl. für Germanistische Linguistik. D-79086 Freiburg, E-Mail: peter.auer@germanistik.uni-freiburg.de

Ә Open Access. (C) 2018 Peter Auer, publiziert von De Gruyter. (c) BY Dieses Werk ist lizenziert unter der Creative Commons Attribution 4.0 Lizenz. 
Regionaldialekten und von dort zu regionalen Standardvarietäten zum Abbau vor allem kleinräumiger Dialektmerkmale. Im Elsass hat hingegen „das Hochdeutsche jegliche Funktion als eine von Kloss (1978) so genannte „Dachsprache" verloren“, wie man schon in einem von Ludwig Eichinger und Robert Hinderling herausgegebenen Handbuch der „mitteleuropäischen Sprachminderheiten“ in Rüdiger Harnischs Beitrag lesen kann (Harnisch 1996: 420). Das Elsässische ist also mangels eines überdachenden Standards ähnlichen Entwicklungen wie in Baden weitgehend entzogen und verändert sich nur wenig. ${ }^{1}$ Unterschiede zwischen den Sprechergenerationen schlagen sich, wie unsere Untersuchung zeigt, nicht in der altersspezifischen Verteilung der Merkmale nieder, sondern radikaler im Wegbrechen der gesamten jüngeren Sprecherschicht.

Die sprachliche Divergenz an der Grenze erscheint deshalb zunächst unabhängig von der Grenzsituation selbst, d. h. sie scheint sich vollständig aus Faktoren zu erklären, die genauso gültig und wirksam wären, wenn Baden und das Elsass nicht aneinander grenzen würden. Allerdings ist diese Sicht besonders für das Elsass zu einfach. Die Vorstellungen (représentations), die die Elsässer von ihrer eigenen Sprache und Sprachregion und deren Verhältnis zum (Standard-)Deutschen wie auch zu den alemannischen Dialekten in Baden haben, sind ja eine der Determinanten der „Abkopplung“ (Hartweg 1983) der elsässischen von der badischen Sprachregion, die sich im für das Elsass typischen sprachlichen Konservatismus niederschlägt. Diese Vorstellungen sind ihrerseits nicht unabhängig von der Wahrnehmung und Bewertung „der Deutschen“ (elsässisch: Schwowe) und natürlich auch „der Franzosen“ (ein Begriff, der sich fallweise auf die Angehörigen der sprachlichen Majorität oder auf die Staatszugehörigkeit bezieht, also die Elsässer mit einschließt). Es lohnt deshalb, solche représentations ${ }^{2}$ genauer zu analysieren. Die einzelnen Gewährspersonen äußern sich in den Interviews über „die Deutschen“ (im Allgemeinen oder auf der anderen Rheinseite, also die Badener) mit Zuschreibungen und Bewertungen, die teils auf eigener Erfahrung, teils eben auf solchen geteilten représentations beruhen. Sie sind ein gesellschaftlich vermitteltes Repertoire

1 Vgl. jedoch Bothorel \& Huck (2001) auf der Grundlage von Daten von 1997 zu Ausgleichsprozessen innerhalb der elsässischen Dialektlandschaft sowie marginalen Einflüssen des Deutschen.

2 Zum Begriff der représentations siehe u. a. Jodelet (1993), die diese als „systèmes d'interprétation régissant notre relation au monde et aux autres“ definiert, die „orientent et organisent nos conduites et les communications sociales“ (1993: 36); zur Anwendung in der Soziolinguistik und den représentations der Elsässer siehe Bothorel-Witz (2008), die ihren diskursiv-emergenten Status betont. Am nächsten ist der Begriff wohl dem der (sprachlichen) Ideologie in der angloamerikanischen Soziolinguistik (etwa: Irvine \& Gal 2000; Kroskrity 2004), obwohl auch Komponenten von „Einstellung“ mitschwingen. 
von (Stereo-)Typisierungen, die auch unabhängig von persönlichen Erfahrungen tradiert und von den Mitgliedern der Gemeinschaft gewusst, wenn auch nicht notwendigerweise verwendet oder gar geglaubt werden.

In diesem Beitrag liegt der Fokus auf der Auswertung der elsässischen Interviews, die in den Jahren 2012 und 2013 durch unsere Elsässer Projektpartner durchgeführt wurden. ${ }^{3}$ Die Auswertung der deutschen Interviews wird an anderer Stelle erfolgen. Ausgangspunkt wird eine Interviewer-Frage sein, die in einem Teil der elsässischen Interviews (nämlich 41 von insgesamt über 140) gestellt wurde. Diese Frage lautete: „Wenn Sie aus irgendeinem Grund gezwungen wären, aus dem Elsass wegzuziehen, würden Sie dann lieber in eine Stadt in Frankreich wie etwa Dijon oder Lyon oder lieber in eine Stadt in Deutschland wie etwa Stuttgart, Karlsruhe oder Freiburg ziehen?“ Im Folgenden werden ausgehend von der Beantwortung dieser Frage einige der (Stereo-)Typisierungen der Deutschen rekonstruiert. Dabei wird sich aber zeigen, dass die relevante Alterität für die Befragten oft nicht nur „die Deutschen“, sondern auch „die Franzosen“ (im Sinne von Angehörigen der Majoritätsgesellschaft, also Nicht-Elsässer) sind. Die sich daraus ergebende doppelte ideologische Abgrenzung kann als Korrelat der Resistenz des Elsässischen gegen sprachlichen Wandel gesehen werden.

Im Folgenden wird zunächst die Frage diskutiert, ob die elsässischen Gewährspersonen die Staatsgrenze und die mit ihr einhergehenden sprachlichen Unterschiede als Kommunikationshindernis sehen. Ich gehe anschließend auf das aus der Beantwortung der „Umzugsfrage“ rekonstruierbare Bild von den Deutschen und die damit verbundenen negativen und positiven Stereotypen ein um abschließend die Selbstkonstruktion der elsässischen Identität zwischen „deutsch“ und „französisch“ zu diskutieren.

3 Die Interviews wurden von Dr. Pascale Erhart (Univ. Strasbourg) durchgeführt und unter der Leitung von Prof. Dr. Dominique Huck (Univ. Strasbourg) transkribiert. Pascale Erhart danke ich auch für verschiedene Hinweise zur Auswertung und zu diesem Beitrag. Das Gesamtprojekt (Frontière linguistique au Rhin Supérieur = FLaRS, Ltg. P. Auer und D. Huck) wurde durch DFG und ANR gemeinsam gefördert. Ziel war einerseits, die dialektalen Veränderungen v. a. im Bereich der Phonologie zu erforschen, andererseits die Vorstellungen von und Einstellungen zur Sprache auf der anderen Rheinseite zu analysieren. 


\section{Die Sicht der Gewährspersonen auf das Elsässische und seine Beziehung zu den deutschen Dialekten}

Obwohl das Elsässische ${ }^{4}$ fraglos sein standarddeutsches „Dach“ verloren hat, wäre es voreingenommen, mit Kloss von einer „dachlosen Außenmundart“ des Deutschen $\mathrm{zu}$ sprechen. Vielmehr hat sich das Elsässische (wie auch einige andere aus deutschen Dialekten entstandene Varietäten, etwa das in Südbrasilien gesprochene „Hunsrückisch“) aus dem Varietätenverbund des Deutschen gelöst, ohne selbst (wie etwa das Luxemburgische) einen eigenen Standard zu entwickeln. Die Rolle des Standards übernimmt das exoglossische Französisch. Soweit das Deutsche im Elsass präsent ist, hat es die Rolle einer schulisch vermittelten Zweit- oder Fremdsprache. „Das Elsässische“ seinerseits wird, wie schon das Glottonym zeigt, nicht als Teil des (plurizentrischen) deutschen Sprachgebiets (also als Dialekt des Deutschen) gesehen, sondern als „unsere Sprache“ und somit als Regionalsprache Frankreichs. (Oft wird es von den Gewährspersonen - meist mit einem neidischen Blick auf das sprachliche Selbstbewusstsein der dortigen bilingualen Gemeinschaft - mit dem Bretonischen verglichen.) Sehr klar drückt das eine jüngere Gewährsperson aus, die sich im folgenden Ausschnitt beklagt, dass die Elsässer, wenn sie elsässisch redeten, von manchen „Franzosen“ aufgefordert würden, sie sollten doch französisch, nicht deutsch sprechen. Sie fährt dann wie folgt fort:

\section{Ausschnitt (1) [1571]}

GP: s gibt euh so euh fàscht e confusion fir mànchi litt zwische s elsässische un s ditsche s gibt ähm so ähm fast eine Verwechselung bei manchen Leuten zwischen dem Elsässischen und dem Deutschen

\footnotetext{
4 Hintergrundinformationen zum Elsässischen lassen sich aus historischer Perspektive bei Huck (2015) finden; zur soziolinguistischen Situation im ausgehenden letzten Jahrhundert siehe Harnisch (1996).

5 In der Transkription sind Phrasengrenzen durch „/“, unverständliche Passagen durch „X“ und in den elsässischen Passagen der accent grave (,à“) das Phonem / / /. I ist die Interviewerin, GP die Gewährsperson. Auf eine typografische Differenzierung des Elsässischen und des Französischen wird verzichtet, weil sie eine dichotomische Kategorisierung der Äußerungen erfordern würde, die sachlich nicht angemessen ist.
} 
I: $\quad \mathrm{mm}$ un fer dich isch $\mathrm{s}$ nit salwe

$\mathrm{m}$, und für dich ist's nicht dasselbe

GP: ah non

I: ja

GP: non non

I: wàs isch no de unterschied/ wàs sin was ist dann der Unterschied/ was sind

GP: euh $\mathrm{s}$ isch doch euh/ $\mathrm{s}$ isch üri sproch ähm es ist doch ähm/ es ist unsere Sprache

I: $\quad \mathrm{mm}$

GP: $\quad \mathrm{s}$ isch $\mathrm{s}$ isch kenn ditsch un mir sin kenn ditsche es ist es ist kein Deutsch und wir sind keine Deutschen

I: ((lacht))

GP: àwwer euh aber ähm

I: $\quad$ stert s dich wenn dir ebber sait euh dü redsch ditsch stört's dich wenn jemand zu dir sagt ähm du redest deutsch

GP: ja

I: ja

GP: ja

I: wie wàrum no/ weil s euh wie warum dann/ weil's ähm

GP: euh pff ich füehl mi frànzesch ähm pff ich fühl mich französisch

I: $\quad \mathrm{mh}$

GP: àwwer euh bon em elsass hànn mir euh e sproch aber ähm naja im Elsass haben wir ähm eine Sprache

I: $\quad \mathrm{mm}$

GP: un die des isch s elsässische und die das ist das Elsässische

I: $\quad \mathrm{mm}$

GP: des isch/ s isch sicher/ s glicht im ditsche àwwer euh das ist/ das ist sicherlich/ es ist dem Deutschen ähnlich aber ähm

I: $\quad \mathrm{mm}$

GP: àwwer mir sin kenn kenn aber wir sind keine keine

I: $\quad$ isch s nit ditsch ist es nicht deutsch

GP: $m^{2} \mathrm{~m}$

I: d'accord

Die Nachfrage der Interviewerin, warum für sie das Deutsche und das Elsässische nicht dasselbe seien, führt die Gewährsperson dazu, etwas für sie Selbst- 
verständliches $\mathrm{zu}$ versprachlichen, von dem sie ausgeht, dass es eigentlich auch von ihrer Gesprächspartnerin qua Zugehörigkeit zur elsässischen Sprachgemeinschaft gewusst werden müsste (vgl. die Partikel doch in der unmittelbaren Antwort: euh s isch doch euh/ s isch üri sproch). Sie tut sich relativ schwer (vgl. die zahlreichen Häsitationen und Reformulierungen), den $\mathrm{Zu}$ sammenhang zwischen Sprachen und nationalen Zugehörigkeiten zu erklären; auch diese Formulierungsschwierigkeiten deuten darauf hin, dass solche sprachideologischen Grundannahmen unter Elsässern weder expliziert noch diskutiert werden müssen. Die schließlich produzierte Explikation bringt die Dinge aber auf den Punkt. Sie bewegt sich in einem nationalsprachlichen Bezugsrahmen: Sprachen gehören zu Nationen, und weil die Elsässer keine Deutschen sind, kann das Deutsche auch nicht die Sprache des Elsass sein ( $s$ isch s isch kenn ditsch un mir sin kenn ditsche). Auch die folgende Interviewfrage, ob und warum es sie störe, wenn sie für eine Deutsche gehalten werde, trifft insofern auf Unverständnis, als auch hier die Antwort für jeden Elsässer und für jede Elsässerin klar sein müsste (vgl. die Interjektion pff): euh pff ich füehl mi frànzesch. Allerdings scheint der Sprecherin bei dieser Antwort zugleich klar zu werden, dass sie dem nationalsprachlichen Diskurs widerspricht: wenn sie Französin ist, müsste sie ja französisch, nicht elsässisch sprechen. Dies führt zu der abschließenden Klärung: em elsass hànn mir euh e sproch/ un die des isch s elsässische/s glicht im ditsche àwwer/ mir sin kenn kenn [ditschi]. Sie macht deutlich, dass die nationale Identifizierung nicht positiv mit dem Französischen als Symbol der nationalen Zugehörigkeit korreliert wird, sondern negativ mit der Differenz zum Deutschen als Symbol der nationalen Zugehörigkeit zu Deutschland.

Allerdings ist die Beziehung zu den alemannischen Dialekten auf der anderen Rheinseite für unsere Gewährspersonen ambivalent. Die ideologische „Abkopplung“ geht nicht so weit, dass die Elsässer die enge sprachliche Verwandtschaft $\mathrm{zu}$ den Dialekten auf der anderen Rheinseite und selbst zum deutschen Standard nicht sehen würden (vgl. im Ausschnitt (1): s glicht im ditsche àwwer euh...). Für die Verständigung mit den Deutschen jenseits des Rheins ist der elsässische Dialekt eine wichtige und effektive Ressource, die auf wahrgenommener und erfahrener sprachlicher Nähe beruht. Zwar haben unsere Gewährspersonen in der Regel nur oberflächliche (auf das Einkaufen beschränkte), nur selten engere Kontakte nach Baden (einige wenige arbeiten in Deutschland oder haben dort gearbeitet, noch weniger haben dort Freunde, selten sind sie an grenzüberschreitenden kulturellen Aktivitäten beteiligt), durchgängig sind sie aber der Meinung, dass die sprachliche Kommunikation im grenznahen deutschen Gebiet für sie kein Problem ist - zumindest wenn beide Seiten Dialekt/Elsässisch sprechen: 
Ausschnitt (2) [1514]

I: àlso reden ihr wenn ihr jetzt niwwer komme oder wenn ihr ditsch muen redde reden ihr ditsch oder

also reden Sie wenn Sie jetzt rüber gehen oder wenn Sie deutsch reden müssen, reden Sie deutsch oder

GP1: ich prowier deitsch ze redde àwwer ich versuch deutsch zu reden aber

I: oder

GP1: oder euh àwwer es isch bstimmt nit richtich im mit denne datif un des dings do des

nicht, ähm aber es ist bestimmt nicht richtig im mit den Dativen und dem Zeug da das

I: $\quad$ un des

GP1: do bin i nit euh/ àwwer sie verstehn mi àlli hein

da bin ich nicht ähm/ aber sie verstehen mich alle, nicht

I: $\quad \mathrm{mm}$

GP1: do isch kein problem/ àwwer wenn s nit geht red i ORTSNAME-isch no geht $\mathrm{s}$ àà

da gibt's kein Problem/ aber wenn's nicht geht sprech ich oRTSNAME-isch ${ }^{6}$ dann geht's auch

I: no gehts àà

Ausschnitt (3) [1612]

GP: $\quad$ wenn $r$ ewer de Rihn gehn/ s esch numme ding hein/ mr vestehn änànder/ nett/ àwer sie redde doch ànderscht

wenn Sie über den Rhein gehen/ es ist nur dings, oder/ wir verstehen einander, nicht/ aber sie reden doch anders

I: $\quad$ redde se andersch wie do reden sie anders als hier

GP: ja ja

I: àwer ihr verstehn eijsch no aber ihr versteht euch noch

GP: verstehsch $\mathrm{s}$ ja/ $\mathrm{s}$ esch oj nett $\mathrm{s}$ ditsche net/ $\mathrm{s}$ esch $\mathrm{s}$ bàdische Du verstehst es, ja/ es ist auch nicht das Deutsche, nicht/ es ist das Badische

I: $\quad$ oui

[...]

I: unn no redde sie àj/ sie redde nett hochditsch/ wa mer newer gehen redde sie ihre ditsch ihre

und dann reden sie auch/ sie reden nicht hochdeutsch/ wenn wir rüber gehen reden sie ihr Deutsch ihre

6 Der Dialekt ihres Wohnorts im Elsass. Der Name wurde aus Anonymisierungsgründen getilgt. 
GP: un mer redde elsassisch und wir sprechen elsässisch.

Ausschnitt (4) [1662]

I: àwer/ redde se/ redde se salwe euh/ uff de ànder sitt vom Rhin wie do enfin

Aber/ reden sie/ reden sie genauso äh/ auf der anderen Seite vom Rhein wie hier oder

GP1: ni/ ni/ nitt gànz/ ni gànz/ àwer wä mr jetz na gehn uff Nejeburg/ hein nein/ nein/ nicht ganz/ nicht ganz/ aber wenn wir jetzt nach Neuenburg ${ }^{7}$ gehn, nicht

I: $\quad \mathrm{hm}$

GP1: des esch gràd nawe àn de granze/ oder euh/ bizell widderscht noch/ euh X

das ist grad dort neben an der Grenze/ oder ähm/ ein wenig weiter nach/ ähm X

I: $\quad \mathrm{hm}$

GP1: wenn dü elsassisch reddsch

wenn du elsässisch redest

I: ja

GP1: sie verstehn di gànz güet

die verstehn dich ganz gut

GP2: e bissel ja

GP1: sie vestehn s güet sie verstehn's gut

GP2: ja ja

GP1: unn no schàffe viel litt im Ditschlànd unn wenn s um s gald geht/ vesteht mr enànde güet

und dann arbeiten viele Leute in Deutschland und wenn's um's Geld geht/ versteht man einander gut

Ausschnitt (5) [1605]

GP: la langue alsacienne se rapproche plus ou moins die elsässische Sprache nähert sich mehr oder weniger

I: ouais

GP: de l'allemand dem Deutschen

I: hànn $\mathrm{r}$ denne indruck haben Sie den Eindruck

GP: mir vestehn se güet ne wir verstehen sie gut, nicht

7 Neuenburg liegt auf der deutschen Rheinseite. 


\section{Ausschnitt (6) [1565]}

I: $\quad$ un wissen ihr wie s isch uf de ander sitt vun de granz/ redde se uf de ander sitt vun de granz/ jetzt ich weis nit in Bàde Bàde oder euh und wissen Sie wie's auf der anderen Seite der Grenze ist/ reden sie auf der anderen Seite der Grenze/ jetzt ich weiß nicht in Baden Baden oder ähm

GP: ((Einatmen))

I: $\quad$ wie redde se derte wie reden sie da

GP: euh ja ich glöb s isch sie euh sie verstehn guet wenn mir uf elsässisch rede

$\overline{a ̈ h m}$ ja ich glaub es ist ähm sie verstehen es gut, wenn wir elsässisch reden

I: $\quad$ ah ja

GP : euh ich glöb sie hàn liewer mir redde elsässisch ähm ich glaub sie haben es lieber wenn wir elsässisch reden

I: ((Lachen/Einatmen))

GP : dàss dàss mir proweere hochditsch [als] dass als dass wir's hochdeutsch versuchen

I : $\quad \mathrm{mm}$

GP : euh ich glöb sie rede ö so s s schwowisch oder euh ähm ich glaub sie reden so schwäbisch oder ähm

I : ouais

GP: oder so

I: $\quad$ ouais $\mathrm{mh} /$ àlso redden se a ke hochditsch ja $\mathrm{mm} /$ also reden sie kein hochdeutsch

GP: nä

Manche Gewährspersonen sagen, dass sie versuchten, „(hoch)ditsch“ zu sprechen, „presque par politesse peut-être/ comme si c'était mieux de parler hochditsch“ (,fast vielleicht aus Höflichkeit, als ob es besser wäre, hochdeutsch zu sprechen`), wie eine Gewährsperson sagt [1592]. In diesem Fall werden die Gebrauchsbedingungen für die Varietäten im elsässischen Repertoire (Französisch als öffentliche und Standardsprache, Elsässisch als Familiensprache im privaten Raum) auf die Situation in Deutschland übertragen, wo dialektale Sprechweisen auch in Teilen des öffentlichen Raums durchaus verbreitet sind. Die Kommunikation im Dialekt wird in diesem Fall als unproblematische Rückfalloption beschrieben (vgl. Ausschnitt (2)). Dominant ist aber die Meinung, dass die Tatsache, dass auch auf der deutschen Seite ein Dialekt gesprochen wird (und nicht „Hochdeutsch“), die Kommunikation erleichtert. Durchgängig wird der alemannische Dialekt jenseits der Grenze als anders, aber ähnlich beschrieben.

Wir können also zunächst festhalten, dass die sprachliche Nähe zwischen der eigenen Sprache und der der deutschen Nachbarn durchaus Teil der repré- 
sentations des Oberrheingebiets ist; die Kommunikation zwischen links- und rechtsrheinischen Bewohnern findet (in der Darstellung der Gewährspersonen) meist im Dialekt statt. Trotzdem ist aber der Rhein als Staatsgrenze nicht nur in Bezug auf die Standardsprachen (Französisch und Deutsch), sondern auch in Bezug auf die Dialekte eine symbolische Grenze: das Elsässische ist Symbol nicht-deutscher (französischer und/oder elsässischer) Identität. Auch wenn die grenzüberschreitenden Kontakte stärker wären, als sie wohl faktisch sind, wäre wohl schon deshalb nicht zu erwarten, dass das Elsässische sich in irgendeiner Weise an den sprachlichen Wandel im badischen Alemannischen anschließen würde.

\section{Nationale und regionale Identitäten}

Auf diesem Hintergrund betrachten wir nun die Beantwortung der „Umzugsfrage“. Die Frage sollte die Gewährspersonen zur Reflexion über ihre Bindung an das Elsass (regionale Identität, Ortsloyalität), aber auch über ihre Beziehung zu Frankreich („den Franzosen“) und Deutschland („den Deutschen“) anregen. Angesichts der grundsätzlich hohen Ortsloyalität der Gewährspersonen, die meist aus ländlichen Gebieten kommen und schon durch die Teilnahme an der Untersuchung ein gewisses Interesse am Elsass und seiner Sprache vermuten ließen, war zu erwarten, dass die Beantwortung den meisten Interviewpartnern schwer fallen würde. Das war auch der Fall; fünf der $41 \mathrm{Be}$ fragten verweigerten die Antwort vollständig; von den übrigen entschied sich eine Mehrheit (66\% der 41 Personen) für den Umzug in eine französische Stadt, $22 \%$ für den Umzug nach Deutschland. ${ }^{8}$ Unter letzteren war allerdings oft die Nähe der deutschen Städte Freiburg und Karlsruhe zum Elsass maßgebend, d.h. indirekt spielte auch hier die elsässische Identität eine Rolle (siehe unten).

Interessanter als diese quantitativen Ergebnisse sind die Antworten selbst und die Art und Weise, wie sie im Interview formuliert werden. Sehr häufig wird die Entscheidung für eine französische Stadt durch nationale Zugehörigkeit begründet, woraus (für die Gewährspersonen) „automatisch“ die Wahl des

8 Der Prozentsatz divergiert übrigens nur wenig von dem der linksrheinischen Befragten. Ein direkter Vergleich ist allerdings nicht sinnvoll, denn für die Elsässer bedeutet der Wechsel nach Dijon/Lyon, die Region der elsässischen Minderheit in Frankreich zu verlassen, während für die Badener der Umzug nach Stuttgart die traditionelle Konkurrenz der Regionen Baden und Schwaben evoziert. Die oberflächlich ähnlichen Prozentzahlen verschleiern auch, dass die Gründe für die Entscheidung jeweils andere sind (vgl. dazu Pfeiffer \& Auer i. V.). 
Orts in Frankreich resultiert (auch wenn dies teils erst nach langem Überlegen und mit verschiedenen „hedges“ passiert). Mit dieser Begründung wird also die nationale Identifizierung mit Frankreich priorisiert.

\section{Ausschnitt (7) [1535]}

GP: $\mathrm{mr}$ sin doch frànzeesch vor àllem wir sind doch vor allem französisch

Ausschnitt (8) [1636]

GP: denn ich bin in Frànkrich bin i d haim euh ich ich geh garn àwwer ich bin frànzesch

denn ich bin in Frankreich bin ich daheim ähm ich ich geh gern aber ich bin französisch

I: $\quad \mathrm{mm}$

GP: un ich bin ((Lachen)) ich bin do d haim un voilà und ich bin ich bin da daheim und so ist es

Ausschnitt (9) [1685]

GP: moi je resterais dans mon pays hein

ich würde in meinem Land bleiben, ne

Ausschnitt (10) [1691]

GP: wil mr frànzos isch/ XX ànfànge/ mr fühlt sich doch ender d heim im Frànkrich

weil man Franzose ist/ XX anfangen/ man fühlt sich doch eher in Frankreich daheim

Diese Begründung (die sich übrigens auch auf der deutschen Seite findet) entspricht der in Ausschnitt (1) bereits explizierten nationalen Identität der Elsässer als Franzosen und Französinnen; sie wird in der Regel als selbsterklärend präsentiert. Teilweise werden (auch oder zusätzlich) sprachliche Gründe genannt, die nur scheinbar mit der oben genannten Rolle des Elsässischen als Kommunikationshilfe in der Interaktion mit den Badenern im Widerspruch steht: es ist etwas anderes, in einem Land vollständig in der Landessprache funktionieren $\mathrm{zu}$ müssen (was die Beherrschung der Standardvarietät in Wort und Schrift impliziert), als sich irgendwie verständigen zu können:

\section{Ausschnitt (11) [1621]}

GP: àwer s einfàchschte wart worschins im Frankri bliwe aber das einfachste wäre wahrscheinlich in Frankreich zu bleiben

I: ja/ wàrum/ waije de/ waije ja/ warum/ wegen der/ wegen

GP: wage de sproch wegen der Sprache 
Ausschnitt (12)[1513]

GP: niwer wodd isch jetzt nit hein

rüber würd ich jetzt nicht wollen

I: nä

GP: mais je ne sais pas pourquoi/ kennt nit saawe wàrum/ XXX/ ùff Karlsruhe wodd isch jetzt nit

aber ich weiß nicht warum/ kann nicht sagen warum/ XXX/ nach Karlsruhe würd ich jetzt nicht wollen

I hm

GP: äh/ isch dat jetzt liwer/ ja/ isch dat liawer im Frankreich blaiwa/ ja/ ob $s$ jetzt de Haut-Rhin isch oder d Bourgogne oder d Champagne/ oder Territoire de Belfort/ i dät liewer do hiwe blaiwe ja äh/ ich würd jetzt lieber/ ja/ ich würd lieber in Frankreich bleiben/ ja/ ob’s jetzt das Oberelsass ist oder die Bourgogne oder die Champagne/ oder das Gebiet von Belfort/ ich würd lieber hier drüben bleiben ja

I: àwer ihr wisse nit wàrùm aber Sie wissen nicht warum

GP: non/ i kànn eich nit saawe wàrùm/ es dät mi à bissel/ $\mathrm{d}$ sprooch kennt i schon a bissel besser wàs s frànzesche ànbelàngt/ ùn dät mi doch à bissel wöhler fiele so im Frànkreich

nein/ ich kann's Ihnen nicht sagen warum/ es wär ein bisschen/ die Sprache könnt ich schon etwas besser was das Französische anbelangt/ und [ich] würd mich doch ein bisschen wohler fühlen so in Frankreich

Der letzte Ausschnitt ist typisch in Bezug auf die Vorsicht und Zurückhaltung, mit der viele der elsässischen Gewährspersonen ihre Präferenz äußern. Sie lässt sich auch als Versuch der Vermeidung diskursiv riskanterer Selbstpositionierungen verstehen. Dahinter versteckt sich wohl eine doppelte Rücksichtnahme auf dominante Diskurse, die im Interview als relevant unterstellt werden: zum einen der Diskurs der politischen Korrektheit, der Kritik an den Deutschen erschwert; zum anderen die mit der Option für Frankreich verbundene Gefahr, im innerelsässischen Diskurs (den die Interviewerin ganz besonders repräsentiert) als jemand mit allzu schwacher elsässischer Identität dazustehen.

Beide Aspekte - die ideologische Abgrenzung gegen „die Deutschen“ und die Identifizierung mit dem Elsass als Minderheit und damit auch gegen die französische Mehrheit - werden jedoch deutlich, wenn man die Begründungen anderer Gewährspersonen für die französische Option betrachtet, die sich etwas mehr exponieren und auf die unterschiedliche „Mentalität“ oder den anderen „Charakter“ der Deutschen verweisen.

Erneut genügt oft bereits dieser Verweis als Antwort, ohne dass begründet würde, worin denn diese Mentalitätsunterschiede bestünden. Worin sie bestehen, wird als bekannt vorausgesetzt: 
Ausschnitt (13) [1512]

I: un wànn ihr jetzt d wàhl hätte/ entweder uff/ ihr muen jetzt unbedingt fort/ täten ihr ender uff Stuttgàrt gehn/ uff Stuttgàrt oder uff Dijon/ oder uff Lyon und wenn Sie jetzt die Wahl hätten/ entweder nach/ Sie müssten jetzt unbedingt fort/ würden Sie lieber nach Stuttgart gehen/ nach Stuttgart oder nach Dijon/ oder nach Lyon

GP1: liawer im Frankreich/ ich àmol lieber nach Frankreich/ ich jedenfalls

GP2: in Frànkreich blaiwe/ nit ins Daitsche in Frankreich bleiben/ nicht ins Deutsche rein

GP1: non/ niwer nit nein/ rüber nicht

I: $\quad$ wàrum

GP2: niwer nit

GP1: pas du tout/ d mentalité/ c'est tout/ ganz und gar nicht/ die Mentalität/ das ist alles

\section{Ausschnitt (14) [1503]}

(Die GP hat zunächst versucht, der Frage auszuweichen, indem sie argumentiert, dass sie nur dorthin gehen würde, wo sie bereits jemand kennt.)

I: àwer wenn ihr jetzt in a ànder lànd sodde/ enfin in a ànder lànd/ nä enfin/ wit von do wag sodde gehn/ täten ihr ender ins Frànkrich aber wenn Sie jetzt in ein anderes Land müssten/ oder in ein anderes Land/ nein ich mein/ weit von hier weggehen müssten/ würden Sie lieber nach Frankreich

GP: in de Süde tät $\mathrm{i}$ gehen in den Süden würd ich gehen

$[\ldots]$

I: àlso in Südfrànkrich

GP: ja/

$[. .$.

I: àwer ich mein kennten $\mathrm{r}$ nit ins Ditschlànd wohne no? aber ich mein könnten Sie dann nicht in Deutschland wohnen?

GP: non/ heinhein

I: däts eich nit euh würde Ihnen das nicht ähm

GP: non non/ non non

I: wàru no warum nicht

GP: jsais pas/ $\mathrm{s}$ isch/ euh/ jsais pas/ $\mathrm{s}$ isch d mentalité wie mr nit gfàllt weiß nicht/ es ist/ ähm/ weiß nicht/ die Mentalität gefällt mir nicht 
In beiden Fällen ist der Verweis auf die andere Mentalität der Deutschen sequenzabschließend (was die Sprecherin GP1 in (13), die für dieses abschließende Statement ins Französische wechselt, überdies noch durch die abschließende Partikel c'est tout markiert). Beide Gewährspersonen in (13) wie auch die Interviewte in (14) haben eine sehr dezidierte Meinung und können sich die Übersiedlung nach Deutschland nicht vorstellen. Über die Gründe dafür sagen sie aber nichts: zu selbstverständlich ist die präsupponierte Ablehnung der deutschen mentalité (und wohl auch zu riskant deren Explikation). Die Präsupposition eines gemeinsamen Wissens über die Mentalitätsunterschiede zwischen Deutschen und Franzosen/Elsässern gilt selbst für eine der Gewährspersonen, die sich für den Umzug nach Deutschland aussprechen (in diesem Fall, weil es von Karlsruhe aus näher ins Elsass wäre als von Dijon):

Ausschnitt (15) [1501]

(Der Sprecher hat schon kurz vorher gesagt, „die mentalität isch doch e bissel ànnersch/ hiwe un driwwe“.)

I: un hàn se au e àndere chàràkter no uf de ànder sitt und haben sie dann auch einen anderen Charakter auf der anderen Seite

GP: ihr hàn's jo gheert vorricht/ hm Sie haben's ja vorhin gehört/ mm

I: sin'r do d'accord mit dàss se sind Sie einverstanden mit der Meinung dass sie

GP: ja ich bin zimlich/ meiner meinung bin ich àllewaj nit allein/ ich hàb gedenkt es gibt emol e gewissi/ e gemeinsàmi identität wenn mal die grenz uf sin/ àwer e Daitscher bleibt e Daitscher/ un Elsasser e Elsaser/ un e Frànzos e Frànzos

Ja ich bin ziemlich/ [mit] meiner Meinung bin ich nicht allein/ ich hab gedacht es gibt einmal eine gewisse/ eine gemeinsame Identität wenn mal die Grenzen auf sind/ aber ein Deutscher bleibt ein Deutscher/ ein Elsässer ein Elsässer/ und ein Franzose ein Franzose.

Wieder ist das apodiktische Statement über die unveränderlichen ethnischen Konstanten der Abschluss des Themas. Zu beachten ist, dass der Sprecher seine Meinung als allgemein geteilte Einschätzung präsentiert und dass sein Kategorisierungssystem explizit „Franzosen“ und „Elsässer“ unterscheidet und damit die nationalstaatliche Binarität der Kategorien unterläuft (vgl. unten, Abschnitt 4).

Manchmal von selbst, manchmal auf Nachfragen der Interviewerin entwickeln sich aus diesen allgemeinen Aussagen zur Mentalität der Deutschen jedoch auch detailliertere Zuschreibungen, von denen man aufgrund ihrer Rekurrenz annehmen kann, dass sie ebenfalls Teil der représentations der Deutschen 
sind. Obwohl sich dies aus den vorliegenden Daten nicht nachweisen lässt, ist anzunehmen, dass sie sich nicht nur bei Elsässern finden lassen, sondern in Frankreich allgemein verbreitet sind.

Im harmloseren Fall wird auf kulturelle Unterschiede im Häuserbau, in der Mode (bei den Frauen; vgl. auch unten, Ausschnitt (20)) oder beim Essen ${ }^{9}$ verwiesen:

\section{Ausschnitt (16) [1527]}

I: $\quad$ awer ihr hàn gsajt sie hàn e ànderi mentalität, wàs meinen $\mathrm{r}$ do demit aber Sie haben gesagt sie haben eine andere Mentalität, was meinen Sie damit?

GP1: sie sin doch àndersch/ $\mathrm{s}$ isch ebs àndersch sie sind doch anders/ es ist was anders

GP2: nä, sie esse nit so wie mir/ ich sieh's jetzt bei de nochbere/ wie mr viel euh mr esse $z$ morjets/ mir esse $z$ middàas/ mir esse $z$ owets/ wenn de sohn in de schul isch esst sie/ tuen sie gràd euh $z$ middàgs euh/ nicht, sie essen nicht so wir wir/ ich seh's jetzt bei den [deutschen] Nachbarn/ die wir viel ähm wir essen morgens/ wir essen mittags/ wir essen abends/ wenn der Sohn in der Schule ist, essen sie/ tun sie ähm mittags ähm

GP1: wie saawe se dann wie sagen sie dann

GP2: s tuet vespere oder so sie vespern oder so

GP1: ((lachend)) vespere

GP2: un euh/ un a $\mathrm{z}$ owets esse/ tut se fer $\mathrm{z}$ owets koch XXX $\mathrm{z}$ owets gross ebs màche/ üsser dàss ebber kommt/ àndersch $d$ (reschter) oder supp un euh voilà enfin und ähm/ und fürs Abendessen/ für abends kochen XXX abends groß was machen/ außer es kommt jemand [zu Besuch]/ sonst (Reste) oder eine Suppe und ähm so halt

Während es sich hier um Unterschiede der Alltagskultur handelt, sind andere Zuschreibungen charakterlicher Art. Auch sie gehören zum Stereotypenvorrat: die Deutschen sind laut, nervig, auftrumpfend, besserwisserisch, aufdringlich, frech, besitzergreifend und sie wollen immer gewinnen. Einige Beispiele:

9 Allerdings werden die oft traditionell thematisierten Unterschiede der Esskultur nicht mehr automatisch als Überlegenheit der elsässischen/ französischen Küche interpretiert. Einige Gewährspersonen berichten, dass sie selbst nach Baden zum Essen fahren (wegen der folkloristisch aufgemachten Lokale, oder wegen der größeren Schnitzel), während andererseits natürlich auch die Deutschen ins Elsass zum Essen kommen. Andere weisen darauf hin, dass sich die Qualität der deutschen Restaurants verbessert habe. 
Ausschnitt (17) [1521]

GP: wäs nit/ àlso/ là franchement/ ich tät lewer uff Lyon wohne/ XXX/ sie hàn à ànder mentalität/ $\mathrm{s}$ isch doch àndersch find $\mathrm{i} / \mathrm{sie}$ hàn ebs àn si wie mr nit/ isch wäs nit/ on se chamaillait toujours gentiment/ àwer sie hàn à ding hein

weiß nicht/ also/ um ehrlich zu sein/ ich würd lieber in Lyon wohnen/ XXX/ sie haben eine andere Mentalität/ es ist doch anders, find ich/ sie haben etwas an sich was wir nicht [haben]/ ich weiß nicht/ man hat sich immer höflich gekabbelt/ aber sie haben sowas an sich, nicht

I: ah ouais

GP: sin à bissel/ im Frànkri sin se moins chiants quand même sind ein bisschen/ in Frankreich sind sie weniger nervig, trotz allem

Dieser Sprecher relativiert seine Aussage über die nervigen Deutschen durch verschiedene rhetorische Strategien wie die Trivialisierung des Konflikts als gentille chamaillerie oder die vergleichsweise Evaluierung der Deutschen zu den Franzosen (die per Inferenz ebenfalls chiants sind, nur eben weniger als die Deutschen). Überdies kommt er zu seiner negativen Charakterzuschreibung erst nach vielen Abbrüchen und Reformulierungen. Die positive(re) Bewertung der Franzosen in der abschließenden Äußerung wird durch Codeswitching ins Französische unterstrichen.

Die beiden Gewährspersonen im folgenden Ausschnitt (ein Ehepaar) sind da wesentlich direkter:

Ausschnitt (18) [1682]

(GP1 hat gerade auf die Frage nach dem Ortswechsel kundgetan, dass sie pour la mentalité nicht nach Deutschland ziehen würde.)

GP1: wenn da kommsch bisch sowieso dr üslander wenn du [zu den Deutschen] kommst, bist du sowieso der ausländer

GP2: bisch üslander/ no isch er frach/gmein

bist ausländer/ dann ist er frech/ gemein

I: $\quad \mathrm{hm}$

GP1: ja

GP2: er word gmein sin er würd gemein sein

GP1: a kleiner ditsch isch gmein ja ein kleiner [= sozial niedrig stehender] Deutscher ist gemein, ja

GP2: un euh wie gsajt conquérant so und äh wie gesagt besitzergreifend und so

GP1: un uffdringlig/ dàs sin se oi/ dàs hà mr gsahn wo mr camping gmàcht hàn/ uffdringlig/ grüsig

und aufdringlich/das sind sie auch/ das haben wir gesehen wie wir beim Camping waren/ aufdringlich/ grausig 
GP2: sinon y'a des gens charmants aussi hein andererseits gibt es auch charmante Leute, nicht

GP1: mr hàn àwer/ so wie jetzt de Martin isch e chàrmànter mensch wir haben aber/ so wie jetzt der Martin, das ist ein charmanter Mensch

GP2: dàdo musch deno in de hautes sphères gehen nit da musst du aber dann in die oberen Schichten gehen, nicht wahr

GP1: awer sonscht aber sonst

[...]

GP2: jo un deno isch er noch fànàtik/ de Ditsch hein ja und dann ist er noch fanatisch/ der Deutsche, nicht

GP1: il est fanatique/ ja/ dàs isch de er ist fanatisch/ ja/ das ist die

[...]

GP2: dàs gsehsch im sport/ XXX wo e match isch das siehst du beim Sport/ XXX wenn ein Spiel ist

Anders als die meisten anderen trifft diese beiden Gewährspersonen die Frage nach den Unterschieden zwischen Deutschen und Franzosen/Elsässern offenbar nicht unvorbereitet; sie haben sich über das Thema anscheinend nicht zuletzt zusammen mit ihrem deutschen Freund Martin schon öfter Gedanken gemacht. Ihre Einstellungen resultieren auch nicht ausschließlich aus den allgemein im Elsass herrschenden représentations der Deutschen, sondern gehen teils auf persönliche Erfahrungen zurück (Camping). Daraus resultiert eine sozial geschichtete Kategorisierung der Deutschen, die offenbar in der Lage ist, den Kontrast zwischen dem „charmanten“ Martin aus „den oberen Schichten“ und den „frechen“ und „gemeinen“ deutschen Kleinbürgern aufzulösen. Aufgrund dieses diskursiven Hintergrunds über die Unterschiede zwischen Deutschen und Franzosen bedarf es keiner großen Überlegungen, um die Fragen der Interviewerin zu beantworten; die Kontraste werden schnell und in großer Übereinstimmung zwischen den beiden Ehepartnern aufgelistet. Dass es sich trotzdem nicht um Einzelmeinungen handelt, zeigt beispielhaft für das Thema „Fanatismus beim Fußball“ der folgende Ausschnitt, in dem sich das Stereotyp des sportfanatischen Deutschen wiederholt:

Ausschnitt (19) [1504]

GP2: sind zwei unterschiedliche welte

I: $\quad$ ah ja/ wàrum

GP2: ça commence par la mentalité/ c'est pas pareil das fängt mit der Mentalität an/ die ist nicht gleich

GP1: ah wie? 
I: ils ont une autre mentalité?

sie haben eine andere Mentalität?

GP2: ouais/ ca se voit au foot/ la rage de vaincre/ ils l'ont plus que nous ja/ das sieht man beim Fußball/ den absoluten Siegeswillen/ den haben sie mehr als wir

Dass Gewährspersonen ohne Zögern sofort stereotypische Zuschreibungen liefern, ist allerdings die Ausnahme. Nicht wenige unserer Gewährspersonen sehen zunächst keine wesentlichen Unterschiede zwischen Deutschen und Elsässern/Franzosen und können höchstens durch mehrfaches Nachhaken dazu gebracht werden, aus ihrem Ressourcenvorrat an Stereotypen zu schöpfen, um sie im Interview zu verwenden; für sie sind sie weniger salient und relevant als für die Gewährspersonen in Ausschnitt (18).

So stellt die Interviewte im folgenden Ausschnitt zunächst (ebenfalls einem verbreiteten Stereotyp folgend) in einem anderen Zusammenhang - Elsässer als Touristen - fest, dass Deutsche in der Öffentlichkeit, z. B. im Restaurant, laut sind und sich auf diese Weise für alle bemerkbar machen, während man als Elsässer eher diskret auftritt. Auf die nachgeschobene Frage, ob es noch andere Charakterunterschiede gäbe, fällt ihr aber zunächst nichts ein. Sie relativiert sogar den genannten Unterschied im Auftreten als „truc de société“: in der Gruppe fühlten sich die Deutschen stark, allein seien sie wohl nicht anders als die Franzosen/Elsässer:

Ausschnitt (20) [1566]

GP: disons contrairement à/ par exemple quand vous avez des Allemands qui viennent chez nous

sagen wir im Gegensatz zu/ zum Beispiel wenn Sie Deutsche haben die zu uns kommen

I: $m$

GP: c'est bruyant das ist laut

I: ah ouais

GP: je crois que que l'alsacien est plus/ se fait plus discret ich glaube dass dass der Elsässer mehr/ sich diskreter aufführt

I: $m$

GP: on revendique pas autant/ disons on revendique on on parle alsacien/ ça oui wir beanspruchen nicht soviel/ sagen wir wir beanspruchen wir wir sprechen elsässisch/ das schon

I: ouais

GP: mais on va pas crier quelque part et se faire euhm euh se faire voir ou se faire entendre ou se faire euh

aber man wird nicht irgendwo rumschreien und sich ähm ähm sich sichtbar machen oder sich hörbar machen oder sich ähm 
[...]

I: d'accord mh et gibt s noch àndere underschiede mit de Ditsche/ sin Ditsche àndersch mit em chàràkter

ok hm und gibts noch andere Unterschiede zu den Deutschen/ sind Deutsche anders in ihrem Charakter?

GP: nä

nein

I: $\quad$ so oder euh

GP: nä

I: non au contraire on a des points communs avec les Allemands umgekehrt haben wir Gemeinsamkeiten mit den Deutschen?

GP: ça je pourrais pas dire je ne fréquente pas assez d'Allemands purs das könnte ich nicht sagen ich hab nicht genug Kontakt mit den richtigen Deutschen

I: non mh par exemple ich mein, hm, zum Beispiel

GP: mais bon c'est aussi un un truc de société c'est-à-dire quand ils sont ensemble ils se sentent forts

aber gut das ist auch ein ein gesellschaftliches Ding das heißt wenn sie zusammen sind dann fühlen sie sich stark

I: ouais

GP: alors que peut-être seuls ils sont comme nous hein während allein sind sie vielleicht wie wir, nicht

I: ouais

GP: parce que nous on va souvent manger en Allemagne euh weil wir gehen öfter nach Deutschland zum Essen ähm

I: $\quad \mathrm{mh}$

GP: ça pose aucun problème das ist überhaupt kein Problem

[...]

GP: c'est c'est c'est pareil das ist das ist dasselbe

I: je veux dire il y a dans les dans la façon de vivre dans enfin dans le ich hab gemeint gibt es bei der bei der Lebensart in ich mein bei dem

GP: non

I: dans les habits les la culture bei der Kleidung, den der Kultur

GP: ah les habits oui c'est pas la France ah die Kleidung ja, das ist nicht [wie in] Frankreich

I: ((lacht))

GP: les habits c'est pas la France ((lacht))

I: ouais ils ont ja, sie haben 
GP: ils ont leurs leurs gouts ((lacht))

sie haben ihren ihren [eigenen] Geschmack

Die Interviewerin muss relativ starke Elizitierungsverfahren anwenden, um die Interviewte zu weiteren Äußerungen über Unterschiede zwischen Elsässern und Deutschen zu bringen. Selbst der Hinweis auf verschiedene Lebensarten führt noch zu einer Verneinung möglicher Unterschiede. Erst als die Interviewerin das Thema Kleidung anspricht, ein sehr starkes Stereotyp über die Deutschen, stimmt die Gewährsperson sofort zu und ist mit der Interviewerin einig, dass der deutsche Kleidungsstil dem französischen (!) nicht entspricht und die Deutschen ihren eigenen, für Französinnen nicht nachvollziehbaren Geschmack hätten. Der Ausschnitt ist nicht zuletzt auch in Bezug auf die Kontrastpaare interessant, die im Gespräch aktiviert werden. Während es zunächst um den Kontrast Deutsche vs. Elsässer geht, wird am Ende des Ausschnitts stillschweigend ein anderes Kategorienpaar relevant gesetzt, nämlich Deutsche vs. Franzosen.

Es ist also auch die Dynamik des Interviews, die für die diskursive Nutzung der Stereotypen von den Deutschen verantwortlich ist. Es gehört zu den Merkmalen der Gattung des sozialwissenschaftlichen Interviews, dass an Punkten weitergefragt wird, an denen im Alltag das Thema beendet wäre. Erst dies führt dazu, dass manche Gewährspersonen Stereotypen über die Deutschen explizit machen. Die Frage der Verwendung solcher Stereotypen für die jeweiligen Zwecke des Gesprächs ist aber unabhängig von ihrer Verfügbarkeit als Teil des gemeinsamen Wissens. Was als Ressource vorhanden ist, muss nicht von jedem auch genutzt werden. Manche Gewährspersonen sind sich überdies der Tatsache bewusst, dass sie auf Stereotypen zurückgreifen müssten, die sich aus ihrer persönlichen Erfahrung heraus nicht bestätigen. Sie verweigern sich der Frage nach einem unterschiedlichen „Charakter“ der Elsässer, Deutschen oder Franzosen. Die gesellschaftliche Verfügbarkeit von représentations als diskursive Ressourcen ist also nicht identisch mit der Frage, ob die befragten Personen glauben, dass „die Deutschen“ die genannten Eigenschaften wirklich „haben“.

Zum Stereotypenrepertoire gehören natürlich auch positive Eigenschaften, die „den Deutschen“ zugeschrieben werden. Obenan steht hier die deutsche rigueur (Strenge, Disziplin), die die Gewährsperson im folgenden Ausschnitt (ein junger Bauer) auch mit persönlichen Erfahrungen belegt:

Ausschnitt (21) [1691]

GP: après tout ce qui est rigueur euh/ la rigueur je vois maintenant dàs siesch maintenant/ on a fait une bstellung là pour les asperges et tout ça letztendlich das was disziplin bedeutet ähm/ die disziplin ich seh jetzt das siehst du jetzt/ wir haben Spargel bestellt und so 
I: $\mathrm{mm}$,

GP: tu/ tu envoies/ deux jours après hesch wàs de brüch hein/ c'est là/ ils t'envoient le truc/ comment tu payes/ pour faire un virement mais c'est tchak tchak/ ils apportent/ c'est emballé c'est pesé

$\mathrm{du} / \mathrm{du}$ schickst es weg/ zwei Tage später hast du was du brauchst, nicht/ es ist da/ sie schicken dir das Zeug/ wie du zahlst/ um eine Überweisung zu machen aber das geht zack zack/ sie bringen es/ es ist eingepackt und gewogen

((Lachen))

GP: minner cousin der màcht $d /$ otto in $d$ garage/ im Ditsche hein/ il emmène hein er kàd/ er kàd/ drackig si hein mein Cousin der bringt sein/ Auto in die Garage/ in Deutschland, nicht/ er bringt es hin, nicht, er kann/ er kann/ dreckig sein, nicht

I: $\mathrm{hm}$

GP: wenn $r$ widder holt/ esch gwascht/ aspiré wenn er ihn wieder holt/ ist er gewaschen/ gesaugt

Die Interjektion zack zack ist ein öfter verwendetes konventionalisiertes Ausdrucksmittel für diese Effizienz der Deutschen (vgl. als Zeichen dieser Konventionalisierung auch den Lautwandel von dt. zack zack zu dem lautmalerisch verstärkten fr. tchak tchak im zitierten Ausschnitt). Es kann, wie in diesem Fall, positiv verwendet werden, ebenso aber auch negativ-bewertend, je nachdem, was der Interviewpartner von der Disziplin der Deutschen hält.

Die kleine Zahl der Elsässer, die sich in der Interviewsituation für einen Umzug nach Deutschland ausspricht, tut dies, wie bereits erwähnt, zu einem Teil aus pragmatischen Gründen: die Nähe der deutschen Städte Karlsruhe und Freiburg zum Elsass würde vergleichsweise die geringere Veränderung des eigenen Lebens mit sich bringen. (Sie gehen von der „postnationalen“ Annahme aus, dass der Umzug nach Deutschland die kleinräumige alltägliche Mobilität über die faktisch kaum existierende Grenze nicht behindern würde, also das Oberrheingebiet eine offene innereuropäische Grenzregion ist.) Soweit die Entscheidung für Deutschland auf einer positiven Einstellung zu den Deutschen (oder Badenern) beruht, sind es in der Regel nicht die stereotypisierten positiven Eigenschaften der Deutschen wie ihre Diszipliniertheit, die dafür ausschlaggebend sind; die Gründe liegen eher in persönlichen Erfahrungen.

Einige der Interviewten entscheiden sich für die deutsche Option allerdings nicht aus pro-deutschen, sondern anti-französischen Gefühlen heraus (Topos der „Unordnung“ in Frankreich vs. deutsche „Ordnung“, [1643]). 


\section{Der „Deutsche im Elsässer“}

Die negative, in einem bestimmten Bereich (nämlich der „Ordnung“ und „Disziplin“) auch manchmal positive Stereotypisierung „des Deutschen“ kommen nicht nur (wenn auch oft verhalten) zum Vorschein, wenn es darum geht zu begründen, warum ein Leben in Deutschland für die meisten befragten Elsässer die schlechtere Option wäre; sie spielt auch eine Rolle, wenn die Elsässer selbst Thema sind. Dann geht es nicht um die Abgrenzung von den Deutschen, sondern um das „Deutsche im Elsässer“. Der rekurrente Topos in den Interviews ist: wir Elsässer verbinden das Beste aus der deutschen und der französischen Welt. Es ist nicht ganz unironisch, dass die mit dem „Deutschen im Elsässer“ verbundenen positiven Selbstzuschreibungen dieselben sind, die bei der ideologischen Konstruktion der Differenz zwischen Deutschen und „uns“ (den Franzosen bzw. Elsässern) den Deutschen zugeschrieben werden.

Wir beginnen mit einem einfachen Beispiel, in dem das Umschlagen vom Kategorienpaar [Franzosen ${ }_{1}$ : Deutsche] in das Kategorienpaar [Franzosen : $_{2}$ : Elsässer] zu beobachten ist:

Ausschnitt (22) [1577]

I: un hàn se aa de salb kàràkter driwwe oder sin se doch e bissel àndersch ingstellt

und haben sie auch denselben Charakter drüben oder sind sie doch ein bisschen anders eingestellt

GP: euhm

I: Ditsche die Deutschen

GP: plus disciplinés disziplinierter

I: ouais

GP: plus disciplinés

I: $\mathrm{mm}$

GP: que nous les Français als wir, die Franzosen

I: $\mathrm{mm}$

GP: et Elsässer hàn viel gerbt vun ne/ ouais zum guete glick und die Elsässer haben viel von ihnen geerbt/ ja, zum guten Glück

I: ah ouais vous trouvez ((Lachen)) ah ja, finden Sie

GP: ((Lachen)) ah oui je trouve

I: ouais 
GP: je trouve qu'il y a quand même euh ce côté germanique qui nous est resté

ich finde, dass uns trotz allem ähm diese germanische Seite geblieben ist

Die Sprecherin beginnt mit der bereits aus Ausschnitt (21) bekannten Gegenüberstellung von Deutschen und Franzosen entlang der Kategorie „Disziplin“. Sie subsumiert sich selbst als Elsässerin klar unter die Kategorie „Franzosen ${ }_{1}$ “ (nous les français). Im zweiten Teil des Ausschnitts verändert sich aber die Perspektive auf die „Elsässer“, deren „germanisches Erbe“ sie den Deutschen annähert. Die Sprecherin ist also zugleich als Französin ${ }_{1}$ von den (disziplinierteren) Deutschen getrennt und als Elsässerin durch ihre Diszipliniertheit mit

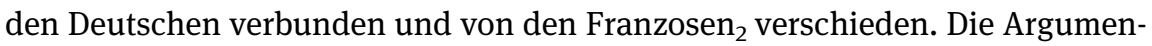
tation ist genetisch (,gerbt“): es gibt ein germanisches Erbe, das den Elsässern „geblieben“ ist, das sie also aus früheren Zeiten in ihre heutige französische Identität hinübergenommen haben.

Das Elsass wird hier aber noch nicht ausdrücklich als Schnittmenge der guten Eigenschaften der Deutschen und Franzosen ${ }_{2}$ dargestellt. Dies geschieht im folgenden Ausschnitt:

\section{Ausschnitt (23) [1596]}

$\mathrm{I}$ : finde $\mathrm{n}$ ihr àss $\mathrm{d}$ ditsche à ànderi mentalität hàn wie mir elsassisch/ äh Elsasser/ y a/ y a une différence

finden Sie dass die Deutschen eine andere Mentalität haben wie wir elsässisch/ ah Elsässer/ gibt's/ gibt's einen Unterschied

GP: ouais sicher/ ouais

I: ouais/ äh/ en quoi/ enfin/ von wie kommt des ja/ äh/ worin/ ich mein/ woher kommt das

GP: ben/ euh/ je pense que nous les Alsaciens on a pris un ptit peu de des Allemands/

naja/ ähm/ ich denke dass wir Elsässer ein kleines bisschen von den Deutschen übernommen haben

I: $\quad \mathrm{mm}$

GP: mais les Allemands ils sont vraiment caractérisés par ordnung quoi aber die Deutschen zeichnen sich wirklich durch Ordnung aus so

[...]

GP: et puis ils sont un peu/ imbus de leur personne euh und dann sind sie ein bisschen/ von sich selbst eingenommen ähm

I: ouais

GP: voilà/ euh/ jtrouve les Alsaciens on a réussi à prendre le bon de l'Allemand so/ ähm/ ich denk die Elsässer haben es geschafft, das Gute vom Deutschen zu übernehmen 
I: $\mathrm{mm} / \mathrm{d}$ 'accord/ ouais

GP: je considère ça comme ça

ich seh das so

I: d'accord/ ouais/ ouais/ et ce serait quoi alors le bon/ c'est le $\mathrm{ok} / \mathrm{ja} / \mathrm{ja} /$ und was wär das dann das Gute/ ist das das

GP: ben au niveau professionnel

naja, auf der beruflichen Ebene

I: ouais

GP: je sais qu'on est beaucoup comparé aux/ Français qui viennent pas de l'Alsace

ich weiß dass wir viel mehr verglichen mit/ den Franzosen die nicht aus dem Elsass kommen

I: $\mathrm{mm}$

GP: dàss mr meh seriös esch dass man seriöser ist

I: $\quad \mathrm{mm}$

GP: dàss meh ordnùng esch in $\mathrm{dr}$ àrweit dass bei der Arbeit mehr Ordnung ist

I: ouais/ mais pas au même point que les Allemands quoi/ enfin/ XXX ja/ aber nicht zum selben Grad wie die Deutschen oder/ halt/ XXX

GP: non/ ouais/ parce qu'apparemment on est quand même accueillants/ frindli

nein/ ja/ weil wir sind vermutlich doch freundlich (frz.)/ freundlich (els.)

I: ouais

GP: euh

I: ouais les Allemands moins du coup/ enfin ja die Deutschen also weniger halt

GP: ouais

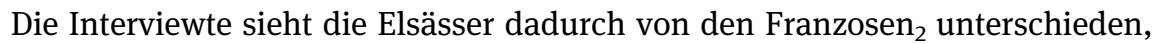
dass sie von den Deutschen die „Ordnung“ (ein Wort, das die Sprecherin auf Deutsch in ihren an dieser Stelle französischen Diskurs einbettet) und „Seriosität“ (wohl im Sinne von „Ernsthaftigkeit“) bei der Arbeit übernommen hätten (on a pris un ptit peu de des Allemands). Aufschlussreich ist die Perspektivierung: die Agentivität liegt bei den Elsässern, die sich das Beste von ihren deutschen Nachbarn ausgesucht und in ihren eigenen Charakter eingebaut haben (les Alsaciens on a réussi à prendre le bon de l'Allemand), dabei aber ihre eigenen guten Eigenschaften (freundlich zu sein) behalten haben. Der französische und der deutsche Kultureinfluss sind also nicht gleichartig: Aus Deutschland wurden positive Eigenschaften der Nachbarn übernommen, die nicht-deutschen positiven Eigenschaften haben die Elsässer schon. 
Der folgende Sprecher verwendet ebenfalls den Topos von den Elässern, die die Vorteile beider Welten, der französischen und der deutschen, vereinen, perspektiviert diese Beziehung zwischen Elsass, Frankreich und Deutschland aber wieder etwas anders:

\section{Ausschnitt (24) [1565]}

I: $\quad$ sin/ gliche d Elsasser/ um umgekehrt gliche d Elsasser meh de Frànzose oder meh in de Ditsche

sind/ sind die Elsässer/ um umgekehrt, sind die Elsässer eher so wie die Franzosen oder eher so wie die Deutschen

GP: ah ja wenn wenn i jetzt in Berlin bin euh: glöw i euh bin i e Frànzos ah ja, wenn wenn ich jetzt in Berlin bin ähm, ich glaub ähm dann bin ich ein Franzose

[...]

I: àwwer do im Elsàss

aber hier im Elsass

GP: ja

I: $\quad$ sin ihr no/ denn wie gsait ihr hànn gsait euh ànderscht wie $d$ Ditsche oder wie wie d Elsasser enfin isch/ wär doch e unterschied zwischem elsässische charakter um Frànzose un de chàràkter vum Frànzos oder sind Sie dann/ weil wie gesagt Sie haben gesagt ähm anders wie die Deutschen oder wie die Elsässer/ also ist/ gäb‘s doch einen Unterschied zwischen dem elsässischen Charakter und dem Franzosen, dem französischen Charakter

GP: ja villicht ja vielleicht

I: $\quad \mathrm{mhm} /$ wàs wär no mhm, was wär dann

GP: euh ich glöb/ ich glöb mir hàn viellicht/ euh ich glöb mir hàn s guete vun euh vun de Schwowe

ähm ich glaub/ ich glaub wir haben vielleicht/ ähm ich glaub wir haben das Gute von ähm von den Deutschen

I: ouais

GP: mir euh ich glàb wenn wenn s euh wenn mir schàffe mien no no no schàffen mir

wir ähm ich glaub wenn wenn’s ähm wenn wir arbeiten müssen dann dann dann arbeiten wir

I: $\mathrm{m}$

GP: euh ich glab ich glab des isch richtich ähm ich glaub ich glaub das stimmt

I: ja

GP: euh un euh euh wàs wàs wàs wàs noch àndersch $\mathrm{X}$ mir hàn s gern wenn s gemietlich ist

ähm und ähm ähm was was was was noch anders [ist] X wir haben es gern wenn es gemütlich ist 
I: $\quad$ mm ja do isch jetzt nit gràd gemietlich mit dem gànze kràmbol $\mathrm{mm}$, ja hier ist es jetzt grade nicht gemütlich mit dem ganzen $\mathrm{Krach}^{10}$

GP: $\mathrm{mm}$ ja ((lacht))

I: ja wie wie meinen ihr des genau ja wie wie meinen Sie das genau?

GP: euh euh $m$ ja gemietlich euh $d$ d des könn ich gàr nit saije uf uf uf frànzesch euh s s euh s mues e so sin m es mues àwwer mir hàn s gern wenn s wenn s euh/ ja pff ich weiß nit es isch nit richtich klàr fir mich äh äh $m$ ja gemütlich äh $d$ d das könnte ich auf französisch gar nicht sagen ähm s s ähm es muß wir sind so es muss aber wir haben's gern wenn's wenn's ähm/ ja pff ich weiß nicht es ist nicht richtig klar für mich

I: $\quad \mathrm{m}$

GP: euhm àwwer mir hànn ö s guete vun de vum Frànkrich ähm aber wir haben auch das Gute von den von Frankreich

I: $\quad$ ah ja

GP: ja e bissel/ doch e bissel euh euh: mir euh làche gar ja, ein wenig/ doch ein wenig ähm ähm wir ähm lachen gern

I: $\quad \mathrm{m}$

GP: $m$ m des isch euh des isch schun guet $\mathrm{hm}, \mathrm{hm}$, das ist ähm das ist schon gut

I: $\quad \mathrm{m} /$ àlso hàn mir s guet von beide sitte $\mathrm{mm}$, also haben wir das Gute von beiden Seiten

GP: ja

I: $m$

GP: ich glöb ja ich glaube ja

I: $\quad \mathrm{m} /$ àlso glichen mir e bissel de Ditsche uf de àrt vun de àrweit $\mathrm{mm}$, also gleichen wir ein bisschen den Deutschen so wie wir arbeiten

GP: ja

I: un de euh Frànzose meh im laawe so und den ähm Franzosen mehr so im Leben

GP: ja ja mir sin seriös ja, ja wir sind seriös

I: $\quad m$

GP: wie wie d Ditsche wie wie die Deutschen

I: $\quad \mathrm{m}$

GP: un euh: àwwer fer s ich glöb mir asse und ähm: aber für's ich glaub wir essen

I: $\quad \mathrm{m}$

10 Die Interviewerin bezieht sich auf das Gewitter während des Gesprächs. 
GP: wie meh wie in Frànkrich

wie mehr wie in Frankreich

I: $\mathrm{m}$, ouais $\mathrm{m}, \mathrm{m}$,

GP: im Ditschland glöw i euh asst mir nit euh so

in Deutschland glaub ich ähm isst man nicht so

I: $\quad \mathrm{m}$, ouais ouais hàn se e ànderi/ isch des e ànderi

$\mathrm{hm}$, ja ja, haben sie eine andere/ ist das eine andere

GP: ah ja euh àm middöj euh sin sin nit viel do wie im Ditschlànd wie àm middöj mit zwei stunge

ah ja, ähm mittags ähm gibt's gibt's nicht viele hier die, in Deutschland, die mittags mit zwei stunden

EX: $m$

GP: pause

I: oui

GP: hàn fer asse haben für's Essen

I: ouais isch des no ebbs wie wie $\mathrm{s}$ ja, ist das dann etwas was was

GP: des isch ich glöb hein des isch meh im Frankrich das gibt's ich glaub, ne, das gibt's mehr in Frankreich

Der Interviewte nimmt zunächst eine wichtige Unterscheidung vor: der Kontrast Elsässer vs. Franzosen ist eine Frage der Granularität. Während aus der Ferne (bei geringerer geografischer Auflösung) die Unterscheidung irrelevant wird und er sich klar als Franzose fühlt, treten auf einer niedrigeren Skalierungsebene die Unterschiede zwischen Elsässern, Deutschen und Franzosen 2 doch zu Tage. Auch diese Gewährsperson ist der Meinung, dass die Elsässer $s$ guete vun euh vun de Schwowe (was für ihn die Ernsthaftigkeit bei der Arbeit ist) und s guete vun de vum Frànkrich (nämlich die Freude am Lachen, die Gemütlichkeit (!) und den Sinn fürs (gute) Essen während einer langen Mittagspause) aufs Beste miteinander kombinieren. Die Interviewerin legt ihm eine Formulierung in den Mund, der er ohne Zögern beistimmt: deutsche Tugenden bei der Arbeit, französische Tugenden beim Leben. Allerdings stellt sich für ihn das Elsass als (passives) Hybrid dar, als Kulturregion, die aus beiden Nachbarkulturen (Frankreich und Deutschland) bestimmte positive Eigenschaften mitbekommen hat. Die Bildlichkeit ist keine agentivische, sondern eher die der Elternschaft: wie ein Kind von den beiden Eltern unterschiedliche Eigenschaften mit in die Wiege gelegt bekommt, so sind für ihn die Franzosen und Deutschen für die verschiedenen positiven Eigenschaften der Elsässer verantwortlich.

Eine letzte Vertreterin der These, dass das Elsass die optimale Kombination aus deutschen und französischen Eigenschaften darstellt, die wir hier betrach- 
ten wollen, ist eine Gewährsperson aus der älteren Generation, die ein Restaurant für deutsche und elsässische Kunden betreibt:

\section{Ausschnitt (25) [1603]}

I: un finden $r$ dàss se driwwe dàss se e àndere chàràkter hàn wie do mir Elsasser und finden Sie dass sie drüben dass sie einen anderen Charakter haben als wir Elsässer?

GP: ich hàb jetzt garn die kültür find i von driwwe ich mag die Kultur, find ich, von drüben ich find sie sin plus/ ender ordonnés àss bi uns ich find sie sind mehr/ ordentlicher als bei uns

I: ouais/ e bissel euh/ ouais/ dans la $\mathrm{ja} /$ ein bisschen ähm/ ja/ im

GP: awer es gibt oi vo àllem/ es gibt oi vo àllem aber es gibt von jeder Sorte welche/ es gibt von jeder Sorte welche

[...]

I: un meinen $r$ dàss mir Elsasser/ hà mir e bissel von dem und meinen Sie dass wir Elsässer/ haben wir ein bisschen davon?

GP: ich glöb mr han devo ich glaub wir haben was davon

I: ouais

GP: $\mathrm{mr}$ sin jo Alemanne wir sind ja Alemannen

I: un die sin/ die Alemanne, die sin und sie sind/ die Alemannen, die sind

GP: do iwwer m Rhin un driwwe gràd so iwwerm Rhin/ mr ha jo die kültür vo ihne/ dis will nit heisse mr si Schwowe hier diesseits vom Rhein und jenseits grad so [wie] diesseits vom Rhein/ wir haben ja ihre Kultur/ das soll nicht heißen, dass wir Deutsche sind

I: füehlen $r$ eich doch àls Frànzos

Sie fühlen sich doch als Franzose

GP: ja àllewaj/ ja àllewaj/ numme jeder mensch kàt siner ding màche/ wàs er euh/ ich hàb jetzt ähm/ ich find jetzt driwwe sin se ender attentif uff de client

ja immer/ ja immer/ jeder Mensch könnte das Seine machen/ was er ähm/ ich hab jetzt ähm/ ich find jetzt dass sie sich drüben mehr um den Gast sorgen

I: $\mathrm{mm} /$ ouais

GP: ass bi uns/ un do derf mr àblöeje als bei uns/ und da dürfen wir uns was abschauen

I: wenn ihr saawe bi uns/ bi uns im Elsàss oder im Frànkrich wenn Sie sagen ,bei uns`/ bei uns im Elsass oder in Frankreich?

((lange Pause)) 
GP: ich find s isch e bissel meh laisser aller (Pause) bis uns

ich find es ist ein bisschen mehr Schlendrian (Pause) bei uns

àlles finde se bissel normàl/ àwer mr muess sich äu miehj ga un ich find dis isch die miehj wo $\mathrm{n}$ sie sich ga

sie finden alles ein bisschen [zu] normal/ aber man muss sich auch Mühe geben und ich find dass sie [die Deutschen] sich die Mühe geben

I: d'accord

GP: so es kommt nix allein/ dis will i sàije

also es kommt nichts von allein/ das will ich sagen

I: d'accord

GP: sie gan sich meh

sie [die Deutschen] geben sich mehr

un dis kà mr ablöeje un kàt e mol guets devon namme

und das kann man sich abschauen und könnte mal [das] Gute davon nehmen

I: $\quad$ ihr namme numme s guete devon hein

Sie nehmen immer das Gute davon, nicht?

GP: ich nimme numme s guete ((lacht))/ ich sàj mr sin prima do/ d Elsasser/ mr namme s guete von de Franzose/ mr namme s guete von de Ditsche/ no kenne mr numme guet si ((lacht))

ich nehm immer das Gute/ ich sag mir wir haben's prima hier/ die Elsässer/ wir nehmen das Gute von den Franzosen/ wir nehmen das Gute von den Deutschen/ dann können wir immer nur gut sein

Die Gewährsperson stellt sich gleich zu Beginn des Ausschnitts als Anhängerin der deutschen (badischen?) Kultur dar und greift zur Begründung auf die bekannten Stereotypen zurück: die Deutschen sind ordentlicher/ordnungsliebender und geben sich bei der Arbeit mehr Mühe. Dabei kommt es über die Kontrastierung auch zu expliziten, negativen Selbstzuschreibungen: zu viel Schlendrian (laisser aller), man gebe sich $\mathrm{zu}$ wenig Mühe. Die Sprecherin macht deutlich, dass sich die Elsässer/Franzosen ${ }_{1}$ etwas von der deutschen Restaurantbranche abschauen könnten.

Die Frage nach den Gemeinsamkeiten zwischen Elsässern und Deutschen bejaht die Interviewte mit Verweis auf die gemeinsame ethnische Zugehörigkeit: diesseits und jenseits der Grenze lebten ja Alemannen. Daraus ergibt sich die Nachfrage der Interviewerin, ob sie mit den Selbstzuschreibungen „Schlendrian“ und „zu wenig Mühe“ spezifisch die Elsässer oder allgemein die Franzosen $_{1}$ meine - denn wenn die Elsässer Gemeinsamkeiten mit den Deutschen haben, müssten sie ja (anders als die Franzosen $_{2}$ ), ebenfalls ordentlich und optimierungsorientiert sein. Diese Frage bleibt allerdings unbeantwortet; es entsteht stattdessen eine lange Pause, die wohl von einer gewissen argumentativen Verwirrung zeugt. 
Am Ende greift die Interviewte auf den Topos vom Elsass als der besten beider Welten zurück, um das Thema abzuschließen: mr namme s guete von de Franzose/ mr namme s guete von de Ditsche. Die Verwendung des Topos ist als konversationelle Strategie erfolgversprechend: er macht keine expliziten negativen, sondern nur positive Zuschreibungen (die negativen Eigenschaften der

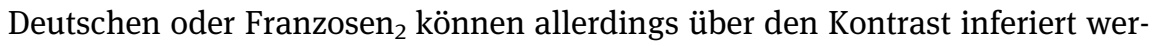
den); zugleich erlaubt er es, die eigene soziale Zugehörigkeit zum Elsass positiv darzustellen. Natürlich baut er auf denselben représentations des deutschen und französischen ${ }_{2}$ „Charakters“ auf, die auch bei der Darstellung des Unterschieds zwischen Deutschen und Franzosen/Elsässern bzw. zwischen Elsässern und Franzosen eine Rolle spielen. Diese werden aber quasi in der positiven Affirmation aufgehoben und münden in eine Eloge der eigenen Identität. Der Topos eignet sich daher auch hervorragend, um Themen abzuschließen. Ihm kann im Elsass kaum jemand widersprechen.

Die Perspektivierung durch die Sprecherin ist, wie in Ausschnitt (23), agentivisch: die Elsässer greifen selbst auf die Nachbarkulturen zurück. Im Gegensatz zur Gewährsperson in Ausschnitt (23) und in Übereinstimmung mit dem Interviewten in Ausschnitt (24) sieht die Sprecherin aber das Elsass als Region zwischen Franzosen 2 und Deutschen.

Trotz des Rekurses auf denselben Topos perspektivieren die drei Sprecher also ihre Aussagen unterschiedlich, wie in den folgenden schematischen Darstellungen zusammenfassend $\mathrm{zu}$ sehen ist:

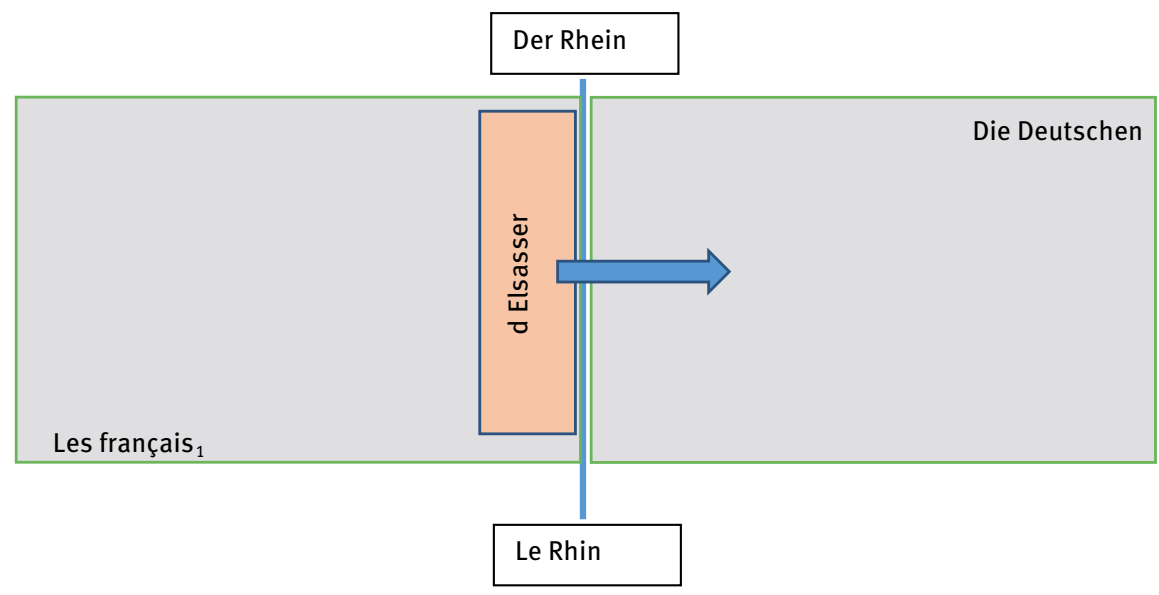

Abb. 1.1: Modell (a), Elsässer aktiv in Bezug auf die Übernahmen aus der deutschen Kultur, zugleich Teil der französischen Kultur (Bsp. 21). 


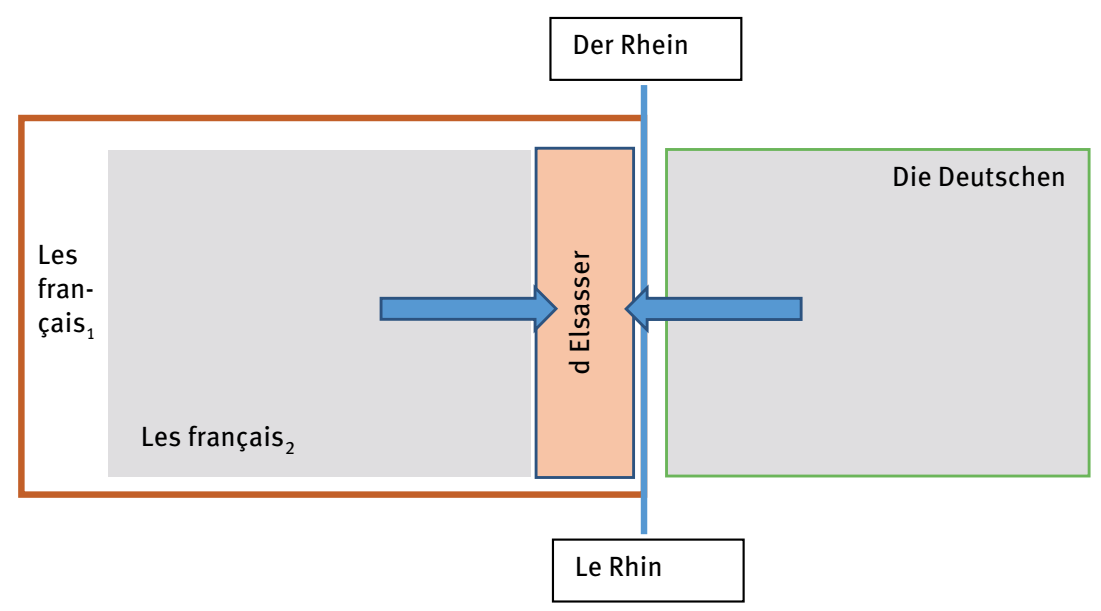

Abb. 1.2: Modell (b), Elsässer passiv mit deutschem und französischem Erbe (vgl. Bsp. 22); bei geringerer Granularität durch die Staatsangehörigkeit mit Frankreich verbunden.

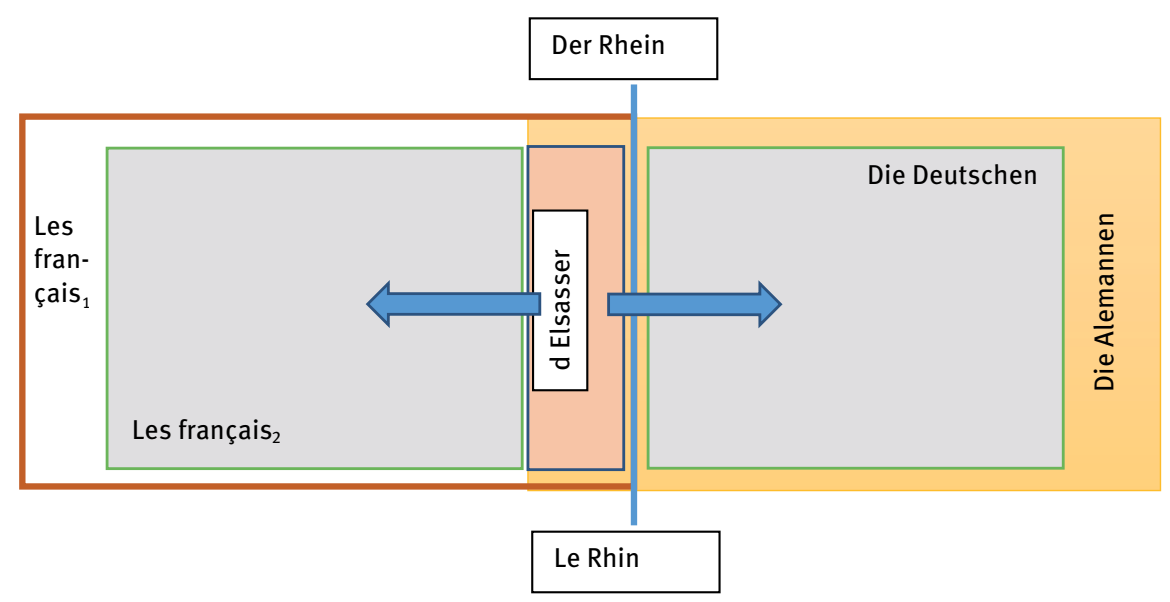

Abb. 1.3: Modell (c), Elsässer aktiv, zwischen Deutschen und Franzosen (vgl. Bsp. 23); durch das alemannische Erbe mit den Badenern, durch die Staatsangehörigkeit mit Frankreich verbunden. 


\section{Abschließende Bemerkungen}

In diesem Beitrag wurden die Stereotypen, die die von uns interviewten elsässischen Dialektsprecher äußern oder erkennen lassen, als Teil der représentations der Elsässer von sich selbst, von den Deutschen (bzw. Badenern) und von den Franzosen (inklusive oder exklusive der Elsässer) untersucht. Diese représentations bilden ein Repertoire von diskursiven Topoi, von denen jeder weiß, dass sie in der Gemeinschaft bekannt sind, und die deshalb in Gesprächen unter Elsässern präsupponiert werden können. Sie können aber auch explizit gemacht und als Ressource für sprachliche Handlungen unterschiedlicher Art verwendet werden.

Stereotype sind ein selbstverständlicher Teil unseres Alltags, ohne den es uns nicht gelingen würde, die Komplexitäten der sozialen Welt, in denen wir leben, zu bewältigen (vgl. Bergmann 2001, Hirschauer 2017). Obwohl Grundlage der sozialen Kategorisierung, sind sie notwendigerweise empirisch falsch, weil sie über abweichende Kategorienmitglieder hinweggeneralisieren. Die Gewährspersonen in unseren Daten sind sich dessen durchaus bewusst. Stereotype müssen deshalb von Meinungen und Einschätzungen unterschieden werden, die auf persönlichen Erlebnissen beruhen; solche persönlichen Erfahrungen können die Stereotypen verstärken oder konterkarieren. Aber auch erfahrungsbasierte „Gegenbeispiele“ setzen stereotypisches Wissen nicht außer Kraft, sondern führen höchstens zu weiteren Differenzierungen, die die Gültigkeit des Stereotyps „retten“ (vgl. die Diskussion zu Beispiel (18)). Solche Erfahrungen sind freilich in unserem Fall aufgrund der meist nur oberflächlichen Kontakte unserer Gewährspersonen mit Deutschen und über die Grenze hinweg nicht häufig. Für die meisten unserer Gewährspersonen ist der Besuch in Deutschland auf ein gelegentliches Einkaufen oder einen Restaurantbesuch beschränkt, der Kontakt im Elsass auf die Sichtung deutscher Autos im Straßenverkehr oder an der Tankstelle bzw. (selten) auf im Elsass lebende und dort bestens integrierte, perfekt frankophone Deutsche.

Das Bild, das sich aus solchen stereotypen Zuschreibungen von „den Deutschen“, „den Franzosen“ und „den Elsässern“ ergibt, muss weder in sich widerspruchsfrei (vgl. den zuletzt diskutierten Ausschnitt (25)) noch frei von Ambiguitäten sein; diese ergeben sich in unserem Fall schon aus der notorischen Unterspezifizierung der Rede von „den Deutschen“ - i. S. v. „Deutsche auf der anderen Seite der Grenze“ (Badener) vs. „Deutsche im allgemeinen“ und der ebenso notorischen Unterspezifizierung der Rede von „den Franzosen“ - i. S. v. „nicht im Elsass geborene/lebende Franzosen“ vs. „Franzosen im allgemeinen“, also einschließlich der Elsässer. Schließlich behauptet die Sicht auf Stereotypen als Teil der représentations, die ich hier verfolgt habe, zwar, 
dass diese im Elsass allgemein bekannt sind, nicht jedoch, dass jeder Sprecher sich mit den in ihnen enthaltenen Zuschreibungen und Bewertungen situationsübergreifend identifiziert, also sie unabhängig von den Kontingenzen der Interaktion für „richtig hält“.

Der Blick auf die représentations ist für interviewbasiert arbeitende Linguisten (und Sozialwissenschaftler) nur durch die Brille dieses definierten Handlungstyps (Gattung) möglich, der eigenen Regeln und Dynamiken folgt. Er macht in Zeiten eines gesteigerten Bewusstseins für political correctness vor allem negative Stereotypisierungen eher unwahrscheinlich; etwas Schlechtes über die Deutschen (oder Franzosen $_{2}$ zu sagen, so wissen natürlich all unsere Interviewpartner, hat keinen großen diskursiven Marktwert. Entsprechend vorsichtig bewegen sie sich in einem schwierigen Gelände. Letztendlich äußern sie sich über „die Deutschen“ so diskret und freundlich, wie es ihrem Autostereotyp entspricht.

Dennoch lässt die Analyse der Daten einige Rückschlüsse zu. Zunächst zeigt die bisherige Auswertung der Daten sehr klar, dass die „europäische“ Vorstellung vom Oberrheingebiet als einer identitätsstiftenden, transnationalen Region, deren Zusammengehörigkeit sich in einer gemeinsamen alemannischen Sprache ausdrückt, für die (dialektsprachigen) elsässischen ${ }^{11}$ Bewohner der grenznahen linksrheinischen Dörfer und Städte keinerlei Realität hat. Die Staatsgrenze am Rhein wird einerseits durch ein solides Bewusstsein von den sprachlichen Unterschieden (trotz struktureller Nähe) und deren nationalsymbolischer Bedeutung ideologisch gestützt, andererseits durch die Konstruktion der mentalité bzw. des „Charakters“ „der Deutschen“, die sich deutlich von der/dem „der Franzosen“ unterscheiden. Dies gilt, obwohl die elsässischen Selbstzuschreibungen einen wesentlichen Anteil der „den Deutschen“ zugeordneten Eigenschaften mit einschließen, wie die Diskussion des Topos vom Elsass als dem Besten aus beiden Welten, der deutschen und der französischen (Abschnitt 5), gezeigt hat.

In der sozialpsychologischen Forschung $\mathrm{zu}$ sprachlichen Einstellungen (vgl. Garrett 2010: Kap. 4 als Überblick) wird zwischen verschiedenen Bewertungsdimensionen unterschieden. Sie werden durch Faktorenanalysen aus einer großen Anzahl von Eigenschaften gewonnen, die einer sprachlichen Varietät im semantischen Differenzial zugeschrieben werden. Oft sind dies die Dimensionen „Überlegenheit“ (mit Eigenschaften wie Status, Macht, Einkommen, Kompetenz), „Attraktivität“ (mit Eigenschaften wie Sympathie und Solidarität) und „Dynamik“ (mit Eigenschaften wie Energie und Einsatzfreudig-

11 Das gilt natürlich auch für die Badener, die hier aber nicht untersucht wurden. 
keit). In soziolinguistischen Untersuchungen zeigt sich üblicherweise, dass der Faktor „Überlegenheit“ mit Varietäten hohen Prestiges (Standardvarietäten) assoziiert wird, der Faktor „Attraktivität“ mit Nicht-Standard-Varietäten. Betrachtet man nun die positiv bewerteten Eigenschaften, die die Elsässer „den Deutschen“ zuschreiben (und die sie zugleich auch für sich reklamieren), so liegen sie auf der „Überlegenheitsdimension“, teils auch auf der DynamikDimension: Disziplin, Ordnung, Effizienz, Engagement. Hingegen liegen die positiven Eigenschaften, die von den Elsässern den Franzosen (und wiederum auch sich selbst) zugeschrieben werden, auf der „Attraktivitätsdimension“ (Freundlichkeit, Entspanntheit, Lebensfreude). Daraus ergibt sich, dass die représentations der Deutschen nicht mit dem Dialekt, sondern höchstens mit dem deutschen Standard assoziiert sind; dieser ist im Elsass aber nicht mehr Teil des Repertoires, weil seine Funktionen durch das Französische abgedeckt werden. Es besteht also keinerlei Grund für eine sprachliche Akkommodation an die regiolektalen Sprechweisen in Baden, deren Sprecher auf der „Attraktivitätsdimension“ ja gerade nicht hoch punkten. Die aufgrund der spärlichen Kontakte über den Rhein hinweg sowieso schon geringen Chancen sprachlicher Konvergenz werden also durch das „Bild des Deutschen“ noch weiter reduziert. Statt Konvergenz hat die Divergenz freien Lauf.

\section{Literatur}

Auer, Peter, Julia Breuninger \& Martin Pfeiffer (2017): Neuere Entwicklungen des Alemannischen an der französisch-deutschen Sprachgrenze im Oberrheingebiet. In Helen Christen, Peter Gilles \& Christoph Purschke (Hrsg.), Räume, Grenzen, Übergänge. Akten des 5. Kongresses der Internationalen Gesellschaft für Dialektologie des Deutschen (IGDD). ZDL Beihefte, 27-44. Stuttgart: Steiner.

Bergmann, Jörg (2001): Kommunikative Verfahren der Konstruktion des Fremden. In Cornelia Bohn \& Herbert Willems (Hrsg.), Sinngeneratoren: Fremd- und Selbstthematisierung in soziologisch-historischer Perspektive, 35-56. Konstanz: UKV.

Bothorel-Witz, Arlette (2008): Le plurilinguisme en Alsace: les représentation sociales (RS) comme ressources ou outils dela description sociolinguistique. Les Cahiers de L'Acedle 5 (1), (= Actes du colloque Acedle Recherches en didactique des langues L'Alsace au coeur du plurilinguisme).

Bothorel-Witz, Arlette \& Dominique Huck (2001): L'allemand en Alsace: Mythe et réalités. In Frederic Hartweg \& Maryse Staiber (Hrsg.), Frontières - Mémoires en hommage à Adrien Finck, 117-140. Straßburg: Presses Universitaires de Strasbourg.

Garrett, Peter (2010): Attitudes to Language. Cambridge: CUP.

Harnisch, Rüdiger (1996): Das Elsaß. In Robert Hindering \& Ludwig M. Eichinger (Hrsg.), Handbuch der mitteleuropäischen Sprachminderheiten, 413-457. Tübingen: Narr.

Hartweg, Frédéric (1983): Tendenzen in der Domänenverteilung zwischen Dialekt und nichtdeutscher Standardsprache am Beispiel des Elsaß. In Werner Besch et al. (Hrsg.), 
Dialektologie. Ein Handbuch zur deutschen und allgemeinen Dialektologie, Bd 2, 1428-1443. Berlin: de Gruyter.

Hirschauer, Stefan (2017): Humandifferenzierung. Modi und Grade sozialer Zugehörigkeit. In Stefan Hirschauer (Hrsg.), Un/doing Difference: Praktiken der Humandifferenzierung, 29-54. Weilerswist: Vebrück Wissenschaft.

Huck, Dominique (2015): Une histoire des langues de l'Alsace. Strasbourg: La Nuée Bleue. Kloss, Heinz (1978): Die Entwicklung neuer germanischer Kultursprachen seit 1800.

2., erweiterte Auflage. Düsseldorf: Schwann.

Irvine, Judith. T. \& Susan Gal (2000): Language ideology and linguistic differentiation. In Paul V. Kroskrity (Hrsg.), Regimes of language: Ideologies, polities, and identities, 35-84. Santa Fe: School of American Research Press.

Jodelet, Denise (1993): Les représentations sociales. Paris: PUF.

Kroskrity, Paul V. (2004): Language ideologies. In Alessandro Duranti (Hrsg.), A companion to linguistic anthropology, 496-517. Malden, MA: Blackwell.

Pfeiffer, Martin \& Peter Auer (i. V.): Erfahrung und Stereotyp an der elsässisch-badischen Grenze - Repräsentationen der Anderen und ihre narrative Verarbeitung. In Nicole Palliwoda, Verena Sauer \& Stephanie Sauermilch (Hrsg.) Politische Grenzen Sprachliche Grenzen? 



\title{
2 "Denn hebbt wi ok mal Platt schnackt.“ Codeswitching in sprachbiographischen Interviews
}

\begin{abstract}
Im Zentrum des Beitrags steht die Frage, welche Funktionen dem Codeswitching in sprachbiographischen Interviews, insbesondere im Kontext von narrativen Identitätskonstruktionen und Positionierungshandlungen, zukommen. Die Interviews wurden im Rahmen des Projekts „Einstellungen gegenüber regionalen Sprachformen in der Großstadt: Niederdeutsch in Hamburg $(\mathrm{NiH})$ “ geführt, in dem das Identifikationspotenzial des Niederdeutschen ermittelt wird.

Als zentrale Aspekte des Codeswitchings haben sich der Ausweis der Sprachkompetenz, die Darstellung der eigenen kommunikativen Überlegenheit sowie der Hinweis auf Informalität und Nähekonstitution herauskristallisiert. Zusätzlich kann Codeswitching der Gesprächsorganisation dienen. Es wird als facettenreiches Instrument eingesetzt, indem die einzelnen Funktionsebenen miteinander verschränkt werden, wobei durch ikonischen Sprachgebrauch die erzählte Situation mit der aktuellen Kommunikationssituation verknüpft wird.
\end{abstract}

Keywords: Codeswitching, Identitätskonstruktion, Narration, Positionierung, sprachbiographisches Interview

\section{1 „Niederdeutsch in Hamburg“: Sprachwahl und Identität}

„Denn hebbt wi ok mal Platt schnackt, aber grundsätzlich ist es schon so, dass hauptsächlich Hochdeutsch gesprochen wird“ (GP39¹). Durch dieses Codeswitching im Kontext eines hochdeutsch geführten sprachbiographischen Inter-

1 GP = Gewährsperson im Interviewkorpus des Projekts „Einstellungen gegenüber regionalen Sprachformen in der Großstadt: Niederdeutsch in Hamburg (NiH)“.

Ingrid Schröder, Universität Hamburg, Institut für Germanistik, Überseering 35, D-22297 Hamburg, E-Mail: ingrid.schroeder@uni-hamburg.de Lara Neumann, Universität Hamburg, Institut für Germanistik, Überseering 35, D-22297 Hamburg, E-Mail: lara.christine.neumann@uni-hamburg.de

Ә Open Access. (C) 2018 Ingrid Schröder und Lara Neumann, publiziert von De Gruyter. (c) BY Dieses Werk ist lizenziert unter der Creative Commons Attribution 4.0 Lizenz. 
views evoziert der Sprecher die referierte Sprachsituation und präsentiert sich als kompetentes Mitglied der Sprechergruppe. Eine erste Analyse narrativer Mittel, die zur Identitätskonstruktion eingesetzt werden, konnte im Rahmen des Projekts „Einstellungen gegenüber regionalen Sprachformen in der Großstadt: Niederdeutsch in Hamburg (NiH)“ bereits exemplarisch aufzeigen, dass Codewechsel ins Niederdeutsche auch Einstellungen gegenüber der verwendeten Sprache offenbaren können. So wird das Niederdeutsche „in emotional aufgeladenen Passagen ikonisch eingesetzt [...], um das referierte Gefühl von Zugehörigkeit zugleich sprachlich umzusetzen“ (Neumann \& Schröder 2017a: 240).

Um das Identifikationspotenzial, das mit dem Niederdeutschen verbunden ist, zu ermitteln, wird im Projekt ein möglicher Zusammenhang zwischen der Sprachwahl Niederdeutsch und einer stadtbezogenen Identitätsstiftung bzw. Identitätswahrung untersucht (zu Projektzielen und zum Projektdesign vgl. Schröder \& Jürgens 2017). Denn obwohl das Niederdeutsche gerade in Hamburg nur noch in geringem Maße in der Alltagskommunikation eingesetzt wird, ${ }^{2}$ spielt es überraschenderweise seit wenigen Jahren in der Öffentlichkeit eine zunehmende Rolle und wird durch entsprechende Namensgebung und Selbstdarstellung von Einrichtungen oder in der lokalen Werbung als Abzeichen für Regionalität bzw. Ortsverbundenheit verstärkt verwendet (vgl. Jürgens 2016).

Um weitere Aufschlüsse über die somit sichtbar werdende Verschiebung von der kommunikativen zur sozialsymbolischen Funktion des Niederdeutschen zu erhalten (vgl. Hess-Lüttich 2004 zur sozialsymbolischen Funktion von Sprache; Bößhenz 2011 zur Dialekt-Standard-Variation; Jürgens 2015: 363-374 zum Niederdeutschen in Hamburg), werden sprachbiographische Interviews mit Gewährspersonen aus fünf Untersuchungsfeldern der Hamburger Öffentlichkeit (Kultur, Medien, Institutionen, Freizeit, Politik) ausgewertet. Das Projekt zielt darauf ab, die Motivation für die Verwendung des Niederdeutschen und für die Auseinandersetzung mit entsprechenden kulturellen und medialen Aktivitäten und Produkten aufzudecken. Darüber hinaus wird untersucht, mit welchen sprachlichen Mitteln personale, soziale und regionale Identitätskonstruktionen in der autobiographischen Erzählung zum Ausdruck gebracht werden.

Im Anschluss an die ersten Beobachtungen zur Rolle der Sprachwahl bei der Identitätskonstruktion soll im Folgenden gezeigt werden, welche Funktionen

2 9,5\% der Bevölkerung in Hamburg beanspruchten 2016 für sich, sehr gute oder gute aktive Niederdeutsch-Kenntnisse zu haben; vgl. Adler et al. (2016: 15). Dass auf die aktive Niederdeutsch-Kompetenz nur in einem geringen Maße zurückgegriffen wird, belegt eine frühere Umfrage von 2007, nach der 54\% derjenigen Befragten, die zumindest einige Wörter sprechen können, vor einem halben Jahr oder früher das letzte Mal Niederdeutsch gesprochen haben; vgl. Möller (2010: 558). 
dem Codeswitching in den hochdeutsch geführten Interviews, insbesondere im Kontext von narrativen Identitätskonstruktionen und Positionierungshandlungen, zukommen. Als Basis für die Analyse soll der Zusammenhang von Identitätskonstruktion und Sprach-/Varietätenwahl erläutert und mit einer Bestimmung von Codeswitching verbunden werden, wobei dessen Funktionen vor allem im Hinblick auf seine identitätsindizierende Rolle im Zentrum stehen (Abschnitt 2). Nach einem Überblick über die vorliegenden Forschungsergebnisse zum hochdeutsch-niederdeutschen Codeswitching (Abschnitt 3) wird das Codeswitching in den sprachbiographischen Interviews des Projekts vornehmlich unter dem Aspekt exemplarisch analysiert, auf welche Weise es einen Beitrag zur narrativen Konstruktion von Identität leistet (Abschnitt 4).

\section{Sprachliche Identitätskonstruktionen und Codeswitching}

Die personale Identität als das ,je spezifische Selbst- und Weltverhältnis sozialer Subjekte“ (Rosa 2007: 47) wird sowohl durch die verschiedenen Rollen, die eine Person im Laufe ihres Lebens einnimmt (personale Teilidentitäten), als auch durch die Zugehörigkeit zu sozialen Gruppen (soziale Identität) geprägt. ${ }^{3}$ Da Identität zu einem erheblichem Maße „durch Sprache und Sprachverwendung konstituiert oder mitkonstituiert wird“ (Thim-Mabrey 2003: 2), kann der Sprachgebrauch bzw. die Sprachwahl - neben anderen Symbolsystemen - Rückschlüsse auf Identitätskonstruktionen erlauben. Sprache wird auf der einen Seite schlechthin als Kristallisationspunkt für Identitätsstiftung beschrieben (vgl. Le Page \& Tabouret-Keller 1985; mit Bezug auf das Niederdeutsche vgl. Scharioth 2015: 36). Dabei wird eine enge Verbindung von Sprache und Identität aufgrund dessen postuliert, dass jede Sprache jeweils spezifische Mittel zur Selbst- und Weltdarstellung bereithalte und darüber hinaus der Sprachgebrauch sozial markiert sei (vgl. Le Page \& Tabouret-Keller 1985: 5). Auf der anderen Seite wird betont, dass Sprachvarianten mit Identitätskonzepten nicht fest verbunden seien, sondern sie vielmehr von den Sprechern verwendet würden, um soziale Bedeutung zu generieren und sich durch stilis-

3 Einen Überblick über verschiedene Theorien bietet Krappmann 2004. Zu den unterschiedlichen Rollen und den damit verbundenen Teilidentitäten vgl. auch Kresić (2006: 6); zur sozialen Identität vgl. grundlegend Tajfel (1981: 255). Auch Regionen „als Handlungs-, Wahrnehmungs- und Bewußtseinsraum konkreter Menschen in ihrer Zeit“ (Flender, Pfau \& Schmidt 2001: 24) dienen als Bezugspunkte sozialer Identität, wodurch regionalen Sprachformen ebenfalls eine identitätsstiftende Funktion zukommen kann. 
tische Praktiken in der sozialen Landschaft zu verorten (vgl. Eckert 2012: 93 f.; zum Konzept der Indexikalität vgl. grundsätzlich Silverstein 2003: 217-222). Vor diesem Hintergrund kann die Frage nach den Funktionen sprachlicher Zeichen und ihrer Varianten nicht generell beantwortet werden, sondern ist immer im jeweiligen (Gesprächs-)Kontext zu betrachten. Dies gilt auch für das Codeswitching, das in Gesprächen als Kontextualiserungshinweis (vgl. Auer 2011: 12) spezifische Identitätskonstruktionen indizieren kann.

Codeswitching soll als der Wechsel von einer Sprache oder Varietät zu einer anderen definiert werden, wobei die beteiligten Sprachen oder Varietäten (im idealisierten Fall) nicht in ihrer Struktur verändert werden (vgl. Lüdi 2004: 342). Diese Definition umfasst das Einfügen einzelner Wörter wie auch ganzer syntaktischer Einheiten in eine andere Sprache oder Varietät sowie den Wechsel von einer Sprache oder Varietät in die andere für längere Äußerungskomplexe (beispielsweise bei Adressierung eines anderen Gesprächsteilnehmers). ${ }^{4}$

Grundsätzlich ist zwischen einem situationellen Codeswitching (vgl. Blom \& Gumperz 1972: 409), das aufgrund äußerer Faktoren wie Adressatenwechsel o. Ä. auftritt, und einem konversationellen Codeswitching (vgl. Gumperz 1982: 59), das einen bestimmten kommunikativen Effekt mit sich bringt, zu unterscheiden. ${ }^{5}$ Codeswitching innerhalb eines Gespräches kann als Kontextualisierungshinweis verstanden werden (vgl. Gumperz 1982: 98), der eine Veränderung des footing mit sich bringt, beispielsweise beim Wechsel zwischen verschiedenen Formalitätsgraden oder zur Signalisierung einer referierten Rolle (vgl. Goffman 1981: 128). Daneben kann Codeswitching auch zur Strukturierung des Diskurses relevant sein: Diskursinduziertes Codeswitching dient der Organisation der Interaktion, teilnehmerinduziertes Codeswitching gibt Hinweise auf Merkmale des Gesprächspartners (vgl. Auer 2009: 491).

In der Forschung ist den kommunikativen Funktionen des Codeswitchings, die der Organisation des Gesprächs dienen, besondere Aufmerksamkeit gewidmet worden (vgl. den Überblick bei Petkova 2016: 55-56; vgl. auch bereits Gumperz 1982: 75-80). Codeswitching kann aber auch expressiv eingesetzt

4 Vgl. den Überblicksartikel von Treffers-Daller 2005. Zu unterschiedlichen Formen des Codeswitchings wie „alternation“, „insertion“ und „congruent lexicalization“ vgl. Muysken 1997, zu Misch- und Übergangsformen vgl. Auer (2009: 506-507); Petkova (2016: 70-73) beschreibt einen „Kreislauf der Kontaktphänomene”. Vgl. zusammenfassend Riehl (2009: 22) und Müller et al. (2015: 11-25). Dem Codeswitching ähnliche Sprachkontaktphänomene wie kleinschrittiges Codemixing und fused lects (vgl. Auer 1999) werden hier nicht behandelt.

5 Blom \& Gumperz (1972: 409) setzen vom situationellen Codeswitching, das den Regeln der Sprechsituation folgt, ein metaphorisches Codeswitching ab, das dem Gesagten eine soziale Bedeutung hinzufügt (dazu auch Blom \& Gumperz 1972: 425). Vgl. Auer (2011: 12), der es generell als Merkmal des Codeswitchings ansieht, dass der Codewechsel als bedeutungsvoll wahrgenommen wird. 
werden, um Einstellungen des Sprechers zum Ausdruck zu bringen (vgl. Appel \& Muysken 1987: 119-120 in Anlehnung an Jakobsons Kommunikationsmodell). Damit steht die Funktion des Codeswitchings als Identitätsmerkmal im Zusammenhang (vgl. Riehl 2009: 26-27). So kann es zur Unterscheidung eines we-Codes und eines they-Codes eingesetzt werden, wobei der we-Code eher informell und der they-Code eher formell markiert ist (vgl. Gumperz 1982: 66). Dabei ist $\mathrm{zu}$ beachten, dass die Zuweisung des we-bzw. they-Codes $\mathrm{zu}$ den verwendeten Sprachen/Varietäten keineswegs von vornherein feststeht, sondern situationsspezifisch variieren kann, abhängig davon, welche Funktionen die Codes im Gespräch erfüllen (vgl. Sebba \& Wootton 1998: 275-276).

Dem Codeswitching kann in Gesprächen eine wichtige Funktion innerhalb

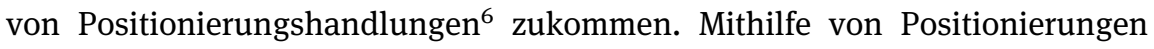
konstruiert ein Sprecher die eigene Rolle im Verhältnis zu anderen Personen, sowohl in der aktuellen Gesprächssituation als auch innerhalb der Erzählung, und teilt seinem Interaktionspartner mit, wie er selbst gesehen werden möchte (Selbstpositionierung) und wie er den Aktionspartner sieht (Fremdpositionierung). Codeswitching kann durch seine expressive Funktion die Positionierung gegenüber dem Gesprächspartner verdeutlichen und solcherart die narrative Konstruktion von Identität unterstützen (zum Konzept der narrativen Identität vgl. u. a. Lucius-Hoene \& Deppermann 2002: 55; zum Projektzusammenhang vgl. Schröder \& Jürgens 2017: 17-20).

Die beschriebenen Zusammenhänge von Identitätskonstruktionen und Codeswitching bilden die Grundlage für die folgende Analyse der Funktionen von Codeswitching in sprachbiographischen Interviews des NiH-Projekts. Auch bei den hier untersuchten bivarietären Niederdeutsch-Hochdeutsch-Sprechern ist keine einfache Zuweisung eines etwaigen we- oder they-Codes möglich, da sie Niederdeutsch zwar in der Regel als Nahsprache konzeptualisieren (vgl. Neumann \& Schröder 2017b; Schröder 2013: 380; zu Niederdeutsch als Nahsprache vgl. auch Stellmacher 1981: 48-49), im Alltag aber, auch in informellen Situationen, überwiegend Hochdeutsch verwenden. ${ }^{7}$ Dies ist auch in der Interviewsituation der Fall.

6 Lucius-Hoene \& Deppermann (2004: 168) bestimmen Positionierung als „die diskursiven Praktiken, mit denen Menschen sich selbst und andere in sprachlichen Interaktionen aufeinander bezogen als Personen her- und darstellen.“

7 Auer (1998: 13) macht darauf aufmerksam, dass insbesondere beim Kontakt von zwei eng verwandten Varietäten die Wahrnehmung der Gesprächsteilnehmer, ob ein oder zwei Codes gegeben sind, von der Wahrnehmung der Analysierenden abweichen kann. Dass eine Unterscheidung auch bei niederdeutsch-hochdeutschen Kontaktphänomenen nicht immer eindeutig zu treffen ist, zeigen Beispiele bei Schröder (2012: 24-27) und Schröder (2015: 35-37, 49); vgl. auch Höder 2011. Solche Fälle finden hier keine Berücksichtigung. 
Aufgrund des Interviewthemas kann Codeswitching als Mittel zur Positionierung insbesondere in Bezug auf die eigene Rolle als kompetenten Niederdeutsch-Sprecher und in Bezug auf die Konzeptualisierung der Sprache als Nahsprache identifiziert werden. Um die Positionierungsverfahren im Einzelnen beschreiben zu können, ist der Varietätenwechsel unter Berücksichtigung weiterer Faktoren wie der Gesprächspartner, der sozialen Rollen, der Situation sowie des Kontextes - sowohl im Rahmen der Interviews als auch innerhalb der Erzählungen - zu beleuchten. Neben den spezifischen Inhalten sind vor allem die expressiven und kommunikativen Funktionen des Codeswitchings in den Blick zu nehmen, um damit zusammenhängende Identitätskonzepte und ihre narrative Konstruktion aufzudecken. Dafür sind die jeweiligen Kontextualisierungen mithilfe einer lokalen Analyse der konversationellen Interaktionen im Einzelfall herauszuarbeiten.

\section{Codeswitching Hochdeutsch - Niederdeutsch}

Bereits mehrfach ist hochdeutsch-niederdeutsches Codeswitching untersucht worden. Als Auslöser konnten Adressaten- und Themenspezifik, Kompetenzunterschiede oder auch als Trigger fungierende sprachliche Elemente herausgestellt werden, als Funktionen wurden vor allem die appellative, die expressive, die metasprachliche und die gesprächsorganisierende hervorgehoben, wie es die folgende Übersicht zeigt:

Auslöser:

- Adressatenspezifik (Herrmann-Winter 1974; Dahl 1974; Johannlükens 1989; Höder 2003; Denkler 2011). Ausschlaggebend für die Sprachwahl ist die sprachliche Kompetenz der Gesprächspartner oder eine spezifische persönliche Beziehung (vgl. Johannlükens 1989: 100-101).

- Themenspezifik (Herrmann-Winter 1974; Dahl 1974; Höder 2003). Dem Hochdeutschen lassen sich Äußerungen und Gesprächssequenzen über eher öffentlichkeits- und institutionsbezogene Themen zuordnen, dem Niederdeutschen Äußerungen über den informellen Nahbereich und über traditionelle Berufsfelder (vgl. Höder 2003: 51-52).

- Kompetenzspezifik, zum Ausgleich von Lücken in der NiederdeutschKompetenz (Höder 2003; Smits 2016).

- Stimulus (Stellmacher 1977; Johannlükens 1989).

Funktionen:

- Appellfunktion, um beim Gesprächspartner Interesse/Aufmerksamkeit zu wecken, an Gefühle zu appellieren oder positive Reaktionen hervorzurufen (Herrmann-Winter 1974; Ziemann 2012). 
- Expressive Funktion, indem Codeswitching ins Niederdeutsche als Ausweis der eigenen sprachlichen Kompetenz genutzt wird (Jürgens 2015; Scharioth 2015; Jürgens \& Schröder 2016), dem Ausdruck von Emotionen oder von Spontaneität dient (Herrmann-Winter 1974; Dahl 1974; Stellmacher 1977; Johannlükens 1989; Höder 2003; Ziemann 2012; Neumann \& Schröder 2017a), eine positive Bewertung des Niederdeutschen signalisiert (Jürgens 2015; Jürgens \& Schröder 2016) und als Identifikationssignal (Stellmacher 1977; Ziemann 2012) genutzt wird.

- Metasprachliche Funktion, mit der die Modalität der Äußerung angezeigt wird, durch Emphase zur Hervorhebung der Relevanz des Gesagten (Johannlükens 1989), durch (Situations-)Komik (Stellmacher 1977; Johannlükens 1989; Scharioth 2015), zur Herstellung von Authentizität (Ziemann 2012; ähnlich auch die Funktion des token codeswitching bei Reershemius 2001) oder als Signal der Informalität (Scharioth 2015).

- Gesprächsorganisierende Funktion, indem Codeswitching der Gesprächsgliederung dient (Denkler 2007, 2011; Ziemann 2012) und für Zitate (Stellmacher 1977; Johannlükens 1989; Ziemann 2012), Beispiele (Stellmacher 1977; Jürgens \& Schröder 2016) oder beim Gebrauch stereotyper Wendungen (Herrmann-Winter 1974; Ziemann 2012) eingesetzt wird.

Der Überblick zeigt, dass eine identitätskonstruierende Funktion bisher nur in wenigen Fällen konstatiert wurde. Während Stellmacher allgemein auf das identitätsstiftende Potenzial des Dialekts als „eines soziolektalen Identifikationssignals“ (Stellmacher 1977: 163) abhebt, bringt Ziemann (2012: 187) die authentizitätsvermittelnde Funktion des Codeswitchings mit der Betonung der Identität als Niederdeutsch-Sprecherin zusammen, also letztlich mit der Anzeige der eigenen Sprachkompetenz. Denkler (2011: 156), der Alltagsgespräche analysiert, misst dem Codeswitching ins Niederdeutsche hingegen kein besonderes Bedeutungspotenzial zu, es trage vielmehr zur Kontextualisierung bei und werde zur Gesprächsorganisation genutzt.

Im Folgenden kann jedoch gezeigt werden, dass in sprachbiographischen Interviews, in denen die Modellierung des eigenen Lebens und der eigenen Sprache das primäre Ziel darstellt, Codeswitching in Positionierungshandlungen expressiv die Selbstdarstellung verstärkt und dazu beiträgt, das Verhältnis $\mathrm{zu}$ anderen Personen zu modellieren. Gleichzeitig übernimmt es auch Kontextualisierungs- und gesprächsorganisierende Funktionen. Die bereits vorliegenden Analysen der NiH-Interviews haben nachweisen können, dass durch Codeswitching die eigene Sprachkompetenz emblematisch belegt wird (vgl. Jürgens \& Schröder 2016: 378; ferner Jürgens 2015: 228) und Niederdeutsch mit dem Ausdruck des Gefühls von Heimat und Geborgenheit verbunden wird 
(vgl. Neumann \& Schröder 2017a: 233). In beiden Beispielen fungiert Codeswitching zugleich als Element der narrativen Identitätskonstruktion und der Positionierung.

\section{Codeswitching in sprachbiographischen Interviews}

Für die vorliegende Analyse wurden die Daten von sechs Gewährspersonen herangezogen. ${ }^{8}$ Im Folgenden werden solche Interview-Passagen näher analysiert, in denen Codeswitiching dazu beiträgt, die eigene Identität narrativ auszudrücken und sich zu positionieren. Es handelt sich um drei Funktionen, die beim Codeswitching zur Darstellung der eigenen Identität abzuheben sind: (1) Codeswitching als Ausweis von Sprachkompetenz; (2) Codeswitching als Hinweis auf Informalität und Nähekonstitution; (3) Codeswitching zur Darstellung kommunikativer Überlegenheit. Bei allen drei Funktionen soll untersucht werden, welche thematischen Bezugnahmen im Zusammenhang relevant sind, auf welche Weise Codeswitching im Rahmen von Positionierungshandlungen eingesetzt wird und ob es zugleich eine gesprächsorganisierende Funktion erfüllt. Zunächst werden relevante Gesprächspassagen ausgewählt, in denen die Sprecher über ihr eigenes Sprachverhalten reflektieren und dabei vom Hochdeutschen ins Niederdeutsche wechseln (und zurück). Diese Passagen werden dann einer kleinschrittigen Analyse unterzogen, bei der das Codeswitching als Kontextualisierungshinweis gewertet wird.

8 Insgesamt sind im Korpus 37 Interviews mit über 1.000 Fällen von Codeswitching enthalten. Nicht alle Passagen sind zur Analyse der Funktionen des Varietätenwechsels und dessen Zusammenhang mit Identitätskonstruktionen gleichermaßen aussichtsreich. Ausgeschlossen werden an dieser Stelle Eigennamen oder Titel (z. B. „Plattdüütsch Root“, „Talk op Platt“, „Tüdelband“), die etwa ein Fünftel der gesamten Fälle ausmachen. Darüber hinaus werden Codewechsel ausgeklammert, die im Zusammenhang mit Sprach- oder Varietätenvergleichen auftreten („Nä, also die sagen hier merkwürdigerweise ((.)) zu ,Bier ' nicht ,Beer‘, sondern ,Bier.“) oder andere Sprachwissensbestände implizieren („Also, es gibt zum Beispiel dieses Wort ,Deuster, hab ich noch nie gehört.“; „Da kann man doch auch ,kookt" schreiben, ne.“). Rund ein Viertel der annotierten Codeswitching-Phänomene sind in einen solchen metasprachlichen Kontext eingebettet. 


\subsection{Codeswitching als Ausweis von Sprachkompetenz}

Fragen zur eigenen Sprachkompetenz oder zum Sprachgebrauch lösen den Wechsel ins Niederdeutsche aus, wodurch die Kompetenz der Sprecher emblematisch zum Ausdruck gebracht wird. Dabei kann die durch Codeswitching demonstrierte Niederdeutsch-Kompetenz als Teil des personalen oder sozialen Identitätskonzepts modelliert werden.

GP30 (weiblich, *1958) hat Niederdeutsch als L1 gelernt und spricht auch gegenwärtig mit ihrer Familie oder Freunden vornehmlich Niederdeutsch. Neben der kommunikativen Bedeutung im Sprachalltag der Gewährsperson kommt dem Niederdeutschen auch ein symbolischer Wert als ihre „Herzenssprache“ zu. Die Beschreibung des niederdeutsch geprägten Alltags geht mit einem Wechsel ins Niederdeutsche einher:

I: $\quad \mathrm{Hm}$, gab es mal so besonders negative Reaktionen darauf, dass Sie Plattdeutsch sprechen oder andersrum auch besonders positive?

GP30: Positive, sehr viel.

I: Ja.

GP30: Sehr, sehr viel, ja. Also negative nur, wenn ich zu schnell gesprochen hab.

I: $\quad$ ((lacht 1sek))

GP30: Beim Theaterstück da ham die/ hat der Regisseur denn manchmal gebrabbelt, der Vorsitzende manchma gebremst, hat gesacht: „Nich so schnell, nich so schnell.“ ((.)) Für mich is dat aber ganz normol. Ik snack jeden Dach Plattdüütsch, wenn ik plattdüütsch Theoter speel. Ik mutt dat nich liern wie ne annere Sprook. ((.)) Nä. Ik snack so.

Für mich ist das aber ganz normal. Ich spreche jeden Tag Plattdeutsch, wenn ich plattdeutsches Theater spiele. Ich muss das nicht lernen wie eine andere Sprache.

I: $\quad($ lacht 1sek))

GP30: Aber für ihn war das denn zu schnell, nä,

I: $\quad$ Okay.

GP30: und dann äh sacht er: „Langsam, das verstehen die nich“, weil die ins Theater gehen, das sind zwar Plattdeutsch-Interessierte, aber nich, wie man denkt, alles Leute, die Plattdeutsch

I: Ja, ja. Klar.

GP30: können, nä.

Als Antwort auf die Frage, welche Reaktionen das Niederdeutsche bei anderen Personen hervorgerufen habe, berichtet GP30, viele positive Rückmeldungen erhalten zu haben. Negative Reaktionen könne GP30 nur für Personen mit niedrigerer Sprachkompetenz konstatieren, die ihr nicht gut folgen konnten 
(„nur, wenn ich zu schnell gesprochen hab“). Diese stellt die Sprecherin, die in ihrer Freizeit in einer plattdeutschen Theatergruppe spielt, durch ein hochdeutsches Zitat des Regisseurs („Nich so schnell, nich so schnell.“) dar. Im Anschluss lenkt GP30 mit der hochdeutschen Präpositionalphrase „Für mich“ den Fokus auf die eigene Person. Die veränderte Perspektive löst einen Wechsel der Sprachform aus. GP30 äußert sich kommentierend zu ihrer Sprachkompetenz und zu ihrem Gebrauch des Niederdeutschen als Alltagssprache, was sie sprachlich durch Codeswitching realisiert („Für mich is dat aber ganz normol.“). Dass das Niederdeutsche mit der personalen Identität der Sprecherin verknüpft ist, wird somit zugleich durch den Inhalt wie durch die Codewahl transportiert. Das Niederdeutsche wird zudem als Muttersprache konzeptualisiert (,Ik mutt dat nich liern wie ne annere Sprook. ((.)) Nä. Ik snack so.“). Durch ein zustimmendes Lachen der Interviewerin wird die niederdeutsche Passage beendet. Als GP30 die Schilderung der Situation wieder aufgreift, wechselt sie zurück ins Hochdeutsche und verwendet erneut ein Fremdzitat.

Dem Codeswitching kommt in dieser Passage außerdem eine gesprächsorganisierende Funktion zu, da es den Übergang zwischen Erzählung und Kommentierung (und zurück) anzeigt. Darüber hinaus kontrastiert GP30 durch den Codewechsel ihr Selbstbild als kompetente Sprecherin, für die Niederdeutsch ein Teil des sprachlichen Alltags („normol“) darstellt (Selbstpositionierung), gegenüber „Plattdeutsch-Interessierte[n]“, die zwar ins niederdeutsche Theater gehen, in Wahrheit jedoch keine kompetenten Sprecher seien (Fremdpositionierung).

Im Gegensatz zu GP30 verwendet GP63 (männlich, *1948), der an einer Hamburger Grundschule als Lehrer arbeitet und sich in einem Verein für die niederdeutsche Sprachpflege engagiert, in seinem Alltag zumeist Hochdeutsch. So sei es bereits „Gewohnheit“, dass GP63 selbst mit seiner niederdeutschkompetenten Ehefrau überwiegend Hochdeutsch spreche. Im Interview wurde nach Situationen gefragt, in denen sich der Sprecher auf Niederdeutsch unterhält.

I: $\quad$ Ähm und äh so mit Freunden oder Nachbarn äh, ((.)) gibt es da noch so im Bekanntenkreis oder auch wenn Sie

GP63: Ja.

I: $\quad$ zum Beispiel mit Leuten aus dem Verein

GP63: Also da spre...

I: abseits des Vereins sprechen sozusagen.

GP63: Då schnackt wi Platt. Da sprechen wir Platt.

I: Okay. Ja. 
GP63: Aber sonst also äh äh dort wo Freunde und Bekannte das Platt können,

I: Hmhḿ.

GP63: da k-kommt auch immer in ne Sequenz irgendwie in der einen oder anderen Weise Plattdeutsch rein.

I: Hmhḿ.

GP63: Und da vermischt sich das auch.

I: Ja.

GP63: Und äh da wo wir wissen, dass da kein plattdeutscher Hintergrund oder norddeutscher Hintergrund is, då versoikt wi dat går ni. da versuchen wir es gar nicht.

Noch bevor die Interviewerin ihren Turn beenden kann, bestätigt GP63, dass er im Bekanntenkreis noch Niederdeutsch spreche, anfangs auf Hochdeutsch („Also da spre...“). Nachdem die Interviewerin die Gebrauchsdomäne spezifiziert hat (,abseits des Vereins sprechen sozusagen“), reformuliert und beendet GP63 seine abgebrochene Äußerung auf Niederdeutsch („Då schnackt wi Platt.“). Der Äußerungsabbruch sowie die Korrektur zugunsten des Niederdeutschen deuten an, dass Codeswitching an dieser Stelle nicht nur eingesetzt wird, um Sprachkompetenz zu belegen, sondern auch, um die referierte Situation durch die Sprachwahl abzubilden. Gleichzeitig wird die soziale Zugehörigkeit der Gewährsperson zur niederdeutschen Sprechergruppe sichtbar, die auch deiktisch indiziert wird (,Då“, „wi“). Inhalt und Sprachwahl korrelieren miteinander, indem der thematisierte Sprachgebrauch niederdeutsch realisiert wird. GP63 switcht im Anschluss zurück ins Hochdeutsche und erläutert den Sprachgebrauch unter Freunden und Bekannten, mit denen ,irgendwie in der einen oder anderen Weise Plattdeutsch“ in das Gespräch eingeflochten werde. Ein erneuter Wechsel ins Niederdeutsche tritt auf, als GP63 den Kontakt mit Personen beschreibt, die ,kein[en] plattdeutsche[n] Hintergrund oder norddeutsche[n] Hintergrund“ aufweisen („då versoikt wi dat går ni“). Im Unterschied zum ersten Wechsel schildert der Sprecher nun, in welcher Situation kein Niederdeutsch gesprochen werde, sodass Inhalt- und Formseite in einem gewissen Kontrast zueinander stehen. Das Niederdeutsche signalisiert hier die soziale Identität. Ihm wird die Funktion eines we-Codes zugewiesen, der im informellen Kontext mit Freunden und Bekannten gebraucht wird und soziale Zugehörigkeit (,wi“) in Abgrenzung zu Hochdeutschsprechern (,då“) konstituiert. Codeswitching wird gesprächsgliedernd eingesetzt, indem es als Rahmen Beginn und Ende eines thematischen Abschnittes markiert. 


\subsection{Codeswitching als Hinweis auf Informalität und Nähekonstitution}

Das Konzept der Nahsprache spielt eine besondere Rolle, um die soziale Zugehörigkeit der Sprechergruppe anzuzeigen. So berichtet GP49 (männlich, *1936) u. a. davon, dass das Niederdeutsche insbesondere im Berufsleben von Vorteil sei und er als Tischler sowohl im Betrieb als auch im Kontakt mit Kunden Niederdeutsch spreche. Im Interview stellt der Sprecher besonders die kontaktfördernde Funktion des Niederdeutschen heraus, nämlich dass durch die Sprache eine „direkte Beziehung“ zum Gesprächspartner aufgebaut werde. An dieser Stelle hakt der Interviewer ein:

I: $\quad$ Und dann is das ne andere ((.)) Nähe oder...

GP49: Andere Nähe, ganz anderes Verhältnis.

I: Ja. ((.)) Ja. Hmhḿ.

GP49: „So nu drink man een“ und „nu mook du dat.“ Erst ma bist du schon im Du. Aber das is man ja auch im Englischen und is ja kein Problem. „So nun trink doch einen“ und „nun mach du das“.

I: $\quad \mathrm{Hm}$.

GP49: Nech, erst ma is man schon im Du ((.)) und dann äh geht das äh/ geht das an sich flotter von/ vonner Hand, nä.

GP49 resümiert seine gesammelten Erfahrungen zuerst auf Hochdeutsch: „Andere Nähe, ganz anderes Verhältnis.“ Nach einer zustimmenden Hörerrückmeldung des Interviewers illustriert GP49 seine Beobachtungen exemplarisch mit einer fingierten wörtlichen Redewiedergabe auf Niederdeutsch, in der aufgefordert wird, Alkohol zu trinken („So nu drink man een“) und etwas zu tun („nu mook du dat.“). Der Wechsel ins Niederdeutsche dient als Belegbeispiel, um die Herstellung von Nähe und Informalität im beruflichen oder öffentlichen Umfeld zu veranschaulichen. ${ }^{9}$ Das Niederdeutsche signalisiert an dieser Stelle eine Veränderung des Kontextes, indem durch das Zitat eine Situation gestaltet wird, in der das Niederdeutsche als we-Code fungiert. Durch die Sprachwahl, durch die Thematisierung des Alkoholtrinkens und durch die Verwendung des Anredepronomens ,du“ wird ein geringer Formalitätsgrad evoziert, der soziale Nähe impliziert. Die Erläuterung, warum das Niederdeutsche eine besondere Nähe herstelle, nimmt GP49 dagegen auf Hochdeutsch vor. Die höhere Formalität der Sprachlage korrespondiert mit der Verbalisierung des Sprachwissens

9 Kurz zuvor wird geschildert, dass die Gewährsperson selbst mit einem Hamburger Bürgermeister Niederdeutsch gesprochen habe. 
(,Erst ma bist du schon im Du. Aber das is man ja auch im Englischen und is ja kein Problem.“).

Das Codeswitching indiziert in diesem Beispiel das Konzept von Nahsprache und trennt gleichzeitig wörtlich zitierte Rede von metasprachlichen Äußerungen. Um Nähe zu Gesprächspartnern herzustellen, kann der Sprecher die soziale Zugehörigkeit zur niederdeutschen Sprechergruppe ausnutzen. Damit positioniert sich GP49 gegenüber dem Interviewer nicht nur als kompetenter Niederdeutsch-Sprecher, sondern auch als kommunikativ versiert und flexibel.

Das Alkoholtrinken als Symbol für einen niedrigen Formalitätsgrad bzw. Vertrautheit wird auch in der gemeinsamen Erzählung des Ehepaars GP03 (weiblich, *1941) und GP04 (männlich, *1938) im Zusammenhang mit der Verwendung des Niederdeutschen thematisiert. Als eine Situation, in der sich das Niederdeutsche als nähestiftend erwiesen habe, schildern GP03 und GP04, dass die Großmutter von GP03 dem späteren Ehemann der Enkelin bei der ersten Begegnung aufgrund seiner Niederdeutsch-Kompetenz besondere Sympathie entgegenbrachte.

GP03: Die wollte uns schon auch/ ((.)) ich

I: $\quad[. .$.

GP03: äh brachte meine Oma dann nach Hause und er kam mit. Also mitm Auto denn ins [Landkreisname]. Und dann äh ging sie ans/ an ihr Schapp/ also an ihren Schrank

I: Hmhḿ.

GP03: un denn hätt se secht: „Wi wüllt mal 'n Lütten neem, nech.“ Un denn:

„Op äh gode Verwandtschaft.“ ((.)) Da war ich ja wütend. und dann hat sie gesagt: „Wir wollen mal einen Kleinen nehmen, nicht.“ Und dann: „Auf äh gute Verwandtschaft.“

GP04: ((lacht 1sek))

GP03: Ich kannte den noch gar nich so lange und sie wollt mich schon verheiraten.

I: $\quad$ ((lacht 1sek))

GP03: ((lacht 3sek)) Aber es war so ne liebe Oma. Also

I: $\quad$ ((lacht 1sek))

GP03: man konnte ihr nich böse sein. Aber da/ also ihn hat sie gleich ins Herz geschlossen. Ja.

Den Wechsel ins Niederdeutsche initiiert GP03 zögerlich, als sie nachzeichnet, wie ihre Großmutter bei sich zu Hause angekommen ,ans/ an ihr Schapp/ also an ihren Schrank“ ging. Hier wird nicht nur die erzählte Situation im Haus der Großmutter aufgerufen, sondern durch das niederdeutsche „Schapp“ zugleich 
die Kenntnis von exklusiv niederdeutschen Wörtern unter Beweis gestellt (Selbstpositionierung). Die Übersetzung des Wortes deutet an, dass GP03 eine geringe Niederdeutsch-Kompetenz bei der Interviewerin annimmt (Fremdpositionierung). Diese wiederum reagiert mit einer zustimmenden Hörerrückmeldung, die der Gewährsperson signalisiert, dass sie ihr folgen könne. Die Sprecherin switcht im Anschluss ins Niederdeutsche, um die erzählte Situation weiter zu reaktivieren (,un denn hätt se secht: ,Wi wüllt mal 'n lütten neem, nech.' Un denn: ,Op äh gode Verwandtschaft.“'). Dass bereits die Ankündigung des Fremdzitats auf Niederdeutsch stattfindet, indiziert, dass die Erzählerin selbst auch als kompetente Sprecherin gesehen werden will (Selbstpositionierung). Im niederdeutschen Zitat wird die zuvor auf Hochdeutsch beschriebene Nähe bzw. Verbundenheit, die das Niederdeutsche stiftet, schließlich lebendig gestaltet und zugespitzt: Die Großmutter, die GP04 offensichtlich erst kennengelernt hat, wollte direkt auf die gute Verwandtschaft anstoßen.

Der Wechsel ins Niederdeutsche dient ähnlich wie bei GP49 zunächst illustrativen Zwecken innerhalb eines Belegbeispiels, kennzeichnet gleichzeitig aber auch den Höhepunkt der Erzählung. Dabei wird - nicht zuletzt auch durch die Thematisierung des gemeinsamen Anstoßens - eine informelle Atmosphäre aufgerufen, sodass das Niederdeutsche auch hier als Nahsprache konzeptualisiert wird. GP03 wechselt zurück ins Hochdeutsche, um die Situation retrospektiv zu kommentieren („Da war ich ja wütend.“) und überspitzt zu paraphrasieren („Ich kannte den noch gar nich so lange und sie wollt mich schon verheiraten.“). Als die Gesprächspartner einvernehmlich lachen, modifiziert die Sprecherin ihre implizite Bewertung („Aber es war so ne liebe Oma. Also man konnte ihr nich böse sein.“), um schließlich den Kern der Geschichte nochmals hervorzuheben („Aber da/ also ihn hat sie gleich ins Herz geschlossen. Ja.“).

\subsection{Codeswitching zur Darstellung kommunikativer Überlegenheit}

Auch die Darstellung der eigenen Durchsetzungsfähigkeit und kommunikativen Überlegenheit kann eng mit der Illustration der Sprachkompetenz verbunden sein. So stellt GP18 (männlich, $\left.{ }^{\star} 1935\right)$ dar, dass seine NiederdeutschKenntnisse seine berufliche Karriere mehrfach befördert haben. Um sich in Berichten über seinen beruflichen Alltag in der Hamburger Behörde als schlagfertig und kommunikativ überlegen darzustellen (vgl. dazu auch Neumann \& Schröder 2017a: 238-239), nutzt GP18 den Wechsel ins Niederdeutsche. 
GP18: [...] Wir komm nach [Ortsname], wollten meinetwegen um zwölf ((...)) bei [Personenname] sein. De/ der hatte den Ökelnamen/ den Spitznamen Heinnäs oder Nasemann. Der hatt so'n Zinken, nä. Na ja, waren irgendwie dor nich Klock twolf, Klock twei. ((.)) Der stand irgendwie aufm Misthaufen, war am Umschaufeln oder was ((.)) und wir kommen aufm Hof, nä. [Vorname] sacht: „Guten Tach“ und „bekannt moken, Herr [Nachname] vom Gänsemarkt“ und so, nä. ((.)) „Wat? Klock twolf hest du secht. Wi hebbt hier toift, dat Eten wäär trecht, aalns wäär mookt und wer kommt nich? Ji baiden. Nu seet man to, dat ji von Hoff koomt. Nu heff ik für euch keine Tiet.“ Soll/ secht [Vorname]: „Wenn't so is, dann koomt wi wedder.“ Ik sech: „Teuf ma af, du.“ ((.)) Ik sech: „Nu ma sinnich. Ik kann nich ((unverständlich 2 sek)) kann nich so wat daför, dat wi nu 'n bitten wat loot sünn, aber de Goosmarkt, de Finanzbehörde, de loot sich von keinen wechschicken. Nu bün ik einma hier un nu ward ok schnackt, ob di dat passt oder nich. Diene Schietgummistevel kannst geern anbehooln, aber diene Fork, de pack ma ut de Hand, nä.“ Hett he mi ankeken, nä, fiel die Kinnlade runter, nä. Na ja, wir waren da nicht um zwölf Uhr, zwei Uhr. ... „Was? Zwölf Uhr hast du gesagt. Wir haben hier gewartet, das Essen war fertig, alles war gemacht und wer kommt nicht? Ihr beiden. Nun seht doch zu, dass ihr vom Hof kommt. Jetzt habe ich für euch keine Zeit.“ Soll/ sagt [Vorname]: „Wenn es so ist, dann kommen wir wieder.“ Ich sage: „Warte mal ab, du.“ Ich sage: „Nun mal langsam. Ich kann nicht ((unverständlich 2 sek)) kann nichts dafür, dass wir nun ein bisschen spät dran sind, aber der Gänsemarkt, die Finanzbehörde, die lässt sich von niemandem wegschicken. Jetzt bin ich einmal hier und nun wird auch geredet, ob es dir passt oder nicht. Deine dreckigen Gummistiefel kannst du gern anbehalten, aber deine Forke lege mal aus der Hand, nä.“ Hat er mich angeschaut, nä.

I: $\quad$ ((lacht 1sek))

GP18: Und denn äh die Forke weg. Na wir ham 'n wunderbares Verhältnis hinterher, wunderbar schnackt ((.)) [...]

Die räumlich-zeitliche Verortung der Episode wird zunächst hochdeutsch vorgenommen (,Wir komm nach [Ortsname], wollten meinetwegen um zwölf ((...)) bei [Personenname] sein.“) und dann niederdeutsch reformuliert, da die zweistündige Verspätung des Erzählers für die Komplikation (Kategorien der Erzählungsstruktur nach Labov \& Waletzky 1967) eine zentrale Rolle spielt. Nachdem der situative Rahmen weiter hochdeutsch gestaltet wird, folgt die Komplikation, nämlich die Ablehnung des Landwirts, das Gespräch mit den Behördenvertretern verspätet zu führen, auf Niederdeutsch. Da GP18 die Anekdote erzählt, um die positive Rolle des Niederdeutschen in seinem beruflichen Alltag $\mathrm{zu}$ illustrieren, ist für die Resolution von Bedeutung, dass die Zurückweisung der Ablehnung niederdeutsch formuliert wurde. Der Sprecher vermag sich mithilfe des Niederdeutsch-Gebrauchs durchzusetzen, nachdem sein Kollege 
schon klein beigeben wollte. Der beim Gesprächspartner ausgelöste Effekt wird zunächst ebenfalls niederdeutsch formuliert („Hett he mi ankeken, nä“), und dann, adressiert an die Interviewerin, die das Gesagte mit einem kurzen Lachen quittiert, hochdeutsch reformuliert („fiel die Kinnlade runter, nä“). Der Sprecher bleibt im Hochdeutschen und wechselt noch einmal emblematisch ins Niederdeutsche mit „schnackt“, um anzuzeigen, dass in späteren Gesprächen Niederdeutsch verwendet wurde. In diesem Zusammenhang wird durch die positive Bewertung des persönlichen Verhältnisses der Verhandlungspartner Niederdeutsch als Nahsprache konzeptualisiert.

Niederdeutsch wird hier für die Wiedergabe des Gesprächs genutzt, bei dem es vor allem darauf ankommt, dass GP18 mit der Sprachwahl ein Überraschungsmoment ausnutzt, da sein Name und seine Funktion hochdeutsch eingeführt wurden („Herr [Nachname] vom Gänsemarkt“), er dann aber niederdeutsch die Gesprächsverweigerung zurückweist. Seine Haltung begründet er durch die eigene amtliche Rolle als Behördenvertreter (,aber de Goosmarkt, de Finanzbehörde, de loot sich von keinen wechschicken“). Gleichzeitig unterläuft er die formulierte soziale Distanz und Asymmetrie mit der Sprachwahl zusätzlich dadurch, dass er den Verhandlungspartner duzt. Die offizielle Rolle als Behördenvertreter wird mit Niederdeutsch als Nahsprache kontrastiert. Dies führt zur Verblüffung des Verhandlungspartners und zugleich zum Einlenken. Die Sprachwahl Niederdeutsch ist für das Verständnis der Pointe wesentlich, setzt aber voraus, dass die Präsupposition, dass Niederdeutsch eine Nahsprache sei, auch von der Interviewerin geteilt wird.

Eine zusätzliche Positionierung wird bei der Verwendung des aus dem Niederdeutschen stammenden Wortes „Ökelnamen“ deutlich. GP18 demonstriert auch hier seine sprachliche Überlegenheit, dieses Mal aber, indem er sich gegenüber der Interviewerin als sprachkompetent zeigt. Er wiederholt das Gesagte auf Hochdeutsch, da er wahrscheinlich davon ausgeht, dass die Interviewerin den Ausdruck nicht versteht.

Insgesamt zeigt sich, dass GP18 seine Niederdeutsch-Kompetenz nutzt, um seine kommunikative Flexibilität und seinen Erfolg in den erzählten Episoden emblematisch darzustellen. Die Aussage wird durch die Sprachwahl illustriert und verstärkt. Die vorgenommenen Positionierungshandlungen finden in zwei Richtungen statt, einerseits in Richtung auf die Personen in den erzählten Ereignissen, denen gegenüber sich GP18 durch seine sprachliche Flexibilität als durchsetzungsfähig und überlegen zeigt, und andererseits in Richtung auf die Interviewerin, der gegenüber er sich ebenfalls als sprachlich kompetent und kommunikativ gewandt positioniert. Auf gesprächsorganisatorischer Ebene wird Niederdeutsch eingesetzt, um die erzählten Episoden sprachlich abzuheben und um durch Zitate einen lebendigen Eindruck von der Originalsituation zu vermitteln. 


\section{Fazit}

Die vorangegangenen qualitativen Analysen konnten exemplarisch verschiedene Funktionen des Codeswitchings aufzeigen, die einen wichtigen Beitrag $\mathrm{zu}$ Identitätskonstruktionen und Positionierungshandlungen leisten: In den sprachbiographischen Interviews haben sich vor allem der Ausweis der Sprachkompetenz, die Darstellung der eigenen kommunikativen Überlegenheit sowie der Hinweis auf Informalität und Nähekonstitution herauskristallisiert.

Zunächst kann grundlegend konstatiert werden, dass der Gebrauch des Niederdeutschen die individuelle Sprachkompetenz im Rahmen der Interviews emblematisch belegt. Selbstpositionierungen als kompetente Sprecher (GP63, GP49, GP03, GP18) bzw. L1-Sprecher (GP30) spielen hier ebenso eine Rolle wie Fremdpositionierung der weniger-kompetenten Interviewer (GP03, GP18). Die Kontrastierung von Kompetenzen spiegelt sich auch in Positionierungshandlungen innerhalb der referierten Situationen. So stellen sich die Gewährspersonen innerhalb von Erzählungen als versierte Niederdeutsch-Sprecher dar und grenzen sich zugleich von nicht- bzw. weniger-kompetenten Personen (GP18) oder Gruppen (GP30, GP63) ab. Die Positionierungshandlungen in der erzählten Situation verschränken sich mit der aktuellen Gesprächssituation, wenn die referierte Selbstdarstellung durch den Wechsel ins Niederdeutsche im Interview reproduziert wird und kommunikative Flexibilität und Überlegenheit abbildet (GP18).

Die soziale Zugehörigkeit zur Sprechergruppe wird darüber hinaus mit Konzeptualisierungen des Niederdeutschen als Nahsprache verknüpft (GP49, GP03, GP18). Diese finden sich in den Schilderungen der Gewährspersonen, in denen beschrieben wird, wie sich das Niederdeutsche als kontaktförderlich erwiesen und Informalität hergestellt habe bzw. als we-Code im Gegensatz zum Hochdeutschen als formellem Code (GP18) benutzt wurde. Dabei sind auch Verknüpfungen zu Tätigkeiten festzustellen, die soziale Nähe indizieren, wie gemeinsames Alkoholtrinken (GP49, GP03 und GP04). Hinsichtlich personaler Identitätskonstruktionen zeigt sich dagegen, dass das Niederdeutsche als Alltags- und Muttersprache konzeptualisiert wird (GP30).

Neben den expressiven Funktionen des Codeswitchings werden gesprächsorganisatorische Effekte sichtbar, wenn dadurch referierte Inhalte und Kommentierungen getrennt werden (GP30), um wörtliche Rede abzusetzen (GP49, GP03, GP18) oder um thematische Abschnitte wie Anfang und Ende (GP63) oder Höhepunkte einer Narrationseinheit (GP03, GP18) zu kennzeichnen. Damit kann die Erzählung lebendig gestaltet und ein authentischer Eindruck der erzählten Situation hervorgerufen werden. 
Deutlich wird die Verschränkung der Funktionsebenen: Codeswitching ist niemals monofunktional zu betrachten, sondern wird von den Sprechern als ein facettenreiches Instrument eingesetzt. Kontextualisierungshinweise dienen dazu, Bestandteile der personalen Identität (Sprachkompetenz) und der sozialen Identität (Konstituierung der Ingroup von Niederdeutschsprechern) aufzurufen, Situationen durch Informalität und Nähe zu kennzeichnen und Positionierungen (Selbstpositionierung als kommunikativ versiert und Fremdpositionierung als weniger sprachkompetent) vorzunehmen. Im Gegensatz zu Analysen in der Gumperz-Tradition (vgl. Abschnitt 2), die auf die Herstellung sozialer Identität durch Codeswitching innerhalb der Kommunikationssituation hinweisen, kann hier gezeigt werden, dass Codeswitching in den Interviews vorrangig zur Darstellung der sozialen Identität dient, indem die Sprecher es ikonisch einsetzen und damit die referierte Kommunikationssituation auch durch die Sprachwahl evozieren. Codeswitching vermittelt einen authentischen Eindruck der erzählten Situation und wirkt durch Gliederungshinweise und die Unterscheidung verschiedener Ebenen im aktuellen Kontext zugleich gesprächsorganisierend. Somit stellt sich Codeswitching als ein komplexes Mehrebenenverfahren zur Konstituierung von sozialer und kommunikativer Bedeutung heraus.

\section{Literatur}

Adler, Astrid, Christiane Ehlers, Reinhard Goltz, Andrea Kleene \& Albrecht Plewnia (2016): Status und Gebrauch des Niederdeutschen 2016. Erste Ergebnisse einer repräsentativen Erhebung. Mannheim: Institut für Deutsche Sprache.

Appel, René \& Pieter Muysken (1987): Language contact and bilingualism. London: Edward Arnold.

Auer, Peter (1998): Introduction. In Peter Auer (Hrsg.), Code-switching in conversation: Language, interaction and identity, 1-24. London: Routledge.

Auer, Peter (1999): From codeswitching via language mixing to fused lects: Toward a dynamic topology of bilingual speech. International Journal of Bilingualism 3 (4), 309-332.

Auer, Peter (2009): Bilingual conversation. In Nikolas Coupland \& Adam Jaworski (Hrsg.), The new sociolinguistics reader, 490-511. London: Palgrave Macmillan.

Auer, Peter (2011): Code-switching/mixing. In Ruth Wodak, Barbara Johnstone \& Paul Kerswill (Hrsg.), The SAGE handbook of sociolinguistics, 460-478. London: Sage.

Blom, Jan-Petter \& John J. Gumperz (1972): Social meaning in linguistic structures: Code switching in Northern Norway. In John J. Gumperz (Hrsg.), Directions in sociolinguistics: The ethnography of communication, 407-434. New York: Holt Rinehart and Winston.

Bößhenz, Katja V. (2011): Die sozialsymbolisierende Funktion der Dialekt-Standard-Variation. Mannheim: Institut für Deutsche Sprache.

Dahl, Eva-Sophie (1974): Interferenz und Alternanz - Zwei Typen der Sprachschichtenmischung im Norden der Deutschen Demokratischen Republik. In Gerhard Ising (Hrsg.), 
Aktuelle Probleme der sprachlichen Kommunikation. Soziologische Studien zur sprachlichen Situation in der Deutschen Demokratischen Republik, 339-388. Berlin: Akademie-Verlag.

Denkler, Markus (2007): Code-switching in Gesprächen münsterländischer Dialektsprecher. Zur Sprachvariation beim konversationellen Erzählen. Zeitschrift für Dialektologie und Linguistik 74, 164-195.

Denkler, Markus (2011): Zum Dialektgebrauch im Westmünsterland. Code-Switching in halböffentlichen Gesprächen. Niederdeutsches Jahrbuch 134, 149-170.

Eckert, Penelope (2012): Three waves of variation study: The emergence of meaning in the study of sociolinguistic variation. Annual Review of Anthropology 41 (1), 87-100.

Flender, Armin, Dieter Pfau, Sebastian Schmidt \& Matthias Weipert (2001): Regionale Identität zwischen Konstruktion und Wirklichkeit: Eine historisch-empirische Untersuchung am Beispiel des Siegerlandes. Baden-Baden: Nomos.

Goffman, Erving (1981): Forms of talk. Philadelphia: University of Pennsylvania Press. Gumperz, John J. (1982): Discourse strategies. Cambridge: Cambridge University Press. Herrmann-Winter, Renate (1974): Auswirkungen der sozialistischen Produktionsweise in der Landwirtschaft auf die sprachliche Kommunikation in den Nordbezirken der Deutschen Demokratischen Republik. In Gerhard Ising (Hrsg.), Aktuelle Probleme der sprachlichen Kommunikation. Soziologische Studien zur sprachlichen Situation in der Deutschen Demokratischen Republik, 75-134. Berlin: Akademie-Verlag.

Hess-Lüttich, Ernest W. (2004): Die sozialsymbolische Funktion von Sprache und Gesellschaft. In Ulrich Ammon, Norbert Dittmar, Klaus J. Mattheier \& Peter Trudgill (Hrsg.), Soziolinguistik. Ein internationales Handbuch der Wissenschaft von Sprache und Gesellschaft. 1 Halbbd. 2., vollständig neu bearb. und erw. Aufl., 91-502. Berlin/ New York: de Gruyter.

Höder, Steffen (2003): „Wi Hamburger schnackt maal so, maal so.“ Hochdeutsch und Niederdeutsch in den Äußerungen bilingualer Sprecher. Korrespondenzblatt des Vereins für niederdeutsche Sprachforschung 110, 47-56.

Höder, Steffen (2011): Niederdeutsch und Norddeutsch: ein Fall von Diasystematisierung. Niederdeutsches Jahrbuch 134, 113-136.

Johannlükens, Ralph (1989): Auslöser und Funktionen niederdeutsch-hochdeutscher Variation. Einige Untersuchungsergebnisse aus der Osnabrücker Region. Niederdeutsches Jahrbuch 112, 92-120.

Jürgens, Carolin (2015): Niederdeutsch im Wandel: Sprachgebrauchswandel und Sprachwahrnehmung in Hamburg. Hildesheim u. a.: Georg Olms.

Jürgens, Carolin (2016): Regionale Identität per Einkaufstüte. Eine Fallstudie zum Enregisterment des Niederdeutschen in Hamburg. In Andreas Bieberstedt, Jürgen Ruge \& Ingrid Schröder (Hrsg.), Hamburgisch. Struktur, Gebrauch, Wahrnehmung der Regionalsprache im urbanen Raum. Frankfurt a. M. u. a.: Peter Lang.

Jürgens, Carolin \& Ingrid Schröder (2016): Sprachstereotype und ihre Realisierungen im Gespräch am Beispiel des Niederdeutschen. In Andreas Bieberstedt, Jürgen Ruge \& Ingrid Schröder (Hrsg.), Hamburgisch. Struktur, Gebrauch, Wahrnehmung der Regionalsprache im urbanen Raum, 345-387. Frankfurt a. M. u. a.: Peter Lang.

Krappmann, Lothar (2004): Identität. In Ulrich Ammon, Norbert Dittmar, Klaus J. Mattheier \& Peter Trudgill (Hrsg.), Soziolinguistik. Ein internationales Handbuch der Wissenschaft von Sprache und Gesellschaft. 1 Halbbd. 2., vollständig neu bearb. und erw. Aufl., 405-412. Berlin/New York: de Gruyter. 
Kresić, Marijana (2006): Sprache, Sprechen und Identität: Studien zur sprachlich-medialen Konstruktion des Selbst. München: Iudicium.

Labov, William \& Joshua Waletzky (1967): Narrative analysis. Oral versions of personal experience. In June Helm (Hrsg.), Essays on the verbal and visual arts. Proceedings of the 1966 Annuals Spring Meeting of the American Ethnological Society, 12-44. Seattle/ London: University of Washington Press.

Le Page, Robert B. \& Andrée Tabouret-Keller (1985): Acts of identity: Creole-based approaches to language and ethnicity. Cambridge: Cambridge University Press.

Lucius-Hoene, Gabriele \& Arnulf Deppermann (2002): Rekonstruktion narrativer Identität. Ein Arbeitsbuch zur Analyse narrativer Interviews. Opladen: Leske + Budrich.

Lucius-Hoene, Gabriele \& Arnulf Deppermann (2004): Narrative Identität und Positionierung. Gesprächsforschung - Online-Zeitschrift zur verbalen Interaktion 5, 166-183.

Lüdi, Georges (2004): Code-Switching/Sprachwechsel. In Ulrich Ammon, Norbert Dittmar, Klaus J. Mattheier \& Peter Trudgill (Hrsg.), Soziolinguistik. Ein internationales Handbuch der Wissenschaft von Sprache und Gesellschaft. 1. Halbbd. 2., vollständig neu bearb. und erw. Aufl., 341-350. Berlin/New York: de Gruyter.

Möller, Frerk (2010): Platt in Hamburg anno 2007. In Wolfgang Müns (Hrsg.), Man mag sik kehrn und kanten, as man will, noch jummer is der'n Eck, wo man ni wen is.

100. Jahrgang der Zeitschrift „Quickborn“. Festschrift, 549-565. Hamburg: Selbstverlag der Vereinigung „Quickborn“.

Müller, Natascha, Laia Arnaus Gil, Nadine Eichler, Jasmin Geveler, Malin Hager, Veronika Jansen, Marisa Patuto, Valentina Repetto \& Anika Schmeisser (2015): CodeSwitching: Spanisch, Italienisch, Französisch: Eine Einführung. Tübingen: Gunter Narr.

Muysken, Pieter (1997): Code-switching processes: Alternation, insertion, congruent lexicalization. In Martin Pütz (Hrsg.), Language choices. Conditions, constraints and consequences, 361-381. Amsterdam: Benjamins.

Neumann, Lara \& Ingrid Schröder (2017a): Identitätskonstruktionen in sprachbiographischen Interviews. Analysen zur Funktion des Niederdeutschen in Hamburg. In Ingrid Schröder \& Carolin Jürgens (Hrsg.), Sprachliche Variation in autobiographischen Interviews. Theoretische und methodische Zugänge, 225-242. Frankfurt a. M. u. a.: Peter Lang.

Neumann, Lara \& Ingrid Schröder (2017b): Zur Bewertung von Niederdeutsch und lokalem Substandard in Hamburg. In Markus Hundt, Christoph Purschke \& Evelyn Ziegler (Hrsg.), Sprachräume: Konfigurationen, Interaktionen, Perzeptionen. Linguistik Online 85 (6), 227-255. http://dx.doi.org/10.13092/lo.85.4088 (letzter Zugriff: 18.1. 2018).

Petkova, Marina (2016): Multiples Code-Switching: ein Sprachkontaktphänomen am Beispiel der Deutschschweiz: Die Fernsehberichterstattung zur »Euro 08« und andere Vorkommenskontexte aus interaktionsanalytischer Perspektive. Heidelberg: Universitätsverlag Winter.

Reershemius, Gertrud (2001): „Token Codeswitching“ and language alternation in narrative discourse: A functional-pragmatic approach. International Journal of Bilingualism 5 (2), 175-194.

Riehl, Claudia M. (2009): Sprachkontaktforschung. Tübingen: Gunter Narr.

Rosa, Hartmut (2007): Identität. In Jürgen Straub, Arne Weidemann \& Doris Weidemann (Hrsg.), Handbuch interkulturelle Kommunikation und Kompetenz. Grundbegriffe Theorien - Anwendungsfelder, 47-56. Stuttgart: Metzler.

Scharioth, Claudia (2015): Regionales Sprechen und Identität. Hildesheim u. a.: Georg Olms. 
Schröder, Ingrid (2012): „Da nicht für.“ Grammatische Kontaktphänomene HochdeutschNiederdeutsch. Sociolinguistica 26 (1), 18-29. https://doi.org/10.1515/soci.2012.26.1.18 (letzter Zugriff: 19.7. 2017).

Schröder, Ingrid (2013): Sprache, Stadt und Stereotyp - Zur sozialsymbolischen Verwendung des Niederdeutschen im urbanen Raum. In Martin Durrell, Hans-Werner Eroms, Elvira Glaser, Wolfgang Haubrichs, Michail L. Kotin, Józef Wiktorowicz \& Ewa Żebrowska (Hrsg.), Diachronische, diatopische und typologische Aspekte des Sprachwandels, 377-382. Frankfurt a. M. u. a.: Peter Lang.

Schröder, Ingrid (2015): Von der Dialektologie zur Regionalsprachenforschung eine norddeutsche Perspektivierung. In Michael Elmentaler, Markus Hundt \& Jürgen E. Schmidt (Hrsg.), Deutsche Dialekte: Konzepte, Probleme, Handlungsfelder; Akten des 4. Kongresses der Internationalen Gesellschaft für Dialektologie des Deutschen (IGDD), 25-57. Stuttgart: Steiner.

Schröder, Ingrid \& Carolin Jürgens (2017): Einstellungen gegenüber regionalen Sprachformen in der Großstadt: Niederdeutsch in Hamburg ( $\mathrm{NiH})$. Eine Projektskizze. In Ingrid Schröder \& Carolin Jürgens (Hrsg.), Sprachliche Variation in autobiographischen Interviews: Theoretische und methodische Zugänge, 11-46. Frankfurt a. M. u. a.: Peter Lang.

Sebba, Mark \& Tony Wootton (1998): We, they and identity: Sequential vs. identity-related explanation in code-switching. In Peter Auer (Hrsg.), Code-switching in conversation: Language, interaction and identity, 262-285. London: Routledge.

Silverstein, Michael (2003): Indexical order and the dialectics of sociolinguistic life. Language \& Communication 23 (3-4), 193-229.

Smits, Tom F. (2016): Code-switching als Sprachverlusterscheinung an der deutschniederländischen Grenze. In Helmut Spiekermann, Line-Marie Hohenstein, Stephanie Sauermilch \& Kathrin Weber (Hrsg.), Niederdeutsch: Grenzen, Strukturen, Variation, 311-336. Wien u. a.: Böhlau.

Stellmacher, Dieter (1977): Studien zur gesprochenen Sprache in Niedersachsen. Eine soziolinguistische Untersuchung. Marburg: Elwert.

Stellmacher, Dieter (1981): Niederdeutsch: Formen und Forschungen. Tübingen: Niemeyer.

Tajfel, Henri (1981): Human groups and social categories: Studies in social psychology. Cambridge: Cambridge University Press.

Thim-Mabrey, Christiane (2003): Sprachidentität - Identität durch Sprache. Ein Problemaufriss aus sprachwissenschaftlicher Sicht. In Nina Janich \& Christiane Thim-Mabrey (Hrsg.), Sprachidentität. Identität durch Sprache, 1-18. Tübingen: Gunter Narr.

Treffers-Daller, Jeanine (2005): Code-Switching/Sprachwechsel. In Ulrich Ammon, Norbert Dittmar, Klaus J. Mattheier \& Peter Trudgill (Hrsg.), Soziolinguistik. Ein internationales Handbuch der Wissenschaft von Sprache und Gesellschaft. 2. Halbbd. 2., vollständig neu bearb. und erw. Aufl., 1469-1482. Berlin/New York: de Gruyter.

Ziemann, Kathleen (2012): Codeswitching zwischen Hochdeutsch und Niederdeutsch. In Barbara Jańczak, Konstanze Jungbluth \& Harald Weydt (Hrsg.), Mehrsprachigkeit aus deutscher Perspektive, 181-194. Tübingen: Gunter Narr. 



\title{
3 Möglichkeiten und Grenzen der quantitativen Spracheinstellungs- forschung
}

\begin{abstract}
Sprecher haben (oft unbewusste) Meinungen und Einstellungen zu Sprachen und Varietäten. Ein Weg, solche Spracheinstellungen zu erfassen, führt über quantitative Verfahren (Fragebögen mit offenen und geschlossenen Fragen), mit denen man Daten großer Gruppen - im Idealfall repräsentativ erheben kann. Im Beitrag werden solche Erhebungen, die am Institut für Deutsche Sprache durchgeführt wurden, vorgestellt, und es werden zwei Instrumente der Spracheinstellungsforschung, die dabei zum Einsatz kamen, näher erläutert: das Allgemeine Sprachbewertungsinstrument (ASBI) und die AttitudesTowards-Languages-Skala (AToL). Außerdem wird gezeigt, wie dialektometrische Auswertungs- und Visualisierungsverfahren auf Einstellungsdaten angewendet werden können.
\end{abstract}

Keywords: empirisch, Fragebogen, Soziolinguistik, Sprache, Spracheinstellungen, Varietäten

\section{Zugänge und Methoden der Sprach- einstellungsforschung}

Unter Spracheinstellungen verstehen wir zu Haltungen verfestigte Meinungen eines Individuums zu Sprache und Sprechern, die mit den jeweiligen individuellen sprachlichen und allgemeinen (tatsächlichen oder vermeintlichen, stabilen oder vagen) Wissensbeständen in Beziehung stehen; als psychische Dispositionen können sie entscheidungs- und handlungsleitend sein; sie können den Sprechern in weiten Teilen unbewusst sein; und sie sind individuell unterschiedlich scharf konturiert. Aus alledem ergibt sich, dass Spracheinstellungen

Astrid Adler, Institut für Deutsche Sprache, Augustaanlage 32, D-68161 Mannheim, E-Mail: adler@ids-mannheim.de

Albrecht Plewnia, Institut für Deutsche Sprache, Augustaanlage 32, D-68161 Mannheim, E-Mail: plewnia@ids-mannheim.de

○ Open Access. () 2018 Astrid Adler und Albrecht Plewnia, publiziert von De Gruyter. (c)BY Dieses Werk ist lizenziert unter der Creative Commons Attribution 4.0 Lizenz. 
ein schwieriges Forschungsobjekt darstellen, sowohl die Gewinnung als auch die Deutung geeigneter Daten ist nicht trivial. Je nach spezifischem Forschungsinteresse wird man unterschiedliche Zugänge $\mathrm{zu}$ wählen haben. Gemeinsam ist aber allen Zugängen, dass sie empirisch basiert sind und dass sie die Unschärfe ihres Objekts reflektieren müssen. Bei der Datengewinnung bewegt man sich, etwas plakativ gesprochen, zwischen zwei Polen: man kann entweder von wenigen Probanden viele und komplexe Einzeldaten produzieren lassen und versuchen, daraus ein möglichst umfassendes Bild zu gewinnen, oder man kann von sehr vielen Probanden Daten erheben, die dann aber von geringerer Komplexität sein müssen. Im ersten Fall ist die Datengrundlage dann beispielsweise eine gewisse Anzahl an gesteuerten Interviews. Rezente Studien mit einem solchen Zugang verfolgen einen eher diskursanalytischen Ansatz, Spracheinstellungen werden dann nicht als psychologisches Phänomen, sondern als in der Interaktion konstruierte Einstellungen und als Positionierungsakte betrachtet (vgl. z. B. Liebscher \& Dailey-O'Cain 2009; Dailey-O’Cain \& Liebscher 2011; König 2014). Der Vorteil solcher Zugänge besteht darin, dass in der Erhebungssituation flexibel reagiert werden kann und in der Folge, je nach Datenmaterial, recht weitreichende Bedingungsanalysen möglich sind. Allerdings sind die Stichproben nicht repräsentativ, ein großes Problem besteht daher darin, dass die jeweiligen Ergebnisse nicht ohne Weiteres generalisierbar sind. Im zweiten Fall liegt genau darin eine besondere Stärke; wenn Erhebungen stark formalisiert und auch replizierbar sind und idealiter eine Stichprobe zur Grundlage haben, die als repräsentativ für eine bestimmte Grundgesamtheit angenommen werden kann, sind die Ergebnisse insgesamt belastbar. Über die Stichprobengröße können auch bestimmte Zufallsunschärfen aufgefangen werden (einzelne merkwürdige Daten liefert die Empirie immer). Wenn über die Stichprobe dann noch hinreichend viele Informationen zur Verfügung stehen, sind beispielsweise auch Analysen möglich, die bestimmte soziodemographische Variablen berücksichtigen. Die Gewinnung großer, repräsentativer Korpora ist jedoch sehr aufwendig; nicht zuletzt diese Tatsache dürfte dafür verantwortlich sein, dass es bislang nur sehr wenige Repräsentativerhebungen zu Spracheinstellungen gibt. 


\section{Aktuelle Spracheinstellungsforschung am Institut für Deutsche Sprache}

\subsection{Repräsentativerhebungen}

\subsubsection{Deutschland-Erhebung 2008}

Mit der Etablierung des (von der Volkswagen-Stiftung geförderten) Projekts „Erkundung und Analyse aktueller Spracheinstellungen in Deutschland“ im Jahre 2008 begann am Institut für Deutsche Sprache (IDS) eine neue Phase der Beschäftigung mit dem Themenkomplex Spracheinstellungen. Materieller Kern dieses interdisziplinär konzipierten Projekts, das in Kooperation mit dem Lehrstuhl für Sozialpsychologie der Universität Mannheim durchgeführt wurde, war eine bundesweite Repräsentativerhebung unter der erwachsenen Wohnbevölkerung in Deutschland ( $n=2004)$; die praktische Durchführung der Erhebung (im Folgenden: Deutschland-Erhebung 2008) erfolgte im Herbst 2008 als Telefonumfrage durch die Forschungsgruppe Wahlen; die Stichprobe wurde nach Geschlecht, Alter, Bildung und Wohnort gewichtet und ist insoweit für die Gesamtbevölkerung repräsentativ. Gegenstand der mit über vierzig inhaltlichen Fragen zu sprachlichen Themen recht umfassend angelegten Befragung waren allgemeine Spracheinstellungen, das Verhältnis zu Deutsch und anderen Sprachen, die Bewertung regionaler Varietäten des Deutschen, Eigenschaftszuschreibungen in Bezug auf Sprachen und Varietäten, Sprecherstereotype, außerdem Sprachwandel und Sprachpolitik. ${ }^{1}$ Einen Überblick über die Erhebung bieten Eichinger et al. (2009) sowie Gärtig \& Rothe (2009), detaillierte Ergebnisse liefern Gärtig, Plewnia \& Rothe (2010); zu Einzelaspekten Plewnia \& Rothe (2009) und Plewnia \& Rothe (2010); zu weiteren Projektergebnissen Eichinger et al. (2012).

\subsubsection{Norddeutschland-Erhebung 2016}

Im Rahmen eines Projekts zum „Sprachlagengefüge in Norddeutschland“, einem Kooperationsprojekt des IDS und des Instituts für niederdeutsche Sprache in

1 Einige der Themen bieten eine Fortschreibung von Teilen einer früheren Repräsentativerhebung des IDS, die bereits 1997 unter Leitung von Gerhard Stickel (dem die DeutschlandErhebung 2008 wesentliche Impulse verdankt) durchgeführt wurde. Diese (von der FritzThyssen-Stiftung geförderte) Befragung wurde von GFM-GETAS als Teil einer Mehrthemenumfrage in direkten Einzelinterviews $(n=2015)$ erhoben; vgl. Stickel \& Volz (1999). 
Bremen, wurde 2016 eine Repräsentativerhebung im norddeutschen Sprachraum durchgeführt (im Folgenden: Norddeutschland-Erhebung 2016). ${ }^{2}$ Auch bei dieser (von der Beauftragten der Bundesregierung für Kultur und Medien geförderten) Erhebung handelt es sich um eine Telefonumfrage unter der norddeutschen Wohnbevölkerung $(n=1632)$, deren praktische Umsetzung wiederum in den Händen der Forschungsgruppe Wahlen lag. Gegenstand der NorddeutschlandErhebung 2016 waren einerseits Kompetenz, Gebrauch und Alltagsrelevanz des Niederdeutschen und andererseits Bewertungen, Einschätzungen und Zuschreibungen zu Niederdeutsch und Hochdeutsch; die ersten Ergebnisse sind bei Adler et al. 2016 publiziert.

\subsubsection{Deutschland-Erhebung 2017/18}

Eine neue bundesweite Repräsentativerhebung hat das IDS im Herbst/Winter 2017/18 in Kooperation mit dem Deutschen Institut für Wirtschaftsforschung (DIW) im Rahmen der Innovationsstichprobe des Sozio-oekonomischen Panels (SOEP-IS) des DIW durchgeführt (im Folgenden: Deutschland-Erhebung 2017/18). Das SOEP ist eine repräsentative Wiederholungsbefragung, die seit 1984 jährlich durchgeführt wird; dabei werden aktuell etwa 30.000 Personen in fast 11.000 Haushalten befragt (Richter \& Schupp 2012). Die Erhebung wird als direkte Befragung durch die TNS Infratest Sozialforschung GmbH, jetzt Kantar Public, durchgeführt; es werden insbesondere Fragen zum Einkommen, zur Erwerbstätigkeit, zur Bildung und Gesundheit erhoben. Seit 2012 gibt es im SOEP die Innovationsstichprobe, die externen Forschern in einem Wettbewerbsverfahren das Einbringen eigener Forschungsfragen ermöglicht; es handelt sich um eine Teilstichprobe der SOEP-Kernerhebung mit etwa 5.500 Personen in rund 3.000 Haushalten, zu denen auch weit zurückreichende Längsschnitt-Daten - nicht nur detaillierte Angaben zur Raumbiographie, sondern z. B. auch soziale Einstellungen - zur Verfügung stehen. In der Erhebungsrunde 2017/18, die in vier Erhebungswellen durchgeführt wird, beteiligt sich das IDS an der Innovationsstichprobe des SOEP mit einem IDSSprachmodul. Dieses Modul soll einerseits Wissen über die tatsächlichen Sprachverhältnisse gewinnen, andererseits wiederum Spracheinstellungen abfragen. Es besteht aus zwei Teilen, zum einen aus einem direkten Interview

2 Diese Erhebung knüpft - außer an die Deutschland-Erhebung 2008 - vor allem an zwei frühere Erhebungen des Instituts für niederdeutsche Sprache aus den Jahren 1984 und 2007 an; vgl. Stellmacher (1987) bzw. Möller (2008). 
(CAPI, computer assisted personal interview) und zum anderen aus einem Onlinefragebogen (CAWI, computer assisted web interview).

Im ersten Teil des IDS-Sprachmoduls geht es insbesondere darum, das Sprachrepertoire der Befragten zu erheben (Erstsprache(n), Fremdsprachenkenntnisse, Dialektkompetenz); hinzu kommt ein Fragenset zu Einstellungen gegenüber Sprachen und Dialekten des Deutschen. Der zweite Teil des IDSSprachmoduls (Onlinefragebogen) ist erheblich umfangreicher, darin werden etwa vierzig Fragen gestellt zu Einstellungen gegenüber dem Deutschen, zu regionalen Varietäten, zu anderen Sprachen, außerdem Fragen zu Mehrsprachigkeit, zu Leichter Sprache, zu Bewertungen von Sprachrichtigkeit, zu Sprachvariation und zur Entwicklung des Deutschen.

Das SOEP eignet sich besonders gut, um unsere Erhebungsinstrumente (vgl. Abschnitt 3) einzusetzen, da es einerseits eine ausgewogene repräsentative Stichprobe der deutschen Wohnbevölkerung darstellt und andererseits ermöglicht, viele Korrelationen mit anderen - auch längsschnittlich vorhandenen - soziodemographischen, ökonomischen und psychologischen Variablen zu berechnen; für die Sprachwissenschaft ist ein solcher Datensatz bislang singulär.

Erste Daten der Deutschland-Erhebung 2017/18 werden im Herbst 2018 vorliegen; voraussichtlich ab Herbst 2019 werden die Daten dann über die Dateninfrastruktur des SOEP im DIW für alle interessierten Forscher offen zugänglich sein.

\subsection{Weitere Studien}

Im Kontext der Deutschland-Erhebung 2008 wurden noch eine Reihe weiterer kleinerer Erhebungen durchgeführt, z. B. mit Erziehern und Eltern in Kindergärten, mit Schülerinnen und Schülern und mit Studierenden. Ziel dieser (nicht-repräsentativen) Unternehmungen war zum einen, in einigen Bereichen, in denen bei der Deutschland-Erhebung 2008 das Entstehen gewisser Unschärfen aus methodischen Gründen nicht zu vermeiden war, zusätzliche Evidenz zu gewinnen (in der Deutschland-Erhebung 2008 konnten nur Personen ab 18 Jahren befragt werden, außerdem ist beispielsweise die Gruppe der befragten Personen mit einer anderen Muttersprache als Deutsch zwangsläufig recht heterogen). Zum anderen ging es darum, in diesen Studien verschiedene Befragungsinstrumente $\mathrm{zu}$ erproben und zu schärfen.

\section{Kindergarten-Studie}

Eine Erhebung wurde im Winter 2011/12 in Kooperation mit dem MercatorInstitut für Sprachförderung und Deutsch als Zweitsprache mit einer Befragung 
in mehreren deutschen, teils mehrsprachigen, Kindergärten durchgeführt (im Folgenden: Kindergarten-Studie); hier ging es um die Erfassung von Einstellungen zu Mehrsprachigkeit sowohl der Eltern als auch der Erzieherinnen und Erzieher. Die Befragung wurde in fünf Kindergärten durchgeführt, zwei davon deutsch-türkisch, zwei deutsch-englisch und einer einsprachig deutsch $(n=100)$; es wurden 32 Erzieher und 68 Eltern befragt (zur Studie und deren Ergebnissen vgl. Rothe \& Wagner 2015).

\section{Schüler-Studie}

Mit einer Erhebung mit Schülerinnen und Schülern der 9. und 10. Jahrgangsstufe wurde gezielt eine Altersgruppe, die mit der Deutschland-Erhebung 2008 nicht erfasst werden konnte, in den Blick genommen. Diese Erhebung (im Folgenden: Schüler-Studie) wurde 2010 und 2011 in Mannheim, an zwei Schulstandorten am Niederrhein sowie im Kreis Steinburg in Schleswig-Holstein mit einem Paper-Pencil-Fragebogen durchgeführt $(n=628)$; Themen waren u.a. Einstellungen zum Deutschen, zu deutschen Dialekten und $\mathrm{zu}$ anderen Sprachen sowie die zugehörigen Sprecherstereotype. Die Schulen waren so gewählt, dass es eine große Zahl an Schülern mit mehrsprachigem Hintergrund gab; Ziel dieser Studie war unter anderem, an einer exemplarischen Stichprobe, in der einige Faktoren (wie z.B. das Alter) konstant gehalten wurden, den Einfluss der Faktoren Herkunft und Mehrsprachigkeit auf Spracheinstellungen zu untersuchen. $\mathrm{Zu}$ den Ergebnissen vgl. Plewnia \& Rothe (2011a) bzw. Plewnia \& Rothe (2015), Plewnia \& Rothe (2012) und Rothe (2012).

\section{Studierenden-Studie}

In einer weiteren Erhebung, die 2010 und 2011 mit Studierenden an vier Universitäten (Bielefeld, Köln, Leipzig und Mannheim; $n=430$ ) durchgeführt wurde (im Folgenden: Studierenden-Studie), ging es ebenfalls um regionale Varietäten des Deutschen, diesmal in erster Linie um Sprachraumwissen bzw. räumliche Konzeptualisierungen. Hier sollten in einer Mental-Map-Aufgabe (ein Instrument, das naturgemäß für eine telefonische Befragung ungeeignet ist) bekannte Sprachräume eingezeichnet werden. Auch hier wurden in der Stichprobe bestimmte Faktoren (Alter, Bildung) im wesentlichen konstant gehalten, während mit dem Erhebungsort der Wohnort der Probanden variierte und damit als bestimmender Faktor untersucht werden konnte. Insofern ergänzt die Studierenden-Studie die Ergebnisse zu Einstellungen zu Dialekten der Deutschland-Erhebung 2008 sowie der Schüler-Studie. Für Ergebnisse vgl. Plewnia \& Rothe (2012), Plewnia (2013). 


\section{Niederdeutsch-Studien}

Zwei weitere Studien, die im Zusammenhang mit der NorddeutschlandErhebung 2016 durchgeführt werden (zu denen allerdings derzeit noch keine Ergebnisse vorliegen), sind hier noch zu nennen: zum einen eine Erhebung in plattdeutschen Kindergärten und Kindertagesstätten, d. h. in solchen Einrichtungen im niederdeutschen Sprachraum, die irgendeine Form von plattdeutschem Sprachangebot haben; hier geht es in erster Linie um Sprachkompetenz und -gebrauch des Niederdeutschen, um pädagogische Konzepte und um Spracheinstellungen zum Niederdeutschen und zum Hochdeutschen; und zum anderen eine Erhebung an plattdeutschen Theatern, d.h. an (vornehmlich kleineren privaten) Bühnen und Laienspielgruppen mit einem niederdeutschen Repertoire; auch hier sollen sowohl die Niederdeutsch-Kompetenzen und die niederdeutsche Sprachpraxis als auch die damit verbundenen Einstellungen und Bewertungen erfasst werden. In beiden Studien erfolgt die Erhebung mittels eines Onlinefragebogens; für die Kindergarten-Erhebung wurden 102 Personen und für die Theater-Erhebungen 751 Personen befragt.

Im Folgenden werden zwei zentrale in den Repräsentativerhebungen sowie in den ergänzenden Studien eingesetzte Instrumente zur Erhebung dargestellt und exemplarisch an Daten aus den Erhebungen und Studien erläutert.

\section{Instrumente der Spracheinstellungsforschung}

\subsection{Allgemeines Sprachbewertungsinstrument (ASBI)}

\subsubsection{Das ASBI-Instrument}

Beim „Allgemeinen Sprachbewertungsinstrument (ASBI)“ handelt es sich um ein Werkzeug zur Erfassung der allgemeinen Einstellungen zu Sprachen und Varietäten. Erschlossen werden diese über die Elizitierung genereller, eher summierender Gefallens- und Sympathiebekundungen, die sehr unspezifisch angesteuert werden. Dieses Instrument kam in den verschiedenen Erhebungen mit bestimmten Modifikationen und jeweils leicht verändertem Fokus zum Einsatz. Dabei können sich die abgefragten Bewertungen auf Sprachen beziehen (Deutschland-Erhebung 2017/18, Schüler-Studie, Kindergarten-Studie), auf regionale Varietäten des Deutschen (Deutschland-Erhebung 2008, DeutschlandErhebung 2017/18, Norddeutschland-Erhebung 2016, Schüler-Studie), auf Hochdeutsch mit einem regionalen Akzent (Deutschland-Erhebung 2008) oder auf Deutsch mit einem fremdsprachigen Akzent (Deutschland-Erhebung 2008). Eine Variante, die wir in der Schüler-Studie und in der Kindergarten-Studie 
erprobt haben, erfragt Wunschsprachen. Die genaue Ausbuchstabierung der allgemeinen Bewertung variiert ebenfalls: in einer Variante wird explizit Sympathie abgefragt, in einer anderen wird nach Gefallen gefragt.

Prinzipiell sind bei Fragebogenerhebungen dieser Art zwei Frageformate möglich: beim geschlossenen Format werden den Probanden vorformulierte Antwortmöglichkeiten vorgegeben, aus denen sie auswählen müssen; beim offenen Format ist nur die Frageformulierung gegeben, die Befragten müssen ihre Antworten frei formulieren. Beide Formate haben ihre Vor- und Nachteile (zu einer Diskussion der Formate vgl. Plewnia \& Rothe 2012: 27-33; dort auch weitere Literatur), beide Formate wurden in unseren Erhebungen angewandt. Eine Variante ist eine Kombination aus beiden Methoden, typischerweise indem bei einer geschlossenen Frage als letzte Antwortoption ein Freifeld vorgesehen ist, das den Befragten eine frei formulierte Antwort ermöglicht. Eine solche Kombination ist jedoch nur in bestimmten Kontexten bei bestimmten Fragetypen sinnvoll, da der kognitive Status von geschlossenen und offenen Antworten nicht identisch ist.

Das geläufigste Format ist das geschlossene. Es hat zum einen praktische Vorteile: dadurch, dass die Antwortkategorien bekannt sind und keinerlei Varianz aufweisen, ist die Auswertung auch bei sehr großen Datensätzen recht unproblematisch. Zum anderen bietet es bestimmte methodische Vorteile: vor allem wird durch die Vorgabe bestimmter Antwortkategorien sichergestellt, dass für diese auch ein Bewertungsergebnis erzielt wird; die Ergebnisse sind dann über alle Befragten vergleichbar, da allen die gleichen Antwortoptionen zur Verfügung standen. Auch ein Vergleich über mehrere Befragungen hinweg ist bei konstant gehaltenen Antwortoptionen problemlos möglich. Ein wesentlicher methodischer Nachteil dieses Verfahrens besteht darin, dass durch die Vorgabe einer geschlossenen Liste an Antwortmöglichkeiten unter Umständen eine Exhaustivität des Feldes suggeriert wird, die keineswegs gegeben sein muss. Eine umso größere Bedeutung kommt daher der alles andere als trivialen (und bisweilen unterschätzten) Herausforderung $\mathrm{zu}$, die vorgegebenen Antwortoptionen zu gewinnen - eine Auswahl, die im Einzelnen gut begründet sein muss.

Insgesamt deutlich seltener kommt das offene Frageformat zum Einsatz. Es ist im Vergleich zum geschlossenen Format in zweifacher Hinsicht anspruchsvoller. Zum einen fordert es in aller Regel die Probanden deutlich stärker, denn zur Beantwortung einer offenen Frage muss der Befragte einen höheren kognitiven Aufwand betreiben, muss sich erinnern, muss Konzepte aktivieren und muss in seinem Wissens- oder Meinungsbestand sein eigenes Referenzsystem aufrufen. Zum anderen ist die Auswertung stets um ein Vielfaches aufwendiger als bei geschlossenen Fragen, weil die gegebenen Antwor- 
ten nachträglich kategorisiert werden müssen und bei der Schaffung von Kategorien und der Zuordnung der Antworten eine Reihe kritischer Entscheidungen zu treffen sind. Andererseits liegt genau darin die Stärke des offenen Formats: während mit einem geschlossenen Format, dessen Antwortoptionen ein Feld inhaltlich abzudecken scheinen, tatsächlich Verzerrungen entstehen können, eben weil die Antwortoptionen doch nicht exhaustiv sind, ermöglicht es das offene Format, gerade weil keine Antwortoptionen vorgegeben sind und die Antworten typischerweise sehr heterogen ausfallen, bestimmte laienlinguistische Konzeptualisierungen und unerwartete Bezugssysteme aufzudecken, die sonst nicht oder nur wenig bekannt sind. Gerade die (arbeitsökonomisch problematische) Heterogenität der Antworten auf eine offene Frage, sowohl in ihrer inhaltlichen Konzeptualisierung als auch in ihrer Granularität, bietet selbst einen Aufschluss; konstruktivistisch gedacht handelt es sich hier schon um diskursive Einstellungsäußerungen (vgl. Soukup 2012; Studler 2014). Auch die Antwort- und Urteilsbereitschaft der Befragten sowie ihre Urteilsproduktivität (vgl. Cuonz 2014) können dazu beitragen, die Organisation von Laienkonzepten über Sprache zu entschlüsseln. Diese - freilich interpretationsbedürftigen Daten entfallen natürlich beim geschlossenen Format.

Für die Deutschland-Erhebung 2017/18 wird in einem Methoden-Split eine Kombination beider Frageformate realisiert. Dazu werden alle Probanden sowohl nach sympathischen und unsympathischen Dialekten als auch nach sympathischen und unsympathischen Sprachen gefragt. Dabei wird die Stichprobe geteilt, die eine Hälfte der Befragten erhält zu den sympathischen und unsympathischen Sprachen die offenen ASBI-Fragen und zu den sympathischen und unsympathischen Dialekten die geschlossenen ASBI-Fragen, die andere Hälfte der Befragten wird hingegen, sozusagen gespiegelt, nach den sympathischen und unsympathischen Sprachen im geschlossenen und dafür nach den sympathischen und unsympathischen Dialekten im offenen Format gefragt. ${ }^{3}$

3 Die Frageformulierungen lauten beim offenen ASBI: „Gibt es Dialekte, die Sie sympathisch finden? Welche sind das?“ und „Und gibt es Dialekte, die Sie unsympathisch finden? Welche sind das?“ bzw. „Gibt es Sprachen, die Sie sympathisch finden? Welche sind das?“ und „Und gibt es Sprachen, die Sie unsympathisch finden? Welche sind das?“. Beim geschlossenen ASBI werden folgende Dialekte abgefragt (in randomisierter Reihenfolge): Bayerisch, Berlinerisch, Hessisch, Hochdeutsch, Kölsch/Rheinisches Platt, Niederdeutsch/Plattdeutsch, Norddeutsch, Österreichisch, Sächsisch, Schwäbisch, Schweizerdeutsch sowie „der Dialekt, den Sie selber sprechen“. Die Auswahl erfolgte auf der Grundlage der Ergebnisse des offenen ASBI bei der Deutschland-Erhebung 2008 und berücksichtigte außerdem systematisch interessante Fälle. Die beim geschlossenen ASBI abgefragten Sprachen sind die folgenden (Reihenfolge ebenfalls randomisiert): Arabisch, Deutsch, Englisch, Französisch, Italienisch, Polnisch, Russisch, Spanisch und Türkisch. 
Der besondere Ertrag dieses Settings liegt darin, dass auf diese Weise ein gezielter Methodenvergleich möglich wird, der es beispielsweise erlaubt, der Relevanz bestimmter Listeneffekte nachzugehen.

\subsubsection{Exemplarische Ergebnisse von ASBI aus den verschiedenen Erhebungen}

\section{Offenes ASBI: Sympathische und unsympathische Dialekte}

Die Wahrnehmung regionaler Variation (und damit verbunden das Wissen um die Existenz einer Dialekt-Standard-Differenz) zählt zu den grundlegenden sprachlichen Alltagserfahrungen von Sprechern des Deutschen. Entsprechend gibt es einen populären laienlinguistischen Diskurs darüber, welche deutschen Dialekte als sympathisch oder unsympathisch angesehen werden. In der Deutschland-Erhebung 2008 wurde mittels des offenen ASBI auf diesen Diskurs rekurriert, indem die Probanden gefragt wurden, welche Dialekte sie sympathisch und welche sie unsympathisch fänden. Das Ergebnis für die sympathischen Dialekte zeigt Abbildung 3.1.

Die meisten Nennungen sind der Kategorie „Norddeutsch“ zuzuordnen, es folgen Bairisch (Bayrisch, Bayerisch) und, mit einigem Abstand, Schwäbisch und Sächsisch. Vorteil und zugleich Nachteil des offenen Frageformats ist es, dass die Probanden aktiv eigene Benennungen vornehmen müssen. Im Falle von Bairisch, Schwäbisch und Sächsisch ist die Nomenklatur vergleichsweise unproblematisch, hier sind die Nennungen sehr einheitlich (es gibt allenfalls spezifizierende Nennungen vom Typ „Niederbairisch“), was auf relativ monolithische Konzepte hindeutet. In Bezug auf Norddeutschland liegen die Dinge etwas anders. Hier gibt es einerseits verbreitet das Niederdeutsche, andererseits werden dort, wo die alten niederdeutschen Dialekte fehlen, die regionalen, standardnahen Umgangssprachen vielfach als Dialekte wahrgenommen. Unter dem Label „Norddeutsch“ sind hier sämtliche Nennungen zusammengefasst, die auf regionale Sprechweisen in Norddeutschland referieren. Diese sind einerseits sehr zahlreich, andererseits in ihrer Form höchst heterogen; das deutet darauf hin, dass der norddeutsche Sprachraum für linguistische Laien durchaus Relevanz besitzt, aber sehr unterschiedlich konzeptualisiert wird (zu diesem Problem vgl. Plewnia 2013: 44-46).

Die Antworten für die unsympathischen Dialekte zeigt Abbildung 3.2. Auffällig ist, dass in beiden Listen die ersten Rangplätze von denselben Dialekten belegt werden; offenbar gibt es Varietäten, „die, positiv wie negativ, stärker als andere zu Bewertungen herausfordern“ (Eichinger et al. 2009: 20), wobei sich die Prominenz von Dialekten nicht ausschließlich auf die zugrundeliegende Sprechergruppengröße zurückführen lässt (zu möglichen Einflussfaktoren vgl. 


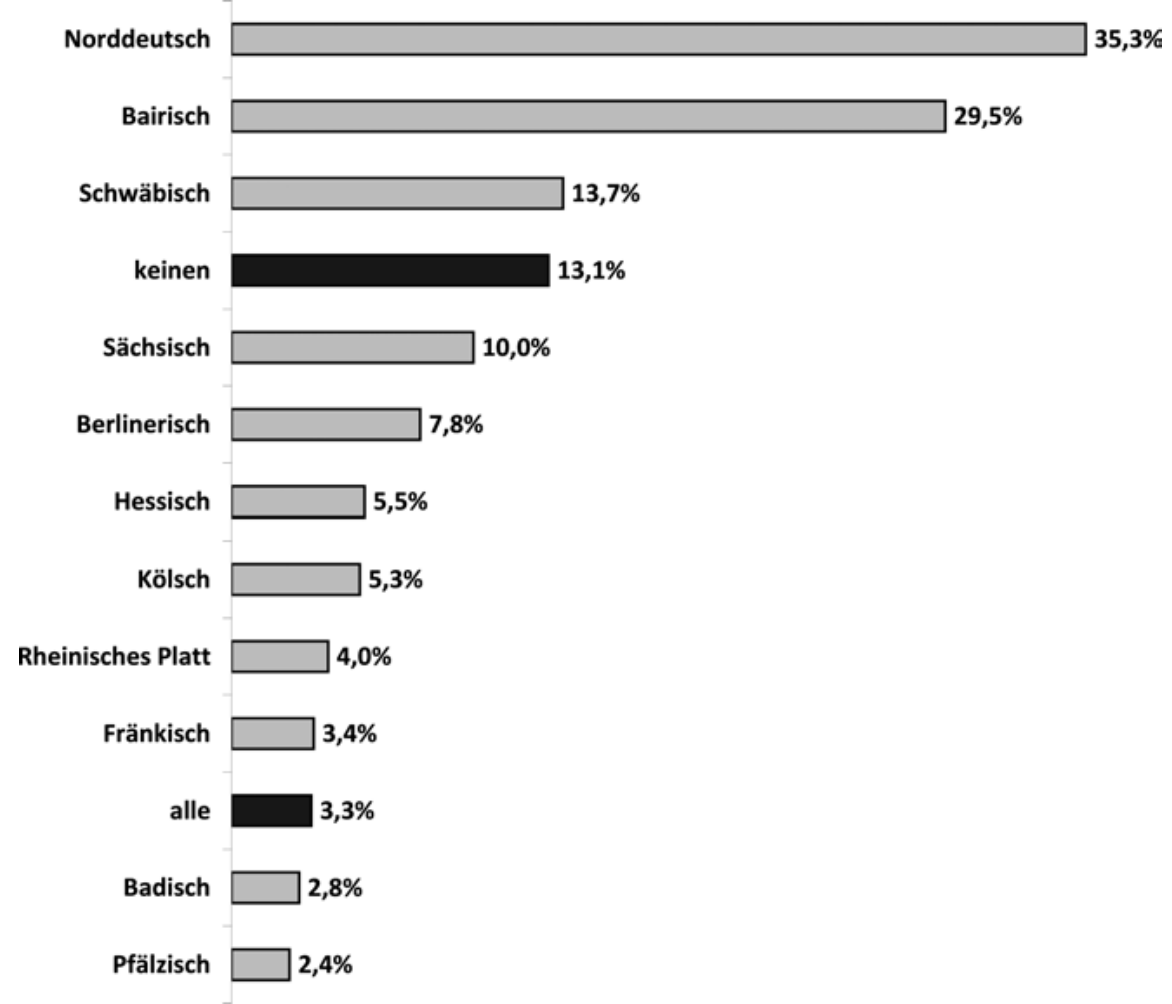

Abb. 3.1: Sympathische Dialekte (gekürzt) (aus: Gärtig, Plewnia \& Rothe 2010: 158).

Plewnia \& Rothe 2012: 46-57). Auffällig ist ferner, dass immerhin ein gutes Drittel der Befragten ausdrücklich angibt, keinen Dialekt unsympathisch zu finden. ${ }^{4}$

4 Dass das offene Frageformat gerade durch seine Offenheit zur Gewinnung weiterer Informationen beiträgt, zeigt auch ein Vergleich mit einer anderen, thematisch ähnlichen Erhebung, die die Gesellschaft für deutsche Sprache mit dem Institut für Demoskopie Allensbach im Frühjahr 2008 durchgeführt hat (vgl. Hoberg et al. 2008). Demnach werden auf die Frage nach sympathischen Dialekten einer vorgegebenen Liste genannt: 1. „Bairisch“: 37 \%, 2. „Norddeutsches Platt (z. B. Hamburger Platt)“: 32\%, 3. „Berlinisch“: 23\%, 4. „Schwäbisch“: 22\%, (...), 7. „Sächsisch“: $11 \%$ (Hoberg et al. 2008: 31). Auf die Frage nach den unsympathischen Dialekten einer Liste werden genannt: 1. „Sächsisch“: 50 \%, 2. „Berlinisch“: 24 \%, 3. „Bairisch“: 19\%, 4. „Schwäbisch“: $14 \%$, (...) 6. „Keinen Dialekt, höre alle gerne“: $12 \%$, (...) 9. „Norddeutsches Platt (z. B. Hamburger Platt)“: $10 \%$ (Hoberg et al. 2008: 32). Ein wesentliches Problem liegt hier in der Auswahl der vorgegebenen Kategorien (deren empirische Fundierung offen bleibt); vgl. kritisch Plewnia \& Rothe (2012: 29-33). 


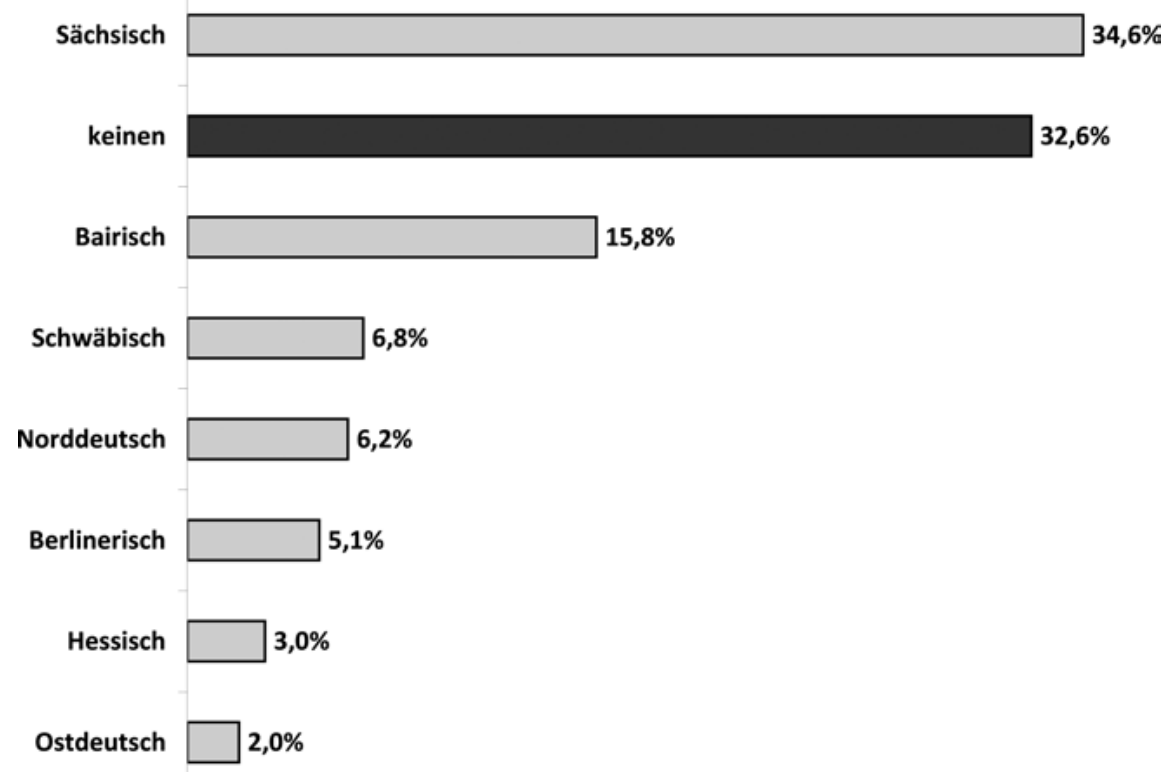

Abb. 3.2: Unsympathische Dialekte (gekürzt) (aus: Gärtig, Plewnia \& Rothe 2010: 163).

In der Deutschland-Erhebung 2017/18 wird, mit einem Jahrzehnt Abstand, wiederum nach sympathischen und unsympathischen Dialekten gefragt. Es wird interessant sein $\mathrm{zu}$ sehen, ob hier in der Diachronie bestimmte Verschiebungen sichtbar werden. Hinzu kommt in der Deutschland-Erhebung 2017/18 der geschilderte Methoden-Split mit einer Kombination von offenem und geschlossenem ASBI, der es erlaubt abzuschätzen, welche Effekte jeweils das Frageformat zeitigt.

\section{Geschlossenes ASBI: Sympathische und unsympathische Sprachen}

Ein Beispiel für den Einsatz des geschlossenen ASBI ist die Kindergarten-Studie. Man kann auf diese Weise die Bewertungen zu einem vorgegebenen Set an Kategorien erheben und gezielt vergleichen. Hier wurde nach der Sympathie für eine Reihe von Sprachen gefragt, und zwar für Arabisch, Deutsch, Englisch, Französisch, Italienisch, Polnisch, Russisch, Spanisch und Türkisch. Im geschlossenen Frageformat ist es leicht möglich, die Antworten bereits zu skalieren (hier auf einer Fünfer-Skala von sehr sympathisch bis sehr unsympathisch). Abbildung 3.3 zeigt die Mittelwerte der Bewertungen der abgefragten Teilstichproben für die Sprachen Russisch, Polnisch, Türkisch und Arabisch (die vor allem als Migrantensprachen eine Rolle spielen). 


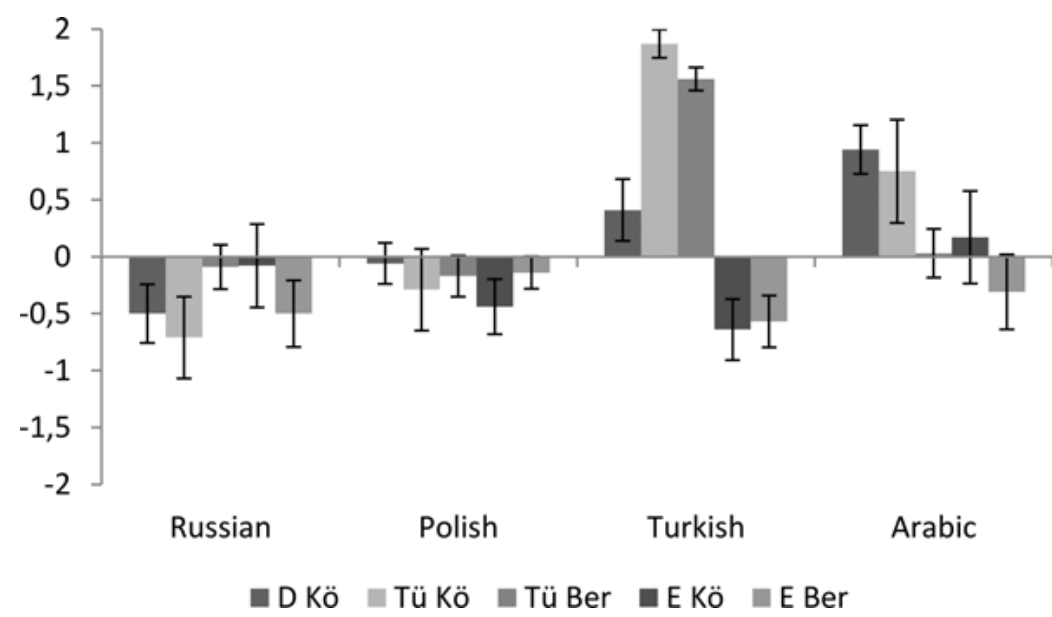

Abb. 3.3: Bewertungen von Sprachen (Mittelwerte) (D: Deutsch, Tü: Türkisch, E: Englisch; Kö: Köln, Ber: Berlin) ${ }^{5}$ (aus: Rothe \& Wagner 2015: 33).

Die verglichenen Teilstichproben sind relativ klein (wie auch an den im Diagramm dargestellten Standardfehlern der Mittelwerte ablesbar ist), insbesondere die Gruppe der Befragten des deutsch-türkisch-sprachigen Kindergartens in Köln, die Mittelwerte dieser Gruppe müssen daher mit Bedacht interpretiert werden. Die Bewertungen für das Russische und das Polnische unterscheiden sich über die Befragten der verschiedenen Kindergärten hinweg kaum (lediglich für das Russische zeigt sich ein - sehr geringer - Gruppenunterschied). Signifikant werden die Unterschiede bei der Bewertung des Arabischen und besonders des Türkischen. Beim Arabischen zeigen die Bewertungsunterschiede kein eindeutiges Muster. Sehr markant sind dafür die Unterschiede zwischen den Bewertungsgruppen in Bezug auf das Türkische: die befragten Personen aus den beiden deutsch-türkisch-sprachigen Kindergärten in Köln und Berlin (die zweite und dritte Teilsäule) bewerten das Türkische deutlich besser als die Befragten aus dem deutschsprachigen Kindergarten in Köln (die erste Teilsäule); noch größer ist der Unterschied zur Bewertung der Befragten aus den deutsch-englisch-sprachigen Kindergärten in Köln und Berlin (vierte und fünfte Teilsäule), die das Türkische deutlich negativer bewerten. Als relevante Einflussfaktoren dürften hier die unterschiedlichen Sprachkonzepte der Kin-

5 Für die Berechnung der Mittelwerte wird für die Antwort „sehr sympathisch“ der Wert 2 gesetzt, für die Antwort „sympathisch“ der Wert 1 usw., für „sehr unsympathisch“ der Wert -2. $\mathrm{Zu}$ jeder Sprache sind in jeweils fünf Säulen die jeweiligen Mittelwerte der fünf Teilstichproben angegeben. 
dergärten, die unterschiedlichen Erstsprachen und die lebensweltliche Verankerung von Mehrsprachigkeit eine wesentliche Rolle spielen.

Ein Vorzug des geschlossenen ASBI (der natürlich mit der Endlichkeit und der Stabilität der Antwortkategorien zusammenhängt) besteht darin, dass sich auch komplexe Ergebnisse sehr gut visualisieren lassen. Insbesondere kann man, wie es hier exemplarisch an Abbildung 3.3 gezeigt wurde, in Kombinationsdiagrammen unterschiedliche Bewertungen über verschiedene Bewertungsobjekte hinweg ebenso wie unterschiedliche Bewertungen eines Bewertungsobjekts durch verschiedene Teilstichproben sichtbar machen.

\section{Geschlossenes ASBI (komplexe Auswertungen): Dialektal gefärbtes Deutsch} Ist - wie im Falle der Repräsentativerhebungen - die Menge der erhobenen Daten groß genug, dann ist es auch möglich, komplexe Zusammenhänge mit den allgemeinen Sprachbewertungen ausfindig zu machen. Das soll an einem Beispiel aus der Deutschland-Erhebung 2008 gezeigt werden. Dort wurde in einer Variante des geschlossenen ASBI nach der Sympathie für dialektal gefärbtes Deutsch gefragt. ${ }^{6}$ Die große Mehrheit der Befragten gibt an, dialektal gefärbtes Deutsch sympathisch (47,9\%) oder sogar sehr sympathisch (15,4\%) zu finden (Gärtig, Plewnia \& Rothe 2010: 156). Was lässt sich nun auf der Grundlage der Stichprobe genauer zum Profil derjenigen Befragten sagen, die angeben, dass sie dialektal gefärbtes Deutsch eher sympathisch finden? Um das herauszufinden, kann man mittels statistischer Tests (über lineare Regression) diejenigen anderen Meinungen und Einstellungen sowie diejenigen weiteren Fragen ausfindig machen, die mit den Antworten auf die Frage nach dialektal gefärbtem Deutsch statistisch im Zusammenhang stehen. Einen solchen statistisch signifikanten Zusammenhang gibt es (1) mit der eigenen Dialektkompetenz und (2) der Häufigkeit des Dialektgebrauchs, (3) mit den Bewertungen von Bairisch und Sächsisch, (4) den allgemeinen Gefühlen gegenüber dem Deutschen, (5) der Position zur Frage des Erhalts von autochthonen Minderheitensprachen in Deutschland, außerdem (6) mit der eigenen Lebenszufriedenheit sowie (7) der Einschätzung der allgemeinen aktuellen Wirtschaftslage. ${ }^{7}$

\footnotetext{
6 Die Frageformulierung lautete: „Vielen Deutschen kann man ihre regionale Herkunft anhören, auch wenn sie keinen ausgeprägten Dialekt oder Platt sprechen. Wie finden Sie solch ein dialektgefärbtes Deutsch?“ mit Antwortmöglichkeiten auf einer Fünfer-Skala von „sehr sympathisch“ bis „sehr unsympathisch“ sowie der zusätzlichen Antwortoption „kommt auf den Dialekt an“. Für detaillierte Auswertungen dieser Frage vgl. Gärtig, Plewnia \& Rothe (2010: 156-158).

7 Die zugrundeliegenden Fragen und die zugehörigen Antworten sowie detaillierte Auswertungen nach Teilgruppen finden sich in Gärtig, Plewnia \& Rothe 2010: 137-145, 146-149, 4797, 24-27, 227-229.
} 


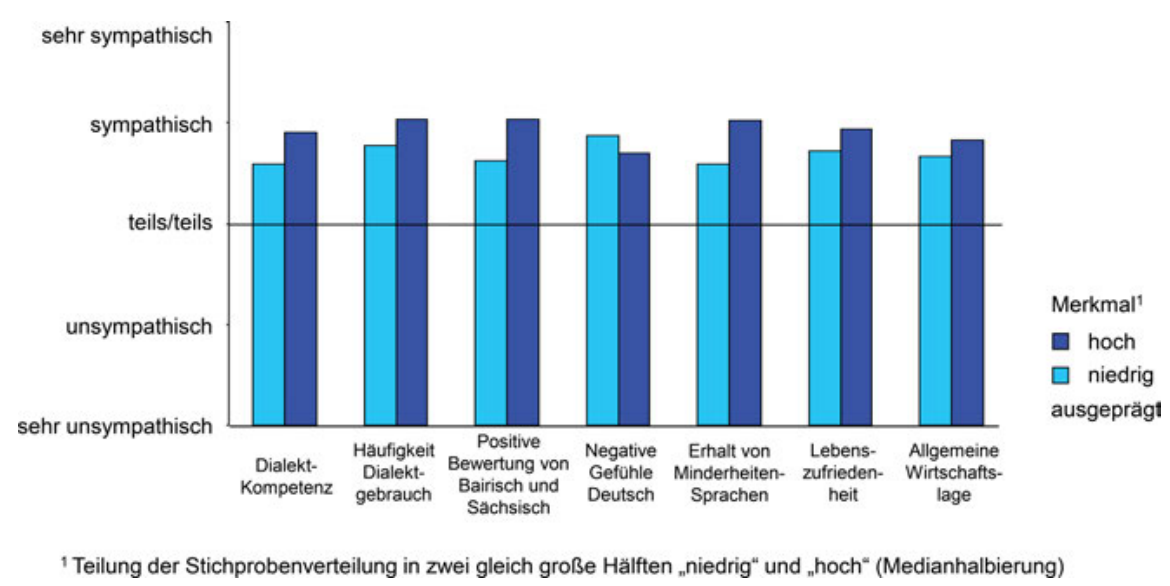

Abb. 3.4: Bewertung von dialektal gefärbtem Deutsch in Zusammenhang mit anderen Merkmalen (aus: Eichinger et al. 2009: 27).

Zur Visualisierung der Ergebnisse wird eine Medianhalbierung vorgenommen, d. h. die gesamte Stichprobe wird für jedes Merkmal entlang der Beantwortung der jeweils ausgewählten Frage, z. B. erstens der eigenen Dialektkompetenz, in zwei Hälften geteilt, eine mit niedriger und die andere mit hoher Ausprägung des jeweiligen Merkmals; die Merkmale sind in der eben genannten Reihenfolge auf der x-Achse des Diagramms abgetragen. Das Resultat einer solchen Darstellung zeigt Abbildung 3.4.

Hier zeigt für die genannten sieben Merkmale jeweils der linke, helle Balken die niedrige und der rechte, dunkle Balken die hohe Ausprägung des Merkmals. Diejenigen, die dialektgefärbtes Deutsch eher sympathisch finden, sind also Personen, die eher eine höhere Dialektkompetenz aufweisen und die ihren Dialekt auch häufiger gebrauchen; es sind Personen, die das Bairische und das Sächsische eher positiv bewerten; in Bezug auf das Deutsche äußern sie eher keine negativen Gefühle; sie sprechen sich eher für den Erhalt von Minderheitensprachen aus; außerdem sind diese Personen mit ihrem Leben eher zufrieden und beurteilen die allgemeine wirtschaftliche Lage in Deutschland eher positiv.

\section{Offenes ASBI (komplexe Auswertungen): Einstellungen zu romanischen Sprachen}

Solche komplexen Analysen lassen sich in ähnlicher Weise auch für Einstellungen, die über das offene ASBI erhoben wurden, durchführen; allerdings kommen dafür andere statistische Tests (mittels logistischer statt linearer Regression) und eine andere Art der Darstellung zum Einsatz; das sei an einem Beispiel 
in Prozent

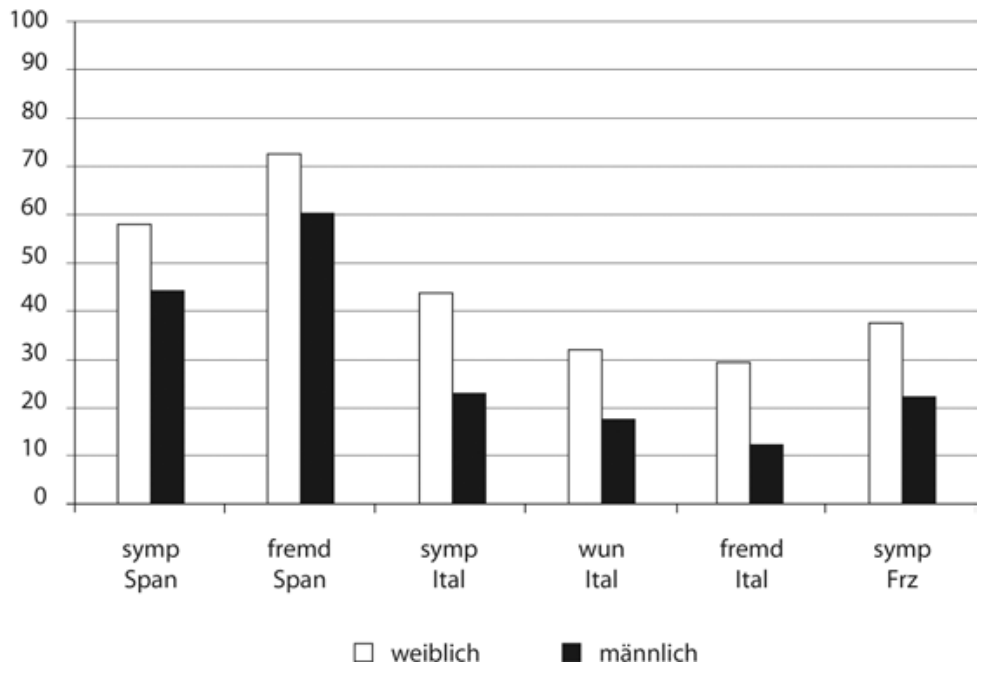

Abb. 3.5: Einstellungen zu romanischen Sprachen (aus: Rothe 2012: 155).

aus der Schüler-Studie erläutert. In der Schüler-Studie (mit 628 Schülerinnen und Schülern der 9. und 10. Jahrgangsstufen an vier Schulstandorten) wurde u. a. nach sympathischen Sprachen gefragt, außerdem danach, welche Fremdsprachen in der Schule gelernt werden sollten; außerdem sollten die Schülerinnen und Schüler Sprachen nennen, die sie gerne können würden (Wunschsprachen). Neben anderen Faktoren, die Einfluss auf die Spracheinstellungen haben, wie etwa das eigene Sprachrepertoire oder die Frage, welche Erstsprache man hat, ist auch das Geschlecht der Befragten ein beeinflussender Faktor. Mit Hilfe einer logistischen Regression kann man den Zusammenhang zwischen einer unabhängigen Variablen (in diesem Fall dem Geschlecht) und einer dichotomischen abhängigen Variablen (hier: Nennung oder NichtNennung einer bestimmten Sprache als sympathische Sprache, als gewünschte Schulfremdsprache oder als Wunschsprache) bestimmen; dazu werden die Antworten auf das offene ASBI als binäre Variablen kodiert. Besonders markante Unterschiede zwischen den Geschlechtern zeigen sich hier in Bezug auf die romanischen Sprachen, wie in Abbildung $3.5 \mathrm{zu}$ sehen ist.

Der jeweils linke, helle Balken zeigt die relativen Nennungen der Schülerinnen, der rechte, dunkle Balken die der Schüler. ${ }^{8}$ Die Schülerinnen nennen durchgängig die romanischen Sprachen Spanisch, Italienisch und Französisch

8 Berücksichtigt wurden dabei nur Schülerinnen und Schüler mit Deutsch als (alleiniger) Erstsprache, um eine Verzerrung durch einen Faktor Mehrsprachigkeit auszuschließen. 
deutlich häufiger als sympathische Sprachen als die Schüler, sie wünschen sich Spanisch und Italienisch signifikant häufiger als Schulfremdsprache als die Schüler, und sie nennen Italienisch signifikant häufiger als Wunschsprache als die Schüler. ${ }^{9}$

\subsection{Attitudes-Towards-Languages-Skala (AToL)}

\subsubsection{Die AToL-Skala}

Das zweite, in unseren Erhebungen systematisch eingesetzte Instrument ist die „Attitudes-Towards-Languages-Skala (AToL)“; sie dient der Erfassung von differenzierteren Spracheinstellungen. Die AToL-Skala ist das erste quantitativ (und auch sprachübergreifend) einsetzbare, validierte Instrument zur Erhebung von Einstellungen gegenüber Sprachen und Varietäten. ${ }^{10}$ Entwickelt und validiert wurde sie in vier Studien im Rahmen des Gemeinschaftsprojekts des IDS mit Lehrstuhl für Sozialpsychologie der Universität Mannheim „Erkundung und Analyse aktueller Spracheinstellungen in Deutschland“ (cf. Schoel et al. 2012; s. o.). Der Einsatz der AToL-Skala als stabiles Instrument über mehrere Erhebungen hinweg ermöglicht aufschlussreiche Vergleiche.

Die AToL-Skala besteht aus mehreren semantischen Differenzialen (z. B. schön - hässlich), die meist mit einer Fünfer-Skala abgefragt werden (z. B. sehr schön, schön, teils/teils, hässlich, sehr hässlich). Im Folgenden soll das Instrument anhand der vier Studien, mittels derer es entwickelt wurde, genauer vorgestellt werden (nach Schoel et al. 2012). Für die erste der vier Studien (Stichprobe: $n=406$; 145 Männer und 261 Frauen, Altersdurchschnitt: 25,3) wurden 51 semantische Differenziale mit bewertenden Items entwickelt. ${ }^{11}$ Diese um-

9 Entsprechende Aussagen lassen sich auch aus den Fragen des in der Schüler-Studie ebenfalls eingesetzten geschlossenen ASBI gewinnen. - Das Bild lässt sich übrigens komplettieren, indem man die Nennungen des Chinesischen als Wunschsprache, als sympathische Sprache und als gewünschte Schulfremdsprache hinzunimmt. Hier zeigt sich der umgekehrte Effekt, Chinesisch wird insgesamt deutlich häufiger von Schülern als von Schülerinnen genannt; zu Einzelheiten vgl. Rothe (2012: 155-157).

10 Bei der AToL-Skala geht es darum, dezidiert Einstellungen gegenüber Sprachen und Varietäten zu erfassen, und nicht die - zweifellos damit in Zusammenhang stehenden - Bewertungen von Sprechern dieser Sprachen und Varietäten. Solche Sprecherstereotype kann man in ähnlicher Weise, aber mit anderen Items erfassen; vgl. dazu z. B. Plewnia \& Rothe (2011b).

11 Die 51 Items waren die folgenden: abwechslungsreich - eintönig, altmodisch - modern, angenehm - unangenehm, anmutig - plump, anziehend - abstoßend, ausdrucksstark - ausdrucksschwach, beständig - unbeständig, beweglich - starr, dunkel - hell, eindeutig - missverständlich, einfach - schwierig, einflussreich - einflusslos, energisch - zurückhaltend, fließend - stockend, 


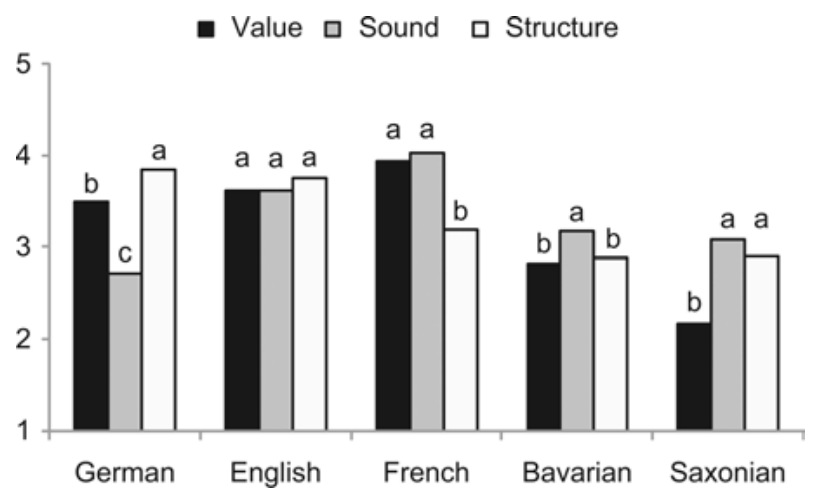

Abb. 3.6: Bewertungen der AToL-Skala, Studie 1 (verschiedene Indizes zeigen signifikante Unterschiede zwischen den Dimensionen pro Sprache an) (aus: Schoel et al. 2012: 27).

fassten sowohl Items des klassischen Instruments von Osgood, Suci \& Tannenbaum (1957) sowie von Crites, Fabrigar \& Petty (1994), Mulac (1975) und Zahn \& Hopper (1985) als auch neue, eigene Items. Die Befragten bewerteten anhand der Items zum einen die Sprachen Deutsch, Englisch und Französisch und zum anderen die zwei deutschen Dialekte Bairisch und Sächsisch. Über eine explorative Faktorenanalyse, in der die Zusammenhänge aller Bewertungen der 51 Items analysiert werden, wurden drei Basisdimensionen ermittelt, die sich mit den Etiketten Wert, Klang und Struktur fassen lassen. Diesen drei Basisdimensionen liegt eine hierarchische Struktur zugrunde: Die Dimension Wert ist eine übergeordnete Dimension, die Dimensionen Klang und Struktur sind dieser untergeordnet und stehen mit ihr in Zusammenhang. Um das Instrument handhabbar zu gestalten, wurden für jede Dimension entsprechend der Faktorladungen der semantischen Differenziale jeweils fünf Items ausgewählt, so dass das Instrument nunmehr aus 15 Items besteht. Abbildung 3.6 zeigt die Ergebnisse für die drei erhobenen Sprachen und die beiden Dialekte; abgetragen sind jeweils die Mittelwerte der aggregierten Basisdimensionen.

flüssig - abgehackt, genau - ungenau, geordnet - durcheinander, gespannt - entspannt, grob fein, hart - weich, hässlich - schön, kalt - warm, klanglos - klangvoll, kraftvoll - kraftlos, langweilig - interessant, laut - leise, lebendig - leblos, lebhaft - ruhig, leicht - schwerfällig, logisch - unlogisch, markant - unauffällig, melodisch - unmelodisch, rau - geschmeidig, regelhaft - regellos, rund - eckig, schleppend - hastig, schnell - langsam, stillos - stilvoll, strukturiert - strukturlos, systematisch - unsystematisch, träge - schwungvoll, überflüssig unersetzlich, übersichtlich - unübersichtlich, umständlich - einfach, unbeliebt - angesehen, unrhythmisch - rhythmisch, verspielt - ernst, wertlos - wertvoll, wichtig - bedeutungslos, widersprüchlich - widerspruchslos, wild - sanft. 


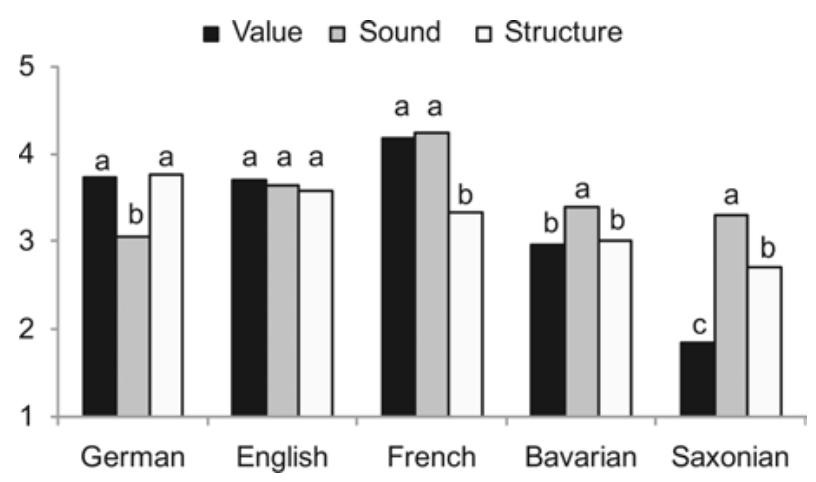

Abb. 3.7: Bewertungen der AToL-Skala Studie 2 (verschiedene Indizes zeigen signifikante Unterschiede zwischen den Dimensionen pro Sprache an) (aus: Schoel et al. 2012: 31).

Für das Deutsche erhalten die Items der Struktur-Dimension (jeweils der rechte, helle Balken) die besten Bewertungen, am niedrigsten werden die zur KlangDimension (jeweils der mittlere Balken) gehörigen Items bewertet. Das Englische wird auf allen drei Dimensionen etwa gleich bewertet; beim Französischen werden die Wert-Dimension (jeweils der linke, dunkle Balken) und die Klang-Dimension höher bewertet als die Struktur-Dimension. Beim Bairischen liegen die Werte für die Klang-Dimension etwas höher als die der Wert-Dimension und der Struktur-Dimension; beim Sächsischen liegen die Items der Wert-Dimension deutlich am niedrigsten. Die höchste Korrelation mit den (über das ASBI erhobenen) allgemeinen Sprachbewertungen ergab sich für die Wert-Dimension; das heißt, dass die Wert-Dimension der AToL-Skala allgemeinen Spracheinstellungsmessungen, wie sie mit dem ASBI vorgenommen werden, konzeptuell am nächsten steht (vgl. Schoel et al. 2012). Die Ergebnisse dieser ersten Studie zeigen, dass das AToL-Instrument geeignet ist, Sprachbewertungen differenziert zu erfassen. Die AToL-Skala wurde in drei weiteren Studien repliziert bzw. modifiziert und validiert.

In der zweiten Studie wurden die Skalendimensionen Wert, Klang und Struktur validiert (Stichprobe: $n=282$; 77 Männer und 205 Frauen, Altersdurchschnitt: 32). Zusätzlich wurden in dieser zweiten Studie auch Sprecherstereotype anhand von Kompetenz- und Wärmeskalen (nach Fiske et al. 2002) abgefragt, die dann mit den AToL-Daten in Beziehung gesetzt werden konnten. Abbildung 3.7 zeigt die Ergebnisse der Bewertungen auf der AToL-Skala für die vorgelegten Sprachen (Deutsch, Englisch, Französisch) und Varietäten (Bairisch, Sächsisch); sie entsprechen weitgehend denjenigen aus der ersten Studie. Auch hier stellen sich wieder sowohl die hierarchische Struktur der Skalendimensionen heraus (mit Wert als übergeordneter Dimension) als auch die 


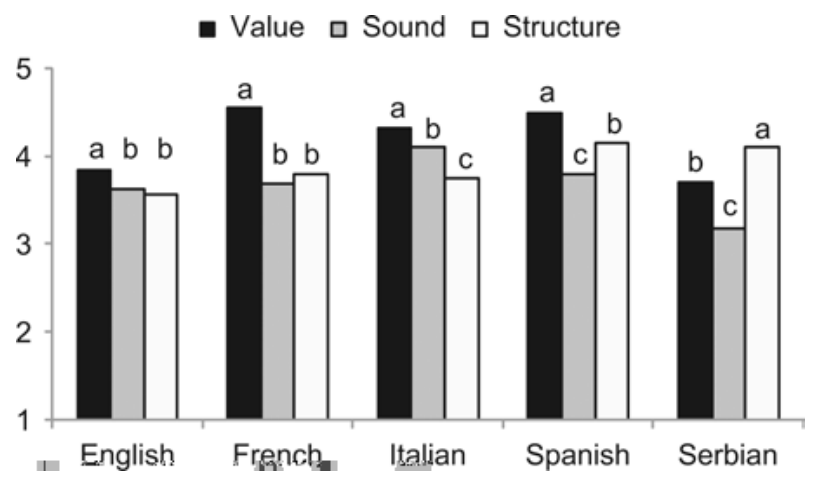

Abb. 3.8: Bewertungen der AToL-Skala, Studie 3 (verschiedene Indizes zeigen signifikante Unterschiede zwischen den Dimensionen pro Sprache an) (aus: Schoel et al. 2012: 34).

offensichtliche konzeptuelle Nähe der Wert-Dimension mit dem allgemeinen Sprachgefallen.

In der dritten Studie wurde die AToL-Skala in fünf anderen Sprachen umgesetzt, nämlich auf Englisch, auf Französisch, auf Italienisch, auf Spanisch und auf Serbisch, und dann Erstsprachlern der jeweiligen Sprachen vorgelegt (Stichprobe: $n=419 ; 136$ Männer und 283 Frauen, Altersdurchschnitt: 30,7; Englisch: 85, Französisch: 77, Italienisch: 69, Spanisch: 63, Serbisch: 125). (Für das Englische und das Französische waren damit auch Vergleiche von Fremdund Selbstbewertungen möglich.) Die Ergebnisse zeigt Abbildung 3.8.

Sowohl Englisch als auch Französisch werden von ihren jeweiligen Sprechern auf der Wert-Dimension besser bewertet als auf den Dimensionen Klang und Struktur (Französisch mit deutlicherem Abstand); darin unterscheiden sich diese Selbstbewertungen von den Fremdbewertungen durch die Deutschsprachigen in den ersten beiden Studien. Ähnlich wie in den ersten beiden Studien sind aber auch hier die Bewertungen über die verschiedenen Dimensionen für das Englische weniger different ausgeprägt als für das Französische. Auch Italienisch und Spanisch werden auf der Wert-Dimension am höchsten bewertet, beim Italienischen folgen Klang und dann Struktur, beim Spanischen Struktur und Klang. Die Bewertungen für das Serbische unterscheiden sich am deutlichsten von den Mustern der anderen Bewertungen, hier wird die StrukturDimension am höchsten bewertet, gefolgt von Wert und Klang. ${ }^{12}$

12 Hinsichtlich der Ursachen für diese Unterschiede lassen sich nur aufgrund dieser Daten keine Aussagen treffen; eine Interpretation dieser Befunde wird sprachkulturelle Kontexte, in diesem Fall etwa das Verhältnis zum Kroatischen, sowie sprachpolitische Diskurse und Fragen sprachlicher Identität zu berücksichtigen haben. 


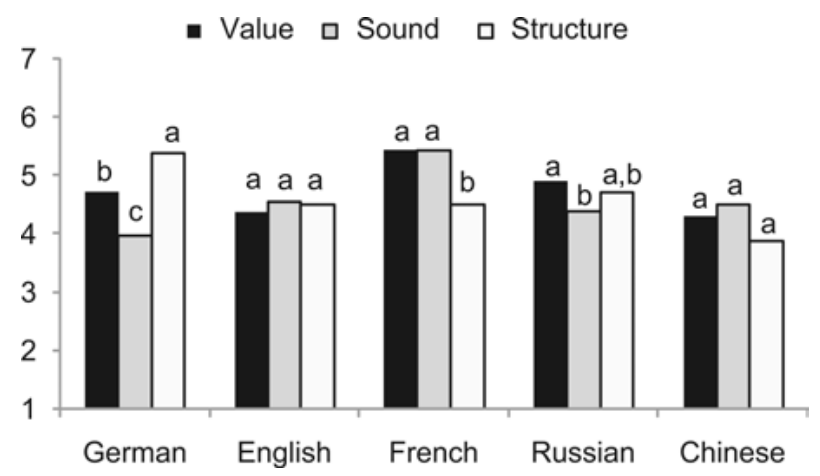

Abb. 3.9: Bewertungen der AToL-Skala, Studie 4 (verschiedene Indizes zeigen signifikante Unterschiede zwischen den Dimensionen pro Sprache an) (aus: Schoel et al. 2012: 38).

Die vierte Studie unterscheidet sich von den vorherigen Studien in einem wesentlichen Punkt: Während bisher nur abstrakt nach Bewertungen von Sprachen bzw. Varietäten gefragt wurde, wurden den Probanden in dieser vierten Studie kurze Sprachproben in deutscher, englischer, französischer, russischer und chinesischer Sprache zur Bewertung vorgelegt (Stichprobe: $n=201$, 59 Männer und 142 Frauen, Altersdurchschnitt: 26,8); die Ergebnisse zeigt Abbildung 3.9.

Bei der Interpretation der Ergebnisse ist zu beachten, dass hier unter Umständen nicht dieselben Dinge abgefragt werden wie bei den vorherigen Umfragen. Prinzipiell muss man davon ausgehen, dass es einen Unterschied macht, ob Probanden abstrakt und kontextfrei nach ihren Einschätzungen gefragt werden, oder ob sie einen gegebenen Stimulus bewerten sollen. In Bezug auf die Bewertung von Sprachproben ist dies aus (mindestens) zwei Gründen relevant: zum einen muss angenommen werden, dass sich eine abgegebene Bewertung nicht nur auf die Sprache oder Varietät bezieht, sondern dass zunächst die konkrete Performanz im Vordergrund steht - und übrigens bisweilen auch der Inhalt, selbst wenn dies in der Frageformulierung dezidiert ausgeschlossen wird und dass damit auch der Sprecher bzw. die Sprecherin bewertet oder zumindest mitbewertet wird, und zwar entlang von Parametern, die mit der Sprache nichts zu tun haben müssen; zum anderen ist nicht immer gewährleistet, dass gegebene Sprachproben genau die als zugehörig gedachten Konzepte der Probanden abbilden; das ist ein Punkt, der insbesondere bei der Bewertung von dialektalen Sprachproben zu berücksichtigen ist (zum Phänomen solcher Zuordnungsirrtümer vgl. z. B. Anders 2010; Schaub 2011).

Dennoch erweisen sich die Ergebnisse dieser vierten Studie als vergleichbar mit den Ergebnissen der ersten beiden Studien; die Bewertungen für Deutsch, Englisch und Französisch sind jedenfalls sehr ähnlich. Für das 
Russische sind die Werte auf der Klang-Dimension die niedrigsten; für das Chinesische lässt sich eine deskriptive Tendenz feststellen, wonach die KlangDimension am höchsten und die Struktur-Dimension am niedrigsten bewertet wird; diese Unterschiede werden jedoch statistisch nicht signifikant. Auch in den Ergebnissen dieser Studie zeigt sich wieder die Hierarchie der Basisdimensionen mit der Wert-Dimension als übergeordneter Dimension.

Insgesamt erweist sich die AToL-Skala als funktionales, leicht einsetzbares und vor allem gut auf große Datenmengen (wie etwa repräsentative Stichproben) anwendbares Instrument, das sich zudem (ggf. mit Anpassungen) gut auf verschiedene Sprachen und Varietäten übertragen lässt. Die drei Basisdimensionen Wert, Klang und Struktur erlauben eine differenzierte Erfassung verschiedener Facetten von Einstellungen; die in diesem Muster hierarchisch übergeordnete Dimension Wert korreliert stark mit allgemeinen Gefallensbewertungen, wie sie mittels des ASBI erhoben werden können.

\subsubsection{Exemplarische Ergebnisse von AToL aus den verschiedenen Erhebungen}

Die AToL-Skala kam in den meisten unserer Erhebungen zum Einsatz; wir konzentrieren uns im Folgenden auf die Repräsentativerhebungen. In der Deutschland-Erhebung 2008 wurde die AToL-Skala eingesetzt, um Bewertungen der deutschen Sprache sowie von Bairisch und Sächsisch zu erfassen, in der Norddeutschland-Erhebung 2016 wurden Hochdeutsch und Plattdeutsch abgefragt, in der Deutschland-Erhebung 2017/18 werden Deutsch, Bairisch, Sächsisch und Plattdeutsch (teils gesplittet) erhoben. Da jedes Mal das gleiche Instrument verwendet wird, sind verschiedene Vergleiche sowohl innerhalb eines Datensatzes als auch über die Erhebungen hinweg möglich; einige Zugänge werden im Folgenden exemplarisch vorgestellt.

Eine naheliegende Gegenüberstellung ist der Vergleich zweier Varietäten, die in einer Erhebung von denselben Probanden bewertet wurden. Abbildung 3.10 zeigt den Vergleich der Bewertungen von Hochdeutsch und Plattdeutsch in der Norddeutschland-Erhebung $2016 .^{13}$

Der Vergleich von Hochdeutsch und Plattdeutsch über die gesamte Stichprobe zeigt, dass die Standardvarietät auf nahezu allen Dimensionen besser bewertet wird. Die größten Unterschiede zeigen sich erwartungsgemäß bei den

13 Für einen entsprechenden Vergleich der Bewertungen von Deutsch vs. Bairisch bzw. vs. Sächsisch, wie er sich aus den Daten der Deutschland-Erhebung 2008 ergibt, vgl. Eichinger et al. (2009: 24). Dort zeigt sich, etwas vergröbert gesagt, dass Deutsch auf der Wert-Dimension und auf der Struktur-Dimension jeweils besser als Bairisch und Sächsisch bewertet wird. 


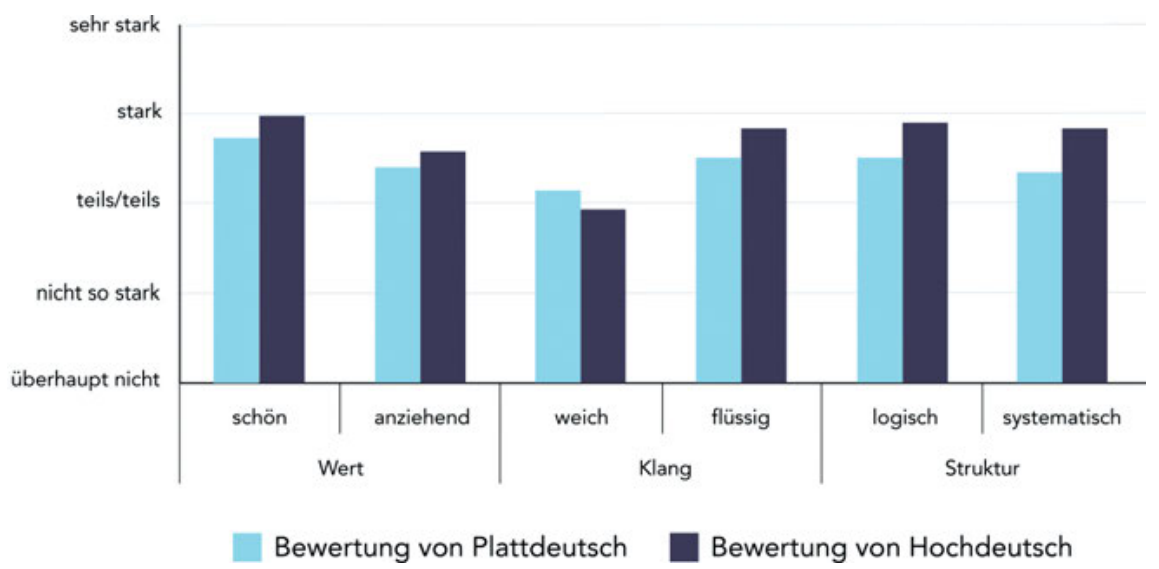

Abb. 3.10: Bewertungen von Plattdeutsch und Hochdeutsch im Vergleich (aus: Adler et al. 2016: 27).

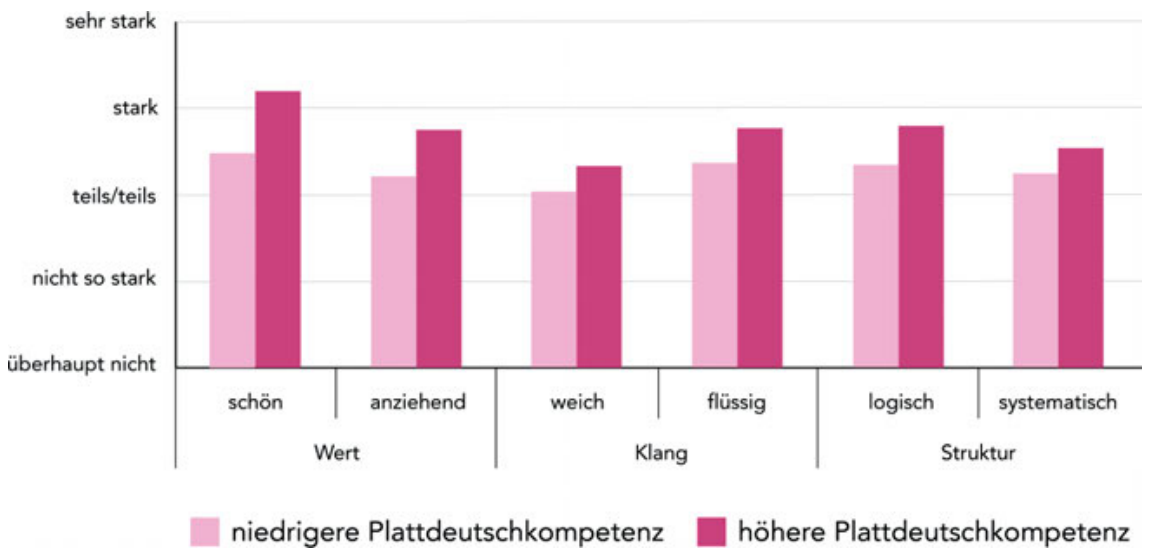

Abb. 3.11: Bewertungen von Plattdeutsch: nach Plattdeutschkompetenz (aus: Adler et al. 2016: 28).

Items der Struktur-Dimension, Hochdeutsch wird als logischer und systematischer bewertet. Plattdeutsch bekommt nur bei einem der beiden Items der Klang-Dimension, bei weich, höhere Werte als Hochdeutsch.

Diese Befunde gelten für die Gesamtmenge der befragten Personen in Norddeutschland. Plattdeutsch wird jedoch nicht von allen Befragten gleich bewertet. Die Bewertungen für Plattdeutsch fallen sehr unterschiedlich aus, wenn man als Kriterium die Plattdeutschkompetenz der Befragten berücksichtigt; einen entsprechenden Vergleich liefert Abbildung 3.11. 


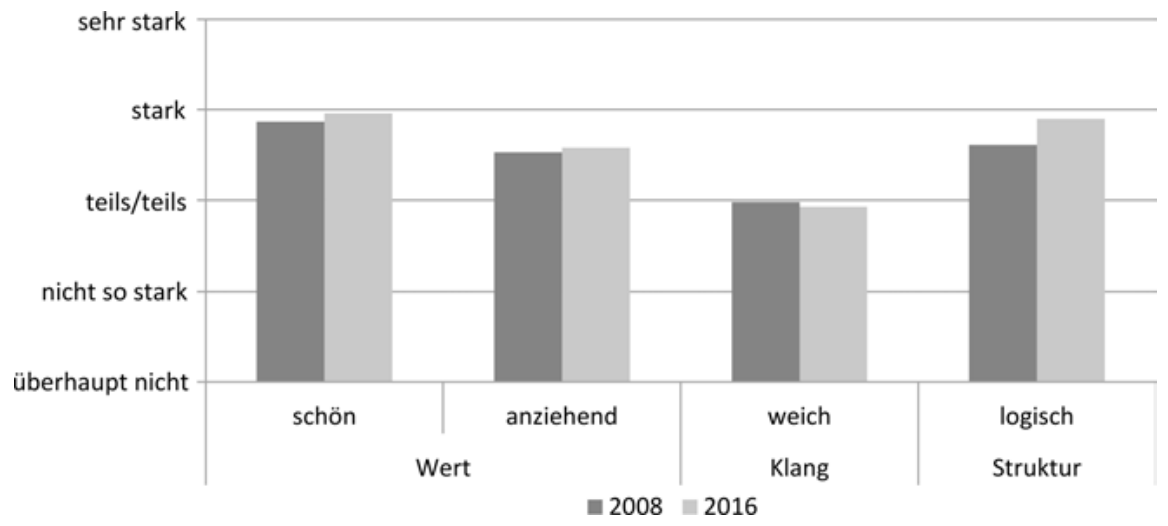

Abb. 3.12: Bewertungen 2008 und 2016: gesamte Stichproben.

Hier wurden mittels einer Medianhalbierung (wie oben bei Abb. 3.4) entlang des Merkmals Plattdeutschkompetenz (gebildet aus den Antworten auf die Fragen, wie gut Plattdeutsch gesprochen wird) zwei Teilstichproben gebildet, zum einen Befragte mit niedrigerer Plattdeutschkompetenz (jeweils der linke, dunkle Balken) und zum anderen Befragte mit höherer Plattdeutschkompetenz (jeweils der rechte, helle Balken). Man sieht, dass die Bewertungen des Plattdeutschen der Personen mit höherer Plattdeutschkompetenz durchgängig höher ausfallen als die Bewertungen derjenigen mit niedrigerer Plattdeutschkompetenz. Besonders groß sind die Bewertungsunterschiede für die Items der WertDimension.

Die Abbildung 3.12 zeigt einen diachronen Vergleich der Bewertungen von Deutsch in der Deutschland-Erhebung 2008 und in der NorddeutschlandErhebung 2016. ${ }^{14}$ Es zeigt sich im diachronen Vergleich, dass die Bewertungen des Deutschen über die acht Jahre, die zwischen beiden Erhebungen liegen, relativ stabil geblieben sind. Relativ hohe Werte ergeben sich in beiden Erhebungen für die Wert-Dimension (hier besonders für das Item schön) und für die Struktur-Dimension (wo die Werte 2016 geringfügig höher liegen); die Werte für die Klang-Dimension liegen im mittleren Bereich. ${ }^{15}$

14 Die Frageformulierungen sind aufgrund des sich jeweils geringfügig unterscheidenden Fragebogenkontexts nicht ganz identisch, 2008 wurde die „deutsche Sprache“ abgefragt, 2016 „Hochdeutsch“.

15 Bei der Klang-Dimension wurde 2016 statt des Items melodisch das Item flüssig eingesetzt, bei der Struktur-Dimension wurde 2016 statt des Items einfach das Item systematisch. Für den diachronen Vergleich bleiben diese beiden Items daher unberücksichtigt. 


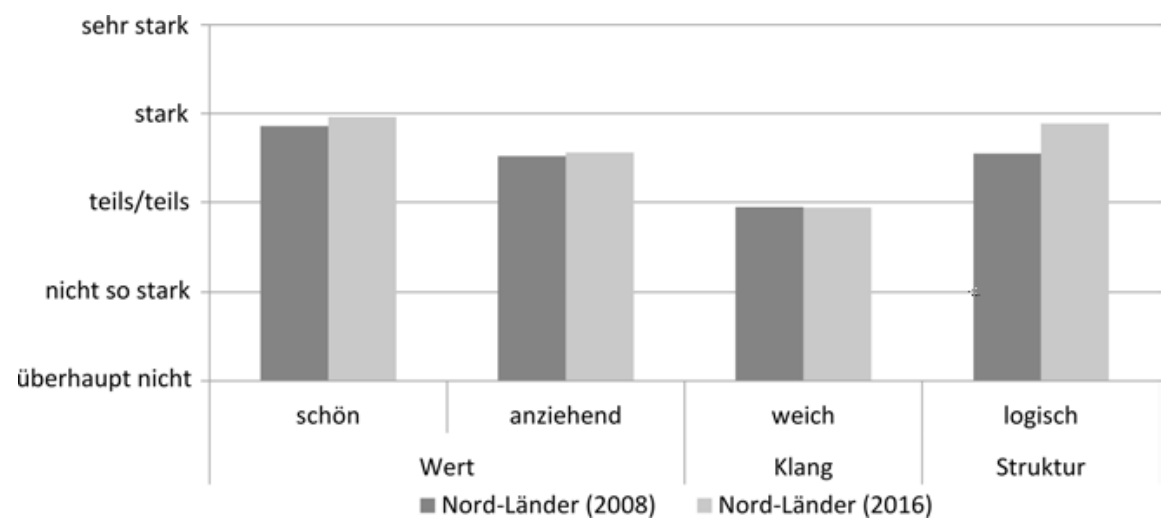

Abb. 3.13: Bewertungen 2008 und 2016: nur Nord-Länder.

Der in Abbildung 3.12 vorgenommene Vergleich der beiden gesamten Stichproben bedarf jedoch der methodischen Absicherung, weil die jeweils zugrundeliegende Grundgesamtheit nicht dieselbe ist. Zwar sind beide Stichproben repräsentativ, die Erhebung von 2008 erfasste jedoch das ganze Bundegebiet, während sich die Erhebung von 2016 nur auf Norddeutschland bezog. Es könnte ja sein, dass die Bewertungen aus Süddeutschland sich erheblich von denjenigen aus Norddeutschland unterscheiden. Man kann den gezogenen Vergleich jedoch in zwei Richtungen absichern: zum einen kann man aus beiden Stichproben nur die deckungsgleichen Erhebungsgebiete betrachten. Und zum andern kann man erhebungsintern die jeweils zugrundegelegten Teilgebiete vergleichen. Sollten sich hier keine erheblichen Differenzen zeigen, kann man davon ausgehen, dass auch der Vergleich der beiden Gesamtstichproben zulässig ist und nicht zu verzerrten Ergebnissen führt.

In der Norddeutschland-Erhebung 2016 wurden die Länder Bremen, Hamburg, Mecklenburg-Vorpommern, Niedersachsen und Schleswig-Holstein flächendeckend und die Länder Brandenburg, Nordrhein-Westfalen und Sachsen-Anhalt jeweils nur in ihren nördlichen Regionen befragt. Da die Daten der Deutschland-Erhebung 2008 für die Bundesland-Ebene zur Verfügung stehen, ist ein Vergleich nur für die fünf norddeutschen, 2016 vollständig erhobenen Länder möglich; Abbildung 3.13 zeigt die Ergebnisse der AToLSkala jeweils für 2008 und für 2016 für die fünf Nord-Länder. Tatsächlich zeigt sich in Abbildung 3.13 praktisch dasselbe Bild wie in Abbildung 3.12. Offenbar macht es keinen Unterschied, ob man nur jeweils die fünf NordLänder betrachtet oder die beiden Gesamterhebungsgebiete miteinander vergleicht. 


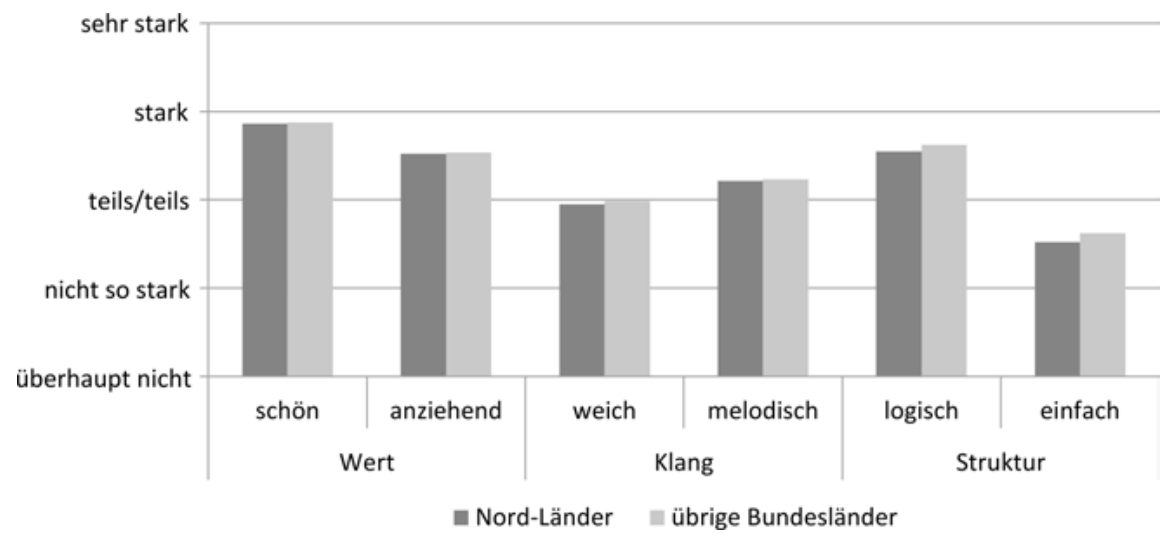

Abb. 3.14: Bewertungen 2008: Nord-Länder und übrige Bundesländer.

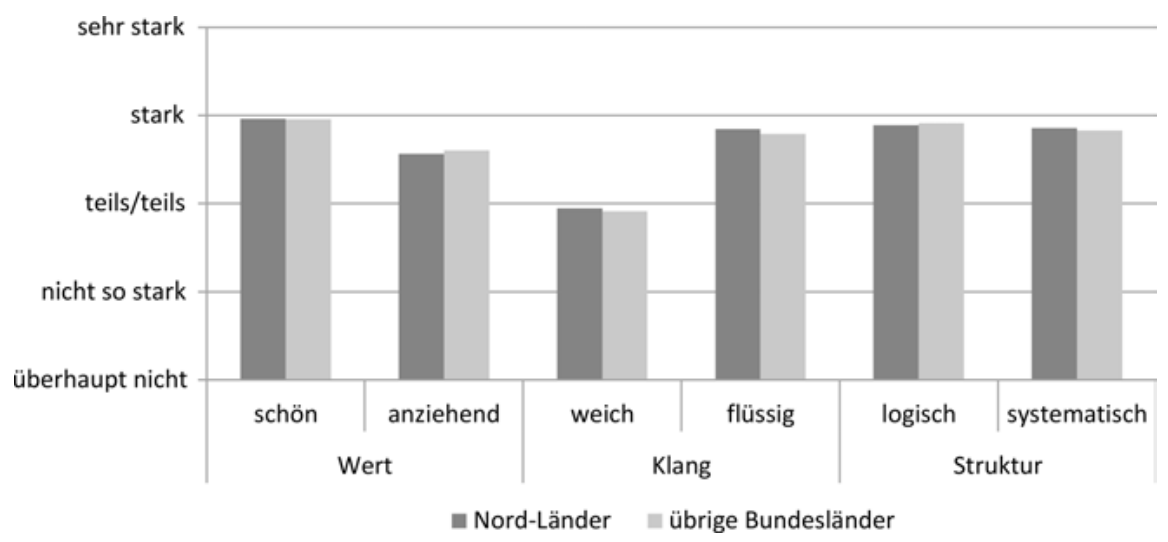

Abb. 3.15: Bewertungen 2016: Nord-Länder und übrige Bundesländer.

Die zweite Absicherung erfolgt jeweils stichprobenintern. Zunächst werden für die Daten der Deutschland-Erhebung 2008 die in Abbildung 3.13 herangezogenen fünf Nord-Länder mit dem übrigen Bundesgebiet verglichen (Abb. 3.14). ${ }^{16}$ Das Ergebnis ist sehr klar. Es zeigt sich, dass die Bewertungen der deutschen Sprache auf der AToL-Skala von der regionalen Herkunft der Befragten unabhängig sind, die AToL-Bewertungen der Befragten aus den nördlichen und den südlichen Bundesländern unterscheiden sich nicht. Abbildung 3.15 bietet denselben Vergleich innerhalb der Norddeutschland-Erhebung 2016.

16 Hier können, weil es sich ja um einen stichprobeninternen Vergleich handelt, wieder alle sechs abgefragten Items der AToL-Skala herangezogen werden. 
Auch hier sieht man, dass sich beim Vergleich der fünf vollständig erhobenen Nord-Länder (Bremen, Hamburg, Mecklenburg-Vorpommern, Niedersachsen und Schleswig-Holstein) mit dem übrigen Erhebungsgebiet (die nördlichen Teile von Brandenburg, Nordrhein-Westfalen und Sachsen-Anhalt) praktisch keine Unterschiede zeigen, dass also die AToL-Bewertungen nicht mit der regionalen Herkunft der Befragten zusammenhängen. Das erlaubt zwei Schlüsse: zum einen kann man sagen, dass der in Abbildung 3.12 vorgenommene Vergleich der beiden Gesamtstichproben von 2008 und 2016 nicht verzerrend war. Und zum zweiten ist mit den in Abbildung 3.12 dargestellten Ergebnissen festzuhalten, dass sich die Bewertungen im Zeitraum von 2008 bis 2016 nicht maßgeblich verschoben haben, die Spracheinstellungen der Deutschen gegenüber dem Deutschen sind also 2016 in etwa die gleichen wie noch 2008. Auch hier werden die Ergebnisse der Deutschland-Erhebung 2017/18, die ja bundesweit angelegt ist und in der neben Deutsch auch Bairisch, Sächsisch und Plattdeutsch abgefragt werden, weiteren Aufschluss bringen und detailliertere Analysen ermöglichen.

\section{Nutzung dialektometrischer Methoden zur Auswertung von Spracheinstellungsdaten}

Einer der wesentlichen Vorzüge unserer großen quantitativen Erhebungen besteht darin, dass die große Menge der zur Verfügung stehenden Daten sehr differenzierte statistische Auswertungen erlaubt. Ab einem gewissen Differenzierungsgrad wird jedoch die Datenbreite und -tiefe, wo sie die Visualisierung komplexer Sachverhalte erfordert, ihrerseits zur Herausforderung. Eine in der Einstellungsforschung bislang noch nicht praktizierte Lösung liegt zumindest in den Bereichen, in denen der Faktor Regionalität eine Rolle spielt, in der Übertragung von Verfahren, die üblicherweise in der Dialektometrie eingesetzt werden (vgl. Goebl 1982, 1984 und z. B. Hansen-Morath 2016). In der Dialektometrie werden die Differenzen bzw. Ähnlichkeiten zwischen objektiven Sprachdaten (etwa bestimmten phonologischen Positionen) gemessen und visualisiert, um areale Verteilungen und etwa Aggregationen zu größeren Räumen zu identifizieren. Wie so etwas für Einstellungs-Daten aussehen kann, soll im Folgenden an Daten aus der Norddeutschland-Erhebung 2016 exemplifiziert werden.

Als Ausgangspunkt wählen wir die Frage nach der aktiven Plattdeutschkompetenz. ${ }^{17}$ Die konventionelle Darstellungsweise ist hier die eines Kreisdiagramms, wie es Abbildung 3.16 bietet.

17 Die Frageformulierung lautete: „Wie gut können Sie selbst Plattdeutsch sprechen?“, mit einer vorgegebenen fünfstufigen Antwortskala. 


\section{Wer spricht Plattdeutsch?}

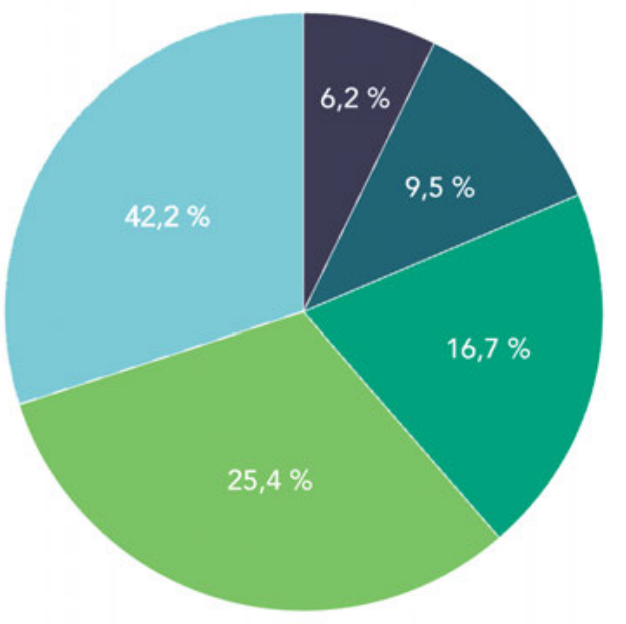

sehr gut

$\square$ gut $\square$ mäßig

nur einige Wörter

gar nicht

Abb. 3.16: Aktive Plattdeutschkompetenz (aus: Adler et al. 2016: 14).

Abbildung 3.16 zeigt die Antworten über die gesamte Stichprobe, bzw. geographisch gesprochen über den gesamten Erhebungsraum. Allerdings ist das Niederdeutsche in Norddeutschland unterschiedlich stark verbreitet, es drängt sich auf, eine Darstellungsform zu suchen, die eine gewisse räumliche Differenzierung sichtbar macht. Abbildung 3.17 zeigt die aktive Plattdeutschkompetenz, differenziert nach Bundesländern.

Hier ist die Fünfer-Skala für jedes Land in einen Hochbalken überführt. Diese Darstellung hat den Vorteil, dass einerseits jedes einzelne Land gut erfassbar ist und dass andererseits die Unterschiede zwischen den einzelnen Ländern gut augenfällig werden. Das Problem ist aber natürlich auch hier, dass die tatsächlichen Verhältnisse durch die Zusammenfassung auf Länderebene stark vergröbert werden. Besonders deutlich wird das im Falle von Niedersachsen, wo mit erheblichen Unterschieden beispielsweise zwischen der Nordseeküste und dem Harz gerechnet werden muss. Wünschenswert ist also eine Darstellung, die eine noch kleinräumigere Differenzierung abbildet. Natürlich könnte man analog zu Abbildung 3.17 auch eine Darstellung liefern, die für alle 101 Kreise des Erhebungsgebiets einzelne Hochbalken liefert (oder auch, in einem ersten Simplifizierungsschritt, für jeden Kreis die entsprechenden Mittelwerte, beispielsweise in einem Balkendiagramm, abbildet). Eine solche Darstellung wäre allerdings genauso unübersichtlich und schwer zu rezipieren wie die zugrundeliegende Tabelle mit 101 Wertzeilen selbst. Wir versuchen daher 


\section{Plattdeutsch sprechen: nach Bundesländern}

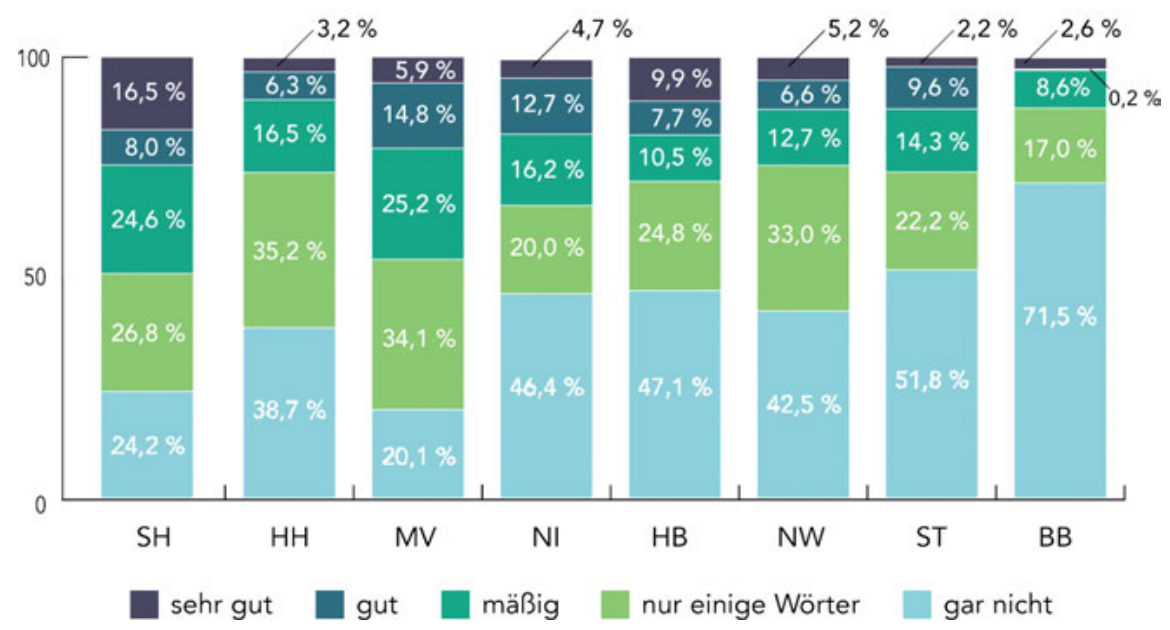

Abb. 3.17: Aktive Plattdeutschkompetenz: nach Bundesländern (aus: Adler et al. 2016: 15).

eine Umsetzung der Daten in einer kartographischen Darstellung. ${ }^{18}$ Für diese Art der Visualisierung gibt es verschiedene Anwendungen; wir nutzen das online frei verfügbar Instrument Gabmap (unter www.gabmap.nl/, letzter Zugriff: 3.1. 2018, vgl. Nerbonne et al. 2011). Abbildung 3.18 zeigt die pro Kreis (durch stilisierte Polygone dargestellt) aggregierten Daten (Mittelwerte) für die aktive Plattdeutschkompetenz.

Je höher die aktive Plattdeutschkompetenz ist, d. h. je besser die Befragten im entsprechenden Kreis Plattdeutsch sprechen, desto dunkler sind die Polygone eingefärbt. Diese Art der Darstellung ermöglicht es, räumliche Verteilungsmuster zu erkennen, die in einer Darstellung wie in Abbildung 3.17 verdeckt bleiben. Man sieht deutlich, dass die Nordseeküste ein Raum mit hoher aktiver Plattdeutschkompetenz ist, während die Werte nach Süden und Südosten hin immer niedriger werden. Insbesondere kann man erkennen, dass zumindest bei dieser Frage - die Bundeslandgrenzen als Ordnungsprinzip irrelevant sind, die Kompetenz-Räume organisieren sich nicht entlang der politischen Grenzen.

18 Dialektometrische Verfahren werden typischerweise auf objektive Daten angewendet und eben bislang noch nicht auf Einstellungsdaten, d. h. subjektive Daten. In diese Richtung weisen aber etwa die dialektometrischen Auswertungen von Vokalperzeptionsdaten von Kendall \& Fridland (2016) oder Stoeckle (2016). 


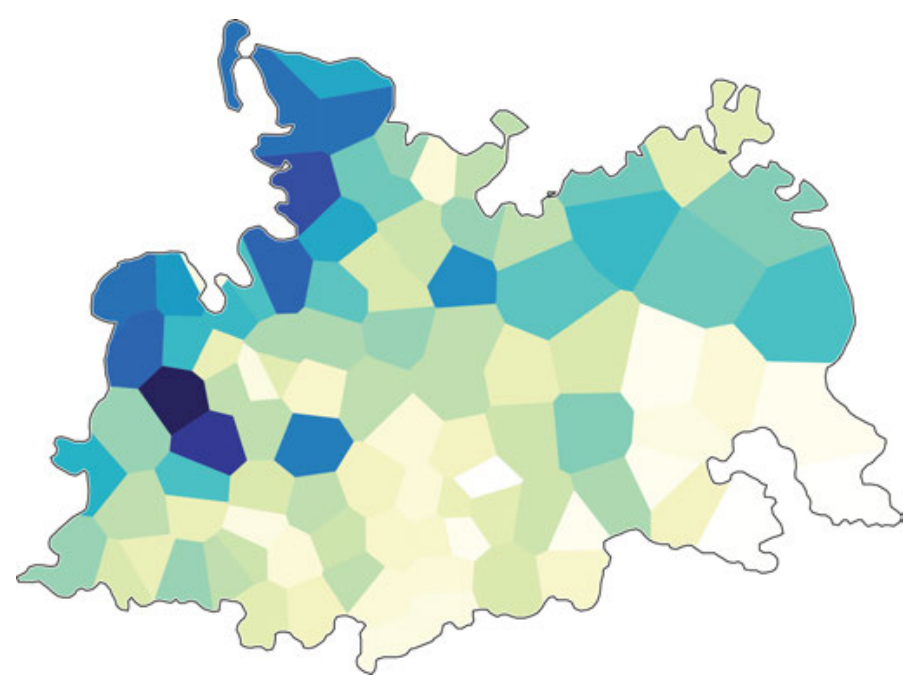

Abb. 3.18: Aktive Plattdeutschkompetenz: nach Kreisen (mit Gabmap).

Eine besondere Stärke von Gabmap liegt darin, dass es sich nicht nur zur einfachen Visualisierung von Daten nutzen lässt, sondern dass es damit auch möglich ist, statistische Berechnungen durchzuführen und deren Ergebnisse dann wiederum auf Raumbilder zu applizieren. Entsprechend lassen sich auch die Daten der AToL-Skala auf diese Weise abbilden. Abbildung 3.19 zeigt beispielhaft die Mittelwerte eines der Wert-Items der AToL-Skala für das Plattdeutsche aus der Norddeutschland-Erhebung 2016, das semantische Differenzial schön/hässlich. Je höher die Bewertungen der Befragten für das Item schön im entsprechenden Kreis ausfallen, desto dunkler sind die Polygone eingefärbt. Die Karte zeigt eine ähnliche Verteilung wie Abbildung 3.18; das entspricht auch den Erwartungen, weil die Bewertung des Plattdeutschen stark mit der Plattdeutschkompetenz korreliert (vgl. oben Abb. 3.11).

In einem weiteren Schritt können nun mittels Gabmap statistische Aggregations-Verfahren angewendet werden, z. B. eine Clusteranalyse oder eine Ähnlichkeitsstrukturanalyse (Multidimensionale Skalierung, MDS). ${ }^{19}$ Clusteranalysen werden verwendet, um Objekte in großen Datenmengen nach ihrer Ähnlichkeit oder Unähnlichkeit zu gruppieren. Dabei werden die untersuchten Objekte so gruppiert, dass einerseits die Unterschiede zwischen den Objekten

19 Natürlich sind solche statistischen Berechnungen auch mit den üblichen Statistikprogrammen wie z. B. SPSS, STATA oder R durchführbar, jedoch ist diese Form der arealen Visualisierung der Ergebnisse damit nicht möglich oder jedenfalls deutlich aufwendiger. 


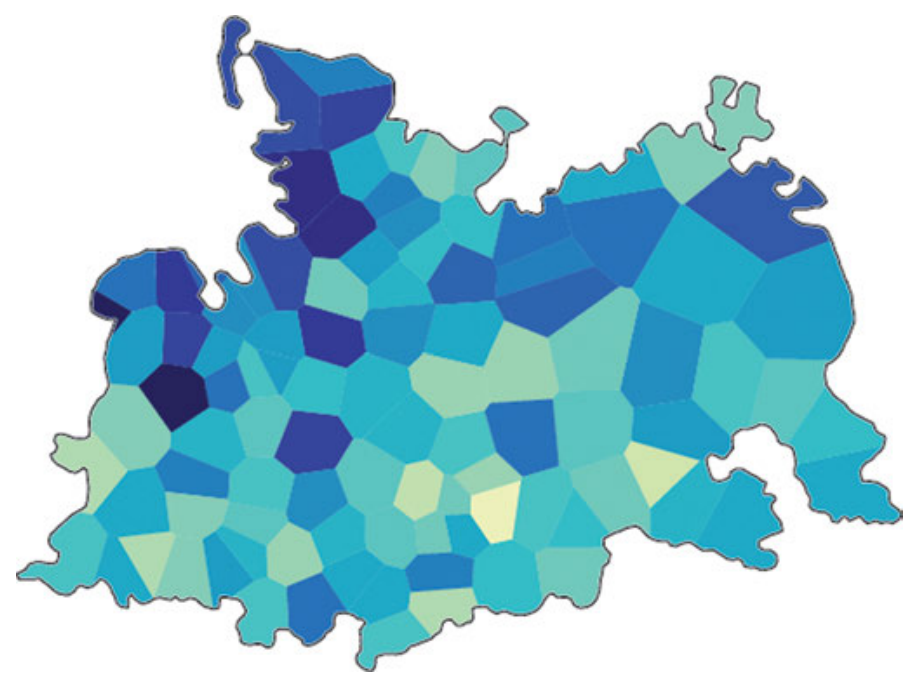

Abb. 3.19: Plattdeutsch/AToL-Item schön: nach Kreisen (mit Gabmap).

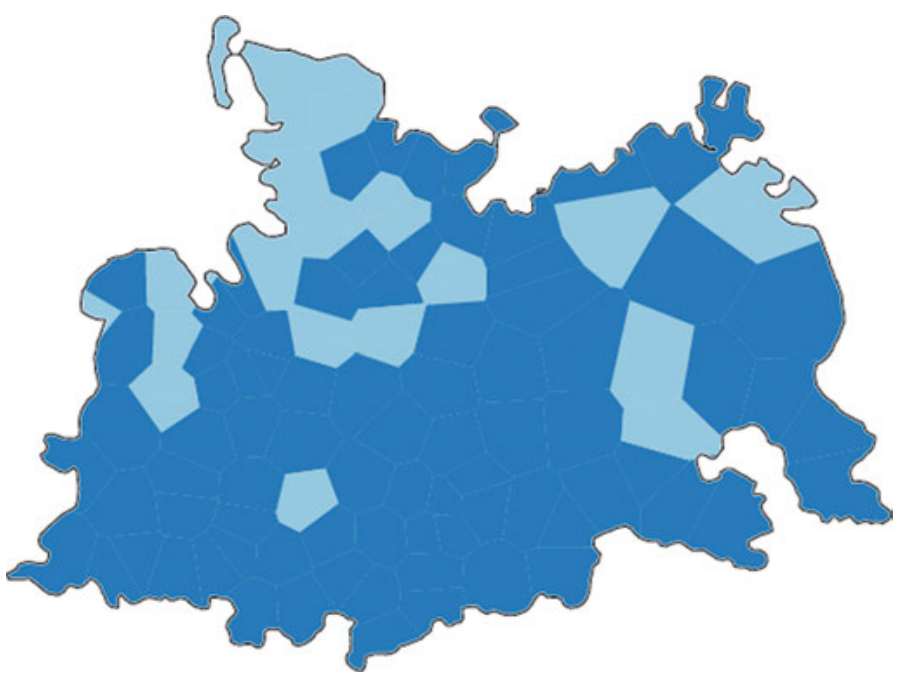

Abb. 3.20: Plattdeutsch/AToL-Item schön: nach Kreisen, Zwei-Cluster-Lösung (mit Gabmap).

innerhalb einer Gruppe bzw. eines Clusters möglichst gering und andererseits die Unterschiede zwischen den Clustern möglichst groß sind. Abbildung 3.20 zeigt eine solche Clusteranalyse für das AToL-Item schön in Bezug auf das Plattdeutsche (hier als Zwei-Cluster-Lösung gerechnet). 
Die Zugehörigkeit der einzelnen Kreise zu einem der beiden Cluster lässt sich an ihrer jeweiligen Einfärbung ablesen. Die beiden gefundenen Cluster ergeben hier ein relativ klares Raumbild, das Ähnlichkeiten zu der Darstellung der Plattdeutsch-Kompetenz nach Kreisen in Abbildung 3.19 zeigt; insbesondere ist die Nordseeküste als markanter Raum identifizierbar. Eine solche Clusteranalyse ist vor allem zweckmäßig, wenn man mehrere Variablen auswerten und in unterschiedlicher Weise kombinieren möchte; für die NorddeutschlandErhebung 2016 werden verschiedene Auswertungen dieser Art vorgenommen; auch für die Deutschland-Erhebung 2017/18 sind solche Analysen vorgesehen.

\section{Resümee}

Nichtlinguisten haben Meinungen über Sprachen und Varietäten, auch wenn ihnen das oft nicht bewusst ist. Ein Weg, solche Spracheinstellungen zu erfassen, führt über quantitative Verfahren, mit denen man Daten großer Gruppen erheben kann. Das ist zwar sehr aufwendig; der Vorteil solcher quantitativen Verfahren besteht aber darin, dass sie nicht nur Einzelmeinungen darstellen oder (plausible Annahmen über) Typen beschreiben, sondern durch die Aggregation überindividueller Daten belastbare und generalisierbare Aussagen erlauben, und dass sie es bei hinreichend großer Datenmenge ermöglichen, Zusammenhänge mit anderen Faktoren zu berechnen. Quantitative Erhebungen dieses Typs sind prinzipiell reproduzierbar, sie bieten damit einerseits Potenzial für Längsschnittdaten und andererseits für Vergleichsstudien etwa auch mit bzw. in anderen Sprachen oder sprachlichen Kontexten. Der Einsatz erprobter, stabiler Instrumente (wie der hier besprochenen ASBI und AToL) erlaubt die vergleichende Analyse einerseits von Teilstichproben innerhalb einer Gesamtstichprobe, andererseits über verschiedene Erhebungen, in denen die Instrumente eingesetzt wurden, hinweg. Zwar ist ein solches Erhebungsformat in der Berücksichtigung diskursiver Kontexte limitiert, durch die Wahl geeigneter Frageformate (offene Fragen) lassen sich aber Daten gewinnen, die als Grundlage für weitere Analysen dienen können. Innovative Darstellungsformate (wie die hier gezeigte Nutzung dialektometrischer Verfahren) erleichtern die Interpretierbarkeit großer Datenmengen.

Da Spracheinstellungen als Teil des Gesamtsets der Einstellungen direkte soziale Relevanz haben, sind Kenntnisse darüber auch außerhalb der Sprachwissenschaft von Belang. Das Wissen um Einstellungen etwa zu anderen Sprachen als Deutsch, zu Lernersprachen oder auch zu Migrantensprachen könnte beispielsweise für Bildungspolitik oder für Integrationspolitik hilfreich sein. 


\section{Literatur}

Adler, Astrid, Christiane Ehlers, Reinhard Goltz, Andrea Kleene \& Albrecht Plewnia (2016): Status und Gebrauch des Niederdeutschen 2016. Erste Ergebnisse einer repräsentativen Erhebung. Mannheim: Institut für Deutsche Sprache.

Anders, Christina Ada (2010): Wahrnehmungsdialektologie. Das Obersächsische im Alltagsverständnis von Laien (Linguistik - Impulse \& Tendenzen 36). Berlin/New York: de Gruyter.

Crites, Stephen L., Leandre R. Fabrigar \& Richard E. Petty (1994): Measuring the affective and cognitive properties of attitudes: Conceptual and methodological issues. Personality \& Social Psychology Bulletin 20 (6), 619-634.

Cuonz, Christina (2014): Sprachliche Werturteile von Laien. Eine sozio-kognitive Analyse (Basler Studien zur deutschen Sprache und Literatur 93). Tübingen: Narr.

Dailey O'Cain, Jennifer und Grit Liebscher (2011): Language attitudes, migrant identities and space. International Journal of the Sociology of Language 212, 91-133.

Eichinger, Ludwig M., Anne-Kathrin Gärtig, Albrecht Plewnia, Janin Roessel, Astrid Rothe, Selma Rudert, Christiane Schoel, Dagmar Stahlberg \& Gerhard Stickel (2009): Aktuelle Spracheinstellungen in Deutschland. Erste Ergebnisse einer bundesweiten Repräsentativumfrage. Mannheim: Institut für Deutsche Sprache, Universität Mannheim.

Eichinger, Ludwig M., Albrecht Plewnia, Dagmar Stahlberg \& Christiane Schoel (Hrsg.) (2012): Sprache und Einstellungen. Spracheinstellungen aus sprachwissenschaftlicher und sozialpsychologischer Perspektive. Mit einer Sprachstandserhebung zum Deutschen von Gerhard Stickel (Studien zur deutschen Sprache 61). Tübingen: Narr.

Fiske, Susan T., Amy J. Cuddy, Peter Guck \& Jun Xu (2002): A model of (often mixed) stereotype content: Competence and warmth respectively follow from perceived status and competition. Journal of Personality and Social Psychology 82 (6) 878-902.

Gärtig, Anne-Kathrin \& Astrid Rothe (2009): Über Liebe zum Deutschen, Sympathie für Dialekte und Sorge um Sprachentwicklung. Was die Menschen in Deutschland über Sprache denken. Sprachreport (3), 2-11.

Gärtig, Anne-Kathrin, Albrecht Plewnia \& Astrid Rothe (2010): Wie Menschen in Deutschland über Sprache denken. Ergebnisse einer bundesweiten Repräsentativerhebung zu aktuellen Spracheinstellungen (amades). Mannheim: Institut für Deutsche Sprache.

Goebl, Hans (1982): Dialektometrie. Prinzipien und Methoden des Einsatzes der numerischen Taxonomie im Bereich der Dialektgeographie (Österreichische Akademie der Wissenschaften. Philosophisch-historische Klasse. Denkschriften 157). Wien: Verlag der Österreichischen Akademie der Wissenschaften.

Goebl, Hans (1984): Dialektometrische Studien anhand italoromanischer, rätoromanischer und galloromanischer Sprachmaterialien aus AIS und ALF. Bd. 1. Tübingen: Niemeyer.

Hansen-Morath, Sandra (2016): Regionale und soziolinguistische Variation im alemannischen Dreiländereck Quantitative Studien zum Dialektwandel. Unveröffentlichte Dissertation an der Albert-Ludwigs-Universität in Freiburg.

Hoberg, Rudolf, Karin M. Eichhoff-Cyrus \& Rüdiger Schulz (Hrsg.) (2008): Wie denken die Deutschen über ihre Muttersprache und über Fremdsprachen? Wiesbaden: Gesellschaft f. dt. Sprache.

Kendall, Tyler \& Valerie Fridland (2016): Mapping the perception of linguistic form dialectometry with perceptual data. In Marie-Hélène Côté, Remco Knooihuizen \& John Nerbonne (Hrsg.), The future of dialects. Selected papers from Methods in Dialectology XV, 173-193. Berlin: Language Science Press. 
König, Katharina (2014): Spracheinstellungen und Identitätskonstruktion. Eine gesprächsanalytische Untersuchung sprachbiographischer Interviews mit DeutschVietnamesen. Berlin: de Gruyter.

Liebscher, Grit \& Jennifer Dailey-O'Cain (2009): Language attitudes in interaction. Journal of Sociolinguistics 13 (2), 195-222.

Möller, Frerk (2008): Plattdeutsch im 21. Jahrhundert. Bestandsaufnahme und Perspektiven. Leer: Schuster.

Mulac, Anthony (1975): Evaluation of the speech dialect attitudinal scale. Speech Monographs 42 (3), 184-189.

Nerbonne, John, Rinke Colen, Charlotte Gooskens, Peter Kleiweg \& Therese Leinonen (2011): Gabmap - A web application for dialectology. Dialectologia (Special Issue II), 65-89. www.publicacions.ub.edu/revistes/dialectologiaSP2011/ (letzter Zugriff: 7.12. 2017).

Osgood, Charles. E., George J. Suci \& Percy H. Tannenbaum (1957): The measurement of meaning. Urbana: University of Illinois Press.

Plewnia, Albrecht (2013): Norddeutsch - Plattdeutsch - Friesisch. Der norddeutsche Sprachraum aus der Sicht linguistischer Laien. In Georg Albert \& Joachim Franz (Hrsg.), Zeichen und Stil. Der Mehrwert der Variation. Festschrift für Beate Henn-Memmesheimer (VarioLingua 44), 43-62. Frankfurt am Main u. a.: Lang.

Plewnia, Albrecht \& Astrid Rothe (2009): Eine Sprach-Mauer in den Köpfen? Über aktuelle Spracheinstellungen in Ost und West. In Albrecht Plewnia (Hrsg.), Deutsche Sprache (2-3). Themenheft: Sprache in Ost und West. Ein Themenheft zum 20. Jahrestag des Mauerfalls, 235-279. Berlin: Schmidt.

Plewnia, Albrecht \& Astrid Rothe (2011a): Spracheinstellungen und Mehrsprachigkeit. Wie Schüler über ihre und andere Sprachen denken. In Ludwig M. Eichinger, Albrecht Plewnia \& Melanie Steinle (Hrsg.), Sprache und Integration. Über Mehrsprachigkeit und Migration (Studien zur Deutschen Sprache 57), 215-253. Tübingen: Narr.

Plewnia, Albrecht \& Astrid Rothe (2011b): Von gebildeten Deutschen, freundlichen Sachsen und temperamentvollen Bayern. Einstellungen zu Varietäten und ihren Sprechern. In Helen Christen, Franz Patocka \& Evelyn Ziegler (Hrsg.), Struktur, Gebrauch und Wahrnehmung von Dialekt. Beiträge zum 3. Kongress der Internationalen Gesellschaft für Dialektologie des Deutschen (IGDD) Zürich 7.-9. September 2009, 179-207. Wien: Praesens.

Plewnia, Albrecht \& Astrid Rothe (2012): „Sprache - Einstellungen - Regionalität.“ In Ludwig M. Eichinger, Albrecht Plewnia, Christiane Schoel \& Dagmar Stahlberg (Hrsg.), Sprache und Einstellungen. Spracheinstellungen aus sprachwissenschaftlicher und sozialpsychologischer Perspektive, 4-134. Tübingen: Narr.

Plewnia, Albrecht \& Astrid Rothe (2015): Spracheinstellungen und Mehrsprachigkeit. Wie Schüler über ihre und andere Sprachen denken. In Rita Brdar Szabó, Elisabeth Knipf-Komlósi \& Roberta Rada (Hrsg.), Zur Rolle und Positionierung des Deutschen in den Ländern Mittelosteuropas. Sprachpolitische Überlegungen. Konferenzband zur Tagung Deutsch 3.0 in Budapest am 15.-16. Mai 2014 (Budapester Beiträge zur Germanistik 72), 57-97. Budapest: ELTE, Germanistisches Institut.

Richter, David \& Jürgen Schupp (2012): SOEP Innovation Sample (SOEP-IS) - Description, structure and documentation. SOEPpaper 463. https://www.diw.de/documents/ publikationen/73/diw_01.c.407141.de/diw_sp0463.pdf (letzter Zugriff: 7.12. 2017)

Rothe, Astrid (2012): Deutsch und andere Sprachen. In Ludwig M. Eichinger, Albrecht Plewnia, Christiane Schoel \& Dagmar Stahlberg (Hrsg.), Sprache und Einstellungen. 
Spracheinstellungen aus sprachwissenschaftlicher und sozialpsychologischer Perspektive, 135-184. Tübingen: Narr.

Rothe, Astrid \& Katarina Wagner (2015): Bilingual Kindergarten programmes. The interaction of language management and language attitudes. In Winifred V. Davies \& Evelyn Ziegler (Hrsg.), Language planning and microlinguistics. From policy to interaction and vice versa, 15-38. Basingstoke: Palgrave Macmillan.

Schaub, Victoria (2011): Beliebt oder nur bekannt? - Einflüsse von Bekanntheit in der sprachdemoskopischen Untersuchung von Einstellungen gegenüber deutschen Dialekten. In Brigitte Ganswindt \& Christoph Purschke (Hrsg.), Perspektiven der Variationslinguistik (Germanistische Linguistik 216-217), 393-417. Hildesheim u. a.: Olms.

Schoel, Christiane, Janin Roessel, Jennifer Eck, et al. (2012): 'Attitudes Towards Languages' (AToL) scale: A global instrument. In Journal of Language and Social Psychology 32 (1), 21-45. Thousand Oaks: Sage Publications.

Soukup, Barbara (2012): Current issues in the social psychological study of 'language attitudes': Constructionism, context, and the attitude-behavior link. Language and Linguistics Compass 6 (4), 212-224.

Stellmacher, Dieter (1987): Wer spricht Platt? Zur Lage des Niederdeutschen heute. Eine kurzgefaßte Bestandsaufnahme. Leer: Schuster.

Stickel, Gerhard \& Norbert Volz (1999): Meinungen und Einstellungen zur deutschen Sprache. Ergebnisse einer bundesweiten Repräsentativerhebung. Arbeitspapiere und Materialien zur deutschen Sprache 3 (amades). Mannheim: Institut für Deutsche Sprache.

Stoeckle, Philipp (2016): Horizontal and vertical variation in Swiss German morphosyntax. In Marie-Hélène Côté, Remco Knooihuizen \& John Nerbonne (Hrsg.), The future of dialects. Selected papers from Methods in Dialectology XV, 195-215. Berlin: Language Science Press.

Studler, Rebekka (2014): „Einige Antworten habe ich contre coeur so angekreuzt“. Zur Relevanz offener Fragen in Fragebogenstudien zu Spracheinstellungen. In Christina Counz \& Rebekka Studler (Hrsg.), Sprechen über Sprache. Perspektiven und neue Methoden der Sprachenstellungsforschung (Stauffenburg Linguistik 81), 169-204. Tübingen: Stauffenburg.

Zahn, Christopher J. \& Robert Hopper (1985): Measuring language attitudes: The speech evaluation instrument. Journal of Language and Social Psychology 4 (2), 113-124. 



\title{
Markus Hundt \\ 4 Wahrnehmungsdialektologie - quo vadis?
}

\begin{abstract}
Nach einer Etablierungsphase ist die Wahrnehmungsdialektologie (anfänglich auch unter anderen Bezeichnungen wie Ethnodialektologie, Alltagsdialektologie, Laiendialektologie, Perzeptionsdialektologie) nun in der deutschsprachigen Areallinguistik angekommen. Der Beitrag verfolgt drei Ziele: Zunächst wird die Entwicklung der Wahrnehmungsdialektologie im deutschen Sprachraum skizziert. In einem zweiten Schritt erfolgt eine Auseinandersetzung mit dem Methodeninstrumentarium der Wahrnehmungsdialektologie. Schließlich werden dann - ausgehend von bestehenden Forschungsdesideraten - Perspektiven für die zukünftige Entwicklung der Wahrnehmungsdialektologie aufgezeigt.
\end{abstract}

Keywords: Areallinguistik, Dialekt, Dialektologie, Erhebungsmethoden, Ethnodialektologie, Laienlinguistik, perceptual dialectology, Sprachbewusstheit, Sprachbewusstsein, Spracheinstellungen, Sprachwissen, Wahrnehmungsdialektologie

\section{Ausgangslage: kleine und große Projekte}

Die Wahrnehmungsdialektologie ist mittlerweile in der deutschsprachigen Areallinguistik angekommen und etabliert. Nach der von Anders (2010b: 18) vorgeschlagenen Unterteilung kann man den Gesamtbereich der areallinguistischen Forschungen in vier Bereiche teilen. Erstens sind die dialektographischen Studien zu nennen, bei denen die Beschreibung der sprachlichen Besonderheiten einzelner Dialekte im Vordergrund steht (Ortsgrammatiken u. Ä.). Zweitens sind die dialektgeographischen Forschungen zu nennen, die in den bekannten Atlasprojekten (z. B. Deutscher Sprachatlas, Deutscher Wortatlas, Atlanten $\mathrm{zu}$ einzelnen Dialektregionen) die geographische Verteilung der Sprechweisen fokussieren. Drittens sind die dialektsoziologischen Studien zu

\footnotetext{
Markus Hundt, Christian-Albrechts-Universität zu Kiel, Germanistisches Seminar, Professur für Deutsche Sprachwissenschaft, Leibnizstraße 8, D-24188 Kiel, E-Mail: hundt@germsem.uni-kiel.de 
nennen, die - seit den 70er Jahren des vergangenen Jahrhunderts - die soziale und situative Stratifikation von Dialekten und die damit verbundenen Konsequenzen für Dialektsprecher in den Blick genommen haben und weiter in den Blick nehmen. Schließlich kann mit dem Aufkommen der Wahrnehmungsdialektologie die vierte Teildisziplin identifiziert werden, in der der Blick des linguistischen Laien auf die Dialekte/Regiolekte und deren Sprecher thematisiert wird. Auch wenn man konzediert, dass es zwischen diesen Bereichen Überschneidungen gibt, lässt sich das Forschungsinteresse der Wahrnehmungsdialektologie insgesamt doch recht klar von den anderen Bereichen abgrenzen.

Das Interesse am linguistischen Laien ist spätestens seit den 90er Jahren des vergangenen Jahrhunderts stetig gewachsen. Mittlerweile liegt auch eine Reihe von Überblicksdarstellungen zur Geschichte der Wahrnehmungsdialektologie im deutschen Sprachraum vor, vgl. z. B. Hundt (2009), Hundt \& Anders (2010), Anders (2010b: 7-55), Anders \& Hundt (2009), Purschke (2011: 146-151). Es genügt daher in diesem Beitrag, die wichtigsten Etappen in Stichworten zu nennen.

Darüber hinaus sollen in diesem Beitrag zwei weitere Ziele verfolgt werden. Zum einen geht es darum, das in zahlreichen Untersuchungen etablierte Methodeninventar der Wahrnehmungsdialektologie daraufhin $\mathrm{zu}$ befragen, welche Vor- und Nachteile mit dem Einsatz der jeweiligen Methoden verbunden sind. Es zeigt sich, dass in der Wahrnehmungsdialektologie - wie in anderen Wissenschaftsdisziplinen auch - die Wahl der Methodik einen erheblichen Einfluss darauf hat, was überhaupt erkannt werden kann (Abschnitt 2). Zum anderen soll in diesem Beitrag darüber nachgedacht werden, in welche Richtung sich die Wahrnehmungsdialektologie im deutschen Sprachraum entwickeln kann und gegebenenfalls auch sollte (Abschnitt 3). Diese Überlegungen sind gespeist sowohl aus den Erkenntnissen, die aus den bisherigen Studien vorliegen, als auch aus den methodischen Problemen, die sich in diesem Bereich ergeben haben.

Zunächst zum Überblick über die Geschichte der Wahrnehmungsdialektologie im deutschen Sprachraum. Diese lässt sich - grob vereinfachend - in vier Etappen untergliedern, die selbstredend nicht trennscharf voneinander geschieden werden können:

1. Etablierung der perceptual dialectology/Vorgeschichte der Wahrnehmungsdialektologie

2. Frühe Studien zu deutschen Dialekten

3. Etablierung im deutschsprachigen Raum

4. Einzelprojekte

1. Die sog. perceptual dialectology wurde maßgeblich durch die verschiedenen Studien von Dennis R. Preston in den wissenschaftlichen Diskurs eingeführt. 
Prestons Untersuchungsgegenstand waren vornehmlich die verschiedenen Formen des US-amerikanischen Englisch und das damit verbundene Laienwissen. Die perceptual dialectology ist damit ein Teil der übergeordneten folk linguistics (Niedzielski \& Preston 2003), die sich generell mit dem Wissen linguistischer Laien zu verschiedenen Varietäten einer Sprache (nicht nur Dialekte) befasst (exemplarisch Preston 1986, 1993, 2002, 2004, 2005, 2010a, b, c). Einen sehr informativen Gesamtüberblick über die Entwicklung der perceptual dialectology im internationalen Kontext bietet das von Preston und Long herausgegebene „Handbook of perceptual dialectology“ (Preston 1999; Long \& Preston 2002; vgl. dazu auch Hundt 2005). Auch in den heutigen Studien finden sich die grundlegenden Fragestellungen und die wesentlichen Methoden dieser Phase wieder. Es ging und geht darum, $\mathrm{zu}$ erforschen, was linguistische Laien über die entsprechenden Dialekte wissen (subjektives Wissen), wie sie Dialektareale verorten und gliedern (mental maps), welche Bezeichnungen sie gegebenenfalls für diese Sprechweisen verwenden, wie sie Differenzen zu Sprechweisen in jeweils benachbarten Räumen bzw. zur jeweiligen Standardsprache einschätzen (correctness) und welche Einstellungen sie gegenüber den Sprechweisen und damit mutatis mutandis auch gegenüber deren Sprechern ${ }^{1}$ (pleasantness) haben. Dieser Forschungsstrang kann als der Teil der Vorgeschichte der deutschsprachigen Wahrnehmungsdialektologie bezeichnet werden, der für die Etablierung der Disziplin im deutschsprachigen Raum die größte Bedeutung erlangen sollte.

2. Das Interesse am linguistischen Laien und an dessen Wissen $\mathrm{zu}$ deutschen Dialekten findet sich vereinzelt natürlich auch in früheren Studien, die der Dialektographie oder der Dialektsoziologie verpflichtet sind. Zum einen ging es darum, Einstellungen gegenüber Dialekten und - soweit dies möglich war die Merkmale, anhand derer Dialekte erkannt und zugeordnet werden konnten, $\mathrm{zu}$ eruieren, um so eventuelle soziale Barrieren sichtbar zu machen, eine geradezu klassische Fragestellung der Dialektsoziologie, vgl. z. B. Ammon (1983), Hundt (1992: 12-26), Vandermeeren (2005), Christen (1998), Siebenhaar (2000, 2002), Hofer (2002, 2004a), Riehl (2000), Tophinke \& Ziegler (2006), Berthele (2002). Zum anderen waren aber auch die mental maps linguistischer Laien Untersuchungsgegenstand, d. h. die Verortung von Dialekten durch linguistische Laien. Die so fassbaren subjektiven Dialekträume unterscheiden sich z. T. erheblich von den objektiven Dialektarealen, die in der Dialekt-

1 In diesem Beitrag wird das generische Maskulinum verwendet. In dieser Form sind alle Geschlechter eingeschlossen. 
geographie festgestellt werden konnten. Eine frühe und wegweisende Studie zur Erhebung von mental maps ist Diercks (1988).

3. In den ersten zehn Jahren des neuen Jahrtausends breitete sich das Forschungsinteresse zur Wahrnehmungsdialektologie immer weiter aus. Nunmehr kann man davon sprechen, dass diese Disziplin auch im deutschen Sprachraum als Teilbereich der Areallinguistik angekommen und etabliert ist. Die vormalige Skepsis gegenüber den naiven, vortheoretischen, aus wissenschaftlich-linguistischer Sicht manches Mal falschen Vorstellungen zu Dialekten ist nun einem erhöhten Interesse gerade für diese Art der subjektiven Daten gewichen. Dies hängt damit zusammen, dass die Rolle linguistischer Laien in den Prozessen des Sprachwandels, bei der Bewertung von Sprechweisen und bei der Frage, welche Rolle Dialekte und Regionalsprachen in der Gegenwart und Zukunft spielen, genauer betrachtet wird. Zudem ergeben sich aus dem Abgleich der objektiv-dialektologischen Daten (Dialektographie, Dialektgeographie) mit den subjektiven Laiendaten an verschiedenen Punkten neue Einsichten. So kann die Wahrnehmungsdialektologie auch Erklärungshilfen für die Dialektographie anbieten, z.B. wenn es um die Entstehung neuer Grenzen (Isoglossen) geht (vgl. z. B. Stoeckle 2014). Zwei Indizien für die Etablierung der Richtung können exemplarisch genannt werden. Zum einen die Tagung „perceptual dialectology - Neue Wege der Dialektologie“, die vom 22. bis 24. Mai 2008 in Kiel stattfand. Die verschiedenen Beiträge, die im Anschluss an diese Tagung publiziert wurden (vgl. Anders, Hundt \& Lasch 2010), zeigen das damalige Spektrum der Forschungsschwerpunkte auf. Zum anderen ist es die Tatsache, dass die Wahrnehmungsdialektologie nach 2012 als eigenständige Sektion in der Internationalen Gesellschaft für Deutsche Dialektologie (IGDD) einen Platz erhielt. In dieser Sektion wurde dann auf dem 5. Kongress der IGDD (Luxemburg 2015) eine Reihe von neuen wahrnehmungsdialektologischen Studien vorgestellt, die in einem eigenen Sammelband (Hundt, Purschke \& Ziegler 2017) publiziert wurden.

Die Vielfalt an Untersuchungsgegenständen und an Untersuchungsmethoden erschwert einen Überblick über das gesamte Gebiet. Allerdings lassen sich drei Themen identifizieren, die immer wieder in den verschiedenen Arbeiten eine Rolle spielen. Dies sind Themen, die auch in den früheren Phasen der Wahrnehmungsdialektologie relevant waren: a) die Frage nach den Einstellungen gegenüber Dialekten und deren Sprechern, b) die Frage danach, welche Merkmale sowohl bei der Dialekterkennung als auch bei der Konzeptkonstitution linguistischer Laien salient sind, und c) die mental maps linguistischer Laien, d.h. die Frage danach, wie und warum linguistische Laien ihre subjektiven Dialekträume konstruieren, welche Dialekte überhaupt bei linguistischen 
Laien in diesen Konzeptualisierungen relevant sind, wo diese Dialekte - etwa im Unterschied zu den objektiven Dialektarealen - verortet werden und auch mit welchen Bezeichnungen diese Dialekte - wiederum ggf. im Unterschied zu den wissenschaftlichen Bezeichnungen - versehen werden.

a) Einstellungen/Prestige/Stigma: Das ursprünglich aus der Sprachbarrierenforschung herrührende Thema (s. o.) erfuhr in den letzten Jahren eine deutliche Erweiterung. In vielen Studien werden Einstellungen gegenüber den eigenen Sprechweisen (Autostereotype), gegenüber fremden Sprechweisen (Heterostereotype) erfasst, und es wird darüber hinaus auch teilweise erhoben, welche Einstellungen Dialektsprecher bei anderen vermuten (vermutetes Heterostereotyp), d.h. „Was denken andere über meinen Dialekt?“. Aus der Vielzahl der Studien, die sich mit Einstellungen gegenüber Dialekten befassen, kann an dieser Stelle nur eine kleine exemplarische Auswahl gegeben werden: zum Sächsischen Anders (2007, 2010b, 2012), Schaufuß \& Siebenhaar (2012), zum Niederdeutschen Adler et al. (2016), Anders (2011a), Arendt (2010a, b, 2011, 2014), Jürgens (2015), Gessinger (2008), Neumann \& Schröder (2017), zu verschiedenen deutschen Dialekten Eichinger et al. (2009, 2012), Gärtig, Plewnia \& Rothe (2010), Plewnia \& Rothe (2009, 2011); zu alemannischen Dialekten (Schweiz) und zum Dialekt-Standard-Kontinuum Cuonz (2014a, b), Oberholzer (2017), Scharloth (2005), Studler (2013, 2014, i. V.), zu Dialekt und Standardsprache in Österreich Goldgruber (2011), Kaiser (2006), zu grundlegenden Fragen der Einstellungsforschung Casper (2002), Cuonz \& Studler (2014), Vandermeeren (2005), Hundt (2011, 2012), Liebscher \& Dailey-O'Cain (2014), Purschke (2014b), Schaub (2011), Soukup (2014), Spiekermann (2009), zur „Sicht von außen“ auf den deutschen Sprachraum z. B. Langer (2010).

b) Die Salienz sprachlicher Merkmale ist ebenfalls ein Dauerbrenner in der Wahrnehmungsdialektologie. Dabei spielt nicht nur die Frage eine Rolle, welche dialektalen Merkmale eine Triggerfunktion bei der Konzeptaktivierung haben, sondern auch grundsätzliche Überlegungen, was man unter dem Begriff der Salienz überhaupt zu verstehen hat. Annäherungen und Konzeptdefinitionen zur Salienz finden sich in Lenz (2010), Purschke (2011, 2014a), Auer (2014), Anders, Palliwoda \& Schröder (2014), Elmentaler, Gessinger \& Wirrer (2010), Gessinger \& Butterworth (2015), Gessinger 2017), Hettler (2014, 2017), Palliwoda \& Schröder (2012, 2016); zur Salienz in Bezug auf einzelne Dialekte vgl. z. B. Guntern (2011), Kiesewalter (2011, 2014). Zur Salienz gehören dabei nicht nur phonologische Merkmale, die i.d. R. im Fokus der Forschung standen, sondern auch prosodische Merkmale, vgl. dazu Gilles (2005), Peters, Auer \& Gilles (2015), Teschke (2009). Schon früh zeigte sich dabei, dass sich Salienz nicht immer an einzelne phonologische Merkmale koppeln lässt, sondern auch in Form von Merkmalsclustern in den Blick genommen werden sollte (vgl. z. B. 
Hundt 1996: 246) und auch dass die von linguistischen Laien als salient verstandenen Merkmale nicht immer der Sprachwirklichkeit entsprechen müssen, d. h. hier wird z. T. auf nicht mehr gebräuchliche Formen zurückgegriffen, z. B. der berühmte s-pitze S-tein im Hamburgischen, Auer (1998, 2014: 14), oder Merkmale assoziiert, die in den vorgespielten Sprechproben überhaupt nicht vorgekommen sind (vgl. z. B. Anders (2007, 2010b).

c) Die mental maps linguistischer Laien werden ebenfalls schwerpunktmäßig untersucht. Sie spielen in vielen Studien eine Rolle, die mit der sogenannten Draw-a-Map-Aufgabe arbeiten, bei der die Gewährspersonen gebeten werden, diejenigen Sprachräume einzuzeichnen, in denen gleich oder ähnlich gesprochen wird. Eine wichtige Studie zu dieser Draw-a-Map-Aufgabe, die methodische Implikationen aufzeigt, ist Lameli, Purschke \& Kehrein (2008). Sie konnten zeigen, dass und inwiefern die bei dieser Aufgabe jeweils vorgegebenen Stimuli (mehr oder weniger Informationen auf den Kartenausschnitten) zu unterschiedlichen Ergebnissen führten. Grundlegende Beiträge zur Frage, wie linguistische Laien ihre subjektiven Sprachräume konzeptualisieren, finden sich in Auer (2004), der verschiedene Raummodellierungen vorstellt (Zentrum-Peripherie vs. Grenze), in Anders (2008) und Hundt (2010), wo Karten- und Kartierungstypen erläutert werden. ${ }^{2}$ Die meisten Untersuchungen im Bereich der Wahrnehmungsdialektologie versuchen, auch die mental maps linguistischer Laien zu erheben, daher soll hier eine kleine Auswahl genügen: Kleene (2015, i. Dr.) zum Bairischen, Palliwoda (2011, 2012), Anders (2010b) zum Sächsischen, Berthele (2006a, b, 2010a, b) zur Schweiz, Hofer (2004b) zum Dreiländereck um Basel. Diese Raumrekodierung erfolgt zwar häufig in Form der Draw-a-Map-Aufgabe. Daneben hat sich aber auch die sog. Pile-sort-Methode etabliert, bei der die Gewährspersonen nicht einfach auf vorgegebenen Karten Räume einzeichnen, sondern bei der jeweils aus einer Anzahl von Orten Stapel gebildet werden, die sprachlich zusammengehörige Gebiete repräsentieren sollen; vgl. zu dieser Methode in der Wahrnehmungsdialektologie Tamasi (2003), Anders (2010b), Elspaß \& Möller (2012: 6. Fragerunde), Kennetz (2010), Schröder (2017, i. Dr.).

4. Die gegenwärtige Forschung in der Wahrnehmungsdialektologie ist über die in den vorigen Abschnitten genannten Projekte und Themen hinaus durch zahlreiche Einzelprojekte geprägt, die größere und kleinere Sprachräume abdecken. Die in den vorigen Etappen genannten Themen spielen dabei natürlich

2 Vgl. zu grundlegenden Aspekten der mental maps Preston (2010c) und weitere Beiträge in Lameli, Kehrein \& Rabanus (2010). 
nach wie vor eine große Rolle. An dieser Stelle können nur exemplarisch wenige Studien genannt werden:

a) Das Kieler DFG-Projekt „Der deutsche Sprachraum aus der Sicht linguistischer Laien“ und die im Anschluss daran entstandenen Beiträge. Dieses Projekt versuchte erstmals, den gesamten deutschen Sprachraum aus wahrnehmungsdialektologischer Perspektive zu untersuchen. Im Rahmen dieses Projekts sind bislang verschiedene Beiträge erschienen: Hundt, Palliwoda \& Schröder (2015a, b, 2017), Palliwoda (2017a, b), Palliwoda \& Schröder (2012, 2016, 2017), Schröder (2015, 2017, i. Dr.), Beuge (2014, 2017), Hoffmeister (2017), Hannemann (2017), Hundt (2017).

b) Im Großprojekt Sprachvariation in Norddeutschland (SiN) steht zwar die Sprachlagenforschung im Fokus, daneben werden aber auch verschiedene wahrnehmungsdialektologische Fragestellungen thematisiert, vgl. z. B.: Elmentaler et al. (2015), Elmentaler (2012a, b), Gessinger \& Butterworth (2015), Scharioth (2012), Butterworth \& Glawe (2011), Gessinger (2008).

c) Das Forschungsprojekt „Länderen - Die Urschweiz als Sprach(wissens)raum“ unter der Leitung von Helen Christen beschäftigt sich ebenfalls mit wahrnehmungsdialektologischen Fragestellungen. Neben der objektsprachlichen Sprachvariation in den Halbkantonen Ob- und Nidwalden wird auch das subjektive Dialektwissen der Gewährspersonen erhoben, vgl. z. B. Christen et al. (2015), Christen (2015, 2014, i. Dr.), Petkova (2017).

d) Der 2016 eingerichtete österreichische Spezialforschungsbereich „Deutsch in Österreich. Variation - Kontakt - Perzeption“ widmet ein ganzes ForschungsCluster mit zwei Teilprojekten wahrnehmungsdialektologischen Fragestellungen. Im Teilprojekt 8 werden „Standardvarietäten aus Perspektive der perzeptiven Variationslinguistik“ (Leitung: Alexandra N. Lenz, Wien) untersucht. Im Teilprojekt 10 geht es um die „Wahrnehmung von Einstellungen zu Varietäten und Sprachen an österreichischen Schulen“ (Leitung: Stephan Elspaß, Salzburg), vgl. www.dioe.at (letzter Zugriff: 4. 12. 2017).

e) Neben diesen größeren Projekten differenziert sich die Wahrnehmungsdialektologie in der Gegenwart immer weiter aus in einer Vielzahl von interessanten Einzeluntersuchungen, die z. T. auch aus größeren Forschungsprojekten hervorgegangen sind. Von diesen können hier nur wenige exemplarisch genannt werden: Berthele (2008), Christen (2008, 2010), Christen, Tophinke \& Ziegler (2005), Cramer \& Montgomery (2016), Cuonz (2014a), Dailey-O’Cain (1999), Davies (2010), Eichinger (2010), Farrús et al. (2006), Funk (2003), Glawe (2013), Hable (2010), Hansen et al. (2012), Harnisch (2004, 2008, 2010a, b, 2015), Harnisch, Frank \& Schnabel (2008), Herrgen (2015), Kehrein (2009, 2012a, b), Kennetz (2008, 2009, 2010), Kleene (2015, i. Dr.), Lameli (2012, 2015), Matthussek (2014), Palliwoda (2013, 2014, i. Dr.), Purschke (2008, 
2010a, b, 2011), Rein (2013), Sauer (2017), Scharioth (2015), Schaub (2011), Schaufuß (2015), Schmidlin (2011), Schnabel (2006), Schneider, Spiekermann \& Till (2011), Siebenhaar (2000), Spiekermann (2010, 2012), Stoeckle (2010, 2012, 2014), Studler (2013, 2014), Twilfer (2012), Wagner (2009), Werth (2007).

Diese Tour de Force durch die Wahrnehmungsdialektologie kann weder einen Anspruch auf Vollständigkeit erheben, noch kann sie der thematischen und auch methodischen Vielfalt der Forschungslandschaft gerecht werden. Allerdings sollte deutlich geworden sein, dass sich die Wahrnehmungsdialektologie aus überschaubaren Anfängen heraus nunmehr zu einer sehr lebendigen und fruchtbaren Teildisziplin der Areallinguistik entwickelt hat, die für die Zukunft noch viele interessante Ergebnisse verspricht.

Das Interesse der Wahrnehmungsdialektologie gilt bekanntermaßen dem linguistischen Laien und dessen Vorstellungen zu deutschen Dialekten und deren Sprechern. Auch wenn bislang noch immer nicht im Detail geklärt ist, was jeweils unter dem linguistischen Laien bzw. unter dem Laienwissen in Bezug auf sprachliche Varietäten genau zu verstehen ist - hier sind weitere Studien auf wissenssoziologischer und variationslinguistischer Basis notwendig -, kann doch festgehalten werden, dass sich das Interesse am Laienwissen zu Dialekten nicht lediglich auf einzelne Sprachsystemebenen bezieht, sondern dass hier alle relevanten Konzeptbestandteile im Blick sind bzw. sein sollten, die zur Strukturierung und inhaltlichen Füllung dessen beitragen, was linguistische Laien mit den Dialekten assoziieren.

Diese umfassen beim derzeitigen Stand der Forschung vier Bereiche:

a) Phonologische/morphologische/syntaktische/lexikalische Merkmale,

b) Verortungen der Sprechweisen im (mental konstruierten) Raum,

c) außersprachliche Merkmale, die mit den Dialekten verbunden werden,

d) Einstellungen gegenüber den Dialekten und deren Sprechern.

Daraus kann man ablesen, dass die Bandbreite dessen, was Wahrnehmungsdialektologen am Laienwissen interessiert, außerordentlich groß ist. Die mittlerweile in großer Zahl vorliegenden Detailstudien fokussieren daher auch jeweils unterschiedliche Aspekte dieser Erkenntnisinteressen.

\section{Methodische Probleme}

Die Vielfalt der bei wahrnehmungsdialektologischen Untersuchungen verwendeten Methoden birgt Vor- und Nachteile gleichermaßen. Zum einen lassen sich natürlich über einen Mehrmethodenzugang differenzierte Erkenntnisse über laienlinguistisches Wissen erheben. Visuelle Stimuli (vorgegebenes 
Kartenmaterial bei der Draw-a-Map-Aufgabe), kognitive Stimuli (z. B. in Tiefeninterviews Fragen nach prominenten Vertretern von Dialekten) und auditive Stimuli (Sprechproben zur Erhebung perzipierter Dialektmerkmale) ergänzen sich gegenseitig. Allerdings haben die bisherigen wahrnehmungsdialektologischen Studien auch gezeigt, dass jede dieser Methoden mit Nachteilen behaftet ist bzw. dass man bei jeder dieser Methoden mit bestimmten Biasfaktoren rechnen muss.

Die klassische Draw-a-Map-Aufgabe muss sich der Frage stellen, welches Kartenmaterial den Gewährspersonen (GPn) vorgelegt wird. Die Studie von Lameli, Purschke und Kehrein ${ }^{3}$ hat deutlich gezeigt, dass sich die Antworten der GPn verändern je nach der Reichhaltigkeit der vorgelegten Grundkarte. Daraus lässt sich nun aber nicht schließen, dass die stimulusärmste Grundlagenkarte die beste für die Draw-a-Map-Aufgabe ist, sondern nur, dass je nach Grundkarte andere Wissensbereiche bei den Probanden fokussiert werden bzw. für diese leichter zugänglich sind.

Bei der Draw-a-Map-Aufgabe ist ferner zu berücksichtigen, dass linguistische Laien über ein vergleichsweise differenzierteres Wissen über ihren eigenen sprachlichen Nahbereich verfügen als über ferner liegende Regionen. Dies ist erwartbar, muss aber insofern bei der Erstellung eines Untersuchungssettings berücksichtigt werden, als jeweils andere Karten zur Verfügung gestellt werden. Soll der linguistische Nahbereich analysiert werden, in dem die GPn in der Regel wesentlich feingliedriger einzelne Sprechweisen voneinander abgrenzen können, dann empfehlen sich Kartenausschnitte mit einem Radius von 50 oder maximal $100 \mathrm{~km}$. Soll ein größerer Sprachraum erfasst werden (Fernbereich), eignen sich andere Karten mit deutlich größerem Maßstab. Wenn nur eine Karte Verwendung findet, ist zu berücksichtigen, dass die GPn dann auf diese eine Karte verschiedene mental maps zu gleicher Zeit einzeichnen: die differenzierteren mental maps der Nahregion (Mikrokartierung) und die gröberen mental maps der sprachlichen Fernregionen (Makrokartierung).

Dies zeigte sich auch im DFG-Projekt „Wahrnehmungsdialektologie“, dem bislang einzigen Projekt, das den Versuch unternommen hat, den gesamten deutschen Sprachraum wahrnehmungsdialektologisch zu erschließen. Dabei wurden sowohl die jeweiligen linguistischen Nahräume (Mikrokartierungen und entsprechende Befragungen) als auch die Fernräume (Makrokartierung und entsprechende Befragungen) in den Blick genommen.

Kognitive Stimuli haben den Vorteil, dass sie das Vorstellungsvermögen der GPn aktivieren und somit helfen können, Wissen über dialektale Varietäten

3 Lameli, Purschke \& Kehrein (2008), Kehrein, Lameli \& Purschke (2010). 
und deren Sprecher zu aktivieren. Solche Stimuli spielen v. a. bei den leitfadengesteuerten Tiefeninterviews eine Rolle. Allerdings gilt für kognitive Stimuli in ähnlicher Form wie für visuelle Stimuli, dass sie mit der jeweiligen Fokussierung auf bestimmte Aspekte andere Aspekte eher in den Hintergrund treten lassen. So fokussiert z. B. die Frage nach prominenten Dialektsprechern i. d. R. Assoziationen $\mathrm{zu}$ einer eher regionalsprachlich ausgeprägten Dialektalität, während die Frage nach „typischen Dialektsprechern in Ihrem Ort“ eher den Fokus auf die kleinräumigere Ortsmundart legen dürfte. Wiederum andere Assoziationen werden durch Fragen nach Erfahrungen mit dem eigenen Dialekt oder mit anderen Dialekten auf In- oder Auslandsreisen geweckt. Das Hauptproblem bei den kognitiven Stimuli dürfte aber das folgende sein: Wenn GPn auf der Basis von kognitiven Stimuli Aussagen zu Dialekten machen, besteht in der Regel nicht die Möglichkeit, diese Aussagen (auch nur ansatzweise) zu objektivieren. D.h. welche konkreten gedanklichen Verknüpfungen die GPn zwischen dem kognitiven Stimulus und dem damit assoziierten Dialekt herstellen, ist ausschließlich aus den Aussagen der GPn rekonstruierbar. Welchen Grad der Dialektalität, welche Verbindung von Person und Dialekt (z. B. bei Fehlzuordnungen von Prominenten zu Dialekten), welche räumlichen Verortungen GPn mit den Dialekten verbinden, und auch was sie genau mit den z. T. genannten assoziierten Dialektmerkmalen meinen, bleibt - wenn man sich allein auf den Einsatz kognitiver Stimuli beschränkt - unklar. Gerade bei den kognitiven Stimuli sollten daher ergänzend auch andere Methoden zum Einsatz gebracht werden, die ggf. erhellend bzw. korrigierend wirken können. So z. B. wenn eine GP im Rahmen der Arbeit mit kognitiven Stimuli einen Dialekt beschreibt und benennt (z. B. als BAYRISCH), sich dann aber beim Einsatz von Sprechproben herausstellt, dass das, was die GP mit BAYRISCH bezeichnet, tatsächlich ein alemannischer Dialekt ist.

Der Einsatz von auditiven Stimuli ist gut etabliert in der wahrnehmungsdialektologischen Forschung. Die Grundidee ist so einfach wie schlüssig. Wenn man den GPn Sprechproben vorspielt und sie danach fragt, was ihnen an diesen Sprechproben auffällt, erhält man - im besten Falle - Auskunft darüber, welche sprachlichen Merkmale diese Dialekte charakterisieren, im Sinne der sprachlichen Salienz. In einem weiteren Schritt können so z. B. Einzelmerkmale auf ihr Salienzpotenzial hin getestet werden, indem den GPn gezielt solche Sprechproben zur Beurteilung vorgelegt werden, die sich systematisch in bestimmten Einzelmerkmalen unterscheiden. ${ }^{4}$

4 Vgl. z. B. die Studie von Steinig (1982) zum palatalisierten $s$ im Schwäbischen; weitere Studien in Hundt (1992: 12-25). 
Ein Nachteil, der mit der Verwendung von Sprechproben verbunden ist, ist die laborartige Fixierung auf jeweils kurze gesprochene Passagen, für die im Vorfeld der Dialektalitätsgrad, die Sprechlage, die Sprechprobenlänge und auch der Inhalt (möglichst ,inhaltfrei' im Sinne von ,nicht durch die Inhalte schon in der Bewertung polarisierend') festgelegt werden muss. Diese Einschränkungen entfernen die Sprechproben jeweils automatisch von natürlichen Gesprächssituationen.

Eine Aufgabe, die mit Sprechproben in der Wahrnehmungsdialektologie ebenfalls häufig verbunden sind, ist die Zuordnung zu einer bestimmten Dialektregion oder zu einem Ort innerhalb eines Dialektgebiets. Hier zeigt sich z. B. bei den Erhebungen im Rahmen des Kieler DFG-Projekts, - dass die GPn in einzelnen Fällen nach dem Ausschlussprinzip zuordneten. D. h. sie konnten zwar die Sprechprobe nicht aufgrund der von ihnen gehörten Dialektmerkmale direkt einer Region zuordnen, wussten aber, dass sie in bestimmte andere Regionen, die bei der Zuordnung ebenfalls zur Auswahl standen, nicht gehören konnte. Die GPn wissen z. B., dass die Sprechprobe nicht zu Potsdam oder Neumünster gehören kann, ordnen dann per Ausschlussverfahren dem übrig gebliebenen Ort/den übrig gebliebenen Orten zu. Das kann passen (muss es aber nicht). Aber: Auch wenn hier passende Zuordnungen vorliegen, ist nicht immer eindeutig erkennbar, dass die GP die Zuordnung aufgrund von sprachlichem Wissen getätigt hat. Aufgefangen werden kann dieser Bias durch Nachfragen der Interviewer, was im Kieler DFG-Projekt auch so praktiziert wurde.

Beim leitfadengesteuerten Interview treten ebenfalls Biasfaktoren auf, die nicht immer vollständig aufgefangen werden können. So spielt die Sprechweise der Interviewer z. T. eine Rolle (Vertrautheit/Nichtvertrautheit mit der Sprechweise der GP), Faktoren der Erhebungssituation (Unterbrechungen des Interviews, Zeitmangel, Motivation der GP etc.), teilweise sind Antworten im Sinne sozialer Erwünschtheit, etwa bei Fragen nach Einstellungen gegenüber Dialekten, zu verzeichnen.

Eine weitere Methode, die bei wahrnehmungsdialektologischen Befragungen zum Einsatz kommt, ist das sog. Pile-Sorting, das der Rekodierung der räumlichen Verortungen von Dialekten durch die GPn dient. Anhand einer Reihe von Karten mit Ortspunkten/Städten sollen die GPn diejenigen zusammensortieren, die ihrer Meinung nach Räume darstellen, in denen gleich oder ähnlich gesprochen wird. Auch hier können sich Probleme ergeben. So ist nicht immer eindeutig zu klären, ob die GPn die Stapelbildung tatsächlich nur aufgrund von sprachlicher Ähnlichkeit vornehmen, oder ob hier nicht auch Stapelbildungen allein aufgrund der geographischen Nähe der Städte zueinander erfolgen, im Sinne des alltagslogischen Schlusses: „Diese Orte liegen nahe beieinander, daher muss dort ähnlich gesprochen werden.“ So können Kon- 
zeptartefakte entstehen. Dass solche Zuordnungen erfolgen, zeigt auch die Tatsache, dass an manchen Stellen diese gebildeten Stapel nicht mit Dialektbezeichnungen versehen werden konnten.

Man kann festhalten: Die Methodenvielfalt in der Wahrnehmungsdialektologie ist insofern von Vorteil, als sie es ermöglicht, die verschiedenen Wissensschichten linguistischer Laien auf verschiedenen Wegen anzusteuern. Zudem ist ein Mehrmethodenzugang immer mit einer längeren Befragungszeit für die GPn verbunden, was es ihnen ermöglicht, schrittweise auf ihr eigenes Wissen zuzugreifen (allmähliche Erschließung des eigenen Wissens). Dabei dürfen jedoch die mit den jeweiligen Methoden verbundenen Nachteile nicht außer Acht gelassen werden, d. h. die durch diese Methoden gewonnenen Ergebnisse sind jeweils kritisch zu hinterfragen.

\section{Zukunftsperspektiven und offene Fragen}

Wie bei jedem interessanten wissenschaftlichen Forschungsgebiet bleibt auch in der Wahrnehmungsdialektologie derzeit noch eine ganze Reihe von Fragen offen. Sowohl in theoretischer Hinsicht $(\mathrm{a}-\mathrm{b})$ als auch mit Blick auf einzelne Untersuchungsgegenstände und Methoden (c-e) lassen sich - exemplarisch Forschungsdesiderata formulieren, die für zukünftige Forschungen in diesem Feld Ausgangspunkte werden könnten.

a) Wie in anderen linguistischen Disziplinen, so sollten auch in der Wahrnehmungsdialektologie zentrale Begriffe gerade in einer sprach- und erkenntnistheoretischen Perspektive genauer unter die Lupe genommen werden, so dass tragfähige, theoretisch fundierte Definitionen für solche Begriffe erarbeitet werden können. Für den Salienz-Begriff wurde und wird diese Diskussion bereits seit einigen Jahren intensiv geführt; s. o. Abschnitt 1. Die Begriffsklärung und -schärfung ist v.a. auch durch die Vorschläge von Purschke und Auer wesentlich vorangekommen. Als abgeschlossen kann diese Diskussion jedoch noch keineswegs betrachtet werden.

b) Noch deutlicher am Anfang der Diskussion steht der Begriff des linguistischen Laien. Zwar wird dieser Begriff in nahezu allen wahrnehmungsdialektologischen Studien vorausgesetzt und verwendet. Eine terminologische, begrifflich-theoretische und auf den Wissensbegriff (der ebenfalls vielschichtig ist) bezugnehmende Definition steht hier jedoch noch aus. Da die Wahrnehmungsdialektologie nur ein Teilbereich der sog. „folk linguistics“ ist, die sich mit dem Laienwissen zu sprachlichen Varietäten insgesamt befasst, ist eine Klärung dessen, was genau unter einem „linguistischen Laien“ und was genau unter „Laienwissen“ $\mathrm{zu}$ verstehen ist, dringlich. Doch bislang werden beide 
Begriffe in Studien vorwiegend auf einem eher vortheoretischen Niveau verwendet, d.h. das, was diese Begriffe genau bezeichnen sollen, wird eher als gegeben und geklärt vorausgesetzt denn in einer stringenten Weise definiert. So ist aus den bisherigen Studien (z. B. DFG-Projekt) deutlich geworden, dass man von dem linguistischen Laien als einer homogenen und vom sog. Experten klar abgrenzbaren Größe wohl kaum wird sprechen können. Das Spektrum zwischen Laie und Experte ist gerade in Bezug auf das Wissen zu sprachlichen Varietäten als ein Kontinuum anzusetzen und nicht als eine Skala mit jeweils klar abgrenzbaren Bereichen. Genauso ist der Begriff des Laienwissens genauer zu fassen. Die Erhebungen des Kieler DFG-Projekts haben z. B. sehr deutlich gemacht, dass linguistische Laien keineswegs über ihr Wissen zu sprachlichen Varietäten in einer Weise verfügen, bei der man sagen könnte „entweder ist es vorhanden, und dann können die GPn auch vollumfänglich darauf zugreifen, oder es ist eben nicht vorhanden“. Der Realität näher kommt ein Ansatz, bei dem von verschiedenen Zugänglichkeitsgraden und von verschieden differenzierten Wissensschichten ausgegangen wird (vgl. Hundt 2017; Hundt, Palliwoda \& Schröder 2015a).

c) Empfehlenswert für zukünftige Studien zur Wahrnehmungsdialektologie im deutschen Sprachraum ist sicherlich eine Fokussierung auf kleinräumige Untersuchungen, da die Erfassung des gesamten deutschen Sprachraums mit einem repräsentativen Ortspunktenetz sehr aufwändig wäre. Idealiter könnten die gesammelten Ergebnisse kleinräumiger Untersuchungen dazu beitragen, den gesamten deutschen Sprachraum in repräsentativer Weise in den Blick zu bekommen. Dieses Vorgehen wäre dann vergleichbar mit den bekannten Atlasprojekten zur deutschen Dialektologie, die durch die Kombination der Ergebnisse aus den verschiedenen Regionalatlanten die Ergebnisse der Erhebungen aus dem 19. Jahrhundert (Deutscher Sprachatlas, Deutscher Wortatlas) ergänzen.

d) Dabei können dann - stärker als bisher - einzelne Interessensschwerpunkte in den kleinräumigeren Untersuchungen genauer untersucht werden: phonologische Analysen, Schibboleths, Einstellungen, räumliche Verortung, außersprachliche Merkmale, Abgrenzungen zu sprachlich benachbarten Räumen. Darüber hinaus sollten dann auch übergreifende Fragestellungen in den Blick genommen werden, die jeweils abhängig sind von der Herkunft der befragten GPn. Ein Beispiel dafür wäre die Konzeptualisierung von „Norden“ und „Süden“ in Bezug auf den deutschen Sprachraum. Aus kursorischen eigenen Beobachtungen und auch aus dem Datenmaterial des Kieler DFG-Projekts ist bekannt, dass sich die Konzepte von „Norden/Norddeutschland“ und „Süden/ Süddeutschland“ z. T. erheblich in Abhängigkeit von der GP-Herkunft unterscheiden. Während für Süddeutsche der „Norden“ bzw. „Norddeutschland“ in sprachlicher Hinsicht bereits nördlich von Frankfurt beginnt, ist in Schleswig- 
Holstein zuweilen die Auffassung zu beobachten, dass der sprachliche Süden eigentlich bereits kurz südlich von Hamburg beginnt. Hier stellt sich nicht nur die Frage, welche Raumkonzepte und Verortungen bei den einzelnen GP-Gruppen relevant sind, sondern auch, warum es zu diesen Einschätzungen kommt.

e) In methodischer Hinsicht hat sich der Einsatz von mehreren, einander ergänzenden Methoden bewährt. Dies sollte auch für zukünftige Studien eine Orientierungsgröße bleiben. Allerdings - dies zeigt die Tatsache, dass linguistische Laien häufig nur allmählich auf die eigenen Wissensbestände zugreifen können - sollte der Einsatz von längeren Tiefeninterviews intensiviert werden.

Der Weg der Wahrnehmungsdialektologie ist somit durch die zahlreichen bislang vorgelegten Studien einerseits gebahnt und andererseits aber auch noch sehr offen. Viele Fragen sind bislang unbeantwortet und harren der genaueren Untersuchung.

\section{Literatur}

Adler, Astrid, Christiane Ehlers, Reinhard Goltz, Andrea Kleene \& Albrecht Plewnia (2016): Status und Gebrauch des Niederdeutschen 2016. Erste Ergebnisse einer repräsentativen Erhebung. Mannheim: Institut für Deutsche Sprache.

Ammon, Ulrich (1983): Soziale Bewertung des Dialektsprechers: Vor- und Nachteile in Schule, Beruf und Gesellschaft. In Werner Besch, Ulrich Knoop, Wolfgang Putschke \& Herbert Ernst Wiegand (Hrsg.), Dialektologie. Ein Handbuch zur deutschen und allgemeinen Dialektforschung. 2. Halbband, 1499-1509. Berlin/New York: de Gruyter.

Anders, Christina A. (2007): Alltagswissen und Einstellungen zum Substandard am Beispiel des Obersächsischen in seiner meißnischen und osterländischen Ausprägung. Deutsche Sprache 35 (2), 173-188.

Anders, Christina A. (2008): Mental Maps linguistischer Laien zum Obersächsischen. In Helen Christen \& Evelyn Ziegler (Hrsg.), Sprechen, Schreiben, Hören. Zur Produktion und Perzeption von Dialekt und Standardsprache zu Beginn des 21. Jahrhunderts, 201-227. Wien: Edition Praesens.

Anders, Christina A. (2010b): Wahrnehmungsdialektologie. Das Obersächsische im Alltagsverständnis von Laien. Berlin/New York: de Gruyter. (Linguistik - Impulse \& Tendenzen 36).

Anders, Christina A. (2011a): „Platt is nich uncool“ - Zu den „coolsten“ und „uncoolsten“ Dialekten des Deutschen und ob das schon immer so war. In Lieselotte Anderwald (Hrsg.), Sprachmythen - Fiktion oder Wirklichkeit?, 117-135. Frankfurt am Main u. a.: Peter Lang.

Anders, Christina A. (2012): „Kaffeesachsen“, „DDR-Sprache“ oder „fichelante Kerlchen“? Ein kognitives Portrait des Obersächsischen. In Rainer Hünecke \& Karlheinz Jakob (Hrsg.), Die obersächsische Sprachlandschaft in Geschichte und Gegenwart, 289-314. Heidelberg: Universitätsverlag Winter. 
Anders, Christina A. \& Markus Hundt (2009): Die deutschen Dialekträume aus der Sicht linguistischer Laien. In Beate Henn-Memmesheimer \& Joachim Franz (Hrsg.), Die Ordnung des Standard und die Differenzierung der Diskurse. Akten des 41. Linguistischen Kolloquiums in Mannheim 2006, 479-502. Frankfurt a. M.: Peter Lang.

Anders, Christina A., Markus Hundt \& Alexander Lasch (Hrsg.) (2010): „perceptual dialectology“: Neue Wege der Dialektologie. Berlin/New York: de Gruyter. (Linguistik Impulse \& Tendenzen 38).

Anders, Christina A., Nicole Palliwoda \& Saskia Schröder (2014): ,in dem moment wo ich es dann erkenne dann ist es auch gleich wieder weg“ - Salienzeffekte in der Sprachperzeption. In: Helen Christen \& Evelyn Ziegler (Hrsg.): Die Vermessung der Salienz(forschung)/Measuring (the Research on) Salience. Linguistik online. Bd. 66 (4), 51-69.

Arendt, Birte (2010a): Niederdeutschdiskurse. Spracheinstellungen im Kontext von Laien, Printmedien und Politik. Berlin: Erich Schmidt. (Philologische Studien und Quellen, Bd. 224).

Arendt, Birte (2010b): Wie Metasprachdiskurse Wirklichkeiten konstruieren. Eine Untersuchung von Spracheinstellungen zum Niederdeutschen. In Ryszard Lipczuk, Jürgen Schiewe, Werner Westphal \& Dorota Misiek (Hrsg.), Diskurslinguistik Systemlinguistik. Theorien, Texte, Fallstudien, 273-287. Hamburg: Dr. Kovac.

Arendt, Birte (2011): Laientheoretische Konzeptionen von Sprache und Dialekt am Beispiel des Niederdeutschen. Eine kontextsensitive Analyse von Spracheinstellungsäußerungen sowie ihre methodologische Fundierung. Niederdeutsches Wort. Beiträge zur niederdeutschen Philologie 51, 133-162.

Arendt, Birte (2014): Qualitative Interviews als interaktive ko-konstruktive Prozesse. Kontextsensitivität in mikroanalytischer Perspektive. In Christina Cuonz \& Rebekka Studler (Hrsg.), Sprechen über Sprache. Perspektiven und neue Methoden der Spracheinstellungsforschung, 7-30. Tübingen: Stauffenburg. (Stauffenburg Linguistik 81).

Auer, Peter (1998): Hamburger Phonologie. Eine variationslinguistische Skizze zur Stadtsprache der Hansestadt heute. Zeitschrift für Dialektologie und Linguistik 65 (2), 179-197.

Auer, Peter (2004): Sprache, Grenze, Raum. Zeitschrift für Sprachwissenschaft 23 (2), 149-179.

Auer, Peter (2014): Anmerkungen zum Salienzbegriff in der Soziolinguistik. Linguistik Online 66 (4), 7-20.

Berthele, Raphael (2002): Attitudes and mental model of language. On the cognitive foundation of sociolinguistic practice. In Gunnstein Akselberg (Hrsg.), Målbryting. Skrifter frå prosjektet Talemålsendring i Norge. Nr. 6: Språkleg identitet og haldning. Bergen: Nordisk institutt, Universitetet i Bergen, 25-66.

Berthele, Raphael (2006a): Wie sieht das Berndeutsche so ungefähr aus? Über den Nutzen von Visualisierungen für die kognitive Laienlinguistik. In Hubert Klausmann (Hrsg.), Raumstrukturen im Alemannischen. Beiträge der 15. Arbeitstagung zur alemannischen Dialektologie auf Schloss Hofen, Lochau (Vorarlberg), 19.-21. September 2005, 163-175. Graz-Feldkirch: Wolfgang Neugebauer.

Berthele, Raphael (2006b): Ort und Weg. Die sprachliche Raumreferenz in Varietäten des Deutschen, Rätoromanischen und Französischen. Berlin/New York: de Gruyter. (Linguistik - Impulse und Tendenzen 16). 
Berthele, Raphael (2008): A nation is a territory with one culture and one language. The role of metaphorical folk models in language policy debates. In Gitte Kristiansen \& René Dirven (Hrsg.), Cognitive sociolinguistics: Language variation, cultural models, social systems, 301-332. Berlin/New York: de Gruyter Mouton.

Berthele, Raphael (2010a): Investigations into the folk's mental models of linguistic varieties. In Dirk Geeraerts, Gitte Kristiansen \& Yves Peirsman (Hrsg.), Advances in cognitive sociolinguistics, 265-290. Berlin/New York: de Gruyter.

Berthele, Raphael (2010b): Der Laienblick auf sprachliche Varietäten. Metalinguistische Vorstellungswelten in den Köpfen der Deutschschweizerinnen und Deutschschweizer. In Christina A. Anders, Markus Hundt \& Alexander Lasch (Hrsg.), „perceptual dialectology“. Neue Wege der Dialektologie, 245-267. Berlin/New York: de Gruyter. (Linguistik Impulse \& Tendenzen 38).

Beuge, Patrick (2014): Was ist gutes Deutsch aus Sicht linguistischer Laien? Zeitschrift für Dialektologie und Linguistik. 81 (2), 129-150.

Beuge, Patrick (2017): Laienlinguistisches Sprachnormwissen. In Markus Hundt, Nicole Palliwoda \& Saskia Schröder (Hrsg.), Der deutsche Sprachraum aus der Sicht linguistischer Laien. Ergebnisse des Kieler DFG-Projektes, 161-181. Berlin/New York: de Gruyter.

Butterworth, Judith \& Meike Glawe (2011): „Wir sprechen hier an und für sich reinrassiges Hochdeutsch“. Zur Erforschung der subjektiven Seite von Sprachverwendung. In Brigitte Ganswindt \& Christoph Purschke (Hrsg.), Perspektiven der Variationslinguistik. Beiträge aus dem Forum Sprachvariation, 371-391. Hildesheim u. a.: Olms. (Germanistische Linguistik 216-217).

Casper, Klaudia (2002): Spracheinstellungen. Theorie und Messung. Heidelberg: K. Casper.

Christen, Helen (1998): Dialekt im Alltag. Eine empirische Untersuchung zur lokalen Komponente heutiger schweizerdeutscher Varietäten. Tübingen: Niemeyer.

Christen, Helen (2008): „...wiu mer das vilich nid I dr ganze schwiz verschteit“ - Empirische Erkundungen zur sozialen Praxis des polydialektalen Dialogs. In Alexandra N. Lenz (Hrsg.), Dialektsoziologie $=$ Sociodialectology $=$ Sociologie du dialecte, 24-47. Tübingen: Niemeyer. (Sociolinguistica 22).

Christen, Helen (2010): Was Dialektbezeichnungen und Dialektattribuierungen über alltagsweltliche Konzeptualisierungen sprachlicher Heterogenität verraten. In Christina A. Anders, Markus Hundt \& Alexander Lasch (Hrsg.), „perceptual dialectology“. Neue Wege der Dialektologie, 269-290. Berlin/New York: de Gruyter. (Linguistik - Impulse \& Tendenzen 38).

Christen, Helen (2014): „Die hiesige Mundart ist nicht ganz so gezogen wie diejenige von Schwyz." Metakommunikate und das Sprachraumwissen von Laien. In Rudolf Bühler, Rebekka Bürkle \& Nina Kim Leonhardt (Hrsg.), Sprachkultur - Regionalkultur. Neue Felder kulturwissenschaftlicher Dialektforschung, 35-54. Tübingen: Tübinger Vereinigung für Volkskunde. (Studien und Materialien des Ludwig-Uhland-Instituts der Universität Tübingen).

Christen, Helen (2015): „Die cheibe Zuger“ oder: Gibt es Zugerdeutsch? In Regula Schmidlin, Heike Behrens \& Hans Bickel (Hrsg.), Sprachgebrauch und Sprachbewusstsein. Implikationen für die Sprachtheorie, 133-154. Berlin u. a.: de Gruyter.

Christen, Helen (i. Dr.): Über Dialektgebrauch und Dialekteinschätzung in einer beweglichen Welt. In Thorsten Burkard \& Markus Hundt (Hrsg.), Sprachmischungen - Mischsprachen. Frankfurt am Main: Lang. 
Christen, Helen, Nadja Bucheli, Manuela Guntern \& Alexandra Schiesser (2015): Ländere ${ }^{\mathrm{n}}$ : Die Urschweiz als Sprachwissensraum. In Roland Kehrein, Alfred Lameli \& Stefan Rabanus (Hrsg.), Regionale Variation des Deutschen. Projekte und Perspektiven, 621-643. Berlin: de Gruyter Mouton.

Christen, Helen, Doris Tophinke \& Evelyn Ziegler (2005): Chat und regionale Identität. In Norbert Richard Wolf \& Sabine Krämer-Neubert (Hrsg.), Bayerische Dialektologie. Akten der Internationalen Dialektologischen Konferenz 26.-28. Februar 2002 in Heidelberg, 425-439. Heidelberg: Winter.

Cramer, Jennifer \& Chris Montgomery (Hrsg.) (2016): Cityscapes and perceptual dialectology. Global perspectives on non-linguists' knowledge of the dialect landscape. Boston, Berlin: de Gruyter Mouton. (Language and Social Life 5).

Cuonz, Christina (2014a): Sprachliche Werturteile von Laien. Eine sozio-kognitive Analyse. Tübingen: Francke. (Basler Studien zur deutschen Sprache und Literatur 93).

Cuonz, Christina (2014b): Was kann die diskursive Spracheinstellungsforschung (nicht)? Methodologische und epistemologische Überlegungen. In Christina Cuonz \& Rebekka Studler (Hrsg.), Sprechen über Sprache. Perspektiven und neue Methoden der Spracheinstellungsforschung, 31-64. Tübingen: Stauffenburg. (Stauffenburg Linguistik 81).

Cuonz, Christina \& Rebekka Studler (Hrsg.) (2014): Sprechen über Sprache. Perspektiven und neue Methoden der Spracheinstellungsforschung. Tübingen: Stauffenburg. (Stauffenburg Linguistik 81).

Dailey-0'Cain, Jennifer (1999): The perception of post-unification German regional speech. In Dennis R. Preston (Hrsg.), Handbook of perceptual dialectology. Vol. 1, 227-242. Amsterdam, Philadelphia: John Benjamins.

Davies, Winifred (2010): Die Rolle (laien-)linguistischer Mythen bei der Reproduktion (sozio-)linguistischer Normen. In Christina A. Anders, Markus Hundt \& Alexander Lasch (Hrsg.), „perceptual dialectology“. Neue Wege der Dialektologie, 385-408. Berlin/ New York: de Gruyter.

Diercks Willy (1988): Mental Maps. Linguistisch-geographische Konzepte. Zeitschrift für Dialektologie und Linguistik 55 (3), 280-305.

Eichinger, Ludwig M. (2010): Kann man der Selbsteinschätzung von Sprechern trauen? In Christina A. Anders, Markus Hundt \& Alexander Lasch (Hrsg.), „perceptual dialectology“. Neue Wege der Dialektologie, 433-449. Berlin/New York: de Gruyter. (Linguistik Impulse und Tendenzen 38).

Eichinger, Ludwig M., Anne-Katrin Gärtig, Albrecht Plewnia, Janin Roessel, Gerhard Stickel, Selma Rudert, Christiana Schöl \& Dagmar Stahlberg (Hrsg.) (2009): Aktuelle Spracheinstellungen in Deutschland. Erste Ergebnisse einer bundesweiten Repräsentativumfrage. Mannheim: Institut für Deutsche Sprache.

Eichinger, Ludwig M., Christiane Schöl \& Dagmar Stahlberg (Hrsg.) (2012): Sprache und Einstellungen. Spracheinstellungen aus sprachwissenschaftlicher und sozialpsychologischer Perspektive. Mit einer Sprachstandserhebung zum Deutschen von Gerhard Stickel. Tübingen: Narr Francke Attempto. (Studien zur deutschen Sprache 61).

Elmentaler, Michael (2012a): Dialectal concepts of space and linguistic variation. In Sandra Hansen, Christian Schwarz, Philipp Stoeckle \& Tobias Streck (Hrsg.), Dialectological and folk dialectological concepts of space. Current methods and perspectives in sociolinguistic research on dialect change, 31-47. Berlin/Boston: de Gruyter.

Elmentaler, Michael (2012b): In Hannover wird das beste Hochdeutsch gesprochen. In Lieselotte Anderwald (Hrsg.), Sprachmythen - Fiktion oder Wirklichkeit?, 101-115. Frankfurt am Main u. a.: Peter Lang. (Kieler Forschungen zur Sprachwissenschaft 3). 
Elmentaler, Michael, Joachim Gessinger, Jens Lanwer \& Peter Rosenberg (2015):

Sprachvariation in Norddeutschland (SiN). In Roland Kehrein, Alfred Lameli \& Stefan

Rabanus (Hrsg.), Regionale Variation des Deutschen. Projekte und Perspektiven, 397-424. Berlin/Boston: de Gruyter Mouton.

Elmentaler, Michael, Joachim Gessinger \& Jan Wirrer (2010): Qualitative und quantitative Verfahren in der Ethnodialektologie am Beispiel von Salienz. In Christina A. Anders, Markus Hundt \& Alexander Lasch (Hrsg.), „perceptual dialectology“. Neue Wege der Dialektologie, 111-149. Berlin/New York: de Gruyter. (Linguistik - Impulse \& Tendenzen 38).

Elspaß, Stephan \& Robert Möller (2012): Atlas zur deutschen Alltagssprache. In http:// www.atlas-alltagssprache.de/ (13.9. 2017).

Farrús, Mireia, Erik Eriksson, Kirk P. H. Sullivan \& Javier Hernando (2006): Dialect imitations in speaker recognition. In Teresa Turell, Jordi Circes \& Maria Spassova (Hrsg.), Proceedings of the 2nd European IAFL Conference on Forensic Linguistics/Language and the Law, 347-354. Barcelona: Iula.

Funk, Edith (2003): „In Lauterbach fängt der Neabl an“ - Welche Sprachunterschiede nehmen Dialektsprecher wahr? In Edith Funk, Stefan Kleiner, Manfred Renn \& Bernadette Wecker (Hrsg.), Sprachgeschichten. Ein Lesebuch für Werner König zum 60. Geburtstag, 43-62. Heidelberg: Universitätsverlag Winter.

Gärtig, Anne-Kathrin, Albrecht Plewnia \& Astrid Rothe (2010): Wie Menschen in Deutschland über Sprache denken. Ergebnisse einer bundesweiten Repräsentativerhebung zu aktuellen Spracheinstellungen. Mannheim: Institut für Deutsche Sprache.

Gessinger, Joachim (2008): Perzeptive Dialektologie und Spracheinstellungsforschung. Niederdeutsches Jahrbuch 131, 133-144.

Gessinger, Joachim \& Judith Butterworth (2015): Salienz als dynamisches und interaktives Konstrukt. In Michael Elmentaler, Markus Hundt \& Jürgen Erich Schmidt (Hrsg.), Deutsche Dialekte. Konzepte, Probleme, Handlungsfelder. Akten des 4. Kongresses der Internationalen Gesellschaft für Dialektologie des Deutschen (IGDD), 259-294. Stuttgart: Steiner.

Gessinger, Joachim (2017): Dimensionen der Wahrnehmung von Varianz. In Markus Hundt, Christoph Purschke \& Evelyn Ziegler (Hrsg.), Sprachräume: Konfigurationen, Interaktionen, Perzeptionen. Linguistik Online 85 (6), 5-28.

Gilles, Peter (2005): Regionale Prosodie im Deutschen. Variabilität in der Intonation von Abschluss und Weiterverweisung. Berlin: de Gruyter. (Linguistik - Impulse und Tendenzen 6).

Glawe, Meike (2013): Individuelle Wahrnehmung der sprachlichen Wirklichkeit in Rödinghausen. Exemplarische Darstellung einer Sprecherbiographie. In Yvonne Hettler, Carolin Jürgens, Robert Langhanke \& Christoph Purschke (Hrsg.), Variation, Wandel, Wissen. Studien zum Hochdeutschen und Niederdeutschen, 221-237. Frankfurt a. M.: Peter Lang. (Sprache in der Gesellschaft. Beiträge zur Sprach- und Medienwissenschaft 32).

Goldgruber, Barbara Elisabeth (2011): Einstellungen zu Dialekt und Standardsprache in Österreich. Eine empirische Untersuchung in Graz und Wien. Dipl.-Arb. Universität Wien. http://othes.univie.ac.at/13724/ (letzter Zugriff: 6.4. 2016).

Guntern, Manuela (2011): Wie wird aus gesprochenem Hochdeutsch gesprochenes Schweizerdeutsch? Zur Salienz von schweizerischen Varianten in der gesprochenen Standardsprache von DeutschschweizerInnen. In Helen Christen, Franz Patocka \& Evelyn Ziegler (Hrsg.), Struktur, Gebrauch und Wahrnehmung von Dialekt, 58-79. Wien: Praesens. 
Hable, Ricarda (2010): Wie stehen Jugendliche zu Dialekten? In Albrecht Greule \& Katja Kessel (Hrsg.), Linguistik zwischen Profis und Laien. Beiträge von Studierenden der Germanistik, 91-106. Meidenbauer: Martin.

Hannemann, Timo (2017): „irgendwas zwischen hochdeutsch und plattdeutsch“. Der norddeutsche Sprachraum in der Wahrnehmung linguistischer Laien. In Markus Hundt, Nicole Palliwoda \& Saskia Schröder (Hrsg.), Der deutsche Sprachraum aus der Sicht linguistischer Laien. Ergebnisse des Kieler DFG-Projektes, 183-212. Berlin/New York: de Gruyter.

Hansen, Sandra, Christian Schwarz, Philipp Stoeckle \& Tobias Streck (Hrsg.) (2012): Dialectological and folk dialectological concepts of space. Current methods and perspectives in sociolinguistic research on dialect change. Berlin; Boston: de Gruyter. (linguae \& litterae 17).

Harnisch, Rüdiger (2004): Junger Dialektwandel in der Bavaria Thuringica. In Maik Lehmberg (Hrsg.), Sprache, Sprechen, Sprichwörter. Festschrift für Dieter Stellmacher zum 65. Geburtstag, 453-463. Stuttgart: Franz Steiner (Zeitschrift für Dialektologie und Linguistik, Beiheft 126).

Harnisch, Rüdiger (2008): Zur Sprachsituation im ehemaligen deutsch-deutschen Grenzgebiet nach 40 Jahren politischer Spaltung. In Peter Canisius \& Erika Hammer (Hrsg.), 50 Jahre Germanistik in Pécs. Akten eines internationalen Kongresses am 5. und 6. Oktober 2006, 83-97. Wien: Praesens.

Harnisch, Rüdiger (2010a): Divergence of linguistic varieties in language and space. In Peter Auer \& Jürgen Erich Schmidt (Hrsg.), Language and space. An international handbook of linguistic variation. Vol. 1: Theories and methods, 275-294. Berlin/Boston: de Gruyter Mouton. (Handbücher zur Sprach- und Kommunikationswissenschaft 30/1).

Harnisch, Rüdiger (2010b): Dialektentwicklung am Rande des Eisernen Vorhangs. Aus Politik und Zeitgeschichte (APuZ) 8, 21-26.

Harnisch, Rüdiger (2015): Untersuchungen zur Sprachsituation im thüringisch-bayerischen Grenzgebiet. In Roland Kehrein, Alfred Lameli \& Stefan Rabanus (Hrsg.): Regionale Variation des Deutschen. Projekte und Perspektiven, 219-240. Berlin: de Gruyter Mouton.

Harnisch, Rüdiger, Frank Reinhold \& Michael Schnabel (2008): Neue Dialektgrenzen an der ehemaligen deutsch-deutschen Grenze? In Peter Ernst \& Franz Patocka (Hrsg.): Dialektgeographie der Zukunft. Akten des 2. Kongresses der Internationalen Gesellschaft für Dialektologie des Deutschen (IGDD) am Institut für Germanistik der Universität Wien, 20. bis 23. September 2006, 203-218. Stuttgart: Franz Steiner (Zeitschrift für Dialektologie und Linguistik, Beihefte 135).

Herrgen, Joachim (2015): Entnationalisierung des Standards. Eine perzeptionslinguistische Untersuchung zur deutschen Standardsprache in Deutschland, Österreich und der Schweiz. In Alexandra N. Lenz \& Manfred M. Glauninger (Hrsg.), Standarddeutsch in Österreich - Theoretische und empirische Ansätze, 139-164. Göttingen: Vandenhoeck \& Ruprecht. (Wiener Arbeiten zur Linguistik 1).

Hettler, Ivonne (2014): Salienz, Bewertung und Realisierung regionaler Merkmale in Norddeutschland. In Helen Christen \& Evelyn Ziegler (Hrsg.): Die Vermessung der Salienz(forschung)/Measuring (the Research on) Salience. Linguistik online, 66 (4), 71-90.

Hettler, Ivonne (2017): Hörer- und Sprechertypen in Bremen und Hamburg. Eine Untersuchung zu Sprachwissen, Sprachwahrnehmung und Sprachgebrauch. In Markus Hundt, Christoph Purschke \& Evelyn Ziegler (Hrsg.), Sprachräume: Konfigurationen, Interaktionen, Perzeptionen. Linguistik online 85 (6), 29-56 
Hofer, Lorenz (2002): Zur Dynamik urbanen Sprechens. Studien zu Spracheinstellungen und Dialektvariation im Stadtraum. Tübingen, Basel: Francke. (Baseler Studien zur deutschen Sprache und Literatur 71).

Hofer, Lorenz (2004a): Spracheinstellungen aus kulturwissenschaftlicher Sicht. In Helen Christen (Hrsg.), Dialekt, Regiolekt und Standardsprache im sozialen und zeitlichen Raum. Beiträge zum 1. Kongress der Internationalen Gesellschaft für Dialektologie des Deutschen, Marburg/Lahn, 5.-8. März 2003, 221-234. Wien: Edition Praesens.

Hofer, Lorenz (2004b): Sprachliche und politische Grenzen im (ehemaligen) Dialektkontinuum des Alemannischen am Beispiel der trinationalen Region Basel (Schweiz) in Karten von SprecherInnen. In Beat Siebenhaar (Hrsg.), Dialektologie des Schweizerdeutschen. Linguistik online 20 (03/04), 23-46.

Hoffmeister, Toke (2017): Der Einfluss der regionalen Herkunft auf das Dialektwissen linguistischer Laien. In Markus Hundt, Nicole Palliwoda \& Saskia Schröder (Hrsg.), Der deutsche Sprachraum aus der Sicht linguistischer Laien. Ergebnisse des Kieler DFG-Projektes, 213-262. Berlin/New York: de Gruyter.

Hundt, Markus (1992): Einstellungen gegenüber dialektal gefärbter Standardsprache. Eine empirische Untersuchung zum Bairischen, Hamburgischen, Pfälzischen und Schwäbischen. Stuttgart: Steiner.

Hundt, Markus (1996): Zum Prestige gesprochener Alltagssprache: Sächsisch und Schwäbisch. Deutsche Sprache 24 (3), 224-247.

Hundt, Markus (2005): Rezension zu Dennis R. Preston (Hrsg.) (1999): Handbook of perceptual dialectology. Volume 1. John Benjamins: Amsterdam, Philadelphia. Daniel Long, Dennis R. Preston (Hrsg.) (2002): Handbook of perceptual dialectology. Volume 2. John Benjamins: Amsterdam/Philadelphia. Beiträge zur Geschichte der deutschen Sprache und Literatur (PBB) 127 (3), 466-481.

Hundt, Markus (2009): Perceptual dialectology und ihre Anwendungsmöglichkeiten im deutschen Sprachraum. In Beate Henn-Memmesheimer (Hrsg.), Die Ordnung des Standard und die Differenzierung der Diskurse. Akten des 41. Linguistischen Kolloquiums in Mannheim, 465-478. Frankfurt am Main u. a.: Peter Lang.

Hundt, Markus (2010): Bericht über die Pilotstudie „Laienlinguistische Konzeptionen deutscher Dialekte“. In Christina A. Anders, Markus Hundt \& Alexander Lasch (Hrsg.), „perceptual dialectology“. Neue Wege der Dialektologie, 179-219. Berlin/New York: de Gruyter. (Linguistik - Impulse \& Tendenzen 38).

Hundt, Markus (2011): Schöner Dialekt, hässlicher Dialekt - Theorien und Methoden der Einstellungsforschung im Bereich der Wahrnehmungsdialektologie. In Michael Elmentaler \& Ulrich Hoinkes (Hrsg.), „Gute Sprache, schlechte Sprache“. Sprachnorm, Sprachwandel und regionale Vielfalt, 77-104. Frankfurt a. M. u. a.: Peter Lang.

Hundt, Markus (2012): Warum gibt es eigentlich „beliebte“ und „unbeliebte“ Dialekte? Theorien und Methoden der Einstellungsforschung im Bereich der Wahrnehmungsdialektologie. In Rainer Hünecke \& Karlheinz Jakob (Hrsg.), Die obersächsische Sprachlandschaft in Geschichte und Gegenwart, 175-222. Heidelberg: Universitätsverlag Winter.

Hundt, Markus (2017): Struktur und Komplexität des linguistischen Laienwissens. In Markus Hundt, Nicole Palliwoda \& Saskia Schröder (Hrsg.), Der deutsche Sprachraum aus der Sicht linguistischer Laien. Ergebnisse des Kieler DFG-Projektes, 121-159. Berlin/ New York: de Gruyter. 
Hundt, Markus \& Christina A. Anders (2010): Die deutschen Dialekträume aus der Sicht linguistischer Laien. In Beate Henn-Memmesheimer \& Joachim Franz (Hrsg.), Die Ordnung des Standard und die Differenzierung der Diskurse. Akten des 41. Linguistischen Kolloquiums in Mannheim 2006, 479-502. Frankfurt a. M. u. a.: Peter Lang.

Hundt, Markus, Nicole Palliwoda \& Saskia Schröder (2015a): Wahrnehmungsdialektologie Der deutsche Sprachraum aus der Sicht linguistischer Laien. Exemplarische Ergebnisse des Kieler DFG-Projekts. In Roland Kehrein, Alfred Lameli \& Stefan Rabanus (Hrsg.), Regionale Variation des Deutschen. Projekte und Perspektiven, 585-620. Berlin: de Gruyter Mouton.

Hundt, Markus, Nicole Palliwoda \& Saskia Schröder (2015b): Der deutsche Sprachraum aus der Sicht linguistischer Laien - das Kieler DFG-Projekt. In Michael Elmentaler, Markus Hundt \& Jürgen Erich Schmidt (Hrsg.), Deutsche Dialekte. Konzepte, Probleme, Handlungsfelder. Akten des 4. Kongresses der Internationalen Gesellschaft für Dialektologie des Deutschen (IGDD), 295-321. Stuttgart: Steiner.

Hundt, Markus, Nicole Palliwoda \& Saskia Schröder (Hrsg.) (2017): Der deutsche Sprachraum aus der Sicht linguistischer Laien. Ergebnisse des Kieler DFG-Projektes. Berlin/New York: de Gruyter.

Hundt, Markus, Christoph Purschke \& Evelyn Ziegler (Hrsg.) (2017): Sprachräume: Konfigurationen, Interaktionen, Perzeptionen. Linguistik online 85 (6).

Jürgens, Carolin (2015): Niederdeutsch im Wandel. Sprachgebrauchswandel und Sprachwahrnehmung in Hamburg. Hildesheim u. a.: Olms. (Deutsche Dialektgeographie 119).

Kaiser, Irmtraud (2006): Bundesdeutsch aus österreichischer Sicht. Eine Untersuchung zu Spracheinstellungen, Wahrnehmungen und Stereotypen. Mannheim: Institut für Deutsche Sprache. (amades 2/06).

Kehrein, Roland (2009): Dialektalität von Vorleseaussprache im diatopischen Vergleich Hörerurteil und phonetische Messung. Zeitschrift für Dialektologie und Linguistik 76 (1), 14-54.

Kehrein, Roland (2012a): Wen man nicht alles für einen Sachsen hält!? Oder: Zur Aktivierung von Sprachraumkonzepten durch Vorleseaussprache. In Rainer Hünecke \& Karlheinz Jakob (Hrsg.), Die obersächsische Sprachlandschaft in Geschichte und Gegenwart, 223-263. Heidelberg: Universitätsverlag Winter.

Kehrein, Roland (2012b): Regionalsprachliche Spektren im Raum - Zur linguistischen Struktur der Vertikale. Stuttgart: Steiner. (Zeitschrift für Dialektologie und Linguistik, Beihefte 152).

Kehrein, Roland, Alfred Lameli \& Christoph Purschke (2010): Stimuluseffekte und Sprachraumkonzepte. In Christina A. Anders, Markus Hundt \& Alexander Lasch (Hrsg.), „perceptual dialectology“. Neue Wege der Dialektologie, 351-384. Berlin/New York: de Gruyter. (Linguistik - Impulse \& Tendenzen 38).

Kennetz, Keith (2008): German and German Disunity. An Investigation into the Cognitive Patterns and Perceptions of Language in Post-Unified Germany. A Dissertation Submitted to the Graduate Faculty of The University of Georgia. Athens, Georgia. https:// getd.libs.uga.edu/pdfs/kennetz_keith_e_200805_phd.pdf. (letzter Zugriff: 1.3. 2017)

Kennetz, Keith (2009): Sächsisch als Verlierersprache. In Beate Henn-Memmesheimer \& Joachim Franz (Hrsg.), Die Ordnung des Standard und die Differenzierung der Diskurse. Akten des 41. Linguistischen Kolloquiums in Mannheim 2006, 503-512. Frankfurt a. M. u. a.: Peter Lang.

Kennetz, Keith (2010): German and German political disunity. An investigation into the cognitive patterns and perceptions of language in post-unified Germany. In Christina A. Anders, Markus Hundt \& Alexander Lasch (Hrsg.), „perceptual dialectology“. 
Neue Wege der Dialektologie, 317-335. Berlin/New York: de Gruyter. (Linguistik Impulse \& Tendenzen 38).

Kiesewalter, Carolin (2011): Zur Salienz remanenter Merkmale des Neuhessischen. In Brigitte Ganswindt \& Christoph Purschke (Hrsg.), Perspektiven der Variationslinguistik. Beiträge aus dem Forum Sprachvariation, 335-371. Hildesheim: Olms. (Germanistische Linguistik). Kiesewalter, Carolin (2014): Salienz und Pertinenz. Zur subjektiven Dialektalität remanenter Regionalismen des Mittelbairischen. In Helen Christen \& Evelyn Ziegler (Hrsg.), Die Vermessung der Salienz(forschung). Measuring (the Research on) Salience. Linguistik Online 66 (4), 111-134.

Kleene, Andrea (2015): Mental Maps des Bairischen und seiner Grenzen. In Michael Elmentaler, Markus Hundt \& Jürgen Erich Schmidt (Hrsg.), Deutsche Dialekte. Konzepte, Probleme, Handlungsfelder: Akten des 4. Kongresses der Internationalen Gesellschaft für Dialektologie des Deutschen (IGDD), 323-340. Stuttgart: Steiner. (Zeitschrift für Dialektologie und Linguistik. Beihefte 158).

Kleene, Andrea (i. Dr.): Perzeptiv-attitudinale Variationslinguistik im bairischen Sprachraum. [Dissertationsschrift Wien].

Lameli, Alfred (2012): Wo vermutet der Westdeutsche eigentlich die sächsische

Sprachlandschaft? Zur Verortung von Regionalsprache durch linguistische Laien. In Rainer Hünecke \& Karlheinz Jakob (Hrsg.), Die obersächsische Sprachlandschaft in Geschichte und Gegenwart, 95-142. Heidelberg: Universitätsverlag Winter.

Lameli, Alfred (2015): Zur Konzeptualisierung des Sprachraums als Handlungsraum. In Michael Elmentaler, Markus Hundt \& Jürgen Erich Schmidt (Hrsg.), Deutsche Dialekte. Konzepte, Probleme, Handlungsfelder: Akten des 4. Kongresses der Internationalen Gesellschaft für Dialektologie des Deutschen (IGDD), 59-83. Stuttgart: Steiner. (Zeitschrift für Dialektologie und Linguistik. Beihefte 158).

Lameli, Alfred, Christoph Purschke \& Roland Kehrein (2008): Stimulus und Kognition. Zur Aktivierung mentaler Raumbilder. Linguistik Online 35, 55-86.

Lameli, Alfred, Roland Kehrein \& Stefan Rabanus (Hrsg.) (2010): Language and Space. An International Handbook of Linguistic Variation. Vol. 2: Language Mapping. Berlin/ Boston: de Gruyter Mouton.

Langer, Nils (2010): Sprechereinstellungen zur Zielsprache im britischen und irischen DaFUnterricht. In Christina A. Anders, Markus Hundt \& Alexander Lasch (Hrsg.), „perceptual dialectology“. Neue Wege der Dialektologie, 409-431. Berlin/New York: de Gruyter. (Linguistik - Impulse und Tendenzen 38).

Lenz, Alexandra N. (2010): Zum Salienzbegriff und zum Nachweis salienter Merkmale. In Christina A. Anders, Markus Hundt \& Alexander Lasch (Hrsg.), „perceptual dialectology“. Neue Wege der Dialektologie, 89-110. Berlin/New York: de Gruyter. (Linguistik Impulse \& Tendenzen 38).

Liebscher, Grit \& Jennifer Dailey-0'Cain (2014): Die Rolle von Wissen und Positionierung bei Spracheinstellungen im diskursiven Kontext. In Christina Cuonz \& Rebekka Studler (Hrsg.), Sprechen über Sprache. Perspektiven und neue Methoden der Spracheinstellungsforschung, 107-122. Tübingen: Stauffenburg. (Stauffenburg Linguistik 81).

Long, Daniel \& Dennis R. Preston (Hrsg.) (2002): Handbook of perceptual dialectology. Vol. 2. Amsterdam, Philadelphia: John Benjamins.

Mathussek, Andrea (2014): Sprachräume und Sprachgrenzen im Untersuchungsgebiet des Sprachatlas von Mittelfranken. Traditionelle Dialektgeographie - Wahrnehmungsdialektologie - Dialektometrie. (OraLingua 7). Heidelberg. 
Neumann, Lara \& Ingrid Schröder (2017): Zur Bewertung von Niederdeutsch und lokalem Substandard in Hamburg. In Markus Hundt, Christoph Purschke \& Evelyn Ziegler (Hrsg.), Sprachräume: Konfigurationen, Interaktionen, Perzeptionen. Linguistik Online 85 (6), 227-255.

Niedzielski, Nancy A. \& Dennis R. Preston (2003): Folk linguistics. Berlin/New York: de Gruyter. (Trends in Linguistics. Studies and Monographs 122).

Oberholzer, Susanne (2017): Sprachgebrauch und Spracheinstellungen in der Deutschschweiz. Pfarrpersonen als sprachbewusste Sprecherinnen und Sprecher im Fokus. In Markus Hundt, Christoph Purschke \& Evelyn Ziegler (Hrsg.), Sprachräume: Konfigurationen, Interaktionen, Perzeptionen. Linguistik Online 85 (6), 127-151.

Palliwoda, Nicole (2011): Die Verortung von Sprachvarietäten im Deutschen. Eine empirische Studie zum Vergleich von „Mental maps“ und Sprachproben. In Brigitte Ganswindt \& Christoph Purschke (Hrsg.), Perspektiven der Variationslinguistik. Beiträge aus dem Forum Sprachvariation, 419-442. Hildesheim u. a.: Olms. (Germanistische Linguistik 216-217).

Palliwoda, Nicole (2012): Laienlinguistische Sprachräume - methodische Aufbereitung anhand von Mental Maps und Sprachproben. In Sabrina Braukmeier, Julia Burkhardt \& Fleur Pfeifer (Hrsg.), Wege in den SprachRaum - Methodische Herausforderungen linguistischer Forschung, 53-67. Leipzig: Peter Lang.

Palliwoda, Nicole (2013): „Ende des Ossis“? Die Priming-Methode innerhalb sprachwissenschaftlicher Analysen. In Yvonne Hettler, Carolin Jürgens, Robert Langhanke \& Christoph Purschke (Hrsg.), Variation, Wandel, Wissen. Studien zum Hochdeutschen und Niederdeutschen, 183-200. Frankfurt a. M.: Peter Lang. (Sprache in der Gesellschaft 32).

Palliwoda, Nicole (2014): Sprachraum Grenze: „Ende des Ossis“? Das Konzept ,Mauer in den Köpfen‘. In Rudolf Bühler, Rebekka Bürkle \& Nina Kim Leonhardt (Hrsg.), Sprachkultur, Regionalkultur. Neue Felder kulturwissenschaftlicher Dialektforschung, 71-95. Tübingen: Tübinger Vereinigung für Volkskunde e. V. (Studien und Materialien des Ludwig-UhlandInstituts der Universität Tübingen).

Palliwoda, Nicole (2017a): Der Einfluss von Grenzen auf die Verortung von Sprachraumkonzepten im Nahbereich - Exemplarischer Einblick in die Mikrokartierung. In Markus Hundt, Nicole Palliwoda \& Saskia Schröder (Hrsg.): Der deutsche Sprachraum aus der Sicht linguistischer Laien. Ergebnisse des Kieler DFG-Projektes, 13-45. Berlin/ New York: de Gruyter.

Palliwoda, Nicole (2017b): Das Ratespiel. Möglichkeiten und Grenzen der Auswertung. In Markus Hundt, Nicole Palliwoda \& Saskia Schröder (Hrsg.): Der deutsche Sprachraum aus der Sicht linguistischer Laien. Ergebnisse des Kieler DFG-Projektes, 83-120. Berlin/ New York: de Gruyter.

Palliwoda, Nicole (i. Dr.): Das Konzept Mauer in den Köpfen. Der Einfluss der PrimingMethode auf die Sprechprobenverortung und -bewertung. Dissertationsschrift, Universität Kiel.

Palliwoda, Nicole \& Saskia Schröder (2012): Wahrnehmungsdialektologie und das Konzept der Salienz. In: Eveline Wandl-Vogt, Stefan Winterstein, Christina Schrödl \& Andrea Singer (Hrsg.): dialekt / dialect 2.0 \& wboe 100. kurzfassungen. Abstracts, 170-172. Wien: Praesens.

Palliwoda, Nicole \& Saskia Schröder (2016): Perceptual dialectology - Speech samples and the concept of salience. Initial findings from the DFG-project "Lay Linguists' perspectives on German regional varieties. Reconstructing lay linguistic conceptualizations of German in a perceptual dialectology approach". In Jennifer Cramer \& Chris Montgomery 
(Hrsg.), Cityscapes and perceptual dialectology. Global perspectives on non-linguists' knowledge of the dialect landscape, 257-274. Berlin/New York: de Gruyter Mouton. (Language and Social Life 5).

Palliwoda, Nicole \& Saskia Schröder (2017): Eine wahrnehmungsdialektologische Betrachtung des bairischen Sprachraums. In Lenz, Alexandra N. et al. (Hrsg.) (2017): Bayerisch-österreichische Varietäten zu Beginn des 21. Jahrhunderts - Dynamik, Struktur, Funktion. Stuttgart: Steiner (Zeitschrift für Dialektologie und Linguistik, Beiheft 167), 133-153.

Peters, Jörg, Peter Auer \& Peter Gilles (2015): Untersuchungen zur Struktur und Funktion regionalspezifischer Intonationsverläufe im Deutschen. In Roland Kehrein, Alfred Lameli \& Stefan Rabanus (Hrsg.), Regionale Variation des Deutschen. Projekte und Perspektiven, 54-80. Berlin/Boston: de Gruyter Mouton.

Petkova, Marina (2017): Linguistic landscapes in der Innerschweiz: Dialekt, Toponyme und heraldische Zeichen als Ausdruck von Raumzugehörigkeit. In Markus Hundt, Christoph Purschke \& Evelyn Ziegler (Hrsg.), Sprachräume: Konfigurationen, Interaktionen, Perzeptionen. Linguistik Online 85 (6), 153-180.

Plewnia, Albrecht \& Astrid Rothe (2009): Eine Sprach-Mauer in den Köpfen? Über aktuelle Spracheinstellungen in Ost und West. Deutsche Sprache 37 (2-3), 235-279.

Plewnia, Albrecht \& Astrid Rothe (2011): Von gebildeten Deutschen, freundlichen Sachsen und temperamentvollen Bayern. Einstellungen zu Varietäten und ihren Sprechern. In Helen Christen, Franz Patocka \& Evelyn Ziegler (Hrsg.), Struktur, Gebrauch und Wahrnehmung von Dialekt. Beiträge zum 3. Kongress der Internationalen Gesellschaft für Dialektologie des Deutschen (IGDD), Zürich, 7.-9. September 2009, 179-207. Wien: Praesens.

Preston, Dennis R. (1986): Five visions of America. Language in Society 15 (2), 221-240.

Preston, Dennis R. (1993): Folk dialectology. In Dennis R. Preston (Hrsg.), American Dialect Research, 333-377. Amsterdam/Philadelphia: John Benjamins.

Preston, Dennis R. (Hrsg.) (1999): Handbook of perceptual dialectology. Vol. 1. Amsterdam, Philadelphia: John Benjamins.

Preston, Dennis R. (2002): Perceptual dialectology. Aims, methods, findings. In Jan Berns \& Jaap van Marle (Hrsg.), Present-day dialectology. Problems and findings, 57-104. Berlin/New York: de Gruyter. (Trends in Linguistics. Studies and Monographs 137).

Preston, Dennis R. (2004): Perceptual Dialectology/Perzeptive Dialektologie. In Ulrich Ammon, Norbert Dittmar, Klaus J. Mattheier \& Peter Trudgill (Hrsg.), Soziolinguistik. Ein internationales Handbuch zur Wissenschaft von Sprache und Gesellschaft. 2., vollständig neu bearbeitete und erweiterte Auflage. 2. Teilband, 1683-1696. Berlin/ New York: de Gruyter. (Handbücher zur Sprach- und Kommunikationswissenschaft 3/2).

Preston, Dennis R. (2005): Dialects across internal frontiers. Some cognitive boundaries. In Markku Filppula, Juhani Klemola, Marjatta Palander \& Esa Penttilä (Hrsg.), Dialects across borders, 121-155. Amsterdam, Philadelphia: John Benjamins.

Preston, Dennis (2010a): Perceptual dialectology in the 21st century. In Christina A. Anders, Markus Hundt \& Alexander Lasch (Hrsg.), „perceptual dialectology“. Neue Wege der Dialektologie, 1-29. Berlin/New York: de Gruyter. (Linguistik - Impulse und Tendenzen 38).

Preston, Dennis R. (2010b): Language, space and the folk. In Peter Auer \& Jürgen Erich Schmidt (Hrsg.), Language and space. An international handbook of linguistic variation. Vol. 1: Theories and methods, 179-201. Berlin/Boston: de Gruyter Mouton. (Handbücher zur Sprach- und Kommunikationswissenschaft 30/1). 
Preston, Dennis R. (2010c): Mapping the geolinguistic spaces of the brain. In Alfred Lameli, Roland Kehrein \& Stefan Rabanus (Hrsg.), Language and space. An international handbook of linguistic variation. Vol. 2: Language mapping. Part I, 121-141. Berlin/ Boston: de Gruyter Mouton. (Handbücher zur Sprach- und Kommunikationswissenschaft $30 / 2$ ).

Purschke, Christoph (2008): Regionalsprachlichkeit im Hörerurteil. In Helen Christen \& Evelyn Ziegler (Hrsg.), Sprechen, Schreiben, Hören - Zur Produktion und Perzeption von Dialekt und Standardsprache zu Beginn des 21. Jahrhunderts, 183-202. Wien: Praesens.

Purschke, Christoph (2010a): Regionalsprachliches Wissen und Perzeption - Zur Konzeptualisierung des Hessischen. In Matthias Katerbow \& Alexander Werth (Hrsg.), Moderne Regionalsprachen als multidimensionales Forschungsfeld, 93-127. Hildesheim u. a.: Olms (Germanistische Linguistik 210).

Purschke, Christoph (2010b): Imitation und Hörerurteil. Kognitive Dialekt-Prototypen am Beispiel des Hessischen. In Christina A. Anders, Markus Hundt, Alexander Lasch (Hrsg.), „perceptual dialectology“. Neue Wege der Dialektologie, 151-178. Berlin/ New York: de Gruyter. (Linguistik - Impulse \& Tendenzen 38).

Purschke, Christoph (2011): Regionalsprache und Hörerurteil. Grundzüge einer perzeptiven Variationslinguistik. Stuttgart: Steiner. (Zeitschrift für Dialektologie und Linguistik, Beihefte 149).

Purschke, Christoph (2014a): „I remember it like it was interesting“: Zur Theorie von Salienz und Pertinenz. In Helen Christen \& Evelyn Ziegler (Hrsg.), Die Vermessung der Salienz(forschung)/Measuring (the Research on) Salience. Linguistik online 66, 31-50.

Purschke, Christoph (2014b): REACT - Einstellungen als evaluative Routinen in sozialen Praxen. In Christina Cuonz \& Rebekka Studler (Hrsg.), Sprechen über Sprache. Perspektiven und neue Methoden der Spracheinstellungsforschung, 123-142. Tübingen: Stauffenburg.

Rein, Charlotte (2013): Wahrnehmung und Verortung regionaler Sprachvarietäten. Sprachraumkonzepte von Schülern aus Dresden und dem Rheinland im Vergleich. München: Akademische Verlagsgemeinschaft.

Riehl, Claudia Maria (2000): Spracheinstellungen und Stereotype im Lichte diskursiver Praxis. In Szilvia Deminger, Thorsten Fögen, Joachim Scharloth \& Simone Zwickl (Hrsg.), Einstellungsforschung in der Soziolinguistik und Nachbardisziplinen, 141-160. Frankfurt am Main: Peter Lang.

Sauer, Verena (2017): „Wenn der Sprache Grenzen gesetzt werden“ - Eine wahrnehmungsdialektologische Untersuchung im Fränkischen und Thüringischen an der ehemaligen deutsch-deutschen Grenze. In Markus Hundt, Christoph Purschke \& Evelyn Ziegler (Hrsg.), Sprachräume: Konfigurationen, Interaktionen, Perzeptionen. Linguistik Online 85 (6), 203-225.

Scharioth, Claudia (2012): Die Diskrepanz zwischen tatsächlichem Sprachgebrauch und normativer Bewertung sprachlicher Merkmale. In Katharina Rosenberg \& Rita Vallentin (Hrsg.), Norm und Normalität. Beiträge aus Linguistik, Soziologie, Literatur- und Kulturwissenschaften, 109-121. Berlin: Logos.

Scharioth, Claudia (2015): Regionales Sprechen und Identität. Eine Studie zum Sprachgebrauch, zu Spracheinstellungen und Identitätskonstruktionen von Frauen in Schleswig-Holstein und Mecklenburg-Vorpommern. Hildesheim u. a.: Olms. (Deutsche Dialektgeographie 120).

Scharloth, Joachim (2005): Asymmetrische Plurizentrizität und Sprachbewusstsein. Einstellungen der Deutschschweizer zum Standarddeutschen. Zeitschrift für Germanistische Linguistik 33 (2), 236-267. 
Schaub, Victoria (2011) Beliebt oder nur bekannt? Einflüsse von Bekanntheit in der sprachdemoskopischen Untersuchung von Einstellungen gegenüber deutschen Dialekten. In Brigitte Ganswindt \& Christoph Purschke (Hrsg.), Perspektiven der Variationslinguistik. Beiträge aus dem Forum Sprachvariation, 393-417. Hildesheim u. a.: Olms. (Germanistische Linguistik 216-217).

Schaufuß, Anja (2015): Regionalsprachlichkeit von Sprechern des Obersächsischen im Dreieck Dresden, Chemnitz, Leipzig. Hörerurteile und phonetischer Abstand im diatopischen Vergleich. In Michael Elmentaler, Markus Hundt \& Jürgen Erich Schmidt (Hrsg.), Deutsche Dialekte. Konzepte, Probleme, Handlungsfelder. Akten des 4. Kongresses der Internationalen Gesellschaft für Dialektologie des Deutschen (IGDD), 359-377. Stuttgart: Steiner. (Zeitschrift für Dialektologie und Linguistik, Beihefte 158).

Schaufuß, Anja \& Beat Siebenhaar (2012): Spracheinstellungen und phonetische Variation als Ausdruck verschwommener Dialektabgrenzung. Zeitschrift für Literaturwissenschaft und Linguistik 166, 88-109.

Schmidlin, Regula (2011): Die Vielfalt des Deutschen: Standard und Variation. Gebrauch, Einschätzung und Kodifizierung einer plurizentrischen Sprache. Berlin/New York: de Gruyter. (Studia Linguistica Germanica 106).

Schnabel, Michael (2006): Dialektspaltung im thüringisch-bayerischen Grenzgebiet am Beispiel des Ortspaars Sparnberg/Rudolphstein. Wie eine politische Grenze zur Sprachgrenze wurde. Zeitschrift für Dialektologie und Linguistik 73 (1), 30-54.

Schneider, Nora, Helmut Spiekermann \& Sarah Till (2011): Laiendialektologische Wahrnehmung schwäbischer Dialekte. In Helen Christen, Franz Patocka \& Evelyn Ziegler (Hrsg.), Struktur, Gebrauch und Wahrnehmung von Dialekt. Beiträge zum 3. Kongress der Internationalen Gesellschaft für Dialektologie des Deutschen (IGDD). Zürich, 7.-9. September 2009, 235-261. Wien: Praesens.

Schröder, Saskia (2015): Mental maps als Zugang zu sprachlichem Wissen. In Robert Langhanke (Hrsg.), Sprache, Literatur, Raum. Festschrift für Willy Diercks, 163-181. Gütersloh: Verlag für Regionalgeschichte.

Schröder, Saskia (2017): Die Verortung der eigenen Sprechweise im Makrobereich durch linguistische Laien. In: Markus Hundt, Nicole Palliwoda \& Saskia Schröder (Hrsg.), Der deutsche Sprachraum aus der Sicht linguistischer Laien. Ergebnisse des Kieler DFG-Projektes, 47-82. Berlin/New York: de Gruyter.

Schröder, Saskia (i. Dr.): Sprachräumliche Praxis. Sprachraumkartierung in der Wahrnehmungsdialektologie. Dissertation. Universität Kiel.

Siebenhaar, Beat (2000): Sprachvariation, Sprachwandel und Einstellung. Der Dialekt der Stadt Aarau in der Labilitätszone zwischen Zürcher und Berner Mundartraum. Stuttgart: Steiner. (Zeitschrift für Dialektologie und Linguistik, Beihefte 108).

Siebenhaar, Beat (2002): Dialektwandel und Einstellung. Das Beispiel der Aarauer Stadtmundart. In Johannes B. Berns \& Kaap van Merle (Hrsg.), Present-day dialectology. Problems and Findings, 313-332. Berlin/New York: de Gruyter.

Soukup, Barbara (2014): Konstruktivismus trifft auf Methodik in der Spracheinstellungsforschung. Theorie, Daten, Fazit. In Christina Cuonz \& Rebekka Studler (Hrsg.), Sprechen über Sprache. Perspektiven und neue Methoden der Spracheinstellungsforschung, 143-168. Tübingen: Stauffenburg. (Stauffenburg Linguistik 81).

Spiekermann, Helmut (2009): Bewertung und Auswahl tertiärer Dialektmerkmale durch dialektologische Laien. In Beate Henn-Memmesheimer \& Joachim Franz (Hrsg.), Die Ordnung des Standard und die Differenzierung der Diskurse. Akten des 41. 
Linguistischen Kolloquiums in Mannheim, 6. 9.-8. 9. 2006. Band 2, 513-527.

Frankfurt a. M. u. a.: Peter Lang. (Linguistik international; 24).

Spiekermann, Helmut (2010): Visualisierung von Dialekten. Ein Beitrag zum Nutzen der Laiendialektologie. In Christina A. Anders, Markus Hundt \& Alexander Lasch (Hrsg.), „perceptual dialectology“. Neue Wege der Dialektologie, 221-244. Berlin/New York: de Gruyter. (Linguistik - Impulse \& Tendenzen 38).

Spiekermann, Helmut (2012): Welche Farbe hat das Sächsische? Ein Versuch zur Visualisierung von Sprachbewertungen und Dialekteinschätzungen. In Rainer Hünecke \& Karlheinz Jakob (Hrsg.), Die obersächsische Sprachlandschaft in Geschichte und Gegenwart, 315-340. Heidelberg: Universitätsverlag Winter.

Steinig, Wolfgang (1982): Zur sozialen Bewertung von drei sprachlichen Varietäten in Schwaben. Wiesbaden: Vieweg.

Stoeckle, Philipp (2010): Subjektive Dialektgrenzen im alemannischen Dreiländereck. In Christina A. Anders, Markus Hundt \& Alexander Lasch (Hrsg.), „perceptual dialectology“. Neue Wege der Dialektologie, 291-315. Berlin/New York: de Gruyter. (Linguistik Impulse \& Tendenzen 38).

Stoeckle, Philipp (2012): The folk linguistic construction of local dialect areas - linguistic and extra-linguistic factors. In Sandra Hansen, Christian Schwarz, Philipp Stoeckle \& Tobias Streck (Hrsg.), Dialectological and folk dialectological concepts of space. Current methods and perspectives in sociolinguistic research on dialect change, 142-163 (app. 271-275). Berlin/Boston: de Gruyter. (linguae \& litterae 17).

Stoeckle, Philipp (2014): Subjektive Dialekträume im alemannischen Dreiländereck. Hildesheim: Olms. (Deutsche Dialektgeographie 112).

Studler, Rebekka (2013): Einstellungen zu Standarddeutsch und Dialekt in der Deutschschweiz. Erste Ergebnisse einer Fragebogenstudie. In Yvonne Hettler, Carolin Jürgens, Robert Langhanke \& Christoph Purschke (Hrsg.), Variation, Wandel, Wissen. Studien zum Hochdeutschen und Niederdeutschen, 201-220. Frankfurt a. M.: Peter Lang. (Sprache in der Gesellschaft. Beiträge zur Sprach- und Medienwissenschaft 32).

Studler, Rebekka (2014): „Einige Antworten habe ich contre coeur so angekreuzt“. Zur Relevanz offener Fragen in Fragebogenstudien zu Spracheinstellungen. In Christina Cuonz \& Rebekka Studler (Hrsg.), Sprechen über Sprache. Perspektiven und neue Methoden der Spracheinstellungsforschung, 169-204. Tübingen: Stauffenburg. (Stauffenburg Linguistik 81).

Studler, Rebekka (i. V.): Zur Genese von Spracheinstellungen am Beispiel des Hochdeutschen und Schweizerdeutschen in der Deutschschweiz. [Habilitationsprojekt].

Tamasi, Susan L. (2003): Cognitive patterns of linguistic perceptions. [Dissertationsschrift. Athens: University of Georgia. http://athenaeum.libs.uga.edu/handle/10724/20928 (letzter Zugriff: 23.10. 2015).

Teschke, Verena (2009): Perzeption prosodischer Merkmale deutscher Regionalsprachen. Der Unterschied zwischen „Identifizierung“ und „Diskrimination“. Sprachwissenschaft 34 (2), 151-185.

Tophinke, Doris \& Evelyn Ziegler (2006): „Aber bitte im Kontext!“. Neue Perspektiven der dialektologischen Einstellungsforschung. In Anja Voeste \& Joachim Gessinger (Hrsg.), Dialekt im Wandel. Perspektiven einer neuen Dialektologie, 205-224. Duisburg: Red. OBST.

Twilfer, Daniela (2012): Dialektgrenzen im Kopf. Der westfälische Sprachraum aus volkslinguistischer Perspektive. Bielefeld: Verlag für Regionalgeschichte. (Westfälische Beiträge zur niederdeutschen Philologie 13). 
Vandermeeren, Sonja (2005): Research on language yttitudes. In Ulrich Ammon, Norbert Dittmar, Klaus J. Mattheier \& Peter Trudgill (Hrsg.), Soziolinguistik. Ein internationales Handbuch zur Wissenschaft von Sprache und Gesellschaft.

2. Teilband. 2. vollständig neu bearbeitete und erweiterte Auflage, 1318-1332. Berlin/ New York: de Gruyter. (Handbücher zur Sprach- und Kommunikationswissenschaft 3/2). Wagner, Melanie M. (2009): Lay linguistics and school teaching. An empirical sociolinguistic study in the Moselle-Franconian dialect area. Stuttgart: Steiner. (Zeitschrift für Dialektologie und Linguistik - Beiheft; 136).

Werth, Alexander (2007): Perzeptionsphonologische Studien zu den mittelfränkischen Tonakzenten. In Jürgen Erich Schmidt (Hrsg.), Zeitschrift für Dialektologie und Linguistik 74 (2/3), 292-316. Stuttgart: Franz Steiner. 


\title{
Evelyn Ziegler
}

\section{Visuelle Mehrsprachigkeit in Migrationsgesellschaften: monolinguale Norm vs. plurilinguale Norm}

\begin{abstract}
Die Linguistic-Landscape Forschung bietet einen neuen Rahmen, um die zunehmende Plurilingualisierung urbaner Räume, als Folge von Migration und Globalisierung, quantitativ und qualitativ zu untersuchen. Das Projekt Metropolenzeichen: Visuelle Mehrsprachigkeit in der Metropole Ruhr widmet sich den verschiedenen Manifestationen öffentlich sichtbarer Mehrsprachigkeit; es ist als Querschnittstudie für die Städte Duisburg, Essen, Bochum und Dortmund konzipiert. In einem multi-facettierten Forschungsdesign, das einen Mehr-Methoden-Ansatz verfolgt, werden Daten visueller Mehrsprachigkeit, metasprachliche Daten zu ihrer Rezeption und Produktion sowie stadtsoziologische Daten analysiert. Folgende Fragen stehen im Mittelpunkt: Welche Formen der Normorientierung (monolingual, multilingual) lassen sich auf Schildern, Aushängen, Aufklebern etc. in verschiedenen Diskurstypen (kommerziell, regulatorisch, infrastrukturell etc.) im öffentlichen Raum der Metropole Ruhr erkennen? Wie werden die Sprachenwahlen und die darin eingeschriebenen Normorientierungen von Befragten mit und ohne Migrationshintergrund wahrgenommen und bewertet? Welche Motive leiten die Produzenten von Schildern und Aushängen etc. bei der Wahl der Sprache(n)?
\end{abstract}

Keywords: Diskurstyp, linguistic landscape, öffentlicher Raum, Spracheinstellungen, Sprachwahlmotive, visuelle Mehrsprachigkeit

\section{Zielsetzung}

Visuelle, d. h. sichtbare Mehrsprachigkeit zeigt sich auf Hinweis-, Informationsund Geschäftsschildern sowie Graffitis. Sie steht in engem Zusammenhang mit Migration und Globalisierung sowie auch mit Regionalisierungstendenzen,

Evelyn Ziegler, Universität Duisburg-Essen, Berliner Platz 6-8, D-45127 Essen, E-Mail: evelyn.ziegler@uni-due.de

Ә Open Access. (c) 2018 Evelyn Ziegler, publiziert von De Gruyter. (c) BY Dieses Werk ist lizenziert unter der Creative Commons Attribution 4.0 Lizenz.

https://doi.org/10.1515/9783110538625-006 
d. h. der Inanspruchnahme kleinräumigerer kultureller Identifikationssymbole wie etwa regionalen Varietäten. Das Projekt „Metropolenzeichen“1 widmet sich diesen verschiedenen Formen und Manifestationen von Mehrsprachigkeit, und zwar bezogen auf den öffentlichen Raum der Metropole Ruhr als bundesweit wichtigster Metropole für Arbeitsmigration. Ein markantes Merkmal der Metropole Ruhr ist der „Sozialäquator A 40“ (Kersting et al. 2009), der die Städte in ethnisch divers und weniger ethnisch divers, arm und weniger arm, gebildet und weniger gebildet teilt. Insofern bietet die Metropole Ruhr die Möglichkeit, zugleich systematisch und vergleichend die zunehmende ethnisch-soziale und sprachliche Heterogenisierung in klar definierten städtischen Teilräumen zu untersuchen.

Ausgangspunkt der folgenden Überlegungen ist ein weites Verständnis von Linguistic Landscape, wie es von Shohamy \& Gorter (2009) vertreten wird und das weit darüber hinaus geht, eine bloße Deskription, d. h. Bestandsaufnahme der verschiedenen Verbreitungs- und Realisierungsformen von sichtbarer Mehrsprachigkeit im öffentlichen Raum zu sein:

it [= linguistic landscape, E. Z.] contextualizes the public space within issues of identity and language policy of nations, political and social conflicts. It posits that LL is a broader concept than documentation of signs; it incorporates multimodal theories to include also sounds, images, and graffiti. (Shohamy \& Gorter 2009: 4)

Charakteristisch für diesen Zugang ist, dass nicht nur die potenziellen Gegenstände der Linguistic Landscape-Forschung weit gefasst werden, sondern auch ein komplexeres Gegenstandsverständnis angestrebt wird, indem dem Verhältnis von Analyse und Kontext (räumlich, sozial, zeitlich) eine große Bedeutung beigemessen wird und auch die den konkreten Sprachwahlen zugrundeliegenden Sprachideologien Berücksichtigung finden. Dementsprechend heißt es im Begleittext der Zeitschrift „Linguistic Landscape. An international journal“:

The dynamic field of Linguistic Landscape (LL) attempts to understand the motives, uses, ideologies, language varieties and contestations of multiple forms of 'languages' as they are displayed in public spaces. ${ }^{2}$

1 Das Projekt „Metropolenzeichen: Visuelle Mehrsprachigkeit in der Metropole Ruhr“ wurde von 2013 bis 2018 vom Mercator Research Center Ruhr gefördert; es ist ein Kooperationsprojekt zwischen der Universität Duisburg-Essen und der Ruhr-Universität Bochum (Leitung: Evelyn Ziegler). Eine Beschreibung des Projektdesigns und der Projektziele findet sich in Ziegler (2013), Projektergebnisse finden sich u.a. in den folgenden Publikationen: Cindark \& Ziegler (2016), Schmitz \& Ziegler (2016), Schmitz (2017, i. Dr.), Wachendorff, Ziegler \& Schmitz (2017), Ziegler, Eickmans \& Schmitz (2017), Ziegler, Schmitz \& Uslucan (i. Dr.), und Eickmans \& Ziegler (2017).

2 Text abrufbar unter: https://benjamins.com/\#catalog/journals/ll/main (letzter Zugriff am 4.7.2017). 
Die Frage, welche Akteure welche Sprache(n) ${ }^{3}$ für welche Zwecke und für welche Adressatengruppe(n) bei der Betextung des öffentlichen Raums verwenden, ist nicht nur eine Frage der Sprach(en)wahl und damit eine Frage der Orientierung an einer monolingualen oder plurilingualen Norm, sondern immer auch eine Frage, die die sozialsymbolische Ebene betrifft. Damit ist gemeint, dass Sprachenwahlen auch etwas darüber aussagen, mit welcher Sprache oder welchen Sprachen Sprecher bzw. hier Textproduzenten identifiziert werden möchten und/oder welche ethnischen bzw. sozialen Gruppen mit der Sprach(en)wahl adressiert werden sollen. Sprachenwahlen sagen damit immer auch etwas über den Status von Sprachen aus und darüber, welche Sprechergruppen sozial anerkannt werden, je nachdem wie inklusiv oder exklusiv die je spezifischen Sprachenwahlen sind.

In diesem Zusammenhang sind die von Spolsky \& Cooper (1991: 81-85) im Rahmen des Language-Management-Ansatzes formulierten Maximen für die Wahl von Sprachen für Texte im öffentlichen Raum instruktiv:

1. Beschrifte einen Aushang in der Sprache, die du beherrschst.

2. Antizipiere die Sprachkompetenz des Adressaten.

3. Beschrifte einen Aushang in deiner eigenen Sprache oder in der Sprache, mit der du identifiziert werden möchtest.

Aus anerkennungstheoretischer Perspektive, d. h. mit Blick auf die Rezpienten, weisen Landry \& Bourhis (1997) darauf hin:

Having one's own language enshrined on most private and government signs should contribute to the feeling that the in-group language has value and status relative to the other languages in the sociolinguistic setting. (Landry \& Bourhis 1997: 27)

Ausgehend von diesem Gegenstandsverständnis, das sowohl die Rezeptionsals auch die Produktionsperspektive berücksichtigt, sollen folgende Fragen behandelt werden:

1. Welche Formen der Normorientierung (monolingual, multilingual) lassen sich auf Schildern, Aushängen, Aufklebern etc. in verschiedenen Diskurstypen (kommerziell, regulatorisch, infrastrukturell etc.) im öffentlichen Raum der Metropole Ruhr erkennen?

2. Wie werden die Sprachenwahlen und die darin eingeschriebenen Normorientierungen von Informanten mit und ohne Migrationshintergrund wahrgenommen und bewertet?

3 Zur Problematik der Identifikation einzelner Sprachen auf Schildern, Aushängen etc. und der Idee von Sprachen als „discrete identities“ vgl. die Diskussion in Gaiser \& Matras (2016). 
3. Welche Motive leiten die Produzenten offizieller und privater Schilder, Aushänge etc. bei der Wahl der Sprache(n)?

\section{Forschungsdesign und Daten}

Die empirische, korpusbasierte Beschreibung und Analyse der schriftsprachlich gestalteten öffentlichen Räume in der Metropole Ruhr basiert auf verschiedenen Datentypen: zum einen auf objektsprachlichen Daten visueller Mehrsprachigkeit $(n=25.504)$ und zum anderen auf metasprachlichen Daten zur Wahrnehmung und Bewertung visueller Mehrsprachigkeit, die in Vor-OrtInterviews $(n=120)$ sowie in einer mehrsprachigen Telefonbefragung ${ }^{4}(n=$ 1.000) erhoben wurden. Zusätzlich wurden metasprachliche Daten erhoben, die die Produzentenperspektive (offizielle und private Akteure) in den Blick nehmen und auf der Grundlage von Leitfaden-gestützen Interviews gesammelt wurden $(n=60)$.

Im Rahmen von Vor-Ort-Begehungen in den Städten Duisburg, Essen, Bochum und Dortmund wurde ein geokodiertes Bilddatenkorpus aufgebaut. Pro Stadt wurden jeweils zwei vorab exakt definierte Bezirke entlang des „Sozialäquators A 40“ jeweils vollständig dokumentiert, d.h. für jedes öffentlich sichtbare Item wurde ein Foto gemacht. Die Bezirke wurden nach stadtsoziologischen Kriterien ausgewählt. Sie zeichnen sich durch eine Mischnutzung aus Wohnen, Einzelhandel und Kleingewerbe aus. Hinsichtlich ihrer ethnischen Zusammensetzung unterscheiden sich diese Stadtteile in je einen

- Stadtteil mit einem hohen Anteil nichtdeutscher Bewohner und relativ homogener Zusammensetzung der ansässigen Bewohner mit Migrationshintergrund (Duisburg: Marxloh, Essen: Altendorf, Bochum: Hamme, Dortmund: Nordstadt; Typ 1).

- Stadtteil mit mittlerem Anteil nichtdeutscher Bewohner und eher heterogener Zusammensetzung (Duisburg: Innenstadt, Essen: Rüttenscheid, Bochum: Langendreer, Dortmund: Hörde; Typ 2).

Zusätzlich wurden folgende infrastrukturelle Einrichtungen berücksichtigt: Hauptbahnhof, Rathaus, Kultureinrichtung (z. B. Dortmunder U-Turm) und eine Kita pro Stadtteil. Die insgesamt 25.504 Bilddaten wurden in eine Bilddatenbank importiert und verschlagwortet (vgl. Mühlan-Meyer \& Lützenkirchen 2017).

$4 \mathrm{Zu}$ den Ergebnissen der mehrsprachigen Telefonbefragung vgl. Ziegler, Schmitz \& Uslucan (i. Dr.). 
Im Folgenden werden die Ergebnisse der quantitativen Analysen der Bilddaten vorgestellt (Abschnitt 3), danach werden die Ergebnisse der Vor-OrtBefragungen der Rezipienten (Abschnitt 4) und die Ergebnisse der Produzentenbefragung präsentiert (Abschnitt 5). Abschließend werden die zentralen Ergebnisse in einer integrierten Analyse zusammengeführt und unter der Frage der monolingualen bzw. multilingualen Normorientierung diskutiert (Abschnitt 6).

\section{Der öffentliche Raum der Metropole Ruhr: eher mono- oder multilingual betextet?}

Die Auswertung aller Sprachvorkommen in der Linguistic Landscape der Metropole Ruhr fasst Tabelle 5.1 zusammen. Berücksichtigt werden die 15 häufigsten Sprachen.

Tab. 5.1: Sichtbarbeit der 15 häufigsten Sprachen und Varietäten in der Metropole Ruhr.

\begin{tabular}{|c|c|c|c|}
\hline & Sprache & Häufigkeit & $\%$ \\
\hline 1 & Deutsch & 18.053 & $66.2 \%$ \\
\hline 2 & Englisch & 5.483 & $20.1 \%$ \\
\hline 3 & Türkisch & 1.122 & $4.1 \%$ \\
\hline 4 & Französisch & 429 & $1.6 \%$ \\
\hline 5 & Italienisch & 379 & $1.4 \%$ \\
\hline 6 & Spanisch & 286 & $1.0 \%$ \\
\hline 7 & Arabisch & 185 & $0.7 \%$ \\
\hline 8 & Latein ${ }^{5}$ & 157 & $0.6 \%$ \\
\hline 9 & Polnisch & 143 & $0.5 \%$ \\
\hline 10 & Deutscher Nonstandard $^{6}$ & 111 & $0.4 \%$ \\
\hline 11 & Niederländisch & 100 & $0.4 \%$ \\
\hline 12 & Chinesisch & 77 & $0.3 \%$ \\
\hline 13 & Japanisch & 76 & $0.3 \%$ \\
\hline 14 & Russisch & 64 & $0.2 \%$ \\
\hline 15 & Griechisch & 53 & $0.2 \%$ \\
\hline
\end{tabular}

5 Latein wird insbesondere im Kontext von Apotheken, Pflegediensten und religiösen Einrichtungen verwendet.

6 Das Ruhrdeutsche ist nicht als eigene Sprache in der Verschlagwortung erfasst, es wurde unter das Label Nonstandard subsumiert. Nonstandard umfasst alle nicht standardsprachlichen deutschen Sprachvorkommen, insbesondere umgangs- und regionalsprachliche Formen. 
Die Auswertung aller Bilddaten für das Ruhrgebiet zeigt, dass zwei Drittel aller Belege auf das Deutsche entfallen (66,1\%), gefolgt vom Englischen (20,1\%) und mit großem Abstand vom Türkischen (4,1\%). Französisch, Italienisch und Spanisch belegen zusammen weitere 4 Prozent aller sichtbaren Texte. Die Sprachen Arabisch, Polnisch, Niederländisch und Chinesisch und alle weiteren in der Datenbank erfassten Sprachen haben Anteile, die alle unter einem Prozent liegen und damit in der Gesamtschau vernachlässigbar sind. Für das Polnische ist dieses Ergebnis bemerkenswert. Immerhin stellt das Polnische die älteste Migrantensprache im Ruhrgebiet dar, und in vielen Stadtteilen bilden die Polen oft die zweitgrößte Gruppe an Nichtdeutschen. Die Vermutung liegt hier nahe, dass diese Gruppe stärker integriert ist und sich an den Werten der Mehrheitsgesellschaft orientiert. Wenig präsent sind auch nonstandardsprachliche Formen des Deutschen. Von den insgesamt 111 Belegen entfallen 45 Belege auf das Ruhrdeutsche, das damit weniger als 0,2\% aller Sprachvorkommen ausmacht (vgl. Ziegler, Eickmans \& Schmitz 2017).

Mit Blick auf die Frage, wie mehr- oder einsprachig das Ruhrgebiet ist, zeigen die Daten aufs Ganze gesehen, dass die Linguistic Landscape der Metropole Ruhr zwar mehrheitlich eine deutsche Sprachlandschaft ist, andere Sprachen als Deutsch aber zu gut einem Drittel die Betextung des öffentlichen Raums prägen. Ein detaillierterer Blick gibt allerdings zu erkennen, dass die Anteile des Deutschen und damit auch die der anderen Sprachen in den untersuchten Stadtteilen variieren, vgl. Karte 5.1.

Insgesamt verdeutlichen die Punktsymbole und ihre unterschiedliche Größe, dass eine größere Diversität in der Bevölkerung sich auch in größerer sprachlicher Diversität widerspiegelt. Gleichzeitig zeigt die Karte, dass das Vorkommen von Deutsch in den nördlich der A 40 gelegenen Stadtteilen Duisburg-Marxloh, Essen-Altendorf und Dortmund-Nordstadt geringer ist als in Bochum-Hamme. Für die Migrantensprachen gibt die quantitative Auswertung zu erkennen, dass Türkisch in Duisburg-Marxloh mit 25,9\% am häufigsten vorkommt. Dieser hohe Anteil lässt sich damit erklären, dass knapp $45 \%$ der nichtdeutschen Bevölkerung eine türkische Staatsangehörigkeit besitzt, der Stadtteil also stark türkisch geprägt ist. Eine weitere Besonderheit von Duisburg-Marxloh ist die Spezialisierung des Einzelhandels auf Brautmode von türkischen Herstellern, die weit über die Stadtgrenzen hinaus Käuferinnen und Käufer anzieht. In Essen-Altendorf und Dortmund-Nordstadt beträgt der Anteil des Türkischen dagegen nur knapp 7\%, ist damit aber immer noch deutlich höher als in den südlich der A 40 gelegenen Stadtteilen Duisburg-Innenstadt (3,1\%), BochumLangendreer (0,9\%) und Dortmund-Hörde (0,9\%). Arabisch, das mit 185 Vorkommen Rang 7 einnimmt (vgl. Tab. 5.1), ist besonders in Essen-Altendorf und in Dortmund-Nordstadt sichtbar. In beiden Stadtteilen erklärt sich das Vorkom- 


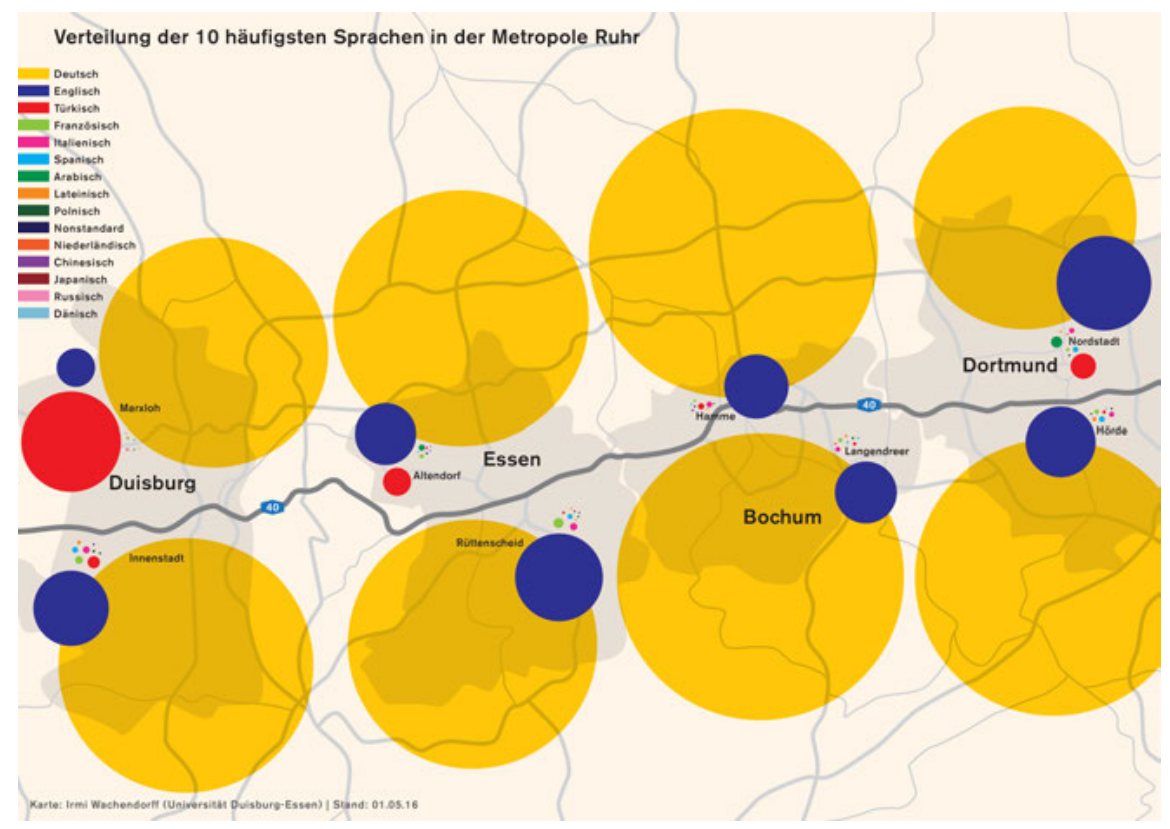

Karte 5.1: Verteilung von visueller Mehrsprachigkeit in der Metropole Ruhr differenziert nach Stadtteilen. ${ }^{7}$

men von Arabisch aus der Bewohnerstruktur. So stellen Stadtteilbewohner mit einer irakischen Nationalität die viertgrößte Zuwanderergruppe in Essen-Altendorf dar. Ähnliches gilt für Dortmund-Nordstadt, wo viele Stadtteilbewohner eine irakische, marokkanische oder libanesische Staatsangehörigkeit besitzen.

In einem nächsten Schritt wurde untersucht, inwieweit sich die Orientierung an einer mono- bzw. multilingualen Norm bei den privaten und offiziellen Akteuren unterscheidet. Die Sprachwahlen auf den offiziellen, d.h. infrastrukturellen und regulatorischen Schildern zeigen, dass $90 \%$ der 2.745 Fotodaten monolingual Deutsch sind. Nur 7,7\% der offiziellen Schilder sind bilingual, weisen also zwei Sprachen auf. Alle zweisprachigen Schilder enthalten Deutsch und werden zu 89,3\% mit Englisch kombiniert, gefolgt von den Sprachkombinationen Deutsch-Türkisch (3,7\%), Deutsch-Französisch (2,1\%) und Deutsch-Arabisch (1,1\%). Auch alle offiziellen dreisprachigen Schilder weisen immer Deutsch auf. Die häufigste Kombination bei den insgesamt 43 im Korpus enthaltenen trilingualen offiziellen Schildern (1,8\%) ist Deutsch-

7 Für die Erstellung der Karte danke ich Irmi Wachendorff/wissenschaftliche Hilfskraft im Projekt „Metropolenzeichen“ an der Universität Duisburg-Essen. 
Englisch-Französisch. Die meisten dieser Schilder befinden sich an Hauptbahnhöfen, wo diese Sprachkombination die Regel ist, vgl. Abbildung 5.1:

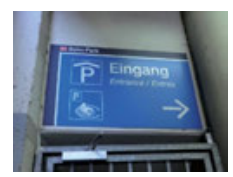

Abb. 5.1: Bildnummer 25021.

Die seltenere Sprachkombination Deutsch-Türkisch-Russisch findet sich auf einer Beschriftung auf einer Mülltonne, die darauf hinweist, dass sie zur Entsorgung von Papier und Pappe dient (Abb. 5.2):

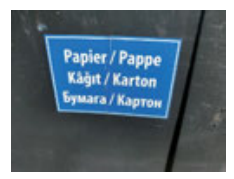

Abb. 5.2: Bildnummer 12128 .

Nur 0,5\% der offiziellen Schilder weisen vier oder mehr Sprachen auf. Diese Schilder enthalten immer Deutsch und Englisch kombiniert mit den Sprachen Französisch (6x), Türkisch (5x), Italienisch (4x), Polnisch (4x), Niederländisch (3x), Russisch (2x) oder Spanisch (1x).

Die Tabelle 5.2 gibt die Gesamthäufigkeiten der Sprachen auf offiziellen Schildern an. Sie zeigt, dass Englisch und Französisch die am häufigsten verwendeten Fremdsprachen sind und die Migrantensprachen mit Werten unter $1 \%$ so gut wie gar nicht vorkommen.

Wie sieht die Sprachenwahl bei nicht-offiziellen, d. h. kommerziellen Schildern aus? Ausgewertet wurden 12.563 Bilddaten. Im Gegensatz zu den offiziellen Schildern sind im kommerziellen Bereich lediglich $71 \%$ der Schilder monolingual, davon $91 \%$ monolingual Deutsch. Bei den übrigen $9 \%$ monolingualen Schildern dominieren Schilder in englischer, türkischer, französischer, italienischer und arabischer Sprache. Die Bandbreite der Sprachen ist bei den kommerziellen Schildern wesentlich höher als bei den offiziellen. Besonders deutlich zeigt sich diese Tendenz bei Aushängen mit dem Hinweis für potenzielle Einbrecher, dass der Geldtresor speziell gesichert sei, ein Einbruch daher sinnlos 
Tab. 5.2: Häufigkeit von Sprachen auf offiziellen Zeichen.

\begin{tabular}{lcr}
\hline Sprache & Zeichenanzahl & \multicolumn{1}{l}{$\%^{8}$} \\
\hline Deutsch & 2.471 & $99,8 \%$ \\
Englisch & 217 & $8,7 \%$ \\
Französisch & 45 & $1,8 \%$ \\
Türkisch & 14 & $0,5 \%$ \\
Italienisch & 8 & $0,3 \%$ \\
Niederländisch & 6 & $0,2 \%$ \\
Russisch & 5 & $0,2 \%$ \\
Sonstige $^{9}$ & 17 & $0,6 \%$ \\
\hline
\end{tabular}

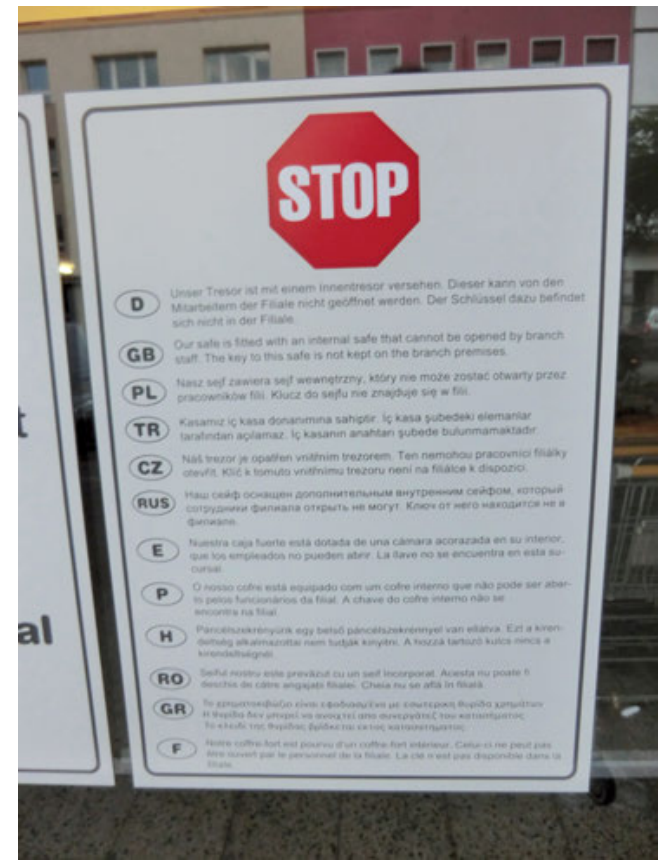

Abb. 5.3: Bildnummer 5005.

sei. Diese Hinweise sind häufig in einer Vielzahl von Sprachen verfasst, wobei der Anteil der Migrantensprachen überproportional hoch ist (vgl. Abb. 5.3).

8 Insgesamt über $100 \%$ aufgrund von Mehrfachvorkommen (mehrsprachige Zeichen).

9 Sonstige: Arabisch (3), Spanisch (3), Koreanisch (3), Latein (2), Nonstandard (1), Portugiesisch (1), Tschechisch (1), Dänisch (1), Schwedisch (1), Sprache unklar (1). 
Insgesamt sind 2.991 (24\%) der kommerziellen Schilder bilingual. Davon enthalten 2.865 Schilder Sprachkombinationen mit Deutsch, 1.714 Schilder Sprachkombinationen mit Englisch, 506 Schilder Sprachkombinationen mit Türkisch, 146 Sprachkombinationen mit Italienisch, 119 Sprachkombinationen mit Französisch, 93 Schilder Sprachkombinationen mit Arabisch, 89 Sprachkombinationen mit Latein, 77 Schilder Sprachkombinationen mit Polnisch und 61 Schilder Sprachkombinationen mit Spanisch, 34 Schilder Sprachkombinationen mit Niederländisch, um die zehn häufigsten Sprachenpaare zu nennen.

Zusammenfassend lässt sich festhalten, dass für offizielle Schilder, Aushänge und Hinweistafeln mehrheitlich die Devise „German only“ gilt. Wenn doch eine andere Sprache als Deutsch verwendet wird, dann in der Regel Englisch, das auch auf nicht-offiziellen Schildern die am häufigsten verwendete Sprache nach Deutsch ist. Im kommerziellen Diskurs ist die Sprachenvielfalt wie zu erwarten - größer. Dabei ist die Migrantensprache, die am häufigsten zusammen mit einer anderen Sprache verwendet wird, das Türkische, das auch insgesamt unter den Migrantensprachen in der Linguistic Landscape der Metropole Ruhr dominiert.

\section{Wahrnehmung und Bewertung der Linguistic Landscape der Metropole Ruhr}

Aktuelle Studien in der Spracheinstellungsforschung betonen die Bedeutung interaktionaler, gesprächsorientierter Zugänge, um einen detaillierten Einblick in die Äußerung von Spracheinstellungen zu erhalten (Tophinke \& Ziegler 2006, 2014; Liebscher \& Dailey O’Cain 2009; König 2014; Bellamy 2016). Dementsprechend wurden für die Analyse der Wahrnehmung und Bewertung visueller Mehrsprachigkeit Vor-Ort-Interviews durchgeführt, auch um eine maximale Gegenstandsnähe zu erzielen. ${ }^{10}$

Die Vor-Ort-Interviews wurden in allen acht Stadtteilen durchgeführt. ${ }^{11}$ In jedem Stadtteil wurden 15 Informanten befragt, sodass insgesamt 120 Vor-Ort-

10 Im Rahmen der Linguistic Landscape-Forschung liegen folgende Untersuchungen zu Spracheinstellungen vor, die in der Regel inhaltsanalytisch orientiert sind: Landry \& Bourhis (1997) und Gillinger et al. (2012) zur Vitalität von Sprachen; Collins \& Slembrouck (2007) zur Bewertung der Sprachkompetenz der Textproduzenten; Aiestaran et al. (2010), Garvin (2010) und Trumper-Hecht (2010) zur Bewertung des pragmatischen, symbolischen, ökonomischen und ästhetischen Wertes von Sprachen.

11 Die Vor-Ort-Interviews wurden von den studentischen Hilfskräften Nilgün Aykut, Sebastian Opara, David Passig, Michael Wentker und der Praktikantin Yvette Rode durchgeführt. 
Interviews vorliegen. Die Länge der Interviews variiert zwischen 3 und $12 \mathrm{Minu-}$ ten. Befragt wurden 65 Männer und 55 Frauen im Alter zwischen 18 und 80 Jahren, davon 49 Personen mit und 71 ohne Migrationshintergrund. Die Interviews wurden auf der Basis eines Interviewleitfadens durchgeführt, der die folgenden Themenblöcke enthält: Wahrnehmung von visueller Mehrsprachigkeit, Stadtteilgeschichte, Bewertung von visueller und gesprochener Mehrsprachigkeit, Funktionen von Mehrsprachigkeit, visuelle Mehrsprachigkeit an öffentlichen Institutionen, Vorzüge und Nachteile von Mehrsprachigkeit. Die Interviews erlaubten aber auch Flexibilität und spontane Anpassung an die Antworten der Befragten. Die Audiodaten der Interviews wurden nach GAT 2 (Selting et al. 2009) als Minimaltranskripte mit dem Partitur-Editor EXMARaLDA transkribiert und anschließend für die Auswertung annotiert.

Die Gesamtheit der Antworten der Befragten (mit/ohne Migrationshintergrund; nördliche/südliche Stadtteile) lassen sich differenziert nach positiver, negativer oder neutraler/unentschlossener Einstellung gegenüber visueller Mehrsprachigkeit wie folgt zusammenfassen, vgl. Tabelle 5.3:

Tab. 5.3: Einstellung der Befragten gegenüber visueller Mehrsprachigkeit differenziert nach nördlichen und südlichen Stadtteilen sowie Migrationshintergrund (MH).

\begin{tabular}{lllllll}
\hline $\boldsymbol{n = 1 2 0}$ & positiv +MH & $\mathbf{- M H}$ & negativ $+\mathbf{M H}$ & $\mathbf{- M H}$ & neutral +MH & $-\mathbf{M H}$ \\
\hline Norden & $66 \%$ & $59 \%$ & $23 \%$ & $23 \%$ & $11 \%$ & $18 \%$ \\
Süden & $56 \%$ & $58 \%$ & $35 \%$ & $36 \%$ & $9 \%$ & $6 \%$ \\
\hline
\end{tabular}

Deutlich wird, dass die überwiegende Mehrheit der Befragten eine positive Einstellung gegenüber visueller Mehrsprachigkeit hat. Ein detaillierter Blick zeigt jedoch, dass sich die Einstellungsprofile der Informanten entlang der NordSüd-Unterteilung unterscheiden und ein komplexes Bild $\mathrm{zu}$ erkennen geben. Während die Befragten mit Migrationshintergrund in den nördlichen Stadtteilen deutlich positiver gegenüber visueller Mehrsprachigkeit eingestellt sind (66\%) als die Befragten ohne Migrationshintergrund (59\%) und auch weniger häufig angeben, keine dezidierte Einstellung $\mathrm{zu}$ diesem Thema $\mathrm{zu}$ haben (11\%), zeigen die Einstellungsprofile der Befragten mit und ohne Migrationshintergrund in den südlichen Stadtteilen eine große Übereinstimmung, wobei die positive Einstellung der Befragten mit Migrationshintergrund um 10 Prozentpunkte niedriger liegt als die ihrer entsprechenden Vergleichsgruppe im Norden. Das bedeutet, dass der Faktor Migrationshintergrund nur bei den Befragten in den nördlichen Stadtteilen einstellungsrelevant ist, bei den Befragten in den Stadtteilen südlich des „Sozialäquators A 40“ dagegen keine Rolle 
spielt. Oder anders formuliert: Arrivierte Migranten nehmen den Habitus der monolingual Deutschen an und äußern im gleichen Maße wie diese Skepsis und Bedenken gegenüber visueller Mehrsprachigkeit.

Wie begründen die Befragten ihre positive oder negative Einstellung? Welche Argumentationsmuster lassen sich erkennen? Die Auswertung der Antworten auf die Frage: „Was halten Sie von mehrsprachigen Schildern an öffentlichen Institutionen (z. B. Rathäusern, Bürgerbüros, Krankenhäusern oder Kindergärten) und die Anschlussfrage: „Welche Sprachen sollten auf mehrsprachigen Schildern an Institutionen sichtbar sein?" sind hier aufschlussreich. So antworteten $63 \%$ der Befragten, dass sie diese Idee „gut finden“, $26 \%$ der Befragten antworteten, dass sie diese Idee ablehnen und $11 \%$, dass sie unentschieden sind. Dabei zeigt sich auch hier, dass die Befragten nördlich des „Sozialäquators A 40“ häufiger eine positive Antwort gaben (73\%) als südlich des Sozialäquators (53\%), wo auch der Diversitätsindex niedriger ist.

Interessante Unterschiede zeigen sich darüber hinaus, wenn man die Argumentationsmuster (vgl. Spitzmüller \& Warnke 2011) untersucht, mit denen für oder gegen visuelle Mehrsprachigkeit im öffentlichen Raum plädiert wird. Für diesen Analyseschritt wurden die in den Interviews geäußerten Argumente zu abstrakteren Argumentationsmustern zusammengefasst. Folgende neun Argumentationsmuster konnten so identifiziert werden:

\section{Funktional-pragmatisches Argument}

Dieses Argument beruht auf der Strategie, auf die Konsequenzen und Ziele, d. h. den Gebrauch und die Funktion von sichtbarer Mehrsprachigkeit zu verweisen. Zentral sind hier Aspekte wie Verständlichkeit, barrierefreie Kommunikation, Information und Orientierung im öffentlichen Raum.

\section{Argument aus der eigenen Erfahrung}

Dieses Argument basiert auf dem Rückbezug auf eigene Erfahrungen in ähnlichen Situationen. Dabei kann sowohl auf individuelle Erfahrungen Bezug genommen werden (,ich“) als auch auf kollektive Erfahrungen („wir“), um auf die eigene soziale Gruppe oder die Mehrheitsgesellschaft zu verweisen oder die Plausibilität der geäußerten Einstellung zu erhöhen. Der Bezug auf solche schon selbst erlebten Situationen soll die Glaubwürdigkeit der eigenen Einstellung verstärken.

\section{Argument der Faktizität}

Das Argument der Faktizität stützt sich auf Aussagen, die keine Möglichkeiten für einen anderen Standpunkt zulassen, sondern die Gegebenheiten als unabänderliche Fakten darstellen. Eine explizite Bewertung wird dadurch vermieden. 


\section{Argument der Beheimatung}

Das Argument der Beheimatung betrachtet die öffentliche Sichtbarkeit von Herkunftssprachen als Symbol für Beheimatung. Damit wird eine emotionale Verbindung mit einem bestimmten Stadtteil, einer bestimmten Stadt oder Region ausgedrückt. Das Beheimatungsargument zeigt eine Beziehung zwischen Ort und Identität dadurch an, dass Orten und ihrer (hier: sprachlichen) Ausgestaltung eine affektive Bedeutung zugeschrieben wird.

\section{Argument der Multikulturalität}

Mit dem Argument der Multikulturalität weisen die Befragten auf die vielen verschiedenen Kulturen, Traditionen und Lebensstile im Stadtviertel hin, die durch mehrsprachige Schilder sichtbar werden können.

\section{Normatives Argument}

Mit dem normativen Argument erheben die Informantinnen und Informanten bestimmte Forderungen und Rechte im Zusammenhang mit Mehrsprachigkeit. Sprachlich werden diese häufig durch die Modalverben „sollen“ oder „müssen“ ausgedrückt.

\section{Integrationsargument}

Das Integrationsargument besagt, dass Mehrsprachigkeit, insbesondere der Gebrauch von Herkunftssprachen im öffentlichen Raum, entweder ein Hinderungsgrund oder aber eine Hilfe für erfolgreiche Integration sein kann.

\section{Affektives Argument}

Affektive Argumente sind solche Argumente, die subjektive Empfindungen und Gefühle gegenüber visueller Mehrsprachigkeit zum Ausdruck bringen. Dafür werden häufig Gefühlsverben wie beispielsweise „fühlen“ verwendet.

\section{Ökonomisches Argument}

Das ökonomische Argument weist auf die Wirtschaftlichkeit und Rentabilität von mehrsprachigen Schildern hin bzw. auf das Gegenteil.

Tabelle 5.4 zeigt die Argumente, die für die Befürwortung von visueller Mehrsprachigkeit am häufigsten gebraucht werden, und zwar differenziert nach Befragten mit und ohne Migrationshintergrund ( $\mathrm{MH})$ : 
Tab. 5.4: Häufigkeit der Argumentationsmuster pro visuelle Mehrsprachigkeit. ${ }^{12}$

\begin{tabular}{lrr}
\hline Argumentationsmuster & - MH & +MH \\
\hline Pragmatisches Argument & $65 \%(81)$ & $50 \%(58)$ \\
Argument aus der eigenen Erfahrung & $8 \%(10)$ & $10 \%(11)$ \\
Faktizitätsargument & $7 \% \quad(9)$ & \\
Argument der Beheimatung & $3 \% \quad(3)$ & $17 \%(20)$ \\
Normatives Argument & & $3 \% \quad(4)$ \\
\hline
\end{tabular}

Die favorisierten Argumentationsmuster geben sowohl Gemeinsamkeiten als auch Unterschiede zwischen den beiden Untersuchungsgruppen zu erkennen. So dominiert in beiden Untersuchungsgruppen das pragmatische Argumentationsmuster mit dem Hinweis auf Aspekte wie Orientierung, Verständigung und Abbau von Sprachbarrieren, wobei die Befragten ohne Migrationshintergrund dieses Argument etwas häufiger verwenden als die Befragten mit Migrationshintergrund.

\section{Transkriptausschnitt 1}

das (.) find ich ganz gut (--)| ja (0.5)| ja für leute die jetzt neu nach deutschland gekommen sind| dann können die sich vielleicht besser (-) orientieren (0.6)| (DoHoe10)

\section{Transkriptausschnitt 2}

würde ja schon barrierefreiheiten ein bisschen ermöglichen| gerade für ausländische mitbürger vielleicht (BoLan4)

\section{Transkriptausschnitt 3}

türk ö almanca bilmeyenler için mesela| ((...)) bizim ihtiyarlar yani mesela bilmiyorlar almancayı (-) coğu|annelerimiz babalarımız bilmiyorlar| onlar için aslında çok iyi olur| (DueMar6)

türkisch ist gut für die, die kein deutsch können, zum beispiel ((...)) unsere alten leute können zum beispiel kein deutsch meistens, unsere mütter und väter können nicht/ für die wäre es eigentlich ganz gut

Insgesamt zeigt sich, dass die Befragten ohne Migrationshintergrund das Bedürfnis von Migrantinnen und Migranten nach Orientierung und Information anerkennen, damit diese im öffentlichen Raum handlungs- und orientierungsfähig

12 Die Tabelle listet nur die vier häufigsten Argumentationsmuster, da die Werte für die anderen Argumentationsmuster zu niedrig sind und daher vernachlässigt werden. 
sind. Die Daten geben außerdem geschlechtsspezifische Unterschiede zu erkennen. Während $76 \%$ der Frauen ihre positive Einstellung mit einem funktionalpragmatischen Argument begründen, sind dies bei den Männern nur $58 \%$. Diese Tendenz lässt sich auch bei den Befragten mit Migrationshintergrund beobachten. Auch hier greifen mehr Frauen (83\%) als Männer (46\%) auf ein funktional-pragmatisches Argument zurück, um ihre positive Einstellung gegenüber visueller Mehrsprachigkeit zu begründen.

Am zweithäufigsten wird von den Befragten mit Migrationshintergrund das Argument der Beheimatung gebraucht. Interessant ist, dass dieses Argument mehrheitlich in den Interviews genannt wird, die auf Türkisch geführt wurden. Aus einer anerkennungstheoretischen Perspektive lässt sich dies so deuten, dass sich diese Informanten in ihrer Besonderheit, eine andere Sprache als Deutsch zu sprechen, wahrgenommen und anerkannt fühlen. Dies wird umso deutlicher, wenn die Befragten zur Versprachlichung ihrer Argumentation auf ihre Herkunftssprache zurückgreifen, d. h. Türkisch wählen. Eine weitere Verstärkung erfährt dieses Antwortverhalten dadurch, dass sich die Befragten häufig eines Vergleichs bedienen, d. h. die Erfahrung der Beheimatung an ihre alte Heimat, die Türkei, binden und so das Spannungsverhältnis zwischen alter und neuer Heimat markieren.

Transkriptausschnitt 4

ne hissediyorum| (0.5) türkiye gibi geliyor| (DueMar15)

Was ich empfinde/ als ob ich in der türkei wäre

Transkriptausschnitt 5

şimdi türkçe gördüğüm bir sokakta| (0.7)| kendimi türkiyede gibi

hissediyorum| (DoNor3)

wenn ich in einer straße türkisch sehe, dann fühle ich mich wie in der türkei

Transkriptausschnitt 6

ist ein schönes gefühl| so man sieht man| ich äh fühle mich so wie in (-)

äh meine heimat| (BoLan5)

Am häufigsten verwenden die Befragten mittleren Alters (30-60 Jahre) das Argument der Beheimatung (14 Nennungen), gefolgt von den Jüngeren (1830 Jahre), die dieses Argument sechsmal nennen. Von den Älteren (über 60 Jahre) wird dieses Argument so gut wie gar nicht verwendet, nur ein Informant beruft sich auf dieses Argument. Offensichtlich verbindet diese Altersgruppe Gefühle der Beheimatung nicht mit der öffentlichen Sichtbarkeit ihrer Herkunftssprache - oder sie äußert diesen Zusammenhang nicht. 
Wie argumentieren die Befragten, die sich gegen mehrsprachige Schilder im Kontext von Bahnhöfen, Rathäusern, Museen und Kindergärten aussprechen? Tabelle 5.5 zeigt die Argumente, die für die Ablehnung von visueller Mehrsprachigkeit am häufigsten gebraucht wurden, und zwar differenziert nach Befragten mit und ohne Migrationshintergrund:

Tab. 5.5: Häufigkeit der Argumentationsmuster contra visuelle Mehrsprachigkeit.

\begin{tabular}{|c|c|c|}
\hline Argumentationsmuster & $-\mathrm{MH}$ & $+\mathrm{MH}$ \\
\hline Normatives Argument & $29 \%(23)$ & $32 \%(21)$ \\
\hline Pragmatisches Argument & $23 \%(18)$ & $23 \%(15)$ \\
\hline Integrationsargument & $12 \% \quad(9)$ & \\
\hline Argument aus der eigenen Erfahrung & $9 \% \quad(7)$ & \\
\hline Affektives Argument & & $14 \% \quad(9)$ \\
\hline Ökonomisches Argument & & $9 \% \quad(6)$ \\
\hline
\end{tabular}

Deutlich wird, dass in beiden Untersuchungsgruppen normative und pragmatische Argumente bei der Ablehnung visueller Mehrsprachigkeit überwiegen. Viele Befragte ohne Migrationshintergrund, die sich auf das normative Argument stützen, indizieren ihre Einstellung auf der sprachlichen Ebene durch die Verwendung des Modalverbs „sollen“ und deuten damit an, dass sie den Erwerb des Deutschen als eine Bringschuld der Migratinnen und Migranten verstehen. Es wird gefordert, dass Migranten der „deutschen Sprache mächtig sein sollten“. Zum Teil wird diese Forderung auch als Bedingung für bestimmte Rechte formuliert. Nimmt man das Integrationsargument hinzu und betrachtet es auch als ein im weiteren Sinne normatives Argument (im Sinne einer Sollund Ziel-Erwartung), dann wird deutlich, wie stark die Forderung nach sprachlicher Integration in der Mehrheitsgesellschaft ausgeprägt ist, vgl. die folgenden Transkriptausschnitte:

Transkriptausschnitt 7

da halte ich eigentlich gar nichts von| ich denke wenn man in deutschland lebt| sollte man auch der deutschen sprache mächtig sein| (-) nel ((...)) (BoLan11)

\section{Transkriptausschnitt 8}

wir leben hier in deutschland| entweder lernen sie deutsch| auch lesen (---)| oder sie gehen wieder (0.8)| (BoLan14) 
Auch viele Befragte mit Migrationshintergrund untermauern ihre Ablehnung visueller Mehrsprachigkeit mit einem normativen Argument, vgl. die folgenden Transkriptausschnitte:

Transkriptausschnitt 9 halt wir leben in deutschland| so wie gesagt habe| (0.4) halt äh| wir müssen uns ja hier anpassen| (DueMar12)

Transkriptausschnitt 10 wer hier äh $<<$ lachend $>$ nach deutschland kommt $>\mid$ (-) dann soll er (.) deutsch können|(DueDel15)

Darüber hinaus zeigt sich, dass sich die Rangfolgen bei den weiteren Argumentationsmustern deutlich unterscheiden. Während die Befragten ohne Migrationshintergrund auch auf das Argument aus der eigenen Erfahrung zurückgreifen, begründen die Befragten mit Migrationshintergrund ihre ablehnende Haltung gegenüber visueller Mehrsprachigkeit $u$.a. auch mit affektiven und ökonomischen Argumenten.

Transkriptausschnitt 11

finde ich unfair| die werden bevorzugt behandelt die türken (0.7)| (DueMar13)

Transkriptausschnitt 12

ama başka dilde gördüğüm zaman o zaman| (0.5)| kendimi yabancı

hissediyorum| (--) äm| äm| rusca| äm| fransızca ingilizce gördüğüm zaman| (1.0)| ( )| o zaman hiç benimsemiyorum yani| (0.8)| hoşuma gitmiyor| (DoNor3)

aber wenn ich in einer anderen sprache als deutsch sehe dann| ich fühle mich fremd| wie ein ausländer| russisch| wenn ich französisch englisch sehe| dann fühle ich mich gar nicht angesprochen also| ich mag es nicht|

Transkriptausschnitt 13

die höheren betriebskosten| weil die faulen maler oder beschilderer da mehr zeit dann brauchen| ja um diese sachen da anzubringen| (BoHam15)

Unabhängig von der Bewertung und Argumentation stellt sich schließlich die Frage, welche Sprachen auf Schildern von Institutionen sichtbar sein sollten. Die Antworten sind in Tabelle 5.6 zusammengefasst.

Die Tabelle zeigt, dass sich 44,2\% der Befragten für Englisch aussprechen, wenn es darum geht, neben Deutsch noch eine andere Sprache für Beschilderungen an öffentlichen Institutionen zu wählen. Auf Platz 2 folgt Türkisch, das 
Tab. 5.6: Nennungen von sichtbaren Sprachen auf Schildern.

\begin{tabular}{lcc}
\hline Sprache & Anzahl der Nennungen & Anzahl in \% \\
\hline Englisch & 53 & $44,2 \%$ \\
Türkisch & 43 & $35,8 \%$ \\
Französisch & 18 & $15 \%$ \\
Arabisch & 12 & $10 \%$ \\
Spanisch & 8 & $6,7 \%$ \\
Italienisch & 7 & $5,8 \%$ \\
Griechisch & 5 & $4,2 \%$ \\
Polnisch & 5 & $4,2 \%$ \\
Russisch & 5 & $4,2 \%$ \\
Bulgarisch & 4 & $3,3 \%$ \\
Chinesisch & 3 & $2,5 \%$ \\
Sonstige & 15 & $12,5 \%$ \\
\hline
\end{tabular}

35,8\% der Befragten für ein mehrsprachiges Schild wählen würden, danach Französisch (15\%) und Arabisch (10\%). Dieses Ergebnis deutet einerseits auf eine Tendenz zur „Eigengruppenfavorisierung“ hin (vgl. Ziegler, Schmitz \& Uslucan i. Dr.), d.h. dass die Befragten mit Migrationshintergrund ihre eigene Herkunftssprache bevorzugen, weil sie eine positive Wahrnehmung ihrer Herkunftssprache anstreben. Andererseits bildet das Antwortverhalten der Befragten auch die Rolle des Arabischen als lingua franca in der arabischen Welt ab, ein Effekt, der durch die aktuelle Fluchtzuwanderung noch verstärkt wird. Die Tatsache, dass Englisch vor allen anderen Sprachen rangiert, ist ihrem Status als internationale Sprache geschuldet. Die häufigere Nennung des Französischen lässt sich damit erklären, dass Französisch eine Schulfremdsprache ist und darüber hinaus auch ein hohes Prestige genießt. Sind dies Indizien für einen „selektiven Multilingualismus“ (Haarmann 1991'13)? Vergleicht man das Spektrum der genannten Sprachen mit der Diversität der Bevölkerung, lautet die Antwort auf die obige Frage Ja. Damit ist aber noch nicht geklärt, was die zugrundeliegenden Selektionskriterien sind. Diese können durchaus heterogen sein. Ist es z. B. das Kriterium des Kulturprestiges, das Kriterium der Tradition oder der politischen Aktualität, das Kriterium der Macht des Einflusses (z. B. Größe der Bevölkerungsgruppe) oder sind es ökonomische Kriterien? All das ist offen. Zudem stellen die genannten Kriterien keine unabhängigen Variablen dar, sondern können interferieren. Damit zusammenhängend liegt die Vermu-

13 Es ist darauf hinzuweisen, dass der Begriff „selektiver Multilingualismus“ zwar von Haarmann (1991) übernommen wurde, hier aber inhaltlich anders gefüllt wird. 
tung nahe, dass die Gründe für die Nennung wie auch Nichtnennung von einzelnen Sprachen durchaus unterschiedlich sind.

\section{Sprachwahlmotive der Produzenten für die Betextung der Linguistic Landscape der Metropole Ruhr}

Welche Motive sind für die Produzenten von Schildern, Aushängen etc. leitend für die Sprachwahl ${ }^{14}$ Warum entscheiden sich Geschäftsleute, ihre Läden und Restaurants mehrsprachig oder einsprachig zu beschildern? Mit welchen Funktionen werden die jeweiligen Sprachenwahlen verbunden? Befragt ${ }^{15}$ wurden 61 private Agenten, ${ }^{16}$ (43 Männer und 18 Frauen). Das Alter der Befragten variierte zwischen 20 und 60 Jahren. 38 der Befragten hatten einen Migrationshintergrund (in den meisten Fällen einen türkischen Migrationshintergrund). Dies zeigt, dass sich mehr Befragte mit Migrationshintergrund für ein Interview zur Verfügung stellten als Befragte ohne Migrationshintergrund, für die das Thema der Befragung anscheinend weniger interessant und relevant ist. Die Länge der Interviews variierte zwischen 2 Minuten und 30 Minuten. Die Interviews wurden mehrheitlich auf Deutsch geführt (44), 12 Interviews wurden auf Türkisch geführt und 2 Interviews auf Französisch. ${ }^{17}$

Die Ausgangsfrage lautete: „Warum haben Sie sich für ein mehrsprachiges/ einsprachiges Schild entschieden? Warum haben Sie gerade diese Sprache(n) gewählt?“ Die Auswertung zeigt, dass bestimmte Motive besonders häufig genannt wurden. Dies betrifft das funktional-pragmatische Motiv und das sozialsymbolische Motiv. Sie entsprechen den oben in Abschnitt 1 genannten Maximen von Spolsky/Cooper (1991):

- Beschrifte einen Aushang in der Sprache, die du beherrschst (,sign writer's skill condition“, Spolsky \& Cooper 1991: 81).

$14 \mathrm{Zu}$ den wenigen Untersuchungen, die auch die Produzentenseite berücksichtigen, zählen Malinowski (2009); Akindele (2011) und Lou (2016).

15 Die Befragungen wurden von der Projektmitarbeiterin Tirza Mühlan-Meyer, der BAKandidatin Vanessa Angenendt und den Hilfskräften Nilgün Aykut und Michael Wentker durchgeführt.

16 Die Antworten der offiziellen Agenten (z. B. Vertreter kommunaler Einrichtungen) werden hier aus Platzgründen nicht diskutiert.

17 Die Zahl der Interviews ist niedriger als die Zahl der Befragten, da an zwei Interviews mehrere Befragte teilnahmen. 
- Antizipiere die Sprachkompetenz des Adressaten („presumed reader’s condition“, Spolsky \& Cooper 1991: 83).

- Beschrifte einen Aushang in deiner eigenen Sprache oder in der Sprache, mit der du identifiziert werden möchtest („symbolic value condition“, Spolsky \& Cooper 1991: 84).

Diese Motive ergeben in der Summe mehr als 85\% aller Nennungen. Wesentlich seltener wird ein normativ orientiertes Motiv genannt oder die eigene Sprachkompetenz als Motiv für die Sprach(en)wahl angeführt. Da die Befragten häufig nicht nur ein Motiv, sondern mehrere Motive nannten, ist die Summe der Motive der Sprachwahlen höher als die Gesamtzahl der Befragten. Insgesamt zeigt sich, dass 97,4\% der Befragten mit Migrationshintergrund ihr Geschäft, Restaurant etc. mehrsprachig ausschildern, während dies in der Gruppe der Befragten ohne Migrationshintergrund nur für $30 \%$ gilt. Tabelle 5.7 fasst die Ergebnisse der Analyse der Motive für mehrsprachige Beschilderungen zusammen, und zwar für beide Untersuchungsgruppen:

Tab. 5.7: Häufigkeit der von den privaten Produzenten genannten Motive für eine mehrsprachige Beschilderung.

\begin{tabular}{lll}
\hline Motiv & Befragte + MH & Befragte -MH \\
\hline funktional-pragmatisch & $55(50 \%)$ & $4(57 \%)$ \\
sozialsymbolisch & $38(35 \%)$ & $3(43 \%)$ \\
normativ & $13(12 \%)$ & \\
Sprachkompetenz & $3(3 \%)$ & \\
\hline
\end{tabular}

\section{Funktional-pragmatisches Motiv}

Das funktional-pragmatische Motiv wird von den Produzentinnen und Produzenten mehrsprachiger Beschilderung verwendet, um auf die Funktionen der Beschilderung zu verweisen. Im Vordergrund steht dabei das Informationsmanagement. Es gilt die Regel, ein Schild in derjenigen Sprache zu verfassen, von der der Produzent annimmt, dass der potenzielle Kunde sie versteht („presumed reader’s conditon“, Spolsky \& Cooper 1991: 83), wie die folgenden Beispiele (Transkriptausschnitte 14, 15) illustrieren.

Transkriptausschnitt 14

wir haben da die sprachen (--) hm (-) mehr oder weniger den möglichkeiten angepasst die wir haben| und ähm (0.6)| mit englisch (---)| italienisch (-)| kommt man eigentlich sehr weit (--)| in der gastronomie| (EsRue6, +MH) 


\section{Transkriptausschnitt 15}

außerdem|ähm obwohl es wenig ist|haben wir auch deutsche kunden| aus diesem grund haben wir uns auch für die deutsche sprache entschieden| (DueMar1, $+\mathrm{MH}$ )

\section{Sozialsymbolisches Motiv}

Das sozialsymbolische Motiv wird von den Produzentinnen und Produzenten mehrsprachiger Schilder verwendet, um deutlich zu machen, womit sie identifiziert werden möchten und wogegen bzw. gegen wen sie sich abgrenzen möchten. Dazu zählt die Identifikation mit einer bestimmten Sprache, einem bestimmten Herkunftsland, einer Herkunftsregion oder einem Herkunftsort. Darüber hinaus wird das sozialsymbolische Motiv auch genannt, wenn es darum geht, Authentizität zu vermitteln, wie der folgende Transkriptausschnitt illustriert. Befragt wurde ein Italiener, der in der Duisburger Innenstadt eine Kaffeerösterei und ein Café betreibt. Das Geschäft ist in Italienisch und Deutsch beschildert. Die Verwendung des Italienischen hat für den Befragten eine große Bedeutung, da er damit potenziellen Gästen signalisieren kann („nach außen strahlen“; Z. 43), dass in seinem Café „wirklich italiener“ arbeiten (Z. 45) und original italienische Produkte angeboten werden. Dies ist ihm vor allem in Abgrenzung zu jenen Gastronomie-Betrieben wichtig, die sich als „Italiener“ bezeichnen, aber nicht von Italienern betrieben werden (Z. 39-40).

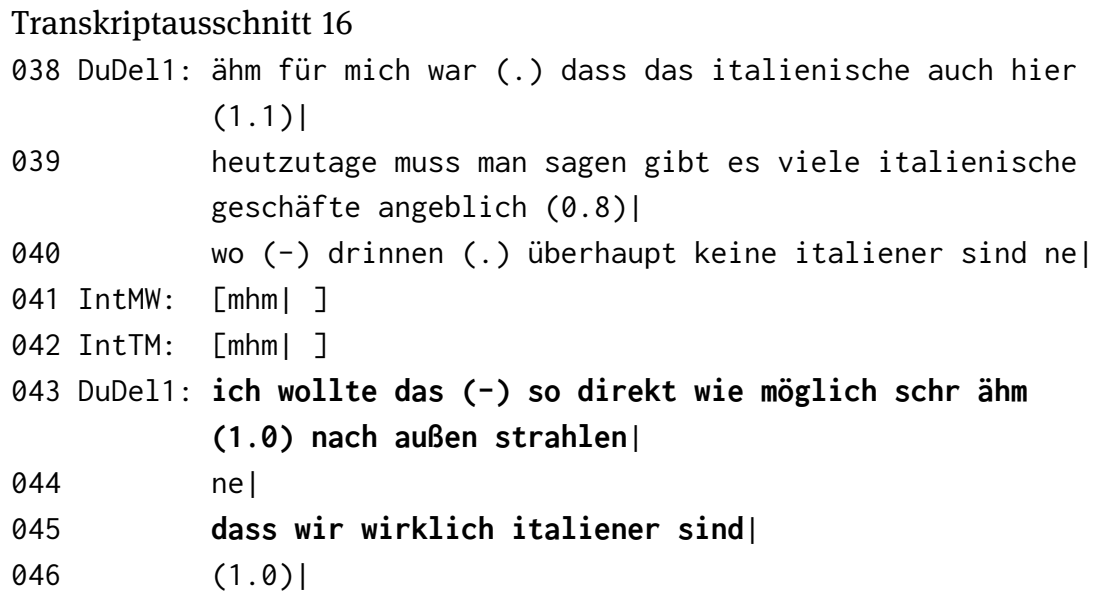

In Beispiel 17 wird dagegen deutlich, dass - insbesondere für die Befragten ohne Migrationshintergrund - die Wahl von anderen Sprachen als Deutsch 
auch aus Prestigegründen erfolgt. In diesen Fällen wird die Fremdsprache, z. B. Französisch oder Englisch, häufig zu emblematischen Zwecken eingesetzt. Das bedeutet, dass die entsprechenden Textteile keine Informationen tragen, sondern für Namen (z. B. Geschäftsnamen, Restaurantnamen), Claims (Werbesprüche) oder auch Ritualia (Begrüßungen, Verabschiedungen) verwendet werden (vgl. Kelly-Holmes 2005; Reershemius 2011). Für diese Sprachpraxis sind keine profunden Sprachkenntnisse erforderlich. Vielmehr geht es darum, das kulturelle Kapital der Sprachen zu nutzen und zu verwerten (,commodification of language“, Cameron 2012) und als Kennzeichen von z. B. Modernität, Weltgewandtheit, Kultiviertheit, Jugendlichkeit, Elitismus oder auch Traditionalität einzusetzen. Wie sehr dabei Produktprestige und Sprachprestige verschmelzen können, zeigt der Auszug aus einem Interview mit einem deutschen Antiquitätenhändler in Essen-Rüttenscheid, der sein Antiquariat in Anlehnung an ein berühmtes Kunstmuseum in Paris benannt hat.

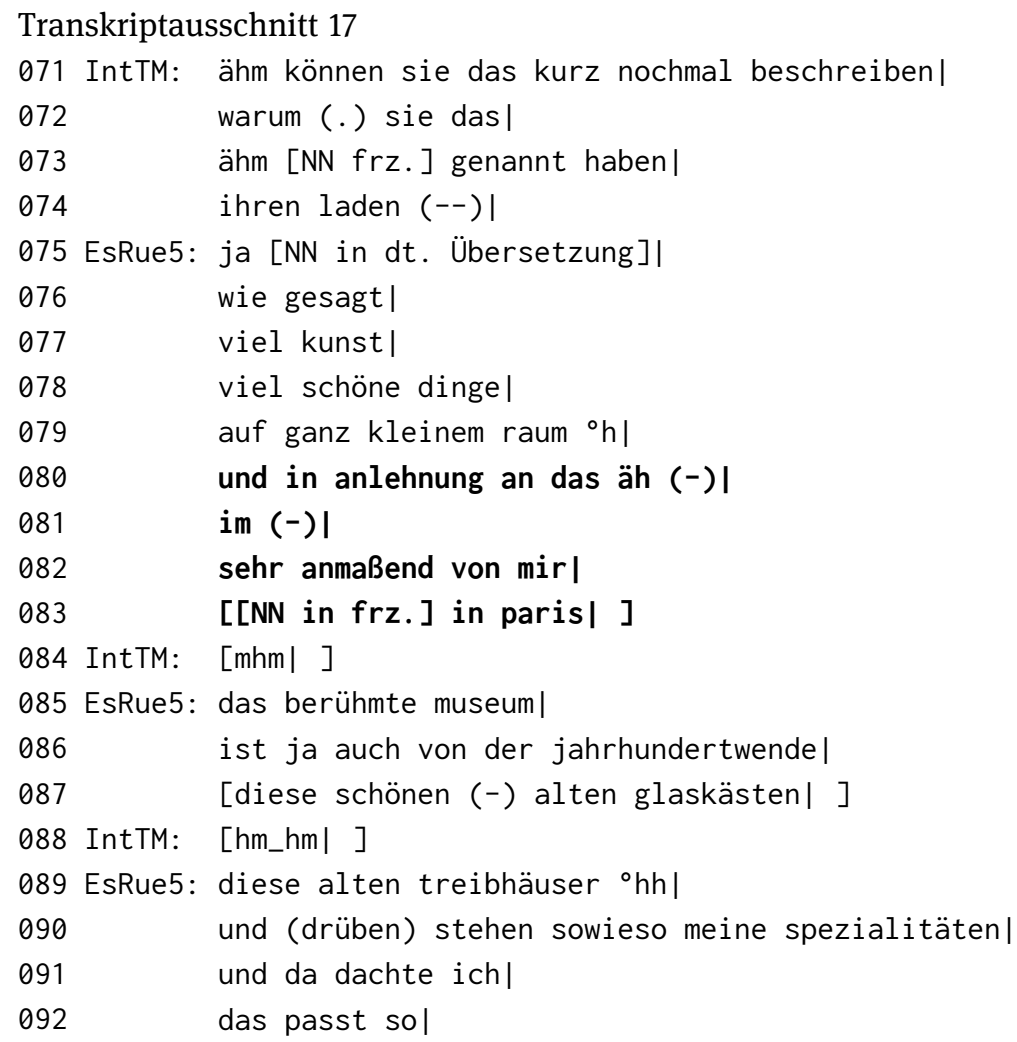




\section{Normatives Sprachwahlmotiv}

Dieses Sprachwahlmotiv wird nur von Befragten mit Migrationshintergrund verwendet. Sie verweisen damit auf faktisch bestehende Normen bzw. gesellschaftliche Erwartungen oder auf epistemische Voraussetzungen. Zur Versprachlichung wird dabei häufig auf die Modalverben „müssen“ und „sollen“ zurückgegriffen, vgl. die folgenden Transkriptausschnitte:

Transkriptausschnitt 18

nein nein nein nein| polnisch und deutsch (--)| deutsch muss alles kennen ja| (EsAlt3)

Transkriptausschnitt 19

(--) ya ama almanyada yaşıyoruz| sonuçta| ähm (.) almanyada (.) şey yaşadığımız için| reklamı mecbur almanca olması lazım| yani türkiyede yaşasan türkçe yazıyorsun| (BoLan4)

ja aber wir leben in deutschland| da wir in deutschland leben| muss die werbung auch auf deutsch sein| also würde man in der türkei leben dann würde man es auf türkisch schreiben| (BoLan4)

\section{Sprachkompetenz als Sprachwahlmotiv}

Während das funktional-pragmatische Motiv die Sprachkompetenzen der Adressaten in den Blick nimmt, bezieht sich das Kompetenzmotiv auf die sprachlichen Fähigkeiten der Produzenten. Mit Spolsky \& Cooper kann davon ausgegangen werden, dass ein Textproduzent generell dazu neigt, ein Schild in der Sprache zu verfassen, die er beherrscht (,sign-writer's skill condition', Spolsky \& Cooper 1991: 81). So antwortet der Inhaber eines türkischen Restaurants auf die Frage, warum er die Sprachen Deutsch, Türkisch und Arabisch zur Beschilderung seines Ladens verwende, dass er diese drei Sprachen „gut sprechen kann“ (Z. 150):

Transkriptausschnitt 20

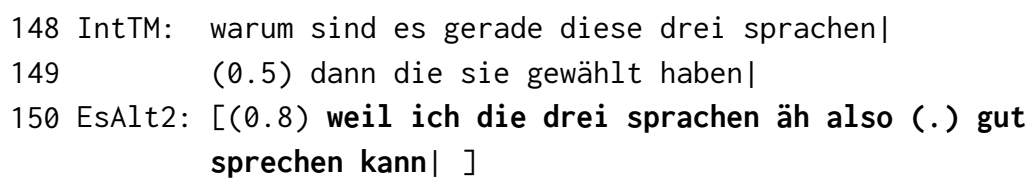

Welche Motive leiten die Befragten, die ihre Geschäfte, Restaurants einsprachig beschildern? Wie erklären sie ihre Ablehnung gegenüber visueller Mehrsprachigkeit? Von den Produzenten mit Migrationshintergrund votierte nur ein einziger Befragter für eine monolinguale Beschilderung. Er nannte dabei sowohl normative als auch funktional-pragmatische Motive, vgl.: 
Transkriptausschnitt 21

008 IntVA: und ähm|

009 wieso gerade deutsch|

010 und nicht noch eine andere sprachel

011 EsRue8: ja weil eben halt hier|

012 IntVA: oder|

013 EsRue8: deutsch gesprochen wird|

...

021 IntVA: und ähm haben sie (-) also hätten sie sich auch vielleicht|

022 also könnten sie sich auch denken dass sie das irgendwie in kroatisch einbinden|

$023 \quad$ oder ${ }^{\circ} \mathrm{h}$ einfach nur deutsch weil|

024 EsRue8: einfach nur deutsch|

025 weil dass ist jal

026 wir sind hier in deutschland|

027 und äh da soll es so bleiben|

028 IntVA: [hm_ml ]

029 EsRue8: [nel ]

030 ich meine was nützt das wenn ich irgendwas auf kroatisch schreibel

031 und kein mensch kann es lesen|

032 außer die paar kroaten|

033 IntVA: hm_ml

034 EsRue8: die hier mal vorbei kommen|

035 äh das ist denke ich mal nicht sinn der sachel

Von den Befragten ohne Migrationshintergrund erklärten dagegen $70 \%$, dass sie ihr Geschäft, Restaurant etc. bewusst einsprachig deutsch beschildern, d. h. mehrsprachige Beschilderungen ablehnen. Am häufigsten wurden dabei funktional-pragmatische Motive genannt (17 Nennungen/74\%), d. h. auf die ausschließlich deutsche Kundschaft und auf externe Vorgaben verwiesen oder die Wahl der Sprachen als schwierig empfunden, vgl.:

Transkriptausschnitt 22

007 DuMar5: also wir werben über (-) ähm (1.1) überwiegend in deutsch|

008 wir haben äh nur deutsche kunden|

$009{ }^{\circ} \mathrm{hh}$ fremdsprachel

010 werben wir nicht| 


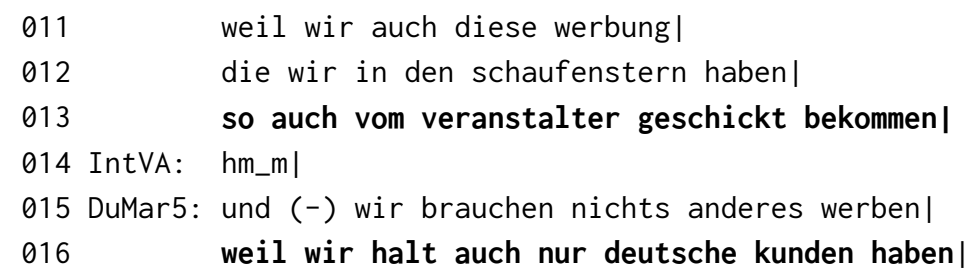

Transkriptausschnitt 23

wie sollte ich mich denn hier präsentieren| in welchen sprachen (0.5)| ich müsste ja hier zwölf sprachen| nach außen me äh äh transportieren (---)| ist ja gar nicht möglich (0.4)| (DueMar3)

Auch normative Motive werden angeführt, allerdings spielen diese mit 5 Nennungen $(21,7 \%)$ eine nur marginale Rolle. Der folgende Interviewausschnitt illustriert diese Motivlage:

Transkriptausschnitt 24

002 IntVA: welche sprachen verwenden sie denn hier|

003 vor allem auf ähm auf schildern|

004 und ähm plakaten|

005 DoNor6: deutsche sprachel

006 Intva: und wieso gerade deutsch|

007 DoNor6: (0.9)।

008 weil ich denkel

009 wir sind nun mal hier in deutschland|

dass wir dann auch die deutsche sprache verwenden müssen|

\section{Fazit}

Wie lassen sich die Ergebnisse der quantitativen Analyse zur Verteilung visueller Mehrsprachigkeit und die Ergebnisse der Adressaten- und Produzentenbefragung im Kontext monolingualer bzw. multilingualer Normorientierung deuten? Die quantitative Analyse der Bilddaten hat gezeigt, dass für die offizielle Beschilderung andere Sprachen als Deutsch kaum eine Rolle spielen, man insofern von einem dominant „monolingualen Habitus“ (Gogolin 2008) sprechen kann. Wenn überhaupt auf andere Sprachen als Deutsch zurückgegriffen wird, dann vorzugsweise auf das Englische, das als lingua franca fungiert. Dagegen sind im kommerziellen Diskurs Migrantensprachen häufiger. Dort, wo 
es um Verbote geht, sind Migrantensprachen sogar überrepräsentiert. Dies deutet auf spezifische Wahrnehmungsstrukturen und verdeckte Vorurteile in der Mehrheitsgesellschaft.

Hier besteht in Anbetracht der aktuellen Migrationssituation Handlungsbedarf für ein nicht-stigmatisierendes Sprachenmanagement, das den ambivalenten Anerkennungsbedürfnissen sowohl nach Besonderheit als auch nach Zugehörigkeit Rechnung trägt, wie die Rezipientenbefragung verdeutlicht. So zeigte sich zum einen, dass die Befragten mehrheitlich ein mehrsprachiges Sprachenmanagement befürworten, insbesondere wenn sie in einem stärker migrationsgeprägten Stadtteil, d.h. nördlich der A 40 leben und einen Migrationshintergrund besitzen. Dabei sind zwei Befunde besonders auffällig: die Eigengruppenfavorisierung und die Einstellung gegenüber dem Arabischen als kommende relevante Migrantensprache. Zum anderen zeigte sich, dass sich die Einstellungen der Befragten ohne und mit Migrationshintergrund in den südlich der A 40 gelegenen Stadtteilen angleichen. Die Befragten mit Migrationshintergrund übernehmen hier die Werte und Normen der Mehrheitsgesellschaft.

Die Ergebnisse der Rezipientenbefragung stehen allerdings in einem interessanten Spannungsverhältnis zu den Ergebnissen, die die Produzentenbefragung zutage gefördert hat. Im Gegensatz zu den Rezipienten sind die Inhaber von Geschäften, Restaurants etc. zurückhaltender gegenüber einer mehrsprachigen Beschilderung eingestellt. Das zeigt vor allen Dingen das Antwortverhalten der befragten Produzenten ohne Migrationshintergrund in den nördlichen Stadtteilen. Diese Befragten lehnen mehrheitlich mehrsprachige Beschilderungen $\mathrm{ab}$, vielleicht weil sie die zunehmende Internationalisierung als Bedrohung empfinden. Inwieweit die genannten funktional-pragmatischen und normativen Motive auch fehlende Fremdsprachenkompetenzen (insbesondere bezogen auf Migrantensprachen) verschleiern sollen (vgl. die „sign writer's skill condition“) und/oder als eine Reaktion auf die zunehmende Diversifizierung der Bevölkerung in diesen Stadtteilen zu deuten ist, müssen Anschlussuntersuchungen zeigen.

Festzuhalten ist daher: Stärker als bisher sollten auch sozialräumliche Faktoren als Makrokontexte für die Ausbildung und Habitualisierung von Einstellungen und Normvorstellungen (vgl. Tophinke \& Ziegler 2006) bei der Untersuchung von Einstellungsäußerungen berücksichtigt werden.

\section{Literatur}

Aiestaran, Jokin, Jasone Cenoz \& Durk Gorter (2010): Multilingual cityscapes: Perceptions and preferences of the inhabitants of the city of Donostia-San Sebastian. In Elana 
Shoamy, Eliezer Ben-Rafael \& Monica Barni (Hrsg.), Linguistic landscape in the city, 219-234. Bristol, Buffalo, Toronto: Multilingual Matters.

Akindele, Dele Olufemi (2011): Linguistic landscape as public communication. A study of public signage in Gaborone Botswana. International Journal of Linguistics. http:// www.macrothink.org/journal/index.php/ijl/article/view/1157/pdf. (letzter Zugriff: 21. 9. 2017).

Bellamy, John (2016): Discussing Ruhrdeutsch: Attitudes towards Spoken German in the Ruhr Region. In Gijsbert Rutten \& and Kristine Horner (Hrsg.), Metalinguistic perspectives on Germanic languages: European case studies from past to present (Historical Sociolinguistics), 185-211. Oxford: Peter Lang.

Cameron, Deborah (2012): The commodification of Language: English as a global commodity. In Terttu Nevalainen \& Elisabeth Closs Traugott (Hrsg.), The Oxford handbook of the history of English, 352-361. Oxford: Oxford University Press.

Cindark, Ibrahim \& Evelyn Ziegler (2016): Mehrsprachigkeit im Ruhrgebiet: Zur Sichtbarkeit sprachlicher Diversität in Dortmund. In Stefaniya Ptashnyk et al. (Hrsg.), Gegenwärtige Sprachkontakte im Kontext der Migration, 133-156. Heidelberg: Winter.

Collins, Jim \& Stef Slembrouck (2007): Reading shop windows in globalized neighborhoods. Multilingual literacy practices and indexicality. Journal of Literacy Research 39 (3), 335-356.

Eickmans, Heinz \& Evelyn Ziegler (2018): Visuelle Mehrsprachigkeit in Dortmund. In Markus Denkler, Dietrich Hartmann \& Heinz Menge (Hrsg.): Dortmund - sprachliche Vielfalt in der Stadt (Niederdeutsche Studien, Bd. 59), 313-339. Köln, Wien: Böhlau.

Garvin, Rebecca T. (2010): Responses to the linguistic landscape in Memphis, Tennessee: An urban space in transition. In: Elana Shohamy, Eliezer Ben-Rafael \& Monica Barni (Hrsg.), Linguistic landscape in the city, 252-271. Bristol: Multilingual Matters.

Gaiser, Leonie \& Jaron Matras (2016): The spatial construction of civic identities: A study of Manchester's linguistic landscapes. http://mlm.humanities.manchester.ac.uk/ wp-content/uploads/2016/12/ManchesterLinguisticLandscapes.pdf (letzter Zugriff: 21. 9. 2017).

Gillinger, Eszter S., Mari Sloboda, Lucija Šimičić \& Dick Vigers (2012): Discourse coalitions for and against minority languages in signs: Linguistic landscape as a social issue. In Durk Gorter, Heiko F. Marten \& Luk Van Mensel (Hrsg.), Minority languages in linguistic landscape, 263-280. Basingstoke: Palgrave Macmillan.

Gogolin, Ingrid (2008): Der monolinguale Habitus der multilingualen Schule. Münster: Waxmann.

Haarmann, Harald (1991): Monolingualism versus selective multilingualism: on the future alternatives for Europe as it integrates in the 1990s. In Sociolinguistica 5, 7-23.

Kelly-Holmes, Helen (2005): Advertising as multilingual communication. Basingstoke: Palgrave Macmillan.

Kersting, Volker, Christian Meyer, Peter Strohmeyer \& Tobias Teerporten (2009): Die A40 Der „Sozialäquator“ des Ruhrgebietes. In Achim Prosses, Helmuth Schneider, Horst A. Wessel, Burkhard Wetterau \& Dorothea Wiktorin (Hrsg.), Atlas der Metropole Ruhr. Vielfalt und Wandel des Ruhrgebiets im Kartenbild, 142-145. Essen: Emons Verlag.

König, Katharina (2014): Spracheinstellungen und Identitätskonstruktion: Eine gesprächsanalytische Untersuchung sprachbiographischer Interviews mit DeutschVietnamesen. Berlin: de Gruyter.

Landry, Rodrigue \& Richard Y. Bourhis (1997): Linguistic landscape and ethnolinguistic vitality: An empirical study. Journal of Language and Social Psychology 16, 23-49. 
Liebscher, Grit \& Jennifer Dailey-0'Cain (2009): Language attitudes in interaction. Journal of Sociolinguistics 13 (2), 195-222.

Lou, Jackie J. (2016): The linguistic landscape of Chinatown. A sociolinguistic ethnography. Bristol: Multilingual Matters.

Malinowski, David (2009): Authorship in the linguistic landscape: A multimodal performative view. In Elana Shohamy \& Durk Gorter (Hrsg.), Linguistic landscape. Expanding the scenery, 107-125. New York, London: Routledge.

Mühlan-Meyer, Tirza \& Frank Lützenkirchen (2017): Visuelle Mehrsprachigkeit im Ruhrgebiet eine Projektpräsentation. Zum Aufbau und den Funktionen der Bilddatenbank „Metropolenzeichen“. Zeitschrift für angewandte Linguistik 66, 79-98.

Reershemius, Gertrud (2011): Reconstructing the past? Low German and the creating of regional identity in public language display. Journal of Multilingual and Multicultural Development 32 (1), 33-54.

Schmitz, Ulrich (2017): Linguistic Landscapes im Ruhrgebiet: Internationalismus und Lokalkolorit. In Lieselotte Anderwald \& Jarich Hoekstra (Hrsg.), Sprache als Marke, Talisman, Tourismusmagnet: Zum Enregisterment von sprachlicher Variation, 163-187. Frankfurt a. M.: Peter Lang.

Schmitz, Ulrich (2018): Im Raume lesen wir die Macht. Zeichen der Macht im öffentlichen Raum des Ruhrgebiets. In Sascha Michel \& Steffen Pappert (Hrsg.), Multimodale Kommunikation in öffentlichen Räumen. Texte und Textsorten zwischen Tradition und Innovation, 133-158. Stuttgart: ibidem. (Perspektiven Germanistischer Linguistik 13).

Schmitz, Ulrich \& Evelyn Ziegler (2016): Sichtbare Dialoge im öffentlichen Raum. Zeitschrift für germanistische Linguistik 44 (3), 469-502.

Selting, Margret et al. (2009): Gesprächsanalytisches Transkriptionssystem Gat 2. Gesprächsforschung. Online-Zeitschrift zur verbalen Interaktion 10, 353-402. www.gespraechsforschung-ozs.de (letzter Zugriff: 21. 9. 2017).

Shohamy, Elana \& Durk Gorter (Hrsg.) (2009): Linguistic landscape: Expanding the scenery. New York, London: Routledge.

Spolsky, Bernard \& Robert L. Cooper (1991): The languages of Jerusalem. Oxford: Clarendon Press.

Tophinke, Doris \& Evelyn Ziegler (2014): Spontane Dialektthematisierung in der Weblogkommunikation: Interaktiv-kontextuelle Einbettung, semantische Topoi und sprachliche Konstruktionen. In Christina Cuonz \& Rebekka Studler (Hrsg.), Sprechen über Sprache, 205-242. Tübingen: Stauffenburg Verlag.

Tophinke, Doris \& Evelyn Ziegler (2006): „Aber bitte im Kontext“: Neue Perspektiven in der dialektologischen Einstellungsforschung. In Anja Voeste \& Joachim Gessinger (Hrsg.), Dialekt im Wandel. Perspektiven einer neuen Dialektologie (Osnabrücker Beiträge zur Sprachtheorie 71), 203-222. Duisburg: Gilles \& Francke.

Trumper-Hecht, Nira (2010): Linguistic landscape in mixed cities in Israel from the perspective of ,walkers': The case of Arabic. In Elana Shohamy, Eliezer Ben-Rafael \& Monica Barni (Hrsg.), Linguistic landscape in the city, 235-251. Bristol, Buffalo, Toronto: Multilingual Matters.

Wachendorff, Irmi, Evelyn Ziegler \& Ulrich Schmitz (2017): „Graffitiscape im Ruhrgebiet“. In Stephan Müller (Hrsg.), Graffiti: Auf- und Inschriften in sprach- und literaturwissenschaftlicher Perspektive, 154-208. Wien: Vienna University Press.

Spitzmüller, Jürgen \& Ingo Warnke (2011): Diskurslinguistik. Eine Einführung in Theorien und Methoden der transtextuellen Sprachanalyse. Berlin: de Gruyter. 
Ziegler, Evelyn (2013): Metropolenzeichen: Visuelle Mehrsprachigkeit in der Metropole Ruhr (Forschungsnotiz). Zeitschrift für germanistische Linguistik 41 (2), 299-301.

Ziegler, Evelyn, Heinz Eickmans \& Ulrich Schmitz (2017): Innere Mehrsprachigkeit in der Metropole Ruhr. In Peter Gilles, Helen Christen \& Christoph Purschke (Hrsg.), Räume - Grenzen - Übergänge: 5. Kongress der Internationalen Gesellschaft für Dialektologie des Deutschen. ZDL Beihefte, 347-374, 406-408. Stuttgart: Steiner. Ziegler, Evelyn, Ulrich Schmitz \& Haci-Halil Uslucan (i. Dr.): Attitudes towards visual multilingualism in the linguistic landscape of the Ruhr Area. In Martin Pütz \& Neele Mundt (Hrsg.): Linguistic landscapes and superdiversity in the city. Berlin: de Gruyter. 



\title{
Stefan Kleiner und Ralf Knöbl
}

\section{Zur Aussprache nicht haupttoniger Vorsilben mit 〈e〉 in Lehnwörtern im deutschen Gebrauchsstandard}

\begin{abstract}
Vortoniges <e> in Lehnwörtern in offenen Silben (demonstrieren, Elefant) ist in den traditionellen deutschen Aussprachewörterbüchern durchgängig mit gespanntem/geschlossenem [e] kodifiziert. Die Auswertung von insgesamt 17 entsprechenden Belegwörtern aus dem Korpus „Deutsch heute“ zeigt für den deutschen Gebrauchsstandard jedoch eine ausgeprägte Variation zwischen den Lauttypen [e], [ع] und [ə], die je nach Lexem in ganz unterschiedlichen Anteilen vorkommen. Als Erklärungsansätze für das differierende Variationsverhalten lassen sich Faktoren wie Wortakzentmuster, Folgekonsonanz, Formalitätsgrad und semantisch-morphologische Durchsichtigkeit der Wortbildung anführen. Außerdem zeigt die Variation auch eine ausgeprägte diatopische Dimension: Während im Norden Deutschlands, aber auch im mittelbairisch geprägten Sprachraum und in der Ostschweiz die [e]-Aussprache dominiert, überwiegen in der südlichen Mitte und im Südwesten Deutschlands, im südbairisch geprägten Sprachraum und vor allem in der Westschweiz Belege mit [ع]-Aussprache. Die Ergebnisse von „Deutsch heute“ zeigen sich in ähnlicher Weise auch in zusätzlich ausgewerteten Sprachdaten (Nachrichtensendungen, FOLK-Korpus).
\end{abstract}

Keywords: deutscher Sprachraum, Gebrauchsstandard, Lehnwort, Nebenton, regionale Variation, Standardaussprache, Vokalismus

Die Aussprachewörterbücher des Deutschen ${ }^{1}$ geben für die Aussprache des <e> geschriebenen Vokals in der nicht den Hauptakzent tragenden ersten Silbe von Lehnwörtern wie demonstrieren, elektronisch, Elefant, reparieren oder

1 Alle Lautschriftbelege in diesem Beitrag stammen aus Duden 6 (2015). In anderen Aussprachewörterbüchern (Duden 6 2005; DAW 2009) verzeichnete Formen unterscheiden sich, soweit nicht ausdrücklich vermerkt, nicht hinsichtlich des hier untersuchten Phänomens.

Stefan Kleiner, Institut für Deutsche Sprache, R5 6-13, D-68161 Mannheim, E-Mail: kleiner@ids-mannheim.de

Ralf Knöbl, Institut für Deutsche Sprache, R5 6-13, D-68161 Mannheim,

E-Mail: knoebl@ids-mannheim.de

Ә Open Access. (C) 2018 Stefan Kleiner und Ralf Knöbl, publiziert von De Gruyter. (c) BY Dieses Werk ist lizenziert unter der Creative Commons Attribution 4.0 Lizenz. 
Respekt konsequent die Realisierung mit geschlossenem/gespanntem [e] vor: [demən'strirrən], [elck'tro:nI]], [elə/e'fant], [repa'rirrən], [re'spckt]. Die Zuweisung des Vokals geschieht dabei in den vier erstgenannten Fällen aufgrund der Silbenstruktur: Weil jeweils ein einfacher Konsonant folgt, also eine offene Silbe vorliegt, ist der Vokal als [e] kodifiziert. Der morphologische Status der ersten Silbe, d.h. ob lediglich eine Vorsilbe wie [e-] in Elefant oder ein (aus dem Lateinischen stammendes) Präfix wie de- bzw. re- vorliegt, ist hier unerheblich; er kann allerdings bei mehrfacher Folgekonsonanz relevant werden: In einem Fall wie Respekt wird ebenfalls ausschließlich [e] kodifiziert, obwohl sowohl die Silbifizierung [re.'spekt] als auch [res.'pekt] denkbar sind, was im letzteren Fall aufgrund der geschlossenen Silbe in der Regel in den meisten deutschsprachigen Regionen mit einer offenen/ungespannten Aussprache des ersten <e> einhergehen müsste: [res.'pckt]. In Einklang mit dieser morphologisch gesteuerten Regel werden z. B. die Präfixe de- und des- in den Aussprachewörterbüchern unterschiedlich behandelt: deskriptiv, despektierlich werden als [deskrıp'ti:f], [despek'ti:e्रlıç] kodifiziert, desorientiert, desinfizieren

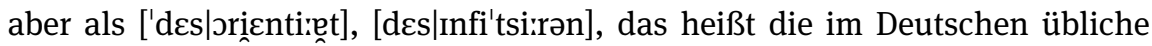
Höherrangigkeit morphologischer Strukturen, die phonologische Prozesse (hier die Bindung/Resilbifizierung) unterbindet, kommt auch in diesem Fall zum Tragen. ${ }^{2}$ Ausnahmen, bei denen möglicherweise der tatsächliche Sprachgebrauch berücksichtigt wurde, gibt es allerdings: So wird Restaurant mit [re-] kodifiziert, für das etymologisch zugehörige, aber semantisch abweichende restaurieren ist jedoch wieder [re-] verzeichnet, weil hier das lateinische Präfix re- ,wieder' auch in der Aussprache durchscheinen soll (obwohl der Stamm -staur-im Deutschen, anders als z. B. -spekt-, nicht als eigenständiges Morphem existiert). ${ }^{3}$

Anders verhält es sich in Wörtern, in denen auf <e > eine Doppelkonsonantenschreibung folgt, wie in Terrasse oder Hellene. Hier setzen die Kodizes in Analogie $\mathrm{zu}$ den Verhältnissen in betonten Silben, wo Schreibungen wie <ll>, $<\mathrm{rr}>$, <ff $>$ in Stelle, sperren, offen eindeutig indirekte Anzeiger der Kürze/ Ungespanntheit des vorhergehenden Vokals sind, für die Nebensilbe iden-

2 In Duden 6 (2005) waren zusätzlich noch die alternativen Varianten ['dezoricntientext], [dezinfi'tsi:rən] gebucht, d.h. mit Resilbifizierung, durch die der Sibilant in den Silbenanlaut gerät und stimmhaft wird. Varianten dieser Art kommen am ehesten noch in der Schweiz vor (dort allerdings mit stimmlosem [z] und offenem $[\varepsilon]$ - dazu s. u.), als überregionale Standardaussprache des Deutschen kann man sie jedoch nicht bezeichnen - darum wurden die Varianten in Duden 6 (2015) gestrichen.

3 Dies gilt allerdings nur für DAW und Duden 6 (2005), in Duden 6 (2015) ist auch für restaurieren die Variante [res-] mit offenem Vokal verzeichnet. 
tische Regularitäten an. Dementsprechend bilden Hellene und der Vorname Helene in allen Kodizes das Minimalpaar [hc'le:nə] vs. [he'le:nə]. Becker (2012: 89) merkt hierzu an: „Ein Teil dieser Gegenbeispiele sind Erfindungen der Aussprachewörterbücher, nämlich die sogenannten ,gespannten Kurzvokale‘ [...] Die Aussprache $B[\supset]$ tanik ist völlig unauffällig, vielleicht sogar die normale, ebenso [hع'le:nə] oder gar [hə'le'nə] für die Helene mit einem $l$. Wenn überhaupt haben wir es hier mit einem Fall von stellungsbedingter Allophonie zu tun: Unbetonte Vokale in geschlossenen Silben sind eher ungespannt, in offenen Silben eher gespannt.“

Da es sich demnach um ein silbenbezogenes Variationsphänomen handelt, finden sich die für vortoniges <e> geschilderten Variationsverhältnisse zwischen gespannten und ungespannten Vokalen in Auftaktsilben in vergleichbarer Weise auch bei den anderen Vokalen: ${ }^{4}<\mathrm{i}>$ Zitrone [tsi'tro:nə], [tsı'tro:nə], Situation [zitua'tsio:n] - [zitua'tsio:n], <0> botanisch [bo'ta:nIf] - [bo'ta:nIf], Pro-

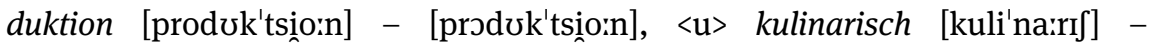
[kvli'na:rI]], <y> hybrid [hy'britt] - [hy'britt], <ö> ökonomisch [øko'no:mif] [œko'no:mi]]. Auch bei unbetontem vortonigem <a> wie in Kakerlake, Rabatt wäre grundsätzlich mit einer entsprechenden Variation zu rechnen, da aber die gespannte und die ungespannte Variante in der überregionalen Standardaussprache (und den regionalen Gebrauchsstandards) in aller Regel dieselbe Vokalqualität aufweisen, fallen beide Varianten lautlich in [a] zusammen (so auch Becker 1998: 87).

\section{Empirischer Teil}

Im vorliegenden Beitrag sollen die oben dargestellten Regularitäten der deutschen Aussprachekodizes sowie die kritischen Bemerkungen dazu von phonologischer Seite auf den empirischen Prüfstand gestellt werden.

\subsection{Auswertung der Daten von „Deutsch heute“}

Zu diesem Zweck wurde zunächst das am IDS im Rahmen des Projekts „Gesprochenes Deutsch“ (vormals „Variation des gesprochenen Deutsch“) im ganzen

4 Vennemann (1991: 234-235) beschreibt die Variationsverhältnisse folgendermaßen „one further property of Standard German [...] there is a strong tendency for pretonic smooth syllables to change to abrupt cut; this is especially noticeable if such syllables are rhythmically prominent („,secondary accent“)“ Der Wechsel zum scharfen Silbenschnitt ist demnach ursächlich für das Auftreten der ungespannten Vokale. 
deutschen Sprachraum erhobene Korpus „Deutsch heute“ konsultiert. Vor allem aus der Wortliste, aber auch aus den Lesetexten und der Bildbenennungsaufgabe wurden insgesamt 17 Wörter dieses Typs jeweils für die 670 aufgenommenen Schüler extrahiert und die Aussprache des <e > in der ersten Silbe analysiert. Außerdem wurden mit dem projektinternen Medienkorpus sowie dem FOLK- und Pfeffer-Korpus ${ }^{5}$ drei weitere Korpora in die Untersuchung einbezogen. Bei der ohrenphonetischen Annotation wurden für alle untersuchten Belegwörter einheitlich vier Lauttypen differenziert: geschlossenes [e], halbgeöffnetes $[\mathrm{e}] /[\varepsilon]$, offenes $[\varepsilon]$, zentralisiertes [ə].

Folgende Belegwörter aus dem Korpus „Deutsch heute“ wurden für diesen Beitrag ausgewertet (Korpusteil in allen Fällen genannt, die nicht aus der Wortliste stammmen):

demoralisiert, demonstrieren, dementieren, detailliert (Wortliste + Lesetext), Delegation, Elefanten (Lesetext), Elektroinstallateur, elektronisch, evangelisch, Methode, Regierung, Revier, repariert (Lesetext), repräsentativen (Lesetext), Respekt, relativ, Telefon (Bildbenennung). ${ }^{6}$

\subsubsection{Variationsmuster und ihre Einflussfaktoren}

Die Belegwörter in Abbildung 6.1 sind aus Übersichtlichkeitsgründen nach abnehmender Häufigkeit der kodifizierten Variante [e] sortiert. Als generelles Ergebnis lässt sich zunächst festhalten: Es zeigt sich alles andere als ein homogenes Bild, die Variationsspanne zwischen den verschiedenen Belegwörtern ist extrem groß und reicht von $86 \%$ kodexkonformen [e]-Realisierungen bei demoralisiert bis zu lediglich $2 \%$ bei Respekt. Die Häufigkeit des Lauttyps $[\varepsilon]$ verhält sich dabei in der Mehrzahl der Fälle invers zu [e], wobei Respekt, repariert und Revier mehr oder weniger deutliche Ausnahmen zu dieser Regularität bilden, weil sie erhebliche Anteile an [ə]-Realisierungen aufweisen (die bei Respekt als einzigem hier untersuchten Belegwort sogar eine klare Mehrheit stellen). Offensichtlich neigt das <e > im Präfix re- stärker als andere <e $>$ in Vorsilben zu zentralisierter Aussprache und verhält sich damit ähnlich wie das native Präfix ge-; in Respekt gehen die Resultate tatsächlich noch deutlich

5 Vgl. http://agd.ids-mannheim.de/folk.shtml bzw. http://agd.ids-mannheim.de/PF--_extern.shtml 6 Die restlichen im Korpus vorhandenen Belegwörter dieses Typs sind Medizin (Lesetext), Medizinerin (Lesetext), Regie (Wortliste + Lesetext), Telefon (Wortliste), telefonieren, telefoniert (Lesetext), Zeremonie. Sie wurden nicht mehr einbezogen, weil keine zusätzlichen Erkenntnisse durch ihre Auswertung zu erwarten waren. 


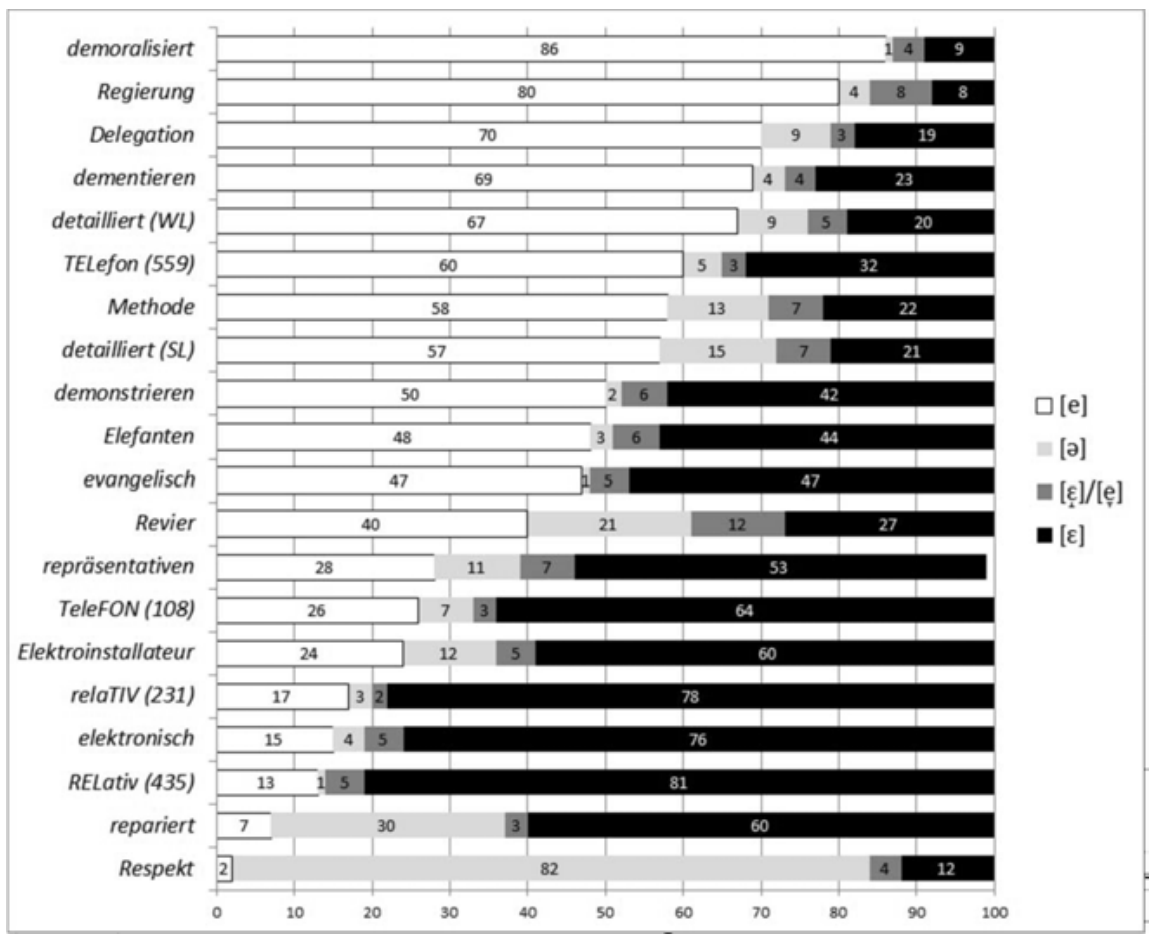

Abb. 6.1: Prozentualer Anteil der vier unterschiedenen Lauttypen für alle 17 untersuchten Belegwörter mit prätonischem <e> (sortiert nach abnehmender Häufigkeit des kodifizierten Lauttyps [e]).

darüber hinaus, weil [ə] in diesem Fall auch in Süddeutschland die dominante Variante ist, obwohl in diesem Raum <e> im Präfix ge- regelmäßig als allenfalls leicht zentralisierter vorderer Vokal [ẹ] realisiert wird (vgl. z. B. König 1989: 317).

Im Vergleich der re-Wörter Regierung, Revier, repräsentativen und repariert lässt sich eine erhebliche Zunahme der [ $\varepsilon$ ]-Anteile von $8 \%>27 \%>53 \%>60 \%$ feststellen. Dafür lassen sich exemplarisch mehrere mögliche Einflussfaktoren anführen, die in ähnlicher Weise auch bei den anderen Belegwörtern wirksam sind, nämlich das Wortakzentmuster, die jeweilige Folgekonsonanz, der Formalitätsgrad (isoliert vs. eingebettet im Satz) und die semantisch-morphologische Durchsichtigkeit der Wortbildung.

Faktor Wortakzentmuster: Regierung und Revier haben den Hauptakzent auf der direkt nachfolgenden, repräsentativen und repariert erst auf der übernächsten Silbe, was zu einem Nebenakzent auf dem initialen re- führt und mit einer Zunahme an $[\varepsilon]$-Belegen einhergeht. Dass das Wortakzentmuster einen Einfluss auf die segmentelle Variation hat, lässt sich auch am Wortpaar elektro- 
nisch und Elektroinstallateur ablesen. Allgemein gehören bei beiden die $[\varepsilon]$ Werte mit zu den höchsten im ganzen Korpus, aber bei elektronisch, bei dem der Hauptakzent erst auf der dritten Silbe liegt, sind sie noch über 15 Prozentpunkte höher als bei Elektroinstallateur, das auf der direkten Folgesilbe seinen Hauptakzent hat, $d . h$. bei Letzterem tritt die gespannte Variante [e] signifikant häufiger auf. ${ }^{7}$ Dass dieser Effekt auch bei anderen Vokalen wirksam ist, illustriert ein ebenfalls im Korpus „Deutsch heute“ erhobener, vergleichbarer Variationsfall bei $\langle 0\rangle$ : Kollege und kollegial wurden in dieser Abfolge direkt hintereinander vorgelesen. Dabei entfielen auf Kollege mit dem Hauptakzent auf der direkt anschließenden Silbe $88 \%$ [o]-Belege, wohingegen bei kollegial mit dem Hauptakzent auf der übernächsten Silbe nur mehr $68 \%$ der Probanden die [o]Variante realisierten (dazu $30 \%$ [0]). Der Effekt des Wortakzentmusters ist hier mit 20 Prozentpunkten Abstand sogar noch deutlicher. Darüber hinaus dürfte aufgrund der direkten Abfolge der beiden Wörter zusätzlich die Vokalrealisierung des vorhergehenden Kollege eingewirkt haben, d.h. unbeeinflusst läge vermutlich der [o]-Wert bei kollegial nochmals niedriger.

Faktor Folgekonsonanz: In Regierung und Revier folgen [g] und [v], also Leniskonsonanten, in repariert und repräsentativen [p] und [pr], also Fortiskonsonanten bzw. ein Konsonantencluster. Hier spiegeln sich Regularitäten, wie sie auch in Tonsilben zu finden sind, wo vor Leniskonsonanten in der Regel gespannte, vor Fortiskonsonanten bzw. Konsonantengruppen ungespannte Vokale artikuliert werden (vgl. Fn. 9).

Faktor Formalitätsgrad: Regierung und Revier wurden als Einzelwörter in der Wortliste erhoben, repariert und repräsentativen im Lesetext, $d$.h. es ist bei Letzteren zum einen mit einer etwas geringeren Artikulationspräzision zu rechnen, zum anderen kann durch die Einbettung in einen Satz die bei isoliertem Vorlesen stärker bewusste Silbifizierung eher in den Hintergrund geraten. Detailliert war sowohl in der Wortliste als auch in einem Lesetext enthalten, so dass hier der Einfluss des Faktors Textsorte bzw. Formalitätsgrad isoliert werden kann. Die Unterschiede sind letztlich nicht groß, aber es ist im Lesetext immerhin ein um 10 Prozentpunkte niedrigerer Wert der [e]-Varianten zu verzeichnen, der allerdings kaum mit einem Anstieg von $[\varepsilon]$, sondern vor allem mit einer Zunahme von [ə] um 6 Prozentpunkte einhergeht - was sich gut mit der textsortenbedingten Abnahme der Artikulationspräzision in Einklang bringen lässt.

7 Warum elektronisch und Elektroinstallateur diese außerordentlich hohen [c]-Werte aufweisen, ist damit natürlich nicht erklärt. Eventuell liegt eine Art vokalharmonischer Einfluss des $<\mathrm{e}>$ der Folgesilbe vor, das wegen der geschlossenen Silbe ganz überwiegend als $[\varepsilon]$ realisiert wird. In dementieren und Respekt folgt zwar ebenfalls [ $\varepsilon$ ], aber hier sind offensichtlich andere Faktoren relevanter (vgl. dazu die entsprechenden Anmerkungen im Text). 
Ein weiterer, wichtiger Einflussfaktor lässt sich aus dem Variationsverhalten der Wörter mit de-Präfix ableiten, die semantisch-morphologische Durchsichtigkeit: In demoralisiert hat das Präfix de- eine eindeutig negierende Funktion, zudem ist der Wortstamm -moral- auch in Moral bzw. in Ableitungen wie moralisch unpräfigiert im Deutschen vorhanden (und in häufigem Gebrauch), die Wortbildung demoralisiert ist darum voll durchsichtig. Deutlich weniger durchsichtig ist dagegen dementieren, dessen unpräfigierter Stamm bzw. Ableitungsbasis nur im bedeutungsmäßig fernstehenden mental auftritt. Ähnlich liegt der Fall bei demonstrieren, zu dem kein *monstrieren o. Ä. existiert (auch die etymologische Beziehung zur im kirchlichen Kontext auftretenden Monstranz dürfte wohl synchron kaum eine Rolle spielen) und bei dem das häufige Kopfwort Demo zusätzlich zur Verschleierung der morphologischen Grenze beiträgt. Und auch wenn es die Taille gibt, wird detailliert kaum als dazu semantisch-funktional passende Präfixbildung erkannt werden (zumal es sich um eine Entlehnung aus dem Französischen handelt). In dementieren, detailliert und demonstrieren ist die semantische Funktion von de- also deutlich verdunkelt bzw. gar nicht vorhanden.

Relativ und Telefon wurden trotz der Tatsache, dass sie einen variablen Wortakzent aufweisen und in unseren Daten die Mehrzahl ihrer Belege auf der ersten Silbe akzentuiert sind (die Belegzahlen sind in Abb. 6.1 in Klammern angegeben), in die Untersuchung einbezogen, um feststellen zu können, ob auch hier die Position des Wortakzents einen Einfluss auf die Realisierung des Vokals der Auftaktsilbe hat. (Direkt folgender vs. erst in der übernächsten Silbe folgender Hauptakzent wurde oben ja bereits als wichtiger Einflussfaktor festgestellt.) Bei relativ wirkt sich die Position des Wortakzents offensichtlich nur marginal aus, denn bei Erstsilbenakzent liegt der Anteil von [ $\varepsilon$ ] um lediglich 3 Prozentpunkte höher. Ganz anders gelagert ist der Fall bei Telefon, wo die Variantenverhältnisse je nach Akzentsitz sehr unterschiedlich ausfallen: Beim Akzentuierungstyp TeleFON beträgt der Anteil von [ع] $64 \%$ und ist damit doppelt so hoch wie bei TELefon mit akzentuierter erster Silbe, wo umgekehrt die [e]-Anteile statt 26\% 60\% betragen. Dieses scheinbar von den Verhältnissen bei relativ völlig verschiedene Verhalten lässt sich allerdings eher auf die ungleichmäßige regionale Verbreitung der betreffenden Akzentuierungsvariante als auf eine Auswirkung des Wortakzents selbst zurückzuführen, denn die Variante TeleFON ist überwiegend in Österreich und Südtirol belegt (relaTIV ist an sich häufiger und vor allem gleichmäßiger über das Sprachgebiet verteilt), wo [ع] allgemein überdurchschnittlich häufig ist im Vergleich zum Gesamtkorpus. (Von den neun TeleFON-Belegen in Norddeutschland hat keiner die Variante $[\varepsilon]$.) 


\subsubsection{Diatopische Auswertung}

Im Folgenden soll die diatopische Dimension der Variation bei der Realisierung der unbetonten <e> in der Auftaktsilbe beleuchtet werden. Dazu werden exemplarisch die drei Belegwörter demonstrieren, repariert und demoralisiert herausgegriffen und sprachgeographisch kartiert, weil sie einerseits ein jeweils sehr unterschiedliches diatopisches Variationsverhalten aufweisen und damit andererseits auch prototypisch für andere Belegwörter mit ähnlichen Variantenhäufigkeiten stehen. Abschließend werden in einer Überblickskarte die Daten aller 18 ausgewerteten Belegwörter aggregiert und damit systematische regionale Unterschiede bei diesem Phänomen nachgewiesen.

Das Verb demonstrieren wurde im Rahmen der Wortliste erhoben. In Abbildung 6.1 weist es, auf das Gesamtkorpus gesehen, einen mittleren Rang auf, die Variantenhäufigkeiten sind relativ ausgeglichen mit einem leichten Übergewicht zugunsten von [e]. Auf der Karte (Abb. 6.2) ist jedoch auf den ersten Blick zu erkennen, dass die Varianten sich diatopisch keineswegs gleichmäßig verteilen, wie man aufgrund der Gesamtwerte erwartet hätte, sondern dass es erhebliche geographische Unterschiede bei ihrer Verteilung gibt. Ganz Norddeutschland (tatsächlich ungefähr ab nördlich der Benrather Linie) bildet ein geschlossenes Gebiet, in dem fast ausschließlich die Variante [e] belegt ist. Ein weiteres großflächiges Areal mit ganz überwiegender [e]-Aussprache findet sich in Altbayern und im größten Teil des benachbarten Österreich (überwiegend mittelbairisch), wobei vor allem in der Steiermark und in Tirol (inklusive Südtirol) mehrheitlich offene Artikulation vorkommt. Weiter westlich dominiert vor allem in der Ostschweiz (und Liechtenstein) wieder [e] (Vorarlberg ist hier uneinheitlich), und es ist außerdem noch am ganzen westlichen Saum Deutschlands in der Mehrheit (von Freiburg bis Aachen), ebenso in Ostbelgien und in Luxemburg.

Der mit offenem Vokal $[\varepsilon]$ realisierte Lauttyp ist in allen übrigen Gebieten die Mehrheitsvariante, konkret in einem zusammenhängenden Areal, das den ganzen mitteldeutschen und den größten Teil des westlichen oberdeutschen Raums umfasst, zusätzlich noch das Ostfränkische. Ein besonders kompaktes Areal von $[\varepsilon]$-Belegen findet sich in der Westschweiz, wo keine einzige [e]-Lautung belegt ist.

Die nur leicht geöffnete Variante $\left[\varepsilon_{\perp}\right] /[$ e ] streut großflächig im $[\varepsilon]$-Gebiet, Konzentrationen lassen sich - erwartungsgemäß ${ }^{8}$ - nicht erkennen. SchwaBelege kommen nur sporadisch vor.

8 Der Lauttyp [ع] $] /\left[e_{T}\right]$ ist bei der ohrenphonetischen Annotation als Sammelbecken für lautliche Realisierungen benutzt worden, die nicht mit Sicherheit den Typen $[\varepsilon]$ oder [e] zuordenbar waren. Eine spezifische Raumbildung war daher nicht zu erwarten. Er ist wohl, auch wegen des Vorkommens überwiegend im [c]-Areal, als dessen Realisierungsvariante zu interpretieren. 


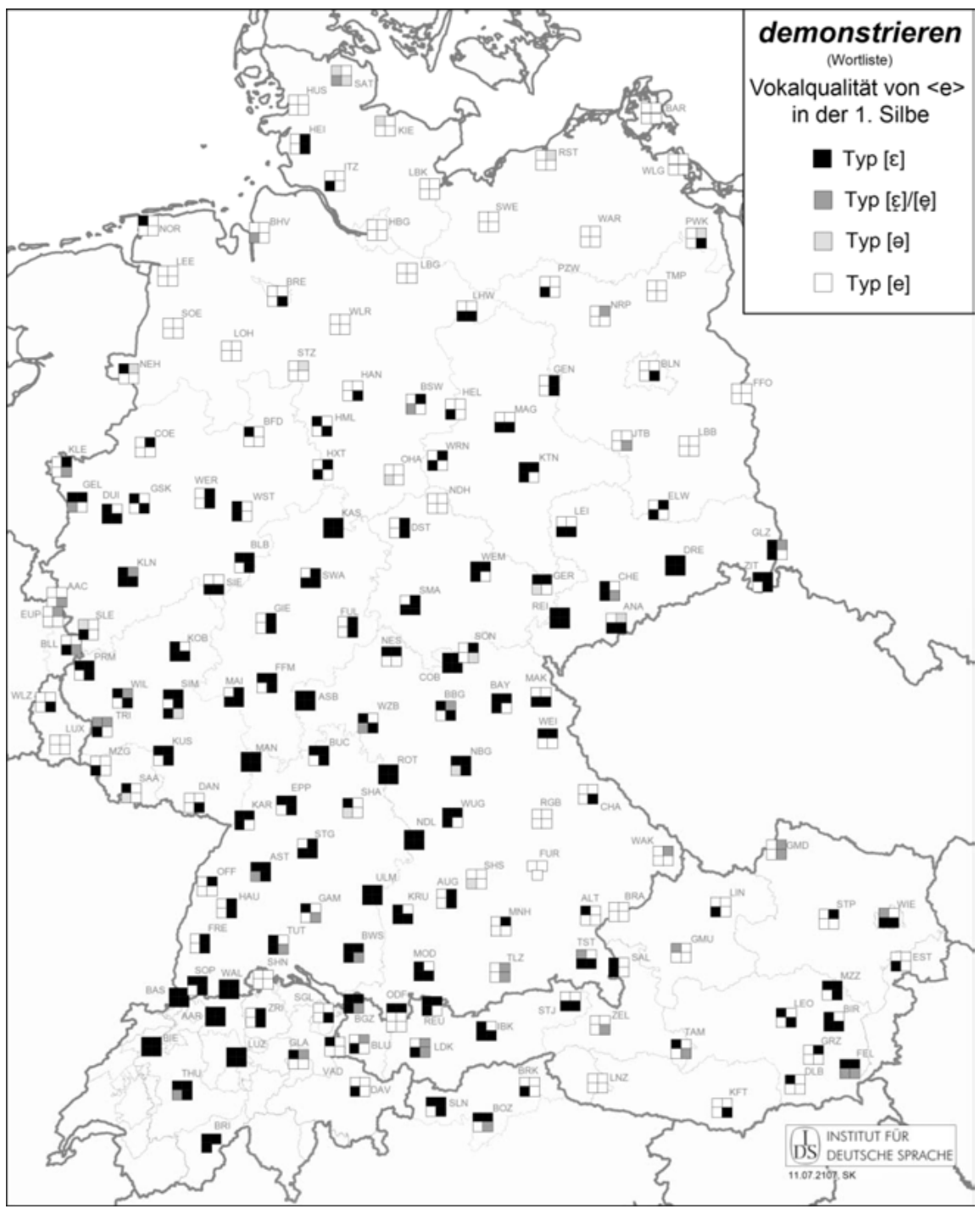

Abb. 6.2: Vokalqualität von <e in der ersten Silbe von demonstrieren.

Als Ergebnis ist festzuhalten, dass die Aussprache des <e > der ersten Silbe von demonstrieren eine sehr ausgeprägte raumspezifische Verteilung der beiden Hauptvarianten [e] und $[\varepsilon]$ aufweist, die sich interessanterweise recht gut mit bekannten sprachgeographischen Räumen (bzw. Grenzen) zur Deckung bringen lässt. Eine im Wesentlichen ähnliche regionale Verteilung der Varianten 
zeigt sich auch für die Aussprache des <e> der ersten Silbe in Elefanten, Telefon, repräsentativen und evangelisch. Bei Letztgenanntem neigt der ostmitteldeutsche Raum allerdings überwiegend zur [e]-Aussprache, was mit der Realisierung des folgenden $\langle\mathrm{v}>$ in evangelisch korreliert, das in diesem Gebiet mehrheitlich als Lenis [v] realisiert wird - anders als in Süd- und Westdeutschland, wo [f]-Aussprache (und [ع]) vorherrscht (vgl. Kleiner 2014: 280). Diese Korrelation deutet darauf hin, dass folgende Fortiskonsonanz ein begünstigender Faktor für das Auftreten der Variante $[\varepsilon]$ ist, was sich gut mit den allgemeinen Tendenzen in der deutschen Tonsilbe in Einklang bringen lässt, wo die ungespannten (Kurz-)Vokale auf geschlossene Silben beschränkt sind, wobei silbenschließende Obstruenten in der Silbengelenkposition (bei Mehrsilbern) aus sprachhistorischen Gründen in aller Regel Fortes sind. ${ }^{9}$

Als zweites Beispiel ist in Abbildung 6.3 die Vokalqualität von <e > in repariert in ihrer diatopischen Variation dargestellt. Im Vergleich zu demonstrieren zeigt die Aussprache des <e> der ersten Silbe im Part. Perf. repariert (erhoben im Lesetext im Satz Die Speichen an meinem Fahrrad sind endlich repariert worden.) deutlich andere Variationsverhältnisse: ${ }^{10}$ Die kodifizierte Variante [e] kommt insgesamt nur auf $7 \%$, dafür liegen die Anteile von [ $[\varepsilon]$ und [ə] mit $60 \%$ bzw. 30\% wesentlich höher. Auf das Kartenbild hat diese Verschiebung bei der Variantenhäufigkeit nur teilweise Auswirkungen, denn die grundsätzliche Trennung des nord- und mitteldeutschen Raums bleibt weiterhin bestehen, allerdings tritt bei repariert im Norden fast flächendeckend [ə] an die Stelle von [e], und die $[\varepsilon]$-Einsprengsel nehmen deutlich zu. In Altbayern und Österreich wird das bei demonstrieren übliche [e] hingegen weitgehend durch $[\varepsilon]$ ersetzt, ein gewisser Grundbestand an [e]-Varianten bleibt jedoch bestehen. Vergleichbares gilt für die Ostschweiz. In Luxemburg und Ostbelgien sind die Verhältnisse ähnlich wie in Norddeutschland, d. h. Wechsel zu [ə] herrscht vor, allerdings bleiben hier die [e]-Belege häufiger.

Als drittes Beispiel wird demoralisiert kartiert (Abb. 6.4), weil es von allen untersuchten Belegwörtern den höchsten Wert an kodifikationskonformem [e] und mithin einen extrem niedrigen Wert an $[\varepsilon]$-Belegen aufweist; es steht hier stellvertretend für einen minimalen Variationsfall.

9 Vgl. z. B. Becker (2012: 95): „das Hochdeutsche meidet stimmhafte Obstruenten und /r/ nach Kurzvokal.“ Fälle wie Robbe, Kladde sind mehrheitlich durch Entlehnungen - v.a. aus dem Niederdeutschen - hinzugekommene, vergleichsweise seltene Ausnahmen. Nur einige <gg>Fälle wie Roggen, Egge sind sprachhistorisch gesehen echtes Hochdeutsch.

10 Die bei diesem Belegwort vor allem in der Mitte Deutschlands häufiger notierte Transkription [e] ( $4 \%$ der Gesamtbelege), wurde - aufgrund der Öffnungsgrads - als wohl durch partielle antizipatorische Assimilation an das [a] der Tonsilbe entstandene allophonische Variante $\mathrm{zu}[\varepsilon]$ interpretiert und, aus Vergleichbarkeitsgründen, bei der Kartierung genauso wie $[\varepsilon]$ symbolisiert. 


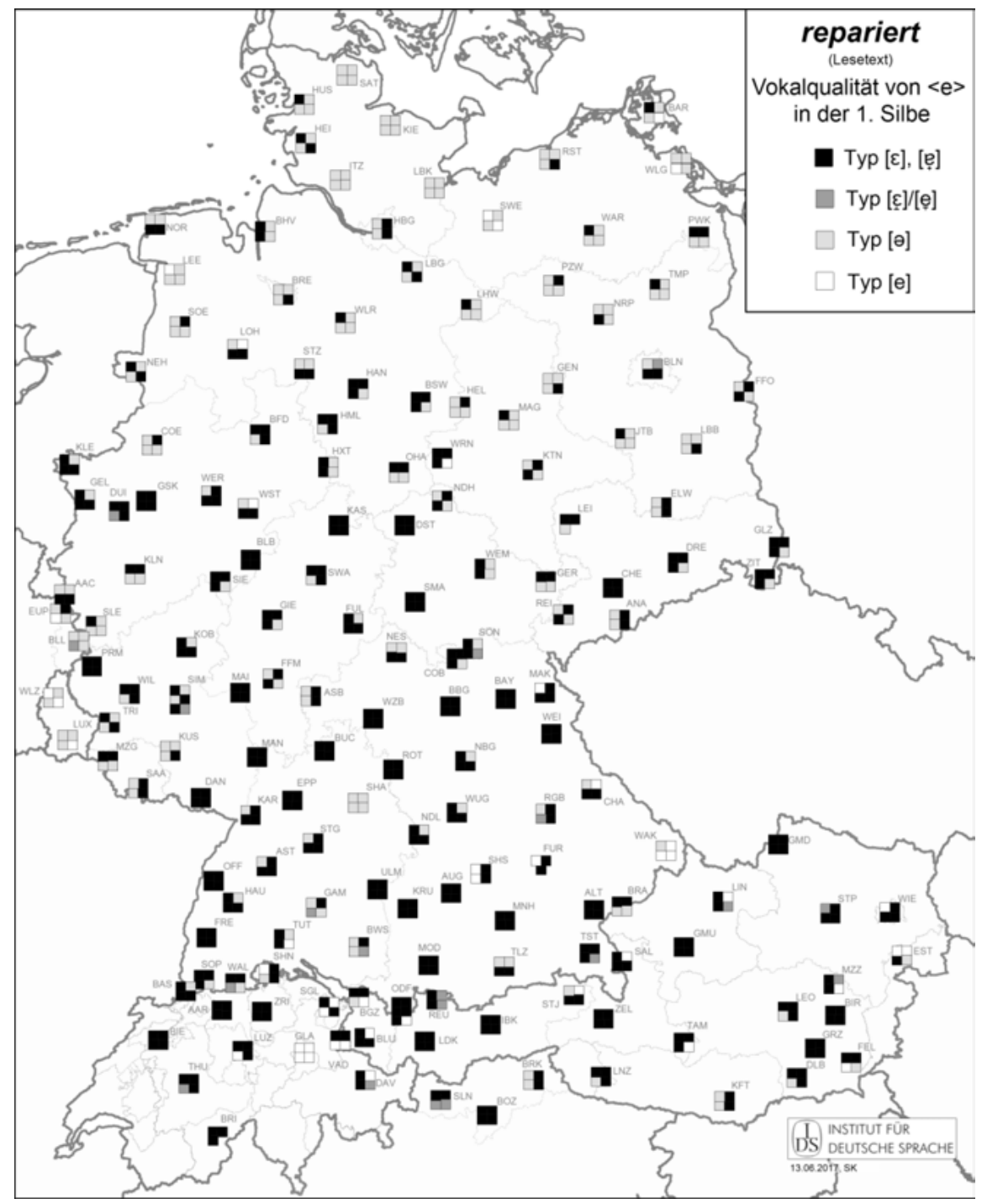

Abb. 6.3: Vokalqualität von <e in der ersten Silbe von repariert.

Das Kartenbild zeigt entsprechend nur noch ein kleines kohärentes Areal, in $\operatorname{dem}[\varepsilon]$ dominiert: die Westschweiz. Überall sonst sind $[\varepsilon]$-Formen nur verstreut belegt, allenfalls im Nordwesten von Baden-Württemberg scheint sich noch eine gewisse Häufung abzuzeichnen. Die Streubelege sind dabei im Wesentlichen auf diejenigen Gebiete beschränkt, in denen bei demonstrieren der Lauttyp $[\varepsilon]$ großflächig auftritt. 


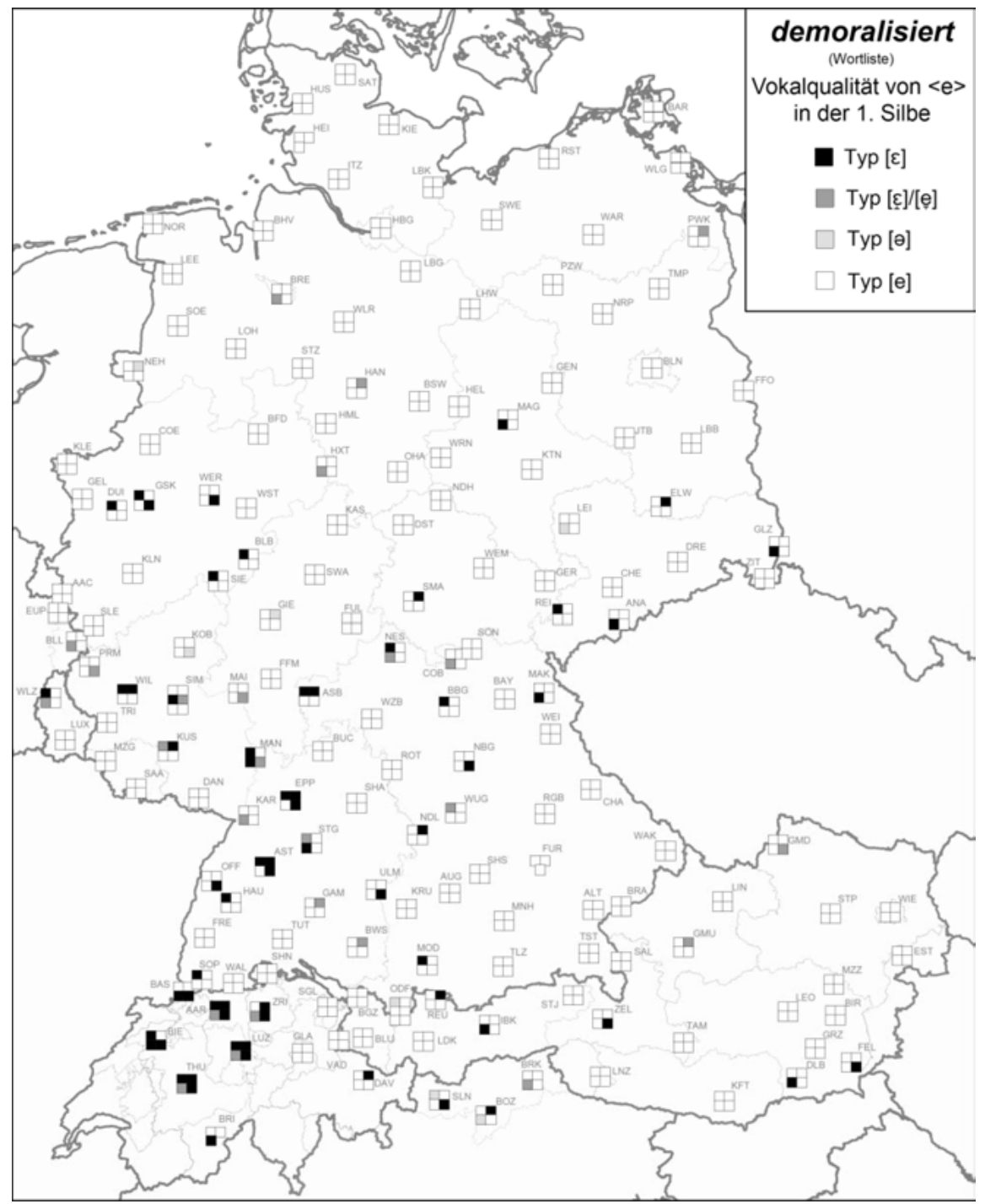

Abb. 6.4: Vokalqualität von <e> der ersten Silbe in demoralisiert.

Um aus den zum Teil recht heterogenen Einzelkarten ein Gesamtbild zu generieren, sind die Einzelergebnisse auf Abbildung 6.5 zusammengefasst worden. Für jede/n Sprecher/in ist in einem Tortensymbol der Anteil der jeweiligen Transkriptionen für alle 18 hier untersuchten Belegwörter abgebildet. Die Farbgebung ist dabei wie auf den Einzelkarten, das auffälligere Schwarz wurde für die nicht kodifizierte Variante [ع] gewählt, Weiß steht für [e], Graustufen mar- 


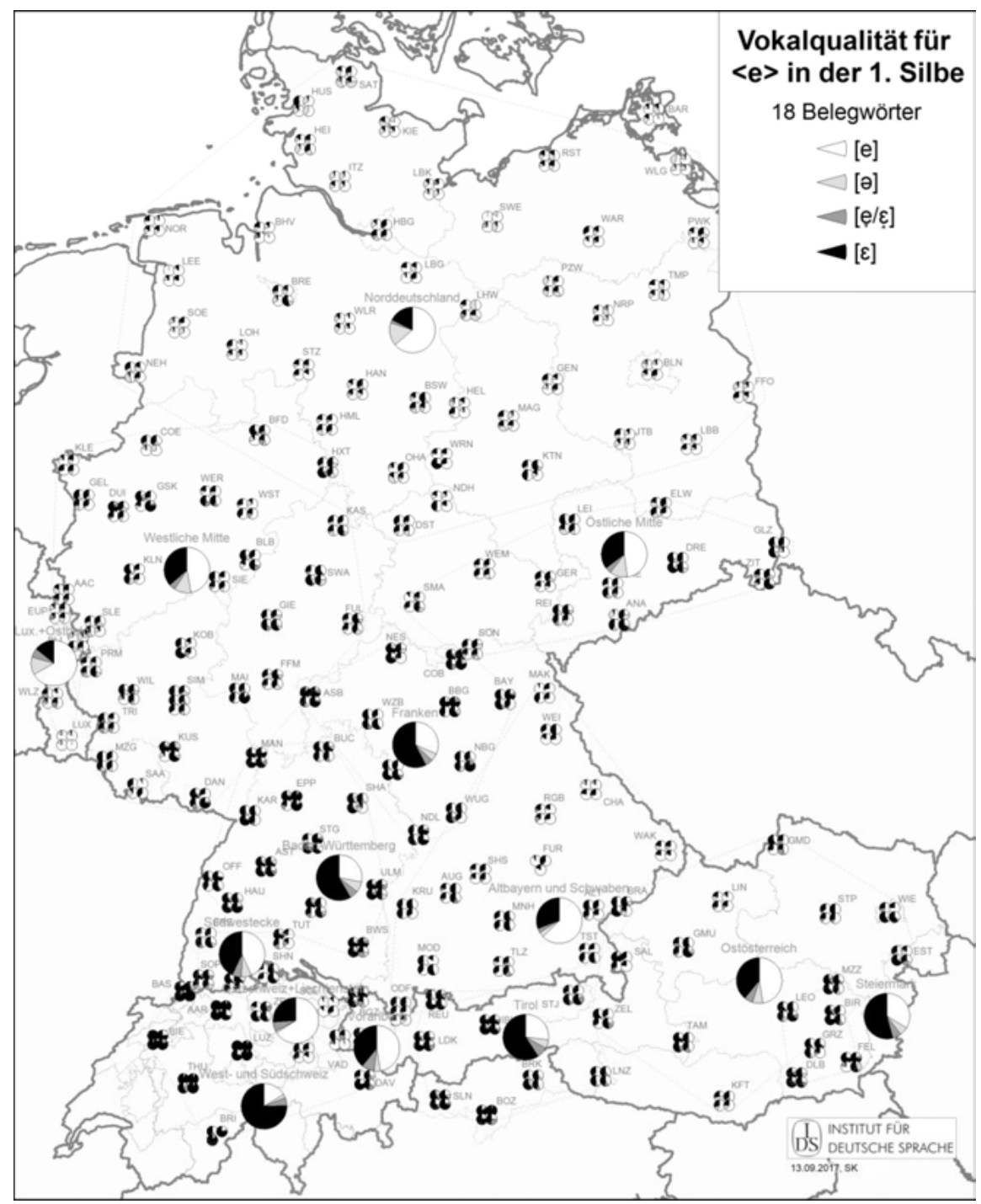

Abb. 6.5: Vokalqualität für 〈e〉 in der ersten Silbe akkumuliert für alle 18 Belegwörter. Individuelle relative Häufigkeiten und Zusammenfassung nach Arealen.

kieren [ə] und [ẹ/દ్s]. Zusätzlich zu den Symbolen bei den einzelnen Sprechern wurden anhand von Ähnlichkeiten bei den Variantenfrequenzen und in Anlehnung an (sprach-)geographische Strukturen Räume festgelegt, für deren Erstreckung die Variationsverhältnisse mittels großer Tortengrafiken zusammengefasst dargestellt werden. 
Anhand der Variantenrelationen lassen sich im Wesentlichen drei Arealtypen differenzieren - wobei sich im Süden kleinräumigere Raumstrukturen abzeichnen: Im ganzen Norden Deutschlands, in Luxemburg und Ostbelgien sowie im Süden und Osten Bayerns dominiert der Lauttyp [e] zu ungefähr zwei Dritteln. In den beiden erstgenannten Arealen ist [ə] der zweithäufigste Lauttyp, in Bayern dagegen kommt [ə] kaum vor, hier entfällt das restliche Drittel fast ausschließlich auf $[\varepsilon]$. In der Mitte Deutschlands - Ost und West unterscheiden sich nicht wesentlich voneinander -, in Ostösterreich und Vorarlberg sowie in der Südwestecke Deutschlands stellt der Lauttyp [e] ungefähr die Hälfte der Belege, [c] kommt hier auf ungefähr 40\%. Im dritten Arealtyp schließlich ist der Lauttyp [ $\varepsilon]$ mehr oder weniger deutlich in der Mehrheit: In der Steiermark kommt er auf die Hälfte, in Tirol, Franken und im größten Teil von Baden-Württemberg auf annähernd $60 \%$, und in der Süd- und Westschweiz lassen sich für den Typ [E] mit gut $75 \%$ die mit Abstand höchsten Werte feststellen.

Aufgrund der Tatsache, dass hier für alle SprecherInnen die aggregierten Transkriptionen von 18 Belegwörtern vorliegen, werden auch Unterschiede im individuellen Sprachverhalten sichtbar. Auffällig sind besonders Individuen, deren Sprachverhalten sich deutlich von dem der anderen am selben Ort oder in der näheren Umgebung unterscheidet. So fallen zum Beispiel in Duisburg, Gelsenkirchen, Mainz, Wernigerode oder Bremen Individuen (DUI1, GSK4, MAI4, WRN4, BRE4) durch viel höhere [ $\varepsilon]$-Werte auf als in den betreffenden Regionen durchschnittlich üblich. Umgekehrt haben in Kusel, Eppingen oder SchwäbischHall (KUS3, EPP3, SHA4) Einzelne hohe Anteile an [e]-Aussprachen, obwohl in diesem Raum an sich [ $\varepsilon]$ dominiert. Dass offensichtlich auch ein solcher vom regionalen Usus systematisch divergierender Sprachgebrauch möglich ist, spricht dafür, dass es sich um ein recht unauffälliges lautliches Phänomen handelt, dessen Variation den meisten SprachteilnehmerInnen nicht bewusst sein dürfte.

Die Gesamtanteile betragen $48 \%$ für [ع], $37 \%$ für [e], $10 \%$ für [ə] (wovon fast die Hälfte allein auf Respekt zurückgeht) und 5\% für $[\underset{T}{e} / \varepsilon]$.

\subsection{Weitere Korpusauswertungen}

Um über die im Rahmen des Atlas zur Aussprache des deutschen Gebrauchsstandards (AADG) üblichen Analysen in diesem Beitrag hinauszugehen, wurden neben dem „Deutsch heute“-Korpus noch zwei weitere Datenquellen herangezogen, in denen ebenfalls die Variation der unbetonten Auftaktvokale bei <e>-Schreibung ausgewertet wurde: 1. Aus dem in den Jahren 2003/2004 im Projekt „Variation des gesprochenen Deutsch“ zusammengestellten Medien- 
Tab. 6.1: Lautliche Realisierung von <e in der Auftaktsilbe bei häufiger vorkommenden Wörtern in 14 Nachrichten- und Informationssendungen.

\begin{tabular}{|c|c|c|c|c|c|}
\hline Wort & Belegzahl & [e] & {$[\varepsilon]$} & [ع]/[e्̦] & [ə] \\
\hline Regierung- & 56 & $91 \%$ & $5 \%$ & $4 \%$ & \\
\hline Demokrat- & 15 & $80 \%$ & $7 \%$ & $13 \%$ & \\
\hline Spezial- & 5 & $60 \%$ & $40 \%$ & & \\
\hline Deleg- & 4 & $50 \%$ & $50 \%$ & & \\
\hline General- & 13 & $31 \%$ & $62 \%$ & $7 \%$ & \\
\hline Elefant- & 7 & $29 \%$ & $71 \%$ & & \\
\hline Revision & 4 & $25 \%$ & $75 \%$ & & \\
\hline Republik- & 4 & $25 \%$ & $75 \%$ & & \\
\hline Demonstr- & 10 & $20 \%$ & $80 \%$ & & \\
\hline Reform- & 26 & $4 \%$ & $62 \%$ & & $35 \%$ \\
\hline Referendum & 11 & & $100 \%$ & & \\
\hline
\end{tabular}

korpus wurden die 14 enthaltenen Nachrichten- und Informationssendungen untersucht (davon acht Ausgaben der ARD-Tagesschau). 2. Aus den über die DGD 2.0 öffentlich zur Verfügung stehenden Korpora wurden das FOLK- und das Pfeffer-Korpus ausgewertet. ${ }^{11}$ Aus Gründen der Übersichtlichkeit wurden nur Lexeme berücksichtigt, die mindestens vier Mal im jeweiligen Korpus belegt sind. Die Belege sind in Tabelle 6.1, wie in Abbildung 6.1, nach abnehmender Häufigkeit der Variante [e] gereiht. ${ }^{12}$

Die in den Nachrichten- und Informationssendungen $\mathrm{zu}$ beobachtende Variation der Aussprache des <e > in der Auftaktsilbe weist an mehreren Stellen Parallelen zu derjenigen im Korpus „Deutsch heute“ auf (vgl. Abb. 6.1): Regefolgt von einem Lenisplosiv und dem Hauptakzent in Regierung kommt auch hier auf die höchsten [e]-Werte, wohingegen Reform und Referendum mit folgendem Fortiskonsonant am anderen Ende der Skala liegen. Dabei ist in Reform - ähnlich wie in repariert in „Deutsch heute“ - für ungefähr ein Drittel der Belege Zentralvokal [ə] notiert worden und für annähernd zwei Drittel $[\varepsilon]$; [e] kommt nur einmal vor - obwohl der Hauptakzent auf der Folgesilbe liegt.

11 Für die - sehr zeitaufwendige - Auswertung dieser Daten danken wir unseren Praktikantinnen Thalia Kimmel, Kristina Becker und Stefanie Prochazka.

12 In den Tabellen 6.1 und 6.2 sind Lexeme, die morphologische Ableitungen desselben Stamms darstellen (wie Demokratie, Demokraten, demokratisch), unter einem Stichwort zusammengefasst worden, was durch einen Bindestrich angedeutet wird. Die Wortakzentstruktur verändert sich in den meisten Fällen durch die morphologische Abwandlung nicht, nur bei wenigen Ausnahmen verschiebt sich der Hauptakzent von der dritten auf die vierte Silbe (Republik - Republikaner). 
Dass Referendum zu $100 \%$ als $[\varepsilon]$ realisiert wurde, wird durch immerhin elf vorliegende Belege gesichert, womit zufällige Schwankungen - anders als bei den nur je vier Mal belegten Revision und Republik - eine geringe Rolle spielen dürften. Allerdings sind höhere Belegzahlen bei einem relativ kleinen Korpus nicht automatisch eine Garantie für höhere Validität, denn es kommt genauso auf die Belegverteilung innerhalb des Korpus an. Im Gegensatz zur Regierung, die erwartungsgemäß in fast allen Nachrichtensendungen vorkommt, sind alle elf Instanzen von Referendum, weil es sich schlicht um ein wesentlich seltener in Nachrichten auftretendes Wort handelt, auf eine einzige Tagesthemen-Sendung begrenzt. Immerhin steuern sowohl der Moderator als auch der Auslandskorrespondent und der Kommentator Belege bei, so dass die Aussagekraft doch weitgehend gewahrt bleibt. Abhilfe könnte hier allenfalls ein umfangreicheres Korpus schaffen. Insofern sind zwar die hohen $[\varepsilon]$-Werte von Demonstr-, Elefant- und General- bemerkenswert und passen in den ersten beiden Fällen ungefähr zu den Verhältnissen im Korpus „Deutsch heute“ (Demokrat- hat hingegen einen auffällig hohen [e]-Anteil), trotzdem bleibt die Aussagekraft aufgrund der geringen Korpusgröße und den damit einhergehenden niedrigen Belegzahlen begrenzt.

In Tabelle 6.2 sind die ausgewerteten Belege aus dem FOLK- und dem Pfeffer-Korpus aufgeführt. Die durchschnittlichen Belegzahlen in diesen beiden Korpora liegen pro Wort deutlich über denen des Medien-Korpus. Auffällig an der Lexemkonstellation ist, dass von den 14 hier aufgrund ihrer hohen Belegzahl ausgewerteten Wörtern zehn mit dem Präfix re- beginnen. Dies lässt sich als klarer Hinweis auf eine allgemein hohe Frequenz von Wortformen mit dem Präfix re- in der Alltagssprache interpretieren, die vielleicht mit erklären kann, warum die Variante mit Reduktionsvokal [ə] sich gerade bei diesem Präfix so stark häuft - hier im FOLK- und Pfeffer-Korpus ebenso wie in den „Deutsch heute“-Daten (vgl. Abb. 6.1).

Die Variationsverhältnisse zeigen insgesamt eine eindeutige Präferenz für die nicht kodifizierten Varianten $[\varepsilon]$ und [ə], deren Werte im Schnitt über denen von „Deutsch heute“ liegen. Dies gilt auch für das direkt vergleichbare Telefon, das hier auf $56 \%$ [ع]-Anteil kommt (bei einer hohen Zahl von 50 Belegen), wohingegen in „Deutsch heute“ lediglich $37 \%$ so annotiert wurden. Nur bei Medizin und regional ist die Variante [e] in der Summe der beiden hier ausgewerteten Korpora (deutlich) in der Mehrheit. Allerdings kann auch hier die Korpuszusammensetzung einen Einfluss auf die Ergebnisse haben. So stammen alle 50 Belege für Telefon aus den FOLK-Daten. Die Aufnahmen des FOLK-Korpus sind jedoch - im Gegensatz zum Pfeffer- oder „Deutsch heute“Korpus - regional alles andere als homogen verteilt. Vielmehr konzentriert sich eine erhebliche Zahl von ihnen bisher - aus rein erhebungspraktischen 
Tab. 6.2: Lautliche Realisierung von 〈e〉 in der Auftaktsilbe bei häufiger vorkommenden Wörtern im FOLK- und Pfeffer-Korpus.

\begin{tabular}{|c|c|c|c|c|c|}
\hline Wort & Belegzahl & [e] & {$[\varepsilon]$} & {$[\varepsilon] /\left[e_{T}\right]$} & [ə] \\
\hline Medizin- & 16 & $88 \%$ & $12 \%$ & & \\
\hline regional- & 14 & $79 \%$ & $21 \%$ & & \\
\hline Telefon- & 50 & $42 \%$ & $56 \%$ & $2 \%$ & \\
\hline Reduktion- & 8 & $38 \%$ & $63 \%$ & & \\
\hline reduzier- & 46 & $33 \%$ & $43 \%$ & $13 \%$ & $11 \%$ \\
\hline Revision- & 11 & $27 \%$ & $64 \%$ & & $9 \%$ \\
\hline Redaktion- & 8 & $25 \%$ & $50 \%$ & & $25 \%$ \\
\hline religiös- & 15 & $27 \%$ & $53 \%$ & & $27 \%$ \\
\hline Republik- & 17 & $18 \%$ & $53 \%$ & $12 \%$ & $18 \%$ \\
\hline Therapie- & 18 & $17 \%$ & $72 \%$ & $11 \%$ & \\
\hline Melodie- & 10 & $10 \%$ & $90 \%$ & & \\
\hline Redakteur- & 20 & $10 \%$ & $65 \%$ & $5 \%$ & $20 \%$ \\
\hline Religion- & 31 & $7 \%$ & $65 \%$ & $3 \%$ & $26 \%$ \\
\hline Referat- & 19 & $5 \%$ & $63 \%$ & $5 \%$ & $26 \%$ \\
\hline
\end{tabular}

Gründen - auf Mannheim oder dessen nähere Umgebung. Entsprechend kommen auch viele der Telefon-Belege aus der rheinfränkischen Sprachregion, die nach Ausweis des auf Abbildung $6.5 \mathrm{zu}$ erkennenden Kartenbilds zu den Sprachräumen gehört, in denen die [ $\varepsilon]$-Variante überdurchschnittlich häufig ist - was eine plausible Erklärung für die um mehr als 20 Prozentpunkte höheren $[\varepsilon]$-Werte liefert.

\section{Zusammenfassung}

Die empirische Überprüfung der Aussprache von $<\mathrm{e}>$ in unbetonten Auftaktsilben mithilfe verschiedener IDS-Korpora (schwerpunktmäßig „Deutsch heute“) hat Erkenntnisse geliefert, die dazu geeignet sind, sowohl die in den Aussprachewörterbüchern praktisch ausschließlich verzeichnete [e]-Aussprache erheblich zu relativieren als auch die Beobachtungen von phonologischer Seite (Vennemann 1991; Becker 1998, 2012), dass in dieser Position ungespannte Vokale, in unserem Fall $[\varepsilon]$, weithin gebräuchlich seien, zu präzisieren.

Das Hauptergebnis, das sich aus dem „Deutsch heute“-Korpus ableiten lässt, ist, dass die Variation eine ganz wesentliche diatopische Komponente aufweist: Nicht nur im übernationalen Vergleich - den Vennemann (1991) bzw. Becker (1998) ohnehin nicht im Blick hatten -, sondern auch innerhalb Deutschlands ist je nach Region die Verwendungswahrscheinlichkeit der Vari- 
anten [e], [ع] oder [ə] völlig unterschiedlich: Im Norden und im Südosten dominiert tatsächlich das in den Kodifikationen fast ausschließlich verzeichnete gespannte [e], und es erscheint darum nicht angemessen, dieses Sprachverhalten mit Becker (1998) als primär orthographieinduziert und bildungssprachlich abzutun. ${ }^{13}[ə]$ lässt sich im Wesentlichen als Variante von [e] ansehen, die regional schwerpunktmäßig in Norddeutschland vorkommt, aber vor allem in geschlossenen Silben beim Präfix re- auch in anderen Regionen verstärkt auftritt. Demgegenüber sind die Süd- und Westschweiz, Südwestdeutschland, Franken und Tirol Gebiete, in denen die ungespannte Artikulation [ $\varepsilon]$ der Normalfall ist, wobei in der Schweiz sogar in morphologisch-semantisch voll durchsichtigen Fällen wie demoralisiert $[\varepsilon]$ vorherrscht. Bemerkenswert ist auch, dass bei diesem Phänomen eine klare innerschweizerische Isoglosse besteht, da in der Ostschweiz in praktisch all diesen Fällen gespanntes [e] üblich ist.

Es konnte anhand der Daten aus dem Medienkorpus bestätigt werden, dass die Variante $[\varepsilon]$ auch bei professionellen Sprechern in Gebrauch ist. In spontansprachlichen Daten, wie sie anhand des Pfeffer- und des FOLK-Korpus ausgewertet wurden, sind $[\varepsilon]$-Formen im Durchschnitt sogar noch in deutlich höherer Frequenz belegt als in den „Deutsch heute“-Daten - was allerdings auch ein Artefakt der regional ungleichmäßigen Verteilung der Aufnahmen sein kann.

Schließlich ließen sich aus dem Vergleich der Variationsmuster der einzelnen Lexeme mehrere innersprachliche, die Variation steuernde Einflussfaktoren herausarbeiten:

1. Semantisch-morphologische Durchsichtigkeit: Mit zunehmender Durchsichtigkeit der Auftaktsilbe steigt die Häufigkeit der [e]-Aussprache, d.h.

13 Becker (1998: 91) führt zur Stützung seiner Argumentation unter anderem ein Zitat aus Klaus Kohlers Einführung in die Phonetik des Deutschen (Kohler 1977: 149) an: „Da die Realisierung als offener oder geschlossener Vokal von einer orthographischen Konsonantenfolge abhängt [...], liegt hier eine typische Schriftaussprache des Aussprachewörterbuchs und derjenigen wenigen vor, die sich mit viel Mühe daran halten. Die wirklich gesprochene Sprache kennt den Unterschied nicht.“ Pikanterweise revidiert Kohler in der 2. Auflage seiner Einführung seine vormalige Meinung praktisch vollumfänglich (Kohler 1995: 142): „[...] haben umfangreiche Korpusuntersuchungen etabliert, daß die in der Hochlautungsdiskussion aufgestellten sog. gespannten Kürzen nicht nur an artifizielle orthographische Konventionen gebunden sind [...], sondern durchaus mit einer lautlichen Differenzierung in der Sprechrealität konform gehen.“ - was Becker (1998) interessanterweise nicht zur Kenntnis genommen hat. Die Sprachdaten, die Kohler zur Änderung seiner Meinung inspiriert haben, dürften indes in wesentlichen Teilen (wie das unter seiner Leitung erhobene Kiel-Korpus) primär norddeutscher Provenienz gewesen sein - und decken sich deshalb gut mit den für diese Region ermittelten Ergebnissen aus dem Korpus „Deutsch heute“. Auf das Deutsche insgesamt übertragbar sind sie, wie die hier durchgeführte Auswertung zeigt, jedoch nicht. 
wenn ein Präfix wie re-/de- vorliegt, der präfigierte Wortstamm zusätzlich auch unpräfigiert existiert und das Präfix eine klare Semantik hat (re,wieder', de- ,Gegenteil').

2. Wortakzentmuster: Folgt der Hauptakzent in der Folgesilbe, begünstigt das die Variante [e], folgt er in der übernächsten oder späteren Silbe, d. h. hat die Auftaktsilbe einen Nebenakzent, wird häufiger $[\varepsilon]$ gesprochen.

3. Folgekonsonanz: Folgender Leniskonsonant (= Tendenz zu offener Silbe) begünstigt die Aussprache als [e], folgender Fortiskonsonant (= Tendenz zu geschlossener Silbe) führt zu mehr [c]-Varianten.

4. Formalitätsgrad: Wortliste vs. Lesetext vs. Spontansprache: Es besteht eine Tendenz zur Zunahme von $[\varepsilon]$ mit abnehmendem Formalitätsgrad der Äußerung.

Auch wenn die vier genannten Einflussfaktoren sicher einen wesentlichen Teil der in den ausgewerteten Daten vorgefundenen Variation erklären können, erhebt die Zusammenstellung keinen Anspruch auf Vollständigkeit.

Es ist letztlich keine große Überraschung, dass sich die sprachliche Realität auch bei der hier untersuchten Variation der Aussprache von <e > in Auftaktsilben als deutlich komplexer erweist, als sie entweder von den Kodizes dargestellt oder von Phonologen - im Wesentlichen aufgrund eigener exemplarischer Beobachtungen - beschrieben wurde (wobei die Annahmen von Becker (1998, 2012) und Vennemann (1991) in ihrem Kern auf jeden Fall zutreffen). Da sich das Duden-Aussprachewörterbuch seit der 7. Auflage (2015) dezidiert an der Sprachrealität orientiert, ergibt sich aus den hier gezeigten Ergebnissen zwangsläufig der Auftrag, in der kommenden Auflage die Variation in den vortonigen Auftaktsilben in weit größerem Umfang als bisher zu berücksichtigen.

\section{Literatur}

AADG = Kleiner, Stefan (2011 ff.): Atlas zur Aussprache des deutschen Gebrauchsstandards (AADG). Unter Mitarbeit von Ralf Knöbl. http://prowiki.ids-mannheim.de/bin/view/ AADG/ (letzter Zugriff: 27.11. 2017).

Becker, Thomas (1998): Das Vokalsystem der deutschen Standardsprache. Frankfurt u. a.: Peter Lang.

Becker, Thomas (2012): Einführung in die Phonetik und Phonologie des Deutschen. Darmstadt: Wissenschaftliche Buchgesellschaft.

Duden 6 (2005) = Duden Band 6 (2005): Das Aussprachewörterbuch. Bearbeitet von Max Mangold. 6. erweiterte Auflage. Mannheim u. a.: Dudenverlag.

Duden 6 (2015) = Duden Band 6 (2015): Das Aussprachewörterbuch. Bearbeitet von Stefan Kleiner, Ralf Knöbl \& Max Mangold. 7. komplett überarbeitete und aktualisierte Auflage. Berlin: Dudenverlag. 
DAW $=$ Krech, Eva-Maria, Eberhard Stock, Ursula Hirschfeld \& Lutz Christian Anders (2009): Deutsches Aussprachewörterbuch. Mit Beiträgen von Walter Haas, Ingrid Hove \& Peter Wiesinger. Berlin, New York: de Gruyter.

Kleiner, Stefan (2014): Die Kodifikation der deutschen Standardaussprache im Spiegel der faktischen Variabilität des Gebrauchsstandards. In Albrecht Plewnia \& Andreas Witt (Hrsg.), Sprachverfall? Dynamik - Wandel - Variation. (= Jahrbuch des Instituts für Deutsche Sprache 2013), 273-298. Berlin, Boston: de Gruyter.

König, Werner (1989): Atlas zur Aussprache des Schriftdeutschen in der Bundesrepublik Deutschland. Band 2: Tabellen und Karten. Ismaning: Hueber.

Kohler, Klaus (1977): Einführung in die Phonetik des Deutschen. Berlin: Erich Schmidt. Kohler, Klaus (1995): Einführung in die Phonetik des Deutschen. 2. neubearb. Aufl. Berlin: Erich Schmidt.

Vennemann, Theo (1991): Syllable structure and syllable cut prosodies in modern Standard German. In Pier Marco Bertinetto, Michael Kenstowicz \& Michele Loporcaro (Hrsg.), certamen phonologicum II. Papers from the 1990 Cortona Phonology Meeting. Torino: Rosenberg \& Sellier. 


\title{
Winifred V. Davies
}

\section{Sprachnormen in der Schule aus der Perspektive der „Critical Language Awareness"}

\begin{abstract}
Das Konzept „language awareness“ (Sprachbewusstheit, Sprachreflexion) ist auch in Deutschland weitverbreitet und kommt in schulischen Richtlinien und theoretischen soziolinguistischen Schriften oft vor. Einige Soziolinguisten wie Norman Fairclough haben aber argumentiert, dass dem Konzept der kritische (soziale, ideologische) Aspekt fehlt und dass es auf einigen Annahmen über sprachliche Variation basiert, die nicht kritisch genug unter die Lupe genommen werden. In diesem Beitrag untersuche ich exemplarisch eine Auswahl an sprachwissenschaftlichen Schriften und schulischen Richtlinien aus Deutschland, um festzustellen, inwiefern Faircloughs Behauptung stimmt. Im Vordergrund stehen zwei geläufige Annahmen über sprachliche Variation: (i) das Angemessenheitsmodell ist weniger normativ als ein Richtigkeitsmodell, und (ii) Standarddeutsch ist unerlässlich für erfolgreiche gegenseitige Verständigung.
\end{abstract}

Keywords: Angemessenheit, Dialekt, gegenseitige Verständigung, kritische Sprachbewusstheit, kritische Sprachreflexion, Sprachbewusstheit, Sprachreflexion, Standarddeutsch

\section{Einleitung}

Den Ausgangspunkt für diesen Beitrag stellen Arbeiten von britischen SoziolinguistInnen wie Norman Fairclough (z. B. Fairclough 1992a), Roz Ivanič (z. B. Ivanič 1988; Janks \& Ivanič 1992) und Marilyn Martin-Jones (z. B. Bhatt \& Martin-Jones 1992) zum Thema „kritische Sprachbewusstheit“ ${ }^{1}$ (critical language awareness, CLA) dar. Der Begriff Sprachbewusstheit bezieht sich auf die ex-

1 In deutschsprachigen Arbeiten gibt es keine eindeutige Entsprechung für „language awareness“. Man findet die Termini Sprachbewusstheit, Sprachbewusstsein und Sprachreflexion, wobei letzterer in Lehr- und Bildungsgplänen relativ oft vorkommt.

Winifred V. Davies, Aberystwyth University, Department of Modern Languages, Aberystwyth, SY23 3DY, Wales, UK, E-Mail: wid@aber.ac.uk

Ә Open Access. (C) 2018 Winifred V. Davies, publiziert von De Gruyter. (c) BY Dieses Werk ist lizenziert unter der Creative Commons Attribution 4.0 Lizenz. 
plizite Lenkung der Aufmerksamkeit der SprachbenutzerInnen auf die Strukturen und Funktionen von Sprache - normalerweise, aber nicht ausschließlich, im Klassenzimmer. Fairclough und andere argumentierten aber, dass diesem Ansatz der kritische Aspekt fehlte, und plädierten in ihren eigenen Arbeiten dafür, die Einbettung von Sprache in einen spezifischen gesellschaftlichen Kontext einschließlich von Machtstrukturen und Ideologien deutlicher hervorzuheben und weitverbreitete theoretische Annahmen über Sprache, Sprachvariation und Kommunikation kritischer zu hinterfragen. In diesem Beitrag wird untersucht, inwieweit dieser Ansatz auch in Deutschland zu finden ist. Aus Platzgründen konzentriert sich der Beitrag auf die Bundesrepublik und die Lage in den anderen deutschsprachigen Ländern bleibt unberücksichtigt (das gilt auch für die ehemalige DDR). Es geht hier übrigens nicht um die Rezeption spezifischer Arbeiten zum Thema CLA in Deutschland, sondern darum, $\mathrm{zu}$ untersuchen, welche Nachweise für eine ähnlich kritische Hinterfragung von theoretischen Annahmen man aufdecken kann. Zwei geläufige und weitverbreitete Annahmen stehen im Fokus des Beitrags: (1) das Angemessenheitsmodell sprachlicher Variation ist weniger normativ als ein Richtigkeitsmodell; (2) erfolgreiche Kommunikation kann nur mittels der Standardsprache gelingen. Eine exemplarische Auswahl an sprachwissenschaftlichen und -didaktischen Schriften wird analysiert, um aufzudecken, wie mit diesen Annahmen umgegangen wird. Weiter werden Richtliniendokumente in Form von Lehrund Bildungsplänen für das Fach Deutsch an bundesdeutschen Gymnasien und den von der deutschen Kultusministerkonferenz herausgegebenen Bildungsstandards für das Fach Deutsch unter demselben Blickwinkel untersucht. Liddicoat (2013: 11) weist darauf hin, dass Richtliniendokumente wie Bildungspläne und -standards Werte und Annahmen über den Gegenstand, mit dem sie sich beschäftigen, enthalten, (re)produzieren und vermitteln. Sie sind in eine bestimmte sprachliche Kultur eingebettet und spiegeln das (dominante) Wertesystem dieser Kultur wider (vgl. Schiffman 1996: 59). Dabei ist nicht zu übersehen, dass der Inhalt der Richtlinien zwar einerseits von den Lehrpersonen in die Praxis umgesetzt werden muss, diese Dokumente andererseits aber nicht von jeder Lehrperson gleich interpretiert und umgesetzt werden (vgl. Liddicoat 2013: 10; Farrell \& Tan Kiat Kun 2007). Manchmal lassen die Dokumente den Lehrpersonen einen gewissen Spielraum, indem der Inhalt relativ abstrakt bzw. vage gehalten wird, manchmal nehmen die Lehrpersonen sich den Spielraum, auch wenn das von den VerfasserInnen der Richtlinien nicht vorgesehen ist. Im Folgenden werden, wie oben schon erwähnt, diese ausgewählten theoretischen Schriften und Richtlinien einer Analyse unterzogen, wohl wissend, dass von diesen Vorlagen nur bedingt auf die Umsetzungspraxis der Lehrenden geschlossen werden kann. 


\section{Zwei Annahmen über Sprache und Sprachvariation}

\subsection{Annahme 1: Das Angemessenheitsmodell ist weniger präskriptiv/normativ und ist sachlicher als ein Richtigkeitsmodell}

\subsection{1 „Angemessenheit“ im sprachwissenschaftlichen Diskurs}

Eine der wichtigsten und am weitesten verbreiteten Annahmen, die von Fairclough (z. B. 1992b) und anderen VerfechterInnen der CLA (z. B. Cameron 2012) einer Kritik unterzogen werden, ist die Annahme, dass das Modell der situativen „Angemessenheit“ von Sprache weniger präskriptiv und sachlicher ist als Modelle, die auf Richtigkeit bzw. Korrektheit basieren. ${ }^{2}$ Fairclough und seine KollegInnen schreiben über die Lage in Großbritannien, das Modell der situativen Angemessenheit von Sprachvariation ist aber auch seit Jahrzehnten in Deutschland bekannt. Barbara Sandig (1973) fasst es wie folgt zusammen:

[D]en richtigen Sprachgebrauch gibt es nicht. Es gibt nur verschiedene Arten von Sprachgebrauch, die als funktionale Stile in bestimmten Kommunikationssituationen kommunikativ angemessen sind und/oder aufgrund sozialer Normen erwartet werden. (Sandig 1973: 33)

Dieser Ansatz basiert auf der Differenz-Konzeption sprachlicher Variation, die im Gegensatz zur Defizit-Hypothese besagt, dass alle Varietäten, solange nicht das Gegenteil bewiesen ist, in ihren Ausdrucksmöglichkeiten und in ihrer logischen Analysekapazität einander funktional äquivalent sind (vgl. Dittmar 1980: 128). Gleichzeitig können die VerfechterInnen dieser Konzeption nicht übersehen, dass Varietäten von der Gesellschaft unterschiedlich bewertet werden. Dittmar (1980) verlangt ein kritisches Herangehen an diese sozialen Bewertungen, z. B. die feste Überzeugung, dass regionale, nicht standardsprachliche Varietäten eher in informellen, privaten und/oder nicht-offiziellen Domänen angemessen sind, und die Anerkennung der sozio-historischen Bedingungen, durch die eine bestimmte Auswahl an sprachlichen Formen und Praktiken als Standardsprache legitimiert und institutionalisiert wurde (vgl. Dittmar 1980: 128-131). Auch Cameron (2012) kritisiert den oft unkritischen

2 Vgl. Cameron (2012: 235). 
Gebrauch des Angemessenheitsdiskurses von seiten einiger VertreterInnen der Differenz-Konzeption:

the way [they] use the language of appropriateness has the effect of treating norms as facts, of obscuring their contingency and thus of blunting critical responses to them. The alternative is to make clear that while norms materially affect people's behaviour [...], these norms are open to challenge and to change. (Cameron 2012: 235)

\subsection{2 „Dialekt als Chance“?}

Seit den 1980er Jahren findet man in der deutschen Sprachwissenschaft Bemühungen, die früher (auch unter Fachleuten) weit verbreitete Vorstellung von „Dialekt als Problem“ durch das positiver konnotierte Konzept von „Dialekt als Chance“ zu ersetzen. Klotz \& Sieber (1993) beschreiben diese Entwicklung wie folgt:

In den siebziger Jahren standen die Diskussionen um Dialektdidaktik weitgehend unter der Forderung, die Nachteile, welche Dialektsprecher gegenüber den anderen Schülern hätten, mit unterrichtlichen Arrangements zu kompensieren bis hin zur radikalen Forderung der „Ersetzung des Dialekts durch die Einheitssprache“ [...]. Demgegenüber sind in die neuere Diskussion Aspekte hinzugekommen, die Dialektvoraussetzungen nicht einfach negativ als Handicap bestimmen, sondern auch nach Chancen und Bildungsmöglichkeiten suchen, die mit der Verfügungskompetenz über dialektale Register verbunden sind [...]. (Klotz \& Sieber 1993: 8)

Aus dieser Perspektive sind nichtstandardsprachliche Varietäten als Ressource anstatt als Problem zu betrachten. In der Regel bedeutet dies: (i) Der regionale Dialekt als identitätsstiftender Faktor, der Auskunft über die regionale und soziale Herkunft der Sprechenden gibt und verwendet werden kann, um Solidarität mit anderen Menschen aus demselben Ort/von derselben sozialen Gruppierung zu zeigen; (ii) Mehrsprachigkeit als wertvolle Ressource: SprecherInnen einer nichtstandardsprachlichen Varietät haben ein zusätzliches Register, das in einigen Situationen angemessener ist als die Standardsprache (vgl. Bücherl 1993: 72-76).

\subsubsection{Dialekt doch als „Problem“?}

In Deutschland reden LaienlinguistInnen aber nach wie vor von „Dialekt als Problem“, wobei nicht immer klar ist, was sie konkret unter „Dialekt“ verstehen. Im Sprachgebrauch von Laien kann sich der Begriff sowohl auf eine Varietät, 
die ExpertInnen als Basisdialekt bezeichnen würden, beziehen, als auch auf eine Sprachlage, die sie als standardnahen Regiolekt einstuften. Ein paar neuere Beispiele für „Dialekt als Problem“ sind Maas (2013) und von Wrangel (2005), die über die „Probleme“ von DialektsprecherInnen in Schwaben berichten, die Kurse bei der Sprecherzieherin Ariane Willikonsky belegen, um Hochdeutsch $\mathrm{zu}$ beherrschen.

Obwohl man, wie Klotz \& Sieber zu Recht behaupten, auch in der älteren Fachliteratur Hinweise auf regionale, nichtstandardsprachliche Varietäten bzw. Varianten als „Problem“ findet, ist diese Perspektive in der Sprachwissenschaft und Sprachdidaktik nicht mehr geläufig. Wenn nichtstandardsprachliche Varietäten in der aktuellen sprachwissenschaftlichen Literatur doch als problematisch beschrieben werden, dann wird deutlich zwischen Dialekt als sozialem Problem (z. B. negative Attitüden seitens einflussreicher Gatekeeper) und Dialekt als sprachlichem Problem unterschieden und es werden Forschungsergebnisse berücksichtigt, die zeigen, dass der Dialektgebrauch nicht pauschal als problematisch betrachtet werden kann. Rosenberg (1993: 24) stellt fest: „Nicht, ob jemand Dialekt spricht, ist also das Problem, sondern ob jemand nur (oder fast nur) Dialekt spricht!“ In der sprachwissenschaftlichen Literatur finden wir auch eine begriffliche Schärfe, die im Diskurs von NichtexpertInnen oft fehlt, d.h. der Begriff „Dialekt“ wird explizit definiert und nicht undifferenziert für den ganzen Varietätenraum zwischen Standardsprache und Basisdialekt verwendet.

NichtlinguistInnen nehmen oft an, dass Menschen, die eine nichtstandardsprachliche Varietät zuerst erworben haben, Probleme beim Erwerb der Standardsprache haben, vor allem in deren schriftlicher Form, weil sie Interferenzfehler machen. SprachwissenschaftlerInnen haben aber gezeigt, dass nicht alle Probleme auf diese Sprechergruppe beschränkt sind. Obwohl Ammon (1982: 36) noch die Position vertritt, dass: „Die Einheitssprache sich unmittelbarer und leichter in die geschriebene Sprache umsetzen läßt“, weist Barbour (1987: 234) hingegen schon darauf hin, dass nicht einmal die Sprachformen von hoch gebildeten und sozial erfolgreichen MittelschichtsprecherInnen hundertprozentig den Regeln der geschriebenen Standardsprache entsprechen. Auch Rosenberg zeigt schon 1986, dass sowohl SchülerInnen aus Hannover, die Standarddeutsch sprechen, als auch dialektsprechende SchülerInnen aus Berlin Fehler in einem Testdiktat machen, und er folgert daraus, dass „[d]er Bereich, der unter den Verschlußlauten am häufigsten zu Fehlern führt, [...] die Stimmhaftigkeitskorrelation [ist]. Diese Schwierigkeiten sind allgemeiner umgangssprachlicher Natur“ (Rosenberg 1986: 222). Inzwischen liegen viel mehr Arbeiten zum gesprochenen Deutsch vor (z. B. Schwitalla 2012; Schneider 2016), was dazu geführt hat, dass heute stärker zwischen (vermeintlichen) 
Fehlern im Schriftdeutschen, die als Interferenz aus anderen Varietäten ausgelegt werden können, und (vermeintlichen) Fehlern, die „typisch“ für gesprochenes Deutsch sind, differenziert werden kann.

\subsubsection{Kritik des Angemessenheitsmodells?}

Auch wenn inzwischen viele sprachwissenschaftliche Studien vorliegen, die der Vorstellung von „Dialekt“ als problematisch und minderwertig entgegenwirken, kann trotzdem nicht behauptet werden, dass das Angemessenheitsmodell ernsthaft in Frage gestellt wurde. Die sozialen Strukturen und die Ideologien, die die Angemessenheitsurteile über verschiedene sprachliche Varietäten untermauern (,nichtstandardsprachliche Varietäten sind nur in weniger prestigereichen, d. h. nicht formellen, nicht öffentlichen, nicht offiziellen Situationen, angemessen“3) werden selten einer ausführlichen Kritik unterzogen. Auch Rosenberg, der dialektsprechende Kinder fördern will, schreibt:

Didaktisches Ziel [d.h. des Deutschunterrichts, W. D.] [muss] eine funktionale „innere Mehrsprachigkeit“ sein, die Kinder zur situationsadäquaten Varietätenverwendung [erzieht], ohne ihnen ihre dialektale Primärvarietät austreiben zu wollen. (Rosenberg 1993: 16)

Diese Ansicht ist nicht untypisch. Rosenberg weist weiter darauf hin, dass die meisten Bemühungen, den Dialektgebrauch auf weitere Domänen auszudehnen, gescheitert sind (Rosenberg 1993: 27, 45). Er behauptet auch, das Konzept einer „funktionalen Mehrsprachigkeit“, d. h. die Verteilung von Varietäten nach Funktion (= das Angemessenheitsmodell), sei relativ verbreitet (im Vergleich zu den 1970er Jahren), meint aber, dass sich dieses theoretische Konzept kaum auf die alltägliche Praxis der Lehrenden ausgewirkt habe (Rosenberg 1993: 47).

Rosenberg (1993) zeigt eine bestimmte Ambivalenz, was Standardsprache und Dialekte betrifft. Einerseits schreibt er: „Selbstverständlich sollte die normale Unterrichtssprache des Deutschlehrers die Standardsprache sein“ (Rosenberg 1993: 76), gleich danach aber schreibt er: „Unter dem Gesichtspunkt des situativen Sprachgebrauchs muß Dialekt im Unterricht als ein positives ,Mehr“ begriffen werden“ (Rosenberg 1993: 76). Die „funktionale Mehr-

3 Obwohl klar ist, dass Prestige kein eindimensionales Konzept ist (vgl. Milroy 1999: 37-39) und nichtstandardsprachliche Varietäten kaum überlebt hätten, wenn sie nicht auch einen bestimmten Grad an Prestige unter ihren SprecherInnen genießen würden, ist weithin unstrittig, dass die Domänen, die mit Macht assoziiert werden, im Großen und Ganzen der Standardsprache vorbehalten sind. 
sprachigkeit“ (Rosenberg 1993: 46) wird als erzieherisches Ziel vorgestellt, das helfen könnte, das sprachliche Selbstbewusstsein von DialektsprecherInnen zu stärken; es ist aber schwer zu begreifen, wie er als ein „Mehr“ präsentiert werden kann, wenn er in der Praxis als nur in nicht prestigereichen und weniger wichtigen Situationen angemessen angesehen wird und wenn klar ist, dass SchülerInnen, die Standarddeutsch sprechen, ihren Sprachgebrauch nicht oder nicht im selben Grad anpassen müssen. Es kann sein, dass StandardsprecherInnen in einigen Situationen darunter leiden, dass sie keinen Dialekt sprechen, es ist aber viel unwahrscheinlicher, dass sie deswegen finanziell oder materiell leiden. Diese „Lücke“ in ihrem Repertoire wird kaum negative Folgen für ihren materiellen Wohlstand haben, umgekehrt aber können Menschen, die Standarddeutsch nicht beherrschen, Benachteiligungen erfahren. ${ }^{4}$ Beim Ansatz „Dialekt als Chance“ wird normalerweise davon ausgegangen, dass die Standardsprache auch zum Repertoire der DialektsprecherInnen gehört bzw. gehören soll; umgekehrt wird selten vorgeschlagen, dass Menschen, die nur Hochdeutsch sprechen, ihr Repertoire dadurch erweitern sollten, dass sie einen Dialekt lernen sollten. ${ }^{5}$

\subsection{5 „Angemessenheit“ in schulischen Richtlinien}

Für diesen Beitrag wurden alle aktuellen Lehrpläne für das Fach Deutsch an Gymnasien in Deutschland durchgesehen. ${ }^{6}$ Dabei hat sich gezeigt, dass alle auf dem Angemessenheitsmodell sprachlicher Variation basieren und darauf hinweisen, dass SchülerInnen lernen sollten, verschiedene Varietäten (,,Sprachen in der Sprache“, wie dies in den nationalen Bildungsstandards für die Sekundarstufe I bezeichnet wird) „angemessen“ $\mathrm{zu}$ verwenden (Termini wie „situationsgerecht/-angemessen/-gemäß/-bezogen“ oder ,adressatengerecht/ -bezogen“ kommen auch vor). In den Bildungsstandards im Fach Deutsch für

4 Vgl. Hommel (2015).

5 Die Lage in anderen deutschsprachigen Ländern, vor allem in der Schweiz, ist sicher anders: In der Schweiz zum Beispiel gibt es Sprachkurse, in denen man einen schweizerdeutschen Dialekt lernen kann, z. B.: https://www.klubschule.ch/Angebote/Sprachen/Deutschkurse/ Schweizerdeutsch (letzter Zugriff: 11.1. 2018).

6 Die einzelnen Bundesländer sind für den Inhalt der Lehrpläne und die Zulassung von Schulbüchern zuständig, obwohl die Kultusministerkonferenz, die Ständige Konferenz der BildungsministerInnen der Länder, für das notwendige Maß an Gemeinsamkeit in Bildung, Wissenschaft und Kultur in Angelegenheiten von länderübergreifender Bedeutung sorgt. Sie verfasste z. B. die Bildungsstandards für den Mittleren Schulabschluss und die Bildungsstandards für die Allgemeine Hochschulreife: https://www.kmk.org (letzter Zugriff: 24. 5. 2017). 
die allgemeine Hochschulreife (2014: 15) steht: „Die Schülerinnen und Schüler handeln in persönlichen, fach- und berufsbezogenen und öffentlichen Kommunikationssituationen angemessen und adressatengerecht"; die meisten Lehrund Bildungspläne geben diesen Inhalt entweder mit denselben oder mit ähnlichen Worten wieder, z. B. im Curriculum für das Gymnasium in Niedersachsen (2009: 14): „Die Schülerinnen und Schüler [...] äußern sich in komplexen Kommunikationssituationen sachangemessen und artikuliert, situations- und adressatengerecht.“

Interessanterweise gab es schon in früheren Fassungen von Lehrplänen konkretere Hinweise auf die angemessene Verteilung von Dialekt und Standardsprache mit ganz konkreten Beispielen für ,angemessenen“ Gebrauch, z. B.:

Die Schüler und Schülerinnen erkennen die Bedeutung der Standardsprache für die überregionale Verständigung und den Eigenwert der Mundart als regional begrenzter Sprachform. (Bildungsplan für das Gymnasium Baden-Württemberg. Deutsch 1994: 428)

Hier werden die Standardsprache und der Dialekt als funktional klar getrennte Varietäten mit ganz unterschiedlichen Kommunikationsradien dargestellt. Im selben Dokument steht weiter:

Inhalt: Mundart und Standardsprache; Großgliederung des deutschen Sprachgebiets; Funktionen der Mundart (Zusammengehörigkeitsgefühl, Gefühlswerte, Anschaulichkeit); Funktionen der Standardsprache (in Staat und Verwaltung, Handel und Verkehr, Wissenschaft, Literatur, Medien). (Bildungsplan für das Gymnasium Baden-Württemberg. Deutsch 1994: 428)

Die Komplementärverteilung auf den öffentlichen/privaten und den formellen/ informellen Bereich wird anhand von konkreten Beispielen veranschaulicht, was wir im aktuellen Bildungsplan für das Gymnasium in Baden-Württemberg (2016: 31) nicht finden. Hier steht viel abstrakter: „Die Schülerinnen und Schüler können [...] sprachliche Äußerungen mündlich und schriftlich situationsangemessen und adressatengerecht formulieren (zum Beispiel Gesprächsbeiträge)." Es wird anscheinend den Lehrkräften mehr Freiraum bei der Entscheidung darüber, woraus „angemessene“ Äußerungen bestehen, belassen. Ähnliche Formulierungen finden sich in allen analysierten Lehrplänen. Wie oben schon angedeutet, können amtliche Richtlinien von den Lehrpersonen, die sie in die Praxis umsetzen sollen, unterschiedlich ausgelegt werden, und es ist wahrscheinlich, dass Lehrkräfte die Angemessenheit verschiedener Varietäten durch die Brille ihrer eigenen Biographie und des lokalen Kontexts bewerten (vgl. Davies 2011: 29). Die meisten Lehrkräfte, die ich für die in Davies (2000) beschriebene Studie befragte, hielten es nicht für wünschenswert, den Gebrauch des Dialekts in weiteren Domänen zu fördern (vgl. oben, Rosenberg 1993), und hinterfragten die traditionelle Domänenverteilung zwischen Stan- 
dardsprache und Dialekt nicht. Sie waren der Meinung, der Dialekt sei nur für bestimmte stereotype Gesprächsthemen (z. B. Mundartdichtung) oder für Sprechereignisse, die am Rand des ,wirklichen“ Unterrichts stattfinden (z. B. persönliche Gespräche) angemessen oder für Themen, die als weniger kopflastig angesehen werden (sexuelle Aufklärung wurde als Beispiel angeführt). Im folgenden Abschnitt werden wir sehen, dass Materialien, die an Schulen verwendet werden sollen, diese Verteilung heute noch kaum hinterfragen. Ich kann mich nicht auf eine vollständige und repräsentative Analyse von Schulbüchern berufen, verweise aber auf Maitz (2015), der zeigt, dass der Dialektgebrauch - sogar in Bayern, das als Hochburg des Dialekts betrachtet wird - in den Schulbüchern sehr oft ziemlich pauschal als unangemessen abgetan wird. Eine Lehrkraft, die die konventionelle situative Verteilung von Dialekt und Standardsprache hinterfragen wollte, fände in diesen Schulbüchern keine Stütze.

\subsection{6 „Angemessenheit“ in Schulbüchern}

Wie wir gerade gesehen haben, wird der Gebrauch von nichtstandardsprachlichen Varietäten immer noch meistens in einigen stereotypen Situationen als angemessen angesehen. Dies wird von Maitz (2015) bestätigt. Seine Durchsicht von Lehrbüchern, die im Deutschunterricht an bayerischen Schulen gebraucht werden, zeigt:

Wenn Dialekte überhaupt behandelt werden, so handelt es sich in den allermeisten Fällen um Dialektgedichte, Witze/Comics, Märchen oder Lieder, die dann von den Schülern ins Standarddeutsche übersetzt werden sollen. (Maitz 2015: 213)

Es ist enttäuschend, dass sich anscheinend seit Neulands (1979) Untersuchung von Schulbüchern wenig geändert hat. Es ist plausibel, dass solche Darstellungen bei SchülerInnen den Eindruck erwecken bzw. konsolidieren, dass sprachliche Variation innerhalb der deutschen Sprache hierarchisch strukturiert ist und gewisse Varietäten, vor allem die Standardsprache, angesehener und erwünschter sind als andere. Wenn diese Situation mit Hinweis auf die Maxime der situativen Angemessenheit begründet wird und die ungleichen Machtverhältnisse zwischen verschiedenen Varietäten und deren SprecherInnen nicht angesprochen werden, fehlt der kritische Aspekt der Sprachbewusstheit/Sprachreflexion.

\subsubsection{Zwischenfazit zu Annahme 1}

Aus der Perspektive der CLA ist der Angemessenheitsdiskurs kritikwürdig, weil er ein Lippenbekenntnis zum Liberalismus und zum Pluralismus ablege (,,alle 
Varietäten, auch nichtstandardsprachliche, haben ihren Platz“), in Wahrheit jedoch die Dominanz von Standardvarietäten unangetastet lasse (vgl. Fairclough 1992b: 43). Gesellschaftliche Unterschiede würden unter den Teppich gekehrt, indem davon ausgegangen werde, dass es einen gesellschaftlichen Konsens darüber gebe, welche Varietät in welcher Situation angemessen sei. Ein kritischer Ansatz würde expliziter darauf hinweisen, dass einige SprecherInnen mehr als andere von den Normen für „angemessenes“ sprachliches Verhalten profitieren (vgl. Bourdieu 1991: 21; Tollefson 1991: 77). In den oben analysierten Schriften und Richtlinien kommt das Modell zwar oft vor (alle Lehrpläne stützen sich darauf), ich habe aber keine Hinweise auf das, was Sealey (1999: 88) „the non-consensual dimensions of language“ nennt, gefunden.

\subsection{Annahme 2: Erfolgreiche gegenseitige Verständigung kann nur mittels der Standardsprache erreicht werden}

\subsubsection{Hintergrund}

Um den dominanten Status und die „Angemessenheit“ der Standardsprache in öffentlichen/formellen/offiziellen Situationen zu legitimieren, beruft man sich regelmäßig auf die größere kommunikative Reichweite dieser Varietät und auf deren Notwendigkeit für die gegenseitige überregionale Verständigung, wie z. B. im Vorwort der 6. Auflage des Duden 9. Richtiges und gutes Deutsch (2007: 5): „[u]nsere Gesellschaft [kommt] nicht ohne eine normativ geregelte Standardsprache aus. Diese ist eine unverzichtbare Grundlage für die allgemeine Verständigung“. ${ }^{7}$ Die Wichtigkeit der Standardsprache für die allgemeine Verständigung wird aber oft eher behauptet als nachgewiesen, wie in diesem Abschnitt besprochen wird. Laut Coupland (2000: 632) fällt es sogar SprachexpertInnen schwer, sich von Perspektiven und Ansätzen zu befreien, die von der Existenz einer (schriftlichen) Standardvarietät als Leitvarietät in vielen, wenn auch nicht allen europäischen Gesellschaften geprägt sind. Vielleicht ist deswegen die implizite Akzeptanz von Standardvarietäten als „natürlicher“ bzw. „nötiger“ soziolinguistischer Realität auch von SprachwissenschaftlerInnen relativ selten hinterfragt worden (vgl. Coupland 2000: 632). Im deutschen Kontext sehen wir zum Beispiel, dass man sich relativ selten kritisch mit der Annahme, gegenseitige Verständigung könne nur mittels der Standardsprache

7 Interessanterweise steht dieser Satz nicht mehr in der jüngsten Auflage von 2016. 
gesichert werden, auseinandergesetzt hat. ${ }^{8}$ Im Gegenteil wird die Notwendigkeit der allgemeinen Verständigung oft als Grund angeführt, warum man die Standardsprache beherrschen muss. Ein Beispiel ist das Vorwort des Duden 9, das oben zitiert wurde; ein zweites finden wir bei Bayer (1984):

Die Fähigkeit zum verständlichen, präzisen und situationsangemessenen expliziten Ausdruck von Gedanken und zu entsprechendem Verstehen ist eine der Voraussetzungen für die Teilnahme an politischen und kulturellen Prozessen, die in einem demokratischen Staat unabdingbar notwendig ist, und nicht zuletzt auch für die sprachliche Bewältigung einer Vielzahl alltäglicher Situationen und für berufliches Fortkommen.

Eine überregionale, syntaktisch und semantisch ausgebaute Standardsprache ist Voraussetzung für die in einem pluralistischen demokratischen Staat notwendige Kritik und Verständigung zwischen den einzelnen Gruppierungen (Altersgruppen, Parteien, Verbänden, usw.).

Wenn es auch außer Zweifel steht, daß Dialekte, Schicht- und Gruppensprachen v.a. wichtige emotionale und beziehungsstiftende Funktionen haben und der Standardsprache in speziellen Teilbereichen sogar überlegen sein können, so kann unter demokratischer Zielsetzung dennoch auf die kulturschließende und integrative Funktion der Standardsprache nicht verzichtet werden. Dialekte, Schicht- und Gruppensprachen sind als Ergänzungen zur Standardsprache (auch im Unterricht) nützlich und wünschenswert; sie können diese aber nicht ersetzen, ohne daß die Gesellschaft in eine große Zahl partikulärer Gruppen ohne die Möglichkeit differenzierter gegenseitiger Verständigung zerfällt. (Bayer 1984: 318-319, Hervorhebung W. D.)

Geiger (2009), Kratzer (2012), Maitz (2015) und Maitz \& Foldenauer (2015) liefern weitere Beispiele. In den von Maitz \& Foldenauer (2015) untersuchten bayerischen Schulbüchern für das Fach Deutsch wird der Dialekt oft als Quelle kommunikativer Probleme dargestellt, die nur durch das Übersetzen in die Standardsprache gelöst werden können. Kratzer (2012) berichtet über den Steuerberater Markus Zwicklbauer, der, als er zu Gast bei der ARD-Talkshow „Hart, aber fair“ auftrat, seine Argumente in einem Deutsch mit (meiner Meinung nach „leichter") bairischer Färbung vortrug. Der Moderator Frank Plasberg forderte ihn aber auf, Hochdeutsch zu sprechen, damit die ZuschauerInnen ihn verstünden.

\subsubsection{Welche Faktoren beeinflussen die Verständnissicherung?}

Anhand der gerade besprochenen Beispiele erkennen wir zwei Problemkomplexe, mit denen man sich kritischer auseinandersetzen könnte. Erstens wäre es sicher angebracht, der subjektiven Dimension der Verständigung bzw. der

8 Ich beziehe mich hier wieder nur auf Deutschland und bin mir bewusst, dass diese Behauptung sicher nicht pauschal für den ganzen deutschsprachigen Raum gilt. 
Verständnissicherung mehr Aufmerksamkeit zu schenken, und zweitens wird angenommen, dass die Standardsprache eine Varietät sei, die von allen SprecherInnen problemlos verstanden werde. König (1978: 135) weist aber auf Studien hin, die feststellten, dass Nachrichtensendungen in Standarddeutsch nicht von allen ZuhörerInnen verstanden wurden bzw. nur teilweise verstanden wurden (vgl. auch Maitz 2015: 214). Ammons (1979: 36) Annahme, dass man mit Standarddeutsch „mühelose Verständigung im ganzen deutschen Sprachgebiet“ erreichen könne, legt auch nahe, dass die Standardsprache eine Art monolithische und neutrale Varietät sei und dass die Bedeutungen von Begriffen wie Freiheit, Demokratie, Sozialismus, die in Wörterbüchern der Standardsprache stehen, einem gesellschaftlichen Konsens unterliegen, anstatt dass sie die Interessen von bestimmten Gesellschaftsgruppen widerspiegeln und mit anderen Bedeutungen konkurrieren. Wachs (1982) hat ganz früh diese Annahme des überregionalen kommunikativen Radius der Standardsprache kritisch hinterfragt:

Selbst der zunächst plausible Verweis auf die überregionale Reichweite der Standardvarietät wirkt wenig überzeugend, da Ammon den Fehlschluß macht, den regional begrenzten Geltungsbereich der Dialekte mit deren kommunikativer Reichweite gleichzusetzen. Diese Sichtweise basiert zum einen auf der Annahme, daß Dialekt und Standard geschlossene Systeme darstellen, darüber hinaus werden die Variationsbreite der Sprecher und ihre Verstehensbereitschaft vernachlässigt [...]. (Wachs 1982: 332)

Sie bringt hier zwei wichtige Argumente an: Erstens sind Standarddeutsch und die Dialekte keine geschlossenen Systeme, und sehr oft bewegen sich die SprecherInnen entlang eines Kontinuums zwischen Dialekt und formellem Standard, abhängig von der Situation (vgl. Bellmann 1983; Durrell 1999). Zweitens weist das Wort „Verstehensbereitschaft“ auf die Rolle subjektiver Faktoren (Einstellungen) bei der erfolgreichen Verständigung. Die Wichtigkeit der Verstehensbereitschaft [Hervorhebung W. D.] wird des weiteren durch Wolffs (1959) Arbeit in Nigeria sowie Haugens (1966) Untersuchungen in Skandinavien untermauert. Wolff (1959: 35-39) beobachtete, dass SprecherInnen von Varietäten, die sprachlich engstens verwandt waren, trotzdem oft behaupteten, sie verstünden die jeweils andere Varietät nicht, wobei SprecherInnen der prestigereicheren Varietät eher dazu tendierten zu behaupten, die andere Varietät nicht zu verstehen als umgekehrt. Haugen (1966: 280) stellt fest, dass der Wille zu verstehen eine zentrale Rolle bei der Überwindung von Verständigungsproblemen zwischen SprecherInnen unterschiedlicher Varietäten spielt. Bourdieu bekräftigt dies und legt auch den Einfluss von sozialen Faktoren offen, wenn er bemerkt, dass nicht jedem aufmerksam zugehört wird, unabhängig davon, wie kompetent er sich ausdrückt: 
The competence adequate to produce sentences that are likely to be understood may be quite inadequate to produce sentences that are likely to be listened to, likely to be recognised as acceptable in all the situations in which there is occasion to speak. [...] social acceptability is not reducible to mere grammaticality. (Bourdieu 1991: 54-44)

Dies unterstreicht die außersprachlichen Aspekte der Verständigung, die bei der Diskussion über die gegenseitige Verständlichkeit zwischen Varietäten und darüber, welche Bedingungen die erfolgreiche Kommunikation erfüllt, nicht vergessen werden dürfen.

Dabei darf nicht unerwähnt bleiben, dass einige SprachwissenschaftlerInnen der Meinung sind, dass der Beitrag von außersprachlichen Faktoren zum Gelingen der Kommunikation zu hoch eingeschätzt wird, z. B. Lesley Milroy (1984). Sie zeigt in diesem Aufsatz, dass der sprachliche und außersprachliche Kontext den Sprechenden nicht immer hilft, kommunikative Hürden zu überwinden, auch wenn der Wille zu verstehen existiert. Die Frage, wie viel Einfluss außersprachliche Faktoren wie Einstellungen auf die Verständlichkeit ausüben, beschäftigt ForscherInnen immer noch, z. B. das Projekt von Charlotte Gooskens an der Universität Groningen: „Mutual intelligibility of closely related languages in Europe: linguistic and non-linguistic determinants“. ${ }^{9}$ Die Ergebnisse von Studien mit SprecherInnen von skandinavischen Sprachen sind nicht eindeutig und widersprechen sich zum Teil (vgl. Schüppert, Haug Hilton \& Gooskens 2015; Schüppert \& Gooskens 2011). Wie Schüppert, Haug Hilton \& Gooskens (2015: 213) schreiben, ,the causal relationship of attitude and intelligibility is still a matter of discussion." Wir sollten deshalb potenzielle Kommunikationsprobleme zwischen SprecherInnen verschiedener Varietäten nicht vorschnell herunterspielen, ich würde aber argumentieren, dass wir die Annahme, dass alle DeutschsprecherInnen die Standardvarietät problemlos verstehen, auch kritisch hinterfragen und empirisch untersuchen sollten, anstatt sie einfach als gegeben bzw. als common sense vorauszusetzen.

\subsubsection{Schulische Richtlinien}

In den Bildungsstandards im Fach Deutsch für den Mittleren Schulabschluss (2004: 8) finden sich folgende Sätze: „[Die Schülerinnen und Schüler] benutzen die Standardsprache. Sie achten auf gelingende Kommunikation und damit auch auf die Wirkung ihres sprachlichen Handelns“. In den Bildungsstandards im Fach Deutsch für die Allgemeine Hochschulreife (2014: 20) steht im

9 www.let.rug.nl/gooskens/?p=project (letzter Zugriff: 14.9. 2017). 
Abschnitt 2.5 „Sprache und Sprachgebrauch reflektieren“: „Die Schülerinnen und Schüler können [...] Bedingungen gelingender Kommunikation analysieren, auch auf der Basis theoretischer Modelle“. Es ist lobenswert, dass SchülerInnen dazu angeregt werden sollen, darüber nachzudenken, wie man eine gelingende Kommunikation erzielt. Die Nebeneinanderstellung von „Standardsprache benutzen“ und „gelingende Kommunikation“ legt aber nahe, dass die zwei Konzepte für die AutorInnen untrennbar miteinander verbunden sind, was auf eine relativ restriktive Vorstellung von gelingender Kommunikation verwiese.

Wie wir im Bourdieu-Zitat oben gesehen haben, spielen außersprachliche Faktoren anscheinend eine wichtige Rolle in der Kommunikation, und es wird den Äußerungen einiger sozialer Gruppen mehr Aufmerksamkeit geschenkt als anderen, auch wenn jede Gruppe die „richtigen“ bzw. „korrekten“ sprachlichen Formen verwendet. Folglich genügt es nicht, das Konzept der gelingenden Kommunikation nur unter sprachlichen Aspekten zu besprechen. Was die sprachlichen Aspekte angeht: In der Schule wie in der Gesellschaft allgemein wird davon ausgegangen - manchmal nur implizit - dass die Beherrschung der Standardsprache zur Grundlage des kommunikativen Erfolgs gehört und dass ein Repertoire ohne Standardsprache ein unvollständiges ist. Deshalb gehört die Vermittlung einer „richtigen“ bzw. „korrekten“ Standardsprache immer noch zu den wichtigsten Aufgaben des Deutschunterrichts, auch wenn Konzepte wie „situationsgerecht“ bzw. „-angemessen“ inzwischen auch ihren festen Platz in den Curricula haben (vgl. Davies \& Langer 2014). Wie wir oben bei der Diskussion zum Thema Dialekt als Chance sahen, kommt selten jemand auf die Idee, ernsthaft dafür zu plädieren, dass ein Repertoire auch durch den Erwerb eines nichtstandardsprachlichen Dialekts bereichert werden könnte. Die US-amerikanische Soziolinguistin Rosina Lippi-Green (2012: 71-74) spricht von Kommunikation als kollaborativem Vorgehen, das am besten funktioniere, wenn alle Gesprächsteilnehmenden aktiv daran mitwirkten und bereit seien, ihren Teil der Kommunikationslast („,communicative burden“) zu übernehmen. Sie argumentiert, es sei unfair und ungerecht zu verlangen, dass sich einige soziale Gruppen (z. B. DialektsprecherInnen) mehr anstrengen sollten als andere, indem erwartet wird, dass sie sich an den Sprachgebrauch der StandardsprecherInnen anpassen, aber nicht umgekehrt. Andersson \& Trudgill (1990: 170) schlagen im britischen Kontext vor, die Schulen könnten einen Beitrag zur faireren Verteilung der kommunikativen Last leisten, indem sie im Unterricht nicht nur auf die kommunikative Rolle der Akkommodation an die GesprächspartnerInnen weisen, sondern auch indem sie die passive Dialektkompetenz aller SchülerInnen ausbauen. Akkommodation braucht nicht unbedingt $\mathrm{zu}$ bedeuten, dass SprecherInnen in eine andere Varietät switchen, sondern könnte auch dadurch erreicht werden, dass sie langsamer sprechen und 
einige Schnellsprechformen (z. B. Elisionen und Assimilationen) vermeiden, wenn sie sich mit GesprächspartnerInnen, die eine andere Varietät sprechen, unterhalten (vgl. Andersson \& Trudgill 1990: 170). Auch in Deutschland könnte der Ausbau der passiven Dialektkompetenz u. a. dadurch erzielt werden, dass SchülerInnen sich Hörbeispiele in verschiedenen Akzenten und Dialekten anhören.

Sieber \& Sitta (1986: 82, 172) sind der Meinung, Lehrende könnten SchülerInnen zu mehr Toleranz erziehen, wenn sie im Unterricht Sprachverständlichkeitsnormen anstelle von Sprachrichtigkeitsnormen vermittelten. Damit das gelingt, müssen die Lehrenden den SchülerInnen auch vermitteln, dass Verständlichkeit nicht nur durch die Auswahl der „richtigen“ bzw. „passenden“ sprachlichen Varianten erzielt wird, sondern von psychologischen und sozialen Faktoren abhängt. Die Bedeutung außersprachlicher Faktoren für eine gelingende Kommunikation wurde in diesem Beitrag schon mehrmals angesprochen. LippiGreen (2012: 324-326) beschreibt Studien, die zeigen, wie schwer sie wiegen können. Sie berichtet z. B. über eine Fallstudie in den USA, die aufdeckte, dass auch wenn Menschen amerikanisches Standardenglisch sprechen, sie trotzdem diskriminiert werden, wenn sie Mitglieder bestimmter sozialer bzw. ethnischer Gruppen sind. Noch beunruhigender sind die Ergebnisse einer anderen Studie: ZuhörerInnen bildeten sich ein, SprecherInnen hätten einen nichtstandardsprachlichen amerikanischen Akzent, nachdem ihnen gesagt wurde, die SprecherInnen seien aus Asien. Als sie denselben Akzent hörten und glaubten, die SprecherInnen seien KaukasierInnen, nahmen sie keinen nicht-standardsprachlichen/nichtmuttersprachlichen Akzent wahr (vgl. Lippi-Green 2012: 9296). ${ }^{10}$

\subsubsection{Zwischenfazit zu Annahme 2}

Die gerade besprochenen Studien zeigen, dass gelingende Kommunikation ein komplexes Phänomen ist, das nicht in einem Leerraum stattfindet, sondern immer in gesellschaftlichen Kontexten, die durch ungleiche Macht- und Prestigeverteilung und individuelle und kollektive Vorurteile gekennzeichnet sind. Selbst (gesprochene) Standardvarietäten sind kontextgebunden: Auch wenn sie häufig als stilistisch und regional neutral beschrieben werden (z. B. Klein 2013: 29), nehmen die SprecherInnen sie nicht unbedingt auch so wahr. Bei einigen Sprechergruppen und/oder in bestimmten Regionen können die Stan-

10 Es wäre bestimmt interessant, solche Studien auch in anderen Sprachgemeinschaften zu replizieren. 
dardvarietät bzw. als standardorientierte wahrgenommene Varietäten als hochgestochen, gezwungen und übertrieben empfunden werden und keinesfalls als unauffällig und neutral (vgl. Davies 1995: 91-100, 111-117). Auch wenn der Einfluss von außersprachlichen Faktoren auf das Gelingen von Kommunikation nicht ganz eindeutig ist, ist es im Expertendiskurs unbestritten, dass solche Faktoren doch eine Rolle spielen, und das wäre ein wichiges Thema für die Schule. Die Annahme, dass nur Standarddeutsch die gegenseitige Verständigung sichern kann - ohne Bezug auf die soziale Einbettung solcher Annahmen könnte kritischer behandelt werden, auch gelegentlich von Seiten der Sprachwissenschaft.

\section{Fazit}

In diesem Beitrag wurde argumentiert, dass zwei geläufige Annahmen über die Sprachvariation in Deutschland kritischer hinterfragt werden könnten, damit deren soziale und ideologische Dimension deutlicher an den Tag kommt. Ausgangspunkt der Überlegungen war die Forderung von VerfechterInnen der CLA, SoziolinguistInnen sollten stärker der Tatsache Rechnung tragen, dass Macht in der Gesellschaft ungleich verteilt ist und dass sich diese Ungleichheit in der Verteilung von sprachlichen Praktiken und in deren unterschiedlicher Bewertung durch die Gatekeeper widerspiegelt (Fairclough 1992b: 48; Bourdieu 1991). Die traditionelle Rollenverteilung zwischen Standardsprache und Dialekten/ nichtstandardsprachlichen Varietäten, auf der das Angemessenheitsmodell fußt, wird in den in diesem Beitrag exemplarisch durchgesehenen Schriften und Richtlinien selten einer radikalen Kritik unterzogen. Das Thema Sprachwandel steht in den meisten Curricula, und die Bildungsstandards für den Mittleren Schulabschluss sehen vor, dass die SchülerInnen in der Lage sein sollen, „sprachliche Gestaltungsmittel in ihren Wirkungszusammenhängen und in ihrer historischen Bedingtheit [zu] erkennen“ (2004: 14). Es wird aber in der hier analysierten Auswahl an Schriften nicht erwähnt, dass den SchülerInnen bewusst gemacht werden sollte, dass Sprach(gebrauchs)normen ein Konfliktgegenstand im Spannungsfeld sozialer Machtinteressen sind. Die von vielen für sehr kritisch und radikal gehaltenen Hessischen Rahmenrichtlinien Deutsch (Christ u. a. 1974), die den privilegierten Status des Standarddeutschen und die Homogenität der Sprachgemeinschaft relativ früh hinterfragten, zeitigten anscheinend keine Breitenwirkung. Was die Rolle von verschiedenen Varietäten bei der gegenseitigen Verständigung angeht: Es wäre im Sinne der CLA, wenn die außersprachlichen, sozialen (und psychologischen) Faktoren, die 
eine gelungene Kommunikation bestimmen, stärker berücksichtigt würden, sowohl in theoretischen Schriften als auch in den schulischen Richtlinien.

Auch wenn die Lehrpersonen und die VerfasserInnen von bildungspolitischen Richtlinien eine kritischere bzw. radikalere Position einnehmen wollten, müssen sie natürlich immer berücksichtigen, dass es zu ihren Aufgaben gehört (v. a. zu den Aufgaben der Lehrkräfte), die SchülerInnen auf das Leben in einer Gesellschaft vorzubereiten, in der der Gebrauch von Sprache entgegen bestimmten Konventionen, d.h. der „unangemessene“ Gebrauch, Benachteiligungen bringen kann. Eine kritische Sprachbewusstheit kann den Menschen jedoch helfen zu erkennen, dass es doch möglich ist, sprachliche Konventionen $\mathrm{zu}$ hinterfragen, und dass einiges, was als common sense und unumstritten und deshalb als nicht anfechtbar ausgegeben wird, doch zum Gegenstand der kritischen Auseinandersetzung werden kann.

\section{Literatur}

Ammon, Ulrich (1979): Regionaldialekte und Einheitssprache in der Bundesrepublik Deutschland. International Journal of the Sociology of Language 21, 25-40.

Ammon, Ulrich (1982): Zum Versuch der soziolinguistischen Legitimation sozialer Sprachungleichheit - am Beispiel von Schullehrzielen in der Bundesrepublik Deutschland - kritische Anmerkungen. In Piet van de Craen \& Roland Willemyns (Hrsg), Sociolinguistiek en Ideologie, 29-57. Brussels: Universiteit Brussel.

Andersson, Lars-Gunnar \& Peter Trudgill (1990): Bad language. Harmondsworth: Penguin.

Barbour, Stephen (1987): Dialects and the teaching of a standard language: Some West German work. Language in Society 16, 227-244.

Bayer, Klaus (1984): Mündliche Kommunikation. In Hopster, Norbert (Hrsg.), Handbuch $>$ Deutsch<. Sekundarstufe 1, 307-333. Paderborn: Ferdinand Schöningh.

Bellmann, Günter (1983): Probleme des Substandards im Deutschen. In Klaus J. Mattheier (Hrsg.), Aspekte der Dialekttheorie, 105-130. Tübingen: Niemeyer.

Besch, Werner, Ulrich Knoop, Wolfgang Putschke \& Herbert Ernst Wiegand (Hrsg.) (1983): Dialektologie. Ein Handbuch zur deutschen und allgemeinen Dialektforschung. Berlin: de Gruyter.

Bhatt, Arvindh \& Marilyn Martin-Jones (1992): Whose resource? Minority languages, bilingual learners and language awareness. In Norman Fairclough (Hrsg.), Critical language awareness, 285-301. London/New York: Longman.

Bourdieu, Pierre (1991): Language and symbolic power (edited and introduced by John B. Thompson). Cambridge: Polity Press.

Bücherl, Rainald (1993): Dialekt als Chance. In Peter Klotz \& Peter Sieber (Hrsg.), Vielerlei Deutsch, 68-77. Stuttgart: Klett.

Cameron, Deborah (2012): Verbal hygiene. 2. Aufl. London/New York: Routledge.

Christ, Hannelore u. a. (1974): Hessische Rahmenrichtlinien Deutsch. Analyse und Dokumentation eines bildungspolitischen Konflikts. Düsseldorf: Bertelsmann.

Coupland, Nikolas (2000): Sociolinguistic prevarication about „standard“ English. Journal of Sociolinguistics 4, 622-634. 
Davies, Winifred V. (1995): Linguistic variation and language attitudes in MannheimNeckarau. Stuttgart: Steiner.

Davies, Winifred V. (2000): Linguistic norms at school: A survey of secondary-school teachers in a central German dialect area. Zeitschrift für Dialektologie und Linguistik 67, 129-147.

Davies, Winifred V. (2011): What (socio-)linguistic competences should we expect from teachers? Some suggestions inspired by German-speaking Switzerland. German as a Foreign Language 2, 16-39.

Davies, Winifred V. \& Nils Langer (2014): Die Sprachnormfrage im Deutschunterricht: das Dilemma der Lehrenden. In Albrecht Plewnia \& Andreas Witt (Hrsg.), Sprachverfall? Dynamik - Wandel - Variation, 299-321. Berlin/New York: de Gruyter.

Dittmar, Norbert (1980): Soziolinguistik. 4., korrigierte Aufl. Königstein/Taunus: Athenäum. Duden 9. Richtiges und gutes Deutsch. (2007): 6. Aufl. Mannheim: Duden.

Duden 9. Richtiges und gutes Deutsch. (2016): 8. Aufl. Berlin: Duden.

Durrell, Martin (1999): Standardsprache in England und Deutschland. Zeitschrift für germanistische Linguistik 27, 285-308.

Fairclough, Norman (Hrsg.) (1992a): Critical language awareness. London/New York: Longman.

Fairclough, Norman (1992b): The appropriacy of appropriateness. In Norman Fairclough (Hrsg.), Critical language awareness, 33-56. London/New York: Longman.

Farrell, Thomas S. C. \& Serena Tan Kiat Kun (2007): Language policy, language teachers' beliefs, and classroom practices. Applied Linguistics 29/3, 381-403.

Geiger, Raphael (2009): Dialekt an Schulen. Bloß nicht nach Niederbayern! Der Spiegel. 14. August 2009. http://www.spiegel.de/lebenundlernen/schule/dialekt-an-schulenbloss-nicht-nach-niederbayern-a-638568.html (letzter Zugriff: 7.12. 2013).

Haugen, Einar (1966): Semicommunication: The language gap in Scandinavia. Sociological Enquiry 36, 280-297.

Hommel, Eva-Maria (2015): Hochdeutsch-Training. „Bei Ihrem Dialekt hat der Konkurrent bessere Chancen“. Der Spiegel. 15. Mai 2015. www.spiegel.de/karriere/dialekte-wieman-fuer-den-beruf-hochdeutsch-lernt-a-1033679.html (letzter Zugriff: 12. 9. 2017).

Ivanič, Roz (1988): Critical language awareness in action. Language Issues 2, 2-7.

Janks, Hilary \& Roz Ivanič (1992): Critical language awareness and emancipatory discourse. In Norman Fairclough (Hrsg.), Critical language awareness, 305-331. London/New York: Longman.

Klein, Wolf Peter (2013): Warum brauchen wir einen klaren Begriff von

Standardsprachlichkeit und wie könnte er gefasst werden? In Jörg Hagemann, Wolf Peter Klein \& Sven Staffeldt (Hrsg.), Pragmatischer Standard, 15-33. Tübingen: Stauffenburg.

Klotz, Peter \& Peter Sieber (Hrsg.) (1993): Vielerlei Deutsch. Stuttgart: Klett. König, Werner (1978): dtv-Atlas zur deutschen Sprache. 2. Aufl. München: dtv. Kratzer, Hans (2012): Deutsch können nur die anderen. Süddeutsche Zeitung. 30. Dezember 2012. http://bit.ly/2qodGsV (letzter Zugriff: 16. 5. 2017).

Liddicoat, Anthony J. (2013): Language-in-education policies. The discursive construction of intercultural relations. Bristol u. a.: Multilingual Matters.

Lippi-Green, Rosina (2012): English with an accent. Language, ideology and discrimination in the United States. 2. Aufl. London/New York: Routledge.

Maas, Marie-Charlotte (2013): Die Frau, die wo den Kurs gibt. UniSpiegel 1/2013, 28.

Maitz, Péter (2015): Sprachvariation, sprachliche Ideologien und Schule. Zeitschrift für Dialektologie und Linguistik 82 (2), 206-227. 
Maitz, Péter \& Monika Foldenauer (2015): Sprachliche Ideologien im Schulbuch. In Jana Kiesendahl \& Christine Ott (Hrsg.), Linguistik und Schulbuchforschung. Gegenstände Methoden - Perspektiven, 217-233. Göttingen: V \& R unipress.

Milroy, James (1999): The consequences of standardisation in descriptive linguistics. In Tony Bex \& Richard J. Watts (Hrsg.), Standard English: The widening debate, 16-39. London/New York: Routledge.

Milroy, Lesley (1984): Comprehension and context: Successful communication and communication breakdown. In Peter Trudgill (Hrsg.), Applied sociolinguistics, 7-31. London: Academic Press.

Neuland, Eva (1979): Dialekt in Sprachbüchern. Wirkendes Wort 29, 79-93.

Rosenberg, Peter (1986): Der Berliner Dialekt und seine Folgen für die Schüler. Tübingen: Niemeyer.

Rosenberg, Peter (1993): Dialekt und Schule: Bilanz und Aufgaben eines Forschungsgebiets. In Peter Klotz \& Peter Sieber (Hrsg.), Vielerlei Deutsch, 12-58. Stuttgart: Klett.

Sandig, Barbara (1973): Zur historischen Kontinuität normativ diskriminierter syntaktischer Muster in spontaner Sprechsprache. Deutsche Sprache 1, 37-57.

Schiffman, Harold F. (1996): Linguistic culture and language policy. London: Routledge.

Schneider, Jan Georg (2016): Syntax der gesprochenen Sprache und Kodifizierung. In Wolf Peter Klein \& Sven Staffeldt (Hrsg.), Die Kodifizierung der Sprache. Strukturen, Funktionen, Konsequenzen, 272-284. Würzburg (= WespA: Würzburger elektronische sprachwissenschaftliche Arbeiten 17).

Schüppert, Anja \& Charlotte Gooskens (2011): Investigating the role of language attitudes for perception abilities using reaction time. Dialectologia II, 119-140.

Schüppert, Anja, Nanna Haug Hilton \& Charlotte Gooskens (2015): Swedish is beautiful, Danish is ugly? Investigating the link between language attitudes and spoken word recognition. Linguistics 53, 375-403.

Schwitalla, Johannes (2012): Gesprochenes Deutsch: Eine Einführung. 4., neu bearb. und erw. Aufl. Berlin: Erich Schmidt.

Sealey, Alison (1999): Teaching primary school children about the English language: A critique of current policy documents. Language Awareness 8, 84-97.

Sieber, Peter \& Horst Sitta (1986): Mundart und Standardsprache als Problem der Schule. Aarau, Frankfurt am Main: Sauerländer.

Tollefson, James W. (1991): Planning language, planning inequality: Language policy in the community. London/New York: Longman.

Wachs, Inge (1982): Dialekt als Sprachbarriere? Soziolinguistische Überlegungen zur Berücksichtigung regionaler Variation in der Schule. Linguistische Arbeiten Berichte, H. 18, Sept. 1982, 235-265.

Wolff, Hans (1959): Intelligibility and interethnic attitudes. Anthropological Linguistics 1, 34-41.

Wrangel, Cornelia von (2005): Wenn der Schwabe zu sehr leidet - muß er Hochdeutsch lernen. Damit läßt sich Geld verdienen. Frankfurter Allgemeine Sonntagszeitung 47, 27. November 2005.

\section{Lehr- und Bildungspläne, auf die im Text verwiesen wird}

Bildungsstandards im Fach Deutsch für den Mittleren Schulabschluss (2004): München: Wolters Kluwer Deutschland GmbH. 
Bildungsstandards im Fach Deutsch für die Allgemeine Hochschulreife (2014): München: Wolters Kluwer Deutschland GmbH.

Bildungsplan für das Gymnasium Baden-Württemberg. Deutsch (1994):

Villingen-Schwenningen: Neckar-Verlag.

Bildungsplan des Gymnasiums Baden-Württemberg. Deutsch (2016): Villingen-Schwenningen: Neckar-Verlag.

Lehrplan Deutsch. Gymnasium. Rheinland-Pfalz (1984): Grünstadt: Emil Sommer Verlag für das Schulwesen.

Lehrplan Deutsch (Klassen 5 - 9/10). Hauptschulen, Realschulen, Gymnasien, Regionale Schulen, Gesamtschulen. Rheinland Pfalz. (1998): Grünstadt: Emil Sommer.

Niedersachsen. Deutsch. Kerncurriculum für das Gymnasium - gymnasiale Oberstufe, die Gesamtschule - gymnasiale Oberstufe, das Fachgymnasium, das Abendgymnasium, das Kolleg (2009): Hannover: Unidruck. 
Elisabeth Knipf-Komlósi

\title{
8 Das Dilemma zwischen Norm und Variation im Deutschunterricht in Ungarn - und wie Lehrende darüber denken
}

\begin{abstract}
Im vorliegenden Beitrag geht es um die umstrittene Frage der Sprachnorm im Unterricht für Deutsch als Fremdsprache sowie für Deutsch als Minderheitensprache in Ungarn. Eine empirische online-Befragung unter Deutschlehrerinnen und -lehrern hat ergeben, dass selbst die Lehrenden die Frage der Sprachform nicht in ihrer Komplexität und Anwendbarkeit differenziert im Unterricht handhaben. Vor allem geht es um das Festhalten an einer präskriptiven Vermittlung der Sprachnorm sowie um die Probleme, die sich im Unterricht durch die medialen Unterschiede (gesprochenes und geschriebenes Deutsch) ergeben.
\end{abstract}

Keywords: bilinguale Schulen mit Deutsch in Ungarn, Entwicklung der Normfrage im Unterricht, gesprochenes und geschriebenes Deutsch, sprachliche Handlungskompetenz, Sprachnorm und Variation

\section{Aspekte des Hintergrunds}

Es ist nicht überraschend, dass die im Titel genannten Schlüsselbegriffe „Norm“ und „Variation“ seit geraumer Zeit einen hervorgehobenen Platz in der angewandten Forschung zum Deutschen, insbesondere bezogen auf den Deutschunterricht, einnehmen (vgl. Neuland 2006; Durrell 1999; Ziegler 2011; Hennig 2012; Davies \& Langer 2013). Auch gegen Ende des zweiten Jahrzehnts des 21. Jahrhunderts hat das Thema noch Aktualität, da auch die Lehrenden des Deutschen im DaF-Bereich im Ausland (vgl. Durrell 2006; Stridova 2015; Davies i. d. Bd.) aufgrund ihrer Unterrichtserfahrungen sich immer wieder die Fragen stellen: „Wie ist es um die Norm des Deutschen in unseren Tagen bestellt? Wie viel Variation sollen unsere Schüler im Deutschunterricht kennen lernen?“ Das

Elisabeth Knipf-Komlósi, Germanistisches Institut, Eötvös Loránd Universität, 1088 Budapest, Rákóczi út 5, Ungarn, E-Mail: knipfe@freemail.hu

Ә Open Access. (C) 2018 Elisabeth Knipf-Komlósi, publiziert von De Gruyter. (c)B(BY Dieses Werk ist lizenziert unter der Creative Commons Attribution 4.0 Lizenz.

https://doi.org/10.1515/9783110538625-009 
Spannungsverhältnis zwischen Norm und Variation im Unterricht kommt in der Regel auf, wenn sich gesellschaftlich-soziale Umbrüche in einer Gesellschaft zeigen, wie das z. B. in den 1990er Jahren in Mittelosteuropa der Fall war, oder auch bei internationalen Sprachstandserhebungen in bestimmten Schulstufen, wenn die Sprachkompetenz der Lernenden das von ihnen erwartbare Niveau nicht erreicht (z. B. PISA-Tests). Warnende Stimmen über eine abnehmende Akzeptanz von Normen in der Gesellschaft, auch in der Sprache, laienhafte Sprachkritiken über einen Prestigeverlust der Standardsprache, eine große Varianz im Deutschen, die in der breiten Öffentlichkeit z. B. im Ausland nicht entsprechend thematisiert wurde und daher nicht allgemein bekannt ist, all das sind Umstände, die einen Teil der dortigen Deutschlehrkräften verunsichern, die - und das sollte unbedingt berücksichtigt werden - in einem anderssprachigen Umfeld und bei verhältnismäßig geringen authentischen sprachlichen Impulsen Lernende aller Stufen in Deutsch unterrichten. Gleichzeitig fühlt sich ein anderer Teil engagierter Deutschlehrer durch die Vielzahl von Text- und Sprachhandlungsmustern des Gegenwartsdeutschen verunsichert, weil sie mit dem Spannungsverhältnis zwischen dem in der Schule unterrichteten sog. Schuldeutsch und der Sprachwirklichkeit ${ }^{1}$ konfrontiert werden.

Die grundlegende Frage der Lehrenden, was und wie und mit welchem erwartbaren Ergebnis unterrichtet werden soll, umfasst sowohl den Gegenstand des Unterrichts, die deutsche Sprache, gleichzeitig hat die Frage auch einen didaktischen Aspekt, denn mit dem Was und dem Wie wird auch das Thema des Normativen angesprochen. Diese Fragen der Lehrkräfte führen auch dazu, ihre soziale Rolle im Sinne von Ammon (1995) als Normautoritäten bewusster wahrzunehmen und dem Prozess des Unterrichtens eine erhöhte Aufmerksamkeit zu schenken. In sprachlichen Zweifelsfällen im Unterricht geraten eben die Lehrenden in eine Entscheidungssituation: Welche Form ist richtig, welche ist falsch in der gegebenen Situation? Lehrende haben viele Möglichkeiten, Fragen dieser Art zu beantworten, z. B. zahlreiche einschlägige Nachschlagewerke heranzuziehen, Konsultationen mit muttersprachlichen Lektoren, mit Gastlehrern, bei Fortbildungen zu führen etc. Kurz gefasst, die Verantwortung der Lehrenden ist in ihrer sozialen Rolle als Normvermittler komplexer geworden und in vielen Fällen auch nicht leicht zu erfüllen.

Deutsch wird in Ungarn je nach Schultyp als Fremdsprache und als Minderheitensprache in allen Schulstufen - von der ersten Klasse der Grundschule bis zum Abitur - unterrichtet. Dieser Unterricht blickt in Ungarn aufgrund der

1 In dieser Hinsicht ist die Situation der Deutschlehrenden auf dem deutschen Sprachgebiet, die in der Sprachwirklichkeit selbst leben, selbstverständlich völlig anders. 
mehr als tausendjährigen historischen und kulturellen Verbundenheit mit dem deutschen Sprachraum auf eine lange und gut bewährte Tradition zurück, ${ }^{2}$ was jedoch nichts an der Tatsache ändert, dass die Fremdsprachenkenntnisse eines Durchschnittsungarn immer noch sehr niedrig sind. Deutsch nimmt zur Zeit in Ungarn in der Fremdsprachenwahl nach Englisch den vornehmen zweiten Platz ein, wobei dieser Umstand auf viele Faktoren zurückzuführen ist und immer auch von dem jeweiligen gesellschaftlich-politischen und sozialen Kontext des Landes abhängig ist. Unter einem sozialen Aspekt betrachtet bestehen in den letzten Jahren zwei relevante Motivationsfaktoren für die ungarischen Arbeitnehmer, die deutsche Sprache zu erlernen: Die vorübergehende oder auch langfristige Erwerbsmöglichkeit im deutschsprachigen Ausland (in der Bundesrepublik Deutschland, in Österreich, in der Schweiz) sowie die Möglichkeit, mit soliden Deutschkenntnissen eine Anstellung bei einem deutschsprachigen Unternehmen im Lande (in Ungarn) zu bekommen. Sowohl im schulischen Deutschunterricht als auch an diversen Abendkursen und Sprachschulen ist immer wieder die Frage zu hören, welches Deutsch, gemeint ist meistens welche Varietät, unterrichtet werden soll, wieviel Toleranz von Normabweichungen heutzutage denn zugelassen werden darf. Mit welchem Deutsch sind die künftigen Lerner und auch Arbeitnehmer am besten beraten und für den Sprachgebrauch vorbereitet? Diese praktischen Fragen zeigen nicht nur einen verantwortungsvollen Umgang mit dem Lehrgegenstand Deutsch, sondern können auch auf eine Unsicherheit der Fragenden/Lehrenden hindeuten, oder sie können als Symptom gesehen werden, dass die bislang gelernte und praktizierte Norm der Standardsprache/Hochsprache des Deutschen aufgrund der in letzter Zeit erscheinenden Veränderungen in der Sprache ins Wanken geraten sind. Ist diese vermeintliche oder reale Unsicherheit überhaupt berechtigt? Zur Beantwortung dieser Fragen sollen einige Merkmale der im Titel genannten Schlüsselbegriffe in einem ungarischen Kontext beleuchtet werden. Empirisch untermauert werden diese Ausführungen durch eine Fragebogenerhebung zum Thema Variation und Normempfinden, die kürzlich unter Deutschlehrerinnen und -lehrern in Ungarn durchgeführt wurde. In meinem Beitrag werde ich auf folgende inhaltliche Punkte eingehen:

- die Norm-Problematik in der Deutschlehrerausbildung (DaF und DaM) (Abschnitt 2),

- die Entwicklung der Norm im Deutschunterricht in Ungarn (Abschnitt 3),

- Auswertung der empirischen Angaben zur Akzeptanz einer Variation und zum Normempfinden praktizierender Deutschlehrender (Abschnitt 4).

2 Vgl. Müller (2010) zum Stand des Deutschunterrichts in Ungarn. 


\section{Zur Norm-Problematik in der Deutschlehrer- ausbildung}

Im alltäglichen Sprachgebrauch macht man sich selten Gedanken über die Normen der Sprache, die man eben gebraucht, höchstens tun dies Fremdsprachenlerner, die sich noch unsicher in ihrem Sprachgebrauch fühlen. Mit sprachlichen Normen kommt man eigentlich erst in Berührung, wenn man gegen diese verstößt, wenn sie verletzt werden. Und wem passiert das nicht ab und $\mathrm{zu}$ ? Im Lehrerberuf gehören solche Fälle zum Unterrichtsalltag, in deren Kontext im Mündlichen und Schriftlichen der Lernenden häufig Ungereimtheiten, Normverstöße, d. h. Fehler, auftreten. Im glücklichen Fall werden diese vor Ort geklärt, mit dem Lehrer und/oder der Gruppe besprochen, meistens auch korrigiert, oder in letzter Instanz werden Sanktionsmittel eingesetzt, die den Lehrerinnen und Lehrern zur Verfügung stehen. Diese Situationen verlangen von den Lehrenden nicht nur ein notwendig vorauszusetzendes hohes Sprachvermögen und eine Reihe von überzeugenden Argumenten zum sprachlichen Problem, sondern auch eine mehr oder weniger ausgeprägte Sprachbewusstheit, die den Lernprozess unterstützen kann. Von Seiten der Lernenden tauchen bei Sanktionen auch viele Fragen auf, die der Lehrende meistens vor Ort zu beantworten oder beim Korrigieren der schriftlichen Arbeiten zu begründen hat.

Der Begriff der Sprachnorm, der äußerst polysem ist, gilt schon seit langem als Forschungsgegenstand nicht nur in der Sprachwissenschaft, sondern auch in der Didaktik (vgl. Feilke 2015). Sprachnormen sind das Ergebnis einer langen historischen Entwicklung, entstanden durch eine Auswahl aus mehreren grammatischen Möglichkeiten, die in anderen Varietäten, Kontexten evtl. heute noch auftreten (können). Es kann auch die Frage gestellt werden, ob es beim Normbegriff um die Korrektheit von sprachlichen Normen oder um einen korrekten Sprachgebrauch geht ${ }^{3}$ (vgl. Bartsch 1987: 5). Im Allgemeinbewusstsein sind Normen primär inhaltlich festgelegte Regulative für die einzelnen Sprachebenen, die orthographische, orthoepische, grammatische (morphologische, syntaktische), und die lexikalisch-semantische Ebene. Gloy (1988) bezeichnet sie als konventionelle, gesetzte Normen, die als Ordnungsprinzip beim Sprachgebrauch des Individuums oder der Sprachgemeinschaft mehr oder weniger verbindlich sind. Doch wie sieht das bei einer so heterogenen Sprache wie dem Deutschen aus, mit so vielen Subsystemen und Äußerungsvarianten auf den

3 Im Deutschunterricht in einem nicht deutschsprachigen Land interessiert vor allem jener Aspekt der Sprachnorm, der direkte Auswirkungen auf den Sprachgebrauch der Lernenden hat. 
einzelnen Ebenen, insbesondere in der gesprochenen Sprache, wo durch die starke Betonung der Varianz der Normativitätsaspekt etwas in den Hintergrund geraten ist? So entstand schon vor fast zwei Jahrzehnten der Bedarf einer anderen Sicht auf die Norm und auf deren Legitimierung:

Von besonderer Bedeutung erscheinen neben den kodifizierten und statuierten Normen nun die Entwicklungen der ,subsistenten' Normen, jener normativen Erwartungshaltung, um deren Institutionalisierung und Legitimierung im Rahmen konkurrierender Normierungshandlungen gerungen wird. (Neuland 1998: 8)

So ist es zu begrüßen, dass nun in der einschlägigen Fachdiskussion der letzten Jahre bereits das Begriffspaar statuierte (explizite) und subsistente (verdeckte, implizite) Normen im Mittelpunkt der Diskussion steht (vgl. Gloy 2004; Ziegler 2011). Diese zwei Typen von Normen sind eng miteinander verflochten, doch in ihrer Entstehung und Funktion unterschiedlich, und sie werden auch auf unterschiedliche Weise gehandhabt. Die statuierten Normen sind kodifizierte und tradierte Normen, die als sichere Orientierung für Lehrende und Lernende dienen, die primär durch den Sprachgebrauch selbst und selbstverständlich in der Schule unterrichtet werden und hohes Prestige genießen. Die verdeckten, subsistenten Normen erscheinen in konkreten Sprachgebrauchssituationen, beziehen sich auf sprachliche Handlungsroutinen, und da sie nicht geregelt und kodifiziert sind, besitzen sie kein hohes Prestige, zumal sie noch als Abweichungen von der Standardnorm betrachtet werden (vgl. Ziegler 2011: 70 ff.). Die beiden Normen umfassen somit den Gegenstand sowie dessen Anwendung, den Sprachgebrauch. Feilke (2015: 118) erweitert den Horizont der Normen um eine didaktische Dimension und hebt drei Ebenen des Problems hervor, nämlich die Normativität in der Didaktik, die Normativität des Fachs, d. h. die sprachlichen Normen, und zuletzt nimmt er noch Normen für den Unterricht an. Bei letzteren geht es um „Normen als kommunikatives und kognitives Regulativ für Lehr- und Lernprozesse“. Es bedarf keiner besonderen Einsicht, dass in einem modernen Deutschunterricht über die gesetzten Normen hinaus - die als der Gegenstand des Unterrichts sowieso im Vordergrund stehen - auch die Gebrauchsnormen sowie jenes kommunikative und kognitive Regulativ ${ }^{4}$ im Unterricht eine wichtige Rolle einnehmen müssen, denn das primäre Ziel der Lehrenden wie Lernenden ist es, sich einen in vielen Situationen handhabbaren Sprachgebrauch anzueignen. So kann man mit Durrell (2012: 103) einverstanden sein, dass

4 Die von Feilke genannte dritte Norm verstehe ich als Teil der versteckten (subsistenten) Normen auf der Metaebene. 
die ausschließliche Vermittlung der herkömmlichen hochsprachlichen Normen heutzutage nicht mehr für die Vermittlung umfassender fremdsprachlicher Kompetenzen ausreicht, denn die Lernenden müssen sich auch die Fähigkeit aneignen, sich an einer Vielfalt sprachlicher Handlungen in angemessener Weise zu beteiligen.

Der Begriff der Sprachnorm ist ein relationaler Begriff, der erst durch eine Inbezugsetzung mit komplementären Begriffen sein volles Bedeutungsspektrum erhält. So spricht man über eine Norm im Zusammenhang mit dem Sprachgebrauch eines Individuums, einer Gemeinschaft, vielmehr muss aber der Begriff der sprachlichen Variation sowie die Wahl von möglichen Optionen in der Sprachhandlung vor dem Hintergrund eines Norm-Begriffs diskutiert werden. Das bestehende Spannungsverhältnis zwischen Normbefolgung und Normabweichung, auch der Normtoleranz, und dergleichen wichtige Zusammenhänge können erst durch ihre Beziehung zum Normbegriff erläutert und ins rechte Licht gerückt werden.

Es kann davon ausgegangen werden, dass angehende Lehrerinnen und Lehrer des Deutschen - auch im Ausland - sich im Laufe ihrer Ausbildung nicht nur die Sprache als Kommunikationsmittel auf einem entsprechend hohen Niveau sowie eine linguistische Kompetenz und damit die gesetzten Normen, besonders in den Bereichen der Orthographie und Grammatik, aneignen. Aufgrund ihres kompetenten Umgangs mit der Sprache und den sprachlichen Handlungsmustern kennen sie auch die Normen des Sprachgebrauchs, vor allem jene, die durch Kommunikationssituationen in Lehrbüchern vermittelt werden. Darüber hinaus ist auch anzunehmen, dass sie sich in fachlichen Diskussionen zum Thema der sprachlichen Norm der Gegenwart im Laufe ihres Studiums oder bei Fortbildungen damit auseinandergesetzt haben. ${ }^{5}$ Trotz dieser Annahmen kann jedoch nicht behauptet werden, dass im Curriculum der Deutschlehrerausbildung (in Ungarn) die Frage der Norm eine besondere Gewichtung bekäme. Dies kann mehrere Gründe haben:

1. Die Lehrenden haben nicht genügend Informationen über die Veränderungen der Gebrauchsnormen der Gegenwart; die wenigen haben sie oft nur mühsam selbst zusammentragen. Der variable Sprachgebrauch als Begriff ist ihnen zwar vertraut, doch dass jede sprachliche Äußerung durch die Situation, Funktion, Gesprächspartner etc. motiviert ist, bleibt noch ein theoretisches Konstrukt.

5 Z.B. in Form von philologischen Modulen, wie Sprachsystem und Sprachwandel, Variation und Varietäten des Deutschen, die Soziolinguistik des Deutschen und dergleichen mehr thematisieren (die Beispiele stammen aus dem Curriculum des Germanistischen Instituts der Eörvös-Lorand-Universität Budapest). 
2. Die Sensibilität der Deutschlehrenden im Ausland für die breitgefächerte Vielfalt sprachlicher Handlungs- und Textmuster der Gegenwart kann bei weitem nicht so ausgeprägt sein wie die der Lehrenden im Sprachgebiet. Demzufolge ist ihr Variations- und Normempfinden selbstverständlich auch different.

3. Aus o.g. Gründen steht im Zentrum des DaF-Unterrichts überwiegend noch die Vermittlung der kodifizierten Normen, ${ }^{6}$ auch wenn eine gewisse Lockerung in dieser Hinsicht bereits wahrnehmbar ist.

Glücklicherweise kommt sowohl in der Deutschlehrerausbildung als auch in Fortbildungen seit den 90er Jahren das Konzept der sprachlichen Variation immer mehr an, doch ist diese sprachliche Variation mit all ihren Facetten keine selbst erlebte Sprachwirklichkeit für die Lehrenden, da diese Inhalte nicht genügend diskutiert und didaktisch aufbereitet sind. Die Regeln des Sprachsystems der Standardsprache, die expliziten Normen, sind in Lehr- und Sprachbüchern sowie Grammatiken systematisiert, übersichtlich dargestellt und didaktisch gut aufbereitet. In der Lehrerausbildung für Deutsch bilden diese kodifizierten Normen eine unentbehrliche Grundlage, die im Schulunterricht didaktisiert und mehr oder weniger leicht vermittelbar sind. Im Vergleich dazu sind die verdeckten, impliziten Normen nicht als Regelwerk erlernbar, sie sind auch in schulischen Kommunikationssituationen nicht so einfach erfahrbar, denn oft fehlt es an konkreten Sprechanlässen und Kommunikationssituationen, die - wie das aus den Erfahrungen der Lehrenden hervorgeht - im Fremdsprachenunterricht nicht so leicht gemeistert werden können. ${ }^{7}$ Es geht eben um jene Routinen, denen im heutigen Fremdsprachenunterricht genauso viel Relevanz zukommen sollte wie den Regularitäten des Sprachsystems. Bedenken werden diesbezüglich auch im Vorwort des Tagungsbandes zur IDSJahrestagung 2009 formuliert, nämlich dass es noch unklar ist „,wie ein Konzept zum Sprachgebrauch und seiner Ermittlung auszusehen habe, um mit der Vorstellung von Regularitäten kompatibel zu sein“ (Eichinger 2009: 2). Dass eine Lücke zwischen Sprachgebrauch und Norm nicht nur im DaF-Bereich besteht, beweisen auch sprechsprachliche Formen im Alltagsdeutsch wie z. B. is statt ist, soner statt so einen, inner Klasse statt in einer Klasse und dergleichen mehr. Normen werden im Alltagsleben nicht immer konsequent durchgesetzt, und das Prinzip der sprachlichen Korrektheit und das der systematischen Konsequenz (Busse 2006: 4) sind zwar Grundelemente einer sprachnormativen

6 Das wird ja in den meisten Sprachprüfungen (in Ungarn) noch verlangt, erwartet.

7 Lehrende beklagen sich oft darüber, dass es ziemlich schwer ist, auch bei geeigneten Sprechanlässen die Lernenden zu einem freien Gespräch im Unterricht anzuregen. 
Haltung, die jedoch „keineswegs eine kulturhistorische Selbstverständlichkeit“ (Busse 2006: 3) sind. Diese Haltung beruht auf den Einstellungen der Mitglieder der Gesellschaft zur ihrer Sprache, die dann als Haltung auch für DaF-Lerner beispielgebend wirkt. Ziel des Unterrichts sollte sein, den Lernenden bestimmte, im sprachlichen Alltag wirkende Gebrauchsnormen von Muttersprachlern zu erschließen und zu vermitteln. Die Erschließung der Gebrauchsnormen führt über die Erfahrung mit der Kultur und Sprache des Deutschen. Angesichts dieser Zielstellung sollte eine grundlegend andere Sicht in der Deutschlehrerausbildung auf curricularer Ebene ansetzen, und die relevanten sozialen Normen der Kultur der betreffenden Sprache sollten in den Unterricht so eingepasst und vermittelt werden, dass diese die Aufmerksamkeit der Lernenden wecken und zum besseren Verständnis und einer allmählichen Adaption des sich verändernden Sprachgebrauchs beitragen können. Die Verwirklichung dieser erstrebenswerten Ziele liegt in Händen der Lehrenden, die folgende Prozesse durchlaufen haben:

1. Nach einer ziemlich lange währenden Tradition von Gewöhnung an die präskriptiven Normen wurde eine Kompetenz im schriftsprachlichen Bereich erreicht, „die es auch ermöglicht, dass sich die Sprecher eine Domäne entsprechender Mündlichkeit dazu erobern“ (Eichinger 2011: 13). Der gesprochene Standard, der kodifiziert und relativ invariant ist, bedeutet für die Lehrenden eine sichere Basis, auf die sie in Zweifelsfällen oder bei Veränderungen zurückgreifen können. Doch die im gesprochenen Deutsch wahrnehmbaren lockereren Formen, die Destandardisierungstendenzen, zeigen, dass die Normen der Schriftlichkeit und Mündlichkeit nicht gleichgesetzt werden dürfen (vgl. Ziegler 2011: 71), denn in der geschriebenen Sprache herrschen Normen stärker als in der gesprochenen.

2. Der Gebrauchsstandard, der von regional- und alltagssprachlicher Variation und Abweichungen vom Standard stark beeinflusst ist und der vor allem durch die Medien bekannt und verbreitet wurde, ist nicht einheitlich und nicht kodifiziert. Lehrende halten sich eher an kodifizierte Normen als an Abweichungen, Veränderungen und Variation, weil sie sich die Frage stellen: Woher nehme ich die Informationen darüber, wie flexibel die Sprachnormen sein können und wieviel Variation im Unterricht zugelassen werden darf? Die „Wechselbeziehung zwischen den Sprachnormen (Kodex-orientiertes Sprachverhalten) einer Standardvarietät und dem alltäglichen, aber auch öffentlich-offiziellen Sprachgebrauch einer Gemeinschaft in Wort und Schrift (usage-based Agieren), in dem sich neu herausbildende subsistente Regularitäten oder Normen widerspiegeln“ (Felder 2003: 482), darf nicht ignoriert werden.

3. Zuletzt können die Verschiebungen und Überschneidungen zwischen Gesprochenem und Geschriebenem Probleme im Unterricht bereiten, bzw. 
der Umstand, dass der Sprachgebrauch maßgebend von kommunikativsituativ-funktionalen Parametern bedingt wird, die die jeweilige sprachliche Gestaltung und damit einhergehend auch die soziale Rolle des Individuums in der Gesellschaft bestimmen.

\section{Zur Entwicklung der Norm im DaF und DaM in Ungarn}

Zum besseren Verständnis der Norm- und Variationsfrage im Deutschunterricht erhalten wir interessante Einblicke, wenn wir die Entwicklung der Norm im Deutschunterricht in Ungarn kurz skizzieren. Nachdem zu Beginn der 1960er Jahre in Ungarn erneut ein Deutschunterricht an Schulen zugelassen wurde, konnten auch zur gleichen Zeit zweisprachige Minderheitengymnasien für die deutsche Minderheit gegründet werden. Der einzige Zugang zur deutschen Sprache und Kultur bildete für Ungarn in jener Zeit die sozialistische DDR und ihre Sprache, zumal diese deutsche Sprache auch als „westliche Sprache“ an den Schulen neben der obligatorischen Pflichtsprache Russisch zugelassen war. Diese Varietät des Deutschen hatte sowohl im DaF und im Minderheitenunterricht, als auch in der universitären Ausbildung, in der germanistischen Forschung und in einer damals noch spärlichen deutschsprachigen Pressetätigkeit schlechthin die Norm der deutschen Sprache vorgegeben. ${ }^{8}$

In diesen Jahrzehnten des Deutschunterrichts ging es vor allem um die Dominanz der expliziten Normen, die in der Schule und in der Lehrerausbildung vermittelt und abverlangt wurden. Über sprachliche Variation wurde damals nicht explizit gesprochen, ${ }^{9}$ höchstens konnte eine Variation auf der phonetischen Ebene wahrgenommen werden, z. B. dadurch, dass man gelegentlich von nativen Deutsch-Sprechern aus der DDR eine sächsisch geprägte Ausspracheform hören konnte. Erst nach der Wende in den 90er Jahren, durch die in Ungarn freizügigeren Reisemöglichkeiten, durch die Möglichkeit, deutschsprachige Medien (Satellitenfernsehen) zu empfangen, die sich nach der Wende in allen Altersstufen der Ungarndeutschen großer Beliebtheit erfreuten, sowie durch die intensiv gepflegten Städte-, Orts- und Schulpartnerschaften zu den

$8 \mathrm{Im}$ DaF wie im Minderheitenunterricht wurde die gleiche Methode verwendet, da die Schüler mit Minderheitenabstammung die Schule größtenteils ohne Vorkenntnisse in Deutsch begonnen haben.

9 In Heidolph et al. (1981) „Grundzüge einer deutschen Grammatik“ wurden allerdings in vielen grammatischen Bereichen grammatische Varaitionsmöglichkeiten dargestellt. 
deutschsprachigen Ländern konnte eine persönliche Erfahrung mit der sprachlichen Vielfalt und Variation des Alltags, eine Sprachwirklichkeit, ein Eintauchen in die sprachliche Welt der deutschen Sprache mit all ihren Facetten, hautnah erlebt werden.

Die jahrzehntelang verinnerlichte verhältnismäßig einheitliche Norm schien stabile Nachwirkungen im Deutschunterricht und bei den Lehrenden im Deutschunterricht und bei den Lernenden $\mathrm{zu}$ haben. In den vergangenen Jahrzehnten bis zur Wende sind einige Generationen der Deutschlehrenden mit einer variationsfreien Norm ausgebildet worden. Praktiziert wurde im Deutschunterricht überwiegend eine grammatisch-übersetzende Methode, die eben die Vermittlung der expliziten, nicht aber die der verdeckten Sprachgebrauchsnormen anstrebte.

Mit der kommunikativen Wende im DaF-Unterricht seit den späten 1980er Jahren erfolgte eine gewisse Lockerung im Deutschunterricht, indem das freie, hemmungslose Sprechen als Motto galt, oft ohne konsequente Rücksichtnahme auf die Akzeptabilität der grammatischen Regeln. Das bedeutete zwar didaktisch einen großen Schritt vorwärts, dennoch konnte das Ineinandergreifen der Vermittlung der beiden Normen nicht erreicht werden. In der Gegenwart ist aus den Zielvorgaben des Deutschunterrichts für Minderheiten und auch aus denen des DaF-Bereichs ersichtlich, dass im Deutschunterricht angestrebt wird, die beiden Normen, sowohl die gesetzten als auch die subsistenten Normen, $\mathrm{zu}$ verzahnen. Im Folgenden wird in einem kurzen Auszug aus den beiden Lehrplanvorgaben ein Einblick gegeben, der große Ähnlichkeiten hinsichtlich spezieller Ziele erkennen lässt:

Die Regelungen für den Deutschunterricht der bilingualen deutschen $\mathrm{Na}$ tionalitätenschulen in Ungarn („Wurzeln und Flügel“), die von einem Expertenteam 2010 erstellt wurden, umfassen folgende Bereiche:
a) Sprachliche Handlungskompetenz
b) Landeskundlich-interkulturelle Kompetenz
c) Linguistische Kompetenz
d) Methodenkompetenz
(Wurzeln und Flügel 2010: 4)

Die bilingualen Nationalitätenschulen ${ }^{10}$ streben an, dass sie neben allgemeinen Kenntnissen, die auch im DaF-Bereich erwähnt werden, spezielle zusätzliche, die Sprache, Kultur und Geschichte der deutschen Minderheit in Ungarn thematisierende Inhalte als Lehrstoff aufnehmen, die deutlich andere Schwerpunkte als der Gemeinsame Europäische Referenzrahmen setzen. Exemplarisch her-

10 Der Zahl nach sind es 11 Gymnasien und 47 Schulen. 
vorgehoben seien z. B. die Anforderungen in Klassenstufe 10, wo innerhalb der sprachlichen Handlungskompetenz eine allgemeine Interaktionsfähigkeit angestrebt wird, die u. a. Folgendes erwartet:

der Schüler/die Schülerin kann

- deutschsprachige Medien zur Informationsbeschaffung bzw. zur Kontaktaufnahme mit anderen einsetzen,

- sprachliches Handeln auf antizipierende Kommunikationssituationen einstellen,

- sprachlich flexibel in Präsentationssituationen agieren und eine Mitteilungsabsicht differenziert realisieren,

- elektronische Kommunikationsformen ohne nennenswerte Barrieren nutzen.

(Wurzeln und Flügel 2010: 56)

In diesen Formulierungen erkennen wir einen hohen Maßstab: eine anspruchsvolle Sprach- und Kommunikationsfähigkeit, in der neben der Interaktionsfähigkeit auch subsistente Normen zum Tragen kommen. Noch eindeutiger wird auf die Sprachgebrauchsnormen unter dem Kapitel Kommunikationskultur (Wurzeln und Flügel 2010: 57) eingegangen:

Der Schüler/die Schülerin kann

- in variabler, angemessener Form das Wort ergreifen oder gar abgeben und Redemittel einsetzen, um während des Redebeitrags Zeit zu gewinnen,

- Kommunikationssituationen einschätzen und seine eigenen Sprachhandlungen im Rahmen der üblichen Konventionen angemessen anpassen,

- Anderen sprachlich und formal differenzierte Rückmeldungen (feedback) geben,

- Registerverwendung und -wechsel von Kommunikationspartnern erkennen und der Situation und Rollenverteilung entsprechend angemessen sprachlich handeln ...

(Wurzeln und Flügel 2010: 57)

Dieser Teil fokussiert unmissverständlich jene subsistenten Normen, die auf der Grundlage linguistischer Kompetenzen, die durch die vermittelten deskriptiven statuierten Normen geprägt sind, unvermeidlich als Anforderungen in einer bilingualen Bildungsform erwartet werden können.

Der in Ungarn für die Fremdsprachen konzipierte nationale Rahmenlehrplan fokussiert ausführlich die gesetzten Normen, räumt aber auch den subsistenten einen gebührenden Platz ein, wie ein Ausschnitt aus dem Rahmenlehrplan zeigt:

Die Zielvorgabe bei der Vermittlung im Fremdsprachenunterricht ist die Herausbildung einer kommunikativen Kompetenz, die sich aus drei Elementen zusammensetzt: der sprachlichen, der soziolinguistischen und der Textkompetenz. Die sprachliche Kompetenz beinhaltet die lexikalischen, grammatischen, semantischen, phonetisch-phonologischen, morphologischen und orthographischen Kenntnisse bzw. die zu ihrem Gebrauch notwendigen Fertigkeiten bzw. Basisfähigkeiten. Die Sprachkompetenz wird durch das Verstehen 
des gehörten und geschriebenen Textes, durch die Sprechfertigkeit, die Interaktion bzw. durch die Entwicklung der Schreibfähigkeit aktiviert.

Die soziolinguistische Kompetenz bedeutet die Kenntnis jener gesellschaftlichen Gewohnheiten und Regeln, die den Erfolg der Kommunikation maßgebend beeinflussen und die vom Lehrenden aufgrund der Abweichung von der muttersprachlichen Kultur bei den Lernenden bewusst gemacht werden muss (z. B. Höflichkeitsformen, sprachliche Rituale, Anredeformen, Körpersprache, Humor, Stilschichten, Dialekte). Im Bereich der Textkompetenz werden Kenntnisse zum Aufbau von Texten, Textkohärenzmittel im Gesprochenen und Geschriebenen sowie Regeln der Interaktion bzw. typische Elemente verschiedener Textsorten angeeignet. Die Sprache ist von der Kultur, durch die sie entstand und durch die sie besteht, nicht zu trennen. (Idegennyelvi kerettanterv 2012; Übersetzung E. K.)

An dieser Stelle soll kurz auf die Rollen und Leitfunktionen der Lehrenden in diesem komplexen Aufgabenbereich hingewiesen werden. Bezugnehmend auf die Konstellation des von Ammon erstellten sozialen Kräftefeldes der Norm mit den vier Akteuren, soll die flexible und wechselnde soziale Rolle der Lehrenden im Deutschunterricht am Beispiel Ungarns gezeigt werden.

Von den 1960er bis in die 1980er Jahre, in einer Zeit, als die deutsche Sprache in Ungarn noch keine so große Rolle im Alltag spielte, hatten Lehrende des Deutschen und auch anderer „westlicher Fremdsprachen“ mehrfache Funktionen zu erfüllen. Zunächst galten sie als Vermittler der Sprachkodizes, gleichzeitig - mangels weniger Präsenz einer Sprachwirklichkeit - auch als Normautoritäten, und manchmal mussten sie in der Funktion als Sprachexperten auftreten. $\mathrm{Zu}$ einer direkten Bezugnahme auf Modelltexte und Modellsprecher gab es äußerst beschränkte Möglichkeiten, es herrschte eine Dominanz in der Vermittlung von expliziten Normen in der Erwartung, dass diese durch ausreichende Einübung (z.B. Drillaufgaben) im Späteren zu einer korrekten Anwendung führen werden. Bei einer Veränderung der Kräfteverhältnisse im Normen-Modell - in Gang gesetzt durch gesellschaftlich-politische Umbrüche verlagerten sich jedoch die Kompetenzbereiche und sozialen Rollen der einzelnen Akteure und damit auch ihr Wirkungsbereich. In der Aufbruchphase in Mittel- und Osteuropa nach der Wende, zur Zeit der Öffnung zum deutschsprachigen Raum in Europa, als den Deutschlernenden durch persönliche Erfahrungen z.B. die Sprache des westlichen Nachbarn, das österreichische Deutsch, oder die sprachliche Situation der Schweiz, die mediale Diglossie der Schweiz, bekannt wurde, sowie durch Reisen in die deutschsprachigen Länder die große Vielfalt der Sprachlandschaften in der Bundesrepublik von Lehrenden wie Lernenden erlebt wurde, änderten sich die Verhältnisse. Die Kodizes behielten ihre Gültigkeit in Form der gesetzten Normen, doch durch die westdeutsche Fachliteratur wurde v. a. der plurizentrische Charakter des Deutschen hervorgehoben. Durch die Medien lernte man andere, variable sprachliche Muster der Modellsprecher und neue Modelltexte kennen, man gewöhnte sich 
an das Deutsch der Nachrichtensprecher und Fernsehmoderatoren, die man als authentische Sprecher des Deutschen auch in ihrer Funktion als Normautoritäten betrachten konnte. Die multifunktionalen Aufgaben der früheren Deutschlehrerinnen und -lehrer als Normvermittler verringerten sich. Das sprachliche Eintauchen in die variable deutsche Sprache der Gegenwart führte zu einer im Sprachbewusstsein verankerten Diskrepanz zwischen Norm und Varianz, sowohl bei den Lehrenden als auch den Lernenden. In der Gegenwart ist auch erhöht mit den Auswirkungen der elektronischen Kommunikation zu rechnen, die einen unmittelbaren und unübersehbaren Einfluss auf den Sprachgebrauch sowie auf die Normvorstellungen der Sprachteilhaber welchen Alters auch immer - ausüben kann. Die Aufgabenbereiche im Ammonschen Modell haben sich verlagert, wodurch auch eine Neuorientierung für Lehrende und Lernende in ihrer Einstellung zum Deutschen einhergeht.

\section{Auswertung der Fragebogenerhebung}

Um neuere Angaben zum Norm-Variation-Dilemma zu erhalten, wurde im Frühjahr 2017 eine Umfrage unter Deutschlehrerinnen und -lehrern durchgeführt. ${ }^{11}$ Von den Antwortgebern waren 91\% Frauen und 9\% Männer. Als Muttersprache gaben $85 \%$ der Befragten Ungarisch an, $10 \%$ bezeichneten sich als zweisprachig (Ungarisch und Deutsch bzw. deutscher Dialekt), 5\% nannten Deutsch als Muttersprache.

Hinsichtlich des Wohnortes verteilen sich die Gewährspersonen gleichmäßig (siehe Abb. 8.1).

Die beteiligten Lehrenden repräsentieren das volle Spektrum der Schulstufen des Deutschunterrichts (siehe Abb. 8.2), auf diese Weise kann ein nicht repräsentativer, doch symptomatischer Eindruck über die Sichtweise der Lehrenden $\mathrm{zu}$ den befragten Themen vermittelt werden.

Ein Teil der Ergebnisse, die auf die Schlüsselbegriffe Norm und Variation zugeschnitten waren, wird in diesem Beitrag das erste Mal vorgestellt. ${ }^{12}$ Hierbei geht es um die Frage nach der Notwendigkeit der Variationsvielfalt im Unterricht, um eine freie Formulierung der Befragten zum Begriff Norm sowie um

11 Die Erhebung wurde online durchgeführt, an 138 Deutschlehrende verschickt, davon wurden 83 ausgefüllte Fragebögen zurückgeschickt: 51 aus dem Minderheitenunterricht, 32 kamen aus dem DaF-Bereich. An dieser Stelle sei allen bereitwilligen Lehrenden herzlich für die Antwort und Unterstützung gedankt! Ein Dank geht an Frau Dr. Marta Müller für die Hilfe bei der technischen Durchführung der Erhebung.

12 Aus Umfangsgründen können hier nicht alle Fragen des Fragebogens ausgewertet werden. 


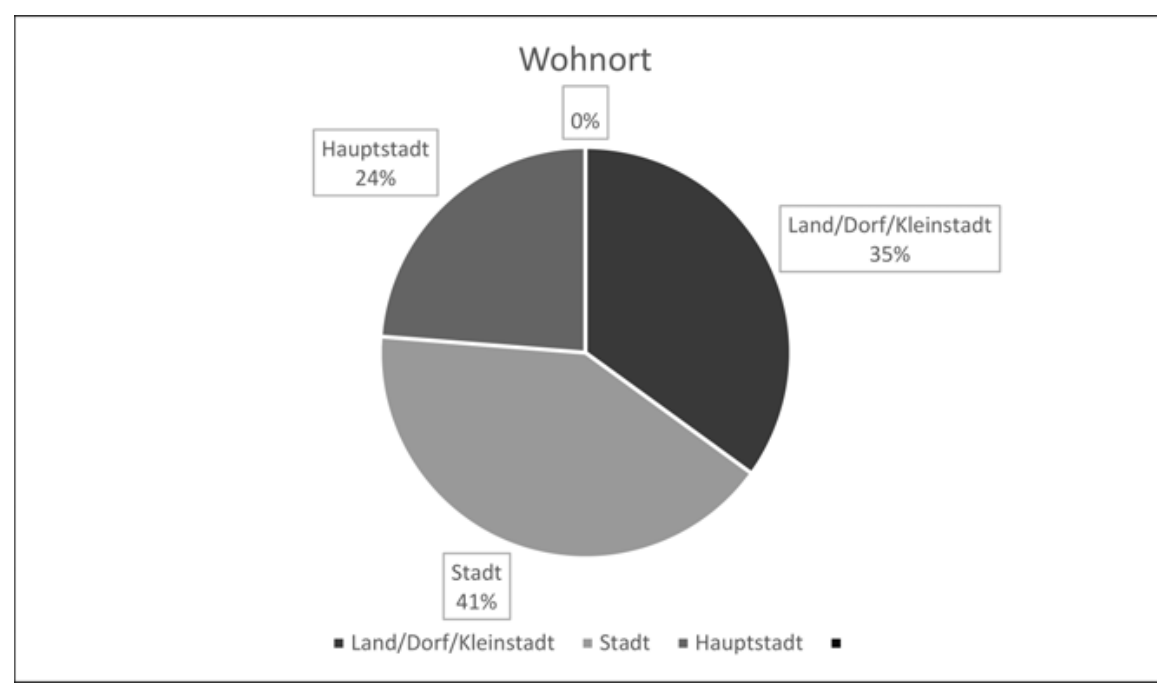

Abb. 8.1: Wirkungsorte (Schulen) der Befragten.

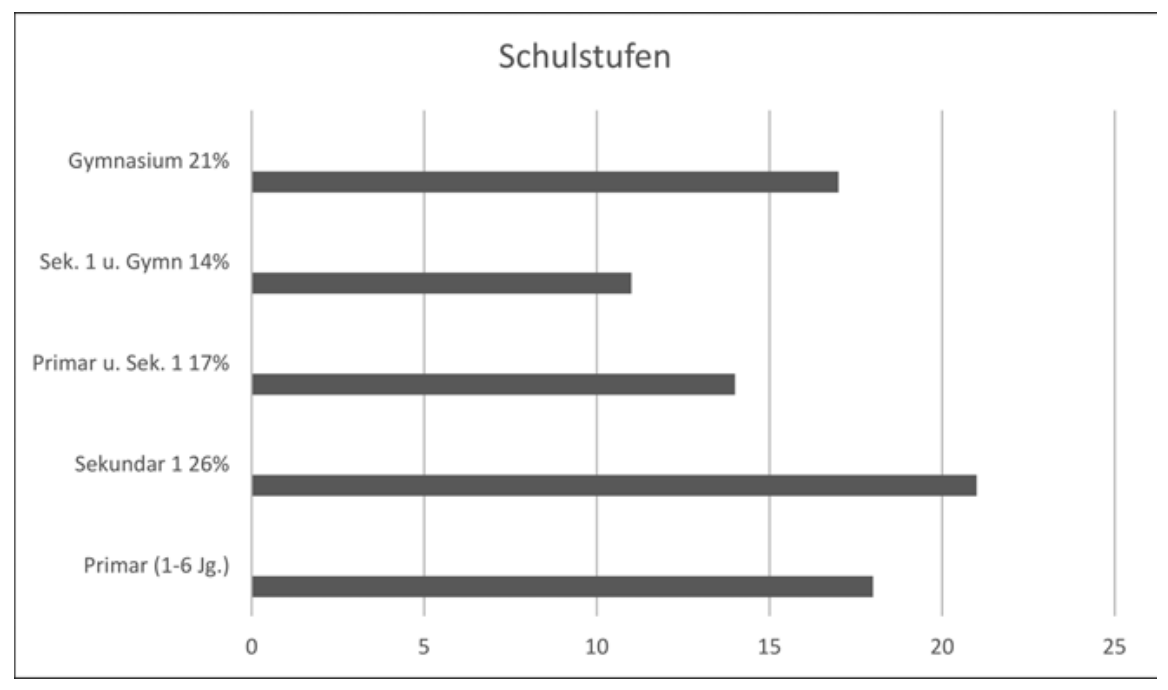

Abb. 8.2: Lehrende in den einzelnen Schulstufen.

die im Deutschunterricht berücksichtigten Unterschiede der medialen Formen. Vor zehn Jahren wurde zum Thema der Variation im Deutschunterricht in Südungarn in Lehrerfortbildungsgruppen eine Erhebung durchgeführt. Damals haben wir unter DaF- und DaM-Deutschlehrenden einen in schriftlicher Form vor- 
gelegten Fragebogen abgefragt. ${ }^{13}$ Die sprachliche Variation hatte in jener Zeit in ihren Anfängen bereits eingesetzt, doch konnte sich das Thema eher in der Lehrerausbildung durchsetzen und etablieren, viel später erst an Schulen und Gymnasien. Einerseits gab es vor zehn Jahren noch keine Unterrichtserfahrungen zum Thema, und es fehlte eine didaktische Aufbereitung dazu, die m.E. heute noch immer nicht ganz entstanden ist. Damals zeigte sich, dass das Thema Variation für Lehrende im Minderheitenbereich eher vertraut und bekannt war als im DaF-Bereich, weil - so zumindest die Einschätzung der Befragten - die Minderheiteninhalte mit der Kenntnis der deutschen Dialekte in Ungarn selbst das Vorhandensein einer Variation voraussetzen, obwohl die traditionelle Auffassung über die dialektale Diversifikation in unseren Tagen eher eine marginale Rolle spielt (vgl. Eichinger 2011: 9). So zeigten die Ergebnisse von vor zehn Jahren, dass bis zu 75\% der Lehrer - auch Minderheitenlehrer - das Thema Variation im schulischen Unterricht eher meiden oder sich davor scheuen, das Thema im Unterricht anzuschneiden (vgl. Davies 2006: 490). Die Begründung dazu lautete tatsächlich, dass durch das Aufzeigen einer sprachlichen Vielfalt und mehrerer Optionen in der sprachlichen Formulierung die Schüler in ihren bisher erworbenen Kenntnissen verunsichert würden. Ein anderer Aspekt könnte auch die Angst der Lehrenden selbst vor der Vermittlung der sprachlichen Vielfalt gewesen sein.

In der aktuellen Abfrage zeichneten sich auf die Frage: „Wird in ihrem Deutschunterricht die Vielfalt des Deutschen angesprochen/erwähnt (mehrere deutschsprachige Länder mit eigenständigen nationalen Varietäten, mehrere Dialekte, mehrere Varietäten)?“ eindeutig positive Ergebnisse und somit eine Wandlung in der Einstellung der Lehrenden ab: Von 83,5\% der jetzt Befragten wurde die Frage bejaht, lediglich 16,5\% haben mit einem Nein geantwortet. Das beweist, dass sich in den letzten zehn Jahren im Deutschunterricht ein Sichtwechsel bezüglich der überholten Homogenitätsannahme des Deutschen vollzogen hat, gleichzeitig eine Öffnung für ein in Ungarn erst seit den 1990ern erschienenes Thema des plurizentrischen und polyarealen Deutschen stattgefunden hat. Der hohe Prozentsatz lässt darauf schließen, dass diese Frage für den Deutschunterricht in der Gegenwart eine hohe Relevanz und Aktualität besitzt, und auch darauf, dass eine didaktische Aufbereitung des Themas im Gange ist, auch dank der zahlreichen elektronischen Möglichkeiten zur Darstellung von mündlichen und schriftlichen Sprachproduktionen, die man in den Klassen den Lernenden zeigen und erlebbar machen kann.

13 Die Ergebnisse wurden in einem Sammelband zur Variation von Neuland (2006) im Aufsatz von Berend \& Knipf-Komlósi (2006) publiziert. 
Auf die Fragen, ${ }^{14}$ warum es für die Lehrenden wichtig sei, diese Vielfalt des Deutschen den Lernenden zu zeigen, und zu welchem Zweck das wichtig ist, wurden von $84,0 \%$ positive und viele praxisbezogene, äußerst reflektierte Antworten gegeben; dabei spiegeln diese Antworten ganz unterschiedliche Sichtweisen der Lehrenden wider. Im überwiegenden Teil der Antworten wird für den Unterricht der Variation plädiert, doch die angeführten Argumente sind breit gestreut. Die Rolle der Variation im Unterricht scheint unbestritten zu sein, die Antworten zeugen von einer variationsbewussten Einstellung der Lehrenden, die größtenteils auch darauf zurückzuführen ist, dass 63,3\% bereits während des Studiums über das Thema Variation und Varietäten gelernt haben, teils aus Überlegungen der Nützlichkeit dieser Sprache (Ausland, Arbeit), doch es gab auch Meinungen wie, weil es Spaß macht, viele Varietäten kennenzulernen, wenn man mit Muttersprachlern kommunizieren will. Eine kleine Auswahl der frei formulierten Antworten soll exemplarisch die Argumentation der Lehrenden veranschaulichen:

Es ist wichtig, wegen dem regelmäßigen Kontakt mit Muttersprachlern aus verschiedenen Regionen. Die Behandlung des Themas ergibt sich daher von selbst.

Ich finde es wichtig, weil sie in den deutschsprachigen Staaten diese treffen werden. Von den Bekannten, die im Ausland arbeiten, hören sie öfter anderes Deutsch und manchmal stellen sie auch Fragen dazu.

Ein wenig Skepsis gegenüber der Dominanz des Standarddeutschen klingt auch mit: „weil man mit der im Unterricht angeeigneten Standardsprache auf deutschem Sprachgebiet nicht weit kommt. Man muss damit im Klaren sein, dass die Variationsbreite des Deutschen Normalität ist“.

Minderheitenlehrerinnen und -lehrer betrachten die sprachliche Variation als zum Minderheitenunterricht gehörend und als ein Merkmal des Deutschen, das eben im Ungarischen keine Relevanz besitzt: „weil unsere Schule eine Nationalitätenschule ist und weil einige Familien ins Ausland umziehen oder im Ausland arbeiten“, „da wir deutsche Nationalitätenschule sind“, „weil das auch in der Sprache der deutschen Minderheit reflektiert ist“.

Ungeachtet der positiven Einstellungen zur Anerkennung der Varietätenvielfalt bleibt eine Betonung der Dominanz des Standarddeutschen: „Es ist wichtig zu wissen, dass in den Schulen meistens Hochdeutsch unterrichtet wird, im Alltag wird das kaum gesprochen. In verschiedenen deutschsprachigen Ländern werden Dialekte, Varietäten gesprochen, trotzdem kann man mit guten Deutschkenntnissen sich gut auskennen“.

14 Die Fragen lauteten: Finden Sie es wichtig, dass in der Schule, in ihrem Unterricht die Variation des Deutschen thematisiert wird? und Begründen Sie, warum Sie es als wichtig erachten? 
Bei den 16,0\% der Nicht-Befürworter der Variationsvielfalt im Unterricht werden folgende Argumente angeführt, die teils objektiv sind: „weil es für das Abitur nicht wichtig ist“" (was tatsächlich der Wahrheit entspricht), teils werden aber subjektive Annahmen geäußert, die dem Thema ausweichen wollen: „wir kommen gar nicht dazu, haben keine Zeit“, „für meine Schüler ist die Aneignung des Hochdeutschen auch eine Herausforderung“, „Hochdeutsch ist das Unterrichtsziel“. Abgelehnt wurde die Frage vor allem aufgrund mangelnder Materialien: „in den Schulbüchern findet man kein genügendes Material zum Thema“, „in den Lehrbüchern gibt es keine Varianten“, was ebenfalls der Realität entspricht.

Es wurde auch abgefragt, ob und welche Texte welcher nationalen Standardvarietät im Unterricht herangezogen werden. $\mathrm{Zu}$ erwarten war, dass die überwiegende Mehrheit mit bundesdeutschen Texten arbeitet (91,0\%), doch erstaunlicherweise wurden auch mit einer geringeren Häufigkeit halbjährlich etwa zwei bis drei Texte zum österreichischen Deutsch herangezogen. Texte zum Schweizerhochdeutschen sind äußerst spärlich vorgekommen (1,5\%).

Ein zentraler Punkt im Zusammenhang mit der Variation ist mit den medialen Unterschieden, der gesprochenen und geschriebenen Sprache, zu erklären. Durch den Einfluss der Medien und die Wirkungen der Globalisierung entsteht eine Vielfalt von sprachlichen Mustern, in denen Züge der Schriftlichkeit und Mündlichkeit aufeinandertreffen. Aus den Antworten geht hervor, dass über 90 \% der Lehrenden im Deutschunterricht erkannt haben, dass bei den mündlichen und schriftlichen Produktionen der Lernenden unterschiedliche Maßstäbe bei der Bewertung angesetzt werden müssen. Die Argumente sind verständlicherweise pädagogisch-didaktischer Natur: „frei sprechen lassen, motiviert, keine Hemmungen entstehen lassen“, „,beim Sprechen ist Verständlichkeit wichtiger als Richtigkeit“, „damit ich ihnen nicht die Lust zum Sprechen nehme“, „beim Sprechen brauchen sie Mut und Kompetenzgefühl, deshalb nur sprechen lassen“, etc.

Bei einem geringeren Teil der Antworten scheinen die Konzepte der Distanzund Nähesprache und deren didaktisch-psychologische Aspekte, die Konzepte Korrektheit und Verstehen, im Sprachbewusstsein der Lehrenden fest verankert zu sein: „weil in der mündlichen Sprachproduktion nicht nur die Sprachrichtigkeit dominiert, sondern Kommunikationsfähigkeit, Ausdrucksvermögen“, „die Korrektur der schriftlichen Fehler kann beim Lernprozess helfen, die Schüler können ihre Fehler sehen und aus den Fehlern lernen“, „in Schrift muss man konsequenter sein, alle Fehler, die man entdeckt, sollen korrigiert werden“, „wenn man redet, nur die Fehler, die das Verständnis stören“, „die Gelegenheit ist anders, schriftlich hat man Zeit, mündlich nicht und es ist störend, wenn man ständig korrigiert wird“ etc. 
Im Fragebogen wurde auch nach der von der Standardnorm abweichenden Gebrauchsnorm gefragt, z. B. ob die Lehrenden es akzeptieren, wenn im Unterricht von den Schülern auch eine lockere Umgangssprache gebraucht wird. 70,0 \% der Befragten bejahten dies. Die Antworten darauf sind allerdings wieder facettenreich, so wird die Nützlichkeit genannt: „ja, weil sie die Umgangssprache mehr benutzen, im beruflichen Leben, bei Auslandsreisen“; eindeutig tritt die Diskrepanz zwischen Lehrbuchdeutsch und der Sprachwirklichkeit hervor: „es ist adäquater, wenn man natürlich wirken möchte, dann sollte man nicht nur Lehrbuchdeutsch verwenden“, ,ja, bis zu einem gewissen Grad sollen sie auch aktuelles Deutsch sprechen, der ganze Unterricht geht in die Richtung: Du sollst dich verständigen können, das wie ist zweitrangig“, „die Umgangssprache können sie später erlernen“.

30,0 \% der Lehrenden verneinten die obige Frage und äußerten ihre Bedenken, die eher auf eine konservativere Einstellung hinweisen. Es sind Lehrende, die eine coole, lockere Sprachform ihrer Schüler eher skeptisch sehen: „bei Sprachprüfungen, Kontrollarbeiten bedeutet das Nachteil ...“, „,wenigstens in der Schule sollen sie aufpassen und auf die Grammatik achten“, „im Unterricht wird die Sprachrichtigkeit gefördert“, „nein, in den Medien hört man heutzutage fast nur noch das coole, lockere Neudeutsch, die Schüler sollten aber auch einen Bezug zur klassischen Hochsprache haben“, „wie in der Muttersprache, sollte man sich zuerst die geregelte Sprache aneignen“.

Zum Schlüsselbegriff dieses Beitrags, zur Normfrage, die von den Lehrenden selbst und frei formuliert werden sollte, entstanden variationsreiche Antworten, die eine tiefe Einsicht in die Denkweise der Lehrenden gaben. Aus den Antworten wurde ersichtlich, dass die Normfrage in der Deutschlehrerausbildung, in Fortbildungen öfter diskutiert werden sollte und eine größere Relevanz bekommen muss, um zum Beispiel über Normtoleranz, Normabweichung, Variation etc. nachzudenken. Es entstanden Antworten, die davon zeugen, dass das Problem des Normempfindens der Befragten kein abgeklärtes Konzept aufzeigt: „die Norm ist identisch mit Standarddeutsch“, „Norm ist vorgegebene Regeln in Orthographie“, „Hochdeutsch, literarische Sprache“, „die Norm ist grammatisch korrekt, aber es ist kein Problem, wenn man Dialekt erkennen kann“, „was in der Schule in Deutschland und Österreich unterrichtet wird, ist die Norm“, „Duden Grammatik, in Zweifelsfällen schlage ich da nach, die Lösungen, das halte ich für korrekt, das ist für mich Norm“, „wie Deutsch richtig gesprochen und geschrieben wird“, „der richtige Gebrauch sprachlicher Mittel, die Gesamtheit der Regeln ist Norm“, „Norm ist, was im Alltag gesprochen, verstanden wird“, „was durch unsere Lehrbücher vermittelt wird, Hochdeutsch“, „die Norm von Deutschland“, „die Standardvarietät des Bundesdeutschen mit süddeutscher Färbung, die in Ungarn an Schulen unterrichtet wird. 
Grundlage sind dafür Schulbücher, Primärwerke, die man früher oder später in die Hände bekommt. Dialektale Ausdrücke sind in diesen kaum aufzufinden.“

\section{Fazit und Ausblick}

Der Beitrag summiert einen Einblick darüber, wie Lehrende des Deutschen in Ungarn über die sprachliche Norm und deren Vermittlung denken bzw. wie sie das Verhältnis zwischen Norm und sprachlicher Variation in der Unterrichtspraxis sehen und beurteilen. Die Lehrenden haben in ihrem Kontext (DaF und DaM in Ungarn) themenrelevante informative und aufschlussreiche Antworten bei der Onlinebefragung gegeben, die von einer vorhandenen Sprachbewusstheit zeugen. Im Vergleich zur schriftlichen Abfragung zur Variation vor zehn Jahren, als mehrheitlich skeptische und ablehnende Antworten zur Darstellung der Variation im Unterricht entstanden, sind diese Antworten in der aktuellen Abfragung einvernehmlich positiv und zustimmend: Die überwiegende Mehrheit befürwortet die Behandlung und Erläuterung des Themas der sprachlichen Variation im Deutschunterricht ab der 5. Klasse. ${ }^{15}$ Für Lehrende an Minderheitenschulen ist das Thema Variation gleichzusetzen mit der dialektalen Diversifikation der ungarndeutschen Dialekte.

Aus den frei formulierten Meinungen der Befragten geht aber auch hervor, dass Lehrende im Unterrichtsalltag die Diskrepanz zwischen den Inhalten und Normen, die sie sich während ihrer Ausbildung angeeignet haben, die Regeln und Regularitäten des Systems und der Teilsysteme sehen und erfassen, doch die Konzepte im Unterricht noch nicht umsetzen können. Die meisten Schwierigkeiten im Umgang mit Norm und Variation ergeben sich im Deutschunterricht in Ungarn m. E. aus folgenden Problemen:

- die Auffassung der Norm als etwas Starres, das Festhalten an präskriptiven Vorstellungen,

- das Erkennen und die Handhabung der medialen Verschiebungen und Überlappungen von sprachlichen Äußerungen in der Unterrichtspraxis (man wird danach beurteilt, wie man sich äußert) und das Erkennen, dass nicht nur Sprache unterrichtet wird, sondern Sprache und Kultur zusammen gehören,

- die didaktische Aufbereitung der sprachlichen Varianz auf allen (oder mind. wesentlichsten) Ebenen des Sprachsystems und deren Präsentation in den benutzten Lehrmaterialien,

15 In der Grundschule, in der Anfangsphase des Fremdsprachenlernens selbstverständlich noch nicht. 
- der Leistungsdruck der Lehrenden und Lernenden im Ausland, ein möglichst einwandfreies Standarddeutsch zu vermitteln bzw. zu erlernen und im Abitur und den sonstigen Prüfungen gute Ergebnisse zu erreichen.

In Anbetracht der Antworten der Lehrenden, die sehr offen und reflektiert sind und von einer Sprachaufmerksamkeit zeugen, sollte m. E. in der Deutschlehrerausbildung ein Sichtwechsel einsetzen, der folgende Punkte bezüglich der Norm zum Überlegen bietet. Die sprachliche Norm reagiert aufgrund ihrer historischen Entstehungsgeschichte langsamer auf Veränderungen der Sprache. Dennoch stellt sie einen Konsens dar, der ein Gerüst der gesetzten, kodifizierten Normen ergibt, die in bestimmten Varietäten und Textsorten nicht außer Acht gelassen werden können. Der Sprachgebrauch einer Gemeinschaft oder von Individuen ist dagegen flexibel, baut teils auf die gesetzten Normen auf, aber lässt mehr Varianz zu, die vor allem durch mediale, funktionale, situative, regionale Faktoren der Sprachwirklichkeit als Sprachgebrauchsbedingungen entstehen. Die Veränderungen, die hinsichtlich der Normerwartungen eindeutig auffallen, sind mit Worten von Eichinger: ,... standardsprachliche Mündlichkeit hat an Natürlichkeit gewonnen, standardsprachliches Auftreten kennt nicht mehr nur eine unbestritten akzeptable Form“ (2005: 380). Dies zu verstehen und zu berücksichtigen sollte Teil jeder Deutschlehrerausbildung werden. Genauso wie sich die Sprachhandlungsmuster von Zeit zu Zeit ändern, genauso vollzieht sich eine Variabilität der Textsorten der Gegenwartssprache. Wann, auf welcher Schulstufe und auf welche Weise diesen Veränderungen Rechnung getragen wird, ist eine Frage der Lehrplangestaltung der einzelnen Schultypen im betreffenden Land und Frage der Gestaltung der lokalen Lehrpläne.

Statuierte Normen bilden schon jahrzehntelang das Kernstück des Fremdsprachenunterrichts und in der Deutschlehrerausbildung, demgegenüber stand die Vermittlung der Routinen, die für den Sprachgebrauch unentbehrlich sind, lange Jahrzehnte im Schatten der Unterrichtspraxis. An diesem Punkt scheint mir der Terminus technicus der transitorischen Norm von Feilke (2015) sehr angebracht zu sein, der andeutet, dass die im Unterricht vermittelten Normen als Übergangsnormen betrachtet werden sollen, die sich erst im Laufe des Lernprozesses bei den Lernenden stabilisieren und sich zu einer richtigen Gebrauchsnorm entwickeln werden. Aus dieser Sicht betrachtet, wird die Lücke zwischen gesetzten Normen und Gebrauchsnormen etwas geringer ausfallen.

\section{Literatur}

Ammon, Ulrich (1995): Die deutsche Sprache in Deutschland, Österreich und in der Schweiz. Das Problem der nationalen Varietäten. Berlin/New York: de Gruyter. 
Bartsch, Renate (1987): Sprachnormen. Theorie und Praxis. Tübingen: Niemeyer (Konzepte der Sprach- und Literaturwissenschaft 38).

Berend, Nina \& Elisabeth Knipf-Komlósi (2006): Sprachliche Variation als Herausforderung für den Deutschunterricht in Osteuropa. In Eva Neuland (Hrsg.), Variation im heutigen Deutsch. Perspektiven für den Sprachunterricht, 161-175. Frankfurt a. M.: Peter Lang.

Busse, Dietrich (2006): Sprachnorm, Sprachvariation, Sprachwandel. Überlegungen zu einigen Problemen der sprachwissenschaftlichen Beschreibung des Deutschen im Verhältnis zu seinen Erscheinungsformen. Deutsche Sprache 34 (4), 314-333.

Davies, Winifred (2006): Normbewusstsein, Normkenntnis und Normtoleranz von Deutschlehrkräften. In Eva Neuland (Hrsg.), Variation im heutigen Deutsch. Perspektiven für den Sprachunterricht, 483-491. Frankfurt a. M.: Peter Lang.

Davies, Winifred \& Nils Langer (2013): Die Sprachnormfrage im Deutschunterricht: das Dilemma der Lehrenden. In Albrecht Plewnia \& Andreas Witt (Hrsg.), Sprachverfall? Dynamik-Wandel-Variation, 299-321. IDS-Jahrbuch 2013. Berlin: de Gruyter.

Durrell, Martin (1999): Standardsprache in England und Deutschland. Zeitschrift für germanistische Linguistik 27, 286-308.

Durrell, Martin (2006): Deutsche Standardsprache und Registervielfalt in DaF-Unterricht. In Eva Neuland (Hrsg.): Variation im heutigen Deutsch. Perspektiven für den Sprachunterricht, 111-122. Frankfurt a. M.: Peter Lang.

Durrell, Martin (2012): Zur Relativierung von hochsprachlichen Normen in der deutschen Sprache der Gegenwart. Der Blick von außen. In Susanne Günthner et al. (Hrsg.), Kommunikation und Öffentlichkeit: Sprachwissenschaftliche Potenziale zwischen Empirie und Norm, 89-105. (= Reihe Germanistische Linguistik 296). Berlin/Boston: de Gruyter.

Eichinger, Ludwig, M. (2005): Standardnorm, Sprachkultur und die Veränderung der normativen Erwartungen. In Ludwig M. Eichinger \& Werner Kallmeyer (Hrsg.): Standardvariation. Wie viel Variation verträgt die deutsche Sprache?, 363-380. IDS-Jahrbuch 2004. Berlin: de Gruyter.

Eichinger, Ludwig M. (2009): Begrüßung: Vom grammatischen Wissen und seiner vernünftigen Verwendung. In Marek Konopka \& Bruno Strecker (Hrsg.), Deutsche Grammatik: Regeln, Normen, Sprachgebrauch, 1-8. Berlin: de Gruyter.

Eichinger, Ludwig M. (2011): Normprobleme, oder: Variation ist sinnvoll. Überlegungen zum heutigen Deutsch. Akademie der Wissenschaften und der Literatur Mainz. Stuttgart: Franz Steiner Verlag.

Feilke, Helmuth (2015): Transitorische Normen. Argumente zu einem didaktischen Normbegriff. Didaktik Deutsch 2 (20), 115-135.

Felder, Ekkehard (2003): Das Spannungsverhältnis zwischen Sprachnorm und Sprachvariation als Beitrag zu Sprach(differenz)bewusstheit. Wirkendes Wort 53, 473-498.

Gloy, Klaus (1998): Zur Realität von Sprachnormen. In Der Deutschunterricht 50 (3/98), 14-23.

Gloy, Klaus (2004): Norm. In Ulrich Ammon et al. (Hrsg.), Sociolinguistics. Soziolinguistik. Bd. 1., 392-398. Berlin, Boston: de Gruyter

Gloy, Klaus (2008): Sprachnormierung und Sprachkritik in ihrer gesellschaftlichen Verflechtung. In Werner Besch, Anne Betten, Oskar Reichmann \& Stefan Sonderegger (Hrsg.): Sprachgeschichte. Ein Handbuch zur Geschichte der deutschen Sprache und ihrer Erforschung. 2., vollst. neu bearb. und erw. Auflage. Band. 1, 396-406. Berlin, New York: de Gruyter.

Heidolph, Karl-Erich, Walter Flämig \& Wolfgang Motsch (1981): Grundzüge einer deutschen Grammatik. Berlin: Akademie-Verlag. 
Hennig, Mathilde (2012): Was ist ein Gramatikfehler? In Susanne Günthner et al. (Hrsg.): Kommunikation und Öffentlichkeit: Sprachwissenschaftliche Potenziale zwischen Empirie und Norm. 121-148. Tübingen: Niemeyer.

Hundt, Markus (2009): Normverletzungen und neue Normen. In Marek Konopka \& Bruno Strecker (Hrsg.), Deutsche Grammatik: Regeln, Normen, Sprachgebrauch, 117-141. Berlin: de Gruyter.

Idegennyelvi kerettanterv (2012). [Rahmenlehrplan für die Fremdsprachen]

Müller, Márta (2010): Die Situation des Deutschunterrichts in Ungarn. In Frank Kosztrzewa, Roberta Rada \& unter Mitarbeit von Elisabeth Knipf-Komlósi (Hrsg.), Deutsch als Fremdund Minderheitensprache in Ungarn. Historische Entwicklung, aktuelle Tendenzen und Zukunftsperspektiven, 74-96. Hohengehren: Schneider Verlag.

Nemzetei Alaptanterv (2012). In Magyar Közlöny 55. szám. jun. 4. [Nationaler Grundlehrplan] Neuland, Eva (1998): „Sprachnormen“ - kein Thema mehr? Der Deutschunterricht 50 (3/98), 4-13.

Neuland, Eva (2006): Variation im heutigen Deutsch. Perspektiven für den Sprachunterricht. Frankfurt a. M.: Peter Lang

Stridova, Nela (2015): DaF-Lehrer als Normautorität am Beispiel eines tschechischen Gymnasiums. In Katerina Sichova, Reinhard Krapp, Paul Rössler \& Vit Dovalil (Hrsg.), Standardvarietät des Deutschen. Fallbeispiele aus der sozialen Praxis, 117-133. Berlin: Logos Verlag.

Wurzeln und Flügel. Rahmenlehrplan für den Deutschunterricht der bilingualen deutschen Nationalitätenschulen in Ungarn (2010) Landesselbstverwaltung der Ungarndeutschen. Wurzeln und Flügel. Kompetenzmodell für den Deutschunterricht der bilingualen deutschen Nationalitätenschulen in Ungarn (2010), Landesselbstverwaltung der Ungarndeutschen

Ziegler, Evelyn (2011): Subsistente Normen und Sprachkompetenz: ihre Bedeutung für den Deutschunterricht. Bulletin suisse de linguistique appliquée 94, 69-85. 


\title{
Rüdiger Harnisch \\ 9 Reanalyse durch Varietätenkontakt - Morphogenese durch Hyperkorrektion
}

\begin{abstract}
Dieser Beitrag steht im Kontext eines aktuellen DFG-Projekts zur Typologie und Theorie der Remotivierung. An einer besonderen Form der Reanalyse sprachlicher Einheiten, der Hyperkorrektion, wird gezeigt, wie diese struktur- und einheitenschaffend wirkt. Hyperkorrektion spielt sich zwischen oft vertikal geschichteten - Varietäten ab. Als deren auslösende und steuernde Kraft wird Analogie ausgemacht. Die Proportionengleichungen, die dieser sprecherpsychischen Aktivität zugrundeliegen, operieren also nicht nur intrasystemisch, sondern auch dia-systemisch, über Varietätengrenzen hinweg. Die Hauptdomäne von Hyperkorrektion ist Phonologie. In diesem Beitrag steht dagegen Morphologie, gewonnen aus amorphischer Lautsubstanz oder vermeintlichen morpho-phonologischen Verschmelzungen, als Produkt hyperkorrigierender Prozesse im Mittelpunkt.
\end{abstract}

Keywords: Analogie, dia-systemische Hyperkorrektion, morphologische Reanalyse (formale Resegmentierung, semantische Remotivierung)

\section{Herleitung des Gegenstands aus einem Fallbeispiel}

Mit folgendem Fall, der sich in den späten 1970er, frühen 1980er Jahren an einer Stadtrandschule in Bayreuth zugetragen hat, soll in das Thema eingeführt werden. Gleichzeitig wird an diesem Vorkommnis die Alltagsrelevanz des Phänomens in Gebieten mit innerer Mehrsprachigkeit und seine soziale Dimension sichtbar: ${ }^{1}$

Renate, ein Bauernkind, geht in eine erste Klasse zusammen mit Kindern, die weitgehend hochdeutsche Umgangssprache (in mehr oder weniger starker fränkischer Färbung) spre-

1 Die Begebenheit schildert Hinderling (1982: 110, Anm. 22). Kursivsetzungen sind aus dem zitierten Original übernommen. Ludwig M. Eichinger wird sich an Örtlichkeit und Zeitumstände aus seiner damaligen Ansässigkeit am betreffenden Stadtrand von Bayreuth erinnern.

Rüdiger Harnisch, Lehrstuhl für Deutsche Sprachwissenschaft, Universität Passau, D-94030 Passau, E-Mail: ruediger.harnisch@uni-passau.de

Ә Open Access. (c) 2018 Rüdiger Harnisch, publiziert von De Gruyter. (c) BY Dieses Werk ist lizenziert unter der Creative Commons Attribution 4.0 Lizenz.

https://doi.org/10.1515/9783110538625-010 
chen, während sie echten oberfränkischen Dialekt spricht. In den ersten Wochen bekommen die Kinder die Aufgabe, verschiedenfarbige geometrische Figuren nach der Form zu ordnen. Allen Kindern außer Renate gelingt dies mühelos. Renate ordnet statt nach der Form konsequent nach der Farbe. Die Lehrerin ist sehr unwillig, schimpft mit dem anscheinend verstockten oder einfach dummen Kind und erteilt die neue Aufgabe. Jetzt bitte nach der Farbe ordnen. Das Ergebnis entspricht dem vorherigen: Alle können die Aufgabe, Renate ordnet konsequent nach der Form. Der Beweis ihrer Zurückgebliebenheit scheint erbracht. Und dabei gehört nur wenig Spürsinn dazu, um zu entwirren, was hier kommunikativ vorgegangen (bzw. in die Binsen gegangen) ist. Die Lehrerin verlangt die Klassifizierung nach der [foam] 'Form'. Renate $m u \beta$ dies analysieren als [foam] = /forbn/ $<$ mhd. varwen = 'Farbe'. 'Form' lautet bei ihr [foam]. Wir haben also ein Minimalpaar von [foam] 'Farbe' $\neq$ [foam] 'Form'. Die zweite Fehlleistung Renates ist vermutlich nicht mehr sprachlich zu interpretieren. Da der Sprecher des Ostfränk. „weiß“, daß [m] u.U. durch Assimilation aus /bn/ zustandekommt (man vergleiche [gla:m] gegenüber [glabd]), wird Renate erkennen können, daß Farbe ihr /forbn/ ist. Aber nachdem das erste Mal die nächstliegende Analyse so danebenging, hat sich Renate vermutlich eine Strategie zurechtgelegt: „Es ist immer gerade anders, als man denkt“. Infolgedessen hängt indirekt auch die zweite Fehlleistung mit der eindeutig sprachlich bedingten ersten zusammen. Das Beispiel macht darüber hinaus deutlich, wie sehr das Kind durch seine Situation „gefordert“ wird, worin freilich auch eine Chance liegt, - wenn man ihr sie nicht verpatzt.

An diesem Vorkommnis ist zu sehen:

- Der Grund für die Fehlleistung ist, dass zwei kontrastierende Varietäten ein und derselben Sprache in Kontakt kommen: Hochdeutsch und - hier oberostfränkischer - Dialekt.

- Dieser Kontakt der kontrastierenden Varietäten wird zum Konflikt, weil beide Sprecherseiten ein Defizit haben: Die Schülerin erkennt nicht die Domänenspezifik bei der Aufgabenstellung, denn sie hält die vermutlich auf Hochdeutsch vorgebrachte Arbeitsanweisung für eine dialektale, die es als ,richtig', d.h. hochdeutsch, zu interpretieren gilt. Die Lehrerin erkennt nicht das Potenzial zum Missverständnis, das in diesem VarietätenKontakt steckt. Immerhin hat die Schülerin der Lehrerin voraus, dass sie um die Tücken innerer Mehrsprachigkeit weiß - wenn sie ihnen letztlich auch selber zum Opfer fällt.

- Die Varietäten werden von beiden Seiten sozial bewertet: Die Lehrerin ist auf die Standardnorm und ihre Erfüllung fixiert. Die Schülerin übersetzt zumindest in dieser Anforderungssituation - den Dialekt (mit dem sie wahrscheinlich schon Stigmatisierungs-Erfahrungen gemacht hat) in die Prestige-Varietät, was zu der Hyperkorrektur ${ }^{2}$ führt, die allein ihre Fehlleistung erklären kann.

2 Im Folgenden wird terminologisch zwischen Hyperkorrektion als Vorgang (psycholinguistische Operation) an sich und Hyperkorrektur als konkretem (sprachlich materialisiertem) Fall von Hyperkorrektion unterschieden. 
- Bestimmte diasystemische Konstellationen varietätenspezifischer systemischer Merkmale sind für solche sozial bedingten Korrekturvorgänge besonders anfällig. Am geschilderten Fall wird deutlich, dass Übergänge von der Formen- zur Lautebene (und umgekehrt) besonders intrikat sind, gerade weil die Sprecher von diesen Übergängen „wissen“, z. B. in der von Hinderling oben beschriebenen Weise, „daß [m] [...] durch Assimilation aus /bn/ zustandekommt.“ Hinzuzufügen wäre, dass dieses /bn/ wiederum meistens durch Suffigierung eines morphischen $-n$ an einen auf...$b$ - auslautenden Stamm entsteht. Die Übersetzung des - unter normativen Aspekten stigmatischen - opaken [m] des Dialekts in die - in normativer Perspektive prestigeöse - transparente /...b-n/-Struktur des Standards ist also mit einer (Wieder-) Herstellung von Morphologie verbunden.

Solche Reanalyse-Prozesse, die eine morphologische Struktur hervorbringen und die nur denkbar sind, wenn von einem innersprachlich mehrsprachigen Sprecher Merkmale der einen Varietät in Merkmale der andern übersetzt werden, stehen im Mittelpunkt dieses Beitrags. Die Überlegungen dazu sind aus der Arbeit im aktuellen Passauer DFG-Projekt zur Typologie und Theorie der Remotivierung (TheoRem) hervorgegangen. Hyperkorrektion stellt eine der Formen des umfassenderen Projektgegenstands, der Reanalyse, dar. Reanalyse wird hier als Sammelbegriff für formale Resegmentierung und semantische Remotivierung verstanden, die, dem Zeichencharakter der betroffenen Einheiten geschuldet, nicht voneinander zu trennen sind. ${ }^{3}$ Wo, wie bei einer Übersetzung von dialektal Form in hochdeutsch Farb-en im eingangs geschilderten Fall, morphologische Struktur entsteht, ist/wird sie inhaltlich belegt. ${ }^{4}$

Um die Besonderheit der Reanalysen zeigen zu können, die nur auf der Basis von Interpretationen der diasystemischen Beziehungen zwischen unterschiedlichen Varietäten zustande kommen (Abschnitt 4), sollen vorher im Kontrast dazu Reanalysen vorgeführt werden, die innerhalb ein und derselben Varietät, also intra-systemisch, vorgenommen werden (Abschnitt 2). Ausführungen zum Zusammenhang zwischen diasystemischer Reanalyse und Hyper-

3 Siehe den programmatischen Untertitel des Sammelbands von Harnisch (2010a), in dem es um Typen formaler Resegmentierung und semantischer Remotivierung geht. Darin hat Eichinger (2010) Überlegungen zu sprecherseitigen Strategien angestellt, die sich als „Remotivierungstendenzen“, so der Untertitel seines Beitrags, beschreiben ließen. Da sein Beispielbereich die Aktion Mein schönstes deutsches Wort war, in deren Rahmen Laien über Sprache reflektierten, konnte er reanalytische Prozesse aufzeigen, die von bewussterer Art sind als die in vorliegendem Beitrag behandelten.

4 In Bezug auf das Suffix im Beispiel entweder als stammbildendes (deklinationsklassenanzeigendes) oder flexivisches. Vgl. Harnisch (2004a: 224) mit ähnlichen Beispielen. 
korrektion (Abschnitt 3) leiten zum Abschnitt 4 über. Nach ergänzenden Ausführungen zur rein phonologischen Hyperkorrektion (Abschnitt 5) wird in Abschnitt 6 ein Fazit gezogen, das Analogie als treibende Kraft reanalytischer Vorgänge im Allgemeinen und hyperkorrigierender Vorgänge im Besonderen begreift und proportionale Gleichungen als deren Mechanismus herausstellt.

\section{Intrasystemische morphologische Reanalysen}

\subsection{Resegmentierung und Remotivierung lautlicher Substanz aus morphologisch ungegliederten Einheiten}

Sprecher reanalysieren sprachliche Einheiten natürlich nicht nur dann, wenn, wie im einleitenden Beispiel, Formenvarianten „in Kontakt“ treten, die unterschiedlichen - hier diastratisch geschichteten - Varietäten angehören. Vielmehr werden Reanalysen auch - vor allem sogar - varietäts-intern, also innerhalb der Systeme dieser Varietäten selbst, vorgenommen. So begegnen wir bis in die historische standardsprachliche Lexikographie hinein einer Deutung von Falter als ${ }^{\star}$ Falt-er : falt-en (Grimm \& Grimm 1862: 1302) analog zu Halt-er: halt-en ${ }^{5}$, innerhalb dialektaler Systeme Reanalysen wie von unser $>{ }^{\star}$ uns-er/uns-e/uns-es analog mein-er/mein-e/mein-es (Harnisch 2004a: 215) oder Restrukturierungen innerhalb transitorischer Systeme wie dem Erstspracherwerb in der Art von sauber > *saub-er (Komparativ) mit den Rückbildungen ${ }^{\star}$ saub (Positiv) und $a m{ }^{\star}$ saub-st-en (Superlativ) analog zu klein-er : klein : am klein-st-en (Harnisch 2007: 7). ${ }^{6}$ Für alle diese Varietäten sind auch systeminterne Reanalysen zu beobachten, die zur Abspaltung ${ }^{7}$ von Proklitika bzw. von phrasensyntaktisch im schwachtonigen Schatten von Autosemantika stehenden Funktionswörtern führen, etwa bei Eigennamen des Typs standardsprachlich Zwieselburg >

5 Falter ist ,falsch“ segmentierend „[a]bgelöst aus mhd. vîvalter“, das aus der Reduplikationsform vîval- (vgl. lat. papil[io]) und dem Reflex eines Suffixes ahd. *-tra (vgl. germ. *fîfal-drôn) besteht (nach Seebold 2011: 275).

6 Strukturell vergleichbar ist ein Beispiel, das Kalau (1984: 151) anführt: mit heiser Stimme 'mit heiserer Stimme'. Hier dürfte aus dem Monomorph heiser ein Komparativ *heis-er reanalysiert und daraus ein Positiv ${ }^{\star}$ heis rückgebildet worden sein. Das -er des Belegs ist dann als korrektes - Flexionsmorphem aufzufassen (heis-er ${ }_{\text {D.sg.f. }}$ ), nicht als pure Auslautsubstanz des Adjektivs, der kein Suffix / ein Nullsuffix folgt ( ${ }^{\star}$ heiser- $\emptyset_{\text {D.Sg.f. }}$ ). Zu Recht kommentiert Kalau aber, dass der Fall „nicht als mundartbedingt interpretiert werden“ könne. Er ist systemintern erklärbar und wird deshalb hier unter 2.1 eingruppiert.

7 Nach Jespersen (1925: 370-373) „Ausscheidung“ (Übersetzung für seinen englischen Terminus „secretion“). 
z' Wieselburg > Wieselburg analog zu z'Wien ' $\mathrm{zu}$ (= in) Wien', dialektal Zwerenain $>z$ ' Werenain $>$ Werenain ${ }^{8}$ analog zu $z$ 'Minga 'zu (= in) München' oder kindersprachlich Diana > die Jana analog zu die Julia.

Wichtig ist für diesen unter 2.1 behandelten Typus, dass das angegebene Analogon jeweils innerhalb derselben Varietät $\mathrm{zu}$ finden ist.

\subsection{Resegmentierung und Remotivierung vermeintlicher morpho-phonologischer Verschmelzungen}

Werden bei den unter 2.1 analysierten Fällen Morpheme aus der lautlichen Substanz der zugrundeliegenden, vorher unsegmentierten Einheiten erst gewonnen, handelt es sich im Folgenden um die Wiederherstellung einer vermeintlich verlorengegangenen morphologischen Struktur samt deren morphologischen Segmenten. Im Trailer zum Tatort-Krimi Lauf des Todes hieß es im Norddeutschen Rundfunk am 22. Januar 2004 gegen 23:30 Uhr: „So viel beredetes Schweigen ist schwer zu ertragen.“ Hier wurde analog gesandt : gesendet das fossilierte und morphologisch opake beredt in die transparente Form bered-et wiederaufgelöst. Dieser Typus ist jedoch selten, weil es nur wenige solche syteminternen Strukturvarianten wie hier die Partizipialformen mit oder ohne Verschmelzung von Stammauslaut ...d mit homorganem Suffix $-t$ gibt. Denn eine intrasystemische Morpho-Phonologie darf es in normativer Hinsicht eigentlich nicht geben, führt sie doch zu „Formstufen“ weg vom Standard nach ,unten'.9

8 Maurer (1912: 31) und Mayer (1945: 6-7), der das dahinterliegende Phänomen als „Aphärese“ bezeichnet und diese definiert als „Wegfall von Lauten, die zum eigentlichen Wort oder Stamm gehören, in unserem Zusammenhang von solchen Lauten, die irrtümlich als agglutinierte Präpositionen oder Artikel angesehen und in diesem Glauben abgestoßen wurden“ (5). Beide Toponyme, Wieselburg in Niederösterreich und Werenain in Niederbayern, sind zum amtlichen Ortsnamen geworden.

9 Vgl. den sprechenden Titel von Meinhold (1973): „Lautschwächungen und Formstufen“, was man so lesen wird, dass Lautschwächung zu einer Abstufung führt, also diastratisch ,abwärts“ gerichtet ist. Unter dieser Prämisse wäre der hier dargestellte Fall besser im Abschnitt 4.2 zur diasystemisch bedingten hyperkorrigierenden Resegmentierung und Remotivierung vermeintlicher morpho-phonologischer Verschmelzungen aufgehoben. 


\section{Diasystemische Reanalysen und Hyperkorrektion}

Konstitutiv für diasystemische Reanalysen ist Hyperkorrektion ${ }^{10}$, die man sich so erklären kann: Je mehr die Diasystemik der beteiligten Varietäten vertikaler Natur ist (diastratisch), je stärker die beteiligten Varietäten sozial geschichtet sind und je ausgeprägter eine varietätenpragmatische Sprachsituation vorliegt, in der systemische Merkmale der ,niederen' Varietät im Abgleich mit den Merkmalen der ,höheren“ Varietät als „Fehler“ gelten (also als „falsch“ und nicht nur als „anders“), desto wahrscheinlicher entsteht aus der resultierenden Unsicherheit das Bestreben, solche Fehler zu vermeiden. Wenn es nur vermeintliche sind, liegt Hyperkorrektion vor.

Die Diasystemik und ihre normative Bewertung als Voraussetzung von Hyperkorrektion betonend, schränkt Herrgen (1986: 143) ein, dass diese „entweder sprachschichtextern oder einzelsprachextern verursacht ist, nicht aber intern“, das heißt entweder entsteht, wenn unterschiedliche Varietäten einer Sprache (Sprachschichten) oder unterschiedliche (Fremd-)Sprachen kontrastieren. In Anm. 4 weist er darauf hin, dass es zwar auch „sprachsystemintern [...] generalisierende Analogie - etwa im Spracherwerb des Kindes“ - gebe, „die als ,Fehler' korrigiert“ würden (etwa gehte analog zu fragte, Täge analog zu Drähte). „Solche systeminternen Analogien werden jedoch nicht als Hyperkorrektionen bezeichnet" - und nicht als solche behandelt, auch in vorliegendem Beitrag, die Fälle von Abschnitt 2 betreffend, nicht.

10 Zur Differenzierung dialektbedingter Fehler - im Sinne des vorliegenden Beitrags müsste man sagen: varietätenkontakt- und -kontrastbedingter Fehler - siehe Henn (1980: 24-25) und Koller (1991: 5). Henns Typen der (a) „Kontrastnivellierung“, (b) „Kontrastübertreibung“ und (c) „Kontrastverschiebung“ entsprechen Kollers Typen der (a) „Direktanzeige“, (b) „Hyperkorrektur“ und (c) „Fehlkorrektur“, wobei die Typen (b) und (c) jeweils näher zusammengehören, weil sie auf der Basis eines erkannten Varietätenkontrasts korrigierend vorgenommen werden und nicht in dessen Unkenntnis ,passieren“ - vgl. Kollers jeweiliges Terminus-Zweitglied -korrektur bei (b) und (c). Zehetner (1977: 11) unterscheidet dementsprechend auch lediglich „Interferenzfehler“ für (a) und „die sogenannten ,hyperkorrekten“ Formen“ für (b) und (c). Allerdings sind auch die Typen (b) und (c) interferenzbedingt, nur eben auf die beschriebene andere Art und Weise. Im weiteren Verlauf spricht Zehetner dann allerdings von „I: Direktanzeigen“ für (a) und von - (b) und (c) zusammenfassend - „II: Hyperkorrektionen“ (23, unter Sigle F). Diese Zweiteilung nimmt auch Löffler (1980: 97-98) vor, der dann allerdings noch die „Reduktion der eigenen sprachlichen Ausdrucksmöglichkeiten“ („die ,restringierte‘ Sprechweise“) anführt, die bis Null reichen könne: zum „Verstummen“. Auch diese („Hyper“-)Reaktionen geschehen in Kenntnis des Varietätenkontrasts und ,aus Angst vor Fehltritten“, sind also „Korrekturen“. Zu solchen Typologien vgl. das Projekt Fehlerlinguistik im BMBF-Vorhaben zu Strategien der Kompetenzentwicklung in der Lehrerbildung (SKILL), an dem Verfasser beteiligt ist. 
Allerdings gibt es eine Unsicherheitszone, in der man nicht weiß, ob eine systemimmanente Analogie oder ein erkannter Varietätenkontrast die Ursache für die falsche Bildung ist. Wenn Kinder z. B. * gilt-et statt gilt bilden, so kann das durch Anpassung an die sonst im gleichen System geltenden Suffigierungsregeln geschehen (analog zu falt-et), aber auch durch SystemkontrastWissen darüber, dass substandardsprachliche Verschmelzungen der Art falt't standardsprachlich in solche der Art falt-et aufzulösen sind, ein als * gilt't interpretiertes gilt dann entsprechend in * gilt-et.

Auch bei einem anderen Typus von Hyperkorrekturen kann man nicht sicher sein, ob diese durch intra- oder diasystemisch bedingte Fehlanalogien entstehen. Wenn nämlich analog zu hieß, spie usw. ${ }^{\star} v e r s p i e s$ statt verspeiste oder analog zu trägt/trug usw. *frägt/frug statt fragt/fragte oder wenn bei Vorliegen lexikalisch-semantischer Varianten ${ }^{\star}$ geschliffen statt geschleift oder *hing statt hängte verwendet werden, ${ }^{11}$ so kann das entweder durch die systeminterne Wahl einer falschen Klasse (hier der starken statt der schwachen Konjugation) verursacht sein oder aber dadurch, dass man vor dem Hintergrund von notorischen Unsicherheitsfällen wie gewinkt/gewunken im diasystemischen Abgleich vertikal skaliert und sich für die als prestigeträchtiger eingeschätzte starke Variante entscheidet.

\section{Diasystemische morphologische Reanalysen}

\subsection{Resegmentierung und Remotivierung lautlicher Substanz aus morphologisch ungegliederten Einheiten}

In seiner fehleranalytischen Untersuchung unterfränkischer Schülertexte bringt Koller (1991: 68) Beispiele wie *ab statt aber. ${ }^{12}$ Er handelt das unter

11 Belegnachweise für die Beispiele in diesem Absatz: ,dass ich den Inhalt meines Weihnachtstellers nicht so flott wie sie verspies“ (Andere Zeiten Magazin 3/2011, 22) - „die alte Weise [...], die einst mich frug und jetzt mich frägt“ (Richard Wagner, Tristan und Isolde, 3. Aufzug, 1. Szene. In Richard Wagner, Die Musikdramen. München 1978, 373) - „Der Elfenbeinturm ist geschliffen" (Peter Frankenberg in Interview Forschung \& Lehre 7/2011, 520-521, in Überschrift und Text), Leserbrief zu diesem Fehler in Folgeheft 8/2011, 610 - „Ein Kleingärtner hing ein Schild in Richtung Nachbar auf“ (FAS 13.1. 2013) - „Winken konjugiert - In ,Stéphanie von Monaco bekommt einen Clown' (FAZ vom 22. Januar) hat von der Manege ein früherer Ehemann ,gewunken'. Wie konjugieren Sie winken? Winken, wank, gewunken oder winken, winkte gewinkt? Mein ,Duden“ (22. Auflage) schreibt ,falsch: gewunken'. Da ich diese Form schon öfter in der F.A.Z. gelesen habe, vermute ich, dass Ihre Schlussredaktion dies für richtig hält““ (Leserbrief FAZ 3.2. 2016).

12 Auch ${ }^{\star} z$ wisch statt $z$ wischen. 
„Silbenfehlern“ ab und geht davon aus, dass „Endsilben abfallen“, also Apokope (aus fränkischer „Neigung zur Ein-Silbigkeit“) vorliegt. Gegen diesen wenig überzeugenden Erklärungsversuch Kollers wird hier jedoch dafür plädiert, von einer morphologisch motivierten Tilgung auszugehen:

a) entweder in der intrasystemischen Weise, dass aber wie die unter 2.1 behandelten Fälle Falter $>^{\star}$ Falt-er (analog zu Halt-er) u.ä. in ${ }^{\star} a b$-er resegmentiert wird, vielleicht analog zum junktional verwendeten, als gegliedert empfundenen und semantisch ebenfalls einschränkenden auß-er ( : aus?);

b) oder aber in der diasystemischen Weise, dass aber als eine ähnlich inkorrekte ,Steigerung' von $a b$ aufgefasst wird, wie etwa dialektal halt-er eine zur standardsprachlichen Modalpartikel halt darstellt.

Hier wird morphologische Motivierung und Reanalyse nach Art von b) postuliert. $^{13}$

\subsection{Resegmentierung und Remotivierung vermeintlicher morpho-phonologischer Verschmelzungen}

Anders als bei den in Abschnitt 2.2 behandelten Fällen intrasystemischer Reanalyse unterstellter morpho-phonologischer Verschmelzungen verhält es sich beim Typus, der durch das einleitende Beispiel repräsentiert ist. Dieser Typ der vermeintlichen Wiedergewinnung morphologischer Struktur soll im Mittelpunkt dieses Beitrags stehen. Bei ihm werden genau deswegen Reanalysen vorgenommen, weil Erscheinungen zweier unterschiedlicher Varietäten in diasystemische Verbindung gebracht werden. Ohne diese Diasystemik wären die zu beobachtenden Prozesse der (Um-) Interpretation sprachlicher Strukturelemente gar nicht denkbar: Nur wer weiß, dass die standardsprachliche [V:bən]-Kette zur substandardsprachlichen bzw. dialektalen [V:m]-Kette werden kann, kann

13 In Bezug auf zwischen (siehe vorausgehende Anm. 12) bieten sich folgende Erklärungen an: (a) Unsicher, ob zwischen (standardsprachlich) oder zwischer (die ostfränkische Form; dazu Harnisch 2004a: 216, Harnisch 2017: 43) korrekt ist, wird die ,unsuffigierte، Variante zwisch gewählt, bei der man in Bezug auf die Endung nichts falsch machen kann. Hier läge eine diasystemisch begründete Strategie vor. (b) Die Präposition zwisch wird gewählt, weil zwischer als zwisch-er reanalysiert und der Form etwas Komparativisches zugeschrieben wird, so wie hinter als hint-er 'weiter hinten', über als üb-er 'weiter oben' interpretiert werden kann. Vgl. Harnisch (2002: 200) und Harnisch (2017: 44) zur komparativischen Interpretation räumlicher Adjektive auf -er und die Ausführungen von Trost (2010) zu bezeichnenderweise so genannten „Komparativpositiven“. 
aus einer [V:m]-Kette eine [V:bən]-Kette restituieren - berechtigt bei [le:m] > [le:bən], unberechtigt bei dialektal [foam] 'Form' > standarddeutsch * [farbən] 'Farben' (siehe das einleitende Beispiel).

Da sich solche Prozesse vor allem an der Naht von Stammauslaut und Suffix(anlaut) abspielen, sind sie mehr als nur phonotaktischer Natur, betreffen sie doch elementar die Morphotaxe: sowohl deren Verunklarung bei den Verschmelzungsprozessen wie auch deren Wiedersichtbarmachung bei den Versuchen der Sprecher, morphologische Transparenz wiederherzustellen. Weil die Flexionssuffixe in Zahl und Lautung begrenzt sind, handelt es sich zum andern immer nur um wenige typische Lautverkettungen aus Stammauslaut und Suffix(anlaut), die verschmelzungsanfällig und - vermeintlich - entschmelzungsbedürftig sind.

Zahlreiche Belege für hyperkorrigierende diasystemische morphologische Reanalysen aus dem Erstspracherwerb, die dem Typus der ,Entschmelzung ${ }^{14}$ vermeintlicher morpho-phonologischer Verschmelzungsprodukte angehören, finden sich bei Harnisch (2004a: 224): $\operatorname{crem}($ en) wird in *creb-en resegmentiert, Rahm(en) als ${ }^{\star}$ Rab-en - vermeintlich wieder - transparent gemacht. ${ }^{15}$ Erklären lassen sich solche Reanalysen auf zweierlei Weise: Entweder wurde den Kindern hier der Input crem'm bzw. Rahm'm gegeben, was sie als Verschmelzung in der Substandardvarietät aufgefasst und in die als standardsprachlich aufgefasste Form * ${ }^{\star}$ creb-en bzw. ${ }^{\star}$ Rab-en (hyper)korrigiert haben. Oder es wurde der Input crem-en und Rahm-en gegeben, doch haben die Kinder das schon als eine erwachsensprachliche Verdeutlichung aufgefasst, bei der das Suffix nochmals gesetzt wurde, nachdem es vorher vermeintliches Opfer einer Verschmelzung geworden war $\left({ }^{\star}\right.$ creb-en $>$ crem $>$ crem-en). Im letzten Fall hätte man zwei gestufte Hyperkorrekturen: Zum einen wird die unterstellte Kompensationsform crem-en mit ihrer ,nochmaligen' Suffigierung korrigiert, zum andern wird die den Anlass für die Kompensation gebende vermeintliche Verschmelzung creb-en > crem rückgängig gemacht. ${ }^{16}$

14 Ein Zyklus von Wandelprozessen, der ,Entschmelzung، als eine Prozess-Phase enthält und gegenläufig zu dem von Lüdtke (1988) entworfenen Zyklus ist, der Verschmelzung als eine Prozess-Phase enthält, wird in Harnisch (2017: 54) vorgeschlagen.

15 Des Weiteren die Form aufräuben 'aufräumen' (Philipp Harnisch im Alter 4;2, Dezember 1989). Die Verben kommen neben den Infinitiven in den finiten Formen ich mich selber eincreb und ich räub auf vor. Die Schreibungen hier sind orthographisch normalisiert, was der Wiedergabe der Lautung im entscheidenden segmentalen Abschnitt $b(e n)$ aber keinen Abbruch tut. 16 Solche Prozesse, bei denen morphologische Operationen die Ergebnisse morpho-phonologischer Störungen reparieren, sind bei Plank (1985) als „reapplication of morphological rules after phonological rules“ beschrieben. Von „Morphologie nach Phonologie“ spricht Harnisch (1987: Kap. 9). Kindersprachliche Beispiele wie klem (< kleb-en) > klem-en sind bei Harnisch (2004a: 226) erläutert. 
Eindeutiger und direkter ist die Rückumsetzung von $m$ in ben, wenn ein spätalphabetisierter Erwachsener in einem Aufsatztext Arbeitzabent 'Arbeitsamt' schreibt. Auch diese Sequenz -abent '-amt' ist nur so erklärbar, dass aus Kenntnis der Verschmelzung von standardsprachlich ben zu umgangssprachlich $m$ der Umkehrschluss gezogen wird, ein vermeintlich falsches $m$ in Amt zu einem ,korrekten' ben in Abent zurücktransformieren zu müssen (vgl. Harnisch 2006: 12, 9). Dieser Hyperkorrektionsvorgang bleibt allerdings morphemintern, ist also rein phonotaktisch, ohne zu einer Morphogenese (Bildung einer Morphemgrenze zwischen vermeintlichem Stamm und vermeintlichem Suffix) zu führen.

Dies wiederum ist jedoch bei einer scherzhaften hyperkorrekten Rückauflösung (Reanalyse) der Fall, die in Franken geläufig ist und der Verspottung überhochdeutscher Ausdrucksweisen dient: Regenscherben für Regenschirm. ${ }^{17}$ Wer die Verschmelzung von Scherb-en 'Scherben' zum ,vulgären' Scherm kennt, mag leicht das vermeintlich ebenso, vulgäre' Scherm 'Schirm' zu Scherb-en re-meliorisieren (vgl. Harnisch i. V.).

Etwas verdeckter liegen die Ursachen für kindersprachliche Formen wie Däubling/Däublinchen 'Däumling/Däumlinchen'. ${ }^{18}$ Hier wäre eine zugrundeliegende Form ${ }^{\star} D a u b$-en zu rekonstruieren, deren stammbildendes Suffix -en bei derivationeller Suffigierung durch -ling bzw. -linchen ersetzt würde (vgl. Gart-en > Gärt-lein). So könnte man auch die Formen erklären, die Schmeller (1821: 118) aufzählt:

E’rwal (Aermel); Háiwal (Hälmlein), Márwal (Märmel, Marmor), das Páiwal (Pälmlein, Sproße), das Wirwal (Würmlein); (schon in Avent[ins] Chron[ik] Edit. von 1566. fol. 73. heißt es: von dem kleinsten Wirbel bis auf den Menschen).

Normalisiert hätte man es dem oben eingeschlagenen Erklärungsweg nach mit

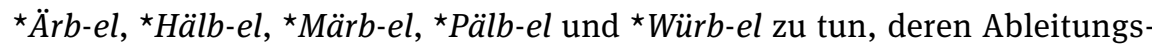
basen aus zugrundeliegendem ${ }^{\star}$ Arb-en, ${ }^{\star} H a l b-e n,{ }^{\star}$ Marb-en, ${ }^{\star}$ Palb-en und ${ }^{\star}$ Wurb-en durch falsche $m>b$-en-Reanalyse aus Arm, Halm, Marm(or), Palm und Wurm rekonstruiert worden wären. ${ }^{19}$

Notorisch für morpho-phonologische Verschmelzung anfällig sind auch Stammauslaute auf ...n, an die - $n$-Suffixe antreten. So werden etwa Possessivartikel wie mein-en/dein-en/sein-en zu mein'n/dein'n/sein'n verschmolzen, die

17 Senkung des Stammvokals $i$ vor $r$ führt zu ostfrk. Scherm. Dieses -e- bleibt als dialektales Spurenelement im Spottbeispiel ${ }^{\star}$ Scherben 'Scherm (= Schirm)' erhalten.

18 Philipp Harnisch (5;6) am 30. April 1991.

19 Schmeller dagegen gibt hier eine rein laut-assimilatorische Erklärung: „m nach $l$ oder $n$ vor der Endsylbe $e l$ oder $l$ lautet gerne wie $w^{“}$. 
sie von den nichtsuffigierten Formen mein/dein/sein nicht unterscheiden. Kalau (1984: 172-173) berichtet von Fällen, dass Nürnberger Schüler statt er hatte sein Fenster offen oder da kommt sein Vater hyperkorrekt schreiben: er hatte ${ }^{\star}$ seinen Fenster offen bzw. da kommt ${ }^{\star}$ seinen Vater. Die sich wohl innerlich vorgesagte richtige Form sein war offensichtlich für falsch erachtet und aus Kenntnis der diasystemischen Verschmelzungsregel seinen > sein (sein'n) $\mathrm{zu}{ }^{\star}$ sein-en rück-,verbessert' ${ }^{\star}$ worden. ${ }^{20}$

Krieg-Holz (2013: 128) behandelt einen weiteren Subtypus, der die „Lautschwächungen“ (Meinhold 1973) im schwachtonigen Endsilbenbereich und damit die morpho-phonologische Variation von Formen mit und ohne Schwa betrifft: eine in stark synkopierenden Dialekten wie dem Bairischen vorzufindende „Hyperkorrektur [...] aus Angst, ein unbetontes - $e$ - auszulassen (z. B. auf der Stiren, beim Nachbaren ${ }^{21}$ )“. Dahinter dürfte die Kontrasterfahrung stecken, dass regional-substandardsprachliches Virn, Nachfahrn usw. gegen ,höheres“ standardsprachliches Viren, Nachfahren mit a steht. Eine ähnliche Folie bildet eine Dialekt-Standard-Variation wie verliern vs. verlieren für die Hyperform ${ }^{\star}$ wanderen statt wandern (129).

Abschließend $z u$ diesem Bereich sei noch auf einen Sondertypus hingewiesen. Bei ihm werden im Gegensatz zu den soeben dargestellten Fällen opake morpho-phonologische Verschmelzungsprodukte nicht unterstellt und können somit auch nicht wiederaufgelöst werden. Sie werden vielmehr als neue Grundmorpheme aufgefasst, denen nun aber die morphologischen Marker fehlen, die Opfer der Verschmelzung geworden waren. An diesen neuen Basen werden dann Korrekturen in Form von Reparaturen vorgenommen, die jene vermissten Morpheme wieder - in Sprechersicht: erst - anfügen. Bülow \& Krieg-Holz (2013: 155-158) behandeln einen Fall ausführlich vor dem Hintergrund der Verstärkungstypologie und -theorie von Harnisch (2004a, 2010a), die im Passauer TheoRem-Projekt mittlerweile als Typologie und Theorie der Remotivierung weiterentwickelt wird. Ähnlich wie der Sprachwandel im Deutschen die Form ge-gessen hervorgebracht hat, werden im Spracherwerb Formen wie *ge-ghabt 'gehabt' gebildet. Die Sprecher finden sich also offensichtlich nicht mit den morphosemantisch unterspezifizierten Verschmelzungsprodukten g'essen bzw. g'habt (aus ge-essen bzw. ge-habt) ab, sondern doppeln das Präfix - in ihrer Sicht: präfigieren erst. Auch so wird durch Reanalyse

20 Ähnlich wie diese ...n-n-Ketten sind ...t-t-Ketten morpho-phonologisch prekär und deshalb für normativ inkorrekte Ver- wie hyperkorrekte Entschmelzung anfällig. Zur Deutungsmöglichkeit von Formen wie gilt-et 'gilt' als diasystemisch induzierte Hyperkorrekturen siehe oben letzten Absatz der Einleitung von Abschnitt 3.

21 Statt Stirn und Nachbarn. 
morphologische Struktur auf der Basis morpho-phonologischer Amorphie aufgebaut. Der Unterschied zwischen beiden Fällen ist aber, dass gegessen als intrasystemische Reparatur erklärt werden kann, weil ge- als Präfix im System vorkommt, die Reparatur zu geghabt aber als diasystemische, hyperkorrigierende erklärt werden muss, da das ge-Präfix in der dialektalen Varietät nicht vorkommen kann, die Reparatur vielmehr das standardsprachliche Element nimmt.

Ein dem hochsprachlichen *ge-essen $>{ }^{\star}$ g'essen $>$ ge-gessen ähnlicher Fall liegt mit der dialektalen Form ostfrk. *ge-hört > *g'hört > ge-ghört 'gehört' ( = 'im Besitz gewesen sein von') vor. ${ }^{22}$ Die hier vorliegende Reanalyse einer ursprünglichen Präfixbildung als neues Grundmorphem wird wortpositionell gespiegelt von der Reanalyse einer ursprünglichen Suffixbildung als neues Grundmorphem: geschob-en-e 'geschobene' > gscho'm- $a$ > gschom-n-a. ${ }^{23}$ Auch diese Re-Interpretation führt zur Morphogenese eines Affixes, hier des ,gedoppelten` - in Sprechersicht: erst gesetzten - Nasalsuffixes.

\section{Ergänzung: Dia-systemische innerphonologische Hyperkorrektion}

In der soziolinguistischen Darstellung von dialektbedingten Fehlern, und darunter Hyperkorrekturen, werden weitaus überwiegend solche behandelt, bei denen der betreffende diasystemische Kontrast der beiden beteiligten Varietäten nicht, wie bei den Fällen von Abschnitt 2 und 4, am Ebenenübergang von der Phonologie zur Morphologie besteht, sondern nur auf einer Systemebene

22 Allerdings enthält schon dessen Grundform ein Präfix (g'hörn 'gehören'). Nach den silbenmorphonotaktischen Regeln auch dieses Dialekts dürfte dieses g'hörn im Partizip Perfekt jedoch nicht nochmals ge-präfigiert werden ( ${ }^{\star} g e$-g’hört) - vergleiche nämlich im selben Dialekt zu gewinna 'gewinnen' das Part.Perf. gewunna (und nicht *ge-gewunna). Unterstützt wird die ,nochmalige‘ Präfigierung bei g'hört aber sicher dadurch, dass das Verschmelzungsprodukt *ge-hört > g'hört zu einer einsilbigem neuen Grundform führt, deren anlautendes $g$ seine morphemische Salienz eingebüßt hat. Synkopiert wird ge- im hier betreffenden Dialekt von Kronach/Oberfranken übrigens nur vor $h$ und Frikativen, ansonsten bleibt das Schwa erhalten. Insofern ist g'hörn mit $h$ doch ein etwas anderer Fall als alle andern Fälle mit anderem Folgelaut (wie obiges gewinna mit $w$ zum Beispiel).

23 Vgl. das bei Harnisch (1984: 167) referierte Beispiel bair. heilig- $\boldsymbol{n}>$ heili' $\eta>$ heiliy- $\boldsymbol{a}$ aus einem Vortrag von Frans Plank. Das - $a$ in heiliy- $a$ ist Allomorphon des $-n$ in heilig- $n$, also tatsächlich das dem entsprechende gedoppelte - in Sprechersicht: erst gesetzte - Suffix. 
(Phonologie ${ }^{24}$, Morphologie, Syntax ${ }^{25}$ ) angesiedelt ist. ${ }^{26}$ Von diesen Systemebenen wiederum ist die lautliche am stärksten betroffen: „Hyperkorrekturen treten zwar potentiell auf allen Systemebenen auf, doch sind phonologische Hyperkorrektionen mit Abstand die häufigsten“ (Herrgen 1986: 178). ${ }^{27}$ Wer beispielsweise auf rein phonologischer Ebene die Beziehungsregeln zwischen standardsprachlichen und substandardsprachlichen Aussprachemerkmalen durchschaut hat und etwa weiß, dass sein bair. Knet hochdeutsch Knecht lautet oder sein fränkisches schlafen bzw. sein thür. Strefen hochsprachlich schleifen bzw. Streifen, wer gar einmal verbessert worden ist, man sage nicht ${ }^{\star} K e c h i n$, sondern Köchin, auch nicht * Teisch, sondern Teich, der kann leicht dem Irrtum unterliegen, es heiße auch ${ }^{\star}$ Veicht statt Veit ${ }^{28}$, ${ }^{\star}$ reifen statt raufen bzw. räufen 'reißen' ${ }^{29}$, ${ }^{\star}$ stöchen statt stechen ${ }^{30}$ oder ${ }^{\star}$ Fleich statt Fleisch ${ }^{31}$.

24 In den Darstellungen der Reihe Dialekt/Hochsprache - kontrastiv, die v. a. auf der Analyse von schriftlichen Schülertexten beruhen, werden lautlich bedingte Fehler zumeist unter der Rubrik „Rechtschreibfehler“ abgehandelt.

25 Einen Schwerpunkt auf syntaktische Interferenzen, darunter Hyperkorrekturen, setzt Henn (1978).

26 Intrasystemische Korrekturen sind eher nicht zu erwarten, da Variation innerhalb eines Systems dem Homogenitätspostulat widerspräche. Wo es trotzdem interne Variation gibt, ist sie als „landschaftlich“, „umgangssprachlich“, „fachsprachlich“ o. ä. eben doch als diasystemisch markiert. Denkbar wären zum Beispiel auf rein lautlicher Ebene aber aus Inkonsequenzen der Norm resultierende, für ,richtiger` gehaltene (oder buchstabilistisch motivierte) Aussprachen wie ${ }^{\star}$ Kile für Chile (weil Kina für China möglich) oder *luthérisch und *haushaltérisch für lútherisch und háushalterisch (weil äthérisch : Äther). Nach dem Duden Aussprachewörterbuch (1990: 478) sind Unterschiede zwischen luthérisch und lútherisch semantisch ('stark orthodox lutheranisch' vs. 'nicht ...') bzw. pragmatisch (,veraltet“ vs. „nicht ...“).

27 „Das hat seinen Grund einmal darin, daß die sprachlichen Kontraste zwischen Dialekt und Standardsprache ,proportional zur Höhe der Beschreibungsebene abnehmen [...]' (Veith 1982: 281). Ein zweiter Grund besteht darin, daß Adaptionsregeln gerade auf der phonologischen Ebene am effektivsten operieren. Beim Phonemsystem handelt es sich um ein kleines, fest strukturiertes Inventar von Einheiten mit hoher Textfrequenz.“ (Herrgen 1986: 178).

28 Dazu vgl. Kranzmayer (1956: 92), Bach (1950: 232), Herrgen (1986: 197).

29 Dieser Fall ist zusätzlich dadurch verkompliziert, dass sowohl ostfrk. [a:] als auch thür. [e:] jeweils eine doppelte sprachgeschichtliche Herkunft aus mhd. ei und ou bzw. aus mhd. ei und öu haben können und synchron sowohl zu hd. ei als auch au im fränkischen Fall bzw. sowohl zu ei und eu/äu im thüringischen Fall in Beziehung stehen können. M. a. W. wird sowohl frk. rafen als auch thür. refen hyperkorrekt mit einem hd. ei-Wort in Verbindung gebracht - statt mit einem $a u$-Wort im fränkischen bzw. mit einem hd. eu/äu-Wort im thüringischen Falle. Diese lautliche Hyperkorrektur dürfte in beiden Fällen unterstützt sein vom Stammvokal ei des hd. lexikalischen Übersetzungsäquivalents reißen.

30 Vgl. Zehetner (1977: 24).

31 Ausführlich zu koronalisierungsbedingten Hyperkorrekturen generell Herrgen (1986). Kanzlerkandidat Schulz vom Niederrhein bringt uns im Wahlkampf 2017 mit seinem Sprechen von den *europäichen statt europäischen Werten wieder Kanzler Kohl vom Oberrhein in Erinne- 
Auf dem Namenschild einer Straße in Bayreuth, mit der der Besitzer der St. Georgener Fayencenmanufaktur Fränkel geehrt werden sollte, stand, er „begleitete verschiedene kommunale Ehrenämter“ statt korrekt „bekleidete“. In diesem Beispiel sind „Direktanzeige“ einer dialektalen Interferenz (anlautendes $k$ vor Konsonant wird * $g$, also $k l->{ }^{\star} g l$-) und „Hyperkorrektur“ (im Verbstamm ${ }^{\star}$ - $t$ - statt richtig - $d$-) vereint: Wer oft genug für sein $d$ gescholten worden ist, wo er $t$ hätte artikulieren sollen, ist geneigt, lieber öfter mal $t$ zu setzen, auch wo $d$ durchaus richtig wäre. ${ }^{32}$

Gerade am nur-phonologischen Typus kann man erkennen, dass Hyperkorrektion keineswegs nur in Richtung vom Substandard zum Standard geht. Vielmehr kann sie durch varietätensystem-übergreifende falsche lautliche Analogien auch vom Standard zum Substandard hin gerichtet sein (vgl. Lenz 2005). Hier gibt es ein Gegenbeispiel zu den gerade beschriebenen fränkischen Re-Fortisierungen vermeintlich falscher Lenes: Der beim Singspiel auf dem Nockherberg seit Jahren den fränkischen Minister und CSU-Politiker Markus Söder spielende Stephan Zinner übergeneralisiert die Regel, dass die hochdeutschen ,harten` Konsonanten (also $p$, $t$ und $k$ ) im Fränkischen ,weich“ ausgesprochen würden, in der Weise, dass er sie auch auf den Fall des anlautenden $k$ vor Vokal überträgt, wo jedoch auch die Franken $k V$ - artikulieren. ${ }^{33}$ So entstehen hyperkorrekt pseudofränkische Aussprachen wie Ganzler, Gabinett oder Gandidat, wo jeweils auch Söder ein Anlaut- $K$ - sprechen würde. Mit seiner Hyperstrategie läge Zinner allenfalls beim Obersächsischen richtig, wo auch anlautendes k- vor Vokal lenisiert wird, z. B. Gatze 'Katze'. Da g im Berlinischen silbenanlautend $\mathrm{zu} j$ wird, kann es nach Herrgen (1986: 192) nun dazu kommen, dass ein Sachse, der einen Berliner imitieren will, jraue *Jatze 'graue Katze' sage, wobei nur das $j$ von jrau stimmt, das $J$ von Jatze jedoch hyperkorrekt ist, weil vom obersächsischen Lautstand ausgehend gebildet.

An der gleichen Stelle referiert Herrgen ein autostereotypisches Beispiel. Demnach ,verspotten Bayern die ,Preußen“, die angeblich [...] ,hyperbairisch“ oans, zwoa, droa zählen.“ Eine andere Quelle für solche hyperdialektale Fehlanalogien sind nicht mehr so dialektfeste jüngere Sprecher in der Anforde-

rung, der ${ }^{\star}$ Gechichte statt Geschichte geschrieben hat - geschrieben im übertragenen Sinne, denn geschrieben hat er so natürlich nicht, nur gesprochen.

32 Es ist bei diesem Beispiel natürlich auch damit zu rechnen, dass zwei Lexem-Gestalten bekleiden und begleiten als Ganze verwechselt und vertauscht wurden, zumal sie Teil eines so oder so wenig durchsichtigen Phraseologismus sind. Doch ist ein solcher Tausch sicher nicht oder nur schwer ohne die im Ostfränkischen herrschenden Lenisierungen denkbar. Den beschriebenen Fall glossiert Siegfried Pokorny in den Meyernberger Informationen 21 von Dezember 1987. 33 Anders als bei anlautendem $k$ mit Folgekonsonant: glaane 'kleiner', Grabbelgrubbm 'Krabbelgruppe' usw. 
rungssituation, ihre Dialektkompetenz zu beweisen. In Harnisch (2004b: 461) wird von Hyperkorrekturen Jugendlicher in einem südthüringischen Ort berichtet, die analog zu Variantenkorrespondenzen wie lafen : laufen ( $<$ mhd. ou) auch *aaf statt auf (< mhd. $\hat{u})$ oder analog Variantenkorrespondenzen wie Maßel : Meißel (< mhd. ei) auch *Amasen statt Ameisen artikulieren, was zwar insofern korrekt wäre, als der Diphthong auch auf mhd. ei zurückgeht, er jedoch im Nebenton steht und im betreffenden Dialekt unter diesen Akzentbedingungen $\mathrm{zu}[ə]$ abgeschwächt ist (['haməsn]). Hyperkorrigierend ist auch die Adaption modernen technischen Wortschatzes an das dialektale Lautsystem, so wenn ein Jugendlicher dort von *tachnischen Geräten spricht (- $a$ - für germ. $\ddot{e})$, nicht, wie es die synchronen Entlehnungsregeln verlangen würden, von technischen (nicht-germ. $e$ / Primärumlaut).

Als nichtphonologisches Beispiel für überdialektale Bildungen könnte man aus der Morphologie die Hyperform bair. Pfiats eich/enk nennen, wo aus dem monomorphen Wortkörper Pfiat des Grußes Pfiat eich/enk 'B'hüt euch [Gott]' der Auslaut ...t resegmentiert und als Suffix der 2. Pers. Pl. remotiviert wird. Dieses (auch) standardsprachliche Suffix wird in hyperdialektaler Weise nun manchmal durch das (ur)bair. Suffix -ts der 2. Pers. Pl. ersetzt - was den ursprünglichen Sinn des Grußes übrigens völlig verdreht, heißt es nun doch falsch *Behütet euch!, statt Behüte euch [Gott]!

An diesen Umkehrfällen wird sichtbar, dass Hyperkorrektion keineswegs eine diastratisch allein vertikal nach ,oben' gerichtete sprecherpsychische Aktivität ist oder sein muss, sondern je nach Zielvarietät so oder so gerichtet sein kann.

\section{Analogie als Antrieb der Reanalyse und Hyperkorrektion}

Zur besseren Übersicht werden alle in den Abschnitten 1 bis 5 behandelten Fälle in den folgenden Tabellen 9.1 bis 9.3 noch einmal aufgelistet und nach Typen gruppiert, die intern wiederum danach differenziert sind, ob es sich um intrasystemische Reanalysen (links in der Tabelle) oder um diasystemische Reanalysen/Hyperkorrekturen (rechts in der Tabelle) handelt. Die Nummerierungen werden von den Abschnitten übernommen, in denen das betreffende Phänomen jeweils dargestellt wurde. Angaben $\mathrm{zu}$ den Varietäten (Standard, bestimmter Dialekt, Kindersprache), in bzw. zwischen denen sich diese Reanalysen abspielen, werden hier nicht gemacht; sie sind den Ausführungen oben zu entnehmen. 
Tab. 9.1: Zielbereich der Reanalyse/Hyperkorrektur: Morphologie aus Lautsubstanz.

\begin{tabular}{|c|c|c|}
\hline \multicolumn{2}{|c|}{$\begin{array}{l}2.1 \\
\text { intra-systemisch begründete Reanalyse }\end{array}$} & $\begin{array}{l}4.1 \\
\text { dia-systemisch begründete } \\
\text { Reanalyse/Hyperkorrektur }\end{array}$ \\
\hline $\begin{array}{l}\text { Falter }>{ }^{*} \text { Falt-er } \\
\text { unser }>\text { *uns-er/uns-e } \\
\text { sauber }>\text { *saub-er/saub } \\
\text { heiser }>\text { *heis-er/heis }\end{array}$ & $\begin{array}{l}\text { Zwieselburg }>{ }^{*} z \text { ' Wieselburg } \\
\text { Zwerenain }>{ }^{*} z \text { ' Werenain } \\
\text { Diana }>\text { *die Jana }\end{array}$ & $\begin{array}{l}a b e r>{ }^{*} a b-e r \gg{ }^{*} a b \\
\text { Korrektur Richtung Dialekt: } \\
\text { pfiat }>{ }^{*} \text { pfia- } t>{ }^{*}-t s\end{array}$ \\
\hline
\end{tabular}

Tab. 9.2: Zielbereich der Reanalyse/Hyperkorrektur: Morphologie aus morpho-phonologischer Verschmelzung.

\begin{tabular}{|c|c|c|}
\hline $\begin{array}{l}2.2 \\
\text { intra-systemisch begründete }\end{array}$ & $\begin{array}{l}4.2 \\
\text { dia-systemisch begrü }\end{array}$ & analyse/Hyperkorrektur \\
\hline $\begin{array}{l}\text { beredt }>{ }^{*} \text { bered-et } \\
\text { gilt }>{ }^{*} \text { gilt-et (oder zu 4.2) }\end{array}$ & $\begin{array}{l}\text { Form }>* \text { Farb-en } \\
\text { Scherm }>* \text { Scherb-en } \\
\text { Daum }>\star \star \text { Daub-en } \\
\text { Arm }>* \star \text { Arb-en } \\
\text { Halm }>* \star \text { Halb-en } \\
\text { Marm- }>* \star \text { Marb-en } \\
\text { Palm }>\star \star \text { Palb-en } \\
\text { Wurm }>* \star \text { Wurb-en }\end{array}$ & $\begin{array}{l}\text { crem }(e n)>{ }^{*} \text { creb-en } \\
\text { räum }(e n)>{ }^{*} \text { räub-en } \\
\text { Rahm }(e n)>{ }^{*} \text { Rab-en } \\
\left(\text { Amt }>{ }^{*} \text { Abent }\right)^{34} \\
\text { sein }>{ }^{*} \text { sein-en } \\
\text { Stirn > Stir-en } \\
\text { wandern }>\text { *wander-en } \\
\text { gilt > gilt-et (oder zu 2.2) }\end{array}$ \\
\hline
\end{tabular}

Zuerst werden die Fälle zusammengestellt, die denjenigen Typus der Reanalyse bzw. Hyperkorrektion repräsentieren, bei dem morphologische Segmente aus lautlicher Substanz gewonnen werden (Tab. 9.1).

In der darauf folgenden Tabelle 9.2 sind die Beispiele versammelt, bei denen durch morpho-phonologische Verschmelzung vermeintlich opak gewordene Formen wieder morphologisch transparent gemacht werden.

Die in Tabelle 9.3 aufgelisteten Fälle sind dadurch gekennzeichnet, dass bei ihnen im Gegensatz zu denen in Tabelle 9.2 das Produkt einer tatsächlichen Verschmelzung hingenommen, das von der Verschmelzung verursachte Fehlen des betreffenden morphologischen Markers aber kompensiert wird.

Schließlich sind zur Ergänzung der Morphologie hervorbringenden Fälle auch noch die Hyperkorrekturen aufgeführt, die sich phonologie-intern abspielen (Tab. 9.4).

34 Ohne Morphogenese. 
Tab. 9.3: Zielbereich der Reparatur: Morphologie nach hingenommener morpho-phonologischer Verschmelzung.

\begin{tabular}{l|l}
\hline intra-systemisch begründete Reanalyse & $\begin{array}{l}\text { dia-systemisch begründete } \\
\text { Reanalyse/Hyperkorrektur }\end{array}$ \\
\hline $\begin{array}{l}\text { ge-essen }>\text { gessen }>\text { ge-gessen } \\
\text { ge-hört > ghört }>\text { *ge-ghört } \\
\text { geschob-en }(-e)>\text { geschom }(-e)>{ }^{*} \text { geschom- } n(-e)\end{array}$ & ge-habt $>$ ghabt $>{ }^{*}$ ge-ghabt \\
\hline
\end{tabular}

Tab. 9.4: Zielbereich der Reanalyse/Hyperkorrektur: Phonologie.

\begin{tabular}{|c|c|c|}
\hline $\begin{array}{l}5[.1] \\
\text { intra-systemisch begründete } \\
\text { Reanalyse }\end{array}$ & \multicolumn{2}{|c|}{$\begin{array}{l}5[.2] \\
\text { dia-systemisch begründete Reanalyse/Hyperkorrektur }\end{array}$} \\
\hline \multirow[t]{3}{*}{$\begin{array}{l}\text { Chile }>{ }^{\star} \text { Kile } \\
\text { lútherisch }>\text { *luthérisch } \\
\text { háushalterisch }>\text { *haushaltérisch }\end{array}$} & \multicolumn{2}{|l|}{$\begin{array}{l}(\text { räufen }>) \text { refen }>{ }^{*} \text { reifen } \\
(\text { raufen }>) \text { rafen }>\text { *reifen } \\
\text { Veit }>\text { *Veicht } \\
\text { stechen }>\text { *stöchen } \\
\text { Fleisch }>\text { *Fleich }\end{array}$} \\
\hline & \multicolumn{2}{|c|}{ Umkehrung der Korrekturrichtung } \\
\hline & $\begin{array}{l}\text { drei }>* \text { droa } \\
\text { auf }>\text { aaf } \\
\text { Ameisen }>{ }^{\star} \text { Amasen } \\
\text { technische }>\text { *tachnische }\end{array}$ & $\begin{array}{l}\text { Kanzler }>\star \text { Ganzler } \\
\text { Kabinett }>\star \text { Gabinett } \\
\text { Kandidat }>\star \text { Gandidat } \\
(\text { Katze }>) \text { Gatze }>\text { *atze }\end{array}$ \\
\hline
\end{tabular}

In den Beispielschilderungen der Abschnitte 2.1-2.2 und 4.1-4.2 wurden diese Reanalysevorgänge explizit als analogische dargestellt. Anhand jeweils einer aus den obigen Typensammlungen ausgewählten Leitform soll das Analogische an diesen Prozessen noch einmal vorgeführt werden (Tab. 2.5). Die aus der Reanalyse bzw. Hyperkorrektion entstandenen Bildungen werden in den proportionalanalogischen Gleichungen ${ }^{35}$ der Tabelle mit *Asterisk versehen und fett gedruckt:

35 Herrgen (1986: 156) verwendet bei seinen Exemplifizierungen eine ähnliche Art von „Dreisatz“. Anstelle der in vorliegendem Beitrag gleich genannten und mit *Asterisk gekennzeichneten Formen setzt er erst ein $x$, das er dann in einer Gleichung $x=\ldots$ auflöst. 
Tab. 9.5: Proportionalgleichungen zu den Typen von Reanalyse/Hyperkorrektion.

\begin{tabular}{|c|c|c|}
\hline $\begin{array}{l}\text { Zielbereich der Reanalyse/Hy- } \\
\text { perkorrektur }\end{array}$ & $\begin{array}{l}\text { intra-systemisch begründete } \\
\text { Reanalyse }\end{array}$ & $\begin{array}{l}\text { dia-systemisch begründete } \\
\text { Reanalyse/Hyperkorrektur }\end{array}$ \\
\hline $\begin{array}{l}\text { Morphologie: Morphemgewin- } \\
\text { nung aus Lautsubstanz }\end{array}$ & $\begin{array}{l}\text { Halter }(: \text { halt-en })>\text { Halt-er } \\
\text { Falter }(: \text { falt-en })>\star \text { Falt-er }\end{array}$ & $\begin{array}{l}\text { halter : halt > halt-er }{ }^{36} \\
\text { aber : }{ }^{\star} a b(<a b-e r)\end{array}$ \\
\hline $\begin{array}{l}\text { Morphologie: von morpho- } \\
\text { phonologischer Basis aus: } \\
\text { „De-Morphonologisierung“ }\end{array}$ & $\begin{array}{l}\text { gesandt : gesend-et } \\
\text { beredt : *bered-et }\end{array}$ & $\begin{array}{l}\text { sterm : sterb-en } \\
\text { Scherm : *Scherb-en }\end{array}$ \\
\hline $\begin{array}{l}\text { Morphologie: Reparatur mor- } \\
\text { pho-phonologischer Störung }\end{array}$ & $\begin{array}{l}\text { kafft }^{37} \text { : ge-kauft } \\
\text { ghört : *ge-ghört }\end{array}$ & $\begin{array}{l}g^{\prime} k a f f t^{38}: \text { ge-kauft } \\
\text { ghabt : *ge-ghabt }\end{array}$ \\
\hline \multirow[t]{2}{*}{ Phonologie intern } & $\begin{array}{l}\text { China : Kina } \\
\text { Chile: }{ }^{*} \text { Kile }\end{array}$ & $\begin{array}{l}\text { Strefen : Streifen } \\
\text { refen (< räufen) : *reifen }\end{array}$ \\
\hline & mit Ziel Substandard: & $\begin{array}{l}\text { eins, zwei : oans, zwoa } \\
\text { drei : *droa }\end{array}$ \\
\hline
\end{tabular}

Hieran erweist sich, dass die von Paul (1920: 106-120) für intra-systemische analogische Angleichungen entwickelten Proportionalgleichungen auch diasystemisch, also varietäten-übergreifend operieren. Das hatte schon Herrgen (1986: 155) herausgestellt: „Paul hat systeminterne Analogie im Auge [...], doch läßt sich der Mechanismus auch systemübergreifend beobachten“. Herrgen zeigte, dass die Proportional-Analogie lautliche Hyperkorrektionen zu erklären vermag. In vorliegendem Beitrag wurde gezeigt, dass dia-systemisch (intervarietär) bedingte Hyperkorrektion und Reanalyse auch die Hervorbringung morphologischer Strukturen und Einheiten erklärt, ob sie nun an affix- bzw. klise-,verdächtiger ' Lautsubstanz ${ }^{39}$ ansetzen oder auf der Basis von vermeintlichen morpho-phonologischen Verschmelzungen operieren. Diese Prozesse der Resegmentierung und Remotivierung sind deutlicher Ausdruck eines sprecherpsychischen Grundbedürfnisses nach mehr Sinn und Struktur, als in den der

36 Kindersprachliche Modalpartikel.

37 Diese ostfrk. Form dürfte unpräfigiert und nicht aus synkopiertem $g$ ' vor $k$-anlautendem Verbstamm entstanden sein, also nicht aus * $g$ 'kafft, denn im betreffenden Dialekt bleibt das ge-Präfix vor $k$ erhalten (z. B. ge-kippt). Zu möglichen Erklärungen präfixloser Perfekt-Partizipien (Tokenfrequenzen bzw. Gebräuchlichkeit, Verbsemantik, z.B. perfektive Bedeutung) vgl. Harnisch (1992: 308-309).

38 Diese bair. Form dürfte aus synkopiertem Präfix $g$ ' vor $k$-anlautendem Verbstamm und Verschmelzung mit ihm entstanden sein, denn im betreffenden Dialekt gibt es keine nichtsynkopierten Formen.

39 Zum Affix-,,Verdacht“ vgl. den Aufsatztitel von Harnisch (2010b). 
Reanalyse zugrundeliegenden Einheiten an sich zunächst steckt. Wo diese Einheiten Angriffspunkte bieten, werden Sie von den Sprechern gerne genutzt, um „konstruktionellen Ikonismus“ herzustellen bzw. ihn vermeintlich wiederherzustellen, so dass man hier von „re-konstruktionellem Ikonismus“ sprechen kann. ${ }^{40}$

\section{Literatur}

Bach, Adolf (1950): Deutsche Mundartforschung. Ihre Wege, Ergebnisse und Aufgaben.

2. Aufl. Heidelberg: Winter.

Bülow, Lars \& Ulrike Krieg-Holz (2013): Verstärkungsprozesse im Variationsbereich zwischen Standard und Substandard. In Rüdiger Harnisch (Hrsg.), Strömungen in der Entwicklung der Dialekte und ihrer Erforschung. Beiträge zur 11. Bayerisch-Österreichischen Dialektologentagung, Passau 2010, 149-159. Regensburg: edition vulpes.

Duden Aussprachewörterbuch. Wörterbuch der deutschen Standardaussprache (1990).

3. Aufl. Bearb. von Max Mangold. Mannheim, Wien, Zürich: Dudenverlag.

Eichinger, Ludwig M. (1988): Die Wörter, die mein Sohn macht. Natürlichkeit und die Wortbildung des Adjektivs. Grazer Linguistische Studien 30, 21-36.

Eichinger, Ludwig M. (2010): „... es müsste sich dabei doch auch was denken lassen.“ Remotivierungstendenzen. In Rüdiger Harnisch (Hrsg.), Prozesse sprachlicher Verstärkung. Typen formaler Resegmentierung und semantischer Remotivierung, 59-86. Berlin, New York: de Gruyter.

Grimm, Jacob \& Wilhelm Grimm (1862): Deutsches Wörterbuch. Dritter Band. Leipzig: Hirzel. Lizenzausgabe München: dtv 1984.

Harnisch, Rüdiger (1984): Das Bairische und die Allgemeine Sprachwissenschaft. [Bericht aus der gleichnamigen Sektion auf der 5. Jahrestagung der Deutschen Gesellschaft für Sprachwissenschaft, Passau 1983.] Deutsche Sprache 12, 166-169.

Harnisch, Rüdiger (1987): Natürliche generative Morphologie und Phonologie des Dialekts von Ludwigsstadt: Die Erprobung eines Grammatikmodells an einem einzelsprachlichen Gesamtsystem. Tübingen: Niemeyer.

Harnisch, Rüdiger (1992): Ein Denkmal der ostfränkischen Volks(schrift)sprache aus dem 17. Jahrhundert. In Andreas Weiss (Hrsg.), Dialekte im Wandel. Referate der 4. Tagung zur bayerisch-österreichischen Dialektologie, Salzburg 1989, 305-326. Göppingen: Kümmerle.

40 So im Aufsatztitel von Harnisch (2004a) und, mit stärkerem Bezug auf einen der Vordenker der Natürlichen Morphologie, Willi Mayerthaler, im Aufsatztitel von Harnisch (2005). Letztgenannter Aufsatz ist auf Fragen des Spracherwerbs spezialisiert. Solche Fragen haben - ebenfalls im theoretischen Rahmen der Morphologischen Natürlichkeit - auch Eichinger (1988) beschäftigt. Zwei Väter haben sich mit der damals aktuellen Natürlichkeitstheorie, offensichtlich empirisch befeuert von Belegen, die ihnen ihre Kinder lieferten, intensiv auseinandergesetzt: der eine, Eichinger, in Sachen Wortbildung, der andere, Harnisch, in Sachen Flexion (siehe Anm. 15 und 18). Der Sonderdruck von Eichinger (1988) ging „Mit väterlichen Grüßen Toni“ an Verfasser. 
Harnisch, Rüdiger (2002): Morphologische Reanalysen bei lokalen Adverbien, Präpositionen und Adjektiven im Thüringischen und Ostfränkischen. In Jan Berns \& Jaap van Marle (Hrsg.), Present-day dialectology. Problems and findings, 193-206. Berlin, New York: de Gruyter.

Harnisch, Rüdiger (2004a): Verstärkungsprozesse. Zu einer Theorie der „Sekretion“ und des „Re-konstruktionellen Ikonismus“. Zeitschrift für germanistische Linguistik 32, 210-232.

Harnisch, Rüdiger (2004b): Junger Dialektwandel in der Bavaria Thuringica. In Maik Lehmberg (Hrsg.), Sprache, Sprechen, Sprichwörter. Festschrift für Dieter Stellmacher zum 65. Geburtstag, 453-463. Stuttgart: Steiner.

Harnisch (2005): Re-konstruktioneller Ikonismus im Spracherwerb. In Gertraud Fenk-Oczlon \& Christian Winkler (Hrsg.), Sprache und Natürlichkeit. Gedenkband für Willi Mayerthaler, 129-136. Tübingen: Narr.

Harnisch, Rüdiger (2006): Fehlerlinguistische Fallstudie zu Texten spätalphabetisierter Erwachsener. Alfa-Forum. Zeitschrift für Alphabetisierung und Grundbildung 62, 8-13, Kopie der analysierten handschriftlichen Texte 7, 14, 21.

Harnisch, Rüdiger (2007): Herstellung von Konstruktionalität - eine Strategie im Erstspracherwerb. Alkalmazott Nyelvtudomány [Hungarian Journal of Applied Linguistics] 7, 5-16.

Harnisch, Rüdiger (Hrsg.) (2010a): Prozesse sprachlicher Verstärkung. Typen formaler Resegmentierung und semantischer Remotivierung. Berlin, New York: de Gruyter (Linguistik - Impulse und Tendenzen, 37).

Harnisch, Rüdiger (2010b): Integration und Isolation von suffixverdächtigen Fremdwörtern das Deutsche in typologischer Perspektive. In Carmen Scherer \& Anke Holler (Hrsg.), Strategien der Integration und Isolation nicht-nativer Einheiten und Strukturen, 105-122. Berlin, New York: de Gruyter.

Harnisch, Rüdiger (2017): Räumliche Ausdrücke in osthochdeutschen Dialekten morphosemantisch, syntaktisch, textfunktionell. In Alexandra Lenz et al. (Hrsg.), Bayerisch-österreichische Varietäten zu Beginn des 21. Jahrhunderts - Dynamik, Struktur, Funktion, 33-58. Stuttgart: Steiner.

Harnisch, Rüdiger (i. V.): Remotivierung in (osthochdeutschen) Dialekten. In Sebastian Kürschner \& Karin Rädle (Hrsg.), Dialektale Daten: Erhebung - Aufbereitung Auswertung. Akten der 13. Bayerisch-Österreichischen Dialektologentagung, Erlangen 2016.

Henn, Beate (1978): Mundartinterferenzen am Beispiel des Nordwestpfälzischen. Wiesbaden: Steiner (Zeitschrift für Dialektologie und Linguistik - Beihefte, 24).

Henn, Beate (1980): Pfälzisch. Düsseldorf: Schwann (= Dialekt/Hochsprache - kontrastiv 7). Herrgen, Joachim (1986): Koronalisierung und Hyperkorrektion. Das palatale Allophon des /ch/-Phonems und seine Variation im Westmitteldeutschen. Stuttgart: Steiner.

Hinderling, Robert (1982): Anpassung und Selbstbehauptung - Zur Bedeutung von Sprache und Sprachen in einer mehrsprachigen Welt. Ergänzte und geringfügig geänderte Fassung des Vortrags, gehalten am 4. Jahrestag der Universität Bayreuth am 27. November 1979. Jahresberichte des Präsidenten [der Universität Bayreuth] 1979/80 [und] 1980/81, 85-111.

Jespersen, Otto (1925): Die Sprache. Ihre Natur, Entwicklung und Entstehung. Heidelberg: Winter.

Kalau, Gisela (1984): Die Morphologie der Nürnberger Mundart. Eine kontrastive und fehleranalytische Untersuchung. Erlangen: Palm \& Enke. 
Koller, Erwin (1991): Fränggisch gschriim? Eine fehleranalytische Untersuchung unterfränkischer Schüleraufsätze. Tübingen: Niemeyer.

Kranzmayer, Eberhard (1956): Historische Lautgeographie des gesamtbairischen Dialektraumes. Wien: Böhlau.

Krieg-Holz (2013): „Nach der Schrift reden“ - Zum Phänomen sprachlicher Abweichung am Beispiel der Überlautung von -e und -en. In Rüdiger Harnisch (Hrsg.), Strömungen in der Entwicklung der Dialekte und ihrer Erforschung. Beiträge zur 11. BayerischÖsterreichischen Dialektologentagung, Passau 2010, 121-131. Regensburg: edition vulpes.

Lenz, Alexandra N. (2005): Hyperdialektismen und Hyperkorrektionen. In Alexandra N. Lenz \& Klaus J. Mattheier (Hrsg.), Varietäten - Theorie und Empirie, 76-95. Frankfurt a. M.: Peter Lang (VarioLingua, 23).

Löffler, Heinrich (1980): Dialektfehler. Ansätze zu einer deutschen „Fehlergeographie“. In Dieter Cherubim (Hrsg.), Fehlerlinguistik. Beiträge zum Problem der sprachlichen Abweichung, 94-105. Tübingen: Niemeyer.

Lüdtke, Helmut (1988): Grammatischer Wandel. In Ulrich Ammon, Norbert Dittmar \& Klaus J. Mattheier (Hrsg.), Soziolinguistik. Ein internationales Handbuch zur Wissenschaft von Sprache und Gesellschaft. 1. Aufl. Teilband 2, 1632-1642. Berlin, New York: de Gruyter (Handbücher zur Sprach- und Kommunikationswissenschaft, 3).

Maurer, G[eorg] (1912): Die Ortsnamen des Hochstifts Passau. Passau: Waldbauersche Buchhandlung.

Mayer, Anton (1945): Heimatliche Beispiele für Agglutination und Aphärese bei Ortsnamen. 2. Folge (= Aus dem Institut für Ostbairische Heimatforschung [Passau], Heft 6).

Meinhold, Gottfried (1973): Deutsche Standardaussprache. Lautschwächungen und Formstufen. Jena: Friedrich-Schiller-Universität.

Paul, Hermann (1920): Prinzipien der Sprachgeschichte. 5. Aufl. Halle/Saale: Niemeyer. Unveränd. Abdruck in 8. Aufl. Tübingen: Niemeyer 1968.

Plank, Frans (1985): On the reapplication of morphological rules and other resolutions of functional conflicts between morphology and phonology. Linguistics 23, 45-82.

Schmeller, Johann Andreas (1821): Die Mundarten Bayerns grammatisch dargestellt. München: Thienemann.

Seebold, Elmar (2011): Kluge. Etymologisches Wörterbuch der deutschen Sprache. 25. Aufl. Berlin, Boston: de Gruyter.

Trost, Igor (2010): Die semantische und die grammatische Sekretion am Beispiel der Komparativpositive. In Rüdiger Harnisch (Hrsg.), Prozesse sprachlicher Verstärkung. Typen formaler Resegmentierung und semantischer Remotivierung, 317-340. Berlin, New York: de Gruyter.

Veith, Werner H. (1982): Theorieansätze einer generativen Dialektologie. In Werner Besch et al. (Hrsg.), Dialektologie. Ein Handbuch zur deutschen und allgemeinen Dialektforschung. Teilband 1, 277-295. Berlin, New York: de Gruyter (Handbücher zur Sprach- und Kommunikationswissenschaft, 1).

Zehetner, Ludwig G. (1977): Bairisch. Düsseldorf: Schwann (= Dialekt/Hochsprache kontrastiv 2). 



\title{
Claudia Maria Riehl
}

\section{Simplifizierungsprozesse revisited}

\author{
Der Abbau der Kasusmarkierung in Sprachkontakt- \\ konstellationen
}

\begin{abstract}
Im Sprachkontakt sind verschiedene Prozesse voneinander zu unterscheiden: Transfer- und Konvergenzprozesse auf den verschiedenen sprachlichen Ebenen sowie Vereinfachungsprozesse im sprachlichen System, die aufgrund der mehrsprachigen Prozessierung von mehreren Sprachen erfolgen. Diese sind von den eigentlichen Sprachkontaktprozessen zu unterscheiden, da sie unabhängig von der typologischen Nähe und Distanz der Kontaktsprache zu beobachten sind. Darunter ist etwa ein Phänomen zu zählen, das in unterschiedlichen Konstellationen im Sprachkontakt auftritt, nämlich das Phänomen des Kasusabbaus, der in vielen Kontaktkonstellationen des Deutschen festgestellt wurde. In dem Beitrag soll versucht werden, aufgrund einer detaillierten Analyse und des intergenerationellen Vergleichs in Daten des Barossadeutschen (Südaustralien) und des Russlanddeutschen festzustellen, ob der Dativabbau in Sprachkontaktsituationen mit den bisherigen Annahmen erklärt werden kann oder ob differenziertere Prozesse berücksichtigt werden müssen.
\end{abstract}

Keywords: gebrauchsbasierte Theorie, Kasusabbau, Simplifizierungsprozesse, Sprachinseln

\section{Ausgangspunkt}

Im Sprachkontakt treten in der Regel unterschiedliche Prozesse auf: Einer der bedeutendsten ist dabei der sog. ,Transfer', ein Prozess, in dem ein bestimmtes sprachliches Element (z. B. ein Wort, ein Laut oder ein Morphem), eine abstrakte sprachliche Struktur (z. B. Aspektmarkierung oder Auslautverhärtung) oder eine Regel (z. B. wann man Futur verwendet) von einer Sprache in die andere übertragen wird. Das bedeutet, man kann konkretes Sprachmaterial, abstrakte Strukturmuster oder Bedeutungen bzw. Gebrauchskontexte für Wörter oder Strukturen von einer Sprache in die andere transferieren (Matras 2009; Riehl 2014).

Claudia Maria Riehl, Ludwig-Maximilians-Universität München, Institut für Deutsch als Fremdsprache, Ludwigstr. 27, D-80539 München, E-Mail: riehl@daf.Imu.de

Ә Open Access. (c) 2018 Claudia Maria Riehl, publiziert von De Gruyter. (c) BY Dieses Werk ist lizenziert unter der Creative Commons Attribution 4.0 Lizenz.

https://doi.org/10.1515/9783110538625-011 
Neben diesen Transferprozessen können in Sprachkontaktkonstellationen weitere Phänomene beobachtet werden, die auf die gleichzeitige Prozessierung mehrerer Sprachen zurückzuführen sind: Da dabei mehr Ausdrucksvarianten aufgerufen werden als bei der Prozessierung von nur einer Sprache, versuchen die Sprecher, die Varianten zu reduzieren, indem sie entweder die sprachlichen Systeme aneinander anpassen (sog. ,Konvergenz') oder aber komplexe Strukturen in einem sprachlichen System vereinfachen. Diese Vereinfachungsprozesse sind von den eigentlichen Sprachkontakterscheinungen $\mathrm{zu}$ unterscheiden, da sie unabhängig von der typologischen Nähe und Distanz der Kontaktsprache zu beobachten sind. Dennoch wirkt der Sprachkontakt insofern ein, als hier in der Sprache bereits angelegte Prozesse beschleunigt werden (vgl. Clyne 1991). Rosenberg (2003) führt dies u.a. auch darauf zurück, dass das Normbewusstsein in der Sprachgemeinschaft abnimmt (Mangel an linguistischem Wissen in Kombination mit Verlust der Sprachloyalität). Simplifizierungsprozesse betreffen besonders die Reduktion komplexer morphologischer Strukturen, wie etwa der Flexionsmorphologie. Ein Faktor, der in diesem Zusammenhang immer wieder ins Feld geführt wird, ist die Lernbarkeit der jeweiligen Strukturen (vgl. die Diskussion bei Rosenberg 2003: 299ff.). ${ }^{1}$ Außerdem besteht ein Zusammenhang zwischen Intensität des Sprachkontakts und morphosyntaktischer Komplexität einer Kontaktvarietät (Maitz \& Németh 2014).

Ein sehr bekannter Vereinfachungsprozess, der in unterschiedlichsten Konstellationen des Sprachkontakts des Deutschen auftritt, ist der Abbau der Kasusmarkierung, namentlich der Abbau der Dativmarkierung. Diese wurde bereits in zahlreichen Kontaktvarietäten des Deutschen belegt (Salmons 1994; Louden 1994; Born 2003; Rosenberg 2003 ff.; Boas 2009; Riehl 2012 u. v. m.).

Die ursprüngliche Annahme (vgl. etwa Louden 1994: 85), dass es sich nicht um eine interne Entwicklung des Deutschen handelt, sondern um einen Konvergenzprozess mit der Kontaktsprache Englisch (im Falle des Pennsylvaniadeutschen), wird etwa widerlegt von Rosenberg (2003), der eine Reduktion der Dativmorphologie auch im Russlanddeutschen feststellt, einer Varietät, bei der die Kontaktsprache (Russisch) über ein sehr ausgebautes System der Kasusmarkierung verfügt. Rosenberg (2003: 294) bezeichnet den Kasusabbau daher als „Ausdruck eines ,typologischen“ Sprachwandels [...], der nicht unmittelbar auf interlinguale und intralinguale Konvergenz zurückgeht.“ In den verschiedenen Beiträgen des „Handbuchs der deutschen Sprachminderheiten in Mittelund Osteuropa“ (Eichinger, Plewnia \& Riehl 2008) wird dieser Befund auch

1 Tatsächlich können Bentz \& Winter (2013) anhand einer statistischen Analyse von 66 Sprachen zeigen, dass Sprachen mit einer hohen Zahl an Zweitsprachlernern zur Reduktion (oder sogar Aufgabe) der Nominalflexion tendieren. 
für Kontaktkonstellationen mit anderen slawischen Sprachen (wie Polnisch, Tschechisch, Ukrainisch und Slowakisch) sowie im Kontakt mit dem Ungarischen, das ebenfalls über ein reiches Kasussystem verfügt, bestätigt.

Allerdings haben bisher nur wenige Studien die Entwicklung des Kasusabbaus in verschiedenen Kontaktkonstellationen miteinander verglichen (vgl. Rosenberg 2003, 2005; Riehl 2010; Yager et al. 2015), um etwa festzustellen, ob es sich bei der angenommenen internen Entwicklung um den gleichen Entwicklungsverlauf handelt oder ob unterschiedliche Entwicklungsverläufe lediglich $\mathrm{zu}$ dem gleichen Ergebnis führen. Außerdem fehlen beim Vergleich unterschiedlicher Konstellationen noch systematische Auszählungen der tatsächlichen Vorkommnisse von Kasussynkretismus; die meisten Untersuchungen beruhen auf qualitativen Ergebnissen. Quantitative Analysen umfangreicherer Korpora (etwa Boas 2009) legen aber dar, dass ein typisches Merkmal sich auflösender Sprachgemeinschaften gerade die hohe Variation ist, und zwar innerhalb der Äußerungen ein und desselben Sprechers und auch zwischen den Sprechern. Das gilt umso mehr beim Vergleich der Daten verschiedener Generationen: Hier kann in verschiedenen Studien nachgewiesen werden, dass der Abbau von einer Generation zur nächsten zunimmt (Huffines 1989; Salmons 1994; Zürrer 1999; Boas 2009; Riehl 2015). Daher soll nun im Folgenden der Dativabbau sowohl im Vergleich unterschiedlicher Kontaktvarietäten des Deutschen als auch pseudolongitudinal quantitativ analysiert werden. Im Mittelpunkt stehen dabei die Auswertungen der pseudolongitudinalen Daten aus dem Barossadeutschen in Südaustralien (Riehl 2015, 2016) und eine neue systematische Auswertung der bisher nur qualitativ analysierten generationenübergreifenden Daten aus dem Korpus zum Russlanddeutschen, das im Rahmen des von Ludwig Eichinger geleiteten DFG-Projektes „Form und Gebrauch des Deutschen in Mittel- und Osteuropa“ erhoben wurde (s.u. 3.2.2). Es soll analysiert werden, ob die Entwicklungspfade des Kasusabbaus in den unterschiedlichen Kontaktvarietäten den gleichen Verlauf nehmen und nur je nach soziolinguistischer Situation unterschiedliche Stadien aufweisen oder ob die Entwicklungspfade sich generell unterscheiden.

\section{Abbau der Kasusmarkierung in Kontakt- varietäten des Deutschen}

\subsection{Referenzvarietät und Pseudolongitudinalstudien}

Ein wichtiges Kriterium, das bei der Analyse des Dativabbaus in Kontaktvarietäten des Deutschen berücksichtigt werden muss, ist, dass auch in vielen Dia- 
lekten des Deutschen bereits ein Synkretismus von Dativ und Akkusativ stattgefunden hat, v. a. im Norden und Osten, während die südlichen Dialekte und Dialekte im mittleren Westen in dieser Hinsicht konservativer sind (vgl. Panzer 1983). Daher kann die Entwicklung in den Minderheitenvarietäten auch vom dialektalen Substrat beeinflusst sein (vgl. Riehl 2016). Allerdings sind die erhobenen Daten häufig schwer zu kategorisieren: in vielen Fällen handelt es sich um Interviews mit einem Sprecher des (Standard-)Deutschen, in denen die Interviewten versuchen $\mathrm{zu}$ akkommodieren und eine distanzsprachliche Varietät $\mathrm{zu}$ verwenden. Diese ist dann wieder vom dialektalen Substrat beeinflusst (vgl. Riehl 2006).

Um daher die Kontaktsituation als zentralen Motor für den Kasusabbau zu definieren, muss der Gebrauch der Kasusformen in einer Minderheitenvarietät über einen längeren Zeitraum und generationenübergreifend untersucht werden. Besonders aussagekräftig ist das Ergebnis dann, wenn der Abbau über Generationen in Sprachinseldialekten erfolgt, die noch eine reiche Dativmorphologie aufweisen, wie etwa das Walserdeutsche im Aosta-Tal, das eine ausgeprägte Dativmorphologie, besonders bei Substantiven im Plural, zeigt (vgl. Zürrer 1999).

Bisherige generationenübergreifende bzw. pseudolongitudinale Studien (etwa von Boas (2009) zum Texasdeutschen, von Zürrer (1999) zum Walserdeutschen und von Riehl (2015, 2016) zum Barossadeutschen) kommen trotz sehr unterschiedlicher Datenbasis zu dem Ergebnis, dass die Dativmarkierung nur noch von der älteren Generation verwendet wird, während jüngere Probanden oblique Formen (die mit den Akkusativ-Formen identisch sind) gebrauchen. Aufgrund der unterschiedlichen Ausgangsituation, d. h. der unterschiedlichen Vitalität der jeweiligen Varietäten, sind hier auch unterschiedliche Belegzahlen in der jeweils ältesten $\mathrm{zu}$ untersuchenden Generation $\mathrm{zu}$ finden. So konnte etwa Zürrer (1999), der drei verschiedene Sprechergenerationen miteinander vergleicht, zeigen, dass die älteste Generation nur einzelne Fälle von Kasussynkretismus aufweist, während die mittlere und jüngste Generation die Dativformen häufig durch Akkusativformen ersetzt. Boas (2009, 2016) und Riehl (2015, 2016) dagegen, die jeweils ihre Daten mit Daten aus früheren Studien verglichen und damit auch eine diachrone Entwicklung nachzeichnen konnten, stellten bereits bei den ältesten Sprechern einen deutlichen Abbau der Dativmarkierung fest (ähnlich auch Salmons 1994).

\subsection{Kontext, Wortart, Frequenz}

Weitere zentrale Aspekte, die bei der Analyse des Kasusabbaus berücksichtigt werden müssen, sind die Abhängigkeit der Markierung vom Gebrauchskontext 
und dem Typus der Konstruktion einerseits und der Häufigkeit der Lexeme und der betroffenen Wortart andererseits. So kann etwa Salmons (1994) zeigen, dass der Dativ im Texasdeutschen v. a. in prototypischen Kontexten verwendet wird (z. B. mit dem Verb helfen). In diesem Zusammenhang spielt auch eine wichtige Rolle, ob der Dativ vom Verb zugewiesen wird oder von der Präposition. Bei Kasusreduktion in Präpositionalphrasen stellt Boas (2009) fest, dass diese nicht nur kontext-, sondern auch item-abhängig ist, da sie bei bestimmten Präpositionen häufiger auftritt als bei anderen. Auch wird bei Wechselpräpositionen keine klare Unterscheidung zwischen Dativ und Akkusativ mehr vorgenommen. Boas (2009) erklärt dies einerseits mit dem Phänomen des Reduktionsprozesses bei Sprachverlust und zum anderen mit einer internen Entwicklung der deutschen Sprache. Ähnliches kann auch Riehl (2015) für das Barossadeutsche zeigen, allerdings hier in Abhängigkeit von bestimmten gebrauchsbasierten Konstruktionen. Die Dativmarkierung wird in abstrakten Schemata wesentlich rascher abgebaut als in konkreten Konstruktionen oder in festen Verbindungen (s. u. Abschnitt 4). In der Studie von Zürrer (1999: 200) spielt die Vorkommenshäufigkeit der Nomina eine entscheidende Rolle: So weisen im Walserdeutschen hochfrequente Lexeme wie töchter (,Tochter'), wetta (,Schwester'), muma (,Tante') viel häufiger Dativendungen auf als weniger gebrauchte Substantive.

Daneben ist auch die Wortart, an der der Dativ markiert wird, von Bedeutung: Hier ist etwa das Pronominalsystem wesentlich robuster gegen den Abbau der Morphologie als Substantive oder Artikel (vgl. Rosenberg 2003). Rosenberg (2016: 198) interpretiert dies dahingehend, dass Flexionsmorpheme eher fusionieren als Einheiten, die als Ganzes lexikalisch gespeichert sind (sog. full listed items), dass Personalpronomina hochfrequent sind, häufig belebte Referenten bezeichnen und häufiger als suppletive Formen gespeichert sind (vgl. es, er vs. ihm, ihn). Außerdem zeigt die syntaktische Reihenfolge im Mittelfeld die unmarkierte Anordnung bekannt vor neu, unbetont vor betont. In dem von Rosenberg (2016) untersuchten Korpus stehen die Dativpronomina überdies überwiegend für eine Rezipientenrolle (Kernbereich ,Empfängerkasus‘).

Die Ergebnisse aus den bisherigen Studien lassen sich wie folgt zusammenfassen:

1. Beim Kasusabbau in Kontaktvarietäten handelt es sich um einen Vereinfachungsprozess und nicht um ein Phänomen der Konvergenz.

2. Das Kasussystem ist am besten in der ältesten Generation erhalten, während jüngere Sprecher bereits eine allmähliche Reduktion der Dativmarkierung zeigen.

3. Man kann im Allgemeinen einen item-abhängigen Gebrauch feststellen: je frequenter (oder prototypischer) ein Lexem oder eine bestimmte Konstruktion ist, desto weniger wird die Dativmarkierung abgebaut. 
4. Der Dativabbau ist an unterschiedliche Wortarten gebunden: Während die Markierung bei Personalpronomina noch weitgehend erfolgt, verschwindet sie beim Artikel, bei Adjektiven und Nomina immer mehr.

\section{Der Dativabbau im Vergleich}

Im Folgenden wird nun der Abbau der Dativmarkierung in zwei unterschiedlichen Kontaktvarietäten, dem Barossadeutschen in Südaustralien und dem Russlanddeutschen (überwiegend der Wolgaregion), miteinander verglichen. Dabei wird zunächst der soziolinguistische Hintergrund der jeweiligen Kontaktsituation dargestellt, im Anschluss daran die Datengrundlage beschrieben und schließlich der Dativabbau in verschiedenen Kontexten und Konstruktionen analysiert.

\subsection{Dativabbau im Barossadeutschen}

\subsubsection{Soziolinguistischer Hintergrund}

Die erste Siedlung im Barossa-Tal, etwa $70 \mathrm{~km}$ nordöstlich von Adelaide (Südaustralien), wurde 1842 gegründet. Die Siedler stammten hauptsächlich aus Nordschlesien sowie der Lausitz und der Region um Posen (vgl. Paul 1965). Allerdings wurden in der Sprachgemeinschaft bereits in den 1960er Jahren keine Dialekte mehr gesprochen, sondern eine auf dem Standarddeutschen basierende Regionalvarietät. Paul (1965: 12) erklärt diese Tatsache mit dem starken Einfluss des lutherischen Schulsystems und dem Sprachgebrauch im religiösen Umfeld.

Im Gegensatz zu anderen deutschen Siedlungen in Australien konnte die deutsche Sprache auch nach 1918, als landesweit die deutsche Sprache als Schulsprache abgeschafft wurde, noch weiter aufrechterhalten werden, zum einen durch den Kontakt der australischen lutherischen Kirche zur evangelischen Kirche in Deutschland, zum anderen, weil noch regelmäßig Gottesdienste in deutscher Sprache abgehalten wurden und auch die Möglichkeit des Besuchs einer Sonnabendschule bestand (vgl. Clyne 1981). Mit dem Beginn des Zweiten Weltkriegs konnte Deutsch nicht mehr im öffentlichen Kontext verwendet werden, und sogar der private Gebrauch wurde eingeschränkt. Dies führte dazu, dass die Verwendungskontexte immer mehr abnahmen und sich ganz auf die Familie und Gespräche mit der älteren Generation beschränkten. 
Eine besondere Einschränkung bestand auch darin, dass die deutsche Sprache nun nicht mehr als Schriftsprache gelernt werden konnte (vgl. Riehl 2016).

Im Gegensatz zu anderen Sprachinseln, in denen wir noch drei Generationen beobachten können, die die Sprachinselvarietät sprechen (etwa in Mittel- und Osteuropa vgl. Eichinger, Plewnia \& Riehl 2008), ist die aktive Sprachkompetenz im Barossadeutschen auf die älteste Generation beschränkt. Da es sich beim Barossadeutschen um eine sterbende Varietät handelt, zeigen die letzten Sprecher sehr viele Muster, die in der Regel bei Sprachgemeinschaften zu finden sind, die zum Sprachwechsel übergehen, wie den Verlust morphosyntaktischer Kategorien oder komplexer syntaktischer Kategorien (vgl. Thomason 2001).

\subsubsection{Datengrundlage}

Zwischen 2009 und 2014 konnten insgesamt 51 Gewährspersonen aufgenommen werden (27 weiblich, 24 männlich). Davon haben aber 14 Probanden nur noch eine passive Kompetenz des Deutschen. Zum Aufnahmezeitpunkt waren die Gewährsleute zwischen 73 und 96 Jahre alt (Durchschnittsalter $=85$ ). Die meisten der männlichen Probanden arbeiteten entweder auf ihren eigenen Farmen oder in anderen Bereichen der Landwirtschaft, in Mühlen oder im Weinbau. Die weiblichen Probanden hatten meist keine berufsspezifische Ausbildung, beinahe alle von ihnen arbeiteten ebenfalls auf der Farm oder waren Hausfrauen, nur zwei von ihnen gingen auf die Highschool. Eine der Probandinnen arbeitete als Krankenschwester. Alle Sprecher sind Mitglieder der Altlutherischen Kirche und besuchen regelmäßig den Gottesdienst. Obwohl viele dem gleichen sozialen Netzwerk angehören, geben sie an, mit Ausnahme einiger Phrasen die deutsche Sprache untereinander nie zu gebrauchen.

Das hier analysierte Korpus besteht aus narrativen Interviews, die jeweils eine Länge zwischen 60 und 120 Minuten aufweisen. Bei manchen Interviews wurden zwei Probanden gemeinsam aufgezeichnet, um eine natürlichere Atmosphäre zu schaffen. Der Großteil der Aufnahmen entstand bei den Probanden zu Hause oder in einem ähnlich entspannten Umfeld. Zahlreiche Fragen betrafen biographische Informationen der Teilnehmer sowie deren Sprachgebrauch, Spracheinstellungen und die Sprachbiographie. Der größte Teil der Interviews besteht jedoch aus freien Erzählungen, in denen die Probanden meist über ihr Leben auf der Farm und die deutsche Gemeinschaft betreffende Bräuche erzählten.

Ergänzend wurde der von Hans Boas in Anlehnung an Gilbert (1972) erstellte Fragebogen zum Texas-German (in leichter Adaption an das australische Englisch und die Gegebenheiten im Barossa-Valley) abgefragt (s. Boas 2009). 
Dieser Fragebogen besteht aus einer Reihe von Einzelwörtern, Phrasen und Sätzen, die aus dem Englischen ins Barossadeutsche übersetzt werden sollten. Allerdings haben nur ein Drittel der Probanden $(n=13)$ den Fragebogen beantwortet, da bei den meisten die Sprachkenntnisse nicht ausreichten.

Wie oben erwähnt (3.1.1), basiert das Barossadeutsche ursprünglich auf einer Form eines Regionaldialekts, der dem Standarddeutschen nahe ist. Deswegen kann in diesem Fall das standarddeutsche Kasussystem als Referenz herangezogen werden. Da nur mehr die älteste Generation das Barossadeutsche beherrscht, ist eine generationenübergreifende Analyse, wie sie etwa Zürrer (1999) unternommen hat, nicht mehr möglich. Deshalb wurden historische Aufnahmen von Michael Clyne aus dem Jahre 1967 mit 79 Sprechern (insgesamt 39 Aufnahmen mit einer Länge von 5-50 min.) ${ }^{2}$ und Daten aus der von Paul (1962, 1965) durchgeführten Studie (Abfrage der Wenkersätze mit 13 Sprechern des Barossadeutschen) herangezogen.

\subsubsection{Der Verlust des Dativs im Barossadeutschen}

Der hier vorgenommenen Analyse der aktuellen Interviewdaten liegt ein Teilkorpus von 20 Sprechern zugrunde, das insgesamt 72.200 Wörter umfasst. Dabei entfällt auf jeden Sprecher eine durchschnittliche Anzahl von 3.830 Wörtern. Im untersuchten Korpus gibt es 1.501 Fälle, in denen im Standarddeutschen der Dativ zugewiesen wird. Allerdings wird davon nur in 457 Fällen die reguläre Dativ-Markierung (30,4\%) realisiert, 941 Fälle zeigen keine oder eine unvollständige Dativ-Markierung (67,7\%), und es treten 103 Akkusativ-Markierungen anstelle von Dativ-Markierungen auf (6,8\%) (s. Tab. 10.1).

Tab. 10.1: Kasus-Markierung in Kontexten, die den Dativ fordern.

\begin{tabular}{lcc}
\hline & Gesamtzahl & Prozent \\
\hline Gesamtzahl der Kontexte, die Dativ fordern & 1.501 & $100.0 \%$ \\
reguläre Dativ-Markierung & 457 & $30.4 \%$ \\
keine oder unvollständige Dativ-Markierung & 941 & $62.7 \%$ \\
Akkusativ- statt Dativ-Markierung & 103 & $6.8 \%$ \\
\hline
\end{tabular}

2 Diese sind in der Datenbank des Gesprochenen Deutsch (DGD) des IDS zugänglich, vgl. dgd.ids-mannheim.de (letzter Zugriff 6.10. 2017). Hier gebührt ein besonderes Verdienst Ludwig Eichinger, der sich für die Überführung der sehr wertvollen Aufnahmen von Michael Clyne an das IDS eingesetzt hat. 
Von 457 Fällen des regulären Dativgebrauchs waren 114 vom Verb zugewiesen, mit einer Ausnahme (dem Weihnachtsmann) handelt es sich dabei stets um Personalpronomina. In den Beispielen, bei denen der Dativ von einer Präposition zugewiesen wird, finden sich Dativmarkierungen in Nominalphrasen am bestimmten Artikel am häufigsten bei Verschmelzungen einer Präposition mit dem Suffix des definiten Artikels, z. B. zur (= zu der), zum (=zu dem), beim (= bei dem), aufm (= auf dem). Allein 44,9\% der Vorkommnisse von durch Präpositionen zugewiesenen Dativ-Markierungen sind diesen Typs $(n=154)$, darunter allein 55 Vorkommnisse (35,7\% aller verschmolzenen Fomen) von zur, entweder in der Konstruktion zur Kirche oder zur Schule. Betrachtet man die verbleibenden Dativformen des bestimmten Artikels in PräpositionalKonstruktionen, findet sich eine aufällige Anzahl in festgeprägten Ausdrücken, z. B. in Kombination mit dem Nomen Krieg: 11 Vorkommnisse mit nach dem oder nachm Krieg und eine mit seit dem Krieg (7,1\% aller Vorkommnisse).

Die Ergebnisse aus den freien Interviews werden durch die Daten der Übersetzungsaufgabe bestätigt: In dieser finden sich 18 Sätze mit Präpositionalphrasen, die im Standarddeutschen den Dativ verlangen: von den insgesamt 216 Vorkommnissen hatten nur $25(=11,5 \%)$ den Dativ markiert. Die meisten Sprecher haben nur eine einzige Form (aufm Boden bzw. aufm floor) mit Dativmarkierung realisiert. Die Ausnahme bildet eine Informantin (MH), die alleine neun standardkonforme Dativmarkierungen verwendet, z. B. auf dem Stuhl, neben dem Baum, unter dem Holzhaufen. Dies lässt sich dadurch erklären, dass die Sprecherin Deutsch liest und gelegentlich auch schreibt (vgl. dazu Riehl 2015, 2016). ${ }^{3}$

Beim Vergleich mit den historischen Daten zeigt sich nun, dass tatsächlich ein weiterer Kasusabbau in der letzten Generation stattgefunden hat: So treten bei von Präpositionalphrasen zugewiesenen Dativen in den gesprochenen Aufnahmen von Clyne noch 44,8\% reguläre Dativ-Markierungen auf gegenüber $13,8 \%$ in den neuen Aufnahmen und in den Übersetzungsaufgaben von Paul 49,4 \% reguläre Markierungen gegenüber 11,5\% in den Übersetzungsaufgaben von 2014 (vgl. auch Riehl 2015).

3 Die Informantin MH sticht heraus, da sie ein sehr emotionales Verhältnis zum Deutschen hat und auch eine hohe Sprachaufmerksamkeit zeigt. Sie berichtet, dass sie über 17 Jahre eine deutschsprachige Zeitschrift abonniert und aufmerksam gelesen hat. Außerdem ist sie seit einigen Jahren mit einigen Bekannten in Deutschland in Kontakt und schreibt ihnen auch Briefe auf Deutsch. 
Tab. 10.2: Vergleich historische Daten - aktuelle Daten.

\begin{tabular}{llllll}
\hline & \multicolumn{2}{l}{ Historische Daten (1962-1967) } & & \multicolumn{2}{l}{ Aktuelle Daten (2009-2014) } \\
\cline { 2 - 3 } \cline { 6 - 6 } & regulär & irregulär & & regulär & irregulär \\
\hline Interviews & $44,8 \%$ & $55,2 \%$ & & $13,8 \%$ & $86,2 \%$ \\
Übersetzungsaufgabe & $49,4 \%$ & $50,6 \%$ & & $11,5 \%$ & $88,5 \%$ \\
\hline
\end{tabular}

Diese Ergebnisse bestätigen einerseits, dass es bereits in der vorhergehenden Generation eine hohe Varianz verschiedener Formen gab, und andererseits, dass die Tendenz des Dativabbaus sich in der heutigen Generation weiter fortgesetzt hat.

Interessant in diesem Zusammenhang ist auch die Entwicklung des Systems der Personalpronomina. Hier zeigt sich eine fortschreitende Tendenz zur Etablierung eines Zwei-Kasus-Systems (Subjekts- und Objektskasus). Die Analyse der Pronominalverwendung ergibt, dass der Dativ vor allem an der 1. Pers. Sg. (mir) (58,4\%) markiert wird, gefolgt von der 1. Pers. Pl. (uns, was sich jedoch morphologisch nicht vom Akkusativ unterscheidet) (25,7\%). Pronomina der 2. Pers. Pl. (dir) machten $8,8 \%$ der Vorkommnisse aus und die 3. Pers. Sg. (ihm oder ihr) 7,1\%. Die 3. Pers. Pl. (ihnen) tritt dagegen überhaupt nicht in der Dativform auf, sondern wird stets durch sie/se ersetzt. ${ }^{4}$

Bis auf eine Ausnahme (hab mich das Messer geholt, GG) werden alle Dativpronomina in der 1. und 2. Pers. Sg. wie im Standarddeutschen gesetzt. Umgekehrt finden sich in $86,5 \%$ aller Fälle, wo das Standarddeutsche das Akkusativpronomen mich bzw. dich fordert, die Dativformen mir und dir. Von den verbleibenden Akkusativpronomina treten die meisten in den Wendungen für mich bzw. für dich (36\%), in Zitaten aus der Bibel oder in hochfrequenten Reflexivkonstruktionen wie hat mich gewundert, hat mich gefreut auf.

Dies deutet auf eine klare Entwicklung zum Ersatz der alten Dativ- und Akkusativ-Distinktion im Bereich der Personalpronomina durch einen obliquen Kasus hin, der sich je nach Person aus den alten Formen des Akkusativs oder Dativs rekrutiert (vgl. Tab. 10.3). Dabei markieren die ursprünglichen Dativformen die 1. und 2. Pers. Sg. (Dat./Akk.) und die ursprünglichen Akkusativformen 3. Pers. Sg. und Pl. (Dat./Akk.) (1. und 2. Pers. Pl. haben ja auch im Standarddeutschen nur eine Form für Dativ und Akkusativ).

Eine Reduzierung der Dativ-Akkusativ-Unterscheidung ist zwar typisch für niederdeutsche Dialekte, ein Einfluss des Niederdeutschen ist aber eher un-

4 Dies deckt sich mit den Beobachtungen von Rosenberg (2016: 203), der in seinen Korpora ebenfalls das Fehlen des Pronomens der 3. Pers. Pl. ihnen bemerkt. 
Tab. 10.3: Restrukturierung der pronominalen Kasus-Markierung im Barossadeutschen.

\begin{tabular}{lll}
\hline & Singular Dat./Akk. & Plural Dat./Akk. \\
\hline 1. Pers. & mir & uns \\
2. Pers. & dir & euch \\
3. Pers. & ihn/sie & sie \\
\hline
\end{tabular}

wahrscheinlich: Zum einen trat dieser Synkretismus in den von der Mehrheit der Siedler mitgebrachten schlesischen und Lausitzer Dialekten nicht auf (s. Weinhold 1853), und der Einfluss des Niederdeutschen war eher gering einzuschätzen (s. Paul 1965). Zum zweiten wird im niederdeutschen Pronominalsystem die oblique Form innerhalb des ganzen Paradigmas (und nicht nur in der 1. und 2. Pers. Sg.) mit dem Dativpronomen markiert (vgl. 3. Sg. em/ehr, 3. Pl. jem) (vgl. Riehl 2016). Somit handelt es sich hier um einen Restrukturierungsprozess des Systems, der ebenfalls durch Sprachkontakt ausgelöst werden kann.

\subsection{Dativabbau im Russlanddeutschen}

\subsubsection{Historischer Hintergrund}

Im Gegensatz zu den Sprachinselvarietäten in Australien sind die deutschen Sprachinseln in Russland wesentlich älter und heterogener. Bereits in den Jahren 1764-67, unter Katharina II., wurden 104 Dörfer an der Wolga gegründet (südlich und nördlich von Saratov). Die meisten Siedler stammten aus Hessen, der Nordpfalz, Nordbayern, Nordbaden und der Fuldaer Gegend. ${ }^{5}$ In Folge kam es zur Entstehung von Ausgleichs- oder Mischmundarten sowie zur Ausbildung einer überregionalen Koiné, dem sog. ,Wolgadeutschen“ (vgl. Berend 2011). In der 1924 gegründeten Wolgarepublik war die deutsche Sprache als Amtssprache zugesichert, diente als Unterrichtssprache, Sprache der Öffentlichkeit und der Medien. Noch in den 1920er Jahren waren die deutschsprachigen Zeitungen

5 Später entstanden Siedlungen in Jamburg, Černigov, Wolhynien und im Schwarzmeergebiet, in denen sich kleinere Gruppen, z. B. Siedler aus dem Danziger Raum, ansiedelten. In den Jahren 1803-1823 (Alexander I.) wurden zur Besiedlung von Bessarabien Deutsche aus Polen, Mecklenburg, Pommern und Westpreußen angeworben, denen noch Siedler unmittelbar aus Südwestdeutschland folgten. 1830-1870 (unter Nikolaus I. und Alexander II.) fand eine Massenansiedlung von Deutschen aus Polen und Galizien in Wolhynien statt (vgl. dazu Berend \& Riehl 2008). 
sehr wenig von der Kontaktsprache Russisch beeinflusst und konnten als Sprachnorm dienen (vgl. Berend \& Riehl 2008).

Mit dem Zweiten Weltkrieg änderte sich die Situation grundlegend: Alle deutschen Siedler im europäischen Teil der damaligen UdSSR wurden nach Kriegsausbruch 1941 nach Sibirien und Mittelasien deportiert. Dies hatte zum einen zur Konsequenz, dass sich in den nun frei werdenden Gebieten Sprecher der Mehrheitsgesellschaft ansiedelten, zum anderen, dass deutschsprachige Personen aus unterschiedlichen Regionen in Kontakt kamen, wodurch es zu einer erneuten Dialektmischung kam (vgl. Blankenhorn 2008: 61). Häufig kam es zu Sprachwechselprozessen, die auch dadurch bestärkt wurden, dass die deutsche Sprache in Folge des Krieges nicht nur einen völligen Prestigeverlust erlitt, sondern teilweise sogar verboten war und der soziale Aufstieg der Minderheiten an die vollkommene Beherrschung des Russischen gebunden war. Viele Deutschsprachige gingen daher auch in der Familie zum Russischen über und gaben das Deutsche (bzw. den deutschen Dialekt) nicht mehr an die Kinder weiter. Doch auch dann, wenn die primäre Sozialisation noch im Dialekt erfolgte, wurde in der zweiten Generation durch die sekundäre Sozialisation die Umgebungssprache Russisch zur primären Varietät. Nach 1990 kam es zwar zu einer Wiederbelebung des Deutschen mit verstärktem Unterricht und neugegründeten kulturellen Institutionen und Verbänden, allerdings erfolgte parallel dazu eine massenhafte sog. ,Spätaussiedlung‘ in die Bundesrepublik Deutschland oder nach Österreich. Diese Faktoren führen dazu, dass die deutsche Sprachgemeinschaft in vielen Gebieten in Auflösung begriffen ist (Riehl 2016; Rosenberg 2016).

\subsubsection{Datengrundlage}

In einem von der Deutschen Forschungsgemeinschaft geförderten Projekt unter der Leitung von Ludwig Eichinger wurden in den Jahren 2000-2002 40 russlanddeutsche Sprecher in der Wolgaregion $(n=12)$, St. Petersburg $(n=9)$ und im Ural (Jekaterinburg, Polevskoj, Krasnotourinsk) $(n=19)$ interviewt. Die Teilnehmer lassen sich aufgrund der jeweiligen politischen und gesellschaftlichen Entwicklung in unterschiedliche Sprechergenerationen einordnen. Zu Generation I wurden Sprecher gerechnet, die vor 1932 geboren worden waren und aufgrund dessen die Möglichkeit hatten, vor der Deportation 1941 eine deutsche Schule zu besuchen, in der sie die deutsche Schriftsprache erwerben konnten. Generation II setzt sich aus Sprechern zusammen, die zwischen 1932 und 1952 geboren wurden, das bedeutet, dass sie Deutsch als L1 in der Familie erworben haben, aber bereits eine russischsprachige Schule besuchten und darüber hinaus in einer Zeit aufwuchsen, in der das Deutsche stark stigmatisiert war. Sprecher der Generation III und IV (zwischen 1952 und 
1975 und nach 1975 geboren) haben in der Regel nicht Deutsch von ihren Eltern gelernt, sondern Deutsch als Fremdsprache erworben. Da das Projekt auf die Generation I und II fokussierte, besteht das Korpus überwiegend aus Informanten dieser beiden Generationen (Generation I: $n=19$, Generation II: $n=12$ ). Die weiblichen Probanden der Studie waren entweder in landwirtschaftlichen Berufen tätig oder als Fabrikarbeiterin bzw. Maschinistin. Lediglich zwei Probandinnen waren in medizinischen Berufen tätig (Apothekerin und CTA). Die Männer arbeiteten als Agraringenieur oder Elektriker, Schlosser, Stahlarbeiter oder Maurer. Zwei von ihnen waren als Lehrer tätig.

Die Vertreter der Generation I sprachen mit den Eltern und teilweise mit den Ehepartnern Deutsch, nur wenige mit den Kindern, darüber hinaus mit Verwandten in Deutschland, im deutschen Kulturzentrum und teilweise mit noch lebenden Geschwistern (oft hier aber eine Mischsprache). Einige hatten auch Kontakte zu Deutschen durch die neuapostolische Kirche und sprachen mit den Priestern Deutsch. Sprecher der zweiten Generation hatten noch teilweise mit den Eltern Deutsch gesprochen, mit den Geschwistern dagegen Russisch oder gemischt. Auch sie haben Kontakte zu Verwandten in Deutschland oder über das Kulturzentrum bzw. die Kirche, allerdings sind die Situationen, in denen sie Deutsch verwenden, äußerst begrenzt (vgl. Riehl 2017). Einige der Sprecher der ersten Generation konnten noch Lesen und Schreiben auf Deutsch in der Schule lernen, etwa die Hälfte unserer Informanten lesen noch deutsche Bücher oder Zeitungen oder schreiben Briefe auf Deutsch. Die Vertreter der zweiten Generation haben dagegen keinen Zugang zur Schriftlichkeit und lesen und schreiben ausschließlich auf Russisch.

Das Korpus besteht aus semi-strukturierten Interviews von einer Länge von 60-120 Minuten. Wie im Barossadeutsch-Korpus adressieren die Fragen die Sprachbiographie, den Sprachgebrauch und Spracheinstellungen. Der Hauptteil bestand auch hier aus freien Erzählungen aus dem Leben der Probanden. In einigen Situationen wurden zwei Personen gemeinsam interviewt; einige Informanten, die gleichzeitig eine Vermittlerfunktion einnahmen, waren in mehreren Interviews anwesend. Die meisten Gespräche wurden bei den Gesprächspartnern zuhause oder in separaten Räumen im Kulturzentrum aufgezeichnet.

Die Probanden im vorliegenden Korpus zum Russlanddeutschen sind insgesamt wesentlich heterogener als die Sprecher des Barossadeutschen, da sie ursprünglich aus unterschiedlichen Dialektregionen stammen. Allerdings sind zwei Aspekte zu beachten: Zum einen wurden die Interviews von Sprechern des Standarddeutschen geführt, so dass die Interviewten sich bemühten, eine möglichst distanzsprachliche Varietät zu verwenden (vgl. Riehl 2006; Berend \& Riehl 2008). Des Weiteren haben sich die meisten Sprecherinnen und Sprecher an das Wolgadeutsche angelehnt (zur Verwendung der wolgadeutschen Koiné vgl. Berend \& Riehl 2008). 


\subsubsection{Dativabbau über Generationen}

Der Analyse liegt ein Teilkorpus von insgesamt 19 Sprechern zugrunde, davon 10, die der ersten, und 9, die der zweiten Generation zuzurechnen sind. Das Korpus beschränkt sich auf die Sprecher aus der Wolgaregion und Sprecher aus dem Ural, die ebenfalls zum Großteil Wolgadeutsch als Ausgleichsmundart untereinander sprechen, um die dialektale Grundlage einigermaßen konstant zu halten. ${ }^{6}$ Insgesamt umfasst das Korpus rund 80.000 Wörter. Im untersuchten Korpus gibt es 1.612 Fälle, in denen im Standarddeutschen der Dativ zugewiesen wird. Davon wird in 913 Fällen die reguläre Dativ-Markierung (56,6\%) realisiert, 699 Fälle zeigen keine oder eine unvollständige Dativ-Markierung (41,0\%), und es treten 37 Akkusativ-Markierungen anstelle von Dativ-Markierungen auf $(2,3 \%)$ (s. Tab. 10.4).

Tab. 10.4: Kasus-Markierung in Kontexten, die den Dativ fordern.

\begin{tabular}{lcc}
\hline & Gesamtzahl & Prozent \\
\hline Gesamtzahl der Kontexte, die Dativ fordern & 1.612 & $100,0 \%$ \\
reguläre Dativ-Markierung & 913 & $56,6 \%$ \\
keine oder unvollständige Dativ-Markierung & 699 & $41,0 \%$ \\
Akkusativ- statt Dativ-Markierung & 37 & $2,3 \%$ \\
\hline
\end{tabular}

Die Verteilung differenziert sich, wenn man die beiden Generationen getrennt betrachtet (vgl. Tab. 10.5). Hier kann man einen deutlichen Rückgang der regulären Dativmarkierung von knapp 20 Prozentpunkten beobachten:

Tab. 10.5: Kasus-Markierung in Kontexten, die den Dativ fordern, nach Generationen.

\begin{tabular}{lcc}
\hline & Generation I & Generation II \\
\hline reguläre Dativ-Markierung & $64,9 \%$ & $45,5 \%$ \\
keine oder unvollständige Dativ-Markierung & $32,7 \%$ & $52,3 \%$ \\
Akkusativ- statt Dativ-Markierung & $2,4 \%$ & $2,2 \%$ \\
\hline
\end{tabular}

6 Aufgrund der in 3.2.1 beschriebenen soziolinguistischen Situation und der durch die Deportation ausgelösten vielfachen Kontaktsituation ist der Dialekteinfluss nicht immer kontrollierbar. Auch ist zu berücksichtigen, dass einige der Sprecher auch Kontakt zum Standarddeutschen haben und in den Interviews versuchten, eine distanzsprachliche Varietät zu sprechen. Da die Aufnahmen ursprünglich soziolinguistischer Natur waren, wurde das nicht kontrolliert. 
Vergleicht man die Daten nach Wortarten und Konstruktionen, so gibt es wenige Belege, bei denen im Standarddeutschen der Dativ in der Substantivgruppe vom Verb zugewiesen wird, nämlich nur 20 im gesamten Korpus. Davon sind 12 analog zum Standarddeutschen als Dativ realisiert, meistens in Kontexten mit prototypischen Verben für Dativ (meinem Vater gesagt, dem Professor gezeigt, der Mutter geholfen). Für den Dativgebrauch in Präpositionalphrasen ergibt sich ein ähnliches Bild wie für die historischen Interviewdaten des Barossadeutschen: Hier zeigen die Daten aus der ersten Generation der Russlanddeutschen sogar noch eine relativ hohe Zahl regulärer Bildungen $(66,0 \%, n=390)$, in der Generation II der Russlanddeutschen werden dagegen exakt die gleichen Werte erzielt wie in den historischen Aufnahmen des Barossadeutschen, nämlich 44,8\% $(n=223)$ reguläre und 55,2\% $(n=275)$ irreguläre Markierung des Dativs, vgl. Tabelle 10.6:

Tab. 10.6: Dativgebrauch in Präpositionalphrasen im Vergleich.

\begin{tabular}{|c|c|c|c|c|c|c|}
\hline & \multicolumn{2}{|c|}{$\begin{array}{l}\text { Barossadeutsch } \\
\text { (Aufnahmen 1967) }\end{array}$} & \multicolumn{2}{|c|}{$\begin{array}{l}\text { Russlanddeutsch } \\
\text { Generation I }\end{array}$} & \multicolumn{2}{|c|}{$\begin{array}{l}\text { Russlanddeutsch } \\
\text { Generation II }\end{array}$} \\
\hline & regulär & irregulär & regulär & irregulär & regulär & irregulär \\
\hline Interviews & $44,8 \%$ & $55,2 \%$ & $66,0 \%$ & $34,0 \%$ & $44,8 \%$ & $55,2 \%$ \\
\hline
\end{tabular}

Damit zeigt sich zwar eine Tendenz des Abbaus der Dativmarkierung zwischen den Sprechern der Generation I und II, aber die Entwicklung ist noch nicht so weit fortgeschritten wie im Barossadeutschen. Hier ist die Generation II auf dem Stand wie gleichaltrige Sprecher des Barossadeutschen vor 50 Jahren. $^{7}$

Die Dativverwendung in vom Verb zugewiesenen Phrasen und in Präpositionalphrasen zeigt sich auch hier vor allem bei hochfrequenten Wörtern (wie Verwandtschaftsbezeichnungen) in Verbindung mit einem Possessivpronomen (mit, bei, von meiner Mutter/Schwester/Tochter, meinem Sohn/Mann etc.). Und ebenfalls ähnlich wie beim Barossadeutschen finden sich eine große Zahl von Dativmarkierungen am bestimmten Artikel am häufigsten bei Verschmelzungen einer Präposition mit dem Suffix des definiten Artikels: Bei den Sprechern der Generation I beträgt dies 33,6\% der korrekten Dativzuweisungen in der Präpositionalgruppe $(n=131)$, bei der Generation II $39 \%$ der korrekten $\mathrm{Zu}$ -

7 Leider lassen sich die Daten aus der 3. und 4. Generation der Russlanddeutschen wiederum nicht damit vergleichen, da diese Sprecher zum Großteil Schulunterricht auf Deutsch hatten oder es nach der Wende im gesteuerten Unterricht wiedererwarben (vgl. Riehl 2017). 
weisungen $(n=87)$. Hier überwiegt aber die kontrahierte Form im (insgesamt 99 Vorkommnisse, davon 11 Vorkommnisse im Dorf) statt wie im Barossadeutschen $z u r$ (s. o.). Das ist dadurch begründet, dass im Russlanddeutsch-Korpus die festen Wendungen zur Kirche, zur Schule jeweils nur zweimal vorkommen, stattdessen verwenden die Sprecher die Wendungen in die Kirche bzw. in die Schule, die den Akkusativ fordern. Hier ist allerdings $\mathrm{zu}$ bemerken, dass die unverschmolzene Form des Lokativs in der Schule ebenfalls sehr frequent ist (42 Vorkommnisse), hier aber manchmal in Variation mit in die Schule (18 Vorkommnisse), bisweilen sogar bei ein und demselben Sprecher (ER, WA, KS). ${ }^{8}$ Wenn man die verbleibenden Dativformen des bestimmten Artikels in Präpositional-Konstruktionen betrachtet, findet sich auch hier eine auffällige Anzahl in festgeprägten Ausdrücken, z. B. in Kombination mit dem Nomen Krieg: 11 Vorkommnisse mit nachm/vorm, am/im Krieg (5\% aller Vorkommnisse). Aber auch hier kann man besonders bei den Sprechern der Generation II bei ein und demselben Sprecher die Variation von nochm Krieg, am Kriech vs. in Kriech und vorn Kriech (KE) beobachten.

Eine weitere Besonderheit lässt sich in diesem Korpus feststellen, und zwar ein Sprachkontaktphänomen, das auch Auswirkung auf die Kasusmarkierung hat: Die Sprecher lassen in einigen Fällen analog zum Russischen den Artikel völlig weg: mit Tochter (MH), von Wolga (HF), aus Krieg (TV). Mit dem Wegfall des Trägers der Flexionsendungen besteht keine Möglichkeit mehr, den Kasus zu markieren, und daher fallen auch diese Beispiele unter fehlende Dativmarkierung. Diese Fälle betragen 5\% in der ersten und immerhin 11,6\% von den fehlenden Dativmarkierungen in der zweiten Generation. Damit lässt sich hier ein Zusammenspiel von Sprachkontakt (Artikellosigkeit) und Vereinfachungsprozessen (Kasusabbau) beobachten.

In Bezug auf das System der Personalpronomina findet sich hier ein völlig anderes Bild als im Barossadeutschen: Wie bereits Rosenberg (2003) für das Russlanddeutsche zeigt, ist hier die Kasusmarkierung noch weitgehend erhalten, allerdings treten verschiedene Variationen auf: einige Sprecher verwenden gelegentlich die Dativpronomina mir, dir für den Akkusativ (das hat mir vieles gekostet, JH), sonst aber wie im Standarddeutschen. Drei Sprecher zeigen gewisse Idiosynkratien: Sprecher HF und FF verwenden häufig die Akkusativformen mich und dich in Dativpositionen: hat mich erzählt (HF), ich helf dich (FF). TM dagegen benutzt auch die Dativformen dir und mir statt der Akkusativpronomina (hat gut mir gekannt). Diese hohe Variation zwischen den Sprechern kann damit erklärt werden, dass sie aus unterschiedlichen Dialektgebieten

8 Daneben finden sich noch fünf Beispiele ohne Artikel (in Schule) sowie zwei unvollständige Äußerungen (in unser Schule). 
stammen (HF, FF aus dem Wolgagebiet, TM aus Wolhynien) und durch die Auflösung von Netzwerken kein Dialektausgleich mehr besteht (vgl. Trudgill 2004). Es bleibt daher bei einer hohen individuellen Variation und idiosynkratischen Verwendungen. Allerdings ist die Verwendung in den von Präpositionen zugewiesenen Fällen noch sehr stabil (86,5\% der Dativmarkierungen sind hier regulär, und zwar in beiden Generationen!). Das gilt auch für das Pronomen der 3. Pers. Pl. ihnen: Hier gibt es nur zwei Abweichungen (mit sie, KA, SM).

\section{Erklärungsmuster}

Für den Abbau der Kasusmorphologie wurde verschiedentlich die sog. Regressionshypothese von Jakobson (1941) angeführt, die besagt, dass Sprachabbau Spracherwerb in umgekehrter Reihenfolge ist. Das würde bedeuten, sprachliche Strukturen, die zuletzt erworben werden, gehen auch am ehesten verloren (Rosenberg 2003; Salmons 1994). Jedoch wurde diese These bereits von Schmid (2002) für individuelle Spracherosion widerlegt. In Riehl (2015) habe ich argumentiert, dass es sich hier vielmehr um eine Regression von EntrenchmentProzessen handelt. Dabei wird davon ausgegangen, dass bei Spracherosion auf die zuerst erworbenen konkreten Konstruktionen, die sich durch eine hohe token-Frequenz auszeichnen (z. B. Mama gibt dem Baby den Ball), länger zugegriffen werden kann als auf die später erlernten abstrakten Konstruktionen (vom Typ $X$ gibt $Y$ [Dat] $Z$ [Akk]) (vgl. Tomasello 2003). Die Tatsache, dass in vom Verb zugewiesenen Konstruktionen die Dativ-Markierung in der Nominalphrase in einer Reliktvarität wie dem Barossadeutschen gänzlich aufgegeben ist, während sie in von Präpositionen zugewiesenen Konstruktionen noch in Resten existiert, kann dadurch erklärt werden, dass verbale Schemata abstrakter sind als präpositionale Schemata und damit früher verloren gehen. Am besten erhalten werden Konstruktionen mit einer hohen Frequenz, die als kompakte Einheiten (sog. frozen units) separat gespeichert sind: Dies wären etwa Beispiele wie zur Schule, zur Kirche.

Diese für das Barossadeutsche dokumentierte Tendenz lässt sich nun auch für das Russlanddeutsche belegen, allerdings zeigt sich hier eine wesentlich höhere individuelle Variation zwischen den Sprechern. Diese Variation lässt sich zum einen damit erklären, dass die Sprecher ursprünglich aus unterschiedlichen Regionen kommen und unterschiedlichen Netzwerken angehören, zum anderen aber damit, dass die Sprache noch nicht zu einer Reliktvarietät reduziert ist. Rosenberg (2016) führt die hohe Variation auch auf die Norminstabilität zurück, die die Sprachinselvarietäten aufweisen. Wie die historischen Daten bzw. Daten der ältesten Sprechergeneration zeigen, ist die Va- 
riation bereits in deren Sprache vorhanden, so dass der Input, dem die nächste Generation ausgesetzt war, schon immer mehrere Varianten ein und desselben Schemas enthielt. So hörten die Sprecher im Spracherwerb sowohl Dativ- als auch Akkusativ-Markierung in ein und derselben Konstruktion wie $X$ liegt auf der Erde vs. $X$ liegt auf die Erde. Daher kann man annehmen, dass die konkurrierenden Konstruktionen, die weniger häufig auftraten, weniger stark im Gedächtnis verwurzelt wurden und damit $\mathrm{zu}$ einem späteren Zeitpunkt nicht mehr zugänglich waren.

Insgesamt wird deutlich, dass sich das Russlanddeutsche in einem noch weniger fortgeschrittenen Stadium der Entwicklung befindet (vgl. die Zahlen in der Präpositionalphrase, bei der Generation II mit der ältesten Generation der Barossadeutschen im historischen Korpus von 40 Jahren früher vergleichbar ist). Das ist sicher zum einen dadurch $\mathrm{zu}$ begründen, dass das Russlanddeutsche - wenn auch in sehr eingeschränktem Rahmen - noch aktiv als Kommunikationsmittel gebraucht wird (vgl. Riehl 2017). Eine weitere Erklärung ist der höhere Anteil an Sprechern, die nicht nur Deutsch lesen, sondern auch schreiben sowie auch Kontakte zu Deutschsprachigen in Deutschland pflegen (z. B. über die neuapostolische Kirche, s. o.). Auf die Bedeutung der Schriftlichkeit in der Kontaktvarietät habe ich in Riehl (2015, 2016) im Zusammenhang mit Entrenchment hingewiesen: Wenn der Sprecher einer Sprache lesen kann, hat er neben den lautlichen Mustern eines Wortes auch seine visuelle Repräsentation gespeichert. Das ist besonders dann von Bedeutung, wenn Wörter phonologisch sehr ähnlich sind, wie das gerade bei den verschiedenen Artikelformen der Fall ist, vgl. z. B. dem und den. Wenn die Sprecher nur einen gesprochenen Input erhalten, können sie die Unterschiede zwischen den und dem oder de (reduzierte Form von die) und der nicht immer wahrnehmen. In diesem Fall verstärkt das visuelle Bild des Wortes, das mit dem Erwerb der Schriftlichkeit einhergeht, die phonologische Repräsentation. Diese These wird dadurch bestätigt, dass die Dativ-Markierung am besten in Konstruktionen erhalten ist, in denen die Markierung phonologisch auffällig ist (wie z. B. in fusionierten Formen wie zur, zum, beim etc.).

Allerdings kann hier auch eine indirekte Auswirkung des Sprachkontakts angenommen werden: Geht man davon aus, dass es sich bei dem Kasusabbau nicht um ein Konvergenzphänomen, sondern um einen typologischen Wandel handelt, dann besteht die Möglichkeit, dass der Sprachkontakt diesen Prozess beschleunigt (Rosenberg 2016: 201). Im Kontakt mit einer nichtkasusmarkierenden Sprache wie dem Englischen könnte daher der Prozess schneller erfolgen als im Kontakt mit einer hochflektierenden Sprache wie dem Russischen.

Trotz großer Ähnlichkeiten in der Entwicklung, v. a. was die Regression von Entrenchmentprozessen angeht, gibt es doch auch Unterschiede zwischen 
dem Kasusabbau im Barossadeutschen einerseits und dem Russlanddeutschen andererseits. Das betrifft zum einen die Entwicklung des Pronominalsystems: Trotz individueller Variation ist die Differenzierung zwischen Dativ- und Akkusativformen im Pronominalsystem des Russlanddeutschen noch erhalten. Hier deutet sich auch in der 3. Pers. Pl. kein Ersatz des Dativpronomens ihnen durch das Akkusativpronomen an. ${ }^{9}$

Im Russlanddeutschen macht sich darüber hinaus ein weiteres Phänomen bemerkbar, das die Entwicklung der Kasusmarkierung maßgeblich beeinflusst, nämlich durch Sprachkontakt bedingtes Weglassen des Artikels analog zum Russischen: in einer Äußerung wie in Dorf ist aufgrund des Abbaus der Nominalflexion eine Kasusmarkierung nicht mehr zu leisten.

\section{Schlussbemerkung und Ausblick}

Wie der Vergleich der beiden Sprachinseln zeigt, folgen die Prozesse des Kasusabbaus ähnlichen Pfaden, befinden sich aber aufgrund unterschiedlicher soziolinguistischer Bedingungen (und eventuell typologischer Unterschiede der Kontaktsprachen, die sich indirekt auf die Geschwindigkeit des Prozesses auswirken) in einem unterschiedlichen Stadium des Vereinfachungsprozesses. In diesem Zusammenhang ist in beiden Sprachkontaktkonstellationen der graduelle Abbau der Kasusmarkierung zunächst in vom Verb zugewiesenen Nominalphrasen, dann in den Präpositionalphrasen zu verzeichnen. Den besten Erhalt der Dativmarkierung zeigen in beiden Konstellationen Konstruktionen, bei denen die Präposition mit dem Artikel verschmilzt.

Dennoch kann man anhand dieses Vergleichs zweier unterschiedlicher Konstellationen zeigen, dass die Entwicklung lediglich an der Oberfläche parallel verläuft. So ist im Russlanddeutschen das Pronominalsystem (trotz individueller Variation bei einigen Sprechern) noch weitgehend erhalten, während im Barossadeutschen die Reduktion auf ein Zwei-Kasus-System fast abgeschlossen ist. Ein weiterer Unterschied besteht in dem durch Sprachkontakt ausgelösten Wegfall des Artikels und damit einhergehender fehlender Möglichkeit zur Kasusmarkierung im Russlanddeutschen.

Grundlegend greift aber für beide Konstellationen der gebrauchsbasierte Ansatz: Abstrakte Konstruktionen (vom Verb zugewiesene Dative) werden vor

9 Diese Befunde stehen im Widerspruch zu den Ergebnissen der Studie von Rosenberg (2016). Allerdings umfasst sein Korpus zum Russlanddeutschen ostniederdeutsche Varietäten, während das vorliegende Korpus westmitteldeutsche und standardnahe Varietäten umfasst. 
konkreten (von Präpositionen zugewiesene Dative) abgebaut. Am stabilsten sind hier die sog. frozen units. Auch hier gibt es interessanterweise Unterschiede, die auf lexikalische Varianten zurückzuführen sind: Während im Barossadeutschen die häufigsten Vorkommnisse bei zur Kirche und zur Schule zu finden sind, werden diese Phrasen im Russlanddeutschen mit in die Kirche und in die Schule ausgedrückt, also mit einer Konstruktion, die den Akkusativ fordert. Die häufigste Konstruktion ist dort im Dorf.

Der hier vorgeführte Ansatz einer generationenübergreifenden Vergleichsstudie, die neben unterschiedlichen Wortarten vor allem auch die unterschiedlichen Kontexte und Konstruktionstypen berücksichtigt, erweist sich somit als fruchtbar für weitere Untersuchungen zur Funktionsweise von Sprachabbauprozessen in Sprachkontaktkonstellationen.

\section{Literatur}

Bentz, Christian \& Odo Winter (2013): Languages with More Second Language Learners Tend to Lose Nominal Case. Language Dynamics and Change 3, 1-27.

Berend, Nina (2011): Russlanddeutsches Dialektbuch: Zur Herkunft, Entstehung und Vielfalt einer ehemals blühenden Sprachlandschaft weit außerhalb des geschlossenen deutschen Sprachgebiets. Halle: Projekte-Verlag Cornelius.

Berend, Nina \& Claudia M. Riehl (2008): Russland. In Ludwig M. Eichinger, Albrecht Plewnia \& Claudia M. Riehl (Hrsg.), Handbuch der deutschen Sprachminderheiten in Mittel- und Osteuropa, 17-58. Tübingen: Narr.

Blankenhorn, Renate (2008): Die russlanddeutsche Minderheit in Sibirien. In Ludwig M. Eichinger, Albrecht Plewnia \& Claudia M. Riehl (Hrsg.), Handbuch der deutschen Sprachminderheiten in Mittel- und Osteuropa, 59-70. Tübingen: Narr.

Boas, Hans C. (2009): The life and death of Texas German (Publication of the American Dialect Society 93). Durham: Duke University Press.

Boas, Hans C. (2016): Variation im Texasdeutschen. Implikationen für eine vergleichende Sprachinselforschung. In Alexandra N. Lenz (Hrsg.), German Abroad. Perspektiven der Variationslinguistik, Sprachkontakt- und Mehrsprachigkeitsforschung, 11-44. Wien: Vienna University Press.

Born, Renate (2003): Regression, convergence, internal development: The loss of the dative case in German-American dialects. In William D. Keel \& Klaus Mattheier (Hrsg.), German Language Varieties Worldwide: Internal and External Perspectives, 151-164. Frankfurt am Main u. a.: Lang.

Clyne, Michael (1981): Deutsch als Muttersprache in Australien. Zur Ökologie einer Einwanderersprache. Wiesbaden: Franz Steiner.

Clyne, Michael (1991): Community Languages. The Australian Experience. Cambridge u. a.: Cambridge Univ. Press.

Eichinger, Ludwig M., Albrecht Plewnia \& Claudia M. Riehl (Hrsg.) (2008): Handbuch der deutschen Sprachminderheiten in Mittel- und Osteuropa. Tübingen: Narr.

Gilbert, Glenn (1972): Linguistic Atlas of Texas German. Austin: University of Texas Press. 
Huffines, Marion L. (1989): Case usage among the Pensylvania German sectarians and nonsectarians. In Nancy C. Dorian (Hrsg.), Investigating obsolescence: Studies in language contraction and death, 211-226. Cambridge u. a.: Cambridge Univ. Press.

Jakobson, Roman (1941): Kindersprache, Aphasie und allgemeine Lautgesetze. Uppsala: Almqvist \& Wiksell.

Louden, Mark L. (1994): Syntactic change in multilingual speech islands. In Nina Berend \& Klaus Mattheier (Hrsg.), Sprachinselforschung. Eine Gedenkschrift für Hugo Jedig, 73-91. Frankfurt am Main u. a.: Lang.

Maitz, Péter \& Attila Németh (2014): Language contact and morphosyntactic complexity: Evidence from German. Journal of Germanic Linguistics 26, 1-29.

Matras, Yaron (2009): Language contact. Cambridge u. a.: Cambridge Univ. Press.

Panzer, Baldur (1983): Formenneutralisationen in den Flexionssystemen deutscher Dialekte. In Werner Besch u. a. (Hrsg.), Dialektologie. Ein Handbuch zur deutschen und allgemeinen Dialektforschung. 2. Halbbd., 1170-1173, Berlin, New York: de Gruyter.

Paul, Peter (1962): Barossadeutsch: Sprachliche Untersuchungen des ostmitteldeutschen Dialektes des Barossatales (Südaustralien). B.A.-Thesis, University of Adelaide.

Paul, Peter (1965): Das Barossadeutsche. Ursprung, Kennzeichen und Zugehörigkeit. Untersuchungen in einer deutschen Sprachinsel. M.A.-Thesis, University of Adelaide.

Riehl, Claudia M. (2006): Sprachwechselprozesse in deutschen Sprachinseln Mittel- und Osteuropas. Varietätenkontakt und Varietätenwandel am Beispiel Transkarpatiens. In Nina Berend \& Elisabeth Knipf-Komlósi (Hrsg.), Sprachinselwelten. Entwicklung und Beschreibung der deutschen Sprachinseln am Anfang des 21. Jahrhunderts, 189-204. Frankfurt am Main u. a.: Lang.

Riehl, Claudia M. (2010): Norm and variation in language minority settings. In Alexandra N. Lenz \& Albrecht Plewnia (Hrsg.), Grammar between Norm and Variation. VarioLingua 40, 275-289. Frankfurt am Main u. a.: Lang.

Riehl, Claudia M. (2012): Deutsch als Reliktvarietät: Der Fall des Barossa-Deutschen (Australien). In Elisabeth Knipf-Komlósi \& Claudia M. Riehl (Hrsg.), Kontaktvarietäten des Deutschen in historischer und gegenwärtiger Sicht, 37-49. Wien: editio praesens.

Riehl, Claudia M. (2014): Sprachkontaktforschung. Eine Einführung. 3. überarb. Aufl., Tübingen: Narr.

Riehl, Claudia M. (2015): Language contact, language attrition, and the concept of relic variety: The case of Barossa-German. International Journal of the Sociology of Language 236, 1-33.

Riehl, Claudia M. (2016): Reliktsprache, Herkunftssprache, Minderheitensprache und neue Mehrsprachigkeit. In Alexandra N. Lenz (Hrsg.), German Abroad. Perspektiven der Variationslinguistik, Sprachkontakt- und Mehrsprachigkeitsforschung, 241-267. Wien: Vienna University Press.

Riehl, Claudia M. (2017): Russian-Germans. Historical background, language varieties, and language use. In Ludmila Isurin \& Claudia M. Riehl (Hrsg.), Integration, Identity, and Language Maintenance in Young Immigrants. Russian Germans or German Russians, 11-40. Amsterdam, Philadelphia: Benjamins.

Rosenberg, Peter (2003): Vergleichende Sprachinselforschung: Sprachwandel in deutschen Sprachinseln in Russland und Brasilien. In Theo Harden \& Elke Hentschel (Hrsg.), Particulae particularum. Festschrift zum 60. Geburtstag von Harald Weydt, 273-323. Tübingen: Stauffenburg.

Rosenberg, Peter (2005): Dialect convergence in the German language islands (Sprachinseln). In Peter Auer, Frans Hinskens \& Paul Kerswill (Hrsg.), Dialect Change: 
Convergence and Divergence in European Languages 221-235. Cambridge u. a.: Cambridge Univ. Press.

Rosenberg, Peter (2016): Regularität und Irregularität in der Kasusmorphologie deutscher Sprachinselvariatäten (Russland, Brasilien). In Andreas Bittner \& Klaus-Michael Köpcke (Hrsg.), Prozesse der Regularität und Irregularität in Phonologie und Morphologie, 177-218. Berlin, New York: de Gruyter.

Salmons, Joseph (1994): Naturalness and morphological change in Texas German. In Nina Berend \& Klaus Mattheier (Hrsg.), Sprachinselforschung. Eine Gedenkschrift für Hugo Jedig, 59-72. Frankfurt am Main u. a.: Lang.

Schmid, Monika (2002): First Language Attrition, Use and Maintenance: The Case of German Jews in Anglophone Countries. Amsterdam, Philadelphia: Benjamins.

Thomason, Sarah G. (2001): Language Contact. An Introduction. Washington: Georgetown University.

Tomasello, Michael (2003): Constructing a language. Cambridge: Harvard University Press.

Trudgill, Peter (2004): New-Dialect Formation: The Inevitability of Colonial Englishes. Cambridge: Harvard Univ. Press.

Weinhold, Karl (1853): Über deutsche Dialektforschung. Die Laut- und Wortbildung und die Formen der schlesischen Mundart. Mit Rücksicht auf verwantes in deutschen Dialecten. Ein Versuch. Wien: Verlag von Carl Gerold und Sohn.

Yager, Lisa, Nora Hellmold, Hyoun-A Joo \& Michael T. Putnam (2015): New Structural Patterns in Moribund Grammar: Case Marking in Heritage German. Frontiers in Psychology 6, 1-9.

Zürrer, Peter (1999): Sprachinseldialekte. Walserdeutsch im Aosta-Tal (Italien). Frankfurt am Main u. a.: Sauerländer. 


\title{
Peter Rosenberg
}

\section{1 Überflutete Sprachinseln: Sprachvariation, Sprachwechsel und Sprachwandel in deutschen Sprachinseln in Russland und Brasilien}

\begin{abstract}
Deutsche Sprachinseln sind heute nahezu überall auf der Welt „untergehende“ Inseln: Ihre Varietäten weisen vielfältige Merkmale des sprachlichen Abbaus und der Reduktion der morphologischen Strukturen auf. Die reguläre und irreguläre Morphologie zeigen jedoch sehr unterschiedliche Entwicklungsverläufe und -geschwindigkeiten: Während die reguläre Morphologie in der Nominalflexion eine rasche Entwicklung zu einem Zwei-Kasus-System (oder sogar zum Einheitskasus) nimmt, ist die irreguläre Morphologie der Personalpronomina stärker differenziert und bewahrt meist Dativformen. Wie lässt sich dies erklären? Welche Rolle spielt der Sprachkontakt dabei? Welche Einflussfaktoren lassen sich feststellen (etwa die Struktur der Sprachinselvarietäten, die Sprechergeneration, das ethnische Zugehörigkeitsempfinden, die nationale Sprachenpolitik)?
\end{abstract}

Keywords: Kasus, Morphologie, Sprachinseln, Sprachkontakt, Sprachwandel

Das Motiv der hier vorgestellten Untersuchung „überfluteter“ Sprachinseln ist die Annahme, dass aus sprachlichem Abbau ebenso viel zu lernen ist wie aus der Entstehung neuer sprachlicher Formen (durch Standardisierung, Koineisierung, Dialektausgleich), die in der Vergangenheit Gegenstand intensiver Forschung war: Der Morphologieabbau erscheint keineswegs chaotisch oder amorph, sondern als strukturiert und gerichtet: Bestimmte Wortarten sind eher der strukturellen Reduktion ausgesetzt als andere, die morphologische „Kernfunktionen“ bewahren.

Zunächst sollen einige Forschungsergebnisse vergleichender Sprachinselforschung vorgestellt werden (Abschnitt 1). Anschließend werden potenzielle Einflussfaktoren hinsichtlich ihrer Erklärungsstärke diskutiert (Abschnitt 2).

Peter Rosenberg, Europa-Universität Viadrina Frankfurt (Oder), Fakultät für Kulturwissenschaften, Große Scharrnstraße 59, D-15230 Frankfurt (Oder), E-Mail: rosenberg@europa-uni.de 


\section{Kasusabbau in deutschen Sprachinseln in Russland und Brasilien}

Gegenstand der hier vorgestellten vergleichenden Sprachinselforschung sind die Entwicklungsverläufe regulärer und irregulärer Morphologie in deutschen Sprachinseln in Russland und Brasilien bei Sprechern unterschiedlicher Ausgangsvarietäten und unterschiedlichen Alters in Sprechsituationen unterschiedlicher Formalität. Ziel ist die Beschreibung der Abbauprozesse der Kasusmorphologie ${ }^{1}$ und ihre Erklärung im Zusammenwirken von Konvergenz, Sprachwandel und Sprachwechsel.

Untersuchungsregionen sind „ethnoterritoriale Kerne“ (Eisfeld 1987: 170) der deutschsprachigen Besiedlung im Süden Brasiliens (Hunsrücker seit 1824, Pommern seit 1858) und im westsibirischen Russland (seit 1892 als russlanddeutsche Tochtersiedlungen in der Altai-Region, Hauptsiedlungsregion in Russland seit den Stalinschen Deportationen). In den Siedlungen bei Pelotas in Rio Grande do Sul dominieren Pommerischsprachige im Kontakt mit HunsrückischSprechern, in Schumanowka im (1991 wiedergegründeten) Deutschen Rayon Halbstadt bei Slawgorod „Plautdietsch“-Sprecher im Kontakt mit „Katholisch“Sprechern. Die Sprachkontaktsituation ist in gewissem Sinne ähnlich: Die Sprechergemeinschaften befinden sich nach Jahren der Repression und gegenwärtigen Liberalisierungen im Niedergang, der Sprachwechsel ist bei den jüngeren Generationen vorangeschritten. Auch die Dialektkontaktsituation ist vergleichbar: Es stehen Ostniederdeutsch-Sprecher (Pomerano, Plautdietsch) im Kontakt mit Westmitteldeutsch-(Hunsrückisch) bzw. WestoberdeutschSprechern („Katholisch“).

Die Sprechweise von 125 Sprechern dreier Generationen $(<40,40-60,>60)$ wurde mit variationslinguistischen Methoden in drei Untersuchungssettings unterschiedlicher Formalität (intendierter Dialekt, Interviewnarrationen, Selbstaufnahmen) in den frühen 1990ern und späten 2000ern erhoben, darunter 16 Sprecher in einer Real-Time-Studie über einen Zeitraum von ca. 15 Jahren. Die soziolinguistischen und Spracheinstellungsdaten wurden mittels eines zweisprachigen Fragebogens (mit 85 Fragen) erhoben.

Die Auswertung der korpuslinguistischen Daten geschieht computergestützt und teilautomatisiert, adaptiert nach dem STTS-Taggingsystem (vgl.

1 Kasusabbau soll hier von Kasussynkretismus unterschieden werden, der zunächst nur Formgleichheit bei Funktionsverschiedenheit beschreibt: „We identify case syncretism when a single inflected form corresponds to two or more case functions.“ (Baerman \& Brown 2013). 


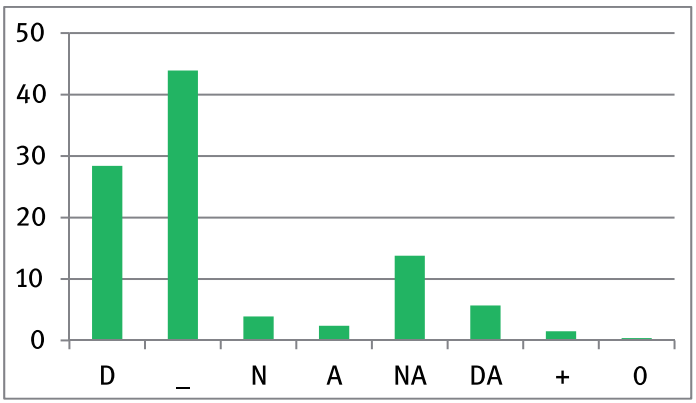

Abb. 11.1: Kasusrealisierung auf Dativ-Input (in \%, $n=6218$ ): Übertragung in intendierten Sprachinseldialekt. ${ }^{2}$

Schiller et al. 1999). Die morphologische Datenbank umfasst ca. 30.000 Typen. ${ }^{3}$ Die Transkription erfolgte mittels EXMARaLDA, die Auswertung mit Exakt und die soziolinguistische Korrelationsanalyse mit Coma. Aktuell liegen Daten zu den ersten beiden Untersuchungssettings vor.

Es zeigen sich in den Daten „selektive“ Abbauprozesse im Bereich der Kasusmorphologie, also der Nominal- und der Pronominalflexion (hier: Personalpronomina): „Während in der Nominalflexion ein weit fortgeschrittener Kasusabbau (in der Regel in Richtung eines Zwei-Kasus-Systems Nominativ $\neq$ Obliquenkasus - meist in der Form des Akkusativs - oder ein EinheitskasusSystem - häufig in der Form des Nominativs) vorherrscht, besteht in der Pronominalflexion oft noch ein Drei-Kasus-Systems (Nominativ $\neq$ Dativ $\neq$ Akkusativ) oder zumindest ein Erhalt von Dativformen im Rahmen eines Zwei-Kasus-Systems (Nominativ $\neq$ Obliquenkasus)“ (Rosenberg 2016: 185).

In der Flexion der Sprachinselvarietäten lässt sich eine deutliche Tendenz zum Kasusabbau im Obliquenkasus erkennen: Weniger als 30\% der Belege realisieren Dativ-Input mit Dativ-Output, Reduktionen der Kasusmorphologie kennzeichnen fast die Hälfte der Belege (Abb. 11.1).

2125 Probanden, 270 kasustragende Elemente, 28.536 types. D = Dativrealisierung, ${ }_{-}=$Reduktion (ohne Kasusendung, z. B. de), N = Nominativ, A = Akkusativ, NA = Nominativ-AkkusativForm (z. B. die), DA = Dativ-Akkusativ-Form (mi/di), + = zusätzliche Form, $0=$ keine Realisierung.

3 Der Umfang erklärt sich aus dem Umstand, dass die Varietäten größtenteils unbeschrieben sind, Mischvarietäten darstellen und die Sprecher sich über die Norm selbst oft nicht mehr im Klaren sind, weshalb weniges als sicher „ungrammatisch“ ausgeschlossen werden konnte. Die Darstellung der Kasusmorphologie verzeichnet Input und Output und ordnet den Output probabilistisch (nach Restriktionen, die aus Verbrektion, Kongruenzforderungen und semantischen Kontextbedingungen abgeleitet wurden). 


\subsection{Reguläre Flexion (Nominalflexion)}

Der Kasuszusammenfall ist in der Nominalflexion hochfrequent: Während er beim Definitartikel geringer ist ( $50 \%$ Dativ-Output), wird beim Adjektiv kaum Dativ markiert, der Output ist bei Dativ-Input und Akkusativ-Input nahezu gleich (Abb. 11.2 und 11.3):
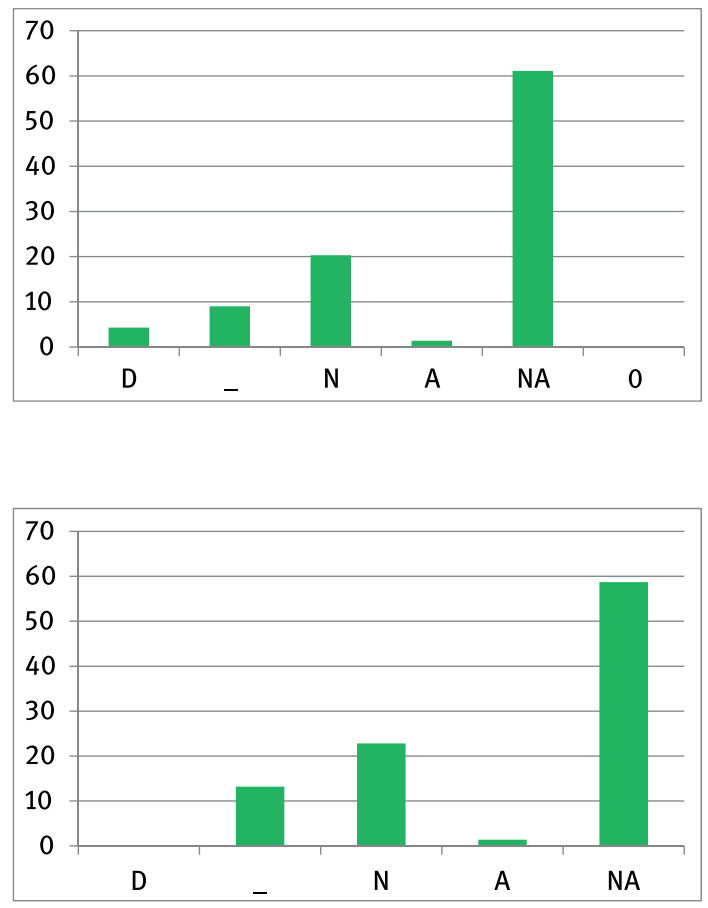

Abb. 11.2: Adjektivflexion: Dativ-Input und Kasusrealisierung (in \%, $n=699$ ).
Abb. 11.3: Adjektivflexion: Akkusativ-Input und Kasusrealisierung (in \%, $n=766$ ).

Im Output wird am häufigsten, soweit ein Objektkasus überhaupt markiert wird, -(e)n verwendet. -(e)n wird als Einheitsform des Objektkasus benutzt, selbst im Neutrum. ${ }^{4}$ Oft wird keinerlei Kasus realisiert: Reduktion sorgt - wie in Abbildung 11.1 deutlich wird - häufig für den Common Case auf das Ein-

\footnotetext{
4 Belege für -en im Neutrum aus den Übersetzungen in den intendierten Dialekt: den brööt ,das Brot', in den huus rinnegåån ,in das Haus hineingehen', den letste jåår ,das letzte Jahr', den schåå ,dem Schaf‘. Die Verwendung von -en kann durchaus als eine jüngere Entwicklung interpretiert werden, soweit Studien hierzu vorliegen. Jedig (1966: 52) berichtete vor 50 Jahren für den bestimmten Artikel im Plautdietsch der Altai-Region: „Der Akkusativ ist beim Maskulinum und Neutrum im allgemeinen vom Dativ verdrängt“. Die Akkusativform werde „fast ausschließlich von Vertretern der älteren Generation gebraucht, bei Vertretern der jüngeren Gene-
} 
heitsflexiv $-e([-ə])$ oder $-\varnothing . .^{5}$ Dies ließe sich markiertheitstheoretisch im Sinne Jakobsons (1936) erklären:

Die höhere Markiertheit des Dativs gegenüber Akkusativ und Nominativ und des Akkusativs gegenüber Nominativ - wird von Jakobson darin gesehen, dass der Dativ - wie der Akkusativ - als „Bezugskasus“ (gegenüber dem Nominativ) die „Unselbständigkeit des Gegenstandes“ anzeige, der Dativ jedoch als „Randkasus“ (gegenüber dem Akkusativ) eine „periphere Stellung“ insofern einnehme, als er den Gegenstand bzw. Teilnehmer einer Handlung oder eines Ereignisses fasse, der weitgehend unberührt von ihren Ergebnissen bleibe (Jakobson 1936: 282).

Die Markiertheitshierarchie enthält beim Aufbau und Abbau sprachlicher Kompetenz ${ }^{6}$ eine für Sprachinseln in Auflösung wichtige Komponente: Markiertheitsabbau als Abbau von Geltungsbeschränkungen läge durchaus im Erklärungsbereich sprachlicher Simplifizierungen. Unmarkiertheit ließe sich auch als Prototypikalität interpretieren: Markiertheitsabbau könnte als Rückführung auf einen Prototypen angesehen werden, Zwischenstufen des Abbaus ließen sich danach als Reduktion des Gebrauchs markierter Elemente auf übliche Verwendungskontexte interpretieren. ${ }^{7}$

ration dagegen findet sie sich fast gar nicht“ (ebd.: 71). 50 Jahre später stellen wir das Gegenteil fest: Es dominiert die Akkusativform (den/'n) bzw. de !

5 Belege aus den Übersetzungen in den intendierten Dialekt: $i k$ hebb löcher in mine nije strömp ,Ich habe Löcher in meinen neuen Strümpfen‘.

6 Waugh/Lafford (2000: 276) sprechen von einer ,association of marked terms with [...] later learning“. Auch Ludwig (2001: 402) nimmt an: „Das merkmallose Glied wird also in der Ontogenese früher erworben“. Die in „Kindersprache, Aphasie und allgemeine Lautgesetze“ von Jakobson (1969) postulierte umgekehrte Reihenfolge des Sprachverlusts ist zunächst nur auf Aphasiker bezogen, wäre für sprachlichen Abbau jedoch durchaus in Rechnung zu stellen.

7 Es ist möglicherweise kein Zufall, dass die Abbaustufen bei Sprechern (und Lernern) der im Rückgang befindlichen Sprachinselvarietät Ähnlichkeiten mit den elementaren Stufen unvollständigen Erwerbs zeigen, die Christine Dimroth für Lerner des Deutschen mit russischer Erstsprache feststellt (Dimroth 2008): Dimroth beobachtet über 1,5 Jahre den Deutscherwerb bei zwei russischen „Quereinsteigerinnen“ unterschiedlichen Alters (8;7 und 14;2 bei Ankunft): Große Unterschiede zeigen sich dort, wo die ältere Lernerin einen unvollständigen Erwerb aufweist, der folgende Bereiche betrifft: „(1) Substantivflexion: Auslassung von Determinierern, besonders Genus-, aber auch Kasusflexion von Pronomen und Determinierern, z. T. auch Kasusmarkierung am Substantiv, (2) Adjektivflexion, (3) Verbzweit, (4) Unregelmäßige Verbalflexion“ (Dimroth 2008: $129 \mathrm{f}$.). In der Adjektivflexion bleibt die ältere Lernerin beispielsweise stecken in einer „Phase, in der - $e$ zur Defaultmarkierung attributiver Adjektive wird und über $90 \%$ aller tokens abdeckt und die zugleich dem Endstand entspricht, weil danach keine Entwicklung mehr stattfindet.“ (Dimroth 2008: 128). Die jüngere Lernerin hingegen produziert am Ende des Beobachtungszeitraums „nur noch zielsprachlich flektierte Adjektive“ (Dimroth 2008: 129). 
In einem soziolinguistischen Sinne wird der unmarkierte Fall auch als der „unauffällige Regelfall“ (Ludwig 2001: 402) gesehen, dem gegenüber der markierte Fall als in bestimmten Kontexten auffällig, häufig als „salient“, gilt. Dabei wird mit dem gemeinsamen Auftreten von „frequency, regularity, normality“ (Waugh \& Lafford 2000: 279) argumentiert. Dies ist nicht unumstritten, da Markiertheit in dieser Bedeutung von manchen als „überdehnt“ angesehen wird. Zumindest handelt es sich um eine empirische Frage, ob Markiertheit mit Salienz korreliert. Ludwig (2001: 402f.) legt dies nahe, wenn er Markiertheit auf den „Ausgangspunkt“ einer leichteren Wahrnehmbarkeit zurückführt, woraus folge: „Das häufigere, einfacher strukturierte und variantenreichere Glied der Opposition ist also der merkmallose Teil“". Frequenz scheint jedoch eher die Folge denn die Ursache von Unmarkiertheit zu sein.

Phonetische Prozesse dürften für die hohe Frequenz des oben als (Objektkasus-)Einheitsflexiv genannten silbischen - $n$ mitverantwortlich sein: $-n$ ist silbisch verwendbar, gut „hörbar“, besitzt insofern Salienzpotenzial, ist von extensiver Funktionalität, es ist als Dentalnasal „natürlich“ kombinierbar mit Dentalflexiven und sonstigen hochfrequenten Dentalendungen (hast, ist, kann; mein, dein, sein; in, an), es lässt sich „längen“ und markiert insofern auch im (gemäßigten) Allegrostil noch (sein-n,), und es dient auch bei weitgehender Reduktion (ähnlich wie auch -ə) der Herstellung des Trochäus bei Einsilblern (vgl. Eisenberg 2004, auch Wegener 1990, 1991). Kurz: Es ist auch phonetisch ein „Vorzugskandidat“ für eine Default-Funktion.

Die Flexive -en (für den Objektkasus) und - $e$ (bei Einheitskasus) sind mit der schwachen Adjektivflexion im Deutschen identisch (Tab. 11.1) und werden stark wie schwach verwendet. Dies lässt sich als Abbau bezeichnen:

Tab. 11.1: Schwache Adjektivdeklination im Deutschen (nach Helbig \& Buscha 2001: 274).

\begin{tabular}{|c|c|c|c|}
\hline Numerus & Kasus & Genus & Flexiv \\
\hline Singular & Nominativ & alle Genera & \\
\hline Singular & Akkusativ & Fem./Neutr. & $-e$ \\
\hline alle anderen & & & $-(e) n$ \\
\hline
\end{tabular}

\subsection{Irreguläre Flexion (Personalpronomina)}

Personalpronomina verhalten sich anders: Während in der Nominalflexion weniger als ein Drittel der Belege auf Dativ-Input Dativ-Output ergeben, zeigt die Flexion der Personalpronomina in der Kasusrealisierung ein völlig anderes Bild: 

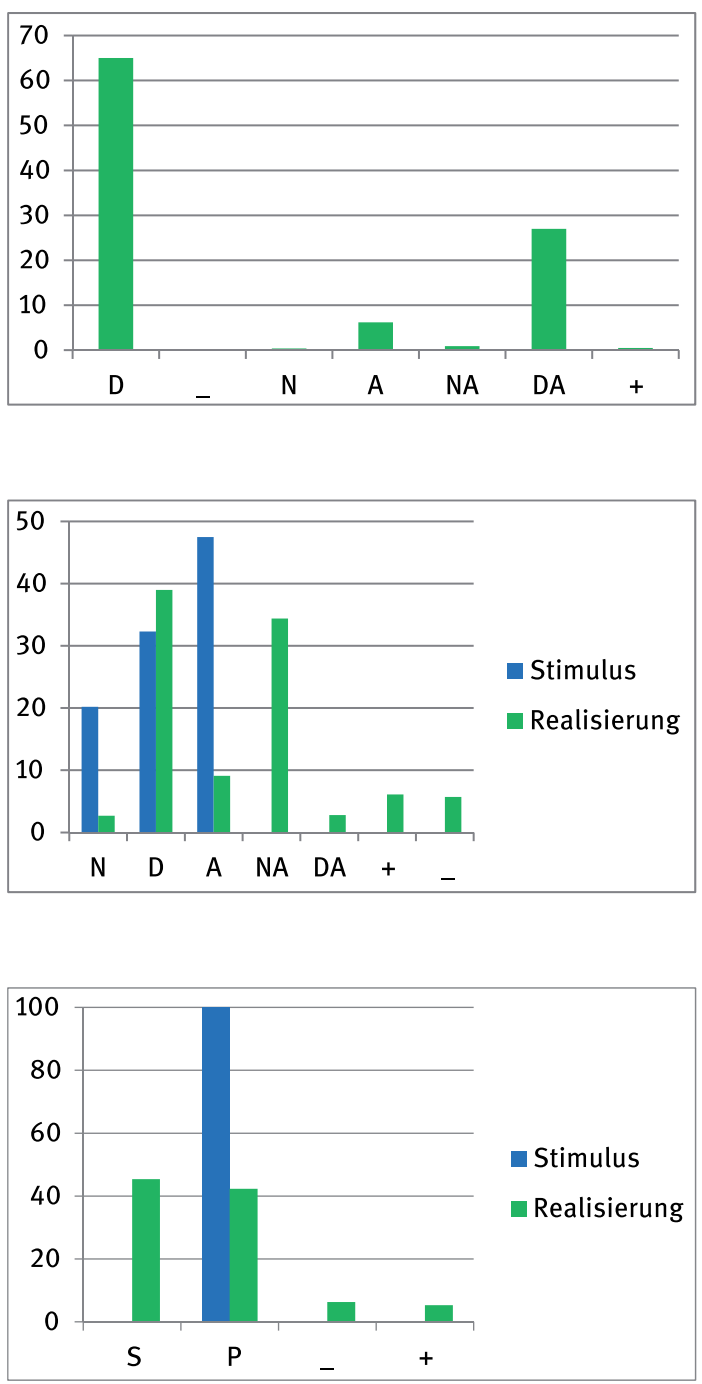

Abb. 11.4: Flexion der Personalpronomina: Kasusrealisierung auf Dativ-Input (in \%, $n=986$ ).

Abb. 11.5: Flexion der Personalpronomina 3. Person Singular: alle Kasus (in \%, $n=1.762$ ).

Abb. 11.6: Flexion der Personalpronomina 3. Person Plural: Numerusrealisierung (in \%, $n=572$ ).

Zwei Drittel realisieren Dativ als Dativ, ein gutes Viertel verwendet die niederdeutsche Objektkasus-Einheitsform mi ,mir/mich', di ,dir/dich' (Abb. 11.4).

Dabei ist es besonders die 3. Person Singular, die maximal kasusdifferenziert wird. Hier übersteigt der Dativ-Output sogar den Dativ-Input (Abb. 11.5), es wird also Dativ als Hauptform für den Objektkasus gewählt.

Der Plural zeigt ein uneinheitlicheres Bild. Er reiht sich in das Gesamtbild der Simplifizierung der Flexionsmorphologie insofern ein, als der Plural durch den Singular ersetzt wird (Abb. 11.6). 
Personalpronomina sind die letzte „Bastion“ der Kasusdistinktion. Dies gilt bekanntermaßen auch in anderen indoeuropäischen Sprachen, die sonst (nahezu) keinerlei Kasusmorphologie mehr aufweisen:

- engl. him/her (aengl. Dativ: him/hire)

- dän. ham/henne (askand. Dativ honum/henni)

- frz. lui (afrz. li, vulgärlat. li, lat. Dativ illi)

Gelegentlich ist bereits darauf hingewiesen worden, die differenziertere Kasusmarkierung in Personalpronomina, besonders die Objektkasusmarkierung in der 3. Person, folge einer bestimmten „Logik“: Rabanus (2008: 274) nennt „Belebtheit“ und „Handlungspotenzial“, die für die stärkere Differenziertheit der 3. Person Singular Maskulinum verantwortlich seien: „Prototypischerweise ist das Agens im Deutschen ein belebtes Maskulinum.“ (Rabanus 2008: 274). Ein Abweichen hiervon mache eine Markierung notwendig: „Wird nun ein typischer Agens- und damit Nominativ-Kandidat als Patiens bzw. vom Verbereignis Betroffener dargestellt, so wird gegen eine Präsupposition verstoßen und dies muss signalisiert werden.“ (Bittner 2002: 216). ${ }^{8}$

Was macht Personalpronomina also resistenter gegen Morphologieabbau?

- Die mit Personalpronomina verbundenen belebten Referenten könnten eine morphologische Unterscheidung syntaktischer Rollen stärker erfordern.

- Ihre hohe Frequenz könnte sie resistenter gegen sprachlichen Wandel machen.

- Sie sind geschlossene und kleinere Klassen, die auf andere Weise lexikalisch gespeichert werden dürften als offene Klassen.

- Personalpronomina sind oft Suppletivformen, die individuell und als ganze Einheiten lexikalisch gespeichert werden. Der Verlust von Kasusmarkie-

8 Angesichts der Sonderrolle, die die 3. Person Singular spielt, ist die Frage interessant, ob es sich bei ihr im eigentlichen Sinne um eine „Person“ handelt: Im WALS wird das Deutsche als eine Sprache klassifiziert, in der die Personalpronomina der 3. Person Singular - bzgl. der Gender-Markierung - mit Demonstrativpronomina verwandt sind: „The question as to whether third person pronouns are related or unrelated to demonstratives appears to form the basis for a typological distinction between two distinct sets of languages. We might call languages in which third person pronouns are related to demonstratives two-person languages, and those in which the two are unrelated three-person languages. Third person pronouns form part of the system of personal pronouns in the latter case but not in the former. This distinction appears to be correlatable with certain other characteristics such as, for example, that

1. gender distinctions among third person pronouns are displayed more frequently by twoperson languages than by three-person languages, and

2. the deictic system is less frequently person-oriented in two-person languages than in threeperson languages.“ (Bhat 2013). 
rungen durch Ersetzung ganzer lexikalischer Einträge (z. B. ihr $\rightarrow$ sie) wäre daher bei Personalpronomina „disruptiver“ (Salmons 1994) als bei Nomina. Full Listing, die Lexikalisierung als ganze Einheit (vgl. Cholewa 1993), wird im Allgemeinen für monomorphematische Wörter und polymorphematische Wörter mit irregulärer Wortbildung bzw. mit weniger produktiven morphologischen Mustern angenommen. Dies könnte auf Personalpronomina zutreffen.

Weshalb wird aber gerade der Dativ markiert?

In den untersuchten Sprachinselvarietäten scheint uns eine kasussemantische Funktion $\mathrm{zu}$ unterliegen: Personalpronomina stehen prototypisch für menschliche „Redegegenstände“. Dative scheinen in den erhaltenen Funktionen auf einen Kernbereich des „Empfängerkasus“ (Rezipient, Benefaktiv/Malefaktiv, Goal) reduziert zu sein: Die in unseren Daten als Dativ realisierten Personalpronomina (ihm/ihr) stehen überwiegend für eine Rezipientenrolle (Gebt sie [die Tasche] ihr zurück!), in geringerer Frequenz besitzen sie eine lokativische (Vor ihm lag ein Hund.) und komitative Bedeutung (Mit ihm fahren wir nicht.). Bezeichnenderweise ist die Dativ-Realisierung auf Dativ-Input zum Ausdruck der Rezipientenrolle gegenüber anderen Kasus am höchsten (85\%), bei lokativischer Funktion sinkt sie auf $64 \%$ (in Komitativfunktion beträgt sie ebenfalls $83 \%$ bei allerdings geringer Belegzahl (Abb. 11.7):

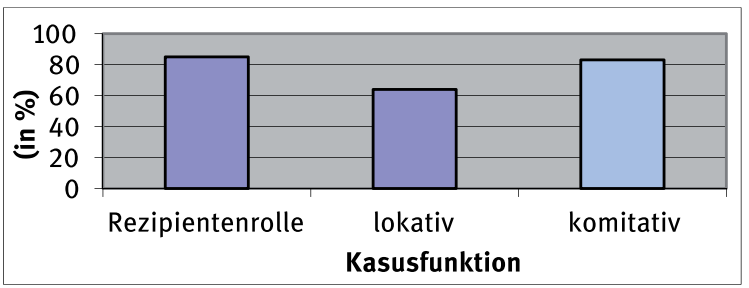

Abb. 11.7: Im Dativ realisierte Personalpronomina 3. Person Singular: Kasussemantik $(n=163)$.

Auch Personalpronomina sind allerdings nicht ausgenommen vom Abbau grammatischer Differenzierung. Sie werden in der Realisierung oft ersetzt durch Demonstrativpronomina, Indefinitpronomina (Abb. 11.8: P_S) und andere Mittel der regulären Flexionsmorphologie.

Bei „Regularisierung“, dem Ersatz von irregulär flektierenden Personalpronomina durch regulär flektierende Formen, folgt die morphologische Markierung dem Muster der Nominalflexion: Es wird bei Dativ-Input -en (z. B. $-e n, d e n)$ oder $-e(-e, d e)$ verwendet und ein Zwei-Kasus- oder EinheitskasusSystem hergestellt. 


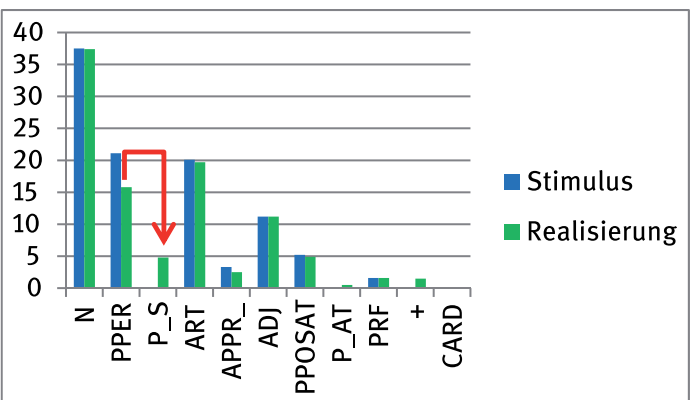

Abb. 11.8: Dativ-Input: Wortartrealisierung (in \%, $n=6218)^{9}$

Fassen wir also die Befunde zur (ir)regulären Morphologie in den untersuchten Sprachinseln zusammen:

1. In der Nominalflexion ist eine Vereinfachung festzustellen, die eine Gegenüberstellung von $-\mathrm{e} \neq-(\mathrm{e}) \mathrm{n}$ bewirkt. Akkusativformen finden sich häufig als Defaults (-en, den, 'n etc.), auch im Neutrum.

2. In der irregulären Morphologie ist der Kasuszusammenfall geringer als in der regulären. Dativformen sind in der Flexion der Personalpronomina hochfrequent. Markiertheitsabbau findet sich in der Nominalflexion, in wesentlich geringerem Maße in der Flexion der Personalpronomina.

3. Resistenz der Kasusmorphologie zeigt sich besonders in den Personalpronomina der 1.-3. Person Singular, die häufig mit belebten Referenten korrelieren. Besonders häufig sind Dativformen in der 3. Person Singular. Hier werden Distinktionen nach kasussemantischen Kernfunktionen (Rezipientendativ) erhalten.

4. Angesichts eines Wortartwechsels von Personalpronomina zu Demonstrativ- und Indefinitpronomina deutet sich eine Abbausequenz an, die zunächst eine Regularisierung und anschließend Morphologieabbau und Substitution, z. B. durch Wortstellung, aufweist.

Nun sind Kasusabbau in der regulären Flexion - im Unterschied zur irregulären Flexion - keine spezifischen Entwicklungen, die nur in Sprachinseln anzutreffen wären. Viele Sprachen des flektierenden Typs weisen eine Entwicklung vom synthetischen („fusionierenden“, Wurzel 1996) zum analytischen Sprachbau auf. Es drängt sich im Gegenteil der Eindruck auf, dass in den

$9 \mathrm{~N}$ = Nomen, PPER: Personalpronomen, P_S = substituierendes Demonstrativ- oder Indefinitpronomen, $\mathrm{ART}=$ Artikel, $\mathrm{APPR}_{-}=$Präposition mit Artikel (Kontraktion), ADJ $=$Adjektiv, P_AT $=$ attributives Demonstrativ- oder Indefinitpronomen, PRF = Reflexivpronomen, $+=$ zusätzliche Form, CARD = Zahlwort (adaptiert nach STTS: vgl. Schiller et al. 1999). 
Sprachinseln - zwar beschleunigt - aber nichtsdestoweniger ein „normaler“, „innerer“ Sprachwandel stattfindet. Weshalb tritt dies aber unter Beschleunigung auf in einer Sprachkontaktsituation, die gekennzeichnet ist durch verbreiteten Sprachwechsel und die Zunahme von Sprechern (der jüngeren Generationen), die allenfalls als Fremdsprachenlerner zu bezeichnen wären?

\section{Mögliche Erklärungen für den Kasusabbau}

Ist die sprachliche Entwicklung in den untersuchten Sprachinseln kontaktinduziert? Handelt es sich unter Umständen um sprachliche Konvergenz?

Konvergenz wäre zu unterscheiden in Konvergenz (besser Advergenz) als Übernahme von Merkmalen der Kontaktsprache und als Übernahme von Merkmalen von Kontaktvarietäten innerhalb der Sprachinseln.

Beides erscheint wenig wahrscheinlich, da die genannten Phänomene in Sprachinseln mit morphologisch reichen wie morphologisch armen Kontaktsprachen sowie in allen Sprachinselvarietäten, in „konservativen“ wie „adaptiven“, in Varietäten mit stärker oder weniger stark differenzierender Morphologie auftreten. Jedoch zeigen die Sprachinseln einige Unterschiede, die im Einzelnen geprüft werden müssen:

\subsection{Einfluss des Sprachkontakts}

Vergleichende Sprachinselforschung bietet das Potenzial, kontaktinduzierte Unterschiede zu identifizieren:

Die Sprachkontaktsituation erweist sich für die russlanddeutschen Sprachinseln als lange Zeit stabiler als für die brasilianischen, aber seit der Aussiedlungsbewegung hat der Sprachwechsel eine extreme Beschleunigung erfahren. Dies zeigt etwa der Vergleich der intergenerationellen Verwendung der Kontaktsprache in der Familiendomäne: Während in den russlanddeutschen Sprachinseln mit den Eltern die Kontaktsprache kaum vorkommt (Abb. 11.9: graue Säulen „gar nicht“), wird sie zum Regelfall mit den ältesten Kindern (Abb. 11.10: rote Säulen „nur“).

Welche Wirkung hat die morphologische Struktur der Kontaktsprachen?

Das Russische besitzt sechs Kasus, die mit wenigen Ausnahmen, in der Nominal- wie in der Pronominalflexion markiert werden - auch unter typischen Reduktionsbedingungen: in mündlicher Rede, im kolloquialen, informellen Stil, unter Allegro-Bedingungen. In den Personalpronomina des Russischen werden alle Kasus unterschieden (Ausnahme: Genitiv und Akkusativ sind im Singular 


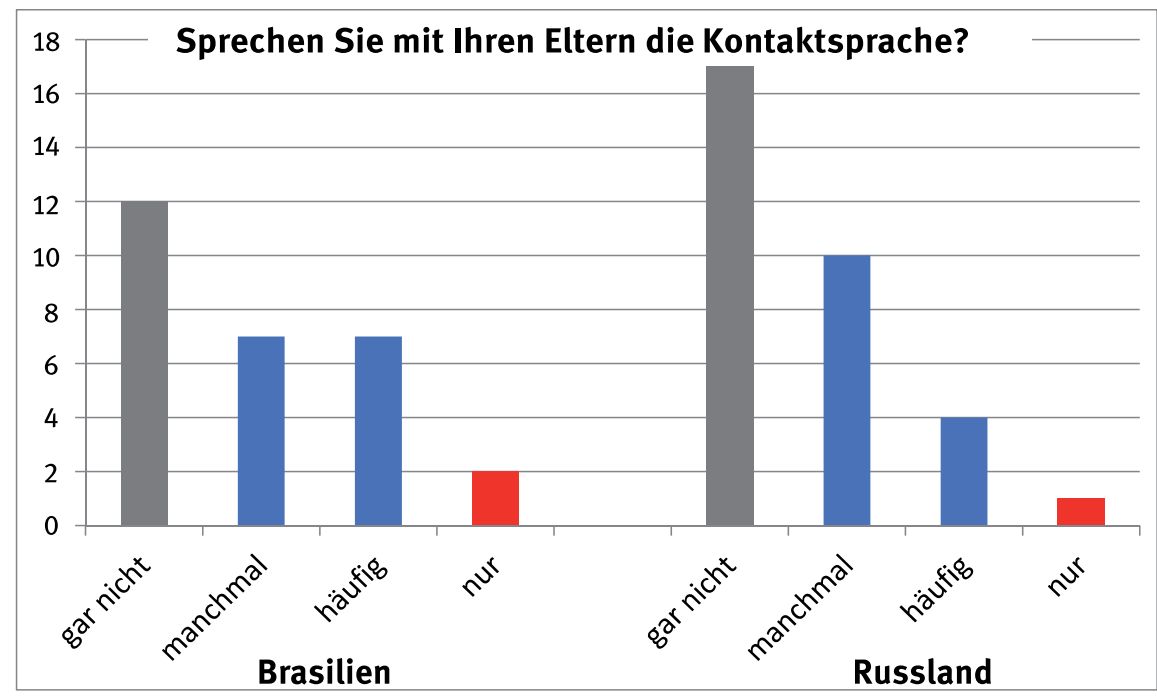

Abb. 11.9: Verwendung der Kontaktsprache mit den Eltern (Anzahl, $n=60$, nur Aufnahmen in 2000ern).

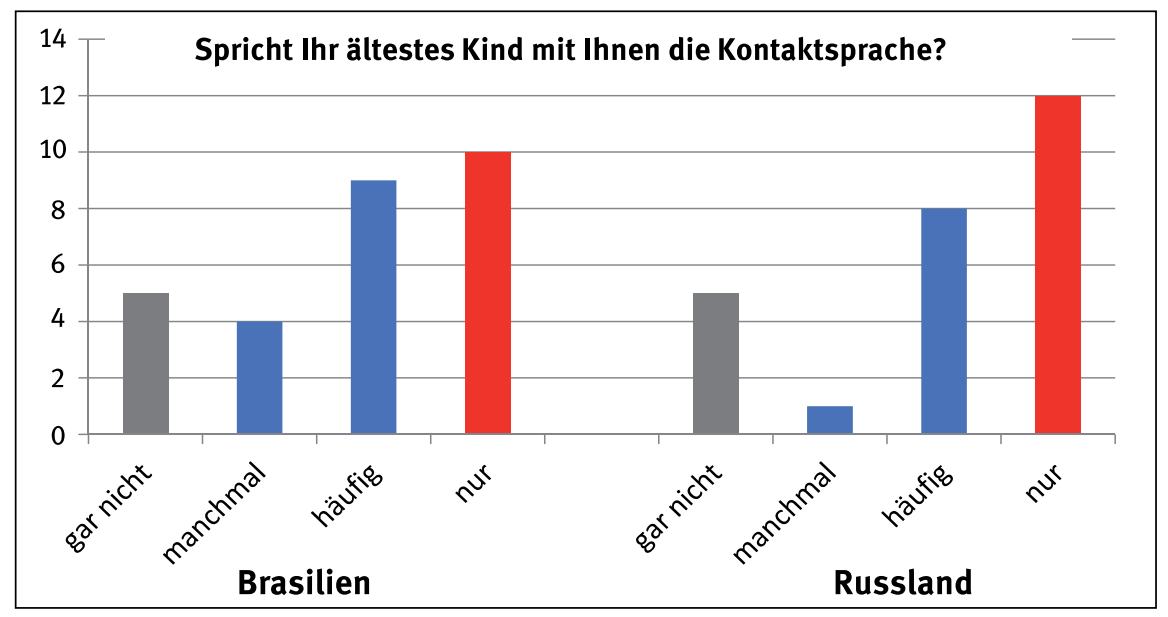

Abb. 11.10: Verwendung der Kontaktsprache durch ältestes Kind (Anzahl, $n=60$, nur Aufnahmen in 2000ern).

formgleich; Dativ und Präpositiv sind formgleich in der 1. und 2. Person Singular und in der 3. Person Singular Femininum; im Plural sind Genitiv, Akkusativ und Präpositiv formgleich). Der Singular zeigt eine stärkere Differenzierung als der Plural, die 3. Person Singular Maskulinum weist die stärkste Differenzierung auf. 
Das brasilianische Portugiesisch kennt Kasus - soweit wir von der Ausdrucksebene sprechen - nur in den Personalpronomina: Während in der 1. und 2. Person (Singular und Plural) nur ein Obliquenkasus markiert wird, werden Dativ und Akkusativ nur in der 3. Person unterschieden: 1. Person Singular: me - mir/mich; 2. Person Singular: te - dir/dich; 3. Person Singular Dativ: lhe ihm, ihr, Ihnen (Singular); 3. Person Singular Akkusativ Maskulinum: (l)o - ihn, es, Sie (Singular); 3. Person Singular Femininum: (l)a - sie, es, Sie (Singular); 1. Person Plural: nos - uns, 2. Person Plural: vos - euch, (Ihnen); 3. Person Plural Maskulinum: (l)os - sie (Plural), Sie (Plural); 3. Person Plural Femininum: (l)as - sie (Plural), Sie (Plural).

Das Konzept Kasus ist damit zweifellos vorhanden. Wir bemerken darüber hinaus, dass die 3. Person eine stärkere morphologische Kasusdifferenzierung aufweist als die 1. und 2. Person in Singular und Plural, so dass nicht nur Dativ und Akkusativ unterschieden werden, sondern im Akkusativ auch die Genera. Akkusativ wird damit stärker differenziert als Dativ. Der Singular zeigt - wie üblich - eine stärkere Differenzierung als der Plural.

Wie oben gezeigt, treten Phänomene des Kasusschwunds in den russlanddeutschen Sprachinseln ebenso auf wie in den brasiliendeutschen Sprachinseln. Auch die soziolinguistischen Daten, die das unterschiedliche Eindringen der Kontaktsprache in die familiäre Domäne zeigen, ergeben keine Unterschiede in der Kasusverwendung. ${ }^{10}$ Ein Transfer der Kasusstruktur aus der Kontaktsprache scheidet also als Erklärung aus, wenn man Erscheinungen eines völligen Zusammenbruchs des Kasussystems bei Angehörigen der jüngsten Generation einmal ausnimmt, die als Zweitsprachlerner (teils sogar eher als Fremdsprachlerner) des Deutschen zu gelten haben.

Eine relevante Einflussgröße ist aber der soziolinguistische Befund einer kontaktinduzierten Schwächung der „Normativität“ in den Sprachinseln, die „innerem“ Sprachwandel mit starker Beschleunigung Raum gibt. Die Struktur dieses Wandels - weitgehende Auflösung der Nominalflexion, Erhalt von Kasusdifferenzierungen im Bereich der belebten Personalpronomina, besonders der 3. Person Singular - scheint am ehesten auf kognitive Erklärungen hinzudeuten.

Dennoch wäre es verfehlt, einen kontaktinduzierten Wandel im strukturellen Sinne generell auszuschließen: Die beobachtete Tendenz einer stärkeren Kasusdifferenzierung in der 3. Person wird ebenso durch die Kontaktsprachen

10 Interessanterweise unterscheiden sich die Altersgruppen wenig in der Kasusrealisierung bei den Personalpronomina. Größere Unterschiede ergeben sich, legt man ein Sprachgebrauchskriterium zugrunde: die intergenerationale Verwendung der Sprachinselvarietät innerhalb der Familie (z. B. im Gespräch mit den Großeltern). Diejenigen, die die Sprachinselvarietät verwenden, realisieren am meisten Dativformen (proportional zur Verwendungshäufigkeit). 
unterstützt. Und auch die „Jakobsonsche“ Reihenfolge im Markiertheitsgefälle zwischen Akkusativ und Dativ wird durch die (russische) Kontaktsprache gestützt. $^{11}$

Unterschiede zwischen den Sprachinseln in Russland und Brasilien sind gleichwohl vorhanden. In den Sprachinseln in Russland wird selbst bei der oben festgestellten „Regularisierung“ durch Wortartwechsel Dativ häufiger erhalten, während in Brasilien der Morphologieabbau häufiger - wie in der Nominalflexion üblich - erfolgt (Abb. 11.11):

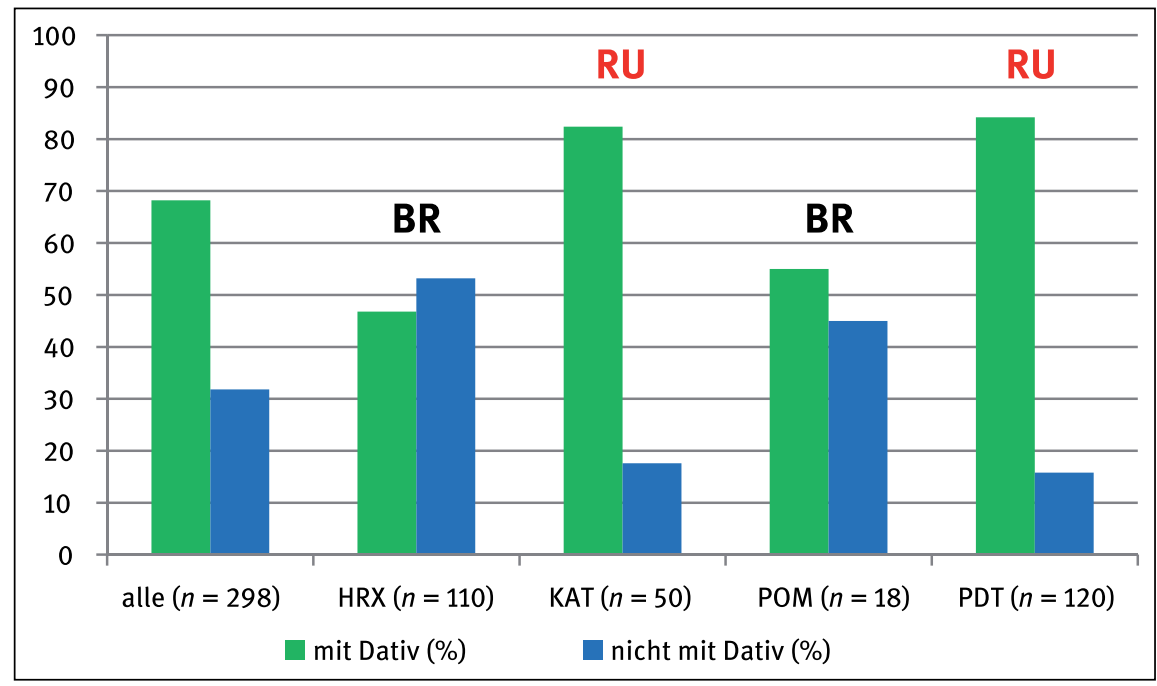

Abb. 11.11: Personalpronomen (mit Dativ-Input), realisiert als Demonstrativ- oder Indefinitpronomen: Kasusverwendung (\%, $n=298$; HRX = Hunsrückisch, KAT = „Katholisch“, POM = Pomerano, PDT $=$ Plautdietsch).

11 Jakobson (1936) sieht - ursprünglich für das Russische - den Nominativ im Rahmen einer „Bezugskorrelation“ als unmarkiert, den Akkusativ ebenso wie den Dativ als markiert und darüber hinaus im Rahmen einer Vollkasus/Randkasus-Korrelation den Akkusativ als unmarkiert und den Dativ als markiert. Der Dativ trägt insoweit eine doppelte Markierung, Akkusativ eine einfache und der Nominativ keine. Nach Jakobson (1936: 282) gilt aber: „Je mehr Korrelationsmerkmale der Kasus in sich trägt, desto vielfältiger wird die Geltung des bezeichneten Gegenstandes in der Aussage beschränkt und herabgedrückt“. Bei sprachlichen Vereinfachungen - etwa im Zuge des durch „Kontaktdruck“ ausgelösten Sprachwandels - werden solche Beschränkungen als erste beseitigt. Daher ist die Reihenfolge der durch Kasusschwund beseitigten Kasusmarkierungen: Genitiv (wenn er je vorhanden war) schwindet als erster, Dativ folgt als zweiter, Akkusativ als dritter - dann existiert nur noch Common Case, das heißt kein (morphologischer) Kasus. 
Hier zeigt sich also möglicherweise eine stärkere Kasusdistinktion, die der morphologischen Differenziertheit der (russischen) Kontaktsprache entspricht.

\subsection{Einfluss des Varietätenkontakts}

Die grammatische Struktur der Sprachinselvarietäten spielt selbstverständlich eine wichtige Rolle. Zudem sind die Sprachgemeinschaften unterschiedlich „adaptiv“: Die Plautdietsch sprechenden Gemeinschaften der Russlandmennoniten sind traditionell stärker als tatsächliche „Gruppen“ (vgl. Brubaker 2007) im Sinne kommunikativer Netzwerke mit eigenen soziokulturellen Normen und eigenständigem Zugehörigkeitsempfinden (,belonging“, Pfaff-Czarnecka 2013) zu sehen. Ihre Sprachinselvarietäten markieren im Sinne des „boundary marking“ (Barth 1969) Differenz auf verschiedenen (soziokulturellen, ökonomischen, religiösen) Ebenen. ${ }^{12}$ In Russland sind in dem untersuchten „Zentraldorf“ die Plautdietsch-Sprecher (zur Zeit der Erhebungen) die Mehrheit und die Autochthonen. Dies gilt auch für die Pomerano-Sprecher in der Pelotas-Region. Allerdings besitzt in Brasilien das Hunsrückische die Funktion einer Koiné (vgl. Altenhofen 1996).

Der Vergleich der Varietäten zeigt durchaus unterschiedlich starken Morphologieabbau. Die Dativrealisierung (D) etwa beim Definitartikel (Abb. 11.12) ist bei den hochdeutschen Varietäten (Katholisch und Hunsrückisch) am höchsten, während die Reduktionsform de (_) bei den ostniederdeutschen Varietäten von gleich hoher oder höherer Frequenz ist. Bei den Personalpronomina (Abb. 11.13) ist die Dativrealisierung ebenfalls bei den hochdeutschen Varietäten etwas höher als bei den niederdeutschen. Die Niederdeutsch-Sprecher, besonders die des Plautdietschen, verwenden - ihrer Ausgangsvarietät entsprechend - mit hoher Frequenz die Objektkasus-Einheitsform mi, di (DA). Hunsrückisch-Sprecher präferieren gelegentlich den Akkusativ im Obliquenkasus des Personalpronomens der 1. und 2. Person Singular mich, dich (A).

\section{Schlussfolgerungen}

Trotz gewisser Unterschiede zwischen den Sprachinselvarietäten in Russland und Brasilien und zwischen den niederdeutschen und hochdeutschen Sprach-

12 Ludwig Eichinger (2003b: 84) spricht diesbezüglich von „Distanzkontrolle“: Sprache markiere sozialsymbolisch die Grenzziehung, weshalb es nicht verwundere, „die sprachliche Konstruktion ideologischer Differenz im harten Kern des Phänomens Sprachinsel zu finden und nicht einfach den sprachlichen Unterschied“. 


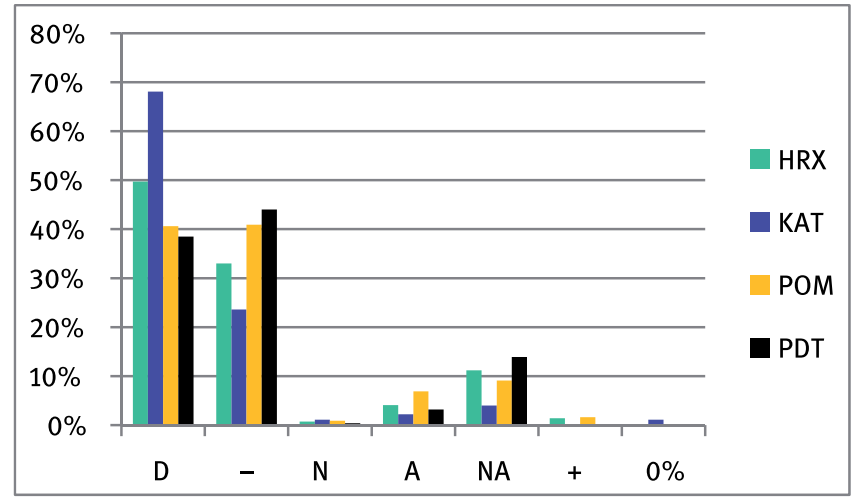

Abb. 11.12: Definitartikel mit Dativ-Input: Kasusrealisierung nach Varietäten $(n=1142)$.

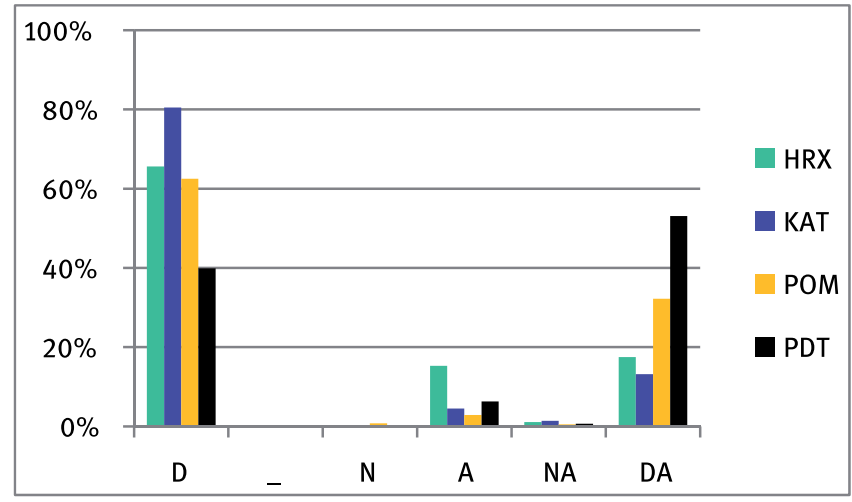

Abb. 11.13: Personalpronomen mit Dativ-Input: Kasusrealisierung nach Varietäten $(n=986)$.

inselvarietäten sind die generellen Tendenzen von einer Uniformität, die Konvergenz als entscheidendes Moment des Kasusabbaus unwahrscheinlich erscheinen lässt. Plausibler scheint eine Erklärung, nach der eine kontaktinduzierte „Erschütterung“ zu einem gerichteten morphologischen Wandel führt, der Vereinfachungen der Kasusmorphologie bewirkt. Diese treten jedoch partiell auch in den binnendeutschen Varietäten auf, entsprechen also der generellen Richtung des Sprachwandels im Deutschen.

Allerdings erfährt das Varietätengefüge in den Sprachinseln eine deutliche Destabilisierung, die sich in beschleunigtem Wandel und weitergehenden Abbauprozessen zeigt. Grammatische Funktionen werden teils durch Wortfolgebeziehungen, teils in Form-Funktions-Mustern nach dem „1:1-Principle“ (vgl. Andersen 1989: 386) realisiert, markierte (Kasus-)Strukturen werden zugunsten 
unmarkierter oder weniger markierter (vgl. Campbell/Muntzel 1989: 189) bzw. zugunsten „natürlicherer“ (vgl. Mayerthaler et al. 1998: 167) Strukturen abgebaut. In der Flexion der Personalpronomina gilt dies nicht oder nur bedingt: Hier sind dem Abbau nach Markiertheit verschiedene Schranken gesetzt. Soweit sich Flexion als resistent erweist, scheint dies einen (kasus-) semantischen Nukleus zu repräsentieren.

Die untersuchten Sprachinselvarietäten sind Varietäten „in obsolescence“ (Dorian 1989). Ihre Sprachgemeinschaften stehen in intensivem Sprachkontakt und sind durch einen Umbau der (immer weniger lokalen) Gruppennetzwerke und die Zunahme von Zweit- und Fremdsprachlernern gegenüber Muttersprachlern gekennzeichnet. Sprache markiert keine in der Alltagspraxis wurzelnde sozialsymbolische Grenzziehung mehr (vgl. Barth 1994: 13; Rosenberg 2015: 161). Die entscheidende soziolinguistische Bedingung scheint uns insofern eine Auflösung der „Normativität“ (Normwissen und Normloyalität), die mit großer Beschleunigung inneren Sprachwandel entlang kognitiver Strukturen freisetzt.

Die von Ludwig Eichinger (2003b: 84) erwähnte „Distanzkontrolle“ setzt voraus: „,in Sprachinseln muss [...] ein so hoher Kohärenzdruck herrschen, dass er die funktional-kommunikativen Nachteile in einer Kosten-Nutzen-Rechnung überwiegt“. Die Aufrechterhaltung der Distanz verdankt sich entweder der „Geographie“ oder einer „Gemeinschaftsideologie [...]. Beide Extreme sind durch die Modernisierung der Welt gefährdet“ (Eichinger 2003b: $84 \mathrm{f}$.). Die infrastrukturelle, administrative, politische, sozioökonomische und soziokulturelle Modernisierung und Vereinheitlichung in Brasilien wie in Russland/UdSSR haben beide „Distanzen“ aufzulösen begonnen: Das meint die Metapher der „überfluteten“ Sprachinsel.

Auch in „untergehenden“ Sprachinseln ist der Morphologieabbau keineswegs amorph, sondern verrät Struktur und bietet uns Erkenntnisse über Kernfunktionen von Sprache im intensiven Sprachkontakt. Und solche Erkenntnisse machen nicht zuletzt „Nutzen und Vergnügen des Vergleichens von Sprachinseln“ aus (Eichinger 2003b: 83).

\section{Literatur}

Altenhofen, Cléo Vilson (1996): Hunsrückisch in Rio Grande do Sul. Ein Beitrag zur Beschreibung einer deutschbrasilianischen Dialektvarietät im Kontakt mit dem Portugiesischen. (= Mainzer Studien zur Sprach- und Volksforschung 21). Stuttgart: Steiner.

Andersen, Roger W. (1989): The „up“ and „down“ staircase in secondary language development. In Nancy Dorian (Hrsg.), Investigating obsolescence. Studies in language contraction and death, 385-394. Cambridge u. a.: Cambridge University Press. 
Baerman, Matthew \& Dunstan Brown (2013): Case syncretism. In Matthew S. Dryer \& Martin Haspelmath (Hrsg.), The world atlas of language structures online. Leipzig: Max Planck Institute for Evolutionary Anthropology. (http://wals.info/chapter/28, letzter Zugriff: 5. 12. 2017).

Barth, Fredrik (1969): Introduction. In Fredrik Barth (1969): Ethnic groups and boundaries. The social organization of culture difference, 9-38. Bergen, Oslo: Universitets Forlaget; London: Allen and Unwin.

Barth, Fredrik (1994): Enduring and emerging issues in the analysis of ethnicity. In: Cora Govers \& Hans Vermeulen (Hrsg.), The anthropology of ethnicity. Beyond „Ethnic Groups and Boundaries“, 11-32. Amsterdam: Het Spinhuis.

Bhat, Darbhe N. S. (2013): Third person pronouns and demonstratives. In Matthew S. Dryer \& Martin Haspelmath (Hrsg.), The world atlas of language structures online. Leipzig: Max Planck Institute for Evolutionary Anthropology. (ttp://wals.info/chapter/43, letzter Zugriff 5.12. 2017)

Bittner, Dagmar (2002): Semantisches in der pronominalen Flexion des Deutschen. Reguläre Irregularitäten: Zur Suppletion im Konzept der natürlichen Morphologie. Zeitschrift für Sprachwissenschaft 21 (2), 196-233.

Brubaker, Rogers (2007): Ethnizität ohne Gruppen. Aus dem Englischen von Gabriele Gockel und Sonja Schuhmacher, Kollektiv Druckreif. (Engl.: Ethnicity without groups. Cambridge, Mass., London 2004: Harvard University Press.) Hamburg: Hamburger Edition HIS.

Campbell, Lyle \& Martha C. Muntzel (1989): The structural consequences of language death. In Nancy Dorian (Hrsg.), Investigating obsolescence. Studies in language contraction and death, 181-196. Cambridge u. a.: Cambridge University Press.

Cholewa, Jürgen (1993): Störungen der lexikalisch-morphologischen Wortverarbeitung bei Aphasie: Ein Literaturüberblick. In: Neurolinguistik 7 (2), 105-126.

Dimroth, Christine (2008): Kleine Unterschiede in den Lernvoraussetzungen beim ungesteuerten Zweitspracherwerb: Welche Bereiche der Zielsprache Deutsch sind besonders betroffen? In Bernt Ahrenholz (Hrsg.), Kinder mit Migrationshintergrund. Spracherwerb und Fördermöglichkeiten, 117-133. Freiburg: Fillibach.

Dorian, Nancy (1989): Introduction. In Nancy Dorian (Hrsg.), Investigating obsolescence. Studies in language contraction and death, 1-10. Cambridge u. a.: Cambridge University Press.

Eichinger, Ludwig M. (1992): Das Deutsche als Minderheitensprache. Eine soziolinguistische Skizze zur Lage des Deutschen in einer Zeit politischen Wandels. Der Deutschunterricht $92(6), 56-69$.

Eichinger, Ludwig M. (1997): Deutsch in weiter Ferne. Die Verbreitung der deutschen Sprache außerhalb des zusammenhängenden deutschen Sprachgebiets: Deutsche Minderheiten. In Gerhard Stickel (Hrsg.), Varietäten des Deutschen. Regional- und Umgangssprachen. Jahrbuch 1996 des Instituts für deutsche Sprache. (= Jahrbuch des Instituts für deutsche Sprache 1996), 155-182. Berlin/New York: de Gruyter.

Eichinger, Ludwig M. (2003a): Der unexotische Blick auf das Fremde: Soziolinguistische Überlegungen zu Datenerhebung und -interpretation. In Klaus J. Mattheier \& William D. Keel (Hrsg.), German language varieties worldwide. Internal and external perspectives. Deutsche Sprachinseln weltweit. Interne und externe Perspektiven, 33-52. Frankfurt am Main u. a.: Peter Lang.

Eichinger, Ludwig M. (2003b): Island Hopping: Vom Nutzen und Vergnügen des Vergleichens von Sprachinseln. In Jannis Androutsopoulos \& Evelyn Ziegler (Hrsg.), „Standardfragen“. 
Soziolinguistische Perspektiven auf Sprachgeschichte, Sprachkontakt und Sprachvariation. (= VarioLingua 18), 83-107. Frankfurt am Main u. a.: Peter Lang.

Eichinger, Ludwig M. (2010): Das Deutsche außerhalb des zusammenhängenden deutschen Sprachraums. In Hans-Jürgen Krumm, Christian Fandrych, Britta Hufeisen \& Claudia Riemer (Hrsg.), Deutsch als Fremd- und Zweitsprache. Ein internationales Handbuch. 1. Halbband. (= Handbücher zur Sprach- und Kommunikationswissenschaft 35/1), 398-405. Berlin, Boston: de Gruyter.

Eichinger, Ludwig M., Albrecht Plewnia \& Claudia Maria Riehl (Hrsg.) (2008): Handbuch der deutschen Sprachminderheiten in Mittel- und Osteuropa. Tübingen: Narr.

Eisenberg, Peter (2004): Grundriss der deutschen Grammatik, Band I: Das Wort. 2. Aufl. Stuttgart: Metzler.

Eisfeld, Alfred (1987): Bleiben die Sowjetuniondeutschen deutsch? In Andreas Kappeler, Boris Meissner \& Gerd Simon (Hrsg.), Die Deutschen im Russischen Reich und im Sowjetstaat. (= Nationalitäten und Regionalprobleme in Osteuropa. 1), 167-177. Köln: Böhlau.

Helbig, Gerhard \& Joachim Buscha (2001): Deutsche Grammatik: Ein Handbuch für den Ausländerunterricht. Berlin usw.: Langenscheidt.

Hinderling, Robert \& Ludwig M. Eichinger (Hrsg.) (1996): Handbuch der mitteleuropäischen Sprachminderheiten. Tübingen: Narr.

Jakobson, Roman (1936): Beitrag zur allgemeinen Kasuslehre. Gesamtbedeutungen der russischen Kasus. In Travaux du Cercle Linguistique de Prague 6: Études Dédiées au Quatrième Congrès de Linguistes, Prague 1936, 240-288. Praha: Jednota Československých Matematiku a Fysiku; Leipzig: Harassowitz.

Jakobson, Roman (1969): Kindersprache, Aphasie und allgemeine Lautgesetze. Frankfurt (Main): Suhrkamp.

Jedig, Hugo (1966): Laut- und Formenbestand der niederdeutschen Mundart des AltaiGebietes. (= Sitzungsberichte der Sächsischen Akademie der Wissenschaften, philologisch-historische Klasse 112, H. 5). Berlin: Akademie-Verlag.

Ludwig, Ralph (2001): Markiertheit. In Martin Haspelmath, Ekkehard König, Wulf Oesterreicher \& Wolfgang Raible (Hrsg.), Language typology and language universals. An international handbook, 400-419. Vol. 1. Berlin, New York: de Gruyter.

Mayerthaler, Willi, Günther Fliedl \& Christian Winkler (1998): Lexikon der Natürlichkeitsheoretischen Syntax und Morphosyntax. Tübingen: Stauffenburg.

Pfaff-Czarnecka, Joanna (2013): Multiple belonging and the challenges to biographic navigation. (= Max-Planck-Institut zur Erforschung multireligiöser und multiethnischer Gesellschaften, Working Paper 13-05). Göttingen: mpimmg.

Rabanus, Stefan (2008): Morphologisches Minimum. Distinktionen und Synkretismen im Minimalsatz hochdeutscher Dialekte. Stuttgart: Steiner. (= Zeitschrift für Dialektologie und Linguistik. Beihefte. 134).

Rosenberg, Peter (2015): Anything goes? The gains and losses of the constructivist view on ethnicity: Some considerations based on German 'language islands' studies. In Peter Rosenberg, Konstanze Jungbluth \& Dagna Zinkhahn Rhobodes (eds.): Linguistic construction of ethnic borders, 149-166. Frankfurt am Main: Lang.

Rosenberg, Peter (2016): Regularität und Irregularität in der Kasusmorphologie deutscher Sprachinselvarietäten (Russland, Brasilien). In Klaus-Michael Köpcke \& Andreas Bittner (Hrsg.), Regularität und Irregularität in Phonologie und Morphologie. Diachron, kontrastiv, typologisch. (= Lingua Historica Germanica 13), 177-217. Berlin: de Gruyter. 
Salmons, Joseph (1994): Naturalness and morphological change in Texas German. In Nina Berend \& Klaus J. Mattheier (Hrsg.), Sprachinselforschung. Eine Gedenkschrift für Hugo Jedig, 59-72. Frankfurt am Main: Peter Lang.

Schiller, Anne, Simone Teufel, Christine Thielen \& Christine Stöckert (1999): Guidelines für das Tagging deutscher Textkorpora mit STTS (Kleines und großes Tagset). Stuttgart, Tübingen: Institut für Maschinelle Sprachverarbeitung, Universität Stuttgart (Seminar für Sprachwissenschaft), Universität Tübingen.

Waugh, Linda R. \& Barbara A. Lafford (2000): Markedness. In Morphologie. Morphology. Ein internationales Handbuch zur Flexion und Wortbildung. An international handbook on inflection and word-formation. Herausgegeben von/Edited by Geert Booij, Christian Lehmann, Joachim Mugdan, Stavros Skopeteas in collaboration with Wolfgang Kesselheim. 1. Halbband/Volume 1. (= Handbücher zur Sprach- und Kommunikationswissenschaft 17), 272-281. Berlin, New York: de Gruyter.

Wegener, Heide (1990): Komplemente in der Dependenzgrammatik und in der Rektions- und Bindungstheorie. Die Verwendung der Kasus im Deutschen. Zeitschrift für germanistische Linguistik 18 (2), 150-184.

Wegener, Heide (1991): Der Dativ - ein struktureller Kasus? In Gisbert Fanselow \& Sascha W. Felix (Hrsg.), Strukturen und Merkmale syntaktischer Kategorien. (= Studien zur deutschen Grammatik 39), 70-103. Tübingen: Narr.

Wurzel, Wolfgang U. (1996): Morphologischer Strukturwandel: Typologische Entwicklungen im Deutschen. In Ewald Lang and Gisela Zifonun (Hrsg.), Deutsch - typologisch. (= Institut für deutsche Sprache. Jahrbuch 1995), 492-524. Berlin, New York: de Gruyter. 
Hans C. Boas und Katrin Fuchs

\title{
12 Zum Einfluss des Standarddeutschen auf das Texasdeutsche im 19. und 20. Jahrhundert: Empirische und methodologische Probleme
}

\begin{abstract}
Der Beitrag beschäftigt sich mit dem Einfluss des Standarddeutschen auf die Entwicklung des Texasdeutschen. Aus soziolinguistischer Perspektive soll gezeigt werden, inwiefern Standarddeutsch im Leben der deutschstämmigen Texaner eine Rolle spielte und ob eine Beeinflussung des Texasdeutschen durch das Standarddeutsche festgestellt werden kann. Darüber hinaus will dieser Beitrag auf empirische und methodologische Probleme aufmerksam machen, die bei der Untersuchung dieses Einflusses auftreten können.
\end{abstract}

Keywords: Sprachkontakt, Standarddeutsch, Texasdeutsch

\section{Einführung}

Dieser Beitrag analysiert das Zusammenwirken von standard- und nichtstandarddeutschen Merkmalen und Sprachkompetenzen in der Konfiguration, Verbreitung und Entwicklung des Texasdeutschen. Ziel unseres Beitrags ist es festzustellen, inwieweit deutschstämmige Texaner im 19. und 20. Jahrhundert Kenntnisse des Standarddeutschen besaßen und inwieweit diese Kenntnisse möglicherweise die Entstehung und Entwicklung des Texasdeutschen beeinflusst haben könnten (siehe Salmons \& Lucht 2006; Boas 2009 und 2016). Unser Ziel ist es zu zeigen, welche empirischen und methodologischen Probleme auftreten, wenn der Status und Einfluss des Standarddeutschen in Texas untersucht werden soll, und wie diese Probleme gelöst werden können.

Anmerkung: Für hilfreiche Kommentare bedanken wir uns bei Stephan Elspaß, Matthias Fingerhuth, Marc Pierce und den Herausgebern.

Hans C. Boas, Department of Germanic Studies, 1 University Station, C3300,

2505 University Ave., The University of Texas at Austin, Austin, TX 78712, U.S.A.,

E-Mail: hcb@mail.utexas.edu

Katrin Fuchs, Department of Foreign Languages and Literatures, 1400 Chapel Drive, Valparaiso University, Valparaiso, IN 46383, U.S.A., E-Mail: katrin.fuchs@valpo.edu

○ Open Access. (๑) 2018 Hans C. Boas und Katrin Fuchs, publiziert von De Gruyter. (c) BY Dieses Werk ist lizenziert unter der Creative Commons Attribution 4.0 Lizenz.

https://doi.org/10.1515/9783110538625-013 
Der Beitrag ist wie folgt gegliedert: Abschnitt 2 gibt einen kurzen Überblick über die Beeinflussung von Nichtstandardvarietäten durch Standardvarietäten. In diesem Zusammenhang soll gezeigt werden, dass es nicht immer möglich ist, eine klare Kausalität in der Sprachentwicklung von Substandardvarietäten unter dem Druck von Standardvarietäten zu etablieren. Abschnitt 3 präsentiert einen kurzen geschichtlichen Überblick über die Entwicklung des Texasdeutschen. Abschnitt 4 diskutiert die Rolle des Standarddeutschen in Texas von der Mitte des 19. bis zur Mitte des 20. Jahrhunderts. Hier sollen besonders die möglichen Ursachen der sprachlichen Variation im Texasdeutschen durchleuchtet werden, insbesondere das Problem der Abgrenzung zwischen Schriftsprache und gesprochener Sprache. Anhand von Daten des Texas German Dialect Projects (TGDP), welche in den Jahren 2001-2017 erhoben wurden, werden zum einen Makrotendenzen der Variation innerhalb des Texasdeutschen in Bezug auf dessen Dialektalität und Standardnähe identifiziert und zum anderen das Vorhandensein einer Standardkompetenz im Standarddeutschen bis zum Dachsprachenwechsel zugunsten des Englischen besprochen. Abschließend (Abschnitt 5) werden einige Anmerkungen zur Datenerhebung und allgemeinen Methodologie gemacht.

\section{Beeinflussung von Nichtstandardvarietäten durch Standardvarietäten}

Wenn man von einer Beeinflussung der Nichtstandardvaritetäten des Deutschen durch die Standardvarietät spricht, muss festgehalten werden, dass die Standardvarietät genuin eine schriftliche Varietät ist, während Nichtstandardvarietäten grundsätzlich eher der Mündlichkeit angehören (Koch \& Oesterreicher 1985: 16). ${ }^{1}$ Auch noch in den Varietäten des 21. Jahrhunderts wird zwischen konzeptioneller Mündlichkeit und Schriftlichkeit unterschieden. Dieser Unterschied drückt sich sowohl in der Mediums- und Registerwahl als auch in der Dichotomie zwischen Nähe und Distanz bzw. Dialekt und Standard aus (Koch \& Oesterreicher 1985: 19). Dementsprechend stellt sich zunächst die Frage, wie viel Einfluss Schriftlichkeit und Mündlichkeit wechselweise aufeinander haben. Zur Verschriftlichung von Mündlichkeit fasst Mihm (2016: $274 \mathrm{ff}$.) die unterschiedlichen Theorien wie folgt zusammen: (1) Die Defizienztheorie, die histori-

1 Die Mündlichkeit der Nichtstandardvarietäten gilt insbesondere seit der Durchsetzung der Standardvarietät in der Schriftlichkeit. Historische Schriftdialekte, die im Frühneuhochdeutschen eigene Standardisierungstendenzen aufwiesen, werden durch interne Standardisierungstendenzen bereits im 17. und 18. Jahrhundert angeglichen (z. B. Möller 2000: 54 f.). 
sche Schreibdialekte als unvollkommene und regellose Vorstufen der modernen Standardvarietät charakterisiert; (2) die Autonomietheorie, die der Schriftsprache eine frühe Fossilisierung und dadurch einen Verlust des Kontaktes zur gesprochenen Sprache attestiert; (3) die Korrespondenztheorie, die geschriebene Sprache als 1:1-Darstellung der historischen gesprochenen Sprache sieht; und (4) die Pioniertheorie, die zunächst von einem Ausgleich der Schreibsprachen ausgeht, der sich dann auf die gesprochene Sprache übertragen hat.

Während Defizienztheorie, Pioniertheorie ${ }^{2}$ und Korrespondenztheorie aus verschiedenen Gründen verworfen wurden, ist die Autonomietheorie die derzeitige Mehrheitsmeinung (Mihm 2016: 274). Nach dieser Theorie kann sich die Schriftsprache komplett unabhängig von der gesprochenen Sprache entwickelt haben und muss diese auch nicht beeinflusst haben. So stellt auch Kyes (1985: 439) hierzu fest: „Orthography neither causes nor prevents sound change, it simply ignores it. The tendency is for the writing system to become increasingly constant, rather than to share in the dynamic fluxion of the spoken language“. Allerdings steht auch diese Theorie im Widerspruch $\mathrm{zu}$ empirisch belegbarer allographischer Variation (Mihm 2016: 274), die sich bis zur Zweiten Orthographischen Konferenz im Jahre 1901 und darüber hinaus gehalten hat.

Dementsprechend orientieren sich einige Untersuchungen an dem Interdependenzstandpunkt von gesprochener und geschriebener Sprache, wie zum Beispiel die Frühneuhochdeutsche Grammatik von Ebert et al. (1993: 13). In der historischen Linguistik hat dies oft methodologische Gründe, da sich eine phonologische Untersuchung grundsätzlich an schriftlichem Material orientieren muss. Allerdings gibt es auch sachliche Begründungen für diese Theorie. Ebert et al. (1993: 14) sagen hierzu:

Es gibt einerseits umfängliche Teile des Schreibsystems, die lautliche Verhältnisse teils im 1:1-Verhältnis spiegeln; und es gibt andererseits umfängliche Teile des Lautsystems, die nur über jahrhundertelange Konstanten des Schreibsystems erklärbar sind. Selbstverständlich schließt der Interdependenzstandpunkt die Existenz schreibunabhängiger Lautungsbereiche und lautungsunabhängige Schreibprinzipien nicht aus.

Dementsprechend kann festgehalten werden, dass es durchaus möglich ist, dass sich eine dialektale oder regiolektale gesprochene Sprache an der geschriebenen Standardsprache orientiert. Ob dies allerdings auch für das Texasdeutsche der Fall ist, wie von Salmons \& Lucht (2006) behauptet, bleibt fraglich. Ausgehend von diesen theoretischen Aspekten wendet sich der nächste Abschnitt der Geschichte des Texasdeutschen ${ }^{3} \mathrm{zu}$.

2 Die Pioniertheorie wurde von Müllenhoff (1863) und Burdach (1884) und später auch noch von Moser (1951) vertreten, gilt allerdings mittlerweile als widerlegt.

3 Dieser Abschnitt beruht auf Boas (2009, 2016). 


\section{Geschichte des Texasdeutschen}

\subsection{Texasdeutsch vor dem Ersten Weltkrieg}

Die Geschichte des Texasdeutschen hat ihren Anfang in den 1820er Jahren, als sich die ersten deutschsprachigen Einwanderer in Texas niederließen, welches damals noch ein Teil von Mexiko war. Nachdem Johann Friedrich Ernst mit seiner Familie 1829 in das südliche Zentraltexas gezogen war, schrieb er 1832 einen Brief an seine Verwandten in Oldenburg, in dem er in den höchsten Tönen über Texas sprach. Dieser Brief wurde nicht nur in der Lokalzeitung veröffentlicht, sondern er wurde auch mit großem Interesse in ganz Deutschland gelesen, was wiederum zu einer großen Begeisterung für Texas führte und immer mehr Deutsche dazu veranlasste, nach Texas auszuwandern. Nachdem Texas 1836 von Mexiko unabhängig wurde, benötigte die junge Republik Einwanderer, um die Infrastruktur aufzubauen, die Landwirtschaft weiter auszubauen, und um Texas gegen Mexiko, welches die Unabhängigkeit nicht anerkannt hatte, verteidigen zu können (Biesele 1930; Kearney 2010). Dies führte in den 1830er und 1840er Jahren zur Einwanderung mehrerer zehntausend zentraleuropäischer Einwanderer, die sich vor allem in Zentraltexas ansiedelten. Eine der größten organisierten Einwanderungswellen wurde durch den sog. Mainzer Adelsverein organisiert, der zwischen 1844 und 1847 mehr als 7.000 deutschen Auswanderern die Reise nach Texas organisierte und unter anderem die Städte New Braunfels und Fredericksburg im sogenannten Hill Country von Zentraltexas gründete.

Der größte Teil der Auswanderer kam aus Nassau, den südlichen Bereichen des Königreichs Hannover, Braunschweig, Nordhessen, Ostthüringen, Oldenburg, Holstein und Westfalen (Gilbert 1978). Einige Einwanderer ließen sich in bereits existierenden Städten wie Galveston, Houston oder San Antonio nieder, die Mehrzahl der Einwanderer entschied sich jedoch dazu, sich in den vom Mainzer Adelsverein neu gegründeten Siedlungen im Texas Hill Country niederzulassen. Während der 1850er Jahre verdoppelte sich die deutschstämmige Bevölkerung in Texas auf mehr als 20.000, und in dem als German belt bekannten Gebiet in Zentraltexas gab es zahlreiche Orte mit deutschsprachigen Zeitungen, Schulen und Kirchen. Der deutschsprachige Bevölkerungsanteil war im texanischen Hill Country sowie in Teilen von Austin County und Colorado County mit 90 bis 99 Prozent am größten, in anderen Gebieten gab es Orte, die zu 10 bis 50 Prozent deutschsprachig waren (neben Englisch, Tschechisch und Spanisch). Der Einfluss der deutschen Einwanderer war so stark, dass die texanische Regierung sogar Gesetzestexte auf Deutsch veröffentlichte. 
Während des amerikanischen Bürgerkriegs kam die deutsche Einwanderung größtenteils zum Erliegen.

Nach dem Ende des amerikanischen Bürgerkriegs 1865 nahm die deutsche Einwanderung wieder drastisch $\mathrm{zu}$, so dass die deutschsprachige Bevölkerung um 1890 mehr als 40.000 betrug. Die meisten Nachkriegseinwanderer siedelten nicht im Texas Hill Country, im Westen von Zentraltexas, sondern hauptsächlich im östlichen Teil von Zentraltexas zwischen Austin, San Antonio, Houston und Dallas. Immer mehr deutschsprachige Texaner zogen auch in die Städte, so dass z. B. um 1880 ein Drittel der Einwohner San Antonios deutschsprachig war (Jordan 1977; Roesch 2012). Viele der deutschsprachigen Gemeinden in Zentraltexas waren bis zum Ende des Ersten Weltkriegs in ihren deutschsprachigen Institutionen engagiert. Neben deutschsprachigen Zeitungen, Schulen und Kirchen gab es in vielen Gemeinden von Zentraltexas deutsche Schützenvereine, Gesangsvereine und Turnvereine, die häufig auch Texaner ohne deutschen Migrationshintergrund aufnahmen, was wiederum zur Integration unterschiedlicher Volksgruppen beitrug (Nicolini 2004). Im Jahr 1917 gab es noch 145 Kirchengemeinden, die Gottesdienste in deutscher Sprache abhielten (Arndt \& Olson 1961: 615; Salmons \& Lucht 2006: 168), und deutschsprachige Schulen sowie eine große Zahl von Gesangs-, Schützen- und Sportvereinen trugen mit ihrer institutionellen Unterstützung dazu bei, dass die deutsche Sprache bis ins 20. Jahrhundert hinein gedieh (Boas 2016). Schätzungen zufolge gab es vor dem Ersten Weltkrieg mehr als 100.000 Deutschsprecher in Texas (Eichhoff 1986).

Der Status des Deutschen in Texas änderte sich drastisch im ersten Viertel des 20. Jahrhunderts. Als 1917 die USA in den Ersten Weltkrieg gegen Deutschland eintraten, wurden im ganzen Land Gesetze erlassen, die das Englische zur alleinigen Unterrichtssprache erklärten. Andere Sprachen durften nicht mehr in der Schule verwendet werden. Dies führte dazu, dass monolinguale deutschsprachige Kinder auf einmal Englisch lernen mussten und, wenn sie in der Schule Deutsch sprachen, bestraft wurden (Heinen 1982; Blanton 2004; Boas 2005). In den USA herrschte während dieser Zeit eine ausgesprochene Deutschfeindlichkeit.

\subsection{Texasdeutsch nach dem Ersten Weltkrieg}

Von den mehr als 100 deutschsprachigen Periodika, die vor dem Ersten Weltkrieg in Texas erschienen (vgl. Arndt \& Olson 1961), mussten zirka zwei Drittel ihren Betrieb während oder kurz nach dem Ersten Weltkrieg einstellen (Salmons \& Lucht 2006; Boas 2009). Deutsche Schützen-, Gesangs- und Turnvereine stellten ihren Betrieb vorübergehend komplett ein oder wechselten 
zum Englischen. Viele deutschsprachige Kirchen wechselten allmählich während der 1920er und 1930er Jahre zum Englischen, viele boten auch parallel gar keine deutschsprachigen Gottesdienste mehr an (Nicolini 2004; Boas 2015). Diese allgemein verbreitete Deutschfeindlichkeit war einer der Hauptgründe, weshalb das Deutsche ab dem Ende des Ersten Weltkriegs aus dem öffentlichen Raum so gut wie verschwand.

Was übrig blieb, waren einzelne Überbleibsel deutschsprachiger Zeitungen und Kirchen, aber ansonsten wurde Deutsch nur noch im privaten Bereich gesprochen. Hierdurch entstand eine Diglossie, in der das Englische die Prestigesprache im öffentlichen Bereich wurde und das Deutsche als Nichtprestigesprache nur noch im privaten Bereich gesprochen wurde (Freunde, Familie, Nachbarn etc.) (Boas 2009; Boas \& Fingerhuth 2017). In den 1930er und 1940er Jahren setzte sich der Niedergang des Deutschen in anderen öffentlichen Domänen wie den Zeitungen und den Kirchen fort, was sich durch unterschiedliche Ursachen erklären lässt.

Erstens gab es in Folge des Ersten Weltkriegs einen erhöhten Assimilationsdruck. Deutschstämmige Familien änderten ihren Namen, damit sie amerikanischer klangen (Schmidt zu Smith, Fuchs zu Fox etc.). Viele deutschtexanische Familien legten Wert darauf, in der Öffentlichkeit Englisch zu sprechen, um nicht aufzufallen (Hawgood 1940). Zweitens führte der Ausbau des Straßennetzes zu größerer Mobilität. Drittens lernten Kinder in der Schule als Folge der Schulgesetze von 1918 nur noch auf Englisch. Dies führte u. a. dazu, dass sie nicht mehr auf Deutsch lesen und schreiben konnten und ihnen daher der Zugang zu deutschsprachigen Zeitungen, Bibeln und Gesangsbüchern nicht möglich war. Als Folge dessen wechselten einige deutschsprachige Zeitungen zum Englischen als Publikationssprache, viele mussten aber wegen drastisch sinkender Abonnenten ihren Betrieb einstellen. Die Neu Braunfelser Zeitung, gegründet 1852, war bis 1957 die letzte deutschsprachige Zeitung in Texas, als sie ihre Publikationssprache komplett auf Englisch umstellte (Nicolini 2004). Ähnliche Entwicklungen lassen sich in den deutschsprachigen Kirchengemeinden feststellen, in denen sich das Englische immer mehr durchsetzte. Städtische Gemeinden vollzogen den Wechsel zum Englischen schneller als ländliche Gemeinden. Einige Gemeinden stellten die Sprache ihrer Gottesdienste gegen Ende des Ersten Weltkriegs von einem Tag zum nächsten komplett um, während andere erst einen englischsprachigen Gottesdienst pro Monat einführten und so über die Jahre hinweg mehr englischsprachige Gottesdienste angeboten wurden, bis es keine deutschsprachigen Gottesdienste mehr gab (Nicolini 2004; Boas 2005). Diese Entwicklung ist in den 1950er Jahren fast komplett zum Abschluss gekommen. Einige Gemeinden boten jedoch auch bis in die 1960er und 1970er Jahre noch einige wenige deutschsprachige Gottesdienste $\mathrm{zu}$ besonderen Festen wie Ostern oder Weihnachten an (Boas 2015). 
Während der Niedergang des Deutschen im öffentlichen Bereich in Texas zwischen 1920 und 1950 relativ schnell ging, blieb der Privatbereich von diesem Wandel zunächst relativ unbeeinträchtigt. Obwohl einige deutschsprachige Eltern sich ab den 1920er Jahren schon bewusst dazu entschieden, ihre Kinder nicht auf Deutsch, sondern auf Englisch zu erziehen, um ihnen eine mögliche Diskriminierung aufgrund ihres deutschen Akzents im Englischen oder ihrer Deutschkenntnisse $\mathrm{zu}$ ersparen, lernten viele dieser Kinder dennoch zumindest eine reduzierte Form des Deutschen, weil ihre Großeltern häufig nur Deutsch sprechen konnten. Aber auch der Privatbereich blieb nicht lange vom Einfluss des Englischen verschont. Die neue Mobilität (Auto, feste Straßen) führte u. a. zu größerem Kontakt zwischen Texasdeutschen und Texanern mit anglo-amerikanischem (sprich: monolingualem englischsprachigen) Hintergrund. In den daraus resultierenden Ehen zwischen Mitgliedern unterschiedlicher ethno-linguistischer Gruppen kam es fast immer dazu, dass das Englische zur Familiensprache wurde, wodurch die Kinder häufig nur Englisch zu Hause lernten.

Viele Texasdeutsche verließen auch für mehrere Jahre ihre deutschsprachigen Gemeinden aufgrund eines Studiums oder Militärdienstes. Letztendlich führte der Zweite Weltkrieg zu einem weiteren Prestigeverlust des Deutschen, und viele deutschsprachige Texaner entschieden sich in Folge dessen, ihre Kinder nur noch auf Englisch großzuziehen. So lässt sich u. a. erklären, dass die 1950er Jahre die letzte Phase darstellen, in der deutsch-texanische Kinder zu Hause noch auf Deutsch erzogen wurden. Der Großteil der 6.000 bis 8.000 heute noch verbliebenen Sprecher des Texasdeutschen ist aber bereits älter als 70 Jahre, was bedeutet, dass das Texasdeutsche innerhalb der nächsten 20-30 Jahre komplett ausgestorben sein wird.

\section{Die Rolle des Standarddeutschen in Texas}

Die Rolle des Standarddeutschen in Texas und sein Einfluss auf die Entwicklung des Texasdeutschen werden in der Literatur recht unterschiedlich bewertet. In diesem Abschnitt vergleichen wir zuerst die Analysen von Salmons \& Lucht (2006) und Boas (2009), die der Rolle des Standarddeutschen recht unterschiedlichen Einfluss beimessen. Dann diskutieren wir den Status und die Rolle des Standarddeutschen im binnendeutschen Sprachgebiet im 19. Jahrhundert, um zu sehen, wie weit eine standardisierte Hochsprache von unterschiedlichen Bevölkerungsschichten in Europa damals beherrscht wurde. 


\subsection{Salmons \& Lucht (2006)}

Salmons \& Lucht (2006) zufolge hat das Standarddeutsche im 19. Jahrhundert das Texasdeutsche relativ stark beeinflusst, weil es ein hohes Prestige genoss und es institutionell verankert war. Auf der Basis von statischen Daten von der Mitte des 19. Jahrhunderts bis zu den 1940er Jahren über die Verbreitung und Leserzahlen deutschsprachiger Zeitungen (siehe u. a. Arndt \& Olson 1961) und Mitgliederzahlen deutschsprachiger Kirchen (siehe u. a. Nicolini 2004) in Zentraltexas argumentieren Salmons \& Lucht, dass das Standarddeutsche weit verbreitet war und ein hohes Prestige genoss. ${ }^{4}$ Außerdem gab es zahlreiche deutschsprachige Schulen, in denen Standarddeutsch die Unterrichtssprache war, unterstützt durch Unterrichtsmaterialien in Standarddeutsch. Um eine korrekte Verwendung des Standarddeutschen sicherzustellen, stellten deutschsprachige Kirchen, Schulen und Zeitungen Pfarrer, Priester, Lehrer und Redakteure aus Deutschland ein.

Diese Beobachtungen führen Salmons \& Lucht (2006: 198) zu folgender These: „[A]s long as German was a common medium of instruction and part of the most rudimentary medium of instruction, active control of Standard German was commonplace, and a full range of styles existed, from standard to dialect.“ Daraus wiederum schließen Salmons \& Lucht, dass „speakers had more knowledge of standard varieties than is appreciated“. Diese Einschätzung wird in einer späteren Publikation zum Wisconsindeutschen wiederholt, in der Wilkerson \& Salmons (2008: 279) folgendes schreiben:

One widespread view, held by Jacob (2002: 78) and Eichhoff (1985: 233-34) (...) is that Standard German was not widely known or used in many communities, especially Low German-speaking ones. The evidence we are aware of point in the opposite direction, suggesting that an American Standard German was widely known and used by many. Some evidence bearing on this is presented in (...) Salmons and Lucht (2006) for Texas.

\subsection{Standardisierung des Deutschen im 19. und 20. Jahrhundert}

In diesem Abschnitt fassen wir Forschungsergebnisse zusammen, die zeigen, dass im Europa des 19. und frühen 20. Jahrhundert die Beherrschung des Standarddeutschen von breiten Bevölkerungsschichten nicht so weit fortgeschritten war, wie allgemein angenommen. Daraus ergibt sich automatisch,

4 Salmons \& Lucht (2006) besprechen auch den Status deutschsprachiger Literatur aus und über Texas sowie die Verbreitung deutschsprachiger Radiosendungen in Texas. 
dass auch in Texas der Einfluss des Standarddeutschen eher gering gewesen sein muss. Dies hat mindestens zwei Ursachen: (1) Die noch nicht ganz abgeschlossene Standardisierung des Standarddeutschen und (2) der relativ geringe Alphabetisierungsgrad der Bevölkerung in Texas.

Nach einer ersten Phase des internen Standardisierungsprozesses ab dem 16. Jahrhundert durch Sprachausgleich ist das 19. Jahrhundert vor allem durch den formellen und daher externen Standardisierungsprozess der Orthographie geprägt (siehe Ludwig 1998; Elspaß 2014). Erst ab der zweiten Hälfte des Jahrhunderts entstand durch gestiegene Alphabetisierung und Textproduktion die Notwendigkeit einer einheitlichen Schriftsprache (Nerius 1975: 59). Wichtige Meilensteine sind die Veröffentlichung von Dudens Vollständiges Orthographisches Wörterbuch der deutschen Sprache im Jahre 1880 sowie die Zweite orthographische Konferenz 1901. Erst im Schuljahr 1903/1904 wurde die allgemeingültige deutsche Standardorthographie, die sich stark an der Sprache der oberen Mittelschicht orientierte, an allen Schulen Pflicht (Nerius 1975: 65). Die von vielen Forschern vertretene Meinung, dass die Standardisierung des Schriftdeutschen zu Anfang des 19. Jahrhunderts abgeschlossen war, wird von Elspaß (2014: 310) in Frage gestellt: „The traditional view that the standardization process of written German was finished by the beginning of the nineteenth century can be contested. In fact, the data suggest that the standardization of German had not been accomplished by the end of that century.“

Inwiefern hängt dies nun mit dem Einfluss des Standarddeutschen auf Nicht-Standardvarietäten zusammen? Wie oben erwähnt, können sich die schriftliche und mündliche Sprache theoretisch durchaus gegenseitig beeinflussen. Dies ist sicherlich der Fall für das gesprochene Deutsch des 20. Jahrhunderts. Seit der zweiten Hälfte des 20. Jahrhunderts ist eine starke Orientierung der Sprechsprache an der standardisierten Schriftsprache unter gleichzeitiger Dialektvermeidung ${ }^{5} \mathrm{zu}$ beobachten (von Polenz 1999: 457). Unterschiedliche Faktoren beeinflussen diese Entwicklung, so etwa die Flucht und Vertreibung nach 1945, gestiegene Mobilität und dadurch Kontakt zwischen den Regionen, die veränderte, standardsprachenorientierte Arbeitswelt (von Polenz 1999: 457) sowie der Einfluss der Kommunikationsmedien Rundfunk und Fernsehen (von Polenz 1999: 338).

Dies ist allerdings nicht in gleichem Maße der Fall für das Verhältnis zwischen Standardsprache und Dialekt im 19. Jahrhundert, welches in Zusammen-

5 Dies ist als generelle Tendenz zu verstehen. In bestimmten Regionen des binnendeutschen Sprachraums ist die Perzeption der Dialektsprecher gegenüber dem Dialekt weiterhin positiv, v.a. in der Schweiz, Österreich und Süddeutschland (von Polenz 1999: 457). Siehe auch www.regionalsprache.de, letzter Zugriff: 14. 9. 2017. 
hang mit der Emigration nach Texas im Fokus steht. Zwar stand das 19. Jahrhundert ganz im Zeichen der Alphabetisierung der Bevölkerung, des Primats der geschriebenen Sprache und der Orientierung am Bildungsbürgertum (von Polenz 1999: 37 f.). Jedoch war das Verhältnis zwischen Mündlichkeit und Schriftlichkeit nicht von derselben Annäherung wie nach 1945 geprägt, sondern eher von einem Nebeneinander mehrerer verschiedener Funktionen und Register, vor allem für große Teile der Unter- und Mittelschichtsbevölkerung. Neuere Untersuchungen zu Texten des 19. Jahrhunderts (wie etwa Elspaß 2005) zeigen, dass alltagssprachliche Texte mit Nähe zur gesprochenen Sprache einen wesentlich größeren Anteil dialektal geprägter Wendungen aufweisen, als dies für die gehobene Schriftsprache angenommen wird (Elspaß 2005: 463f). Die geschriebene Sprache wird hier eher von den jeweiligen gesprochenen Dialekten als von der bildungsbürgerlichen Schriftsprache beeinflusst. ${ }^{6}$

Dies führt auch zu einem weiteren Problempunkt: Dem Vergleich zwischen historischen Schriftdokumenten und gesprochener Sprache. Es ist generell schwierig, geschriebene Sprache in Zeitungsartikeln und Predigten mit dem eigentlichen mündlichen Sprachgebrauch zu vergleichen. Der Vergleich zwischen geschriebenen Dokumenten und dem tatsächlichen Sprachgebrauch ist ein forschungsübergreifendes Problem der historischen Soziolinguistik, die grundsätzlich auf geschriebene Sprache angewiesen ist. Nach HernándezCampoy \& Schilling (2014: 68 f.) lässt sich dieses Problem wie folgt zusammenfassen:

(1) Die geringere Variation an historischen Textdokumenten macht es schwierig, auf den generellen Sprachgebrauch zu schließen. Variation wird hier als Genre-Variation oder als Variation der Autorenschaft verstanden. Dies ist natürlich vor allen Dingen ein Problem für ältere Sprachstufen des Deutschen. Aber auch für das 19. Jahrhundert ist dieser Hinweis angebracht, da von großen Teilen der Bevölkerung immer noch nicht oder zumindest nicht viel geschrieben wird (siehe z. B. Elspaß 2014). Dementsprechend haben wir es bei den Autoren der oben erwähnten Zeitungen und Predigten mit einem Ausschnitt der texasdeutschen Bevölkerung zu tun, von welchem man nur begrenzt auf die Gesamtbevölkerung schließen kann. Für die Leser- und Hörerschaft dieser Texte gilt grundsätzlich, dass Rezeption nicht gleich Produktion ist.

(2) Ein Blick auf die Autoren der texasdeutschen Texte bringt das Problem der Authentizität mit sich, welches Labov (1994: 11) folgendermaßen definiert: „The linguistic forms in such documents are often distinct from the vernacular of the writers, and instead reflect efforts to capture a normative dialect that never was any speaker's native language.“ Das Problem der Authentizität geht

6 Siehe auch Konopka (1996), Takada (1998), Langer (2001) und Elspaß (2015). 
auf die Unterscheidung zwischen geschriebener, standardisierter Sprache und gesprochener, dialektaler Sprache zurück. Wie oben erwähnt, können sich beide gegenseitig beeinflussen. Es kann allerdings nicht automatisch von der standardisierten Sprache eines Textes auf die tatsächliche Sprachrealität geschlossen werden. Es ist durchaus denkbar, dass die Autoren dieser Texte, auch wenn sie erst kürzlich aus dem binnendeutschen Sprachraum nach Texas kamen, Dialekt gesprochen und Standard geschrieben haben. ${ }^{7}$ Dies würde der Lage entsprechen, die Elspaß (2014: 305) für Europa beschreibt:

[P]eople from all ranks of society in the nineteenth century produced a volume of letters which is unprecedented in the history of the German speaking countries. It has to be emphasised that people in these countries never wrote in their local dialect. They used the kind of written German which they had acquired at school, i.e. Schriftdeutsch ('written German'). For most of them the oral form of Schriftdeutsch which they learnt in school and which they heard in church was also their first spoken L2 (as it is still the case in German speaking Switzerland today).

Schließlich kommt ab dem 20. Jahrhundert der Status des Standarddeutschen in Texas als weiteres Problem hinzu. Wie bereits oben erwähnt, herrschte ab den 1920er Jahren in Texas eine ausgesprochene Deutschfeindlichkeit. Dies wirkte sich auch auf die Benutzung des Standarddeutschen aus. Während im 20. Jahrhundert in europäischen deutschsprachigen Ländern die prestigeträchtige, öffentlich genutzte Sprache das Standarddeutsche wurde, welches durch eine Annäherung von privater und öffentlicher Sprache die Dialekte massiv beeinflusste, war dies für das Texasdeutsche nicht der Fall. Wie oben erwähnt stand dem privat genutzten Texasdeutschen nicht das Standarddeutsche, sondern das Standardenglische als öffentliche, prestigeträchtige und geschriebene Sprache gegenüber (Boas und Fingerhuth 2017).

\subsection{Boas (2009)}

Im Gegensatz zu Salmons \& Lucht (2006) argumentiert Boas (2009), dass die Verbreitung des Standarddeutschen in Texas und sein Einfluss auf das Texasdeutsche weitaus geringer waren. Dabei stützt sich Boas u.a. auf die von Elspaß (2002: 60-61) aufgestellte Beobachtung, dass die Standardisierung des geschriebenen und gesprochenen Standarddeutschen im 19. Jahrhundert selbst in Europa noch immer in vollem Gange war. So gab es z. B. in Preußen zwar einige standardisierte Grammatiken und Wörterbücher (Adelung 1782), aber

7 Siehe Methzenthin Raunick (1929) für einen Überblick über deutsch-texanische Literatur. 
diese wurden hauptsächlich an Gymnasien und höheren Schulen verwendet, welche im 19. Jahrhundert vorwiegend von den Kindern der oberen fünf Prozent der Klassengesellschaft besucht wurden (siehe von Polenz 1999). Der Großteil der Kinder besuchte nur Grundschulen, in denen der Einfluss des Standarddeutschen nicht ganz eindeutig zu bewerten ist, da man davon ausgehen kann, dass ein Großteil des Schulunterrichts und der Alphabetisierung ohne Bücher (bzw. nur mit Büchern für Lehrer) stattfand. Auch im Deutschen Reich gab es bis 1902 keine einheitliche Rechtschreibung, bis die von Konrad Duden ab 1880 kodifizierte Rechtschreibung offiziell anerkannt wurde.

Die Tatsache, dass die Standardisierung des geschriebenen Deutsch in Europa erst im frühen 20. Jahrhundert von staatlicher Seite legitimiert wurde, lässt Boas (2009) vermuten, dass ein starker Einfluss eines einheitlichen standardisierten Deutsch auf das Texasdeutsche in der zweiten Hälfte des 19. und im frühen 20. Jahrhundert eher unwahrscheinlich ist. Neben der Entwicklung der deutschen Rechtschreibung in Deutschland diskutiert Boas (2009: 52) auch die Kodifizierung einer einheitlichen hochdeutschen Aussprache, welche zwar mit Viëtors Die Aussprache des Schriftdeutschen (1885) zum ersten Mal publiziert und verbreitet wurde, aber bis zur Mitte des 20. Jahrhunderts nicht wirklich von breiten Bevölkerungsschichten aktiv verwendet wurde (Durrell 1999; Elspaß 2002). Diese Beobachtungen führen Boas (2009) zu dem Schluss, dass, wenn das Standarddeutsche selbst in Deutschland bis ins 20. Jahrhundert nicht weit verbreitet und einflussreich gewesen ist, es dann in Texas mit an Sicherheit grenzender Wahrscheinlichkeit sogar noch weniger weit verbreitet und einflussreich gewesen ist.

Boas (2009) führt einen weiteren wichtigen Punkt an, der den von Salmons \& Lucht (2006) behaupteten starken Einfluss des Standarddeutsche auf das Texasdeutsche in Frage stellt. Die überwiegende Mehrheit der Texasdeutschen wohnte im 19. und 20. Jahrhundert auf dem Land, wo die meisten Kinder nicht mehr als vier bis sechs Jahre Schulunterricht absolvierten. Außerhalb der begrenzten Zeit in der Schule und der Kirche sprachen die Kinder eher Texasdeutsch als Standarddeutsch, so dass höchstens von einer passiven Kenntnis des Standarddeutschen ausgegangen werden kann. Eine Analyse der Transkripte der vom Texas German Dialect Project durchgeführten soziolinguistischen Interviews mit Sprechern des Texasdeutschen führt Boas (2009) außerdem zu dem Schluss, dass selbst die vier bis sechs Jahre Schulunterricht keinen dauerhaft intensiven Einfluss gehabt haben können, da der Schulunterricht nur ca. 9 Monate pro Jahr dauerte und Kinder auch während des Schuljahres häufig abwesend waren, weil sie auf dem Hof mithelfen mussten. 


\section{Probleme der empirischen Datenerhebung und Forschungsdesiderata}

Der letzte Abschnitt hat gezeigt, dass es nicht einfach ist, den Einfluss des Standarddeutschen auf das Texasdeutsche genau zu bestimmen. Dies hängt u. a. mit der Nichtverfügbarkeit von verlässlichen Daten zusammen, die als Grundlage für eine genauere empirische Untersuchung dienen könnten. Wir fassen zunächst zusammen, was bis heute an empirischen Daten als Grundlage von Salmons \& Lucht (2006) und Boas (2009) verwendet worden sind. Dann besprechen wir, welche Daten möglicherweise in Zukunft aufbereitet und verwendet werden könnten, um so den Einfluss des Standarddeutschen auf das Texasdeutsche näher bestimmen zu können.

\subsection{Stand der Forschung}

Die von Salmons \& Lucht (2006) verwendeten Daten beruhen überwiegend auf Statistiken zur Verbreitung und Auflagenstärke deutschsprachiger Zeitungen sowie der Mitgliederzahlen in deutsch- und englischsprachigen Kirchen in Texas. Aus den schrumpfenden Auflagen- und Mitgliederzahlen schließen Salmons \& Lucht, dass (1) der Einfluss des Standarddeutschen auf das Texasdeutsche im Laufe des 20. Jahrhunderts stark nachließ und (2) dass das Standarddeutsche im 19. und frühen 20. Jahrhundert relativ weit verbreitet war (,active control of Standard German was commonplace“, Salmons \& Lucht 2006: 198). Aus unserer Sicht eignen sich die von Salmons \& Lucht verwendeten Daten jedoch nicht gut, um den Status und den Einfluss des Standarddeutschen empirisch zu belegen.

Erstens sind Auflagenzahlen deutschsprachiger Zeitungen in Texas wenig aussagefähig, wenn es um die aktive Beherrschung des Standarddeutschen von Zeitungslesern geht. Die Auflagenzahlen belegen lediglich, wie viele Zeitungen verkauft wurden. Ob und von wem diese Zeitungen gelesen worden sind, lässt sich anhand von Auflagenzahlen jedoch nicht bestimmen. Auch ist nicht feststellbar, ob die Zeitungsleser aktive Kenntnisse des Standarddeutschen hatten, und wenn ja, wie gut diese Kenntnisse waren. Auch der Status der Zeitungen selbst, besonders die Verwendung von fehlerfreiem Standarddeutsch, ist nicht ganz klar. Viele der mehr als hundert deutschsprachigen Zeitungen in Texas wurden in kleineren Städten auf dem Land verlegt. Ein Beispiel ist das Giddings Deutsches Volksblatt, welches von 1899 bis 1949 in Giddings, Texas, erschien. Der Gründer und Herausgeber John A. Proske (18561943) wurde in Deutschland geboren und wanderte mit dreizehn Jahren nach 
Texas aus und besuchte wohl nur sechs Jahre lang die Schule (Garrett 1998: 118). Die von Proske verfassten Lokalnachrichten und von ihm publizierten Anzeigen von 1899-1909 weisen regelmäßig Fehler auf (Verwendung von Kasus, Pluralformen, Genus etc.), die zeigen, dass das Verfassen bzw. das Korrekturlesen nicht immer in fehlerfreiem Standarddeutsch vonstattenging. Ob dies ein Einzelfall ist, lässt sich nicht so einfach feststellen, sondern kann nur im Rahmen einer groß angelegten vergleichenden Analyse texasdeutscher Zeitungstexte beantwortet werden, welche in Zukunft durchgeführt werden sollte. Solch eine Analyse könnte dann darüber Aufschluss geben, inwieweit deutschsprachige Zeitungen in Texas in fehlerfreiem Standarddeutsch gedruckt waren. Aber selbst diese Ergebnisse könnten nicht dazu beitragen $\mathrm{zu}$ verstehen, inwieweit Sprecher des Texasdeutschen auch aktive Kontrolle des Standarddeutschen hatten.

Zweitens haben die Mitgliederzahlen in deutschsprachigen Kirchen nur begrenzten Aussagewert. Sie belegen zwar, wie viele Besucher die jeweiligen Kirchen hatten und dass die englischsprachigen Gottesdienste im Laufe des 20. Jahrhunderts die deutschsprachigen Gottesdienste mehr und mehr ablösten. Aber diese Mitgliederzahlen sagen nichts darüber aus, inwieweit die Pastoren, Pfarrer und Kirchenbesucher aktive Kontrolle des Standarddeutschen hatten. Mit anderen Worten, es kann davon ausgegangen werden, dass die Besucher der deutschsprachigen Gottesdienste mit an Sicherheit grenzender Wahrscheinlichkeit die Lesungen und Predigten verstehen konnten. Ob diese aber auch immer in Standarddeutsch stattfanden, lässt sich aus den Mitgliederzahlen der deutschsprachigen Kirchengemeinden nicht erschließen. Dazu bräuchte man Tonaufnahmen, anhand derer man feststellen könnte, inwieweit die Gottesdienste auf Standarddeutsch stattfanden oder ob diese vielleicht nicht doch in standardnahen Dialekten abgehalten wurden. Selbst wenn wir solche Aufnahmen hätten, ließe sich daraus nicht leicht erschließen, ob die Besucher der deutschsprachigen Gottesdienste selbst aktive Kontrolle des Standarddeutschen hatten. Wilson (1960: 86) schreibt, dass „ministers preach in S[tandard] G[erman] with a very good pronunciation“, aber das sagt uns nichts über die Sprachkenntnisse der Besucher der Kirche, weil man nicht von Rezeption auf Produktion schließen kann.

Drittens untersuchen Salmons \& Lucht (2006) hauptsächlich statistische Daten zur Verwendung des Deutschen in Zeitungen und Kirchen. Strukturelllinguistische Daten werden zwar auch angesprochen, doch kommen diese nur aus literarischen Werken. Wünschenswert wäre die Analyse natürlichsprachlicher Tonaufnahmen gewesen, die zeigen könnten, welche Varietäten des Deutschen in Texas im späten 19. und frühen 20. Jahrhundert tatsächlich gesprochen wurden. Solche Tonaufnahmen gibt es aber leider nicht. Wenn man 
sich aber die Daten in Gilberts (1972) Linguistic Atlas of Texas German genauer anschaut, so entdeckt man fast nur dialektale Merkmale in der Morphologie, der Phonologie, der Syntax und dem Lexikon. Diese Daten können leider keine Auskunft über die aktive Beherrschung des Standarddeutschen geben, aber sie beruhen auf Tonaufnahmen, die in den 1960er Jahren aufgenommen worden sind. Da die von Gilbert aufgenommenen Sprecher zwischen 1890 und 1944 geboren wurden, lässt sich mit Hilfe des „Apparent-Time“-Konstrukts (Sankoff 2006) rekonstruieren, wie sich das Texasdeutsche entwickelt hat. Die von Gilbert aufgenommenen Daten können dann mit den vom TGDP seit 2001 aufgenommenen Tonaufnahmen verglichen werden, um so mit Hilfe des „Real-Time“Konstrukts (Labov 1994) den Sprachwandel im Texasdeutschen zu analysieren (siehe Boas 2009; Boas \& Pierce 2011).

Aber weder die Daten von Gilbert (1972) noch die vom TGDP erhobenen Daten lassen sich verwenden, um die aktive Kontrolle des gesprochenen Standarddeutschen in Texas und den Einfluss des Standarddeutschen auf das Texasdeutsche im späten 19. und frühen 20. Jahrhundert zu untersuchen. Da es keine standarddeutschen Tonaufnahmen aus Texas aus dem späten 19. und frühen 20. Jahrhundert gibt, wird sich wahrscheinlich nie feststellen lassen, ob und in welchem Umfang es wirklich eine aktive Kontrolle des gesprochenen Standarddeutschen in Texas gegeben hat. Mit Hilfe moderner korpuslinguistischer Methoden lassen sich aber eine Reihe elektronischer Ressourcen erstellen, mit denen die Verbreitung bzw. der Einfluss des Standarddeutschen im späten 19. und frühen 20. Jahrhundert genauer bestimmt werden können.

\subsection{Zukunftspläne}

Die wahrscheinlich ergiebigsten Daten lassen sich aus den mehr als hundert deutschsprachigen Zeitungen gewinnen. Einige dieser Zeitungen wie die Neu Braunfelser Zeitung sind bereits digitalisiert ${ }^{8}$ und können transkribiert werden oder mit Hilfe von sog. „Optical Character Recognition“ in ein elektronisches Textkorpus umgewandelt werden, welches dann mit korpus-linguistischen Mitteln und Methoden bearbeitet werden kann. Diese korpuslinguistische Aufbereitung und Bearbeitung kann mit denselben Methoden angegangen werden wie die im Deutschen Textarchiv ${ }^{9}$ enthaltenen historischen Zeitungstexte. Mit automatischen Verfahren zur Tokenisierung, Lemmatisierung und zum

8 Siehe https://texashistory.unt.edu/explore/collections/TDNP/browse/?fq=dc_language:ger (letzter Zugriff: 5. 8. 2017).

9 Siehe www.deutschestextarchiv.de/ (letzter Zugriff: 7. 8. 2017). 
POS-Tagging können die Zeitungstexte dann bearbeitet werden und ähnlich wie im Deutschen Textarchiv mit einer linguistischen Suchmaschine indiziert werden, welche dann komplexe Suchanfragen und Recherchen durchführen kann. Solch ein Korpus kann systematisch nach Daten durchsucht werden, um festzustellen, ob bzw. inwieweit wirklich durchgehend Standarddeutsch in deutschsprachigen texanischen Zeitungen verwendet wurde oder ob die Texte nicht doch Substandard-Merkmale in den Bereichen Morphologie, Syntax und Lexikon aufweisen. Anhand einer solchen Datengrundlage könnte man sich ein klareres Bild davon verschaffen, ob bzw. inwieweit die Leser deutschsprachiger Zeitungen in Texas im späten 19. und frühen 20. Jahrhundert Zeitungen gelesen haben, die wirklich „nur“ Standarddeutsch enthielten.

Solch ein Zeitungskorpus mag zwar darüber Aufschluss geben, welche Sprachnormen in den Zeitungen benutzt wurden, aber es trägt nicht zur Lösung der Frage bei, ob die Sprecher des Texasdeutschen wirklich aktive Kontrolle des Standarddeutschen hatten oder nicht. Dieses Problem spricht auch Elspaß (2014: 309) an, der folgendes feststellt:

If the model is based on norms of usage, one may ask which written language usage has been considered, let alone analysed. It is certainly not a trivial question to ask whether standardisation studies of modern western languages focus on printed language only, or whether they have taken hand-written sources into account.

Um die Frage nach der (schriftlichen) Sprachverwendung zu beantworten, bedarf es daher noch weiterer Ressourcen, nämlich eines Korpus handgeschriebener Dokumente. Auch hier gibt es zahlreiche unterschiedliche Sorten handgeschriebener Dokumente: Zum einen öffentliche Dokumente wie Protokolle und Berichte von Kirchen-, Vereins- und Gemeinderatssitzungen, zum anderen Dokumente aus dem privaten Bereich wie Briefe, Tagebücher und andere persönliche Dokumente, die eine potenzielle Nähe zur gesprochenen Sprache aufweisen. Das TGDP hat seit 2001 zahlreiche solcher Dokumente gesammelt und archiviert, und es gibt auch größere Sammlungen von Auswandererbriefen in der Forschungsbibliothek Gotha (siehe auch Elspaß 2005). ${ }^{10}$ Diese handgeschriebenen Dokumente können mit denselben korpuslinguistischen Methoden bearbeitet und aufbereitet werden wie die im sog. Corpus of Patient Documents, welches $\mathrm{u}$.a. als Datenbasis des Forschungsprojekts Flexible Schreiber in der Sprachgeschichte an der Friedrich-Alexander-Universität Erlangen-Nürnberg dient. ${ }^{11}$ Die digital aufbereiteten Briefe zeigen u.a., dass

10 Siehe www.auswandererbriefe.de/ (letzter Zugriff: 10. 8. 2017).

11 Siehe www.copadocs.de/ (letzter Zugriff: 17. 8. 2017). 
selbst Schreiber einfachster Herkunft in der Lage sind, ihre Varietätenwahl ähnlich wie Sprecher (vgl. Macha 1991) abhängig von den intendierten Rezipienten $\mathrm{zu}$ gestalten, und dass der Sprachgebrauch von Einzelpersonen trotz sehr ähnlicher sozialer Herkunft und vergleichbaren Berufen deutliche Divergenzen aufweisen kann (Schiegg 2015: 196).

Anhand eines vergleichbaren Korpus für das Texasdeutsche ließe sich dann der Sprachgebrauch von Sprechern des Texasdeutschen genauer untersuchen, um festzustellen, inwieweit es wirklich eine aktive Kontrolle des (geschriebenen) Standarddeutschen gegeben hat, und wenn ja, ob diese ein weit verbreitetes Phänomen war, oder ob es auch hier erhebliche Variation gegeben hat, die der Variation des gesprochenen Texasdeutschen, wie sie von Gilbert (1972) belegt ist, entspricht. ${ }^{12}$ Ob sich auf diese Weise herausarbeiten lässt, inwieweit Texasdeutsche Kenntnisse des Standarddeutschen hatten, ist nicht ganz klar und muss der zukünftigen Forschung überlassen werden. Laut Elspaß (2014: 309) könnte dies ein eher schwieriges Unterfangen werden:

The underlying division into 'written language' and 'spoken language' has proved to be simplistic. To be more precise, the dichotomy between the written and the spoken medium must be distinguished from the scalar differences between conceptually oral and conceptually written language - or 'language of immediacy' and 'language of distance' in the terminology of Peter Koch and Wulf Oesterreicher (cf. Koch \& Oesterreicher 1985, 1994). Ignoring this distinction results in the notorious difficulty of establishing which registers of 'written language' and 'spoken language' represent standard language (formal as well as informal registers? only formal registers? informal registers only in the written medium?).

\section{Fazit}

Dieser Beitrag hat gezeigt, dass es momentan nur sehr schwer möglich ist, den genauen Einfluss des Standarddeutschen auf das Texasdeutsche im 19. und frühen 20. Jahrhundert zu belegen. Die von Salmons \& Lucht (2006: 198) vorgeschlagene These, dass ,active control of Standard German was commonplace, and a full range of styles existed, from standard to dialect“", lässt sich anhand der von ihnen verwendeten Daten aus unserer Sicht nicht belegen. Die von Boas (2009) vorgeschlagene These, dass das Standarddeutsche nur einen sehr begrenzten Einfluss auf das Texasdeutsche gehabt hat, beruht auf strukturel-

12 Ob die von Texasdeutschen verfassten handschriftlichen Dokumente wirklich aussagekräftig bezüglich der aktiven Kontrolle des gesprochenen Standarddeutsch sind ist nicht ganz klar, da es sich um sog. Schriftdeutsch handelte. 
len Daten zum gesprochenen Texasdeutschen in Gilbert (1972) und der vom TGDP erhobenen gesprochenen Daten, welche $\mathrm{u}$. a. Anekdoten zum Gebrauch von Dialekt und Standard im frühen 20. Jahrhundert enthalten. Eine genauere Untersuchung der Sprachwirklichkeit der texasdeutschen Sprachgemeinschaft vor über hundert Jahren ist jedoch notwendig, um die von Boas (2009) vorgestellten Thesen $\mathrm{zu}$ verifizieren.

Um das genaue Verhältnis von Standarddeutsch und Dialekt in Texas besser verstehen zu können, haben wir in Abschnitt 5 einen kurzen Katalog von Daten- und Forschungsdesiderata aufgestellt. Dieser Katalog beinhaltet u. a. die Erstellung von historischen digitalen Korpora deutschsprachiger Zeitungen in Texas sowie die Erstellung eines digitalen Korpus handgeschriebener Dokumente. Basierend auf den Texten der sprachlichen Nähe (persönliche Briefe, Tagebücher, etc.) sollte es möglich sein zu sehen, inwieweit Sprecher des Texasdeutschen eine aktive Kontrolle des Schriftdeutschen hatten. Diese Daten bieten der Variationslinguistik und Dialektologie eine neuartige und vielschichtige Quelle, um den Sprachgebrauch der Sprecher des Texasdeutschen im 19. und frühen 20. Jahrhundert hinsichtlich ihrer Fähigkeit zu sprachlicher Flexibilität und ihres Sprachbewusstseins zu untersuchen.

Anhand der handgeschriebenen Dokumente ließen sich dann auch vorsichtige Aussagen zur gesprochenen Sprache machen, die man mit den „Apparent-Time-“ und „Real-Time-“gesprochenen texasdeutschen Daten des TGDP und von Gilbert (1972) vergleichen könnte. Noch genauer wird sich aber der Einfluss des Standarddeutschen in Texas wohl nicht bestimmen lassen.

\section{Literatur}

Adelung, Johann Christoph (1782): Umständliches Lehrgebäude der Deutschen Sprache, zur Erläuterung der Deutschen Sprachlehre für Schulen, Bd. 1 u. 2. Berlin: Voss.

Arndt, Karl J. R. \& May E. Olson (1961): German-American newspapers \& periodicals 1732-1955: History and bibliography. Heidelberg: Quelle \& Meyer.

Blanton, Carlos Kevin (2004): The strange career of bilingual education in Texas, 1836-1981. College Station: Texas A\&M Press.

Biesele, Rudolph (1930): The history of German settlements in Texas, 1831-1861. Austin: Böckmann-Jones.

Boas, Hans C. (2005): A dialect in search of its place - The use of Texas German in the public domain. In Craig Cravens \& David Zersen (Hrsg.), Transcontinental encounters: Central Europe Meets the American Heartland, 78-102. Austin: Concordia University Press.

Boas, Hans C. (2009): The life and death of Texas German. Durham: Duke University Press.

Boas, Hans C. (2015): Linguistic splits along religious lines - The role of language maintenance among Catholics and Lutherans in Texas. In Peter Rosenberg, 
Konstanze Jungbluth \& Dagna Zinkhahn Rhobodes (Hrsg.), Linguistic constructions of ethnic borders, 167-181. Frankfurt/New York: Peter Lang.

Boas, Hans C. (2016): Variation im Texasdeutschen: Implikationen für eine vergleichende Sprachinselforschung. In Alexandra Lenz (Hrsg.), German Abroad. Perspektiven der Variationslinguistik, Sprachkontakt- und Mehrsprachigkeitsforschung, 11-44. Vienna University Press.

Boas, Hans \& Matthias Fingerhuth (2017): „I am proud of my language but I speak it less and less!“ - Der Einfluss von Spracheinstellungen und Sprachgebrauch auf den Spracherhalt von Heritage-Sprechern des Texasdeutschen. Linguistische Berichte 249, 95-121.

Boas, Hans C. \& Marc Pierce (2011): Lexical developments in Texas German. In Putnam, Michael (Hrsg.), Studies on German language islands, 129-150. Amsterdam/ Philadelphia: John Benjamins.

Burdach, Konrad (1884): Die Einigung der neuhochdeutschen Schriftsprache. Einleitung: Das sechzehnte Jahrhundert. In Konrad Burdach (Hrsg.), Vorspiel. Gesammelte Schriften zur Geschichte des deutschen Geistes. Volume 1 (2), 1-33. Halle/Saale: Niemeyer.

Durrell, Martin (1999): Standardsprache in England und Deutschland. Zeitschrift für Germanistische Linguistik 27, 285-308.

Ebert, Robert, Oskar Reichmann, Hans-Joachim Solms \& Klaus-Peter Wegera (1993): Frühneuhochdeutsche Grammatik. Tübingen: Niemeyer.

Eichhoff, Jürgen (1986): Die Deutsche Sprache in Amerika. In Frank Trommler (Hrsg.), Amerika und die Deutschen, 235-252. Opladen: Westdeutscher Verlag.

Elspaß, Stephan (2002): Standard German in the 19th century? (Counter-) Evidence from the private correspondence of 'ordinary people'. In Andres Linn \& Nicola McLelland (Hrsg.): Standardization. Studies from the Germanic Languages, 43-65. Amsterdam/ Philadelphia: Benjamins.

Elspaß, Stephan (2005): Sprachgeschichte von unten. Untersuchungen zum geschriebenen Alltagsdeutsch im 19. Jahrhundert. Tübingen: Niemeyer.

Elspaß, Stephan (2014): Prescriptive norms and norms of usage in nineteenth-century German. In Gijsbert Rutt, Rik Vosters \& Wim Vandenbussche (Hrsg.), Norms and usage in language history, 1600-1900. A sociolinguistic and comparative perspective, 303-320. Amsterdam/Philadelphia: John Benjamins.

Elspaß, Stephan (2015): Private letters as a source for an alternative history of Late Modern German. In Anita Auer, Daniel Schreier \& Richard J. Watts (Hrsg.): Letter Writing and Language Change, 35-52. Cambridge: Cambridge University Press.

Garrett, Daphne Dalton (1998): Giddings Deutsches Volksblatt. 1899-1949. A history of the newspaper and print shop of the Texas Wends. Warda, TX: Garrett Historical Research.

Gilbert, Glenn (1972): Linguistic atlas of Texas German. Austin: University of Texas Press.

Gilbert, Glenn (1978): Origin and present-day location of German speakers in Texas. In Glenn Gilbert \& Jacob Ornstein (Hrsg.), Problems in applied educational sociolinguistics. Den Haag: Mouton, 119-129.

Hawgood, John (1940): The tragedy of German-America: The Germans in the United States of America during the nineteenth century and after. New York: Putnam's Sons.

Heinen, Hubert (1982): German in Texas schools, 1849-1939. Heritage of the Great Plains 15, 11-20.

Hernández-Campoy, Juan \& Natalie Schilling (2014): The application of the quantitative paradigm to historical sociolinguistics: Problems with the generalizability principle. In 
Juan Manuel Hernández-Campoy \& Juan Camilo Conde-Silvestre (Hrsg.), The handbook of historical sociolinguistics, 63-79. Oxford: Wiley-Blackwell.

Jordan, Gilbert (1977): The Texas German language of the Western Hill Country. Rice University Studies 63 (3), 59-71.

Kearney, James (2010): Nassau plantation: The evolution of a Texas German slave plantation. Denton: University of North Texas Press.

Koch, Peter \& Wulf Oesterreicher (1985): Sprache der Nähe - Sprache der Distanz: Mündlichkeit und Schriftlichkeit im Spannungsfeld von Sprachtheorie und Sprachgeschichte. Romanistisches Jahrbuch 36, 15-43.

Konopka, Marek (1996): Strittige Erscheinungen der deutschen Syntax im 18. Jahrhundert. Tübingen: Niemeyer.

Kyes, Robert L. (1985): Principles, traditions, and phonological restoration. Neuphilologische Mitteilungen 86 (4), 433-441.

Labov, William (1994): Principles of linguistic change, Vol. 1: Internal change. Oxford: Blackwell.

Langer, Nils (2001): Linguistic purism in action. How auxiliary tun was stigmatized in Early New High German. Berlin/New York: de Gruyter.

Ludwig, Otto (1998): Alphabetisierung und Volksschulunterricht im 19. Jahrhundert. Der Beitrag der Schreib- und Stilübungen. In Dieter Cherubim, Siegfried Grosse \& Klaus J. Mattheier (Hrsg.), Sprache und bürgerliche Nation: Beiträge zur deutschen und europäischen Sprachgeschichte des 19. Jahrhunderts, 148-166. Berlin: de Gruyter.

Macha, Jürgen (1991): Der flexible Sprecher. Untersuchungen zu Sprache und Sprachbewusstsein rheinischer Handwerksmeister. Köln: Böhlau.

Mihm, Arend (2016): Zur Theorie der vormodernen Orthographien: Straßburger Schreibsysteme als Erkenntnisgrundlage. Sprachwissenschaft 41 (3), 271-309.

Möller, Robert (2000): Rheinische Sprachgeschichte von 1300 bis 1500. In Jürgen Macha, Elmar Neuss \& Robert Peters (Hrsg.), Rheinisch-Westfälische Sprachgeschichte. Köln/ Weimar/Wien: Böhlau.

Moser, Virgil (1951): Frühneuhochdeutsche Grammatik. Bd. 1.3: Lautlehre (Konsonanten). Heidelberg: Carl Winter.

Müllenhoff, Karl (1863): Vorwort. In Karl Müllenhoff \& Wilhelm Scherer (Hrsg.), Denkmäler deutscher Poesie und Prosa aus dem VIII-XII Jahrhundert. Berlin: Steinmeyer.

Nerius, Dieter (1975): Untersuchungen zu einer Reform der Orthographie. Berlin: Akademie. Nicolini, Marcus (2004): Deutsch in Texas. Münster: LIT Verlag.

Polenz, Peter von (1999): Deutsche Sprachgeschichte vom Spätmittelalter bis zur Gegenwart. Band 3: 19. und 20. Jahrhundert: Berlin: de Gruyter.

Roesch, Karen A. (2012) Language maintenance and language death: The decline of Texas Alsatian. Amsterdam/Philadelphia: John Benjamins.

Salmons, Joseph C. \& Felicia A. Lucht (2006): Standard German in Texas. In Linda Thornburg \& Janet Fuller (Hrsg.), Studies in contact linguistics: Essays in honor of Glenn G. Gilbert, 165-186. New York: Peter Lang.

Sankoff, Gillian (2006): Age - Apparent time and real time. Encyclopedia of language and linguistics, 110-115.

Schiegg, Markus (2015): Der flexible Schreiber in der Sprachgeschichte. Zeitschrift für Dialektologie und Linguistik LXXXII (2), 169-204.

Takada, Hiroyuki (1998): Grammatik und Sprachwirklichkeit von 1640-1700. Zur Rolle deutscher Grammatiker im schriftsprachlichen Ausgleichsprozess. Tübingen: Niemeyer. Viëtor, Wilhelm (1885): Die Aussprache des Schriftdeutschen. Leipzig: O. R. Reisland. 
Wilkerson, Miranda \& Joseph Salmons (2008): „Good old immigrants of yesteryear“ who did learn English: Germans in Wisconsin. American Speech 83 (3), 259-283.

Wilson, Joseph (1960): The Texas German of Lee and Fayette Counties. Rice University Pamphlet 47, 83-98. 



\title{
Péter Maitz und Siegwalt Lindenfelser \\ 13 Gesprochenes Alltagsdeutsch im Bismarck-Archipel um 1900
}

\author{
Das Zeugnis regional markierter Superstrateinflüsse \\ in Unserdeutsch
}

\begin{abstract}
Unserdeutsch (Rabaul Creole German) entstand um 1900 an einer katholischen Missionsstation in Vunapope auf der Insel New Britain im Bismarck-Archipel. Seine dominante Substratsprache ist Tok Pisin, das melanesische Pidgin-Englisch, seine Superstratsprache Deutsch. Der Aufsatz versucht das sprachliche Superstrat von Unserdeutsch näher zu bestimmen, d. $h$. die Frage zu beantworten, welches Deutsch von den Missionaren in Vunapope um 1900, am Ort und zum Zeitpunkt der Entstehung von Unserdeutsch, gesprochen wurde. $\mathrm{Zu}$ diesem Zweck werden die als Superstrattransfer aus dem Deutschen erklärbaren, regional markierten linguistischen Strukturmerkmale in Unserdeutsch untersucht und im geschlossenen Sprachgebiet sprachgeografisch lokalisiert. Ergänzt wird diese linguistische Evidenz durch extra- und metalinguistische Evidenz aus einschlägigen, zeitgenössischen Quellen. Die Ergebnisse deuten auf ein vorwiegend nordwestdeutsch-westfälisch geprägtes, insgesamt jedoch heterogenes, standardnahes sprachliches Superstrat hin und widerlegen somit frühere diesbezügliche Aussagen in der einschlägigen Fachliteratur. Und sie zeigen zugleich auch, dass die Analyse von kolonialen und sonstigen Auswanderervarietäten, besonders von solchen, die - wie Unserdeutsch - im Laufe ihrer späteren Geschichte den Kontakt zum sprachlichen
\end{abstract}

Anmerkung: Die Forschungen, die dem Aufsatz zugrunde liegen, werden von der Deutschen Forschungsgemeinschaft (MA 6769/1-1) gefördert. Wir danken Werner König und Lena Schmidtkunz (beide Augsburg) für ihre wertvollen Hinweise sowie Rebecca Karrer und MarcOliver Ubl (beide Augsburg) für ihre Hilfe bei den Literatur- und Datenrecherchen. Unser aufrichtiger Dank gebührt außerdem den Missionaren und Missionsschwestern vom Hlst. Herzen Jesu in Hiltrup (Münster) und Vunapope (East New Britain, Papua-Neuguinea) sowie dem Erzbistum Rabaul (East New Britain, Papua-Neuguinea), die uns Zugang zu ihren Archiven gewährt haben, sowie den zuständigen Archivaren für ihre Hilfe bei den Recherchen.

Péter Maitz, Universität Augsburg, Lehrstuhl für Deutsche Sprachwissenschaft, Universitätsstraße 10, D-86159 Augsburg, E-Mail: peter.maitz@philhist.uni-augsburg.de Siegwalt Lindenfelser, Universität Augsburg, Lehrstuhl für Deutsche Sprachwissenschaft, Universitätsstraße 10, D-86159 Augsburg, E-Mail: siegwalt.lindenfelser@philhist.uni-augsburg.de

Ә Open Access. (†) 2018 Péter Maitz und Siegwalt Lindenfelser, publiziert von De Gruyter. (c) BY Dieses Werk ist lizenziert unter der Creative Commons Attribution 4.0 Lizenz.

https://doi.org/10.1515/9783110538625-014 
Mutterland vollständig verloren haben, zur Rekonstruktion historischer Mündlichkeit wertvolle Daten liefern kann.

Keywords: Alltagssprache, Kreolsprache, Papua-Neuguinea, Sprachgeschichte, Sprachkontakt, Sprachvariation, Superstratsprache, Unserdeutsch

\section{Was wir herausfinden wollen}

Die meisten der als Pidgins und Kreols klassifizierten Sprachen sind Ergebnis der Expansion europäischer Mächte in kolonialen Kontexten (vgl. Velupillai 2015: 99-103). Deshalb basiert die Mehrzahl dieser Sprachen zumindest partiell auf den Sprachen der einstigen europäischen Kolonialmächte, besonders auf dem Englischen, Französischen, Niederländischen, Portugiesischen und Spanischen. ${ }^{1}$ Vereinzelt sind aber auch koloniale Kontaktvarietäten dokumentiert, die aus dem Deutschen hervorgegangen sind. $\mathrm{Zu}$ diesen gehört neben dem inzwischen ausgestorbenen Ali-Pidgin in Papua-Neuguinea (vgl. Mühlhäusler 2012) oder dem Küchendeutsch in Namibia (vgl. Deumert 2009) auch das einst im Bismarck-Archipel entstandene und heute mehrheitlich in Australien gesprochene Unserdeutsch (vgl. Maitz 2016, 2017; Volker 1982), von dem im Folgenden die Rede sein wird.

Die am Entstehungsort einer Pidgin- oder Kreolsprache geläufige koloniale Varietät der dominanten europäischen Sprache, die als Kontaktsprache maßgeblich an der Entstehung des Pidgins/Kreols beteiligt ist, wird in der Literatur meist als Superstratsprache (superstrate), Lexifikatorsprache (lexifier, lexical source language) oder auch Zielsprache (target language) bezeichnet. Da ihre Denotate auf der einen Seite weitgehend identisch sind, werden diese Bezeichnungen häufig synonym gebraucht. Auf der anderen Seite gehen aber ihre Fokusse und Implikationen teilweise erheblich auseinander, auch enthalten sie problematische Übergeneralisierungen, sodass all diesen Termini gegenüber vielfach auch berechtigte Kritik hervorgebracht wird. Der Terminus Superstratsprache reflektiert in erster Linie den sozialen und politischen Status der in Kontakt stehenden Gruppen (vgl. Thomason \& Kaufmann 1988: 116). Die Superstratsprache ist die Varietät der siegreichen (Kolonial-)Macht und damit der macht- und prestigevollen Gruppe (vgl. Arends, Kouwenberg \& Smith 1995: 99), die die lokale(n) Sprache(n) der Region, die Substratsprache(n) näm-

1 Die Vermutung liegt nahe, dass im Laufe der Geschichte weltweit zahlreiche Kontaktsprachen dieser Art entstanden sind, nur mögen viele von ihnen einer vom Westen ausgehenden Beschreibungsperspektive entgangen sein (vgl. Thomason 2001: 163). 
lich, „überlagert“. Die Bezeichnung Lexifikatorsprache trägt der Tatsache Rechnung, dass die Sprache der jeweiligen Kolonialmacht in aller Regel den Großteil des Lexikons einer Pidgin- oder Kreolsprache stellt. Und schließlich lenkt die Bezeichnung Zielsprache den Fokus auf den Erwerbsaspekt und damit auch in die Nähe der sog. Interlanguage-Hypothese (vgl. Plag 2008). Demnach sei die Sprache der Kolonialherren das Lernziel in einer Erwerbssituation gewesen, von dem jedoch das Erwerbsergebnis, die neue, koloniale Kontaktvarietät nämlich, aus welchen Gründen auch immer abweicht.

Ohne die einzelnen Termini kritisch reflektieren und die einschlägigen terminologischen Debatten ${ }^{2}$ innerhalb der Kreolistik ausführlich und systematisch referieren zu wollen, möchten wir an dieser Stelle nur festhalten, dass wir uns im vorliegenden Aufsatz für den Terminus Superstrat entscheiden werden. Gleichwohl gilt auch in unseren Augen, dass die Superstratsprache eines Pidgins oder Kreols nicht zwangsläufig zugleich auch deren Lexifikatorsprache sein muss (vgl. Selbach 2008 am Beispiel von Lingua Franca), während sie außerdem womöglich nie Zielsprache im eigentlichen Sinn war (vgl. Baker 1990; Jansson, Parkvall \& Strimling 2015).

Die Rolle bzw. der Einfluss des Superstrats auf die Struktur einer Kreolsprache wird, abseits der Lexik, unter Kreolisten ${ }^{3}$ sehr unterschiedlich gesehen. M. a. W. besteht kein Konsens, inwieweit normalerweise struktureller Transfer aus dem Superstrat stattfindet. „Substratisten“, etwa Vertreter der Relexifizierungs-Hypothese (Lefebvre 1998), achten den strukturellen Einfluss des Superstrats gering:

[F]rom a typological point of view, PCs [pidgins and creoles, P. M. \& S. L.] resemble their substratum languages in spite of the fact that the phonological representation of their lexicons are derived from their respective superstratum languages. (Lefebvre 2004: 18)

„Superstratisten“ hingegen - wie etwa Chaudenson (2003) oder Mufwene $(2015 b)^{4}$ - schätzen den strukturellen Einfluss des Superstrats als sehr hoch ein:

2 Zur kritischen Auseinandersetzung mit den einzelnen Termini siehe z. B. Baker (1990), Bakker (1995: 39) und (2002: 71), Goodman (1993: 64-65), Jansson, Parkvall \& Strimling (2015), Mufwene (2015a: 348-349), Selbach (2008).

3 Aus platzökonomischen Gründen und verwenden wir bei Personenbezeichnungen das generische Maskulinum und meinen dabei stets gleichermaßen Personen weiblichen und männlichen Geschlechts.

4 In einigen anderen Publikationen, etwa Mufwene (2001), vertritt Mufwene allerdings im Zuge seiner complementary hypothesis einen Mittelweg: den Wettbewerb zwischen Substratund Superstratstrukturen. 
The claim that a creole has typically inherited its grammar from sources other than its lexifier (Holm, 1988; Thomason and Kaufman, 1988; Thomason, 2001) is not backed by the diachronic evidence. Although a creole or a pidgin, often (mis)identified with the basilect, has a grammar very different from the standard variety of its lexifier (called acrolect), it has often been shown that much of its grammar can be traced, at least partly, to some of the nonstandard varieties to which its "creators" were exposed. (Mufwene 2015b: 133, Hervorhebung im Original)

Fest steht, dass der Einfluss des Superstrats sicher nicht auf die Bereitstellung des Lexikons beschränkt ist (vgl. Holm 2000: 172). Zugleich ist es aufgrund von strukturellen Konvergenzen und Ähnlichkeiten häufig schwer bis unmöglich $\mathrm{zu}$ entscheiden, aus welcher der beteiligten Kontaktsprachen eine Struktur transferiert wurde - und ob überhaupt Transfer vorliegt, da auch Reanalysen und Erwerbsuniversalien eine Rolle spielen können (vgl. etwa Frowein 2006).

Im Fall der deutsch-lexifizierten Kreolsprache Unserdeutsch ist die Bedeutung strukturellen Substrattransfers nicht zu bestreiten: Große Teile der Grammatik von Unserdeutsch konvergieren in auffallender Weise mit korrespondierenden Strukturen in seiner Substratsprache Tok Pisin (vgl. Volker 1989; Lindenfelser \& Maitz 2017; Maitz, Lindenfelser \& Volker i. E.). Gleichwohl können zahlreiche Strukturen und Regeln auch plausibel über Superstrattransfer, d. $h$. als Übernahme aus dem Deutschen, mit motiviert werden. In diesem Aufsatz wollen wir jedoch nicht der klassischen - und gewichtigen - Frage nach der historisch-genetischen Erklärung von einzelnen Kreolmerkmalen nachgehen. Uns geht es vielmehr darum, in umgekehrter Herangehensweise die Superstratvarietät von Unserdeutsch, die den sprachlichen Input bei der Genese der Sprache darstellte, möglichst exakt zu bestimmen. M.a.W. wollen wir die Varietät(en) des Deutschen rekonstruieren, die von den Missionaren ${ }^{5}$ in Vunapope im Bismarck-Archipel um 1900, am Ort und zum Zeitpunkt der Entstehung von Unserdeutsch, gesprochen wurde(n). ${ }^{6}$ Dazu werden im Folgenden (a) als Superstrattransfer erklärbare linguistische Strukturmerkmale in Unserdeutsch sowie (b) zur Verfügung stehende, einschlägige historische Quellen näher untersucht.

Diese Richtung der Fragestellung wurde allem Anschein nach bislang systematisch (und dort sehr detailliert) vor allem bei der Bestimmung von Substratvarietäten verfolgt. Die genaue Bestimmung der Superstratvarietät scheint in der Kreolistik - abseits der World Englishes (etwa Hickey 2005) - vor allem nur dann relevant zu werden, wenn mehrere distinkte Sprachen als Superstrat

5 Die Bezeichnung Missionar referiert im Text sowohl auf weibliche als auch auf männliche Ordensangehörige, d.h. auf Missionsschwestern, Patres und auch auf nicht geweihte Ordensbrüder, die in Vunapope tätig waren.

6 Zur Entstehung von Unserdeutsch s. Maitz (2017). 
in Frage kommen (etwa im Fall des spanisch-/portugiesisch-lexifizierten Papiamentu, vgl. bspw. Jacobs 2009). In Fällen eindeutig englisch-, französisch- oder anderweitig lexifizierter Kreols wurde und wird eine genauere Bestimmung des Superstrats hingegen selten versucht.

Eine solche Superstratbestimmung soll im Folgenden für Unserdeutsch geleistet werden. Die Rekonstruktion des sprachlichen Superstrats ist in diesem Fall nicht nur deswegen von Interesse, weil diese Frage bis heute nie systematisch untersucht wurde, sondern vor allem auch deswegen, weil in der Fachliteratur faktisch inkorrekte Annahmen diesbezüglich kursieren. Nachdem diese problematischen Annahmen teils sogar der Erklärung struktureller Merkmale von Unserdeutsch zugrunde gelegt wurden (bspw. Frowein 2006: 28-29), ist die Klärung der Superstratfrage umso dringender. Nicht zuletzt ist die systematische Analyse der Superstrateinflüsse in Unserdeutsch auch für die deutsche Sprachgeschichte und die historische Variationslinguistik von besonderem Interesse. Die als Superstrattransfer erklärbaren Strukturmerkmale in Unserdeutsch liefern nämlich wertvolle und einzigartige empirische Evidenz zur Rekonstruktion von gesprochener deutscher Alltagssprache um 1900, wie sie zuletzt auch von Ganswindt (2017) versucht wurde. Als wichtigste Superstratschicht von Unserdeutsch kann nämlich, so unsere Ausgangshypothese, das naturgemäß regional gefärbte gesprochene Alltagsdeutsch angenommen werden, das von den deutschen Missionaren in Vunapope gesprochen wurde und das somit (neben dem schulischen Schriftstandard) den Großteil des sprachlichen Inputs für die mixed-race Kinder an der Missionsstation ausmachte. Ein bedeutender Teil der Regeln und Strukturen dieser Alltagssprache ist - wie eine Art sprachlicher Einschluss - in Unserdeutsch konserviert worden und bietet heute Einblicke in die regionalen Gebrauchsstandards, die die deutschen Missionare aus ihrer Heimat in den Bismarck-Archipel gebracht haben.

Im Sinne des Gesagten behandelt dieser Aufsatz zwei Forschungsfragen:

(F1) Wo lässt sich das rekonstruierbare Unserdeutsch-Superstrat im geschlossenen deutschen Sprachraum verorten?

(F2) In welchem Segment der Dialekt-Standard-Achse lässt sich das rekonstruierbare Unserdeutsch-Superstrat lokalisieren?

Als empirische Grundlage zur Beantwortung dieser Fragen dienen primär linguistische Daten. Erstens die diatopisch markierten linguistischen Merkmale in den soziolinguistischen Interviews und Fragebuch-Übersetzungen mit Unserdeutsch-Sprechern der letzten, dritten Sprechergeneration (rund 70 Std., erhoben 2014-2017 von Péter Maitz und Craig A. Volker) sowie in den erhalten gebliebenen Interviewresten mit der zweiten und dritten Sprechergeneration 
(ca. 1 Std., erhoben 1979 von Craig A. Volker). ${ }^{7}$ Zweitens wurden auch relevante Schriftzeugnisse auf diatopisch markierte Strukturen hin untersucht und ausgewertet. Hierbei handelt es sich einerseits um in deutscher Sprache, in neuguineischem Standarddeutsch (sic!) also, verfasste Schriftstücke von UnserdeutschSprechern der ersten und zweiten Sprechergeneration, die - im Gegensatz zur heute lebenden, letzten - auch noch eine schriftliche und mündliche Standarddeutschkompetenz hatten (vgl. Maitz 2017; Volker 1982). Andererseits sind auch die wenigen überlieferten Briefe der Herz-Jesu-Missionare selbst ausgewertet worden, die während der deutschen Kolonialzeit in Vunapope im Einsatz waren und somit als die Sprecher des hier zu bestimmenden Unserdeutsch-Superstrats gelten dürfen.

Ergänzend bzw. stützend werden historische Quellen herangezogen, um die regionale Herkunft der Patres, Brüder und Schwestern, die um die Jahrhundertwende in Vunapope tätig waren, im deutschen Sprachraum über deren Geburtsort zu ermitteln. Einerseits handelt es sich dabei um Handbücher mit biographischen Informationen über die Südsee-Missionare (Jassmeier 1971; Mückler 2010, 2014), um historische Studien (v. a. Waldersee 1995; Steffen 2012; Loosen 2014) sowie relevante Hinweise in Publikationen von Herz-Jesu-Missionaren (v. a. Hiltruper Monatshefte; Rascher 1909; Hüskes 1932). Andererseits sind es Archivalien des Generalarchivs der Missionsschwestern vom Hlst. Herzen Jesu in Hiltrup-Münster (GAMS), des Provinzarchivs der Hiltruper Missionare (PAHM) und des Archivs der Erzdiözese Rabaul in Vunapope (AEDR).

Zur arealen Verortung der einzelnen Merkmale werden in erster Linie, soweit vorhanden, einschlägige Sprachatlanten - wie der Atlas der deutschen Alltagssprache [AdA], der Wortatlas der deutschen Umgangssprachen [WdU], der dtvAtlas Deutsche Sprache und der Norddeutsche Sprachatlas [NOSA] - herangezogen. Darüber hinaus und im Fall, dass zum jeweiligen Merkmal keine sprachgeografischen Aussagen zur Verfügung stehen, wird weitere einschlägige Forschungsliteratur miteinbezogen.

Im Aufbau der Studie folgt nun eine knappe Darstellung zum Kontext der Fragestellung: zum soziokommunikativen Kontext der Entstehung von Unserdeutsch sowie $\mathrm{zu}$ bisherigen Hypothesen $\mathrm{zu}$ seinem sprachlichen Superstrat (Abschnitt 2). Darauf folgt nach einer kurzen methodologischen Reflexion die Analyse und Interpretation linguistischer Evidenz zur Rekonstruktion des sprachlichen Superstrats bei der Genese von Unserdeutsch (Abschnitt 3). In Abschnitt 4 wird anschließend die stützende extralinguistische Evidenz vorgestellt und ausgewertet, während im abschließenden Abschnitt 5 die Ergebnisse

7 Zur Methodologie der Datenerhebung und zu den Daten s. Maitz, König \& Volker (2016) und Götze et al. (2017). 
zusammengefasst und im breiteren kreolistischen und variationslinguistischen Kontext interpretiert werden.

\section{Was man schon weiß oder zu wissen glaubt}

Über die soziokommunikativen Hintergründe und Prozesse der Entstehung von Unserdeutsch ist an anderer Stelle bereits ausführlich geschrieben worden (vgl. Maitz 2017; Volker 1982). Daher seien an dieser Stelle nur diejenigen wichtigsten Momente aus dieser Sprachentstehungsgeschichte kurz zusammengefasst, die zur Beantwortung der hier behandelten Forschungsfragen (F1) und (F2) unerlässlich sind und bei unserer Argumentation im Folgenden vorausgesetzt werden.

Unserdeutsch, die einzige deutsch-lexifizierte Kreolsprache weltweit, entstand zu Beginn des 20. Jahrhunderts an der Missionsstation der katholischen Herz-Jesu-Missionare in Vunapope in der damaligen deutschen Südpazifikkolonie Deutsch-Neuguinea. Kinder vorwiegend gemischter (europäischmelanesischer bzw. asiatisch-melanesischer) Herkunft wurden hier in einer Internatsschule gesammelt, erzogen und unterrichtet (vgl. Janssen 1932). Der größte gemeinsame sprachliche Nenner war für diese Kinder bei ihrer Ankunft eine mehr oder minder ausgeprägte Kompetenz in einer frühen Form des Tok Pisin, der Hauptverkehrssprache der damaligen Kolonie und noch heute in Papua-Neuguinea. Tok Pisin diente dann weiterhin der Kommunikation mit Indigenen und Migranten, war den Kindern jedoch im Umgang untereinander untersagt (vgl. Volker 1991: 46). Stattdessen war die Sprache sowohl des Unterrichts als auch des täglichen Umgangs an der Mission das Deutsche, in dem die Kinder eine solide schriftliche und mündliche Kompetenz erworben hatten.

Dieser gesteuert wie ungesteuert verlaufende, immersive Erwerbsprozess des Deutschen hatte eigenartigerweise ${ }^{8}$ zwei Endpunkte (vgl. Maitz 2017). Der übliche Weg über individuelle, instabile Lernervarietäten führte auf der einen Seite zum tatsächlichen Erwerb des Standarddeutschen, auf der anderen Seite aber parallel dazu auch zur Stabilisierung und gruppeninternen Etablierung dieser von umfangreichem L1-Transfer geprägten Lernervarietäten in der primären Funktion als „medium for community solidarity“ (Baker 2000). Die Stabilisierung und Konventionalisierung dieser Lernervarietäten erfolgte offensichtlich relativ früh und zumindest zum Teil auch bewusst und führte zur

8 Für eine Übersicht der typischen und weniger typischen soziohistorischen Umstände der Unserdeutsch-Genese vgl. Lindenfelser \& Maitz (2017). 
Herauskristallisierung einer vereinfachten und restrukturierten Kontaktvarietät, die sich als Pidginoid fassen lässt (vgl. Maitz 2017): ihrer Struktur nach weitgehend anderen Pidgins ähnlich, funktional aber insofern nicht, als sie ausschließlich innerhalb der in-group Kommunikation im Gebrauch war. Infolge der Endogamie der aufwachsenden Missionskinder wurde dieses Pidginoid als Erstsprache an die Folgegeneration weitergegeben, und Unserdeutsch war somit bereits in der Zwischenkriegszeit kreolisiert.

Mit der australischen Okkupation des Kolonialgebiets bei Ausbruch des Ersten Weltkriegs und ganz besonders mit der japanischen Übernahme zu Beginn des Zweiten Weltkriegs nahm die Rolle des Englischen an der Mission stark zu. Im Leben der heutigen, dritten und aller Wahrscheinlichkeit nach letzten Sprechergeneration, ${ }^{9}$ die zum größten Teil mit der Unabhängigkeit Papua-Neuguineas 1975 nach (Ost-)Australien auswanderte und bis heute dort lebt, hat die australisch-englische Sprachdominanz noch weiter zugenommen. Das linguistische Ergebnis ist eine Reihe von englischen Kontakteinflüssen in Unserdeutsch. Davon unabhängig gilt allerdings weiterhin und uneingeschränkt, dass die Lexik von Unserdeutsch zum weit überwiegenden Teil seiner Superstratsprache - dem Deutschen - entstammt, während das strukturelle Profil der Sprache von der Phonologie bis hin zur Syntax in weiten Teilen als Substrattransfer aus Tok Pisin erklärt werden kann (vgl. Lindenfelser \& Maitz 2017; Maitz, Lindenfelser \& Volker i.E.).

Doch welches Deutsch hat nun als Superstrat seine Spuren in der Lexik und zu einem geringeren Teil auch in der Struktur von Unserdeutsch hinterlassen? Die einzigen bislang - wenn auch eher impressionistisch - ausformulierten diesbezüglichen Hypothesen gehen auf Volker (1982) zurück. Er geht zunächst davon aus, dass wahrscheinlich ein Bündel von (kolonial-)deutschen Nonstandardvarietäten als das Superstrat von Unserdeutsch fungierte:

[T]his does not imply that Rabaul Creole German was derived from Standard German. More probably its parents were the various German dialects spoken in the Gazelle Peninsula during the German colonial period. (Volker 1982: 18)

Diese Annahme impliziert, dass für die sprachliche Sozialisation der mixedrace Kinder in Vunapope nicht allein das Deutsch der Schwestern, Brüder und Patres an der Missionsstation eine prägende Rolle spielte, sondern potenziell alle Varietäten, die auf der Gazelle-Halbinsel, im Umfeld der Missionsstation also, gesprochen wurden. Volker muss auf diese Annahme zurückgreifen, um einen scheinbaren Widerspruch auflösen zu können. Dieser ergibt sich aus

\footnotetext{
9 Zu Unserdeutsch als stark gefährdeter Sprache vgl. Maitz \& Volker (2017).
} 
dem Umstand, dass er auf der einen Seite die Missionare ihrer Herkunft nach mehrheitlich dem süddeutsch-österreichischen Raum als Hochburg des Katholizismus zuordnet:

This usage undoubtedly reflects the fact the vast majority of the teachers of the first Vunapope Germans would have come from the Catholic areas of southern Germany and Austria. (Volker 1982: 43)

Dem steht allerdings auf der anderen Seite die Feststellung gegenüber, dass zumindest in der Phonologie die Mehrzahl der Abweichungen vom Standarddeutschen als norddeutsches Merkmal identifiziert werden kann (vgl. Volker 1982: 18) und nur wenige als süddeutsch. Während sich die süddeutschen Varianten nach Volkers Meinung aus der postulierten Herkunft der Missionare aus dem katholischen Süden erklären, werden die norddeutschen Varianten auf zweifache Weise motiviert. Erstens durch die norddeutsche Dominanz in der kolonialen Verwaltung und der Seefahrt, und zweitens dadurch, dass das Deutsch der nicht wenigen niederländischen Missionare an der Missionsstation in Vunapope dem Norddeutschen nähergestanden haben dürfte als dem Süddeutschen (vgl. Volker 1982: 18). Eine auch nur einigermaßen systematische, philologisch gestützte, sprachgeografische Spurensuche wird jedoch von Volker nicht unternommen. Über die oben genannten allgemeinen Behauptungen hinaus finden sich in seiner Arbeit nur sehr vereinzelt Aussagen zu konkreten sprachlichen Merkmalen. Während seine Behauptung zur norddeutschen Dominanz in der unserdeutschen Phonologie nicht belegt und weiter differenziert wird, weist er - und im Anschluss an ihn auch Mühlhäusler (1997: 200) immerhin in Bezug auf das einzige analytische Vergangenheitstempus in Unserdeutsch auf die diesbezügliche Parallelität zu den süddeutschen Dialekten hin (vgl. Volker 1982: 43; s. dazu Abschnitt 3.2). Zusammenfassen lässt sich Volkers Position damit, dass er das sprachliche Superstrat von Unserdeutsch in einem Nebeneinander nord- und süddeutscher Dialekte zu erkennen glaubt, wobei aufgrund der von ihm postulierten Herkunft der Missionare eigentlich eine Dominanz als süddeutsch identifizierbarer Varianten zu erwarten wäre - was sich allerdings so in seinen Daten und Beschreibungen nicht unbedingt zeigt.

Deutlich stärker formuliert wird die These zum Unserdeutsch-Superstrat von Frowein (2006), wenngleich ohne jegliche explizite Beweisführung bzw. Begründung. Er geht klar und eindeutig von einem - nicht weiter spezifizierten - süddeutschen dialektalen Superstrat aus, indem er im Zusammenhang mit einer nicht unproblematischen Argumentation zu unserdeutschen Possessivkonstruktionen feststellt, „[t]he superstrate variants which are assumed to have participated in the Unserdeutsch creolization process [...] were dialects of southern areas“ (Frowein 2006: 29). 
Vor dem Hintergrund dieser - zugegebenermaßen eher vereinzelten und wenig belastbaren - Aussagen soll nun im Folgenden auf solider empirischer Basis das sprachliche Superstrat von Unserdeutsch möglichst genau bestimmt werden. Gleichzeitig sei jedoch betont, dass es uns dabei allein schon aus Umfangsgründen nicht um die exhaustive Inventarisierung und Interpretation aller diatopisch markierten deutschen Elemente in Unserdeutsch geht. Wir wollen und dürfen es nicht riskieren, vor lauter Bäumen den Wald aus den Augen $\mathrm{zu}$ verlieren. Daher wird unser Fokus auf diejenigen fundamentalsten und salientesten Strukturmerkmale in Unserdeutsch gerichtet sein, die in ihrer Kookkurrenz im Idealfall die Konturen derjenigen binnendeutsche(n) Varietät(en) erkennen lassen, die als wichtigste Superstratvarietät(en) an der Entstehung von Unserdeutsch beteiligt war(en).

\section{Was die empirischen Daten zeigen}

$\mathrm{Zu}$ Beginn unserer Analysen sei festgehalten, dass wir die Superstratsprache von Unserdeutsch im Gegensatz zu Volkers Meinung (1982: 18) ausschließlich innerhalb der Grenzen der Missionsstation von Vunapope suchen. Aufgrund der zur Verfügung stehenden Informationen erscheint es nämlich zumindest fragwürdig, in welchen Kontexten und auf welche Art und Weise die Sprache der deutschen Verwaltungsbeamten und Seeleute den Sprachgebrauch der an der Mission in sozialer Isolation lebenden Kinder hätte beeinflussen und prägen können. Wir gehen also davon aus, dass das sprachliche Superstrat von Unserdeutsch mit dem sprachlichen Input identifiziert werden kann, den die mixed-race Kinder von Vunapope in den ersten Jahren bzw. Jahrzehnten des 20. Jahrhunderts von den deutschen Missionaren in Schule und Alltag bekommen haben.

Die Erziehungs- und Bildungsstandards in Vunapope waren - bis hin zur Anstandslehre als Schulfach (vgl. Hiery 2001: 209) - weitgehend von den zeitgenössischen deutschen Bildungs- und Erziehungsnormen geprägt (vgl. Janssen 1932: $151 \mathrm{f}$.). In diesem Sinne können wir hinsichtlich des Grades der diatopischen Markiertheit davon ausgehen, dass das Superstrat von Unserdeutsch im Gegensatz zu Volkers oben zitierter Annahme auf jeden Fall standardnah gewesen sein muss. Die Schriftstandardkompetenz der ersten und zweiten Sprechergeneration von Unserdeutsch ist tatsächlich auch überliefert (vgl. Maitz 2017). Über den schulischen, formellen Schriftstandard hinaus wird jedoch vor allem auch das gesprochene Alltagsdeutsch der Missionare eine dominante Superstratrolle gespielt haben, da ja der Großteil der Alltagskommunikation an der Mission offensichtlich in dieser Sprachlage stattfand. Aufgrund 
der bildungs- und schriftorientierten Lebenswelt der Missionsstation, die mit ihren Internaten und Schulen als das geistige Zentrum der Insel galt (vgl. Gründer 2004: 115), kann davon ausgegangen werden, dass auch dieses gesprochene Alltagsdeutsch standardnah gewesen sein muss.

Es besteht in der Forschung Einigkeit darüber, dass das gesprochene Standarddeutsch vor der Durchsetzung der präskriptiven Standardnormen noch viel mehr als heute - landschaftlich gefärbt war (vgl. Schmidt 2010; Ganswindt 2017). Es stellt sich daher die Frage, welcher regionale Gebrauchsstandard von den Missionaren gesprochen wurde. Bevor wir dieser Frage im Folgenden mit Hilfe linguistischer Evidenz nachgehen werden, können wir aufgrund ordensgeschichtlicher Gegebenheiten bereits erste Hypothesen formulieren. Die Ordensgemeinschaft der Herz-Jesu-Missionare (Missionarii Sacratissimi Cordis, kurz: MSC), die erst 1854 in Frankreich gegründet wurde, hatte das Stammhaus ihrer deutschen Ordensprovinz in Hiltrup bei Münster eingerichtet. Da der Orden bis zum Ausbau der Südseemission zahlenmäßig sehr klein und auch weltkirchlich unbedeutend war (vgl. Steffen 2001: 343 f.), können wir davon ausgehen, dass sein Einzugsgebiet damals regional beschränkt war. In diesem Sinne lässt sich, anders als von Volker (1982: 18) behauptet, ein vorwiegend westfälisch geprägter mündlicher Sprachgebrauch unter den deutschen Missionaren in Vunapope vermuten.

Die Anfänge des Sprachkontakts zwischen den deutschen Missionaren und den mixed-race Kindern von Vunapope gehen auf das letzte Jahrzehnt des 19. Jahrhunderts zurück. Eine systematische sprachgeografische Dokumentation zum landschaftlichen Hochdeutsch der Jahrhundertwende gibt es jedoch - im Gegensatz zu den deutschen Dialekten - leider nicht. Daher werden wir uns bei unserem sprachgeografischen Puzzlespiel zwangsläufig auf spätere bzw. aktuelle linguistische Darstellungen - wie zum Beispiel den AdA (2003 ff.) - beziehen müssen. Ein solches Verfahren setzt natürlich die stillschweigende Annahme einer grundsätzlichen Konstanz der sprachgeografischen Verhältnisse voraus, was zu methodologischer Sorgfalt auffordert. Sofern jedoch zeitgenössische linguistische Evidenz zur Verfügung steht und uns bekannt ist, werden wir selbstverständlich diese bevorzugen.

\subsection{Lautliches}

Vertieft man sich in die Interviewdaten, die zwischen 2014 und 2017 im Rahmen des Augsburger Dokumentationsprojekts erhoben worden sind, so fällt einem die schwache regionale Markiertheit der linguistischen Strukturmerkmale auf, die aus dem Deutschen, d.h. als Superstrateinfluss, erklärt werden können. Diese Standardnähe charakterisiert das gesamte Spektrum des Kreol- 
Kontinuums (vgl. Maitz 2017) und lässt sich im Einklang mit unserer oben formulierten Hypothese als linguistische Evidenz dafür deuten, dass das sprachliche Superstrat von Unserdeutsch im Gegensatz zu früheren Annahmen standardnah gewesen sein muss.

In Bezug auf die segmentale Phonologie muss - in striktem Gegensatz zu Volkers (1982: 18) diesbezüglicher Meinung - grundsätzlich festgehalten werden, dass das Phonemsystem von basilektalem Unserdeutsch weitgehend mit dem von Tok Pisin identisch ist (vgl. Maitz, Lindenfelser \& Volker i. E.). Auf den ersten Blick könnte man bestimmte kookkurrente lautliche Merkmale zweifelsfrei als süddeutsch einordnen. So etwa die Absenz von stimmhaftem [z] (vgl. [si] - ,sie‘, [disc] - ,diese‘), die apikale Realisierung des konsonantischen $r$ (vgl. [kakaruk] - ,Huhn', [friftik] - ,Frühstück'), oder auch die Formen des Personalpronomens der 1. P. Sg. Nom. und Akk. $i$ - [i] und mi - [mi], um nur einige Beispiele zu nennen. Dennoch handelt es sich bei diesen Beispielen, so unsere These, nicht um die Reflexe von süddeutschen lautlichen Regionalismen. Vielmehr sind diese Lautungen im Zusammenhang der Gesamttendenz in basilektalem Unserdeutsch - und in Kreolsprachen allgemein - zu sehen, typologisch unübliche, markierte Vokale und Konsonanten ihrer Lexifikatorsprache abzubauen bzw. zu ersetzen. Die Absenz des stimmhaften [z] oder auch des velaren/uvularen $r$ fügen sich in eine ganze Reihe von am Tok Pisin ausgerichteten Adaptationen standarddeutscher Phoneme (vgl. Maitz, Lindenfelser \& Volker i.E.). Zu diesen Adaptationsphänomenen gehört nicht zuletzt auch der Abbau der standarddeutschen komplementären Allophone [ç] und [x], die in Tok Pisin ebenfalls grundsätzlich nicht vorhanden sind. Dementsprechend werden sie in Unserdeutsch in wortmedialer Onset-Position in der Regel durch [h] substituiert (vgl. [lahen] - ,lachen“, [methen] - ,Mädchen'), in Koda-Position hingegen tendenziell entweder geschwächt oder ersatzlos getilgt: [ni] - ,nicht‘, [ta] - ,Tag‘, [filar] - ,vielleicht‘ etc. In diesem Sinne sind also auch die Formen des Personalpronomens der 1. P. Sg. $i-[\mathrm{i}]$ und $m i-[\mathrm{mi}]$ weniger mit einem süddeutschen Superstrat zu erklären, vielmehr werden sie wie zahlreiche andere, einschlägige Wortformen in basilektalem Unserdeutsch auch - durch folgende Adaptationsregeln erzeugt:

$$
\begin{aligned}
& \text { STD [ç] } \rightarrow \text { UD } \varnothing /{ }_{-} \$ \\
& \operatorname{STD}[\chi] \rightarrow \text { UD } \varnothing /{ }_{-} \$
\end{aligned}
$$

Es gibt im Wesentlichen nur ein einziges, eindeutig als Superstrattransfer erklärbares, segmentalphonologisches Merkmal in Unserdeutsch, das zwar durchaus (bis heute noch) zum gesprochenen Gebrauchsstandard des Deutschen gehört, dennoch aber eine einigermaßen klare regionale Zuordnung ermöglicht. Gemeint ist das im Deutschen als $g$-Spirantisierung bekannte Phänomen bzw. des- 


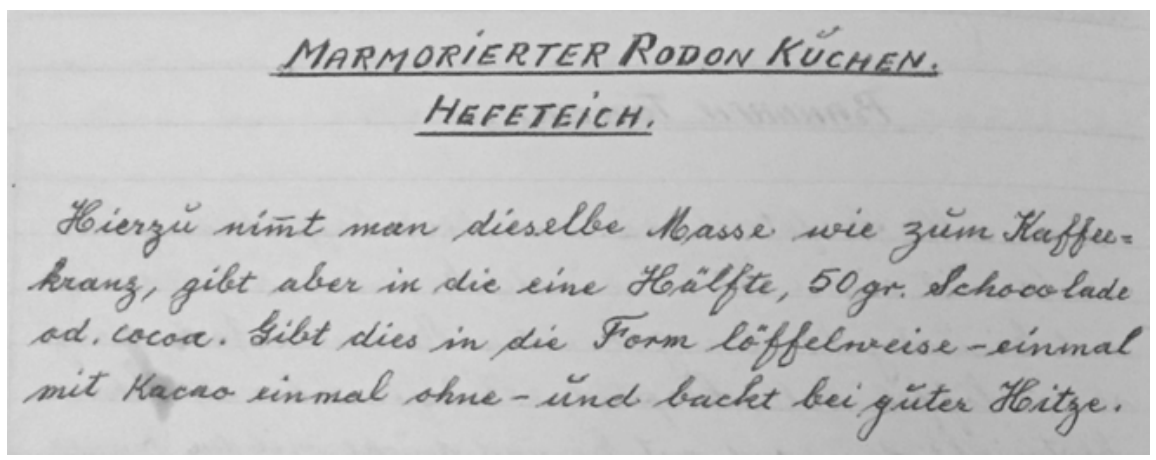

Abb. 13.1: Ausschnitt aus einem handschriftlichen Rezeptbuch eines Unserdeutschsprechers, geschrieben in neuguineischem Standarddeutsch.

sen Reflexe. Die Spuren der $g$-Spirantisierung lassen sich in Unserdeutsch in systematischer Weise erkennen, und zwar in unterschiedlichen Formen, mit unterschiedlichen Reduktionsgraden. Vor allem in meso- und akrolektalen Varietäten, seltener aber auch im Basilekt, bleibt der stimmlose Frikativ am Wortende erhalten, vgl. [kriç] - ,Krieg‘, [tax] - ,Tag‘ etc. Im Basilekt kann er bisweilen artikulatorisch geschwächt erscheinen, in der Regel wird er jedoch vollständig getilgt; vgl. [ta] - ,Tag‘, [gesa] - ,gesagt‘, [tswantsi] - ,zwanzig‘ etc. Reflexe der $g$-Spirantisierung finden sich sogar im Schriftstandard der auch noch im Standarddeutschen kompetenten, zweiten, heute nicht mehr lebenden Sprechergeneration, etwa in Form von Schreibungen wie $<$ Mürbeteich $>$, $<$ Hefeteich>, <Zwiebackteich> etc. (vgl. Abb. 13.1).

Von der $g$-Spirantisierung wissen wir, dass sie in der gesprochenen Alltagssprache vor allem in Mittel- und Norddeutschland großräumig verbreitet ist (vgl. Kleiner 2011 ff.; AdA 2003 ff.: Tag, Zeug, König, wenig, zwanzig). Und da dieses Phänomen in Unserdeutsch, wie gesagt, in systematischer Weise nachgewiesen werden kann, spricht dieser Befund insgesamt dafür, das dominante sprachliche Superstrat von Unserdeutsch in Mittel- und Norddeutschland, auf jeden Fall aber nicht in Süddeutschland zu suchen. Die Daten des NOSA (2015: 261) zeigen für Westfalen selbst in den standardnächsten Sprachlagen eine auch innerhalb Norddeutschlands überdurchschnittlich starke Verbreitung der $g$-Spirantisierung. Diese Daten wiederum unterstützen unsere vorhin formulierte Vermutung, wonach das Alltagsdeutsch der Missionare in Vunapope vorwiegend westfälisch geprägt gewesen sein könnte. ${ }^{10}$

10 Ebenfalls in diese Richtung könnte die tendenzielle Deaffrizierung von anlautendem [pf] in Unserdeutsch gedeutet werden, da dieses Phänomen wiederum gerade in Westfalen auffal- 


\subsection{Grammatisches}

Wie schon im Bereich der Phonologie, so lässt sich auf der Ebene der Grammatik eindeutig feststellen, dass Unserdeutsch keine als Superstrattransfer interpretierbaren Strukturmerkmale aufweist, die man nur bzw. eindeutig aus kleinräumigen, dialektalen Varietäten des Deutschen erklären müsste bzw. überhaupt könnte. Jeder grammatische Transfer aus dem Deutschen kann im Bereich des Gebrauchsstandards lokalisiert werden.

Wie oben bereits erwähnt, weist Volker in seiner Arbeit (1982: 43) auf die Parallele im Tempusgebrauch zwischen Unserdeutsch und den süddeutschen Dialekten hin. Seiner Meinung nach sei das - jenseits von Hochfrequenzverben - einzige, analytische Vergangenheitstempus in Unserdeutsch, das dem deutschen Perfekt entsprechend gebildet werde, mit einem süddeutschösterreichischen Superstrat erklärbar (Volker 1982: 43). Demgegenüber liegt in unseren Augen auch hier - ähnlich zu den lautlichen Parallelen (s. Abschnitt 3.1) - lediglich eine Scheinkorrelation vor. Vor dem Hintergrund jüngst erhobener Daten ist Volker zuzustimmen, dass Unserdeutsch nur bei der Kopula sein sowie bei den Modalverben synthetische Präteritalformen kennt. Im System der Sprache ist tatsächlich nur ein aus dem deutschen Perfekt ableitbares, in seiner Bildungsweise aber teilweise reanalysiertes, analytisches Vergangenheitstempus verankert, dessen Gebrauch jedoch - wie die grammatische Tempusmarkierung in Unserdeutsch überhaupt - hochgradig fakultativ ist (vgl. Lindenfelser \& Maitz 2017). Dieses Tempussystem weist zwar in der Tat Ähnlichkeiten zu süddeutschen gesprochenen Varietäten auf, die Ähnlichkeit scheint aber lediglich eine akzidenzielle zu sein.

Der Abbau von morphologischen Kategorien - wie zum Beispiel auch des Präteritums - gehört zu den wichtigsten Formen zweitspracherwerbsbedingter Simplifizierungen. Dementsprechend ist der Verlust synthetisch markierter, morphologischer Kategorien in den unterschiedlichsten high-contact Varietäten der Welt (Pidgins, Kreols, mixed languages, Lernervarietäten etc.) zu beobachten (vgl. Mühlhäusler 2001; Trudgill 2010: 307 f.; Harnisch 2004: 527). Auch der Abbau bzw. die Meidung des Präteritums ist - neben einer ganzen Reihe von weiteren Kategorienverlusten - keineswegs nur in Unserdeutsch, sondern ebenso auch in anderen high-contact Varietäten bzw. Sprachen anzutreffen, so etwa in Namibian Black German (vgl. Deumert 2003: 578), im Jiddi-

lend stark verbreitet ist (vgl. König 1989: 259). Da jedoch von dieser Deaffrizierungstendenz in Unserdeutsch auch die dentale Affrikate betroffen ist (vgl. etwa [flansur] - ,Pflanzung‘, [susamen] - ,zusammen'; s. dazu Maitz, Lindenfelser \& Volker i.E.), dürfte es sich hierbei durchaus auch um eine am Tok Pisin ausgerichtete Phonemadaptation, d. h. um eine kontaktinduzierte Simplifizierung handeln. 


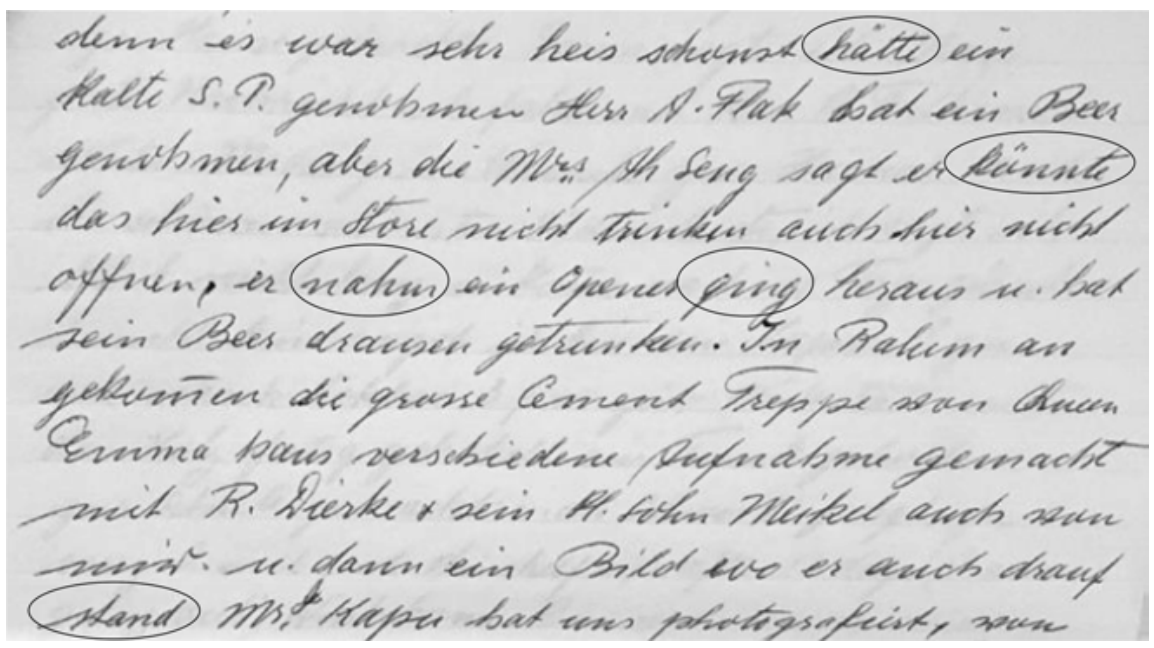

Abb. 13.2: Tagebucheintrag eines Unserdeutschsprechers in neuguineischem Standarddeutsch aus der Nachkriegszeit.

schen (vgl. Timm 1986), im sog. Gastarbeiterdeutsch (vgl. Keim 1978: 156 ff.) oder in Lernervarietäten des Deutschen. Der Erhalt von Präteritalformen bei Auxiliar-, Kopula- und/oder Modalverben, wie wir ihn in allen betroffenen Varietäten vorfinden können, ist unzweifelhaft als sprachunabhängiger Frequenzeffekt zu werten. Vor diesem Hintergrund scheint Volkers Interpretation zum Vergangenheitstempus in Unserdeutsch etwas voreilig und nicht ganz plausibel. Der Verlust des Präteritums bzw. der Erhalt eines einzigen analytischen Vergangenheitstempus in Unserdeutsch fügt sich, wie gesagt, in eine grundlegende, kontaktinduzierte, typologische Simplifizierungstendenz und muss folglich auch im Kontext dieser gesehen und erklärt werden. Dass der Abbau des Präteritums in Unserdeutsch tatsächlich nicht auf die strukturelle Beschaffenheit des sprachlichen Superstrats an der Missionsstation in Vunapope zurückgeführt werden kann, belegt nicht zuletzt auch empirische Evidenz. Im geschriebenen Standarddeutsch von Unserdeutschsprechern der zweiten Generation sind Präteritalformen systematisch belegt (s. Abb. 13.2).

Ein fundamentales Systemmerkmal von Unserdeutsch, das eindeutig auf Superstrattransfer zurückgeführt werden kann und auch eine räumliche Zuordnung im geschlossenen Sprachgebiet ermöglicht, ist die Konstruktion (sein) + $a m /(a n)+V E R B$, die formal im Wesentlichen dem deutschen sog. am-Progressiv entspricht (vgl. Lindenfelser \& Maitz 2017). Diese als Aspektmarker fungierende, stark grammatikalisierte Konstruktion kann (auch) in Unserdeutsch entweder Progressivität - wie in (1) - oder Habitualität - wie in (2) - ausdrücken: 
(1) er is am predig-en $\mathrm{zu}$ mich

3SG.M COP.3SG PROG preach-V to 1sG.ACC

,Er hat auf mich eingeredet (mir eine Predigt gehalten).

(2) jeden tach fi drei wohe i war am spreh-en mit sie every day for three week 1SG COP.PST HAB talk-v with 3sG.F ,Drei Wochen lang habe ich jeden Tag mit ihr gesprochen.'

Die Konstruktion taucht aber auch im geschriebenen deutschen Gebrauchsstandard der Vunapope mixed-race Gemeinschaft, etwa in Privatbriefen, auf:

(3) mama ist immer noch ganz fleißig am einrichten. [Karl Hoerler, 6. 8.1939]

Vor allem hinsichtlich ihres Grammatikalisierungsgrades und ihrer Syntax (bei Objekterweiterung) mag diese Konstruktion nicht unerhebliche, zum Teil offenbar kontaktinduzierte Unterschiede $\mathrm{zu}$ ihrem deutschen Äquivalent zeigen (vgl. Gárgyán 2014; Volker 1982; Lindenfelser \& Maitz 2017). Ihre Form ist aber eindeutig und monokausal als Superstrattransfer motivierbar. Jüngere Erhebungen zeigen, dass diese Konstruktion in der gesprochenen Alltagssprache heute in fast ganz Deutschland, besonders aber im Westen, verbreitet ist (vgl. AdA 2003 ff.: Verlaufsformen). Dies scheint jedoch während der deutschen Kolonialzeit in der Südsee (1884-1914) noch ganz anders gewesen zu sein. Elspaß (2005: 268-275) konnte die am-Konstruktion in der geschriebenen Alltagssprache des (späteren) 19. Jahrhunderts ausschließlich bei Schreibern aus Westfalen und dem Rheinland belegen. Diese historische Evidenz steht sowohl mit unseren Hypothesen in Abschnitt 3 als auch mit unserem lautlichen Befund in Abschnitt 3.1 im Einklang und unterstützt die Annahme, dass das dominante sprachliche Superstrat von Unserdeutsch, d. h. die Alltagssprache in Vunapope um 1900, höchstwahrscheinlich westfälisch, auf jeden Fall aber nordwestdeutsch geprägt gewesen sein muss.

Dafür sprechen nicht zuletzt auch Daten zur Syntax des am-Progressivs in Unserdeutsch. Im Falle einer Objekterweiterung folgt das Objekt in basilektalem Unserdeutsch, wie schon bei Volker (1982: 46) ausgeführt, in aller Regel dem Verb - vgl. (4). Dies entspricht syntaktisch der englischen ProgressivKonstruktion und kann somit als syntaktischer Adstrattransfer erklärt werden. Es muss allerdings auch ein möglicher Substrateinfluss von Tok Pisin in Betracht gezogen werden, da Tok Pisin - noch mehr als Englisch - eine strikte SVO-Sprache ist.

(4) wi war imma am stehl-en alle pomelo 1PL COP.PST always PROG steal-V PL pomelo ,Wir haben ständig Pomelos geklaut.‘ 
Daten aus Sprachlagen in der Nähe des akrolektalen Pols im Kreol-Kontinuum wie in (5) - zeigen jedoch auch das syntaktische Muster des deutschen amProgressivs mit präverbaler Stellung des Objekts:

\section{(5) er is de kartoffel an schäl-en \\ 3SG.M COP.3SG ART.DEF potato PROG peel-V \\ ,Er schält die Kartoffeln.}

Diese syntaktische Konstruktion scheint eindeutig als Superstrattransfer aus dem Deutschen erklärbar zu sein. Objekterweiterte am-Progressive dieses Typs sind im Deutschen bis heute noch regional eingeschränkt verbreitet, wiederum vor allem auch im Nordwesten (AdA 2003 ff.: Verlaufsformen). Vor dem Hintergrund der sprachhistorischen Evidenz von Elspaß (2005) liegt hier somit ein weiterer Hinweis auf ein nordwestdeutsches Superstrat vor. Andererseits zeigt das Vorhandensein dieser Konstruktion in Unserdeutsch zugleich auch, dass der erweiterte am-Progressiv im regionalen Gebrauchsstandard schon um 1900 etabliert war.

Ein weiteres grammatisches Merkmal, das eine sprachgeografische Lokalisierung des sprachlichen Superstrats ermöglichen könnte, stellen die diskontinuierlichen Pronominaladverbien dar. Diese sog. Spaltungskonstruktionen (vgl. Negele 2012: 73) kommen auch in Unserdeutsch vor, sind im Korpus allerdings ausschließlich mit dem Pro-Adverb wo belegt:

\section{a. ein leben wo i war richti froh mit} ART.INDF life that 1SG COP.PST really happy with ,Ein Leben, mit dem (womit) ich richtig zufrieden war.‘

b. ni weiß wo die komm von not know where 3PL come from ,Weiß nicht, woher (wovon) sie kommen.'

Von diesen Spaltungskonstruktionen wissen wir, dass sie sich im 20. Jahrhundert zwar nach Süden ausgebreitet haben (vgl. AdA 2003 ff.: damit, davon, daran (Präpositionaladv.)), ihr Hauptverbreitungsgebiet im 19. Jahrhundert jedoch eindeutig auf den Norden des deutschen Sprachraums beschränkt war (vgl. Negele 2012: 120 f.) - vor allem auch auf Westfalen, aber außerdem darüber hinaus (vgl. Elspaß 2016: 368f.). Speziell zur Verbreitung der Spaltungskonstruktionen mit wo(r)- haben die Korpusuntersuchungen von Negele (2012: 87) eine klare Ballung im westniederdeutschen Raum (inkl. des Niederfränkischen) ergeben. Diese Befunde dürften wieder für ein westniederdeutsches Superstrat sprechen, wenngleich angemerkt werden muss, dass es sich bei den Spaltungs- 
konstruktionen angesichts des intensiven Kontakts von Unserdeutsch zum Englischen nach 1914 prinzipiell ebenso auch um kontaktinduzierte Reflexe des preposition stranding im Englischen handeln könnte.

\subsection{Lexikalisches}

Im Gegensatz zur Phonologie und Grammatik, die beide von umfangreichem Substrattransfer aus Tok Pisin geprägt sind, geht die Lexik von Unserdeutsch zum weit überwiegenden Teil auf Superstrattransfer aus dem Deutschen zurück (vgl. Maitz, Lindenfelser \& Volker i. E.). Aus diesem Grund ist die Wahrscheinlichkeit auf dieser Ebene der Sprache auch deutlich größer, Regionalismen auf die Spur zu kommen, die eine genauere Bestimmung des Superstrats erlauben.

Erstens muss festgehalten werden, dass es auch auf der Ebene der Lexik keine grundlegenden Merkmale in Unserdeutsch gibt, die man nur bzw. zwangsläufig aus kleinräumigen, dialektalen Varietäten des Deutschen erklären könnte bzw. müsste. Zweitens gilt, dass die Lexik von Unserdeutsch im Hinblick auf regional markierte Superstrateinflüsse ein deutlich differenzierteres Bild zeigt, als wir dies im Bereich der Phonologie und der Grammatik gesehen haben. Während die tiefsten - d.h. die phonologischen und grammatischen - Spuren in Unserdeutsch, wie sich gezeigt hat, eindeutig in Richtung Nordwesten, nach Westfalen, führen, so verzweigen sie auf der sprachlichen Oberfläche, in der Lexik, bereits in unterschiedliche Richtungen.

Im Bereich der geschlossenen Wortklassen zeigt sich ein in Bezug auf unsere Fragestellung auffallendes Merkmal bei den Konnektoren in Relativund Temporalsätzen. In Unserdeutsch werden nämlich Relativsätze und Temporalsätze auch - aber keineswegs immer - mit wo eingeleitet; vgl. (7) und (8).

(7) de ganz-e haus wo is auf de straße

ART.DEF whole-ATTR house REL COP.3SG on ART.DEF street ,die ganzen Häuser, die auf der Straße stehen`

(8) wo mein vater tot bishop Scharmach sa $\mathrm{zu}$ mein when 1.sG.Poss father dead bishop Scharmach say to 1.SG.Poss mutter ... mother ... ,Als mein Vater starb, sagte Bischof Scharmach zu meiner Mutter ...

Vom temporalen wo wissen wir im Spiegel jüngerer Erhebungen, dass es in der gesprochenen deutschen Alltagssprache bis heute vor allem im Süden und ganz besonders im Südwesten des deutschen Sprachgebiets verbreitet ist, wäh- 
rend das relative wo in verdichteter Form ausschließlich im Südwesten vorkommt (vgl. AdA 2003 ff.). Somit liegt in diesem Merkmal ein erster linguistischer Hinweis darauf vor, dass es unter den deutschen Stimmen in Vunapope auch südwestdeutsche Klänge gegeben haben muss.

Hinweise hierauf finden sich auch im Bereich der Wortbildung, und zwar beim Diminutivsuffix. In lexikalisierten Diminutiva und bei Appellativen tritt in Unserdeutsch in der Regel das Diminutivsuffix -chen - im Basilekt in der Regel als [hen] realisiert - auf (vgl. 9). Vor allem bei Rufnamen sind jedoch häufiger auch -le-Varianten wie in (10) belegt, die (auch schon) in der geschriebenen Alltagssprache des 19. Jahrhunderts im Südwesten verbreitet waren (vgl. Elspaß 2005: 343; s. auch König, Elspaß \& Möller 2015: 157).

(9) Olga is de swester-hen, ah, hat bruder-hen Olga COP.3SG ART.DEF sister-DIM ah have brother-DIM ,Olga ist die Schwester, ah, sie hat einen Bruder.'

(10) Marie-chen hat immer ge-wein: mama-le papa-le Hannah-le Marie-DIM AUX.PST always PTCP-cry mum-DIM dad-DIM Hannah-DIM David-le

David-DIM

,Mariechen hat immer geweint: Mamale, Papale, Hannahle, Davidle!

Dieser Umstand deutet ebenfalls auf einen Variatätenkontakt unter den Missionaren in Vunapope und auf das Vorhandensein eines - wenn auch nicht dominanten - südwestdeutschen Elements in der Alltagssprache an der Missionsstation um 1900 hin.

Spuren dieses Varietätenkontakts finden sich nicht zuletzt auch unter den Inhaltswörtern, in den offenen Wortklassen. Auf der einen Seite finden sich im Lexikon von Unserdeutsch, das im Vergleich zu seiner Lexifikatorsprache sehr stark reduziert ist, mehrere Lexeme, die sich mehr oder weniger eindeutig im norddeutschen Raum lokalisieren lassen. $\mathrm{Zu}$ diesen gehört etwa das unserdeutsche Wort fegen wie in (11), dessen quellsprachliches Pendant im Deutschen ausschließlich im Norden, dort aber großräumig, verbreitet ist (vgl. AdA 2003 ff.; s. auch König, Elspaß \& Möller 2015: 234).

(11) Joseph muss alle blätt-er und alles auffeg-en Joseph must all leaf.PL-PL and everything sweep_up-V ,Joseph musste alle Blätter und alles auffegen/aufkehren.

Ebenso in Norddeutschland (vgl. DWDS) - und dabei auch in Westfalen bzw. im Münsterland (vgl. Damme 2011: 349) - verbreitet ist das Wort duhn - 
,betrunken', das in Unserdeutsch in der Form dun - [dun] relativ dicht belegt ist; vgl. (12).

(12) die nu singen wenn die bis dun 3PL only sing-v when 3PL COP drunk ,Sie singen nur, wenn sie betrunken sind.

Ausschließlich im Nordwesten Deutschlands, darunter besonders auch in Westfalen, ist das Wort flöten - ,pfeifen' verbreitet, sowohl in dialektalen als auch in standardnahen Sprachlagen bzw. Varianten des gesprochenen Deutsch (vgl. König, Elspaß \& Möller 2015: 213; WdU 1977: 9). Das Vorhandensein dieses Lexems in Unserdeutsch - vgl. (13) - kann als weiterer Hinweis auf ein westfälischnordwestdeutsches, rheinisch-westfälisches Superstrat bei der Entstehung von Unserdeutsch gewertet werden.

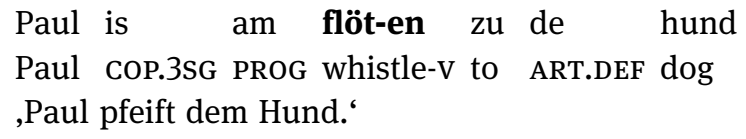

Diese Annahme wird schließlich auch vom unserdeutschen Wort quatschen ,sprechen, plaudern' - wie in (14) - gestützt, dessen quellsprachliche Form in verdichteter Form in Mittel- und Norddeutschland, darunter ganz besonders auch im Rheinland und Westfalen verbreitet ist (vgl. AdA 2003 ff.; über Alltägliches reden).

(14) $\mathrm{du}$ weiß wi quatsch-en around

2SG know 1PL chat-v around

,Weißt du, wir haben herumgeplaudert.‘

Neben all diesen lexikalischen Merkmalen finden sich allerdings in Unserdeutsch andererseits zwar vereinzelt, aber dennoch auch Lexeme, die nur oder tendenziell eher aus dem süddeutschen Raum erklärt werden können. Bei diesen handelt es sich jedoch ausschließlich um anscheinend geringfügig konventionalisierte Lexeme, die nur bei einzelnen Sprechern belegt sind. $\mathrm{Zu}$ diesen gehört zum Beispiel das Wort Bube - ,Junge‘ (vgl. König, Elspaß \& Möller 2015: $166 \mathrm{f}$.$) , das im Gegensatz zur in Unserdeutsch ansonsten üblichen Variante$ Knabe in den Interviewdaten nur äußerst sporadisch - wie in (15) - vorkommt.

(15) die war alle bube von Vunapope 3PL COP.PST all boy from Vunapope ,Sie waren alle Jungen von Vunapope. 
Im Gegensatz zu Knabe, das in standardnahen Sprachlagen des 19. Jahrhunderts anscheinend im ganzen Sprachgebiet verwendet wurde (vgl. Elspaß 2005: 402), ist Bube klar süddeutsch markiert, wenngleich das Wort im 19. Jahrhundert vereinzelt auch noch aus Westfalen belegt ist (vgl. Elspaß 2005: 400).

Und last but not least: Bei lediglich zwei Sprechern ist neben der oben bereits erwähnten Variante quatschen bzw. sprechen auch das Verb schwätzen belegt (vgl. 16), das laut sprachgeografischer Befunde eindeutig und ausschließlich im Südwesten des geschlossenen deutschen Sprachgebiets lokalisiert werden kann (vgl. König, Elspaß \& Möller 2015: 176).

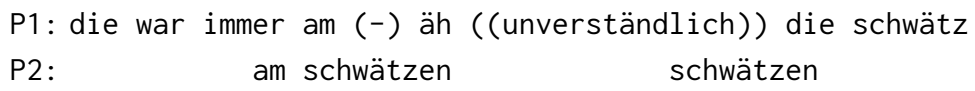

Versucht man nun im Spiegel der oben angeführten empirischen Evidenz eine sprachgeografische Bilanz zu ziehen, so kann folgende Schlussfolgerung versucht werden: Die allermeisten regional markierten Superstrateinflüsse in Unserdeutsch lassen sich eindeutig aus der gesprochenen Alltagssprache im Norden bzw. Nordwesten Deutschlands herleiten. Blendet man die Isoglossen dieser Merkmale übereinander, so kristallisiert sich der geografische Raum von bzw. um Westfalen, d.h. das weitere Umland von Hiltrup, als Überschneidungsgebiet heraus. Eine kleine Anzahl von Merkmalen, die aber eher die Oberflächenschichten von Unserdeutsch, das Lexikon nämlich, betreffen, deutet daneben auf einen geringfügigen süd- bzw. südwestdeutschen Superstrateinfluss hin. Die Tatsache, dass die meisten geografisch lokalisierbaren Superstrateinflüsse aber eindeutig norddeutscher Herkunft sind und unter diesen es zugleich auch mehrere aus den phonologischen und grammatischen Tiefenschichten der Sprache gibt, unterstützt die im Abschnitt 3 formulierte Hypothese, wonach das gesprochene Alltagsdeutsch an der Missionsstation in Vunapope um 1900 vorwiegend westfälisch-nordwestdeutsch geprägt gewesen sein muss.

\section{Was die historischen Quellen zeigen}

Zur regionalen Verortung des Unserdeutsch-Superstrats im geschlossenen deutschen Sprachraum (F1), d.h. zur Bestimmung der sprachlichen Herkunft der Vunapope-Missionare, werden im Folgenden drei Arten von historischer Evidenz herangezogen: (a) die Lage der MSC-Ordenshäuser bzw. Ausbildungsstätten für die Südseemission mit deren Einzugsgebiet, (b) die Geburtsorte der Missionare und schließlich (c) die einschlägigen Hinweise in Heimatbriefen der Missionare. Die Daten können an dieser Stelle nur holzschnittartig umrissen 
werden, eine ausführlichere Auswertung und Darstellung ist in Vorbereitung (Lindenfelser i. V.).

Nachdem das Deutsche Reich im Jahr 1884 Deutsch-Neuguinea zum „Schutzgebiet“ erklärt hatte, lief der dort operierende französische MSC-Orden Gefahr, ausgewiesen zu werden, wenn nicht rasch deutsches Missionspersonal vorgezeigt werden konnte (vgl. Gründer 1982: 49). Die Gründung einer Ordensniederlassung der Herz-Jesu Missionare auf deutschem Boden wurde damit zur dringenden Notwendigkeit, war aber aufgrund des schwierigen deutschfranzösischen Verhältnisses und des antikatholischen Klimas vor Ort (Kulturkampf) erst lange zwölf Jahre später realisierbar. In dieser Übergangszeit wurden daher Deutsche für die Südseemission in den neu gegründeten Ordenshäusern in Salzburg (Österreich), Tilburg (Niederlande) und Antwerpen (Belgien) ausgebildet, insbesondere aber in Antwerpen (vgl. Bender 1932: 198). Eine kleine Gruppe deutscher Kandidaten hatte zuvor bereits die Seminarschule in Issoudun, dem Gründungsort des Ordens im Herzen Frankreichs, besucht. Als 1897 dann das langersehnte erste Ordenshaus auf deutschem Boden in Hiltrup bei Münster eröffnet werden konnte, wurde die Ausbildung für die Südseemission mit sofortiger Wirkung dorthin verlegt und drei Jahre später auch der MSC-Schwesterorden dort gegründet (vgl. Steffen 2001: 453f.). Ab der Jahrhundertwende wurden die deutschen Missionare für Vunapope zum allergrößten Teil von Hiltrup aus entsandt. Interessant aus unserer Sicht ist vor allem das Ordenshaus in Salzburg. Dass im Süden des deutschen Sprachgebiets eine Ausbildungsstätte für die Herz-Jesu-Missionare eröffnet wurde, und diese gerade während der deutschen Kolonialzeit eine wichtige Rolle spielte, dürfte bereits andeuten, womit sich die im vorangehenden Kapitel detektierten süddeutschen Superstrateinflüsse in Unserdeutsch erklären lassen.

Auch die Geburtsorte der Missionare, soweit sie uns vorliegen und eindeutig identifizierbar sind, bestätigen unsere bisherigen Befunde. Ein Datensatz mit 213 Geburtsorten umfasst den Großteil aller deutschsprachigen Missionare, die zwischen 1890 und 1975 (das Jahr der Unabhängigkeit PapuaNeuguineas) die Gazelle-Halbinsel im Bismarck-Archipel bzw. Vunapope erreicht haben. Legt man diesen Datensatz auf die dialektgeografische Gliederung des deutschen Sprachraums um 1900 (vgl. z. B. König, Elspaß \& Möller 2015: 230 f.), so ergibt sich ein eindeutiges Bild (vgl. Abb. 13.3).

Die überragende Mehrzahl dieser Missionare, insgesamt 135 von den 213, entstammt gebürtig dem westniederdeutschen Sprachraum. 73\% von diesen wiederum, insgesamt 98 Missionare, entfallen auf den westfälischen Raum, also das weitere Umland von Hiltrup. Aus den verschiedensten Regionen des angrenzenden westmitteldeutschen Gebiets kommen auch manche Missionare, was aufgrund der geographischen Nähe $\mathrm{zu}$ Westfalen wenig überrascht. Der 


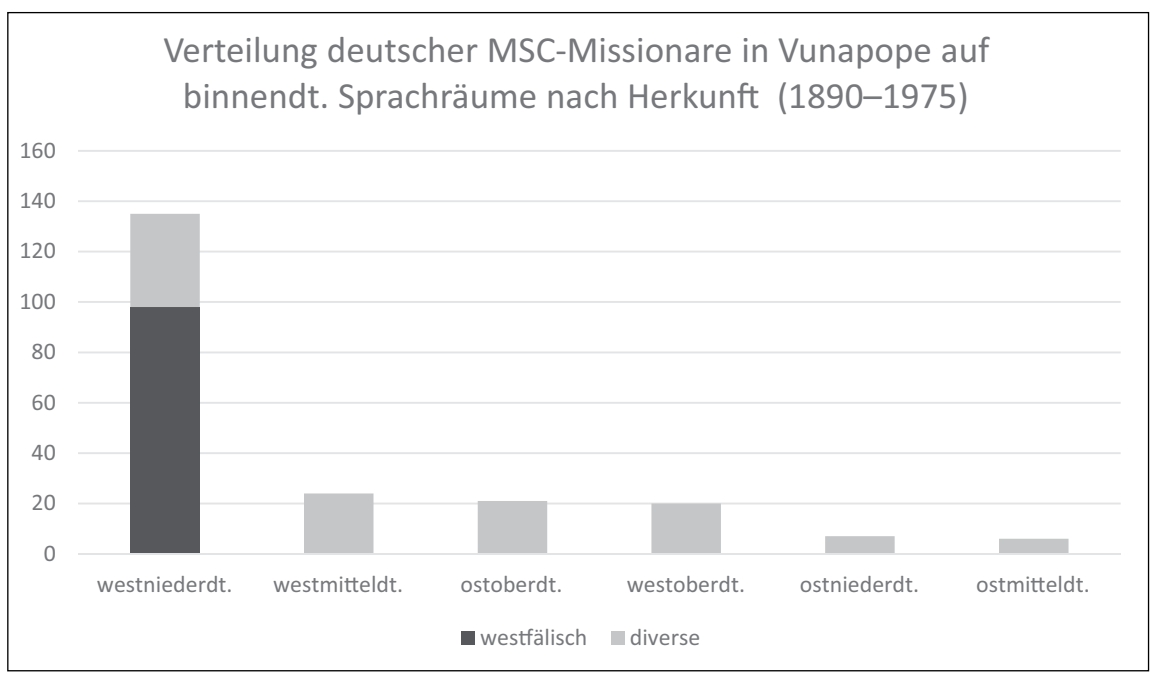

Abb. 13.3: Verteilung der Missionare in Vunapope auf binnendeutsche Sprachräume.

zwar klar untergeordnete, aber doch in den Daten ersichtliche und historisch durch die Salzburger Missionsniederlassung erklärbare Einfluss des oberdeutschen Sprachraums spiegelt sich in der Herkunft von insgesamt 41 Missionaren aus diesem Gebiet (insgesamt knapp doppelt so viel wie aus dem westmitteldeutschen Raum), wobei der ost- und der westoberdeutsche Raum zahlenmäßig gleich stark vertreten sind. Aus dem ostniederdeutschen und ostmitteldeutschen Sprachraum entstammen hingegen im gesamten Untersuchungszeitraum von 85 Jahren insgesamt nur 13 Missionare, was wiederum das beschränkte Einzugsgebiet der Ordenshäuser zeigt (vgl. auch Frings 2000: 70 f.) und zugleich auch das Fehlen der für diesen Raum charakteristischen distinktiven Merkmale in den Sprachdaten erklärt.

Die prozentuale Verteilung ändert sich nicht wesentlich, wenn man die Gruppe der Missionare noch einmal nach Patres und Brüdern versus Schwestern oder nach Ankunftszeiträumen und damit in etwa der Schulzeit der unterschiedlichen Unserdeutsch-Sprechergenerationen subklassifiziert. Von zentraler Bedeutung wäre in diesem Zusammenhang natürlich die Anschlussfrage, welche der Missionare durch ihren Aufgabenbereich bedingt - etwa in Erzieherund Lehrfunktion - besonders engen Kontakt zu den Kindern hatten und damit stärkeren sprachlichen Einfluss ausübten. Durch die Beantwortung dieser Frage dürfte sich auch erklären, warum in den linguistischen Daten der südwestdeutsche sprachliche Einfluss stärker ist als der südostdeutsche, obwohl beide Gebiete unter den Missionaren zahlenmäßig gleich vertreten sind (vgl. 
Abb. 13.3). Die Erklärung hierfür dürfte mit einiger Wahrscheinlichkeit darin bestehen, dass die südwestdeutschen Missionare im Vergleich zu den südostdeutschen einen intensiveren Kontakt zu den mixed-race Kindern hatten und somit einen stärkeren sprachlichen Einfluss ausüben konnten. Die genaue Rekonstruktion der sozialen Netzwerke an der Missionsstation in Vunapope, die zur endgültigen Klärung dieser Frage nötig wäre, steht - soweit sie heute überhaupt noch möglich ist - aus. Die linguistische Evidenz (s. Abschnitt 3) sowie die Herkunft der Missionare deuten jedoch auf jeden Fall eindeutig darauf hin, dass die mixed-race Kinder von Vunapope am meisten mit Missionaren aus dem nordwestdeutsch-westfälischen Gebiet in Kontakt waren.

Die drei gemäß den Herkunftsdaten relevanten Sprachräume (Westniederdeutsch, Westmitteldeutsch, (Ost-)Oberdeutsch) sind auch in den metasprachlichen Aussagen einer Missionsschwester in ihrem Brief an das Mutterhaus in Hiltrup aus dem Jahre 1904 benannt:

Der Frühstückstisch war selbstverständlich so festlich als möglich geschmückt. In der Mitte stand eine hübsche Palme, in welcher die holländische Flagge, von der österreichischen, bayrischen, preußischen und deutschen umgeben flatterte, um so endlich einmal dem langwierigen Sprachenkriege ein Ende zu machen, denn derselbe war bei uns schon sehr heftig entbrannt, weil die Westfalen nicht zugeben wollten, daß sie Skule statt Schule, die Rheinländer Heiten und Waißenkinder, die Bayern k statt $\mathrm{g}$ und so fort sagen. (Brief Sr. Angela nach Hiltrup, Vunapope, 22. Juli 1904. GAMS, Nr. 1566; kursive Hervorhebungen durch uns, P. M. \& S. L.)

Dass unter all diesen Varietäten wiederum der westniederdeutsche bzw. westfälische Einfluss am höchsten einzustufen ist, legen auch weitere Briefe nahe, in denen sich mehrfach explizite Hinweise auf den deutschsprachigen Nordwesten bzw. konkret auf Westfalen finden. So liest man etwa im Bericht einer anderen Schwester über die Anreise von ihr und einer Reihe von Hiltruper Mitschwestern nach Vunapope im Jahr 1904 Folgendes:

Wir hatten uns eine kurze Zeit mit dem hohen Herrn in holländischer Sprache (mit Hilfe unserer westfälischen Mundart) unterhalten, als es an der Tür klopfte. (Bericht Sr. Mathilde, Vunapope, 1904, zit. nach Brief o. V. [vermutl. Sr. Clothilde] nach Hiltrup. GAMS, Nr. 1565; kursive Hervorhebung durch uns, P. M. \& S. L.)

In zwei Briefen aus derselben Zeit ist statt von der „westfälischen Mundart“ etwas breiter von Platt die Rede, was also erneut in den (west)niederdeutschen Raum zielt:

Wollen wir uns miteinander verständigen, so spricht er holländisch und wir platt, was jedesmal wieder zu lachen gibt. (Brief, höchstwahrscheinlich Sr. Elisabeth, an Bord des Dampfers „Sachsen“ nach Hiltrup, 7. Oktober 1902. GAMS, Nr. 1551) 
Wenn ehrw. Schw. Oberin und Schw. Klara Platt sprechen, soll ich dieses Kauderwelsch übersetzen, wobei sie mich natürlich gründlich auslachen. (Brief Sr. Elisabeth nach Hiltrup, Vunapope, 6. März 1904. GAMS, Nr. 1551)

Diese - und weitere - Quellen deuten darüber hinaus auch auf die Rolle niederländischer Missionare in der Südsee-Mission und speziell in Vunapope hin, bei denen im Einklang mit Volker (1982: 18) der Gebrauch einer norddeutsch orientierten L2-Varietät des Deutschen angenommen werden kann. Zweitens zeigen die zitierten Stellen die Dialektkompetenz der norddeutschen Missionare und zugleich auch die Funktionen des Platt in Vunapope. Die Verwendung des Dialekts war anscheinend eine domänenspezifische Akkommodationsstrategie, die die effiziente Kommunikation mit den niederländischen Missionaren ermöglichen sollte. Plattdeutsch dürfte darüber hinaus als informelle in-group Varietät unter den norddeutschen Missionaren verwendet worden sein, nicht jedoch als out-group Varietät mit den mixed-race Kindern, wie dies auch an der Absenz plattdeutscher Superstrateinflusse in Unserdeutsch erkennbar ist. ${ }^{11}$ Und drittens reflektieren die zitierten Briefe die innere Mehrsprachigkeit bzw. den Varietätenkontakt an der Missionsstation, was einen heterogenen sprachlichen Input bei der Entstehung von Unserdeutsch nahelegt und somit mit der in Abschnitt 3 vorgestellten linguistischen Evidenz im Einklang steht.

Die Annahme, dass bei der Entstehung von Unserdeutsch tatsächlich kaum mit dialektalem Input/Superstrat gerechnet werden kann, wird auch von unabhängiger sprachhistorischer Evidenz unterstützt. In und um Hiltrup bei Münster, am Ausbildungsort der Patres, Schwestern und Brüder ab der Jahrhundertwende, machte sich bereits in der zweiten Hälfe des 19. Jahrhunderts eine klare Standardorientierung bemerkbar. Die Stadt nahm diesbezüglich anscheinend eine gewisse Vorreiterrolle in der Region ein:

Der Stadt Münster kommt dabei wohl die wichtigste Neuerungsfunktion zu. Dort sind es insbesondere die Zuwanderer aus nichtwestfälischen Gebieten, das Bildungsbürgertum und soziale Aufsteiger, die ausschließlich Hochdeutsch oder wenigstens eine als solche intendierte Umgangssprache westfälischer Prägung [...] verwenden. (Kremer 2000: 320 f.)

Für die angehenden Missionare sind alle drei genannten Gruppen relevant. Erstens waren unter den Missionaren, wie die Statistik der Herkunftsorte zeigt,

11 Einige wenige Einzelwörter wie vor allem Kopp (auch in Du Kartoffelkopp!) sind zwar auch mit unverschobenen Plosiven in den Interviewdaten belegt, in der überwiegenden Mehrheit der Fälle kommen aber erstens selbst diese Wörter mit verschobenen Konsonanten vor, und zweitens können Formen wie Kopp auch und vor allem um 1900 durchaus im Bereich des regionalen Gebrauchsstandards angesetzt werden. 
durchaus Zuwanderer aus nichtwestfälischen Gebieten vertreten. Zweitens sind die Patres, d. h. die Ordenspriester, klar den bildungsbürgerlichen Schichten der damaligen deutschen Gesellschaft zuzurechnen. Und drittens bedeutete (vor allem) für die Schwestern wiederum der Eintritt in den Missionsorden einen klaren sozialen Aufstieg, da sie meist aus einfachen Verhältnissen kamen und die beruflichen Möglichkeiten für Frauen zu dieser Zeit ohnehin noch höchst eingeschränkt waren.

Die sprachliche Standardorientierung der Ordensleute wird im Spiegel zeitgenössischer Aussagen auch noch durch ihren speziellen Berufsstand erhärtet:

In Westfalen ist das Hoch- und Plattdeutsche getheilt zwischen den gebildeten und den unteren Ständen. Jenes ist ausserdem Sprache der Kirche, der Schule und des Gerichts [...] während sie [Anm.: Landpfarrer, P. M. \& S. L.] im Süden [d.h. in Westfalen, L. K.] auch durch den Sprachunterschied ein priesterliches Ansehen $\mathrm{zu}$ behaupten suchen. (August von Eye 1855: 97, zit. nach Kremer 2000: 319)

Die Tatsache, dass das Hochdeutsche (auch) in Westfalen nicht nur als Schulund Kirchensprache, sondern anscheinend auch als Sozialsymbol innerhalb des Klerus galt, dürfte ebenfalls für die Dialektferne der Intergruppenkommunikation an der Missionsstation in Vunapope sprechen; schließlich waren die Internate von Vunapope in jeder Hinsicht nach dem Vorbild bürgerlicher deutscher Schulen konzipiert. Diese Standardorientierung führte wiederum gewiss zur Nivellierung salienter, regional markierter Nonstandardmerkmale, was auch an den linguistischen Strukturmerkmalen von Unserdeutsch klar erkennbar ist (s. Abschnitt 3).

\section{Was wir herausgefunden haben: Schlussfolgerungen}

Durch unseren triangulativen Rekonstruktionsversuch verfolgten wir das Ziel, das sprachliche Superstrat von Unserdeutsch $\mathrm{zu}$ bestimmen und damit die Beschaffenheit der gesprochenen Alltagssprache an der Missionsstation in Vunapope im Bismarck-Archipel um $1900 \mathrm{zu}$ rekonstruieren. Unsere Analysen haben folgende Antworten auf die in Abschnitt 1 formulierten Forschungsfragen (F1) und (F2) ergeben:

$\left(\mathrm{F}^{\prime}\right)$ Der sprachliche Input, den die mixed-race Kinder in Vunapope von den Missionaren bekommen haben, scheint im Lichte unterschiedlicher Daten und Quellen eindeutig heterogen gewesen zu sein. Linguistische 
Evidenz zeigt einhellig, dass dieser Input in weiten Teilen nord- und vor allem nordwestdeutsch-westfälisch geprägt gewesen sein muss, mit geringeren süd- und vor allem südwestdeutschen Einflüssen.

(F2') Hinsichtlich des Grades der regionalen Markiertheit dieses Inputs deutet alles auf eine klare Dialektferne hin. Neben dem formellen Schriftstandard der Schule kristallisiert sich im Spiegel der Daten ein regionaler Gebrauchsstandard - oder terminologisch anders: landschaftliches Hochdeutsch (vgl. Ganswindt 2017) - als gesprochene Alltagsvarietät zwischen Missionaren und Kindern an der Missionsstation in Vunapope heraus. Die deutlich erkennbare Meidung dialektalen Sprechens in Vunapope scheint dabei vor allem (a) auf die bildungszielbedingte Präferenz des Standards als Prestigevarietät im schulischen Kontext zurückzuführen zu sein, war aber daneben anscheinend auch (b) als bewusste Akkommodationsstrategie unter dem sprachlich heterogenen Missionspersonal intendiert.

Unsere Erkenntnisse lassen somit die meisten diesbezüglichen, eingangs zitierten und diskutierten Aussagen in Volker (1982) und Frowein (2006) als unhaltbar erscheinen. Dieser Widerspruch zwischen unseren und ihren einschlägigen Ansichten dürfte sich dabei zweifelsfrei zum Teil auch durch unsere viel breitere und differenziertere Datenbasis erklären.

Unsere Befunde dürften (mindestens) aus zweierlei Sicht von kreolistischer Relevanz sein. Erstens liefern sie weitere empirische Evidenz dafür, dass wir im Gegensatz zu manchen prominenten Forschermeinungen (vgl. etwa Mufwene 2015a: 349 und 2015b: 133) - das Superstrat von Kreolsprachen nicht immer in kolonialen Nonstandardvarietäten suchen müssen, sondern dieses durchaus auch standardnah bzw. gänzlich im Bereich des (Gebrauchs-)Standards angesiedelt sein kann. Diese Standardnähe des sprachlichen Superstrats kann in unserem Fall eindeutig mit dem schulischen Kontext der Sprachentstehung erklärt werden. Solche Entstehungskontexte sind bei Kreolsprachen zwar tatsächlich unüblich, aber auch über den Fall von Unserdeutsch hinaus durchaus bekannt (vgl. z. B. Ehrhart 1993). Diese Tatsache wiederum deutet auf die Gefahren von - gewiss notwendigen - Verallgemeinerungen in der Kreolistik hin und zeigt, dass (auch) die Annahme von kolonialen Nonstandardvarietäten als Kreolsuperstrate lediglich als statistische Verallgemeinerung verstanden werden darf. Die Entstehungsszenarien und -kontexte von Kreolsprachen können, wie gerade auch das Beispiel von Unserdeutsch zeigt (vgl. Lindenfelser \& Maitz 2017), trotz gewisser grundlegender Ähnlichkeiten so unterschiedlich bzw. unikal sein, dass dies zu größter Vorsicht bei generellen Aussagen auffordert. 
Zweitens zeigt sich auch in unserem Zusammenhang, dass die Rolle von Superstratsprachen, wie u. a. auch Chaudenson (2003) oder Mufwene (2015b: 133) betonen, weit über die Bereitstellung des Lexikons einer Kreolsprache hinausgehen kann. Auch im Fall von Unserdeutsch ist, wie wir in Abschnitt 3 gesehen haben, sowohl Phonologisches ${ }^{12}$ als auch Grammatisches aus dem Superstrat transferiert worden (s. auch Lindenfelser \& Maitz 2017). Der Umfang dieses tiefer gehenden Superstrateinflusses ist jedoch gewiss wieder stark abhängig vom Entstehungsszenario und dem sozialen Kontext der einzelnen Kreolsprachen, sodass Verallgemeinerungen auch in diesem Fall zwangsläufig nur die statistisch gesehen typischen Fälle erfassen können.

Für die Variationslinguistik bzw. die historische Sprachwissenschaft des Deutschen wiederum dürfte unsere Darstellung gezeigt haben, dass die Analyse von kolonialen und sonstigen Auswanderervarietäten, besonders von solchen, die - wie Unserdeutsch - im Laufe ihrer späteren Geschichte den Kontakt zum sprachlichen Mutterland vollständig verloren haben, zur Rekonstruktion historischer Mündlichkeit wertvolle Daten liefern kann.

Und zum Schluss muss noch einmal betont werden, dass es im Rahmen dieses Aufsatzes aus mehreren Gründen nicht möglich war, alle regional markierten Superstrateinflüsse in Unserdeutsch aufzudecken und zu berücksichtigen. Wir mussten und wollten uns hier tendenziell auf die salientesten und grundlegendsten, am meisten konventionalisierten Strukturmerkmale beschränken. Dies bedeutet, dass das von uns gezeichnete Bild den Grundtendenzen eindeutig gerecht werden müsste, von der zukünftigen Forschung jedoch überprüft und in Bezug auf weniger saliente und konventionalisierte Merkmale weiter differenziert werden könnte - und sollte.

\section{Abkürzungen}

$\begin{array}{llll}\text { 1PL } & \text { 1. Person Plural } & \text { COP } & \text { Kopula } \\ \text { 1SG } & \text { 1. Person Singular } & \text { DEF } & \text { definit } \\ \text { 2SG } & \text { 2. Person Singular } & \text { DIM } & \text { Dimuitiv } \\ \text { 3PL } & \text { 3. Person Plural } & \text { F } & \text { feminin } \\ \text { 3SG } & \text { 3. Person Singular } & \text { HAB } & \text { habituell } \\ \text { ACC } & \text { Akkusativ } & \text { INDF } & \text { indefinit } \\ \text { ART } & \text { Artikel } & \text { M } & \text { maskulin } \\ \text { ATTR } & \text { attributiv } & \text { PL } & \text { Plural } \\ \text { AUX } & \text { Auxiliar } & \text { POSS } & \text { Possessivum }\end{array}$

12 Wie zum Beispiel das Phonem / $/$ /, das in Wörtern mit spirantisiertem finalem /g/ realisiert wird. 


$\begin{array}{llll}\text { PROG } & \text { progressiv } & \text { STD } & \text { Standarddeutsch } \\ \text { PST } & \text { Vergangenheit } & \text { TP } & \text { Tok Pisin } \\ \text { PTCP } & \text { Partizip } & \text { UD } & \text { Unserdeutsch } \\ \text { REL } & \text { Relativum } & \text { V } & \text { Verb }\end{array}$

\section{Literatur}

AdA (2003 ff.) = Elspaß, Stephan \& Robert Möller (2003 ff.): Atlas der deutschen Alltagssprache (AdA). www.atlas-alltagssprache.de (letzter Zugriff 27. 9. 2017).

Arends, Jacques, Silvia Kouwenberg \& Norval Smith (1995): Theories focusing on the nonEuropean input. In: Jacques Arends, Pieter Muysken \& Norval Smith (Hrsg.), Pidgins and creoles: An introduction, 99-110. Amsterdam, Philadelphia: Benjamins.

Baker, Philip (1990): Off target? In: Journal of Pidgin and Creole Languages 5, 107-119.

Baker, Philip (2000): Theories of creolization and the degree and nature of restructuring. In: Ingrid Neumann-Holzschuh \& Edgar W. Schneider (Hrsg.), Degrees of restructuring in creole languages, 41-63. Cambridge: Cambridge University Press.

Bakker, Peter (1995): Pidgins. In: Jacques Arends, Pieter Muysken \& Norval Smith (Hrsg.), Pidgins and creoles: An introduction, 25-40. Amsterdam, Philadelphia: Benjamins.

Bakker, Peter (2002): Some future challenges for pidgin and creole studies. In: Glenn Gilbert (Hrsg.), Pidgin and creole linguistics in the twenty-first century, 69-92. New York u. a.: Lang.

Bender, Josef (1932): Missionshilfe in der Heimat. In: Josef Hüskes (Hrsg.), Pioniere der Südsee. Werden und Wachsen der Herz-Jesu-Mission von Rabaul zum Goldenen Jubiläum 1882-1932, 197-205. Hiltrup, Salzburg: Missionare vom hl. Herzen Jesu.

Chaudenson, Robert (2003): La créolisation: théorie, applications, implications. Paris u. a.: Harmattan.

Damme, Robert (2011): Westfälisches Wörterbuch. Band 2. Neumünster: Wachholtz.

Deumert, Ana (2003): Markedness and salience in language contact and second-language acquisition: Evidence from a non-canonical contact language. Language Sciences 25 , 561-613.

Deumert, Ana (2009): Namibian Kiche Duits: The making (and decline) of a Neo-African language. Journal of Germanic Linguistics 21, 349-417.

DWDS = Digitales Wörterbuch der deutschen Sprache. URL: https://www.dwds.de (letzter Zugriff: 17. 9. 2017).

Ehrhart, Sabine (1993): Le créole français de St-Louis (le tayo) en Nouvelle-Calédonie. Hamburg: Buske.

Elspaß, Stephan (2005): Sprachgeschichte von unten. Untersuchungen zum geschriebenen Alltagsdeutsch im 19. Jahrhundert. Tübingen: Niemeyer.

Elspaß, Stephan (2016): Typisch und nicht so typisch Westfälisches in der nicht-dialektalen Alltagssprache. In: Helmut Spiekermann, Line-Marie Hohenstein, Stephanie Sauermilch \& Kathrin Weber (Hrsg.), Niederdeutsch: Grenzen, Strukturen, Variation, 359-382. Wien: Böhlau.

Frings, Bernhard (2000): Mit ganzem Herzen. Hundert Jahre Missionsschwestern vom Heiligsten Herzen Jesu in Hiltrup. Dülmen: Laumann.

Frowein, Friedel M. (2006): Transfer, continuity, relexification and the bioprogram - what the substratist/universalist debate in creolistics implies for modern theories of 
language acquisition. Online Veröffentlichung. URL: http://bit.ly/10WFuWG (letzter Zugriff: 14. 7. 2017).

Ganswindt, Brigitte (2017): Landschaftliches Hochdeutsch. Rekonstruktion der oralen Prestigevarietät im ausgehenden 19. Jahrhundert. Stuttgart: Steiner.

Gárgyán, Gabriella (2014): Der am-Progressiv im heutigen Deutsch. Neue Erkenntnisse mit besonderer Hinsicht auf die Sprachgeschichte, die Aspektualität und den kontrastiven Vergleich mit dem Ungarischen. Frankfurt a. M.: Peter Lang.

Goodman, Morris (1993): African substratum: Some cautionary words. In: Salikoko S. Mufwene \& Nancy Condon (Hrsg.), Africanisms in Afro-American language varieties, 6474. Athens, London: University of Georgia Press.

Götze, Angelika, Siegwalt Lindenfelser, Salome Lipfert, Katharina Neumeier, Werner König \& Péter Maitz (2017): Documenting Unserdeutsch: A workshop report. In: Péter Maitz \& Craig A. Volker (Hrsg.), Language contact in the German colonies: Papua New Guinea and beyond (= Sonderheft von Language and Linguistics in Melanesia), 65-90.

Gründer, Horst (1982): Christliche Mission und deutscher Imperialismus. Eine politische Geschichte ihrer Beziehungen während der deutschen Kolonialzeit (1884-1914) unter besonderer Berücksichtigung Afrikas und Chinas. Paderborn: Schöningh.

Gründer, Horst (2004): Christliche Heilbotschaft und weltliche Macht. Studien zum Verhältnis von Mission und Kolonialismus. Gesammelte Aufsätze. Hrsg. v. Franz-Joseph Post, Thomas Küster \& Clemens Sorgenfrey. Münster: LIT Verlag.

Harnisch, Rüdiger (2004): Morphologie/Morphology. In: Ulrich Ammon, Norbert Dittmar, Klaus J. Mattheier \& Peter Trudgill (Hrsg.), Soziolinguistik. Ein internationales Handbuch zur Wissenschaft von Sprache und Gesellschaft. 2. Auflage. Band 1,522-530. Berlin, New York: de Gruyter.

Hickey, Raymond (2005): English dialect input to the Caribbean. In: Raymond Hickey (Hrsg.), Legacies of colonial English. Studies in transported dialects, 326-360. Cambridge: Cambridge University Press.

Hiery, Hermann Joseph (2001): Schule und Ausbildung in der deutschen Südsee. In: Hermann Joseph Hiery (Hrsg.), Die deutsche Südsee. Ein Handbuch, 198-238. Paderborn: Schöningh.

Holm, John (1988): Pidgins and creoles. Band 1: Theory and structure. Cambridge: Cambridge University Press.

Holm, John (2000): An introduction to pidgins and creoles. Cambridge: Cambridge University Press.

Hüskes, Josef (Hrsg.) (1932): Pioniere der Südsee. Werden und Wachsen der Herz-JesuMission von Rabaul zum Goldenen Jubiläum 1882-1932. Hiltrup, Salzburg: Missionare vom Hlst. Herzen Jesu.

Jacobs, Bart (2009): The origins of Old Portuguese features in Papiamentu. In: Nicholas Faraclas, Ronald Severing, Christa Weijer \& Liesbeth Echteld (Hrsg.), Leeward voices. Fresh perspectives on Papiamentu and the literatures and cultures of the $A B C$ islands. Proceedings of the ECICC-conference. Band 1, 11-38. Curaçao: FPI/UNA.

Janssen, Arnold (1932): Die Erziehungsanstalt für halbweiße Kinder. In: Josef Hüskes (Hrsg.), Pioniere der Südsee. Werden und Wachsen der Herz-Jesu-Mission von Rabaul zum Goldenen Jubiläum 1882-1932, 150-155. Hiltrup, Salzburg: Missionare vom Hlst. Herzen Jesu.

Jansson, Fredrik, Mikael Parkvall \& Pontus Strimling (2015): Modeling the evolution of creoles. Language Dynamics and Change 5 (1), 1-51. 
Jassmeier, Joseph (1971): Missionaries. Who serve or have served: The church and the people of New Britain (Archdiocese of Rabaul). Vunapope: Mission Press.

Keim, Inken (1978): Gastarbeiterdeutsch. Untersuchungen zum sprachlichen Verhalten türkischer Gastarbeiter. Tübingen: Narr.

Kleiner, Stefan (2011 ff.): Atlas zur Aussprache des deutschen Gebrauchsstandards (AADG). Unter Mitarbeit von Ralf Knöbl. URL: http://prowiki.ids-mannheim.de/bin/view/AADG/ (letzter Zugriff: 16.10. 2017).

König, Werner (1989): Atlas zur Aussprache des Schriftdeutschen in der Bundesrepublik Deutschland. Band 2: Tabellen und Karten. Ismaning: Max Hueber.

König, Werner, Stephan Elspaß \& Robert Möller (2015): dtv-Atlas Deutsche Sprache. 18. Auflage. München: Deutscher Taschenbuch Verlag.

Kremer, Ludger (2000): Westfälische Sprachgeschichte von 1850 bis zur Gegenwart. In: Jürgen Macha, Elmar Neuß \& Robert Peters unter Mitarbeit von Stephan Elspaß (Hrsg.), Rheinisch-Westfälische Sprachgeschichte, 315-335. Köln: Böhlau.

Lindenfelser, Siegwalt (i. V.): Sprachentstehung durch Sprachkontakt. Genese und Geschichte von Unserdeutsch (Rabaul Creole German). Dissertation, Universität Augsburg.

Lindenfelser, Siegwalt \& Péter Maitz (2017): The creoleness of Unserdeutsch (Rabaul Creole German): A typological perspective. In Péter Maitz \& Craig A. Volker (Hrsg.), Language contact in the German colonies: Papua New Guinea and beyond. (= Sonderheft von Language and Linguistics in Melanesia), 91-142.

Loosen, Livia (2014): Deutsche Frauen in den Südseekolonien des Kaiserreichs. Alltag und Beziehungen zur indigenen Bevölkerung, 1884-1919. Bielefeld: transcript.

Maitz, Péter (2016): Unserdeutsch. Eine vergessene koloniale Varietät des Deutschen im melanesischen Pazifik. In: Alexandra N. Lenz (Hrsg.), German abroad - Perspektiven der Variationslinguistik, Sprachkontakt- und Mehrsprachigkeitsforschung, 211-240. Göttingen: V \& R unipress.

Maitz, Péter (2017): Dekreolisierung und Variation in Unserdeutsch. In: Helen Christen, Peter Gilles \& Christoph Purschke (Hrsg.), Räume - Grenzen - Übergänge. Akten des 5. Kongresses der Internationalen Gesellschaft für Dialektologie des Deutschen (IGDD), 215-242. Stuttgart: Steiner.

Maitz, Péter, Werner König \& Craig A. Volker (2016): Unserdeutsch (Rabaul Creole German): Dokumentation einer stark gefährdeten Kreolsprache in Papua-Neuguinea. Zeitschrift für germanistische Linguistik 44 (1), 93-96.

Maitz, Péter \& Craig Alan Volker (2017): Documenting Unserdeutsch: Reversing colonial amnesia. Journal of Pidgin and Creole Languages 32 (2), 365-397.

Maitz, Péter, Siegwalt Lindenfelser \& Craig A. Volker (i. E.): Unserdeutsch (Rabaul Creole German), Papua New Guinea. In Hans C. Boas, Ana Deumert, Mark L. Louden \& Péter Maitz (Hrsg.), Varieties of German worldwide. Oxford: Oxford University Press.

Missionare vom heiligsten Herzen Jesu (Hrsg.) (1884-2017): Monatshefte zu Ehren U. L. Frau vom hlst. Herzen Jesu [inzwischen: Hiltruper Monatshefte], 125 Jahrgänge. Hiltrup.

Mückler, Hermann (2010): Mission in Ozeanien (= Kulturgeschichte Ozeaniens, Band 1). Wien: Facultas.

Mückler, Hermann (2014): Missionare in der Südsee. Pioniere, Forscher, Märtyrer. Ein biographisches Nachschlagewerk zu den Anfängen der christlichen Mission in Ozeanien (= Quellen und Forschungen zur Südsee, Reihe B: Forschungen, Band 6). Wiesbaden: Harrassowitz. 
Mufwene, Salikoko S. (2015a): The emergence of creoles and language change. In: Nancy Bonvillain (Hrsg.), The handbook of linguistic anthropology, 345-368. London: Routledge.

Mufwene, Salikoko S. (2015b): Pidgin and creole languages. In: James Wright (Hrsg.), International encyclopedia of behavioral and social sciences. Band 18. 2. Auflage, 133-145. Oxford: Elsevier.

Mühlhäusler, Peter (1997): Pidgin and creole linguistics. 2. Auflage. London: University of Westminster Press.

Mühlhäusler, Peter (2001): Typology and universals of pidginization. In: Martin Haspelmath, Ekkehard König, Wulf Oesterreicher \& Wolfgang Raible (Hrsg.), Language typology and language universals. Band 2, 1648-1655. Berlin, New York: de Gruyter.

Mühlhäusler, Peter (2012): Sprachliche Kontakte in den Missionen auf Deutsch-Neuguinea und die Entstehung eines Pidgin-Deutsch. In: Stefan Engelberg \& Doris Stolberg (Hrsg.), Sprachwissenschaft und kolonialzeitlicher Sprachkontakt. Sprachliche Begegnungen und Auseinandersetzungen, 71-100. Berlin: Akademie-Verlag.

Negele, Michaela (2012): Varianten der Pronominaladverbien im Neuhochdeutschen. Grammatische und soziolinguistische Untersuchungen. Berlin, New York: de Gruyter.

NOSA (2015) = Elmentaler, Michael \& Peter Rosenberg (Hrsg.) (2015): Norddeutscher Sprachatlas. Band 1: Regiolektale Sprachlagen. Hildesheim: Olms.

Plag, Ingo (2008): Creoles as interlanguages: Inflectional morphology. Journal of Pidgin and Creole Languages 23, 109-130.

Rascher, Matthäus (1909): M.S.C. und Baining (Neu-Pommern). Land und Leute. In: Missionare vom heiligsten Herzen Jesu (Hrsg.), Aus der deutschen Südsee. Mitteilungen der Missionare vom heiligsten Herzen Jesu, Band 1. Münster: Aschendorff.

Schmidt, Jürgen Erich (2010): Die modernen Regionalsprachen als Varietätenverbände. In: Peter Gilles, Joachim Scharloth \& Evelyn Ziegler (Hrsg.), Variatio delectat. Empirische Evidenzen und theoretische Passungen sprachlicher Variation. Frankfurt am Main: Peter Lang, 125-143.

Selbach, Rachel (2008): The superstrate is not always the lexifier: Lingua Franca in the Barbary Coast 1530-1830. In: Susanne Maria Michaelis (Hrsg.), Roots of creole structures: Weighing the contribution of substrates and superstrates, 29-58. Amsterdam, Philadelphia: Benjamins.

Steffen, Paul (2001): Die katholischen Missionen in Deutsch-Neuguinea. In: Hermann Joseph Hiery (Hrsg.), Die deutsche Südsee. Ein Handbuch, 343-383. Paderborn: Ferdinand Schöningh.

Steffen, Paul (2012): Die ganzheitliche Evangelisierungsmethode der katholischen Mission und ihr Beitrag zur Landesentwicklung in Papua-Neuguinea. Annales Missiologici Posnanienses 18, 7-56.

Thomason, Sarah G. (2001): Language contact: An introduction. Edinburgh: Edinburgh University Press.

Thomason, Sarah G. \& Terrence Kaufman (1988): Language contact, creolization, and genetic linguistics. Berkeley: University of California Press.

Timm, Erika (1986): Das Jiddische als Kontrastsprache bei der Erforschung des Frühneuhochdeutschen. Zeitschrift für germanistische Linguistik 14 (1), 1-22.

Trudgill, Peter (2010): Contact and sociolinguistic typology. In: Raymond Hickey (Hrsg.), The handbook of language contact, 299-319. Oxford: Wiley-Blackwell.

Velupillai, Viveka (2015): Pidgins, creoles and mixed languages: An introduction. Amsterdam, Philadelphia: Benjamins. 
Volker, Craig A. (1982): An introduction to Rabaul Creole German (Unserdeutsch). Unveröffentlichte Masterarbeit, Universität Queensland. URL: http://bit.ly/2iJoNdX (letzter Zugriff: 21. 7. 2017).

Volker, Craig A. (1989): Rabaul Creole German syntax. Working Papers in Linguistics 21 (1), 153-189.

Volker, Craig A. (1991): The birth and decline of Rabaul Creole German. Language and Linguistics in Melanesia 22, 143-156.

Waldersee, James (1995): „Neither eagles nor saints“. MSC missions in Oceania 1881-1975. With the collaboration of John F. McMahon MSC who wrote the last three chapters. Sydney: Chevalier Press.

WdU (1977 ff.) = Eichhoff, Jürgen (1977 ff.): Wortatlas der deutschen Umgangssprachen. Bd. I/II [1977/78] Bern: Francke; Bd. III [1993] München u. a.: Saur; Bd. IV [2000] Bern, München: Saur. 



\section{Register}

Alemannisch 5-6, 10, 13, 35, 37, 103, 108

Alltagssprache 50, 172, 204, 292, 306, 309$310,317,320,322-323,325,330$

Analogie 158, 219, 222, 224-225, 232-233, 236

Angemessenheit 177, 179, 183-186

Areallinguistik 99, 102, 106

Bairisch 72-73, 76, 80-81, 84, 89, 157, 187

Basilekt 317, 323

Codeswitching 20, 41, 43-54, 58

Dativabbau 241, 243, 246, 250-251, 254

Deutsch als Fremdsprache (DaF) 197, 199, 203-206, 209-211, 215, 241, 253

Deutsch, geschriebenes 197, 294

Deutsch, gesprochenes 159, 170, 181-182, 204, 291, 324

Deutschlehrerausbildung 199, 202-203, 214, 216

Dialekt $6-8,10-11,13-14,38,42,47,67-$ 68, 71-74, 76-77, 80, 83, 99-103, 106109, 177, 180-185, 187-188, 190-192, 208-209, 211-212, 214-215, 220-223, 226-234, 236, 244, 246, 250-252, 254, 264, 266-267, 284-285, 291-293, 296-297, 300, 309, 313, 315, 318, 322, 324, 329, 331

Dialektologie 99, 102, 111, 300

Dialektometrie 89, 91, 94

Diskurstyp 127, 129

Draw-a-Map-Aufgabe 104, 107

Einstellungen 5-7, 21, 25, 37, 41-42, 45, 63-64, 66-69, 76-79, 84, 94, 101-103, 105-106, 109, 111, 137-138, 141-142, 152, 188-189, 204, 209, 211-212, 214

Elsass $5-14,16,18-19,21-22,25,27-29$, 31-32, 34, 36-38

Empirie 36, 63-64, 73, 130, 159, 173, 189, 197, 199, 237, 268, 283, 285, 295, 309, $314,319,325,331$

Erhebungsmethode 99

Ethnodialektologie 99
Fragebogen $63,68,199,209,211,214,247-$ 248

gebrauchsbasierte Theorie 241

Gebrauchsstandard 157, 159, 170, 204, 309, 315-316, 318, 320-321, 329, 331

Handlungskompetenz, sprachliche 197, 207 Hochdeutsch 11, 13, 41, 43, 45-46, 48, 50$52,54-57,66,69,71,84-86,166,181$, 183, 187, 212-214, 219-221, 231-232, 277, 294, 315, 329-331

Hyperkorrektion, dia-systemische 219

Identitätskonstruktion 41-43, 48

Interview, sprachbiographisches 41-43, 45, 47-48, 57

Kasus 243, 248, 250-251, 254, 256, 263, 265-266, 268-269, 271, 273, 275-276, 278, 296

Kasusabbau 241-242, 244-245, 249, 256, 258-259, 264-265, 272-273

Konvergenz 38, 242, 245, 264, 273, 278

Kreolsprache 306-308, 311, 316, 331-332

Laienlinguistik 72, 99

Lehnwort 157

Lernervarietät 311, 318-319

linguistic landscape 127-128

Mehrsprachigkeit, funktionale 182

Mehrsprachigkeit, visuelle 127, 130, 133, 136-143, 151

Mental-Map 68

Morphologie 219, 221, 227, 230-231, 233237, 245, 263-264, 272-273, 297-298

Narration 41

Nebenton 157, 233

Niederdeutsch 41-43, 45-57, 65-66, 69, 71-73, 76, 84-86, 89-94, 103, 166, 250-251, 277, 328-330

Nonstandardvarietät 312, 331

Ә Open Access. (C) 2018 publiziert von De Gruyter. (c) BY Dieses Werk ist lizenziert unter der Creative Commons Attribution 4.0 Lizenz. https://doi.org/10.1515/9783110538625-015 
Norddeutsch $51,66,71-72,87,174,223$, $310,313,323,325,329$

Norddeutschland 65-66, 69, 72, 84-90, 92, $94,105,111,163-164,166,174,317$, 323-324

Norm 127, 129, 133, 149, 152, 179, 186, 197209, 214-216, 231, 265, 277

Oberrheingebiet $5,25,37$

Papua-Neuguinea 305-306, 311

Positionierung 41, 45, 48, 56

Raum, öffentlicher 127-129, 131, 138-140, 288

Remotivierung, semantische 219, 221

Resegmentierung, formale 219, 221

Rheingrenze 5

Sächsisch 71-73, 76-77, 80-81, 84, 89, 103-104, 205

Salienz 22, 102-104, 108, 110, 230, 268, 330,332

Schule, bilinguale 197

semantisches Differenzial 79

Simplifizierungsprozesse 241-242

SOEP (Sozio-oekonomisches Panel) 66-67

Soziolinguistik $6,8,38,63,177,186,202$, 207-208, 230, 243, 246, 254, 259, 264-265, 268, 275, 279, 283, 292, 309

Sprachbewusstheit 99, 177, 185, 193, 215

Sprachbewusstsein 99, 177, 209, 213

Spracheinstellung 63-66, 68-69, 78-79, $89,94,99,127,136,247,253$

Sprachgeschichte 231, 298, 306, 309

Sprachgrenzen 5

Sprachinseln 241, 247, 251, 259, 263-264, 267, 272-273, 275-279

Sprachkontakt 241-242, 251, 256, 258-259, $263,273,279,283,306,315$

Sprachnorm 177, 197, 200, 202, 204, 252, 298

Sprachreflexion 177, 185

Sprachvariation 105, 178-179, 192, 263, 306 Sprachwahlmotive 127, 145, 149

Sprachwandel 65, 192, 202, 229, 263-264, 273, 275, 279, 297
Sprachwissen 99

Standardaussprache 157, 159

Standarddeutsch $8,177,181,183,188,192$, 214, 216, 248, 283, 290, 294-300, 310, 315-317, 319, 333

Standardisierung 263, 290-291, 293-294

Stereotypen 5, 7, 21-22, 24, 33, 36

Substrat 244, 307

Superstrat 305, 307-310, 312-314, 316-318, 320-321, 324, 329-332

Superstratsprache 305-307, 312, 314, 332

Superstrattransfer 305, 308-309, 316, 318322

Texasdeutsch 283, 286-287, 294

Tok Pisin 305, 308, 311-312, 316, 318, 320, 322, 333

Ungarn 197-199, 202-203, 205-208, 211, 214-215

Unserdeutsch 305-306, 308-314, 316-327, 329-333

Unterricht 182, 187, 191, 197-198, 201, 203204, 206, 209, 211-216, 252, 255

Variation 42, 72, 105, 157, 159, 161, 164, 166, 170-171, 173-175, 177-179, 183, 185, 197-199, 202-206, 209-215, 229, 231, 243, 256-259, 284-285, 292, 299

Varietät 8, 13, 37-38, 44-45, 63, 65, 67-69, 72, 79, 81, 83-84, 94, 101, 105-107, 110-111, 128, 131, 179-186, 188-192, 199-200, 202, 205, 211-212, 216, 219224, 226, 230, 233, 242, 244, 247, 252-254, 259, 263, 265, 273, 277-279, 284, 296, 306, 308, 312, 317-319, 322, 328-329

Verständigung $5,10,140,177,184,186-$ 189,192

Vokalismus 157

Wahrnehmungsdialektologie 99-107, 109112

Wortakzent 161, 163

Zwei-Kasus-System 259, 263 


\section{Autorinnen und Autoren}

Astrid Adler, Institut für Deutsche Sprache, Augustaanlage 32, D-68161 Mannheim, E-Mail: adler@ids-mannheim.de

Peter Auer, Albert-Ludwigs-Universität Freiburg, Abtl. für Germanistische Linguistik, D-79086 Freiburg, E-Mail: peter.auer@germanistik.uni-freiburg.de

Hans C. Boas, Department of Germanic Studies, 1 University Station, C3300, 2505 University Ave., The University of Texas at Austin, Austin, TX 78712, U.S.A., E-Mail: hcb@mail.utexas.edu

Winifred V. Davies, Aberystwyth University, Department of Modern Languages, Aberystwyth, SY23 3DY, Wales, UK, E-Mail: wid@aber.ac.uk

Katrin Fuchs, Department of Foreign Languages and Literatures, 1400 Chapel Drive, Valparaiso University, Valparaiso, IN 46383, U.S.A., E-Mail: katrin.fuchs@valpo.edu

Rüdiger Harnisch, Lehrstuhl für Deutsche Sprachwissenschaft, Universität Passau, D-94030 Passau, E-Mail: ruediger.harnisch@uni-passau.de

Markus Hundt, Christian-Albrechts-Universität zu Kiel, Germanistisches Seminar, Professur für Deutsche Sprachwissenschaft, Leibnizstraße 8, D-24188 Kiel, E-Mail: hundt@germsem.uni-kiel.de

Stefan Kleiner, Institut für Deutsche Sprache, R5 6-13, D-68161 Mannheim, E-Mail: kleiner@ids-mannheim.de

Elisabeth Knipf-Komlósi, Germanistisches Institut, Eotvos Lorand Universität, 1088 Budapest, Rakoczi ut 5, Ungarn, E-Mail: knipfe@freemail.hu

Ralf Knöbl, Institut für Deutsche Sprache, R5 6-13, D-68161 Mannheim, E-Mail: knoebl@ids-mannheim.de

Alexandra N. Lenz, Universität Wien, Institut für Germanistik, Universitätsring 1, A-1010 Wien, E-Mail: alexandra.lenz@univie.ac.at

Siegwalt Lindenfelser, Universität Augsburg, Lehrstuhl für Deutsche Sprachwissenschaft, Universitätsstraße 10, D-86159 Augsburg,

E-Mail: siegwalt.lindenfelser@philhist.uni-augsburg.de

Peter Maitz, Universität Augsburg, Lehrstuhl für Deutsche Sprachwissenschaft, Universitätsstraße 10, D-86159 Augsburg, E-Mail: peter.maitz@philhist.uni-augsburg.de

Lara Neumann, Universität Hamburg, Institut für Germanistik, Überseering 35, D-22297 Hamburg, E-Mail: lara.christine.neumann@uni-hamburg.de

Albrecht Plewnia, Institut für Deutsche Sprache, Augustaanlage 32, D-68161 Mannheim, E-Mail: plewnia@ids-mannheim.de

Claudia Maria Riehl, Ludwig-Maximilians-Universität München, Institut für Deutsch als Fremdsprache, Ludwigstr. 27, D-80539 München, E-Mail: riehl@daf.Imu.de

Ә Open Access. (C) 2018 publiziert von De Gruyter. (c) BY Dieses Werk ist lizenziert unter der Creative Commons Attribution 4.0 Lizenz. https://doi.org/10.1515/9783110538625-016 
Peter Rosenberg, Europa-Universität Viadrina Frankfurt (Oder), Fakultät für Kulturwissenschaften, Große Scharrnstraße 59, D-15230 Frankfurt (Oder), E-Mail: rosenberg@europa-uni.de

Ingrid Schröder, Universität Hamburg, Institut für Germanistik, Überseering 35, D-22297 Hamburg, E-Mail: ingrid.schroeder@uni-hamburg.de

Evelyn Ziegler, Universität Duisburg-Essen, Berliner Platz 6-8, D-45127 Essen, E-Mail: evelyn.ziegler@uni-due.de 